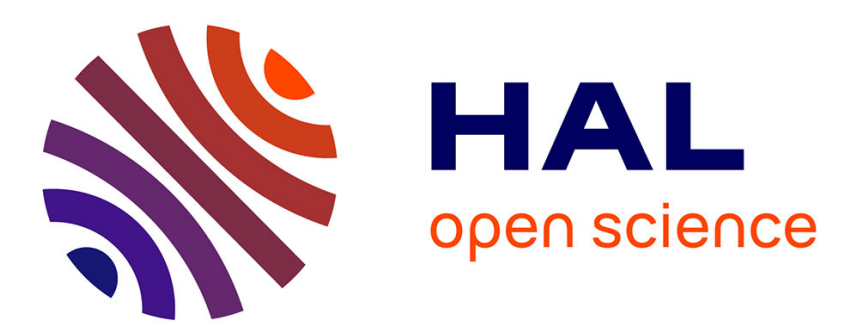

\title{
Creation and orientation of nano-crystals by femtosecond laser light for controlling optical non-linear response in silica-based glasses \\ Jing Cao
}

\section{- To cite this version: \\ Jing Cao. Creation and orientation of nano-crystals by femtosecond laser light for controlling optical non-linear response in silica-based glasses. Material chemistry. Université Paris Saclay (COmUE), 2017. English. NNT : 2017SACLS055 . tel-01988887}

\section{HAL Id: tel-01988887 \\ https://theses.hal.science/tel-01988887}

Submitted on 22 Jan 2019

HAL is a multi-disciplinary open access archive for the deposit and dissemination of scientific research documents, whether they are published or not. The documents may come from teaching and research institutions in France or abroad, or from public or private research centers.
L'archive ouverte pluridisciplinaire HAL, est destinée au dépôt et à la diffusion de documents scientifiques de niveau recherche, publiés ou non, émanant des établissements d'enseignement et de recherche français ou étrangers, des laboratoires publics ou privés. 
universite்

NNT : 2017SACLS055

\title{
THESE DE DOCTORAT
}

\author{
DE \\ L'UNIVERSITE PARIS-SACLAY
}

PREPAREE A

L'UNIVERSITE PARIS-SUD

ECOLE DOCTORALE N 571 : 2MIB

Ecole Doctorale Sciences Chimiques : Molecules, Materiaux, Instrumentation et

BIOSYSTEMES

Chimie

Par

\section{Mme CAO Jing}

Creation and orientation of nano-crystals by femtosecond laser light for controlling optical non-linear response in silica-based glasses

Thèse présentée et soutenue à Orsay, le 3 mars 2017 :

Composition du Jury :

M. POUMELLEC Bertrand Mme FERRARIS Monica

M. SOLIS Javier

M. ALLIX Mathieu

M. MAZEROLLES Léo

M. VAN DER BEEK Kees

M. LANCRY Matthieu
Directeur de Recherche CNRS (ICMMO/UPSud, Orsay)

Professore Ordinario (Politecnico di Torino, Turin)

Research Professor (Instituto de Óptica-CSIC, Madrid)

CNRS Researcher (CEMHTI laboratory, Orléans)

Directeur de recherche (ICMPE, Thiais)

Directeur LSI (Ecole Polytechnique, Palaiseau)

Assistant Professor (ICMMO/UPSud, Orsay)
Directeur de thèse

Présidente du jury

Rapporteur

Rapporteur

Examinateur

Examinateur

Examinateur 



\section{Contents}

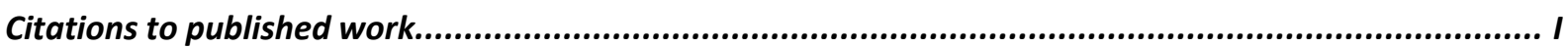

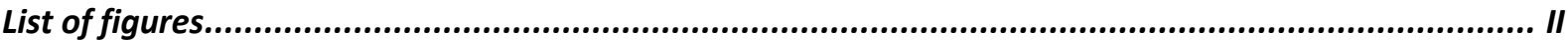

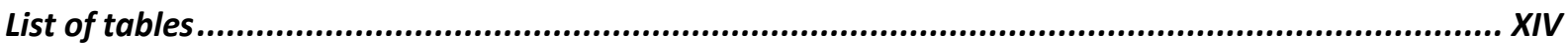

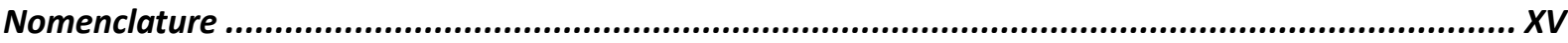

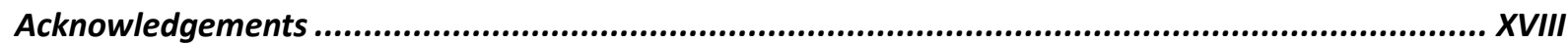

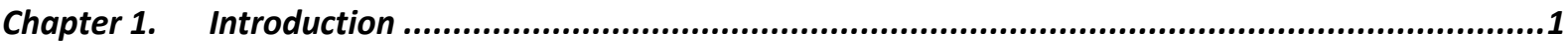

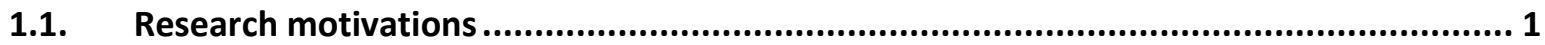

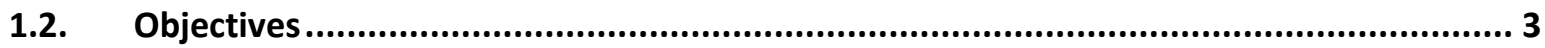

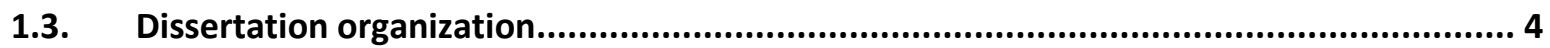

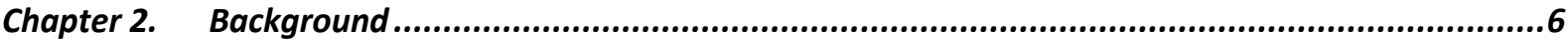

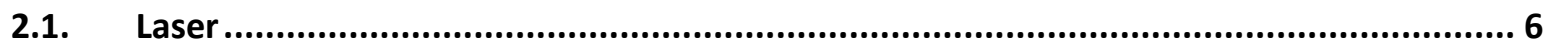

2.1.1. Basic knowledge on laser ................................................................................................. 6

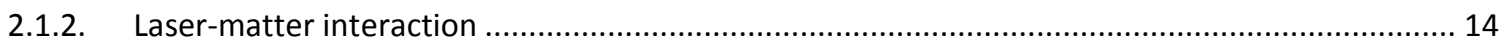

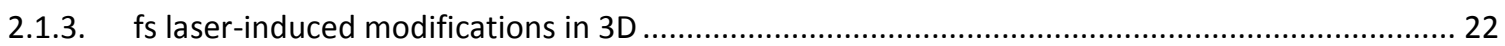

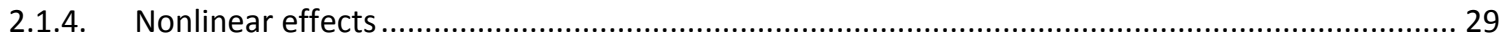

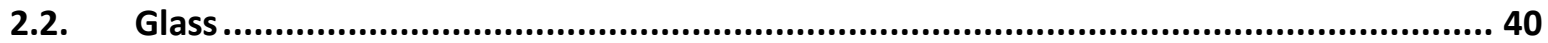

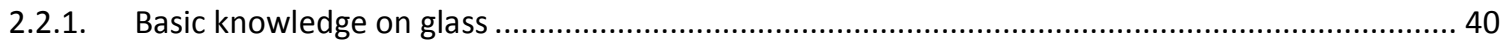

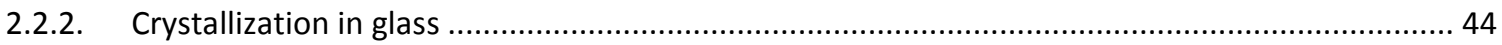

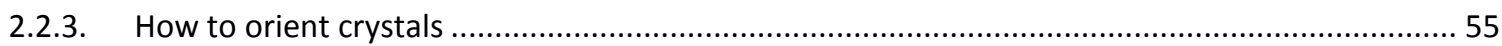

2.2.4. Privious work on fs laser-induced chemical process in glasses ................................................ 56

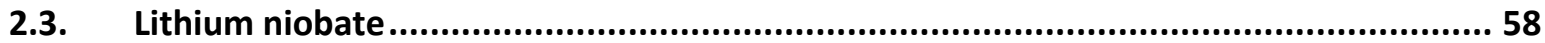

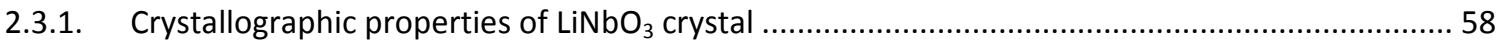

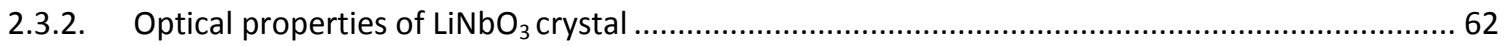

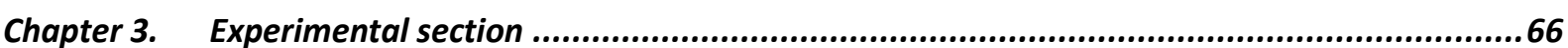

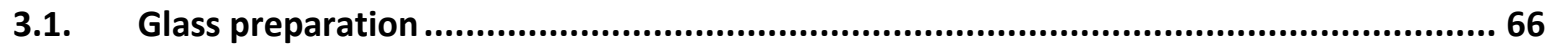

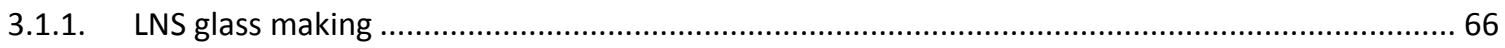

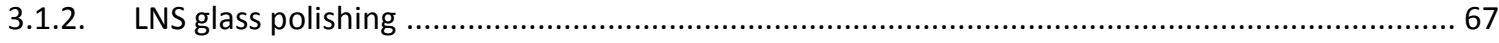

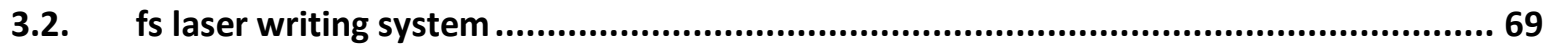

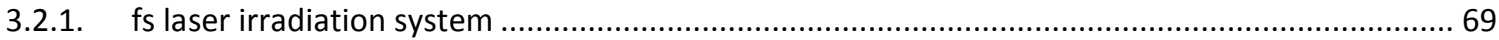




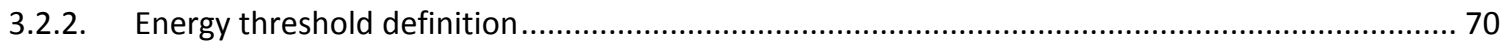

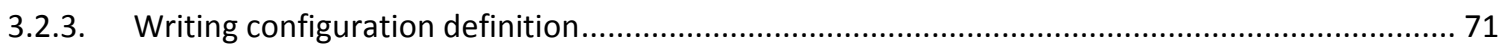

3.3. Thermal modelling in the case of fs laser irradiation................................................... 71

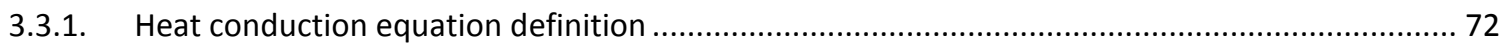

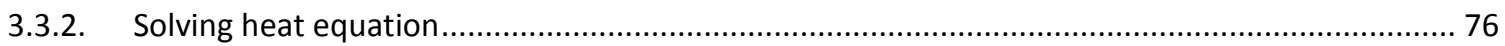

3.4. Observation of fs laser-induced modifications........................................................... 83

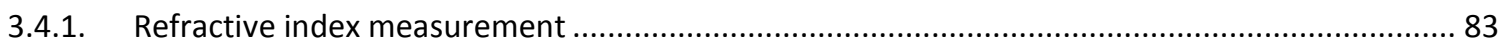

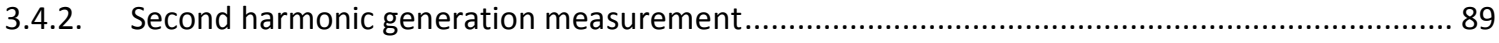

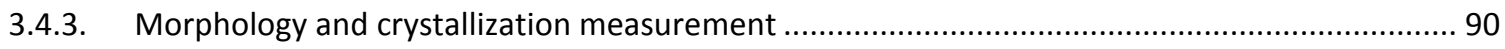

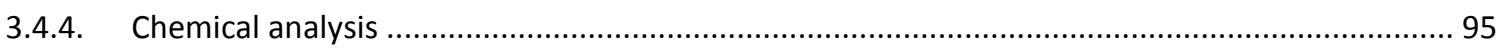

Chapter 4. fs laser-induced modifications in LNS glasses ............................................... 101

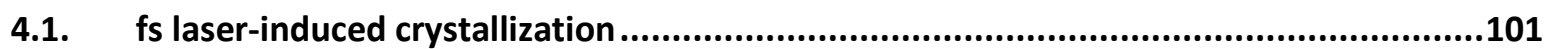

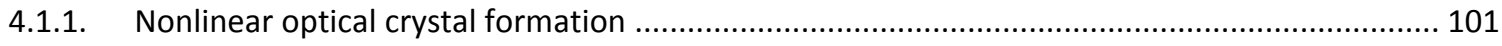

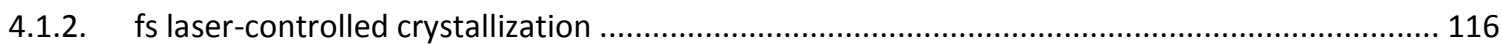

4.1.3. Interpretation of $\mathrm{fs}$ laser polarization controlled nano-crystal orientation ............................. 124

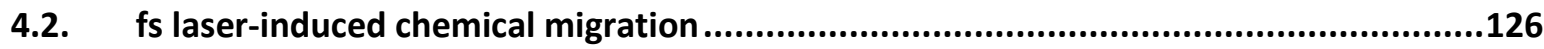

4.2.1. Nanoscale chemical migration observation ............................................................... 126

4.2.2. Interpretation of nanoscale chemical migration ........................................................ 133

4.3. fs laser-induced nanostructures .............................................................135

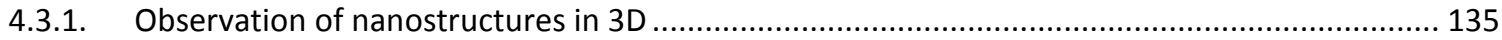

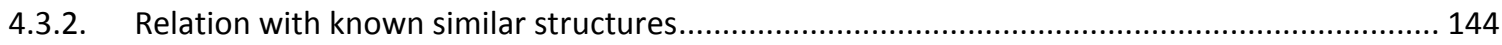

4.3.3. Formation of oriented nanostructure interpretation ....................................................... 145

Chapter 5. fs laser-induced properties in LNS glasses .................................................151

5.1. Second harmonic generation .....................................................................151

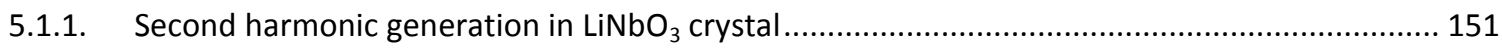

5.1.2. Second harmonic generation observation ........................................................................ 153

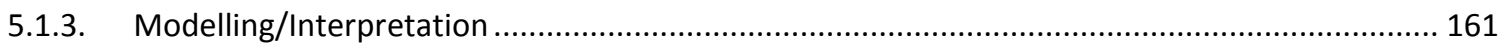

5.2. Refractive index change....................................................................166

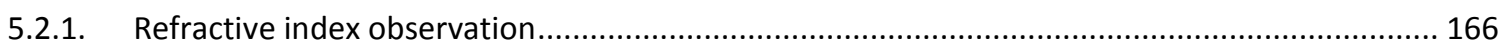

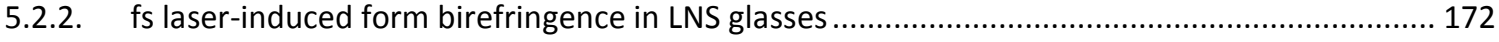

Chapter 6. Conclusions and future works................................................................ 178 


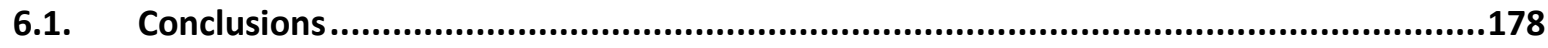

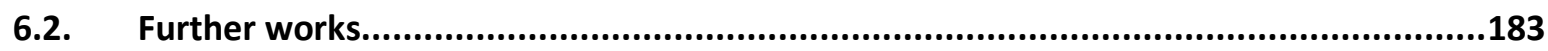

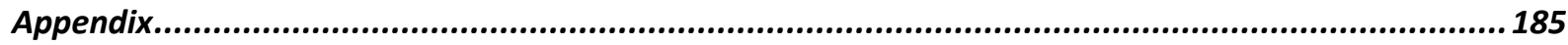

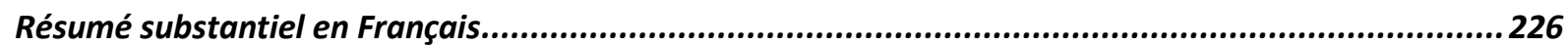

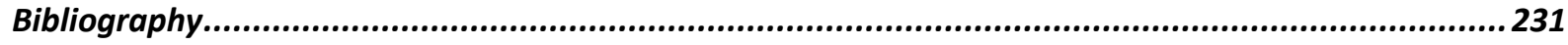

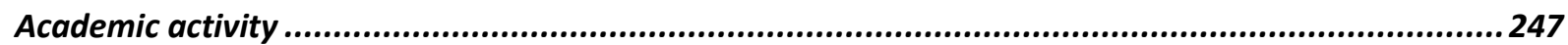

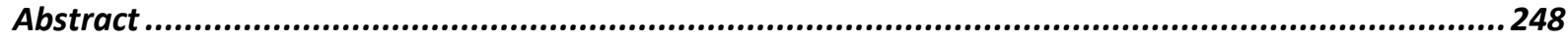




\section{Citations to published work}

Parts of this dissertation are based on the following publications:

1) J. Cao, L. Mazerolles, M. Lancry, F. Brisset, and B. Poumellec, "Modifications in lithium niobium silicate glass by femtosecond laser direct writing: morphology, crystallization, and nanostructure," Journal of the Optical Society of America B, 2017, 34 (1) 160

2) J. Cao, B. Poumellec, L. Mazerolles, F. Brisset, A. L. Helbert, S. Surble, X. He, and M. Lancry, "Nanoscale phase separation in lithium niobium silicate glass after femtosecond laser radiation," Journal of the American Ceramic Society, 2017, 100 (1) 115

3) J. Cao, L. Mazerolles, M. Lancry, D. Solas, F. Brisset, and B. Poumellec, "Form birefringence induced in multicomponent glass by femtosecond laser direct writing," Optics Letters, 2016, 41 (12) 2739.

4) J. Cao, B. Poumellec, F. Brisset, A. L. Helbert, and M. Lancry, "Tunable angular dependent second harmonic generation in glass by controlling femtosecond laser polarization," Journal of the Optical Society of America B, 2016, 33 (4) 741

5) J. Cao, B. Poumellec, F. Brisset, A. L. Helbert, and M. Lancry, "Angular dependence of the second harmonic generation induced by femtosecond laser radiation in silica-based glasses: variation with writing speed and pulse energy," World Journal of Nano Science and Engineering, 2015, 5 (03) 96 (Special Issue on Silica Materials) 


\section{List of figures}

Figure 1 Analysis procedure of the investigation of fs laser-induced modifications in LNS glasses ......

Figure 2 Principle of laser: a) stimulated emission and b) schematic of typical laser set-up

Figure 3 Progress in a) ultra-short pulse generation and b) laser intensity since the first demonstration of laser in 1960, CPA: chirped pulse amplification, ELI: extreme light infrastructure (cited from [42, 45]) .......8

Figure 4 Gaussian beam: a) changes in wave front radius with propagation distance, b) the definition of numerical aperture (after [53]), and c) the embedded Gaussian (cited from [54]).....

Figure $\mathbf{5}$ Schematic of the interface spherical aberration due to the refractive index mismatch along laser propagation direction (after [57]).

Figure 6 Contours of the turbidity (with unit of $\mathrm{m}^{-1}$ ) as a function of the crystal radius and crystal volume fraction (with $\Delta \mathrm{n}=0.1, \overline{\mathrm{n}}=1.7$ and $\lambda_{0}=647 \mathrm{~nm}$ ) (cited from [60]).

Figure 7 Schematic diagram of the photoionization of an electron in an atomic potential for different values of the Keldysh parameter $(\gamma)$. In a solid, the electron is promoted from the valence band to the conduction band, rather than ionized: a) multi-photon ionisation, b) tunnelling ionisation, c) the above processes combines, and d) avalanche ionisation: free carrier absorption followed by impact ionisation (cited from [63])

Figure 8 Different photo-ionisation regimes varying the photon energy and laser intensity. The dashed gray line shows case of $\gamma=1$ for $C_{60}$ ionisation which separates the multi-photon regime $(\gamma>1)$ from the tunnelling regime $(\gamma<<1)$ (cited from [62]) .

Figure 9 a) Calculated evolution of free electron density for a $100 \mathrm{fs}, 1053 \mathrm{~nm}$ pulse (dashed curve) of peak intensity $11.7 \mathrm{TW} / \mathrm{cm}^{2}$ in fused silica. Multi-photon ionization (dotted curve) starts the avalanche; solid curve is total electron density including impact ionization (cited from [65]). b) Absorptivity as function of pulse energy, repetition rate, and pulse duration. Neglecting the laser beam scattering and the reflection at the focus, absorptivity is defined as $1-W_{t} /\left[W_{0}(1-R)^{2}\right]$, where $W_{0}$ is the incident laser power to the work piece, $\mathrm{W}_{\mathrm{t}}$ is the transmitted laser power, and $\mathrm{R}$ is the reflectivity of the beam at the glass surface (after [66])

Figure 10 a) Schematic representation of the dynamics of fs laser interactions with dielectrics. The numbers from 1 to 5 correspond to the process as described within the text (after S. Guizard, LSI, CEA, Saclay France and [6]), and b) timescale of the physical phenomena associated with the interaction of a fs laser pulse with transparent materials (cited from [1])

Figure 11 Observed values of damage threshold at $1053 \mathrm{~nm}$ for fused silica and $\mathrm{CaF}_{2}$. Solid lines are $\tau^{0.5}$ fits to long pulse results. Estimated uncertainty in the absolute fluence is $\pm 15 \%$ (cited from [65]) .....

Figure 12 Symmetry of the excited states of SiODC(II): ${ }^{1} A_{1}$ is the ground state, ${ }^{1} B_{1}$ is the first excited singlet, and ${ }^{1} B_{2}$ is the second excited singlet. $S_{1}$ (corresponding to the excitation band at $5.0 \mathrm{eV}$ ) is related to transition from ${ }^{1} \mathrm{~A}_{1}$ to ${ }^{1} \mathrm{~B}_{1}$ which is related to an electron transiting from an orbital with symmetry a1 to an orbital with symmetry b1, built mainly on the "non-bonding" Si $i_{3 p x}$ atomic orbital (x being perpendicular to the molecular plane). The $S_{0}-S_{1}$ transition is polarized along $x$ direction. $S_{2}$ (corresponding to the excitation band at $6.8 \mathrm{eV}$ ) is related to transition from ${ }^{1} \mathrm{~A}_{1}$ to ${ }^{1} \mathrm{~B}_{2}$ which is related to an electron transiting 
from a a1 orbital to a b2 orbital, built mainly on the $\mathrm{Si}_{3 p}-\mathrm{O}_{2 p}$ anti-bonding molecular orbital in the molecular plan. The transition between $\mathrm{S}_{0}$ and $\mathrm{S}_{2}$ is polarized in the O-Si-O plane (cited from [76]) .........22

Figure $13 \mathrm{fs}$ laser fabricating in 3D: a) a laser damage line, b) the cross section of a line written by moving the sample parallel to the incident laser beam, and c) the cross section of a line written by moving the sample perpendicular to the incident laser beam (after [51])

Figure 14 A schematic showing focused fs laser pulses in bulk fused silica: a) fs laser is focused below the sample surface resulting in a high intensity in the focal volume, b) the energy is nonlinearly absorbed and a free electron plasma is created by multi-photon/tunneling and avalanche photoionization, c) the plasma transfers its energy to the lattice on a $\sim 10 \mathrm{ps}$ time scale resulting in one of three types of permanent modification, and d) fs laser-induced various modifications in glass as a function of pulse energy (after [81])

Figure 15 SEM images of nanostructure for parallel scanning (top) and perpendicular scanning (bottom) after polishing and etching (fused silica). The speed of the sample movement was 6.97, 2.98, 1.25, 0.6, $0.125,0.06,0.023$, and $0.006 \mathrm{~mm} / \mathrm{s}$ from left to right, the right two images are the zoom of SEM images (cited from [92])

Figure 16 Pulse energy versus numerical aperture (NA) and pulse duration: synthetic diagram for a) small pulse duration, b) $160 \mathrm{fs}, \mathrm{c}) 200 \mathrm{fs}$, and d) $250 \mathrm{fs}$ (region II disappears) with silica (cited from [82])

Figure 17 a) Effect of increasing the pulse energy at $30 \mathrm{~mm} / \mathrm{s}$ in fused silica. The patterns were written approximately $200 \mu \mathrm{m}$ below the surface, b) evidences of "frozen-in" bubble coalescence events, and c) qualitative fluid analogy of the laser energy accumulation-discharge process (cited from [97]).

Figure 18 Energy level diagrams of a) second harmonic generation, b) two-photon excited fluorescence, and c) coherent anti-Stokes Raman scattering (after [104]).

Figure 19 Interrelationships of non centro-symmetric crystal classes in both Hermann-Mauguin and Schoenflies symbols (after [109]) 35

Figure 20 Two-dimensional schematic of a) an ordered $\mathrm{SiO}_{2}$ (the forth bonds of the Si project upward or downward from the plane of the illustration) and b) random $\mathrm{SiO}_{2}$ network, the actual relative surface area requirement of the Oxygens is indicated by dashed lines (cited from [116])

Figure 21 a) Phase diagram of silica and b-e) dependence of silica structure on temperature (cited from [117])

Figure 22 Various routes to the glassy state, roughly indicating the energies of the initial states relative to the final glassy state. The route of crystal compression below $T_{g}$ may yield glasses that are thermodynamically distinct from those obtained by the other routes but which may transform to them by way of non-equilibrium first-order transitions (after [118]) .....

Figure $\mathbf{2 3}$ Comparison of the viscosity of several kinds of glasses versus temperature, in silicate systems viscosity attains the largest values for vitreous silica (cited from [119])

Figure 24 ps laser-induced thermo-mechanical processes: a) the formation of the plastic layer due to the elastoplastic wave, b) softening of the plastic region upon heating, c) stress release and density redistribution, and d) the potential effect of a subsequent pulse arriving on $\sim 10 \mu \mathrm{s}$ is represented (after [123]) 
Figure 25 a) The free energy change associated with homogeneous nucleation of a sphere of radius $r(T<$ $T_{m}$ ), and $b$ ) change of $r^{*}$ with the under-cooling of the melt, the asterisk defines critical values (after [124])

Figure 26 a) Sketch of free enthalpy according to configurational parameter and b) a superimposition of the nucleation and growth rates (after [124])

Figure 27 Time temperature transformation curve and continuously cooling transformation curves compared to cooling curves with different cooling rates (cited from [124, 129])....

Figure 28 Phase-equilibrium diagram of $\mathrm{Li}_{2} \mathrm{O}-\mathrm{SiO}_{2}$ (after [130]). Tridymite and cristobalite are high temperature poly morphs of silica (see details in Appendix VIII)

Figure 29 Phase-equilibrium diagram of $\mathrm{Nb}_{2} \mathrm{O}_{5}-\mathrm{SiO}_{2}$ (cited from [135]).

Figure 30 a) Phase-equilibrium diagram for $\mathrm{LiNbO}_{3}$ (cited from $[169,170]$ ) and b) schematic of the furnace for Czochralski growth at $\mathrm{LiNbO}_{3}$ (cited from [171])

Figure 31 Space group and structure in $\mathrm{LiNb}_{3} \mathrm{O}_{8}, \mathrm{LiNbO}_{3}$, and $\mathrm{Li}_{3} \mathrm{NbO}_{4}$ (cited from [173]).

Figure 32 Positions of the lithium atoms (double cross-hatched circles) and the niobium atoms (single cross-hatched circles) with respect to the oxygen octahedra in the a) paraelectric phase and b) ferroelectric phase of lithium niobate, the horizontal lines on the right side stands for the oxygen layers (after [38]); c) hexagonal unit cell of $\mathrm{LiNbO}_{3}$, the Cartesian $\mathrm{x}, \mathrm{y}, \mathrm{z}$ system is used to describe the physical tensor properties of lithium niobate (after [124])

Figure 33 Refractive indices of congruently grown $\mathrm{LiNbO}_{3}$ versus wavelength. The solid curves represent the three-oscillator Sellmeier fitting the data, the measured temperature was $21^{\circ} \mathrm{C}$ (after [183]).....

Figure 34 Pseudo-ternary phase diagram of $\mathrm{Li}_{2} \mathrm{O}-\mathrm{Nb}_{2} \mathrm{O}_{5}-\mathrm{SiO}_{2}$. Black and blue dots are glass, while red and green dots represent crystallization (green and blue dots data are redrawn from [140], black and red dots are cited from [188]) 66

Figure 35 Schematic of interferometer (cited from [191]). 68

Figure 36 Schematic diagram of the fs laser fabrication system (the reference of writing configuration is based on the beam specificities at the origin shown in black Cartesian coordinate system, and changed to green Cartesian coordinate system when arrived in the sample). N.B.: because the number of mirrors on the optical table is odd, the coordinate system changed from right-handed to left-handed. . .70

Figure 37 The comparison of the glass relaxation time and the cooling profile after single pulse fs laser irradiation for pure and doped silica. The cooling profiles with varying incident pulse energy are for pure silica only; $T_{c}$ is the new fictive temperature (after [201]).

Figure 38 Calculated temperature in LNS glass for multi-pulse case in static mode: varying time and repetition rate at $\mathrm{r}=0$. Other parameters: $33 \mathrm{Li}_{2} \mathrm{O}-33 \mathrm{Nb}_{2} \mathrm{O}_{5}-34 \mathrm{SiO}_{2}(\mathrm{~mol} \%), \mathrm{z}=0,0.5 \mu \mathrm{J} /$ pulse 79

Figure 39 Spatial temperature distribution and TTT curve in static mode (after Poumellec et al. ICMMO, France)...

Figure 40 Cooling curves are compared to the CCT domain in scanning mode (after Poumellec et al. ICMMO, France). 
Figure 41 Shape of the crystallized volume according to the pulse energy amplitude in scanning mode, cooling curves are compared to the CCT domain (after Poumellec et al. ICMMO, France)

Figure 42 Schematic diagram of a) Brewster's angle, b) reflection coefficients ( $r$ ) and reflectance (R) as a function of incidence light, the light polarized in the plane of incidence is marked as $p$ wave, the one polarized perpendicular to the plane is indicated as $s$ wave, $n_{1}=1, n_{2}=1.8$, and $R=r^{2}$, and $c$ ) light path of LNS glass refractive index measurement set-up (M: mirror).

Figure 43 a) Polarized light microscopy configuration, red rectangles mark the polarizer and analyzer along light path (cited from [204]), b) birefringent crystal between crossed polarizers, c) wave front propagation in anisotropic crystal, and d) anisotropic crystals interact with polarized light in an optical microscope (cited from [205])

Figure $\mathbf{4 4}$ Working mechanism of first order retardation plate measuring slow axis of sample (cited from [206])

Figure 45 a) Quantitative birefringence microscopy set-up and b) the corresponding schematic diagram (cited from Abrio Product Bulletin, CRI, Inc)....

Figure 46 Second harmonic generation measurement set-up.

Figure 47 a) Schematic representations of scanning electron microscopy. FA: final aperture; SD: solid state backscattered electron detector; EDS: energy-dispersive X-ray spectrometer; WDS: wavelength-dispersive X-ray spectrometer; CRT: cathode ray tube; E-T: Everhart-Thornley secondary/backscattered electron detector, consisting of F: Faraday cage; S: scintillator; LG: light guide; and PM: photomultiplier (cited from [209]), b) processes resulting from electron bombardment (cited from [208]) ..... 91

Figure 48 How electron backscatter diffraction measurements work: a) schematic diagram of the EBSD system, b) the beam-specimen interaction, and c) formation of a backscattered Kikuchi band (cited from [210])

Figure 49 a) Working principle of TEM (cited from [216]), b) bright field, and c) off-axis dark field imaging of TEM (cited from [217])

Figure 50 Illustration of sample dissecting process by FIB: a) the sample was cut along the plane perpendicular to the scanning direction (i.e. $X$ direction) and polished, b) a volume of irradiated part was picked up from the irradiated line, $c$ ) the "picked-up" part was dissected along laser propagation direction (i.e. Z direction) by FIB, and d) image of the irradiated line after FIB treatment.

Figure $\mathbf{5 1}$ Schematic of Ion beam analyses: proton-induced X-ray emission (PIXE), Rutherford backscattering spectrometry (RBS), and proton-induced Gamma-ray emission (PIGE)....

Figure 52 Schematic of different domains of the electron diffusion angle after passing the sample (after [221])

Figure 53 Schematic illustration of the comparison of excited sample volumes from a) electrons in a SEM, b) electrons in a STEM, and c) from proton beams (after [223, 224]) .....

Figure 54 Schematic of a)-d) laser-induced breakdown spectroscopy (LIBS) (cited from [226]), and e) a "traditional" LIBS set-up (cited from [227]).

Figure 55 SEM pictures according to the pulse energy defining three types of material modifications obtained in the plane perpendicular to scanning direction (after HF treatment): a) regime $1, b)$ regime 2 , 
and $\mathrm{c}-\mathrm{d}$ ) regime 3. The color in IPFs (b, c, and d right) is based on $\mathrm{R} 3 \mathrm{c}$ space group, $\mathrm{LiNbO}_{3}$, coding the crystal axis along laser polarization direction. Other parameters: $33 \mathrm{Li}_{2} \mathrm{O}-33 \mathrm{Nb}_{2} \mathrm{O}_{5}-34 \mathrm{SiO}_{2}(\mathrm{~mol} \%), 1030 \mathrm{~nm}$, $300 \mathrm{fs}, 300 \mathrm{kHz}, \mathrm{NA}=0.6$, focus depth $350 \mu \mathrm{m}$ in glass, $5 \mu \mathrm{m} / \mathrm{s}$, pulse energy of a) $0.4, b) 0.7, \mathrm{c}) 1.3$, and d) $1.8 \mu \mathrm{J} /$ pulse, laser polarization (illustrated by $\mathrm{E}$ ) is parallel to scanning direction (i.e. $\mathrm{X}$ direction).

Figure 56 0001-Polar Figure. The color scale refers to multiple of the pole density for random distribution (quoted 1 and green). Other parameters: $33 \mathrm{Li}_{2} \mathrm{O}-33 \mathrm{Nb}_{2} \mathrm{O}_{5}-34 \mathrm{SiO}_{2}$ (mol\%), $1030 \mathrm{~nm}, 300 \mathrm{fs}, 300 \mathrm{kHz}, 0.7$ $\mu \mathrm{J} /$ pulse, NA $=0.6$, focus depth $350 \mu \mathrm{m}$ in glass, $5 \mu \mathrm{m} / \mathrm{s}$, writing laser polarization direction is parallel to $X$ direction and laser propagation direction is along $Z$ direction. 103

Figure 57 a) SEM image in regime 3 and the corresponding and b) IPF of laser track (1.8 $\mu \mathrm{J} /$ pulse). The color in IPF is based on $\mathrm{R3C}$ space group, $\mathrm{LiNbO}_{3}$. Laser polarization is perpendicular to scanning direction. IPF is coding along scanning direction. $\mathrm{E}$ indicates the laser polarization direction. c) SHG microscopy images of irradiated lines at different pulse energies. Other parameters: $33 \mathrm{Li}_{2} \mathrm{O}-33 \mathrm{Nb}_{2} \mathrm{O}_{5}-34 \mathrm{SiO}_{2}(\mathrm{~mol} \%)$, $1030 \mathrm{~nm}, 300 \mathrm{fs}, 300 \mathrm{kHz}, \mathrm{NA}=0.6$, focus depth $350 \mu \mathrm{m}$ in glass, $5 \mu \mathrm{m} / \mathrm{s}$, wring direction is along $X$ direction 104

Figure 58 EBSD maps of cross section of laser tracks for parallel writing configuration, coding the crystal orientation along the directions parallel to $X$ (left), $Y$ (middle), and $Z$ direction (right), respectively (see details in Figure 36). The color in EBSD map is based on $\mathrm{R3C}$ space group, $\mathrm{LiNbO}_{3}$ (inset at the right bottom). Writing configuration considering laser scanning direction and incident laser polarization angle is defined as $(\theta, \phi)$, displayed for each picture. Other parameters: $33 \mathrm{Li}_{2} \mathrm{O}-33 \mathrm{Nb}_{2} \mathrm{O}_{5}-34 \mathrm{SiO}_{2}(\mathrm{~mol} \%), 1030 \mathrm{~nm}$, $300 \mathrm{fs}, 500 \mathrm{kHz}, 5 \mu \mathrm{m} / \mathrm{s}, \mathrm{NA}=0.6$, focal depth $610 \mu \mathrm{m}$ in glass, $0.8 \mu \mathrm{J} /$ pulse. The laser came from the top.

Figure 59 Polar Figures of cross section of laser tracks for parallel writing configuration, coding the crystal orientation along the directions parallel to $X$ (left), $Y$ (middle), and $Z$ direction (right), respectively (see details in Figure 36). Writing configuration considering laser scanning direction and incident laser polarization angle is defined as $(\theta, \phi)$, displayed for each picture. Other parameters: $33 \mathrm{Li}_{2} \mathrm{O}-33 \mathrm{Nb}_{2} \mathrm{O}_{5}-$ $34 \mathrm{SiO}_{2}$ (mol\%), $1030 \mathrm{~nm}, 300 \mathrm{fs}, 500 \mathrm{kHz}, 5 \mu \mathrm{m} / \mathrm{s}, \mathrm{NA}=0.6$, focal depth $610 \mu \mathrm{m}$ in glass, $0.8 \mu \mathrm{J} /$ pulse $\ldots 107$

Figure 60 EBSD maps of cross section of laser tracks for perpendicular writing configuration, coding the crystal orientation along the directions parallel to $X$ (left), $Y$ (middle), and $Z$ direction (right), respectively (see details in Figure 36). The color in EBSD map is based on $\mathrm{R3}$ c space group, $\mathrm{LiNbO}_{3}$ (inset the right top). Writing configuration considering laser scanning direction and incident laser polarization angle is defined as $(\theta, \phi)$, displayed for each picture. Other parameters: $33 \mathrm{Li}_{2} \mathrm{O}-33 \mathrm{Nb}_{2} \mathrm{O}_{5}-34 \mathrm{SiO}_{2}(\mathrm{~mol} \%), 1030 \mathrm{~nm}$, $300 \mathrm{fs}, 500 \mathrm{kHz}, 5 \mu \mathrm{m} / \mathrm{s}, \mathrm{NA}=0.6$, focal depth $610 \mu \mathrm{m}$ in glass, $0.8 \mu \mathrm{J} /$ pulse. The laser came from the top. 108

Figure 61 Polar Figures of cross section of laser tracks for perpendicular writing configuration, coding the crystal orientation along the directions parallel to $X$ (left), $Y$ (middle), and $Z$ direction (right), respectively (see details in Figure 36). Writing configuration considering laser scanning direction and incident laser polarization angle is defined as $(\theta, \phi)$, displayed for each picture. Other parameters: $33 \mathrm{Li}_{2} \mathrm{O}-33 \mathrm{Nb}_{2} \mathrm{O}_{5}-$ $34 \mathrm{SiO}_{2}$ (mol\%), $1030 \mathrm{~nm}, 300 \mathrm{fs}, 500 \mathrm{kHz}, 5 \mu \mathrm{m} / \mathrm{s}, \mathrm{NA}=0.6$, focal depth $610 \mu \mathrm{m}$ in glass, $0.8 \mu \mathrm{J} /$ pulse $\ldots 110$

Figure 62 EBSD maps of cross section of laser tracks for parallel writing configuration, coding the crystal orientation along the directions parallel to $X$ (left), $Y$ (middle), and $Z$ direction (right), respectively (see details in Figure 36). The color in EBSD map is based on $\mathrm{R3c}$ space group, $\mathrm{LiNbO}_{3}$ (inset at the right bottom). Writing configuration considering laser scanning direction and incident laser polarization angle is defined as $(\theta, \phi)$, displayed for each picture. Other parameters: $33 \mathrm{Li}_{2} \mathrm{O}-33 \mathrm{Nb}_{2} \mathrm{O}_{5}-34 \mathrm{SiO}_{2}(\mathrm{~mol} \%), 1030 \mathrm{~nm}$, 
Figure 63 EBSD maps of cross section of laser tracks for perpendicular writing configuration, coding the crystal orientation along the directions parallel to $X$ (left), $Y$ (middle), and $Z$ direction (right), respectively (see details in Figure 36). The color in EBSD map is based on $\mathrm{R}^{3}$ space group, $\mathrm{LiNbO}_{3}$ (inset at the right top). Writing configuration considering laser scanning direction and incident laser polarization angle is defined as $(\theta, \phi)$, displayed for each picture. Other parameters: $33 \mathrm{Li}_{2} \mathrm{O}-33 \mathrm{Nb}_{2} \mathrm{O}_{5}-34 \mathrm{SiO}_{2}(\mathrm{~mol} \%), 1030 \mathrm{~nm}$, $300 \mathrm{fs}, 500 \mathrm{kHz}, 5 \mu \mathrm{m} / \mathrm{s}, \mathrm{NA}=0.6$, focal depth $610 \mu \mathrm{m}, 1.7 \mu \mathrm{J} / \mathrm{pulse}$. The laser came from the top

Figure 64 Polar Figures of cross section of laser tracks for parallel writing configuration, coding the crystal orientation along the directions parallel to $X$ (left), $Y$ (middle), and $Z$ direction (right), respectively (see details in Figure 36). Writing configuration considering laser scanning direction and incident laser polarization angle is defined as $(\theta, \phi)$, displayed for each picture. Other parameters: $33 \mathrm{Li}_{2} \mathrm{O}-33 \mathrm{Nb}_{2} \mathrm{O}_{5}{ }^{-}$ $34 \mathrm{SiO}_{2}$ (mol\%), $1030 \mathrm{~nm}, 300 \mathrm{fs}, 500 \mathrm{kHz}, 5 \mu \mathrm{m} / \mathrm{s}, \mathrm{NA}=0.6$, focal depth $610 \mu \mathrm{m}$ in glass, $1.7 \mu \mathrm{J} /$ pulse...114

Figure 65 Polar Figures of cross section of laser tracks for perpendicular writing configuration, coding the crystal orientation along the directions parallel to $X$ (left), $Y$ (middle), and $Z$ direction (right), respectively (see details in Figure 36). Writing configuration considering laser scanning direction and incident laser polarization angle is defined as $(\theta, \phi)$, displayed for each picture. Other parameters: $33 \mathrm{Li}_{2} \mathrm{O}-33 \mathrm{Nb}_{2} \mathrm{O}_{5}-$ $34 \mathrm{SiO}_{2}$ (mol\%), $1030 \mathrm{~nm}, 300 \mathrm{fs}, 500 \mathrm{kHz}, 5 \mu \mathrm{m} / \mathrm{s}, \mathrm{NA}=0.6$, focal depth $610 \mu \mathrm{m}$ in glass, $1.7 \mu \mathrm{J} /$ pulse. ...115

Figure 66 Structure of the laser trace by scanning electron microscopy: a) SEM image for $0.8 \mu \mathrm{J} / \mathrm{pulse}, \mathrm{b}$ ) the magnification of the part framed in red in a). c) A similar part of the laser track but for $1.4 \mu \mathrm{J} /$ pulse; d) the corresponding IPFs coding crystal direction along the writing laser polarization direction for 0.8 $\mu \mathrm{J} /$ pulse; e) similar information for $1.4 \mu \mathrm{J} /$ pulse. The color in IPF maps (inset at the right bottom) is based on R3c space group, $\mathrm{LiNbO}_{3}$. All scale bars are $1 \mu \mathrm{m}$. Other parameters: $32.5 \mathrm{Li}_{2} \mathrm{O}-27.5 \mathrm{Nb}_{2} \mathrm{O}_{5}-40 \mathrm{SiO}_{2}(\mathrm{~mol} \%)$, $1030 \mathrm{~nm}, 300 \mathrm{fs}, 250 \mathrm{kHz}, 5 \mu \mathrm{m} / \mathrm{s}$, focus depth $300 \mu \mathrm{m}$ in air, writing direction along $45^{\circ}$ and laser polarization direction parallel to $90^{\circ}$

Figure 67 Apparent grain size (diameter) distribution of laser tracks varying pulse energy. Other parameters: $32.5 \mathrm{Li}_{2} \mathrm{O}-27.5 \mathrm{Nb}_{2} \mathrm{O}_{5}-40 \mathrm{SiO}_{2}$ (mol\%), $1030 \mathrm{~nm}, 300 \mathrm{fs}, 250 \mathrm{kHz}, 5 \mu \mathrm{m} / \mathrm{s}$, writing direction along $45^{\circ}$ and laser polarization direction parallel to $90^{\circ}$.

Figure 68 0001-Pole Figure of texture calculated from EBSD-scans i.e. the polar axis from EBSD scans: at pulse energy of a) 0.8, b) 1.4 , and c) $1.8 \mu \mathrm{J} /$ pulse. The milky disks at the centre of the pole figures indicate the part of the distribution that should not be taken into account for SHG interpretation with probe polarization in $\mathrm{XY}$ plane (will be used in Section 5.1.3.1). Green dots illustrate the writing laser polarization and red dots illustrate the writing direction. 118

Figure 69 Structure of the laser trace investigated by SEM. The section was achieved perpendicularly to the scanning direction: a-b) SEM images of glass after irradiation; Inverse pole figure (IPF), coding the crystal orientation along c) writing laser polarization direction, d) scanning direction, and e) along the laser propagation direction; f) apparent grain size (diameter) distribution. The colors in the IPF map are based on $\mathrm{R} 3 \mathrm{C}$ space group, $\mathrm{LiNbO}_{3}$ (inset at the right bottom). Scanning direction is along $45^{\circ}$ and incident laser polarization angle is $135^{\circ}$. All scale bars are $1 \mu \mathrm{m}$.

Figure $70 \mathrm{IPFs}$ of texture calculated from EBSD-scans for configuration $\left(45^{\circ}, 135^{\circ}\right)$ coding the crystal orientation along a) incident laser polarization direction, b) scanning direction, and c) laser propagation direction. The red arrow indicates the absence of crystal with polar axis parallel to incident laser 
polarization direction. The white arrow indicates the crystals with an accumulation of axes close to

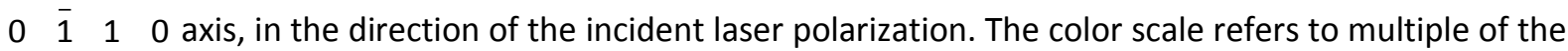
pole density in the case of random distribution (quoted 1 and green). Laser irradiation-configuration considering scanning direction and incident laser polarization angle is defined as $(\theta, \phi)$. 120

Figure 71 0001-Pole Figure of texture calculated from electron backscatter diffraction (EBSD)-scans i.e. the polar axis from EBSD scans for laser radiation-configurations $\left(45^{\circ}, 135^{\circ}\right)$ for a), $\left(45^{\circ}, 45^{\circ}\right)$ for b), $\left(45^{\circ}\right.$, $\left.0^{\circ}\right)$ for $\left.\mathrm{c}\right)$, and $\left(225^{\circ}, 0^{\circ}\right)$ for d). The milky disks at the centre of Pole Figures indicate the part of the distribution that has not to be taken into account for interpretation of SHG angular dependence with polarization in XY plane (will be discussed in Section 5.1.3.1). The color scale refers to multiple of the pole density for random distribution (quoted 1 and green). Laser radiation-configuration considering scanning direction and incident laser polarization angle are defined as $(\theta, \phi)$. Green dots illustrate the writing laser polarization and red dots illustrate the writing direction.

Figure 72 Domain of fs laser-induced crystallization in LNS glass varying pulse energy and repetition rate. Other parameters: $32.5 \mathrm{Li}_{2} \mathrm{O}-27.5 \mathrm{Nb}_{2} \mathrm{O}_{5}-40 \mathrm{SiO}_{2}$ (mol\%), $1030 \mathrm{~nm}, 300 \mathrm{fs}, \mathrm{NA}=0.6$, focus depth $300 \mu \mathrm{m}$ in air, $5 \mu \mathrm{m} / \mathrm{s}$ (the dots are the measured data)

Figure 73 SEM images of laser tracks: a) $500 \mathrm{kHz}, 1.4 \mu \mathrm{J} /$ pulse and b) $100 \mathrm{kHz}, 2.8 \mu \mathrm{J} /$ pulse (after HF treatment). Other parameters: $32.5 \mathrm{Li}_{2} \mathrm{O}-27.5 \mathrm{Nb}_{2} \mathrm{O}_{5}-40 \mathrm{SiO}_{2}$ (mol\%), $1030 \mathrm{~nm}, 300 \mathrm{fs}, \mathrm{NA}=0.6$, focus depth $300 \mu \mathrm{m}$ in air, $5 \mu \mathrm{m} / \mathrm{s}$

Figure 74 Schematic of non-oscillating torque acting on crystal orientation 124

Figure 75 Chemical analysis of irradiated glass by SEM/EDS in bulk sample: a) the SEM image and b) the corresponding chemical profiles across the head of the laser track of Si (blue) and $\mathrm{Nb}$ (red). Other parameters: $33 \mathrm{Li}_{2} \mathrm{O}-33 \mathrm{Nb}_{2} \mathrm{O}_{5}-34 \mathrm{SiO}_{2}$ (mol\%), $1030 \mathrm{~nm}, 300 \mathrm{fs}, 300 \mathrm{kHz}, 0.9 \mu \mathrm{J} /$ pulse, $5 \mu \mathrm{m} / \mathrm{s}$, NA = 0.6, focal depth $350 \mu \mathrm{m}$ in glass 126

Figure 76 Chemical analysis of irradiated glass by WDS microprobe in bulk sample: a) the secondary electron image of the cross section of laser tracks varying the pulse energy (marked with red arrows), the red lines illustrate the elemental analysis line profiles, starting from the red points and b) the corresponding chemical profiles across the laser track of $\mathrm{O}$ (yellow), $\mathrm{Si}$ (blue), and $\mathrm{Nb}$ (red). Other parameters: $33 \mathrm{Li}_{2} \mathrm{O}-33 \mathrm{Nb}_{2} \mathrm{O}_{5}-34 \mathrm{SiO}_{2}$ (mol\%), $1030 \mathrm{~nm}, 300 \mathrm{fs}, 300 \mathrm{kHz}, 0.6 \mu \mathrm{J} /$ pulse, $5 \mu \mathrm{m} / \mathrm{s}$, NA = 0.6, focal depth $350 \mu \mathrm{m}$ in glass

Figure 77 Chemical analysis of irradiated glass by ions beam analysis in bulk sample (beam size $2 \times 2 \mu \mathrm{m}^{2}$ ): a) a camera photo of the cross section of two laser tracks (marked with red arrows, with distance of 30 $\mu \mathrm{m}$, map size $70 \times 34 \mu \mathrm{m}^{2}$ ) and the corresponding elemental distribution mappings of b) Li, c) $\mathrm{Si}$, and d) $\mathrm{Nb}$ of the irradiated glass. Color bar: proportional of the number of counts on $\mathrm{Li}, \mathrm{Si}$ or $\mathrm{Nb}$ atoms. Other parameters: $33 \mathrm{Li}_{2} \mathrm{O}-33 \mathrm{Nb}_{2} \mathrm{O}_{5}-34 \mathrm{SiO}_{2}$ (mol\%), $1030 \mathrm{~nm}, 300 \mathrm{fs}, 300 \mathrm{kHz}, 2.2 \mu \mathrm{J} /$ pulse, $5 \mu \mathrm{m} / \mathrm{s}, \mathrm{NA}=0.6$, focal depth $350 \mu \mathrm{m}$ in glass

Figure 78 a) X-ray absorption spectra (XAS) and b) X-ray absorption near edge structure (XANES) of Nb $\mathrm{L}_{\text {III }}$ edge for glass matrix (black curve), for irradiated area (red curve) and $\mathrm{LiNbO}_{3}$ (green curve). For glass matrix and irradiated area, XANES is recorded from normalized total electron yield (TEY) mode (TEY/I $)$ with InSb (111) monochromator. For crystal $\mathrm{LiNbO}_{3}$, XANES is from X-ray absorption spectrum. TEY/I for irradiated area has been recorded in the cross section of the laser track, perpendicular to scanning direction; other parameters: $33 \mathrm{Li}_{2} \mathrm{O}-33 \mathrm{Nb}_{2} \mathrm{O}_{5}-34 \mathrm{SiO}_{2}$ (mol\%), $1030 \mathrm{~nm}, 300 \mathrm{fs}, 300 \mathrm{kHz}, 1.8 \mu \mathrm{J} /$ pulse, $5 \mu \mathrm{m} / \mathrm{s}$, laser polarization is parallel to scanning direction. The insert is the magnification of irradiated zone (red 
curve) and glass matrix (black curve). The green dashed line is redrawn from previous work [231], left shift $1.4 \mathrm{eV}$.

Figure 79 Chemical analysis of irradiated glass at the nanoscale. a) An overview of the laser-modified; b) the magnification image (green rectangle marks the area for $\mathrm{Nb}$ and $\mathrm{Si}$ mappings). STEM/EDS characterization of irradiated lithium niobium silicate glass in the mapping mode for c) $\mathrm{Nb}$ and d) Si; and e) the line profile crossing alternated bright and dark regions illustrated by dashed lines in the mapping images (from top to bottom). Pulse energy $1.3 \mu \mathrm{J} /$ pulse. Other parameters: $33 \mathrm{Li}_{2} \mathrm{O}-33 \mathrm{Nb}_{2} \mathrm{O}_{5}-34 \mathrm{SiO}_{2}$ (mol\%), $1030 \mathrm{~nm}, 300 \mathrm{fs}, 300 \mathrm{kHz}, 1.3 \mu \mathrm{J} /$ pulse, $5 \mu \mathrm{m} / \mathrm{s}, \mathrm{NA}=0.6$, focal depth $350 \mu \mathrm{m}$ in glass, and laser polarization is parallel to scanning direction (i.e. $\mathrm{X}$ direction). 131

Figure 80 Nanostructure properties of irradiated glass investigated by TEM (bright field mode): a) TEM image of the laser-modified area and b) high resolution TEM showing the diffracting properties of the two zones (the zone in the red rectangle is the one with high contrast to show the crystal lattice) for 1.3, c) 1.8 and d) $0.7 \mu \mathrm{J} /$ pulse. Other parameters: $33 \mathrm{Li}_{2} \mathrm{O}-33 \mathrm{Nb}_{2} \mathrm{O}_{5}-34 \mathrm{SiO}_{2}$ (mol\%), $1030 \mathrm{~nm}, 300 \mathrm{fs}, 300 \mathrm{kHz}, 5 \mu \mathrm{m} / \mathrm{s}, \mathrm{NA}$ $=0.6$, focal depth $350 \mu \mathrm{m}$ in glass, and laser polarization is parallel to scanning direction (i.e. $\mathrm{X}$ direction).

Figure 81 Phase shift interferometry (PSI) images of the polished glass a) before and b) after HF treatment (the right bottom part is a broken edge, used for marking, illustrated by green dashed arrow; the red arrows illustrate the scanning direction (S); $\mathrm{Z}$ is the laser propagation direction; the distance between each line is $50 \mu \mathrm{m}$. Other parameters: $32.5 \mathrm{Li}_{2} \mathrm{O}-27.5 \mathrm{Nb}_{2} \mathrm{O}_{5}-40 \mathrm{SiO}_{2}$ (mol\%), $1030 \mathrm{~nm}, 300 \mathrm{fs}, 250 \mathrm{kHz}, 0.8$ $\mu \mathrm{J} /$ pulse, and $5 \mu \mathrm{m} / \mathrm{s}, \mathrm{NA}=0.6$, focal depth $300 \mu \mathrm{m}$ in air (regime 2). 133

Figure 82 a) fs laser-induced four regions (quoted with A-D) of regime 3 with three clear boundaries (left) and the corresponding IPF showing crystallization and crystal orientation, coding along laser polarization direction (right), b) the magnification of Figure 82a marked with red rectangle, and c) schematic of the laser-induced modulated structures along the propagation direction (the magnified SEM showing the smooth structure line). Other parameters: $32.5 \mathrm{Li}_{2} \mathrm{O}-27.5 \mathrm{Nb}_{2} \mathrm{O}_{5}-40 \mathrm{SiO}_{2}$ (mol\%), $1030 \mathrm{~nm}, 500 \mathrm{kHz}, 300 \mathrm{fs}$, $1.0 \mu \mathrm{J} /$ pulse, $5 \mu \mathrm{m} / \mathrm{s}, \mathrm{NA}=0.6$, focal depth $300 \mu \mathrm{m}$ in air, and laser polarization is parallel to scanning direction (i.e. $\mathrm{X}$ direction). 136

Figure 83 Morphology of the fs laser track, when laser polarization (i.e. $Y$ direction) is perpendicular to scanning direction (i.e. $X$ direction): a) SEM image (left) and the corresponding IPF coding along laser polarization direction (right) for $1.0 \mu \mathrm{J} /$ pulse, b) the magnification of Figure 83a marked with red rectangle, and c) the schematic of the laser-induced structures. Morphology of the laser track varying the pulse energy: d) 0.8 and e) $1.4 \mu \mathrm{J} /$ pulse. Other parameters: $32.5 \mathrm{Li}_{2} \mathrm{O}-27.5 \mathrm{Nb}_{2} \mathrm{O}_{5}-40 \mathrm{SiO}_{2}$ (mol\%), $1030 \mathrm{~nm}$, $500 \mathrm{kHz}, 300 \mathrm{fs}, 5 \mu \mathrm{m} / \mathrm{s}, \mathrm{NA}=0.6$, focal depth $300 \mu \mathrm{m}$ in air

Figure 84 Comparisons of SEM images of irradiated LNS glass a) before and b) after HF treatment. It was observed in the plane perpendicular to scanning direction, E indicates the laser polarization direction, and laser polarization is parallel to scanning direction, laser propagation is along $\mathrm{Z}$ direction. Other parameters: $33 \mathrm{Li}_{2} \mathrm{O}-33 \mathrm{Nb}_{2} \mathrm{O}_{5}-34 \mathrm{SiO}_{2}$ (mol\%), $1030 \mathrm{~nm}, 300 \mathrm{fs}, 300 \mathrm{kHz}, 1.3 \mu \mathrm{J} /$ pulse, $5 \mu \mathrm{m} / \mathrm{s}$, and NA = 0.6, focal depth $350 \mu \mathrm{m}$ in glass, laser polarization direction is parallel to writing direction. 138

Figure 85 Nanostructure formation in LNS glass in the plane perpendicular to laser propagation direction: TEM images of the laser modified area (bright field). a-b) 0.7 , c-d) $1.8 \mu \mathrm{J} /$ pulse. a-c) laser polarization is parallel to scanning direction and d) laser polarization is perpendicular to scanning direction. The insets of a, c-d) are the corresponding SAED patterns. In the c) case for which the texture is well defined, we can see that the crystalline phase produced is $\mathrm{LiNbO}_{3}$. $\mathrm{E}$ indicates the laser polarization direction. Other 
parameters: $33 \mathrm{Li}_{2} \mathrm{O}-33 \mathrm{Nb}_{2} \mathrm{O}_{5}-34 \mathrm{SiO}_{2}$ (mol\%), $1030 \mathrm{~nm}, 300 \mathrm{fs}, 300 \mathrm{kHz}, 5 \mu \mathrm{m} / \mathrm{s}, \mathrm{NA}=0.6$, focal depth $350 \mu \mathrm{m}$ in glass.

Figure 86 fs laser-induced nanostructures investigated in XZ-plane: a) TEM images of the laser trace (the inset is the SAED) for laser polarization direction is parallel to writing direction. b) The similar information for the one when laser polarization is perpendicular to writing direction. In the a) case for which the texture is well defined, we can see that the crystalline phase produced is $\mathrm{LiNbO}_{3}$. Other parameters: $33 \mathrm{Li}_{2} \mathrm{O}-33 \mathrm{Nb}_{2} \mathrm{O}_{5}-34 \mathrm{SiO}_{2}$ (mol\%), $1030 \mathrm{~nm}, 300 \mathrm{fs}, 300 \mathrm{kHz}, 1.8 \mu \mathrm{J} /$ pulse, $5 \mu \mathrm{m} / \mathrm{s}, \mathrm{NA}=0.6$, focal depth 350 $\mu \mathrm{m}$ in glass. Schemes of fs laser-induced nanostructure in 3D for laser polarization is c) parallel and d) perpendicular to writing direction. 141

Figure $\mathbf{8 7}$ fs laser-induced nanoscale chemical migration in LNS glass: a) STEM-HAADF image, elemental mappings of b) $\mathrm{Nb}, \mathrm{c}$ ) $\mathrm{Si}$, and line profile of $\mathrm{Nb}$ (red line) and $\mathrm{Si}$ (green line); $\mathrm{E}$ indicates the laser polarization direction. Other parameters: $33 \mathrm{Li}_{2} \mathrm{O}-33 \mathrm{Nb}_{2} \mathrm{O}_{5}-34 \mathrm{SiO}_{2}(\mathrm{~mol} \%), 1030 \mathrm{~nm}, 300 \mathrm{fs}, 300 \mathrm{kHz}, 1.8$ $\mu \mathrm{J} /$ pulse, $5 \mu \mathrm{m} / \mathrm{s}, \mathrm{NA}=0.6$, and focal depth $350 \mu \mathrm{m}$ in glass. 143

Figure 88 fs laser-induced nanoscale phase separation in LNS glass: a) quality image (IQ), b) IPF (colors are based on $\mathrm{R3}$ c group, $\mathrm{LiNbO}_{3}$, coding along laser propagation direction), and c) composite image: IQ+IPF. Other parameters: $33 \mathrm{Li}_{2} \mathrm{O}-33 \mathrm{Nb}_{2} \mathrm{O}_{5}-34 \mathrm{SiO}_{2}$ (mol\%), $1030 \mathrm{~nm}, 300 \mathrm{fs}, 300 \mathrm{kHz}, 1.3 \mu \mathrm{J} / \mathrm{pulse}, 5 \mu \mathrm{m} / \mathrm{s}, \mathrm{NA}=$ 0.6 , and focal depth $350 \mu \mathrm{m}$ in glass.

Figure $89 \mathrm{fs}$ laser-induced nanogratings in borosilicate glass: the SEM images indicate laser written traces ( $x$-y plane) after polishing and etching, a) 300 P, 1000 P respectively, and b) 2000 P, $130 \mathrm{~nJ}, \mathrm{P}$ denotes the incident pulse number (cited from [90]). 146

Figure 90 Nonlinear optical crystal precipitation by optical floating-zone method: a) diagram of the optical floating zone method set-up (glasses are marked in yellow, the infrared radiation sources in red, and the ellipsoidal mirrors in green), b) a sample photo, c) XRD pattern, and d) SHG image of irradiated glass. .. 147

Figure 91 Microstructure in irradiated LNS glass observed by SEM 148

Figure 92 Spatial crystals distribution of irradiated glass investigated by EBSD: a) Image quality map and b) the corresponding IPF of irradiated LNS glass

Figure 93 Chemical analysis of irradiated glass: a) SEM/EDS taken across the melted (left) and un-melted zone (right), superimposing the SEM image for O (green), Si (red), and Nb (orange) chemical profiles ( 82 points with step $10 \mu \mathrm{m}$ ), b) a photograph illustrating the region for laser-induced breakdown spectroscopy measurement (marked with red rectangle, the scale bar is $2 \mathrm{~mm}$ ) and c) the element distribution from $A$ (un-melted zone) to B (melted zone). 150

Figure 94 The relationship between the probing laser polarization angle and $\mathrm{LiNbO}_{3}$ cell: the probing laser angle $(\alpha)$ varying in xz plane, the probing laser angle $(\beta)$ varying in xy plane, and the probing laser angle $(\gamma)$ varying in yz plane. 151

Figure 95 Normalized SHG intensity (normalized at the largest value) as the function of the probing laser polarization angle $(\alpha)$ varying in xz plane, the probing laser angle $(\beta)$ varying in xy plane, and the probing laser angle $(\gamma)$ varying in yz plane. 152

Figure 96 SHG of irradiated lines varying with pulse energy: a-b) 0.8 , c-d) 1.4 , and e-f) $2.2 \mu \mathrm{J} /$ pulse. The double arrows mark the probing laser polarization. Other parameters: $33 \mathrm{Li}_{2} \mathrm{O}-33 \mathrm{Nb}_{2} \mathrm{O}_{5}-34 \mathrm{SiO}_{2}(\mathrm{~mol} \%)$, $1030 \mathrm{~nm}, 300 \mathrm{fs}, 500 \mathrm{kHz}, \mathrm{NA}=0.6$, focus depth $650 \mu \mathrm{m}$ in glass, $5 \mu \mathrm{m} / \mathrm{s}$, laser polarization direction is 
parallel to writing direction (i.e. $X$ direction). Two lines in each energy group, the distance between each line is $50 \mu \mathrm{m}$.

Figure 97 Normalized SHG intensity of irradiated lines as function of probing laser polarization angle, measured in the $\mathrm{XY}$ plane: the variable parameter is the pulse energy. Other parameters: $32.5 \mathrm{Li}_{2} \mathrm{O}-$ $27.5 \mathrm{Nb}_{2} \mathrm{O}_{5}-40 \mathrm{SiO}_{2}$ (mol\%), $1030 \mathrm{~nm}, 300 \mathrm{fs}, 250 \mathrm{kHz}, \mathrm{NA}=0.6$, focus depth $300 \mu \mathrm{m}$ in air, writing direction is along $45^{\circ}$, and laser polarization is parallel to $90^{\circ}$. 155

Figure 98 Normalized SHG intensity of irradiated lines as function of probing laser polarization angle, measured in the $\mathrm{XY}$ plane: the variable parameter is the writing speed at pulse energy of a) 0.8 and b) 1.4 $\mu \mathrm{J} /$ pulse. Other parameters: $32.5 \mathrm{Li}_{2} \mathrm{O}-27.5 \mathrm{Nb}_{2} \mathrm{O}_{5}-40 \mathrm{SiO}_{2}(\mathrm{~mol} \%), 1030 \mathrm{~nm}, 300 \mathrm{fs}, 250 \mathrm{kHz}, \mathrm{NA}=0.6$, focus depth $300 \mu \mathrm{m}$ in air, writing direction is along $45^{\circ}$, and laser polarization is parallel to $90^{\circ}$. 156

Figure 99 Normalized SHG intensity of irradiated lines as function of probing laser polarization angle, measured in the $\mathrm{XY}$ plane: the writing orientation is the variable parameter for writing speeds at a) 1 and b) $25 \mu \mathrm{m} / \mathrm{s}$. Other parameters: $32.5 \mathrm{Li}_{2} \mathrm{O}-27.5 \mathrm{Nb}_{2} \mathrm{O}_{5}-40 \mathrm{SiO}_{2}$ (mol\%), $1030 \mathrm{~nm}, 300 \mathrm{fs}, 250 \mathrm{kHz}, \mathrm{NA}=0.6$, focus depth $300 \mu \mathrm{m}$ in air, $1.4 \mu \mathrm{J} /$ pulse, and laser polarization is parallel to $90^{\circ}$. 157

Figure 100 Anisotropy magnitude of irradiated lines. Anisotropy magnitude is defined by the following ratio: $\left(S H G_{\max }-S_{H} G_{\min }\right) /\left(\mathrm{SHG}_{\max }+\mathrm{SHG}_{\min }\right)$. The lines are guides for the eyes. Writing direction is along $45^{\circ}$. Other parameters: $32.5 \mathrm{Li}_{2} \mathrm{O}-27.5 \mathrm{Nb}_{2} \mathrm{O}_{5}-40 \mathrm{SiO}_{2}$ (mol\%), $1030 \mathrm{~nm}, 300 \mathrm{fs}, 250 \mathrm{kHz}$, NA $=0.6$, focus depth $300 \mu \mathrm{m}$ in air, writing direction is along $45^{\circ}$, and laser polarization is parallel to $90^{\circ}$. 158

Figure 101 Normalized polarization dependent SHG of irradiated lines: a) normalized SHG intensity as function of probing laser polarization angle $\gamma$, the variable parameter is writing laser polarization angle $\phi$. b) The probing laser polarization angle at the minimum SHG intensity is quoted $\gamma_{\min }$ and maximum SHG intensity is quoted $\gamma_{\max }$ according to $\phi$. Other parameters $32.5 \mathrm{Li}_{2} \mathrm{O}-27.5 \mathrm{Nb}_{2} \mathrm{O}_{5}-40 \mathrm{SiO}_{2}(\mathrm{~mol} \%), 1030 \mathrm{~nm}$, $300 \mathrm{fs}, 250 \mathrm{kHz}, 0.8 \mu \mathrm{J} /$ pulse, $5 \mu \mathrm{m} / \mathrm{s}, \mathrm{NA}=0.6$, focus depth $300 \mu \mathrm{m}$ in air, writing direction is along $45^{\circ}$.

Figure 102 SHG property of irradiated lines varying writing direction $(\theta)$ and writing laser polarization angle $(\phi)$ : a) $\gamma_{\max }$ and b) $\gamma_{\max }-\left(\phi+90^{\circ}\right)$, according to $\theta$ and $\phi$. The probing laser polarization angle at the maximum SHG intensity is quoted $\gamma_{\max }$. Other parameters $32.5 \mathrm{Li}_{2} \mathrm{O}-27.5 \mathrm{Nb}_{2} \mathrm{O}_{5}-40 \mathrm{SiO}_{2}(\mathrm{~mol} \%), 1030 \mathrm{~nm}$, $300 \mathrm{fs}, 250 \mathrm{kHz}, 0.8 \mu \mathrm{J} /$ pulse, $5 \mu \mathrm{m} / \mathrm{s}$, NA = 0.6, focus depth $300 \mu \mathrm{m}$ in air...... 160

Figure $103 \gamma_{\max }-\left(\phi+90^{\circ}\right)$ as a function of the writing laser polarization $\phi$ for two particular writing orientation (The lines are guides for the eyes, where the red line illustrated a weak, while the black one represented a strong departure amplitude of $\gamma_{\max }$ from $\phi+90^{\circ}$, varying with $\phi$ ). Other parameters $32.5 \mathrm{Li}_{2} \mathrm{O}-27.5 \mathrm{Nb}_{2} \mathrm{O}_{5}-40 \mathrm{SiO}_{2}$ (mol\%), $1030 \mathrm{~nm}, 300 \mathrm{fs}, 250 \mathrm{kHz}, 0.8 \mu \mathrm{J} /$ pulse, NA = 0.6, focus depth $300 \mu \mathrm{m}$ in air, $5 \mu \mathrm{m} / \mathrm{s}$...... 161

Figure 104 Experimental data and computered data of a) $0.8 \mu \mathrm{J} /$ pulse and b) $1.8 \mu \mathrm{J} /$ pulse shown in Figure 97. 162

Figure 105 Irradiated lines taken by natural light microscopy (transmission mode) measured in XY plane. Other parameters: $33 \mathrm{Li}_{2} \mathrm{O}-33 \mathrm{Nb}_{2} \mathrm{O}_{5}-34 \mathrm{SiO}_{2}$ (mol\%), $1030 \mathrm{~nm}, 300 \mathrm{fs}, 500 \mathrm{kHz}, \mathrm{NA}=0.6$, focus depth 650 $\mu \mathrm{m}$ in glass, $5 \mu \mathrm{m} / \mathrm{s}$, pulse energy: a) 0.4, b) 0.6, c) 0.8 , d) 1.0 , e) 1.4 , and f) $2.0 \mu \mathrm{J} /$ pulse (two lines in each energy group, the blue arrows indicate the writing orientations), laser polarization direction is parallel to scanning direction. The distance between each line is $50 \mu \mathrm{m}$. 166 
Figure 106 Images of irradiated lines taken between crossed polarizers measured in $\mathrm{XY}$ plane: a) parallel or perpendicular and b) diagonal position with respect to the polarizers. Other parameters: $33 \mathrm{Li}_{2} \mathrm{O}-33 \mathrm{Nb}_{2} \mathrm{O}_{5}-$ $34 \mathrm{SiO}_{2}$ (mol\%), $1030 \mathrm{~nm}, 300 \mathrm{fs}, 500 \mathrm{kHz}, \mathrm{NA}=0.6$, focus depth $650 \mu \mathrm{m}$ in glass, $5 \mu \mathrm{m} / \mathrm{s}$, pulse energy: 0.4 to $2.2 \mu \mathrm{J} /$ pulse with a step of $0.2 \mu \mathrm{J} /$ pulse (from left to right, two lines in each energy group, the blue arrows indicate the writing orientations), writing laser polarization direction is illustrated by $\mathrm{E}$. The distance between each line is $50 \mu \mathrm{m}$. 167

Figure 107 Images taken between crossed polarizers (denoted as A and P, respectively) and full wave retardation plate $\left(\lambda\right.$, double-headed arrow indicates slow optical axis of the retardation plate, $45^{\circ}$ of the polarizer axis) with the irradiated lines oriented at a) $-45^{\circ}$ and b) $45^{\circ}$. Other parameters: $33 \mathrm{Li}_{2} \mathrm{O}-33 \mathrm{Nb}_{2} \mathrm{O}_{5^{-}}$ $34 \mathrm{SiO}_{2}$ (mol\%), $1030 \mathrm{~nm}, 300 \mathrm{fs}, 500 \mathrm{kHz}, \mathrm{NA}=0.6$, focus depth $650 \mu \mathrm{m}$ in glass, $5 \mu \mathrm{m} / \mathrm{s}$, laser polarization direction is illustrated by $\mathrm{E}$. Two lines in each energy group, the distance between each line is $50 \mu \mathrm{m} . . .168$

Figure 108 Digital holographic microscopy images of irradiated lines at varying pulse energy. Other parameters: $33 \mathrm{Li}_{2} \mathrm{O}-33 \mathrm{Nb}_{2} \mathrm{O}_{5}-34 \mathrm{SiO}_{2}$ (mol\%), $1030 \mathrm{~nm}, 300 \mathrm{fs}, 500 \mathrm{kHz}, \mathrm{NA}=0.6$, focus depth $650 \mu \mathrm{m}$ in glass, $5 \mu \mathrm{m} / \mathrm{s}$, laser polarization direction is parallel to writing direction. Two lines in each energy group, the distance between each line is $50 \mu \mathrm{m}$. Writing configuration considering laser scanning direction and incident laser polarization angle is defined as $(\theta, \phi)$, displayed for each picture. 169

Figure $109 \mathrm{fs}$ laser-induced birefringence of irradiated lines in LNS glass varying pulse energy (0.4-2.2 $\mu \mathrm{J} /$ pulse with a step of $0.2 \mu \mathrm{J} /$ pulse, two lines with different scanning orientations for one pulse energy and the scanning directions are illustrated in yellow arrows): a) retardance amplitude image, b) quantitative birefringence microscopy image, c) profiles of the retardance, and d) the slow axis orientation, respectively. The color in QBM image shows the slow axis direction of the irradiated line and the color of the circular legend displays slow axis direction. Other parameters: $33 \mathrm{Li}_{2} \mathrm{O}-33 \mathrm{Nb}_{2} \mathrm{O}_{5}-34 \mathrm{SiO}_{2}$ (mol\%), $1030 \mathrm{~nm}, 300 \mathrm{fs}, 500 \mathrm{kHz}, \mathrm{NA}=0.6$, focus depth $650 \mu \mathrm{m}$ in glass, $5 \mu \mathrm{m} / \mathrm{s}$, laser polarization direction is parallel to writing direction (i.e. $\mathrm{X}$ direction). Two lines in each energy group, the distance between each line is $50 \mu \mathrm{m}$. 170

Figure 110 AOW effect of fs laser-induced birefringence of irradiated lines in LNS glass: a) retardance amplitude image and b) quantitative birefringence microscopy image. The color in QBM image shows the slow axis of the irradiated line and the color of the circular legend displays slow axis direction. The arrows indicate the writing direction. Writing configuration considering laser scanning direction and incident laser polarization angle is defined as $(\theta, \phi)$, displayed for each picture.Other parameters: $33 \mathrm{Li}_{2} \mathrm{O}-33 \mathrm{Nb}_{2} \mathrm{O}_{5}-$ $34 \mathrm{SiO}_{2}$ (mol\%), $1030 \mathrm{~nm}, 300 \mathrm{fs}, 500 \mathrm{kHz}, \mathrm{NA}=0.6$, focus depth $650 \mu \mathrm{m}$ in glass, $5 \mu \mathrm{m} / \mathrm{s}, 0.8 \mu \mathrm{J} / \mathrm{pulse}$, laser polarization direction is parallel to writing direction. Two lines in each energy group, the distance between each line is $50 \mu \mathrm{m}$, red arrows indicate the writing orientation. 171

Figure 111 fs laser-induced birefringence of irradiated line: a) retardance image, b) retardance profile of the irradiated line, and c) QBM image. The color in QBM images displays the slow axis of the birefringence zone. The color of the circular legend presents the direction of the slow axis (right top). Other parameters: $33 \mathrm{Li}_{2} \mathrm{O}-33 \mathrm{Nb}_{2} \mathrm{O}_{5}-34 \mathrm{SiO}_{2}$ (mol\%) , $1030 \mathrm{~nm}, 300 \mathrm{fs}, 300 \mathrm{kHz}, 0.7 \mu \mathrm{J} /$ pulse, $\mathrm{NA}=0.6$, focus depth $350 \mu \mathrm{m}$ in glass, and $5 \mu \mathrm{m} / \mathrm{s}$. 174

Figure 112 Schematic representation of the nanostructure involved in form birefringence with array of parallel plates

Figure 113 fs laser-induced nanostructures investigated by TEM (bright field mode): a) overview of the FIB slice in the XY plan and b) HRTEM image showing a zoom on a single nano-layer (the inset is the SAED pattern) for $33 \mathrm{Li}_{2} \mathrm{O}-33 \mathrm{Nb}_{2} \mathrm{O}_{5}-34 \mathrm{SiO}_{2}$ (mol\%), $1030 \mathrm{~nm}, 300 \mathrm{fs}, 300 \mathrm{kHz}, 0.7 \mu \mathrm{J} /$ pulse, $\mathrm{NA}=0.6$, focus depth 
$350 \mu \mathrm{m}$ in glass, and $5 \mu \mathrm{m} / \mathrm{s}$. c) Schematic illustration of fs laser-induced nanostructures with amorphous "walls" (blue plates, oriented perpendicular to laser polarization direction) and nano-crystals (red plates). The nano-crystal (illustrated by green hexagonal prism) is oriented with polar axis perpendicular to laser polarization direction.

Figure 114 Three regimes of fs laser-induced modifications of irradiated lines in LNS glass as the function of pulse energy (0.3-2.2 $\mathrm{\mu} /$ pulse). Other parameters: $33 \mathrm{Li}_{2} \mathrm{O}-33 \mathrm{Nb}_{2} \mathrm{O}_{5}-34 \mathrm{SiO}_{2}(\mathrm{~mol} \%), 1030 \mathrm{~nm}, 300 \mathrm{fs}$, $300 \mathrm{kHz}, 5 \mu \mathrm{m} / \mathrm{s}, \mathrm{NA}=0.6$, focus depth $350 \mu \mathrm{m}$ in glass 


\section{List of tables}

Table 1 Typical commercial ultra-short pulse laser systems.

Table 2 Applications and proposed nonlinear materials

Table 3 Glass compositions and the formed nonlinear optical crystalline phases by laser irradiation.

Table 4 Methods for glass-ceramics with oriented crystals.......

Table 5 Crystallographic parameters of $\mathrm{LiNbO}_{3}$ crystal. 61

Table 6 Thermal parameters of LNS glasses

Table 7 Parameters of polishing LNS glasses..... 68

Table 8 Thermal properties of LNS glasses for modeling.

Table 9 Refractive index of LNS glasses

Table 10 Statistics of the widths and the lengths of laser tracks for the different writing configurations at low pulse energy 109

Table 11 Statistics of the widths and the lengths of laser tracks for the different writing configurations at high pulse energy. 


\section{Nomenclature}

3D: three dimensions

AOW: asymmetric orientational writing

BF: bright field

$\mathrm{CB}$ : conduction band

$\mathrm{Cl}$ : confidence Index

CCD: charge coupled device

CCR: critical cooling rate

CCT: continuous cooling transformation

CNT: classical nucleation theory

CPA: chirped pulse amplification

CW: continuous wave

DF: dark field

DHM: digital holographic microscopy

DTA: differential thermal analysis

EBSD: electron backscatter diffraction

EDS: energy-dispersive X-ray spectroscopy

FIB: focused ion beam

fs: femtosecond

HF: hydrofluoric acid

IPF: Inverse Pole Figure

IQ: image quality

IR: Infrared

KLM: Kerr-lens mode locking 
Laser: light amplification by stimulated emission of radiation

LC: liquid crystal

LED: light-emitting diodes

LIBS: laser-induced breakdown spectroscopy

LIDT: laser-induced damage threshold

LNS: lithium niobium silicate

Maser: microwave amplification by stimulated emission of radiation

MPI: multi-photon ionization

NA: numerical aperture

NIR: near-Infrared

ns: nanosecond

OIM $^{\mathrm{TM}}$ : orientation imaging microscopy

PF: Pole Figure

PIGE: proton-induced Gamma-ray emission

PIXE: proton-induced X-ray emission

PL: photoluminescence

PMT: photomultiplier tube

ps: picosecond

PSI: phase shift interferometry

RBS: Rutherford backscattering spectrometry

RGB: red-green-blue

SAED: selected area electron diffraction

SEM: scanning electron microscopy

SA: spherical aberration 
SHG: second harmonic generation

SPM: self-phase modulation

STE: self-trapped exciton

STEM-HAADF: scanning transmission electron microscopy-high angle annular dark-field

$\mathrm{T}_{\mathrm{c}}$ : Curie temperature

TEM: transmission electron microscopy

TEY: total electron yield

$\mathrm{T}_{\mathrm{g}}$ : glass transition temperature

$\mathrm{T}_{\mathrm{m}}$ : melting temperature

TTT: time temperature transformation

QBM: quantitative birefringence microscopy

UV: ultraviolet

VB: valence band

WDS: wavelength dispersive spectroscopy

XANES: X-ray absorption near edge structure

XAS: X-ray absorption spectroscopy

XPS: X-ray photoelectron spectroscopy

XRD: X-ray diffraction 


\section{Acknowledgements}

I am grateful to my supervisor M. B. Poumellec for the support and encouragement during my $\mathrm{PhD}$ research. I appreciate his capable of exploring the world. It is fantastic being with him to discover the secret behind the thing.

It is a pleasure to thank M. M. Lancry for the help in fs laser fabrication, SHG measurement, and useful discussions. I would like to thank M. F. Brisset for the SEM, SEM/EDS, and EBSD measurements, M. L. Mazerolles for the nanostructure investigation, M. E. Leroy for the measurement of WDS, STEM-HAADF, and STEM/EDS, M. T. Baudin, M. D. Solas, Mme A-L Helbert, and M. K. Verstraete for the EBSD analysis, M. P. Kazansky for the access to QBM and DHM measurements, Mme S. Surble for the chemical analysis, Mme D. Vantelon and M. B. Lassalle for the XAS experiment, Mme M. Leon-Pichel for UV-Vis attenuation measurement, Mme D. Dragoe for XPS analysis, M. J.-B. Sirven for the LIBS measurement, M. P. Berthet, Mme N. Ollier, Mme S. Gautier, and Mme T. Nait for glass making, M. R. Saint-Martin for XRD and optical floating-zone experiment, and Mme X. He for the discussion about fs laser writing. Your help makes possible to unveil the secret of crystallization process in glass by fs laser radiation.

I am sincerely grateful to my friends Mme S. Cao, M. Z. Song, M. F. Shao, M. Z. Cui, and Mme Y. Wang who help me a lot in France.

I greatly appreciate the continued support by my family, especially the one from my husband, Jianrong, who makes our life full of fun and encourage.

Finally, I am grateful to the support provided by China Scholarship Council and Université Paris Sud.

May I have the courage to have the life I loved.

Jing CAO

Université Paris-Sud, Orsay, France

January 10, 2017 


\section{Chapter 1. Introduction}

\subsection{Research motivations}

Femtosecond ( $\mathrm{fs}, 1 \mathrm{fs}=10^{-15} \mathrm{~s}$ ) laser direct writing in bulk transparent material has generated substantial interest, due to its unique properties and promising applications in optical waveguides, data storage, and microfluidic system [1-3]. Remarkably, it is an excellent method to obtain modifications in three dimensions (3D) with micron or nano-scale spatial resolution in single-step processing $[4,5]$. Therefore, it meets the requirements for device miniaturization and multi-functionalization.

In practice, an intense fs laser pulse is focused inside a transparent material, placed on a computer-controlled 3D motion stage. At certain irradiation conditions, through the nonlinear absorption of photons [6], various modifications can be achieved in material by adjusting the parameters such as material properties [7], irradiation parameters [8], or translation speed of the substrates [9]. By mechanically moving the motion stage during radiation, modifications can be realized in 3D [10]. In that case, to have a clear picture of the fs laser-induced modifications in material is of significance in fs laser direct writing.

Exposure of silica-based glasses to fs laser irradiation induces numerous novel phenomena after initial multi-photon absorption [11]. To date, various permanent changes including space charge migration [12], stress field creation [13], and crystalline phase transformation [14] have been reported. Accurate control of fs laser-induced modifications of glass provides a significant approach to explore ultra-fast laser-matter interaction and fabricate functional devices, such as low-loss waveguides [15], computer memory [16], and nonlinear optics [17]. Understanding the phase transformation in glass and the morphology of related nanostructure after fs laser irradiation is of great importance for fabricating functional optics, in which glass crystallization is involved to obtain nonlinear optical properties.

Obtaining nonlinear optical property in materials by fs laser irradiation has received intensive attention due to the potential applications in active optical devices (e.g. frequency converters) [18]. There are various approaches to achieve nonlinear optical property in glasses by fs laser radiation, such as morphological modification of embedded metallic nanoparticles [19], space charge migration [20], or laser-induced crystallization [21]. Among them, precipitating nonlinear optical crystal inside glasses has attracted much attention. At certain repetition rate (ca. $>200$ 
$\mathrm{kHz}$ ), pulse energy could be deposited at the focal point in materials, causing obviously thermal accumulation effect [22]. This can result in a temperature high enough and a duration long enough to induce crystallization [14, 23]. In addition, the crystallized region can be spatially selected by controlling the laser focused position movement [10].

fs laser-induced nonlinear optical crystals inside glass can date back to 2000, when Miura et al. [24] reported the space-selective growth of $\beta-\mathrm{BaB}_{2} \mathrm{O}_{4}$ inside $\mathrm{BaO}-\mathrm{Al}_{2} \mathrm{O}_{3}-\mathrm{B}_{2} \mathrm{O}_{3}$ glass at the focal point. To date, various choices of nonlinear optical crystals, including $\mathrm{Ba}_{2} \mathrm{TiSi}_{2} \mathrm{O}_{8}$ [10], $\mathrm{LaBGeO}_{5}$ [25], $\mathrm{LiNbO}_{3}$ [17], and $\mathrm{Sr}_{2} \mathrm{TiSi}_{2} \mathrm{O}_{8}$ [26] have been reported. Generally, nonlinear optical crystals bear higher second order nonlinearities than metallic nanoparticles.

However, there are several issues to be considered for fabricating nonlinear optical crystal based optical devices.

1) Because the refractive index difference between polycrystalline (e.g. $\mathrm{LiNbO}_{3} \sim 2.3$ ) and glass matrix (e.g. $\mathrm{SiO}_{2} \sim 1.45$ ) is large, to obtain transparent devices, the size of poly crystal has to be much smaller than the wavelength to maintain the scattering loss at a low level [27]. Generally, the crystalline size should stay below $\lambda / 10$ to maintain the transparency of composite materials. Yet, to maintain nonlinear optical crystal phase with high SHG property, the size of crystal must be larger than 100 nm [28].

2) To permit a coherent nonlinear optical response from the assembly of nonlinear crystals, it is necessary to control the crystal orientation [29]. For example, for $\mathrm{LiNbO}_{3}$, the SHG reaches the maximum when probing laser polarization is parallel to polar axis. Stone et al. [14] have reported the nonlinear optical crystal with polar axis parallel to scanning direction. However, for second harmonic generation (SHG) waveguides, it requires polar axis of nonlinear optical crystal to be perpendicular to scanning direction. Therefore, it is urgent to control the crystal orientation. It is worth noting that the emission from a nano-crystal can lose its phase relation with the other nano-crystals due to multiple scattering [30].

3) fs laser-induced nanostructure in glass represents a significant route to design and fabricate architectures in optics [2]. A nanostructure was discovered in silica glass [31], and then investigated deeply by several teams $[5,32,33]$. This nanostructure consists in series of nanoplanes perpendicular to the laser polarization direction [2]. Recently, the material systems have been extended to multicomponent glasses including borosilicate glasses [7], germanium dioxide glass [34], $\mathrm{TiO}_{2}$-containing silicate glasses [7, 35], and alkali-free aluminoborosilicate 
glass [36]. However, none of them have been pointed out nonlinear optical crystal embedded in such nanostructures. Therefore, it is meaningful to explore whether it is possible to obtain nanostructure consisting of nonlinear optical crystals, especially textured nano-crystals by fs laser irradiation. It will open a new front in multifunctional device fabrication.

Lithium niobium silicate (LNS) glass has attracted great interest due to its optical transparency [37], wide range of chemical compositions, and favorable laser-induced crystallization [18]. Moreover, the formed nonlinear crystal (i.e. $\mathrm{LiNbO}_{3}$ ) owns unique properties such as high Curie temperature [38] and large nonlinear coefficient [39].

The previous reports $[17,18,23]$ have shown that it is possible to obtain nonlinear optical crystals in LNS glass system by fs laser irradiation. Yonesaki et al. [18] demonstrated that in LNS glass fs laser irradiation induced the migration of glass constitute cations, locally modified chemical composition, and drove crystalline phase precipitation at micrometre scale. $\mathrm{A} \mathrm{LiNbO}_{3}$ phase was reported when crystallization occurred on sub-micro or nanometer scales, but not the silica rich counterpart [23]. Therefore, we wonder whether a phase separation really occurs and if silica could be included in $\mathrm{LiNbO}_{3}$ structure as $\mathrm{LiNbO}_{3}$ exhibits quite a large departure from stoichiometry (as a matter of fact, $\mathrm{LiNbO}_{3}$ shows a large departure of stoichiometry when doped with transition metal elements [40]). In addition, the modifications (e.g. whether there is an fs laser-induced nanostructure) in this glass system are not clear. So far, there is no systematic report about mastering the SHG in this glass system.

\subsection{Objectives}

The aim of this research is to make a contribution to the elaboration of nonlinear optics in glass by fs laser direct writing. We investigated systematically the crystallization inside LNS glasses induced by fs laser irradiation and explored how to master the fs laser-induced material modifications, such as crystallization, nanostructure, and the corresponding optical properties.

1) Clarify the regimes of $\mathrm{fs}$ laser-induced modifications (i.e. morphology, nanostructure, and crystallization) in LNS glasses varying with pulse energy and writing configuration (laser polarization direction versus scanning one) to give a clear picture of fs laser-LNS glass interaction (e.g. which regime is suitable for crystallization);

2) Determine the chemical redistribution and the evolution of the glass microstructure according to the laser parameters; 
3) Control the size and orientation of fs laser-induced nonlinear optical crystal (i.e. $\mathrm{LiNbO}_{3}$ );

4) Explore the optical properties (e.g. SHG and form birefringence) due to the above architecture, and find the relationship between fs laser-induced modifications and SHG properties or birefringence;

5) Investigate the mechanisms behind the fs laser-induced properties in LNS glasses.

\subsection{Dissertation organization}

The organization of this dissertation follows the analysis procedure of the investigation of $\mathrm{fs}$ laser-induced modifications in LNS glasses, shown in Figure 1.

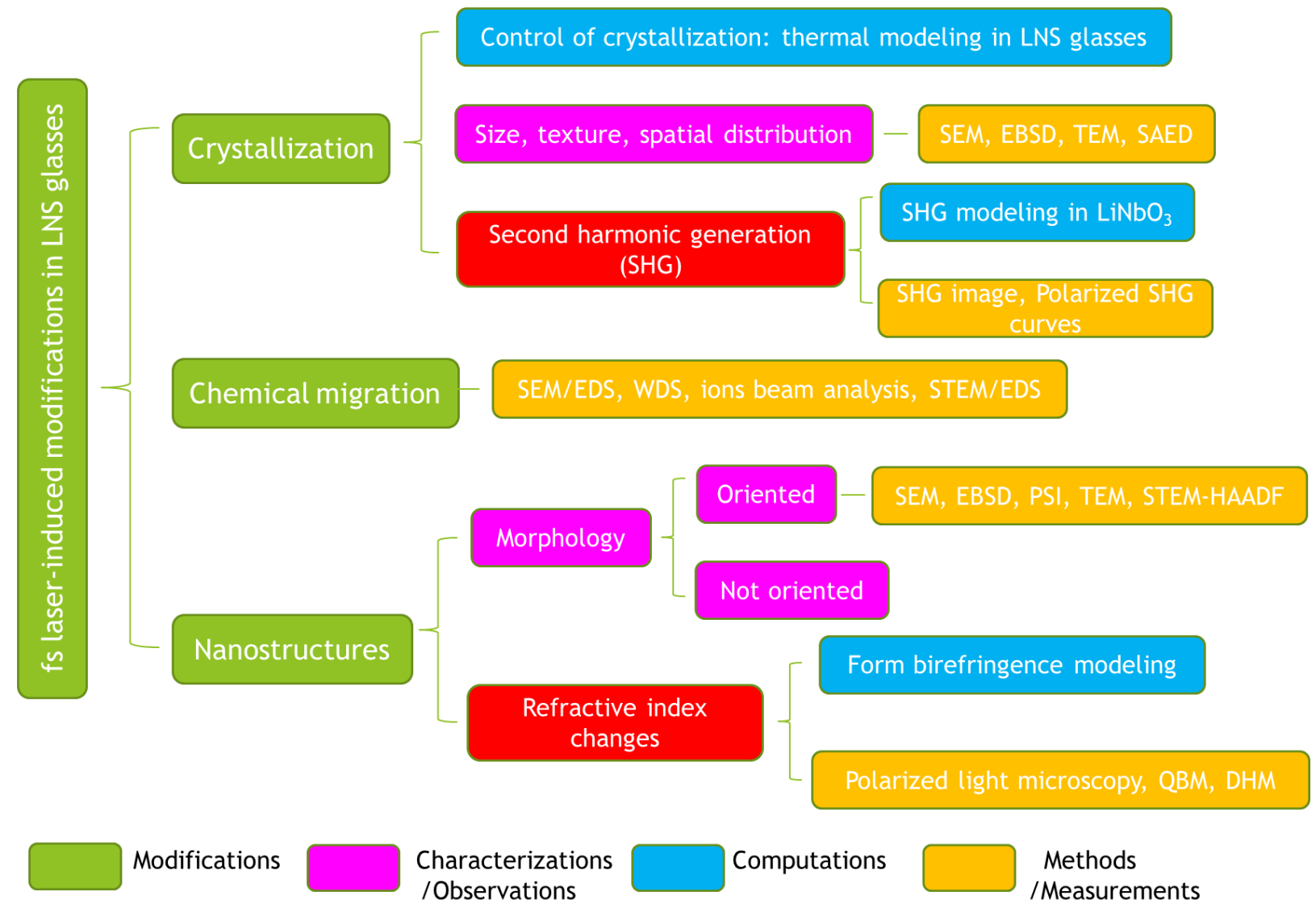

Figure 1 Analysis procedure of the investigation of $\mathrm{fs}$ laser-induced modifications in LNS glasses

Chapter 1: the introductory chapter consists of the thesis synopsis, including the motivation, the definition of the issues motivating the research work, aim to this research, and a brief overview of recent achievements in this field.

Chapter 2: the background section, describes a basic knowledge about laser, laser-matter interaction, nonlinear optics, and crystallization. Firstly, the history of laser is presented. Then typical used fs laser systems are listed to have a clear picture of their applications. The ultra-fast 
laser-induced modifications inside materials are listed. The basic knowledge of crystallization in glasses is overviewed. Finally, the properties of lithim niobate (e.g. crystallographic and optical properties) are also mentioned.

Chapter 3: this section considers methodology, providing information about glass making, glass polishing, experimental design (e.g. the laser writing system), and the corresponding characterizations (e.g. SHG measurement). In addition, a thermal modelling is conducted to provide essential information for optimizing fs laser irradiation conditions for $f s$ laser-induced modification (e.g. crystallization) in LNS glasses.

Chapter 4: the experimental section is about fs laser-induced modifications in LNS glasses. fs laser was used to control the crystallization and nanostructure in LNS glasses in single-step processing. The crystal size, orientated crystals (or texture) can be mastered simply by adjusting the laser parameters such as pulse energy and laser polarization. Remarkably, a self-organized nanostructure, with chemical changes of nonlinear optical crystals and amorphous phases, has been observed.

Chapter 5: this section is devoted to display the fs laser-induced properties in LNS glasses, including SHG and refractive index change. The regime (e.g. the pulse energy, repetition rate, and other parameters) for obtaining adjustable SHG and form birefringence is presented. In addition, the mechanism behind the phenomena is proposed.

Chapter 6: the concluding chapter and an outlook on further basic research and technique development in fs laser micromachining, and the possible extension to other glass systems. 


\section{Chapter 2. Background}

\subsection{Laser}

This section intends to give a clear picture of the development of laser and nonlinear optics. It contains the following three parts. The first part is about laser history including the concept, theory, and development of various kinds of lasers. In addition, the basic knowledge about Gaussian beam and some linear effects (e.g. spherical aberration) are presented. The second part is about the mechanisms of the interaction between ultra-fast laser and matter, including nonlinear absorption and relaxation mechanism. Some information about 3D fs laser fabricating is listed. The last part is about nonlinear optics, the theoretical basis for nonlinear effects, especially about SHG.

\subsubsection{Basic knowledge on laser}

\subsubsection{Basic historical facts}

Laser (light amplification by stimulation emission of radiation) is mentioned by G. Gould in 1957. It was once joked as "a solution looking for a problem". However, in a sense, it has shaped the development of civilization in optical field.

Laser has fantastic properties such as high monochromaticity, wide tuning wavelength range, high coherence, high degree of collimation, and small spot size. Because of the above unique properties, it has a wide range of applications in material processing, communication, nonlinear optics, displays, and medical surgery. Some milestones along laser development and remarks on laser safety are given in Appendix I and II, respectively.

Einstein proposed the stimulated emission, which is the basic theory of laser. As illustrated in Figure $2 \mathrm{a}$, stimulated emission is a process by which an incident photon with certain energy (i.e. $\left.h v_{0}=E_{1}-E_{0}\right)$, can stimulate an electron in an excited state (i.e. $E_{1}$ ) to a lower energy level (i.e. $E_{0}$ ). The new created photon will have the same frequency, direction, phase, and polarization as the incident photon. Based on the above analysis, population inversion and initial photons are the main requirements for laser.

The schematic of typical laser set-up is presented in Figure $2 \mathrm{~b}$. Three are three basic components for a laser system: 
1) Pumping source: an external energy supply such as electric current, flash tube, laser diode, and chemical reaction (e.g. Nd:YAG solid state rod can be activated by laser diodes);

2) Gain medium: an active medium, amplifying the circulating light. Generally, three or fourlevel laser systems are applied to realize population inversion. Nd-doped glass is a commonly used amplifier material in the high-power energy pulsed laser.

3) Optical resonator: generally made of two parallel mirrors. One is total reflect and the other is partially reflective mirror (or output coupler). Light can circulate between these two mirrors.

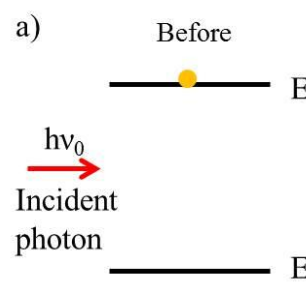

Electron in excited state $\mathrm{E}_{1}-\mathrm{E}_{0}=\mathrm{h} \mathrm{v}_{0}$

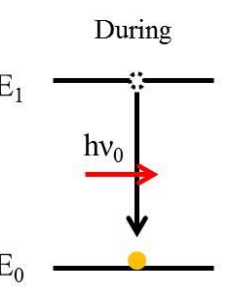

促

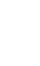

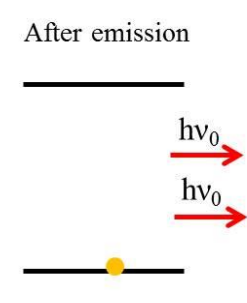

Electron in ground state b)

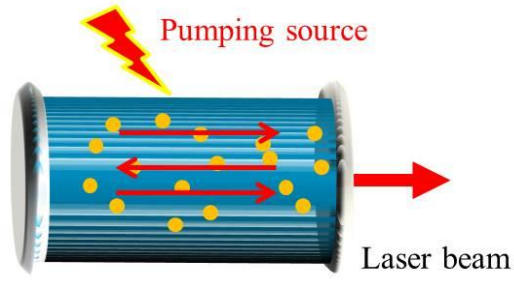

Gain medium

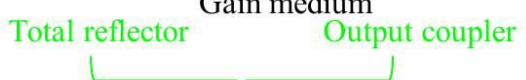

Optical resonator

Figure 2 Principle of laser: a) stimulated emission and b) schematic of typical laser set-up

In 1960, T. H. Maiman fabricated the first laser using a ruby crystal pumped by a xenon flash lamp. However, the beam quality and the reproducibility of the laser were not so nice. Since then, various new types of lasers were invented and the laser performance was improved. In particular, the ultra-short pulses lasers open a new way of the laser material processing (e.g. at nanoscale) [41]. The comparison (i.e. operating wavelength, advantages, disadvantages, and applications) of different types of lasers is shown in Appendix III.

So far, high laser energy has been achieved in long pulse laser system. Large laser peak power has been increased by decreasing the laser pulse duration. In addition, by reducing the laser wavelength, higher energy photons can be produced (e.g. X-ray laser).

\subsubsection{Ultra-fast laser}

Ultra-fast lasers generate optical pulses in the picosecond (ps, $1 \mathrm{ps}=10^{-12} \mathrm{~s}$ ) and fs range. Such short pulses can be obtained form lasers by modelocking [42]. Ultrafast lasers own features such as ultra-short pulse duration, high pulse repetition rate, broad spectrum, and high peak intensity. Figure 3a displays the progress in generating ultra-short pulse since the first demonstration of laser in 1960 . In the 70 s and 80 s, sub-ps pulses were acheieved by passively 
modelocked dye lasers. Until the end of $80 \mathrm{~s}$, when a solid-state laser material, Ti:sapphire, was discoved that owned the necessary broad gain bandwidth to support fs pulses. Figure $3 b$ illustrates the development of laser intensity from 1960. Such high peak intensity sources can achieve 'non-thermal' ablation, leading to the higher quality of ablated patterns in materails than the ones obtained by nanoseconde (ns) pulses.

Recently, ultra-fast laser has extended to attosecond (as, 1 as $=10^{-18} \mathrm{~s}$ ) scale. Attosecond physics is the one of electrons in motion at atomic and molecular length scales. The physics of this short pulses is based on nonlinear, nonperturbative laser-atom interaction [43]. This topic opens up new frontiers in the field of ultra-fast laser-matter interaction [44].
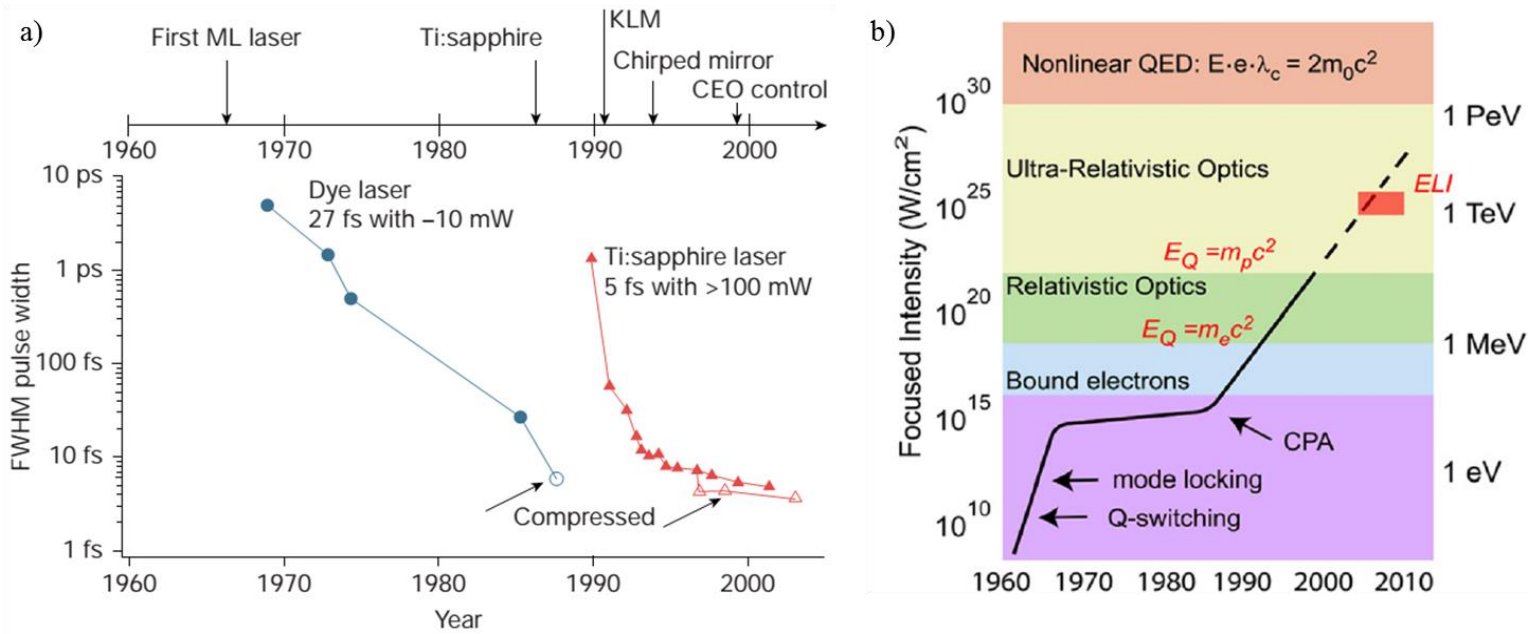

Figure 3 Progress in a) ultra-short pulse generation and b) laser intensity since the first demonstration of laser in 1960, CPA: chirped pulse amplification, ELI: extreme light infrastructure (cited from $[42,45]$ )

The typically solid state laser material saturation fluence is 1 and $10 \mathrm{~J} / \mathrm{cm}^{2}$ [46]. Pulse of $\mathrm{ns}$ duration or longer can be efficiently amplified without damaging the laser amplifier. Amplifying the short pulses directly in the laser amplifier can lead to damages in the amplifier, because the intensity is so high that nonlinear effects occur (e.g. self-focusing). The capability of laser materials for energy storage, generating high energy pulses pumping, and generating high peak power are presented in Appendix IV.

Ultra-fast high-power laser science came with chirped pulse amplification (CPA) technique [47, 48]. It enables the amplification of ultra-short (few $\mathrm{fs}$ ) laser pulses to high energy levels $\left(>10^{20}\right.$ $\mathrm{W} / \mathrm{cm}^{2}$ ). CPA enables lowering the peak power during amplification by temporally stretching the pulses (i.e. laser pulse duration varies from about $100 \mathrm{fs}$ to several $\mathrm{ns}$ ). For details please see Appendix IV. Besides the higher peak power achieved by CPA technique, it can also miniaturize laser system, resulting in a compact high power laser system. 
Table 1 lists the typical commercial ultra-short pulse laser systems.

Table 1 Typical commercial ultra-short pulse laser systems

\begin{tabular}{llll}
\hline Laser system & Typical parameters & Comments & Refs \\
\hline $\begin{array}{l}\text { Regenerative amplified } \\
\text { Ti:sapphire laser }\end{array}$ & high pulse energies $(\mu \mathrm{J}-\mathrm{mJ})$ & kHz repetition rates & [49] \\
$\begin{array}{l}\text { Oscillator-only } \\
\text { Ti:sapphire laser }\end{array}$ & low energy $(\mathrm{nJ})$ & high repletion rates $(\mathrm{MHz})$ & {$[49]$} \\
$\begin{array}{l}\text { Yb:KGd(WO})_{2} \text { laser } \\
\text { oscillator }\end{array}$ & $1030 \mathrm{~nm}, 80 \mathrm{fs}$ & high-power diode-pumped & [50] \\
Yb-doped fiber amplifier & $\begin{array}{l}1030 \mathrm{~nm}, \sim 300 \mathrm{fs}, \text { high pulse } \\
\text { energy }(\mathrm{nJ}-\mu \mathrm{J}), \mathrm{high} \text { repletion }\end{array}$ & compact, stable operation & [49] \\
& rate $(100 \mathrm{kHz}-\mathrm{MHz})$ & & \\
&
\end{tabular}

The aim of this thesis is to target 3D modifications of optical properties within the glasses (i.e. below the glass surface). That is, glasses do not have any linear absorption at the wavelength of the fs laser. So this needs some visual or infrared (IR) wavelength to create nonlinear multiphotons ionization process with at least 3 photons [1]. Visual or near-infrared (NIR) photons are better to achieve a good 3D confinement of the laser-matter interaction [51]. This implies to use Ti: sapphire laser at $800 \mathrm{~nm}$ [24] or Yb-doped fiber amplifier fs lasers at $1030 \mathrm{~nm}$ like our laser system (see details in Appendix V). In addition, high repetition rate laser is needed to work in the heat accumulation regime to crystallize LNS glass [23]. So a commercial Yb-doped fiber amplifier fs laser (Satsuma, Amplitude Systèmes Ltd.) emitting at 1030 nm with $100 \mathrm{kHz}-\mathrm{MHz}$ repetition rate is used here.

\subsubsection{Gaussian beam}

Figure 4a displays a continuous wave (CW) Gaussian beam propagating along $\mathrm{z}$ direction $(\mathrm{z}=0$ for the focal point of the focused laser), the fundamental Gaussian beam (lowest order TEM $\mathrm{O}_{00}$ mode) is given as [52]

$$
\begin{gathered}
E(r, z)=E_{00} \frac{\omega_{0}}{\omega(z)} \cdot \exp \left[\frac{-r^{2}}{\omega^{2}(z)}\right] \cdot \exp \left[-i \frac{k r^{2}}{2 r(z)}\right] \cdot \exp \{-i[k z-\Phi(z)]\} \\
\phi(z)=\arctan \left(z / z_{R}\right), \quad R(z)=z\left[1+\sqrt{1+\left(z_{R} / z\right)^{2}}\right], \quad \omega(z)=\omega_{0} \sqrt{1+\left(z / z_{R}\right)^{2}}, \quad z_{R}=\pi \omega_{0}^{2} / \lambda, \quad \theta=\lambda / \pi \omega_{0} \\
I(r, z)=\frac{\varepsilon_{0} c n}{2} \cdot E(r, z) \cdot E(r, z)^{*}=I_{00}\left(\frac{\omega_{0}}{\omega(z)}\right)^{2} \exp \left(\frac{-2 r^{2}}{\omega^{2}(z)}\right), \quad \text { with } \quad I_{00}=\frac{\varepsilon_{0} c n}{2} E_{00}^{2}
\end{gathered}
$$


Where the beam has circular symmetry, $r$ is the radial distance from the beam centre axis, $z$ is the axial distance from the "beam waist", $E_{00}$ is the amplitude of the electric field at the origin, $\omega_{0}=\omega(0)$ is the "beam waist" at the focus, the radial size at the narrowest point, $\omega(z)$ is the radium of the beam at distance $z$ from the waist, $R(z)$ is the radius of curvature, $k$ is the wave number, $\phi(z)$ is the Gouy phase shift, $z_{R}$ is Rayleigh length, $\lambda$ is the laser wavelength, and $\theta$ is the beam spreading angle, or divergence (with units of radians).

a)

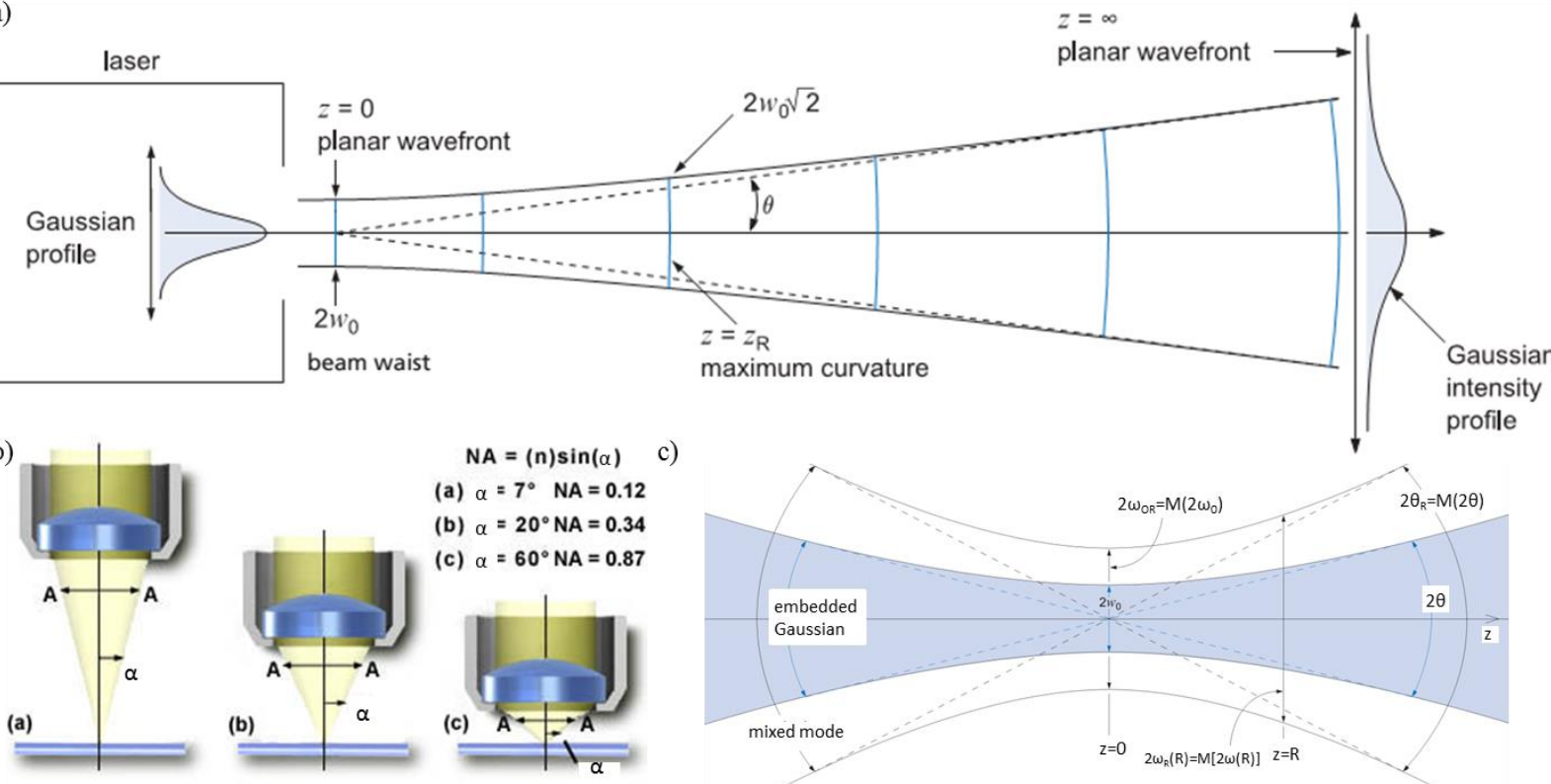

Figure 4 Gaussian beam: a) changes in wave front radius with propagation distance, b) the definition of numerical aperture (after [53]), and c) the embedded Gaussian (cited from [54])

As presented in Figure $4 a$, at $z_{R}$, the spot size is $\sqrt{2} \omega_{0}$, the $1 /$ e position of the electric field and the centre intensity is reduced to half the maximum value. $z_{R}$ is a variable to show how far the beam is collimated. When $z$ is far from the beam waist, the relationship between $\omega(z)$ and $z$ becomes linear

$$
\omega(\mathrm{z}) \approx \omega_{0}\left(\frac{\mathrm{z}}{\mathrm{z}_{\mathrm{R}}}\right)
$$

We can see that, when $\omega \rightarrow \omega_{0}, R \rightarrow \infty$, and $\theta \rightarrow 0$ is the one of perfectly collimated beam with no diffraction effect; when $\theta \rightarrow$ constant and $\omega \rightarrow \theta_{z}, R \rightarrow z$, is the one of a geometrical beam spreading from a point source.

The Rayleigh range has the relationship with the numerical aperture (NA) as

$$
\mathrm{z}_{\mathrm{R}}=\frac{\omega_{0}}{\mathrm{NA}}, \quad \omega_{0}=\frac{\lambda}{\pi \mathrm{NA}}
$$

Where NA $=\mathrm{n} \sin \alpha$ (illustrated in Figure $4 \mathrm{~b}$ ). NA is a parameter to measure the ability to collect light. So the lens can affect the beam focal spot size, shape, and energy redistributions. 
However, as shown in Figure $4 c$, the real beam waist $\left(\omega_{O R}\right)$ is $M$ times larger than the one of the Gaussian beam $\left(\omega_{0}\right)$, and the real beam divergence $\left(\theta_{R}\right)$ is $M$ times larger than the one of the Gaussian one $(\theta)$. But they own the same radius of curvature and Rayleigh length. The product of the beam waist radius and the far-field divergence are constant as the beam propagates through an optical system. The ratio of the beam parameter production of the real beam one to a Gaussian beam one is given as

$$
\mathrm{M}^{2}=\frac{\omega_{\mathrm{OR}} \theta_{\mathrm{R}}}{\omega_{0} \theta}=\frac{\pi \omega_{\mathrm{OR}} \theta_{\mathrm{R}}}{\lambda}
$$

$\mathrm{M}^{2}$ (or beam quality factor) is dimensionless and can be used to define the degree of variation of a beam from an ideal Gaussian beam. For theoretical Gaussian beam, $M^{2}=1$ and for real beam, $M^{2}>1$. Generally, $M^{2}$ increases with the increasing output power. For high-power laser system, the by-product of lasing in solid state media (e.g. heat) has an important effect on laser performance. The wave-front distortion occurs due to thermal lensing effect [55].

In fact, due to the focusing mode of laser beam, together with non-linear effects (e.g. selffocusing), the precise measuring of spot size and energy density inside glass is difficult or even impossible. The concept of embedded Gaussian (illustrated in Figure 4c) is useful as a construct to assist with both laboratory measurements and theoretical modelling.

For the laser used in the thesis, $\mathrm{M}^{2}$ is less than 1.2 (see details in Appendix V), which is nearly prefect. The laser is working in $\mathrm{TEM}_{00}$ mode and generating an appropriate Gaussian beam.

\subsubsection{Spherical aberration}

For a perfect beam focusing, the parallel light rays can be focused on a single point. However, the spherical surface of a lens can induce spherical aberration (SA). The parallel light rays pass through the central part of the lens focus further than the ones passing through the edges of the lens, leading to many focal points along light propagation direction. Further the rays from the lens centre is, larger the error is. To avoid SA, aspheric lens is used to focus beam in this thesis.

SA can also be due to refractive index mismatch (e.g. the air-silica interface) [56]. As shown in Figure 5, the central region of light is focused shallower than the adjacent one. This interface SA enlarges the focal spot (i.e. extension of the focal point) along laser propagation direction. The focal displacement $(\delta)$ is given as

$$
\delta=\left|O F_{0}\right|\left(\sqrt{\frac{n^{2}-N A^{2}}{1-N A^{2}}}-n\right), \quad N A=n_{0} \sin \theta
$$


Where $F_{0}$ is the geometrical focus in air, $F_{1}$ is the focus point under paraxial approximation, $F_{3}$ is the focus of the marginal rays (i.e. ray 3 ), $O$ is the crossing point of the light axis and the interface, $\mathrm{n}$ is the sample refractive index, and $\mathrm{n}_{0}$ is imaging medium refractive index between the objective front lens and the sample, which is equal to $\sin \theta$ here ( $\theta$ is in degree). The focusing depth in sample is defined as $d=n_{1 O F} l$.

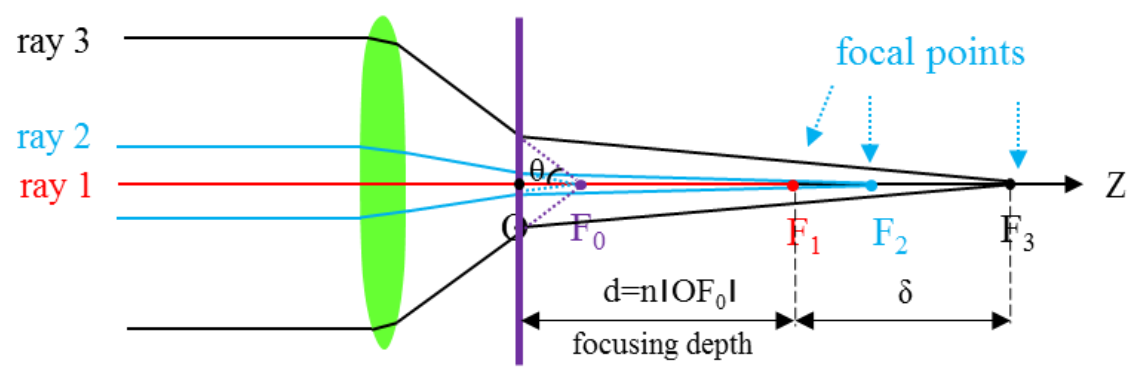

air-sample interface

Figure 5 Schematic of the interface spherical aberration due to the refractive index mismatch along laser propagation direction (after [57])

SA influences the light intensity distribution along the laser propagation direction. At high laser power, a long plasma channel along the laser incident direction can be observed and the length of this channel increases with the focusing depth [57]. The electric field distribution in material for the paraxial approximation is given in $[58,59]$. SA has an influence on the threshold pulse energy for fs laser-induced modification as a function of the focusing depth [56].

\subsubsection{Linear volume scattering}

Scattering is a phenomenon when a wave (wavelength of $\lambda$ ) interacts with a particle in a way that removes energy in the directional propagation wave and transfers it to other directions. There are two essentially types of scattering: elastic scattering (in which the wave energy is not changed) and inelastic scattering (in which the wave energy is changed). Mie scattering and Rayleigh scattering are two kinds of elastic scattering.

Mie scattering is the one when the size of the particle radius is close to or larger than $\lambda$, most of the incident light scatters in the forward direction. Rayleigh scattering occurs when the particles are much smaller than $\lambda$ (i.e. $<\lambda / 10$ ). The scattered light in the light forward and reverse direction of the degree is the same. In the direction perpendicular to the incident light has the lowest degree.

For glass ceramic materials, the attenuation of light because of scattering is dependent on the difference in refractive index of the two phases (i.e. $\Delta n=n_{2}-n_{1}$ ) and the size and distribution of 
crystals in the glass [60]. S. Hendy [60] computed the scattering intensity and found that the attenuation of light because of scattering (i.e. turbidity) can be given as

$$
\frac{14}{15 \pi} \varphi(1-\varphi) k^{8} R^{7}\left(\frac{\Delta \mathrm{n}}{\bar{n}}\right)^{2}
$$

Where $\varphi$ is the mean crystalline volume fraction, $k$ is the wavevector of light, $R$ is the average radius of the crystals in the glass, and $\bar{n}$ is the mean refractive index of the medium.

Based on this modelling, contours of turbidity as a function of radius and crystal volume fraction are displayed in Figure 6 . There is a strong dependence of the turbidity on the average radius of the crystals in the glass (i.e. $\propto R^{7}$ ) at a given crystalline volume fraction.

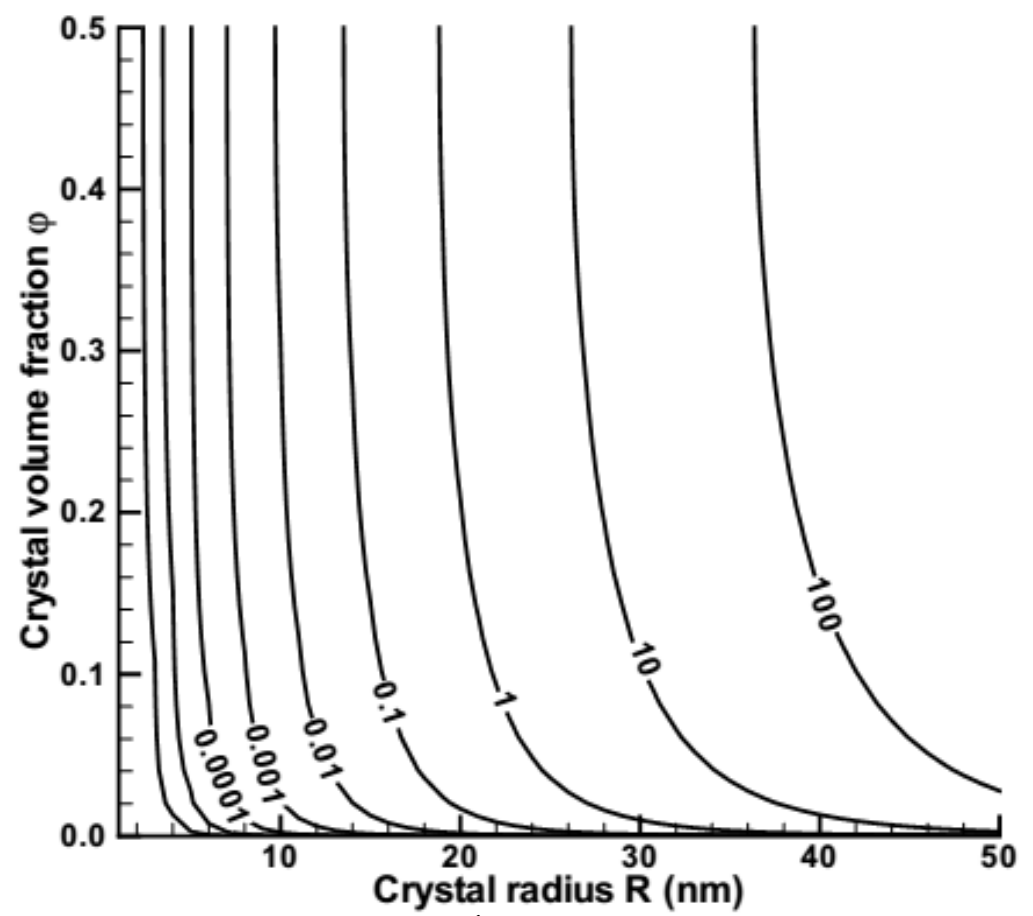

Figure 6 Contours of the turbidity (with unit of $\mathrm{m}^{-1}$ ) as a function of the crystal radius and crystal volume fraction (with $\Delta \mathrm{n}=0.1, \overline{\mathrm{n}}=1.7$ and $\lambda_{0}=647 \mathrm{~nm}$ ) (cited from [60])

In our case, $\mathrm{LiNbO}_{3}$ is obtained embedded in amorphous phases (i.e. $\mathrm{SiO}_{2}$ ). Because the refractive index difference between these two compositions is large (i.e. 2.3 and $\sim 1.45$ respectively), to obtain transparent devices, the size of crystal has to be much smaller than $\lambda$ to maintain the low scattering loss. The crystalline size should stay below $\lambda / 10$ to maintain the transparency of composite materials. 


\subsubsection{Laser-matter interaction}

Ultra-fast laser-matter interaction differs from the ones of long pulses or CW lasers due to its nonlinear characteristics. fs laser has the unique properties such as short duration of the pulse, great peak power (sufficient to induce direct bond breaking), the excitation is heat adiabatic process, and many dielectric media are transparent to the laser wavelength (i.e. red-NIR spectral zone) [41]. In this section, firstly the nonlinear absorption (e.g. multi-photon Ionisation and tunnelling ionisation) is discussed. Then several nonlinear optical effects, which play an important role in the fs laser-matter interaction and the further laser micro-machining in transparent materials, are presented.

\subsubsection{Free electron formation}

Wide-band gap $\left(E_{g}\right)$ materials are transparent in the visible to near infrared (ca. 390-1100 nm) at low-intensity light radiation, because the incident photon energy is smaller than material band gap energy (i.e. $h v_{0}<E_{g}$ ). Take fused silica for example (i.e. $E_{g}=9 \mathrm{eV}, 1 \mathrm{eV}=1.60 \times 10^{-19} \mathrm{~J}$ ). A laser beam with wavelength of $1030 \mathrm{~nm}$ (i.e. $\mathrm{hv}_{0}=1.2 \mathrm{eV}<9 \mathrm{eV}$ ) cannot be absorbed in the linear interaction regime. However, at high-intensity laser radiation, it is possible to promote electrons to higher energy states by nonlinear absorption, which mainly contains two approaches [61].

1) Multi-photon ionization (MPI) is achieved mainly at short wavelength and low field. As shown in Figure $7 a$, it is a process occuring when $m h v>E_{g}$, where $m$ is the number of absorption photons. For example, at least 8 photons (with laser wavelength $1030 \mathrm{~nm}$ ) are needed to promote an electron from valence band (VB) to conduction band (CB) in fused silica. The density of electrons in the $\mathrm{CB}$ depends strongly on the laser intensity (I) as following

$$
\left(\mathrm{d} \rho_{\mathrm{e}} / \mathrm{dt}\right)_{\mathrm{MPI}}=\delta_{\mathrm{m}} \mathrm{I}^{\mathrm{m}}
$$

Where $\mathrm{t}$ is the time and $\delta_{\mathrm{m}}$ is the multi-photon absorption coefficient for $\mathrm{m}$-photon absorption.

This formula can also be rewritten as follows, leading to a linear dependence on a double logarithmic scale [62].

$$
\log \left(d \rho_{e} / d t\right)_{M P I}=m \cdot \log \left(\delta_{m} l\right)
$$




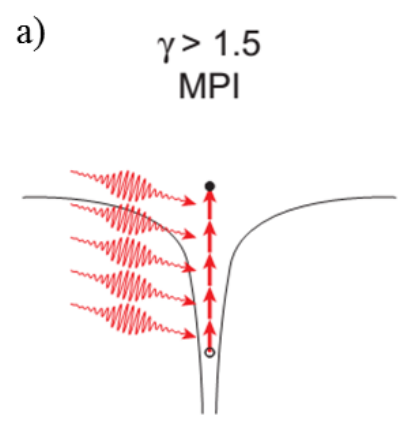

b)

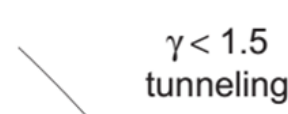

d)
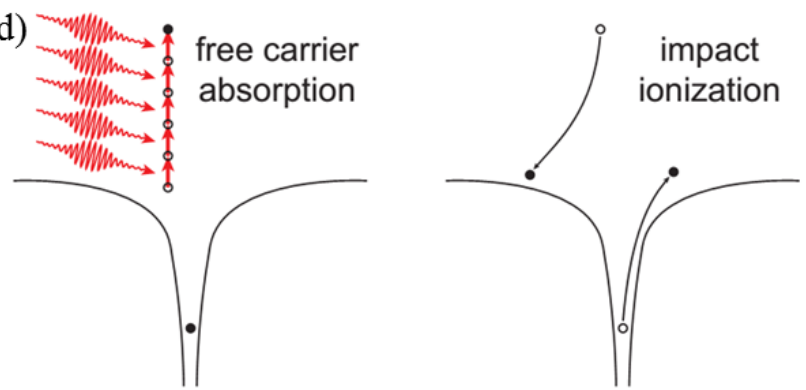

tunneling

c) $\quad \gamma \sim 1.5$

intermediate

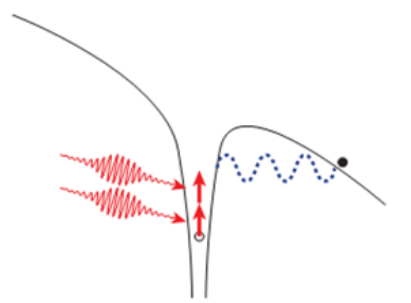

Figure 7 Schematic diagram of the photoionization of an electron in an atomic potential for different values of the Keldysh parameter $(\gamma)$. In a solid, the electron is promoted from the valence band to the conduction band, rather than ionized: a) multi-photon ionisation, b) tunnelling ionisation, c) the above processes combines, and d) avalanche ionisation: free carrier absorption followed by impact ionisation (cited from [63])

2) Tunnelling ionization occurs mainly at low frequency and strong field. As illustrated in Figure $7 \mathrm{~b}$, the band gap of the material is distorted due to the application of a strong laser electric field. The potential barrier between the CB and VB is reduced. In that case, electron can escape from their bound states by direct band transitions. It is worth noting that unlike MPI, the ionizaiton in strong fields occurs very quickly, in which the electron escaping at a time much shorter than the laser period [64].

The Keldysh parameter $\gamma$ was used to define the transition between the above two different ionisation regimes

$$
\gamma=\omega / \omega_{\mathrm{t}}=\frac{\omega}{\mathrm{e}} \sqrt{\frac{\mathrm{mcn} \varepsilon_{0} \mathrm{E}_{\mathrm{g}}}{\mathrm{I}}}
$$

Where $\omega$ is the laser frequency, $\omega_{t}$ is the frequency of electron tunnelling through a potential barrier, $m$ is the electron mass, and $n$ is the refractive index of the material.

When $\gamma$ > 1.5, MPI dominates; for $\gamma \ll<1.5$ tunnelling is dominating; and for $\gamma \sim 1.5$, the above processes combine (Figure 7c) [63]. For fused silica $\left(E_{g}=9 \mathrm{eV}\right)$, the MPI is dominating. The $E_{g}$ of $\mathrm{LiNbO}_{3}$ is $3.7 \mathrm{eV}$. It indicates that, comparing with fused silica, the weight of tunnelling is increasing. Different photo-ionisation regimes according to photo energy and laser intensity are illustrated in Figure 8. 


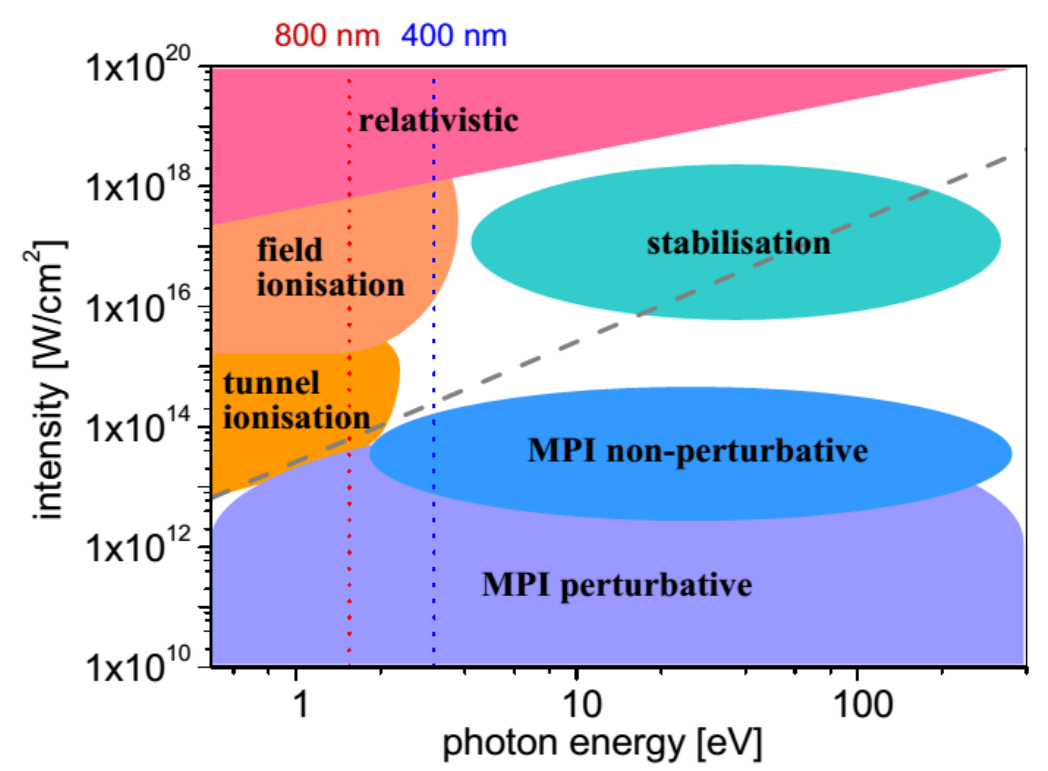

Figure 8 Different photo-ionisation regimes varying the photon energy and laser intensity. The dashed gray line shows case of $\gamma=1$ for $C_{60}$ ionisation which separates the multi-photon regime $(\gamma>1)$ from the tunnelling regime $(\gamma<<1)$ (cited from [62])

In addition to these direct excitations of electrons by laser electric filed, avalanche ionization also plays an important part in the process of the energy deposition from light to material. This process is triggered by seeded excited electrons that have been promoted to the CB by MPI or tunnelling ionization, or the intrinsic defects that can be easily ionized by the process such as thermal excitation [63]. These electrons can absorb photons linearly, resulting in their energy larger than the minimum CB (i.e. free-carrier absorption, shown in Figure $7 d$ left). This electron with enough energy $\left(2 E_{g}\right)$ can ionize electron in $V B$ (i.e. impact ionization, illustrated in Figure $7 d$ right), knocking the electron from the $\mathrm{VB}$, thus leading to two electrons at the minimum of the $\mathrm{CB}$. This process occurs if the laser electric field is present. It is dependent linearly on laser intensity (I). The density of electrons in the CB due to this process grows as

$$
\left(\mathrm{d} \rho_{\mathrm{e}} / \mathrm{dt}\right)_{\text {avalanche }}=\alpha \mid \rho_{\mathrm{e}}
$$

Where $\alpha$ is the avalanche ionization coefficient.

Based on MPI, the energy required to span the band gap is depending on the material $E_{g}$. However, the optical breakdown threshold is nearly not dependent on the $\mathrm{E}_{\mathrm{g}}$ and the threshold intensity, increasing by only about a factor of 2 [1]. This is mainly related to the avalanche photon-ionization, which is linearly depending on the laser intensity. In that case, it is flexible to choose the materials to interact with fs laser. 
Figure 9a illustrates the electron density evolution for $100 \mathrm{fs}$ duration at $11.7 \mathrm{TW} / \mathrm{cm}^{2}$. $\mathrm{MPI}$ is strongly laser intensity dependent. The electron production occurs principally at the laser pulse peak. MPI produces a substantial number of free electrons with only a small collisional avalanche is required to achieve critical density $\left(\sim 10^{21} \mathrm{~cm}^{-3}\right)$. Critical density will not be produced until late in the pulse, and only this last part of the laser pulse will lead to any strong absorption or reflection [65].
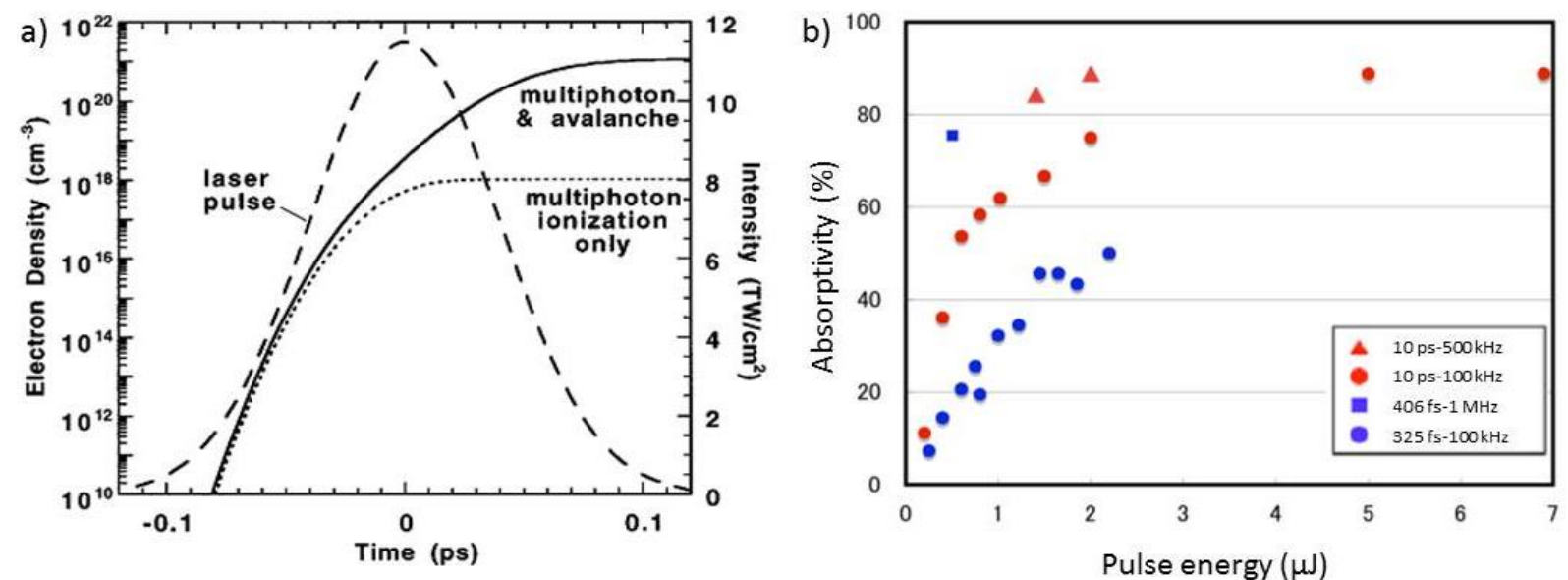

Figure 9 a) Calculated evolution of free electron density for a $100 \mathrm{fs}, 1053 \mathrm{~nm}$ pulse (dashed curve) of peak intensity $11.7 \mathrm{TW} / \mathrm{cm}^{2}$ in fused silica. Multi-photon ionization (dotted curve) starts the avalanche; solid curve is total electron density including impact ionization (cited from [65]). b) Absorptivity as function of pulse energy, repetition rate, and pulse duration. Neglecting the laser beam scattering and the reflection at the focus, absorptivity is defined as $1-W_{t} /\left[W_{0}(1-R)^{2}\right]$, where $W_{0}$ is the incident laser power to the work piece, $W_{t}$ is the transmitted laser power, and $\mathrm{R}$ is the reflectivity of the beam at the glass surface (after [66])

Nonlinear absorption can affect the glass absorptivity of ultra-short pulses [66]. As shown in Figure $9 b$, the absorptivity increases with the increase of pulse energy, which can be explained that photoionization rate increases with the increase of laser intensity. For the $0.5 \mu \mathrm{J}-100 \mathrm{kHz}$ case, even the laser intensity of $325 \mathrm{fs}$ one is larger than that of $10 \mathrm{ps}$ one, the 10 ps absorptivity is 2-3 times larger than that of $406 \mathrm{fs}$ pulses. This is due to $10 \mathrm{ps}$ pulse duration is long enough for the electron density growth in the $C B$ through avalanche ionization. At $0.5 \mu \mathrm{J}, 406 \mathrm{fs}-1 \mathrm{MHz}$ one is $\sim 4$ times larger than that of $325 \mathrm{fs}-100 \mathrm{kHz}$ one, which is because the temperature at the laser irradiated zone just before the pulse arrival (i.e. the $1 \mathrm{MHz}$ one is larger than the $100 \mathrm{kHz}$ one), determining the thermally excited electrons density in $\mathrm{CB}$. Higher absorptivity at $1 \mathrm{MHz}$ is due to the larger thermally excited electrons number in the CB from the VB [67]. In addition, at $500 \mathrm{kHz}$, the 10 ps pulses absorptivity can reach as large as $90 \%$, indicating the electron density grows until the critical plasma density, and efficiently absorbs laser energy by free-carrier absorption. This high absorptivity also indicates that the critical electron density was reached at very beginning of the pulse duration, at most 1-2 ps [66]. So, for 10 ps pulses, higher absorptivity 
can be achieved even at lower repetition rate, because much longer time is available for the electron density growth through the avalanche ionization. However, higher repetition rate is important for enhancing absorptivity for sub-ps pulses.

\subsubsection{Relaxation of photo-excited electrons}

The mechanism of the fs laser-induced modifications in dielectrics is still an open question. The widely-accepted one is that excited electron-ion plasma produced at the focal volume by fs laser during excitation and the subsequent dissipation of the energy into the lattice leads to various modifications (e.g. refractive index changes).

a)

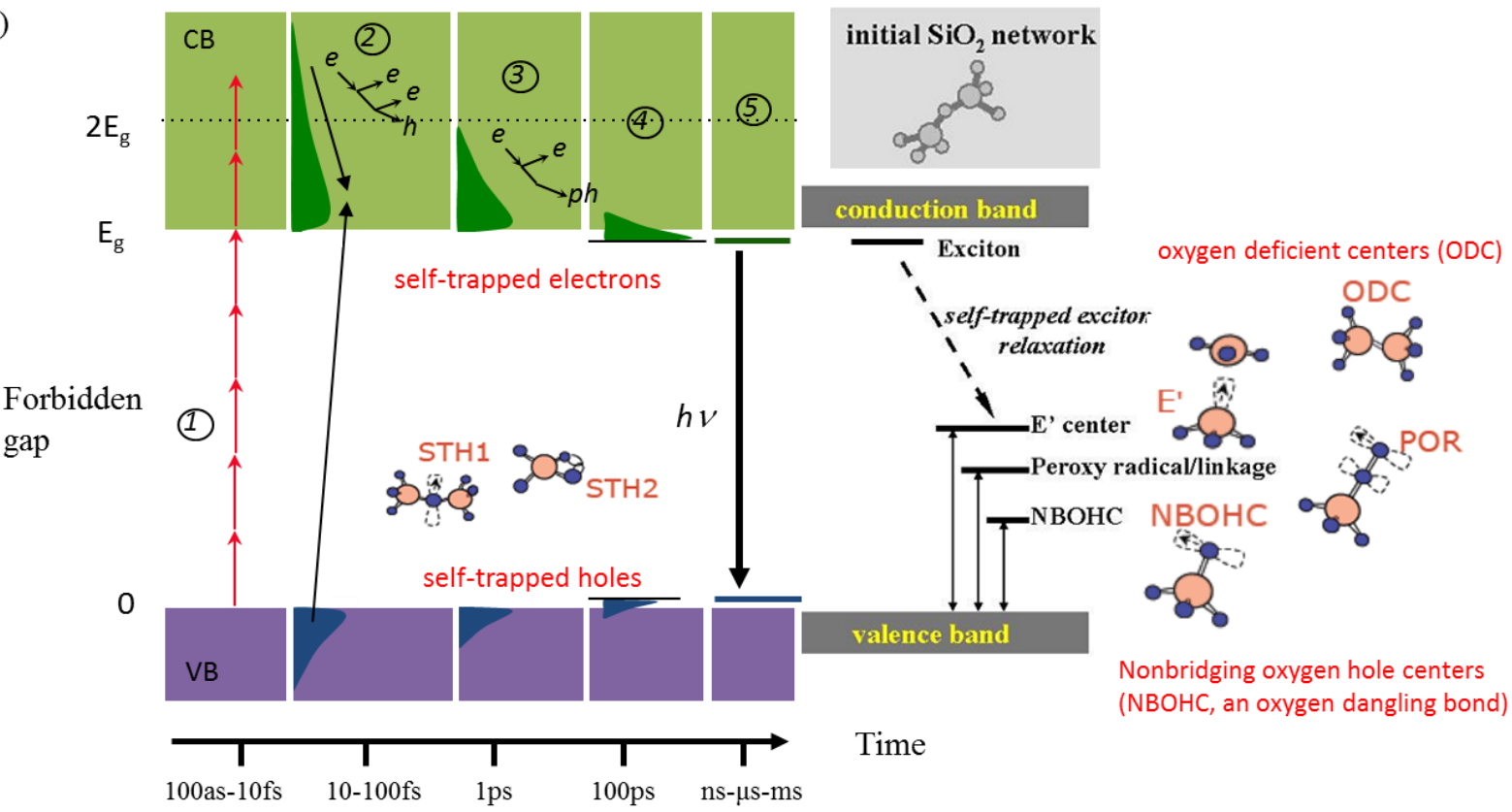

b)

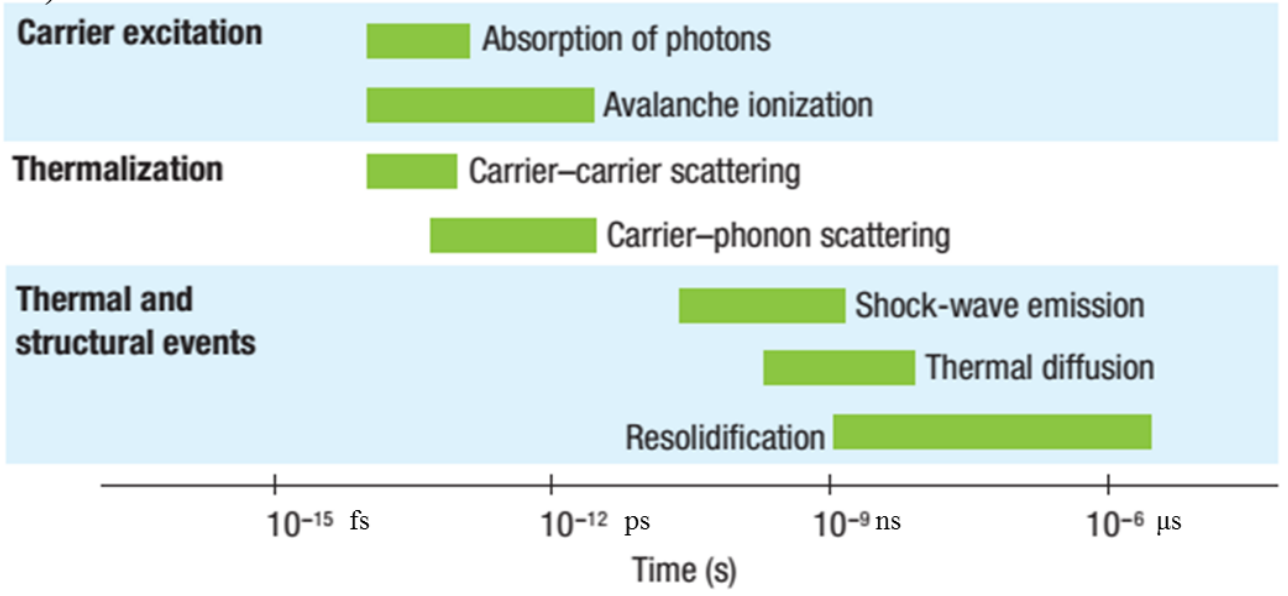

Figure 10 a) Schematic representation of the dynamics of $f$ laser interactions with dielectrics. The numbers from 1 to 5 correspond to the process as described within the text (after S. Guizard, LSI, CEA, Saclay France and $[6])$, and b) timescale of the physical phenomena associated with the interaction of a fs laser pulse with transparent materials (cited from [1]) 
Figure 10a displays the process of the interaction between fs laser and dielectrics. For convenience, Figure $10 \mathrm{~b}$ lists the timescale of the relevant physical phenomena associated with fs laser interaction with transparent materials. Note that although the light absorption occurs at the fs timescale, the material can continue to undergo changes $\mu$ s later [1].

1) Laser absorption (e.g. MPI) produces free electron plasma in the $\mathrm{CB}$ and holes in the VB.

2) The formed plasma is then heated by laser electro-magnetic field through free carrier absorption or the electron density grows through avalanche ionization until the plasma frequency, given as

$$
\sqrt{\frac{\rho_{\mathrm{e}} \mathrm{e}^{2}}{4 \pi^{2} \varepsilon_{0} \mathrm{~m}}}
$$

reaches the light frequency.

The excited electrons distribute energy to other electrons (i.e. carrier-carrier scattering) within a timescale of $10-100$ ps, resulting in the energy redistribution between the excited carriers. So, after the fs laser irradiation, electron-hole pairs are created.

3) The electron plasma relaxes by coupling with the lattice (lattice heating).

In contrast to the ns laser pulses, the ultra-short laser pulses can transfer from the laser-excited electrons to the lattice after the laser pulse is completed [66].

The timescale for the electron energy transfers to the lattice is around tens of ps. In that case, the laser pulse duration $(\tau)$ has an important effect in the material modifications. When $\tau$ is at the same time scale, the laser energy can be deposited to the lattice during irradiation (i.e. carrier-phonon scattering), and the excited phonons can transfer the energy to the nonirradiated part by thermal diffusion. If the temperature in the affected area is high enough, permanently damages occur.

As shown in Figure 11, typically, the damage threshold observed for the surface of material (e.g. fused silica) is proportional to $\tau^{0.5}$ when $\tau>10 \mathrm{ps}$, indicating the transfer of electron kinetic energy to the lattice and diffuse during the laser pulse [65]. In addition, short pulse $(\tau<10 \mathrm{ps})$ damage can be confined into a small zone (i.e. at the peak of the Gaussian beam), where the laser intensity is sufficient to produce MPI. Because there is not sufficient time for lattice coupling, no collateral damage occurs, and the damaged region is much smaller with short pulses than with long pulses [65]. 


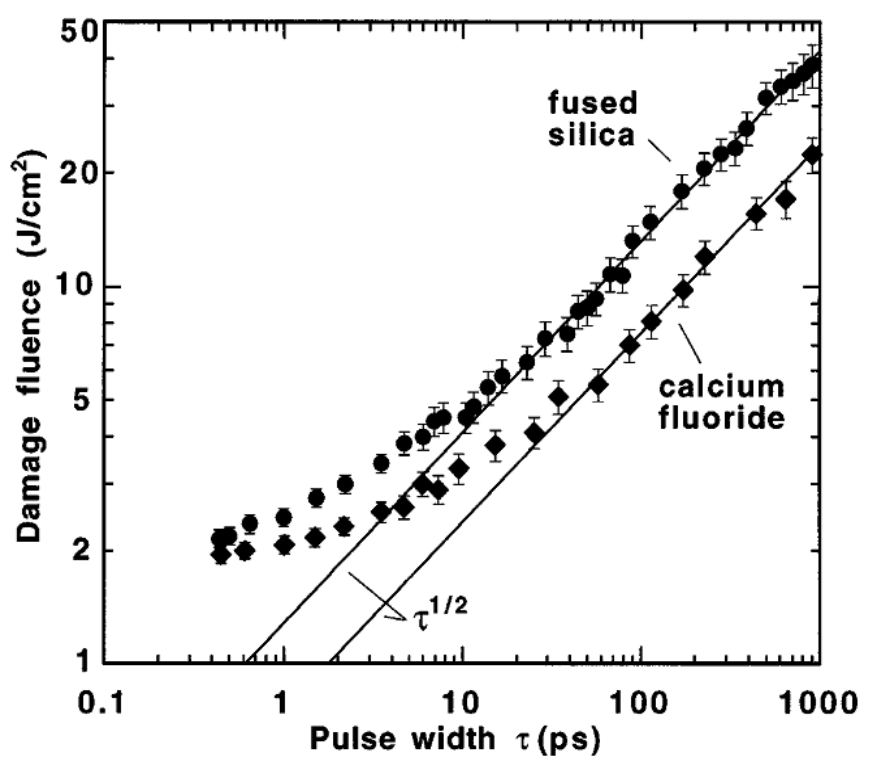

Figure 11 Observed values of damage threshold at $1053 \mathrm{~nm}$ for fused silica and $\mathrm{CaF}_{2}$. Solid lines are $\tau^{0.5}$ fits to long pulse results. Estimated uncertainty in the absolute fluence is $\pm 15 \%$ (cited from [65])

4) Exciton is a bound state of an electron and an electron hole, being attracted to each other by the electrostatic Coulomb force. It is an electrically neutral quasi particle, existing in semiconductors. Self-trapped excitons (STEs) can be formed in a few ps.

In crystals (or in glasses) excitons interact with phonons, the lattice vibrations. A photon with energy near $E_{g}$ can be absorbed, creating a loosely bound Wannier-Mott exciton in the network [68]. Self-trapping results in dressing excitons with a dense cloud of virtual phonons which strongly suppresses the ability of excitons to move across the lattice [69]. In simpler terms, this means a local deformation of the crystal lattice around the exciton. It should be of atomic scale, of about an electron-volt below the CB.

5) Then STE's annihilate and relax of energy as light or to the lattice again or produce point defects and structural modifications.

The exciton could either recombine, or by a phonon-assisted process drop to a slightly lower energy state, named as STE, or combine non-radiatively back into the ground state. This STE occurs in the network structure as a point defect pair [70]. Excited electrons and holes relax into STE by electron-phonon coupling at the time scale of $100 \mathrm{fs}$. Decay of STE can be achieved by radiative recombination with photon emission with a lifetime of ns at room temperature, or a non-radiative way by coupling with lattice phonons, or transforming into point defects [71].

The time for heat diffusion is $1 \mu \mathrm{s}$, at certain high repetition rate (ca. $>100 \mathrm{kHz}$ ), the thermal accumulation occurs. In that case, the pulse energy of fs laser could be deposited at the focal 
point inside transparent materials, causing evidently thermal accumulation effect and the heataffected zones were minimized. By this means, high precision sub-micrometre resolution spatial controlled modification in 3D in buck material is realized. In comparison with typical 3D material processing techniques (e.g. photolithography), fs laser micro-machining is a rapid, flexible, and clean fabrication, without using toxic photo resist comparing with photolithography. The comparison of a several common micro- and nano-fabrication technologies is shown in Appendix VI.

\subsubsection{3. fs laser-induced defects}

As shown in Figure 10a right, $\mathrm{SiO}_{4}$ tetrahedron is the structural unit of silica, in which the Si atom is bonded to four $\mathrm{O}$ atoms with $\mathrm{O}-\mathrm{Si}-\mathrm{O}$ angle of $109.5^{\circ}$. The formation of defect starts from the relaxation of STEs, for instance, E' center (oxygen vacancies with a trapped hole or 3-foldcoordinated silicons) and nonbridging oxygen hole center (NBOHC, an oxygen dangling bond). By adding an interstitial $\mathrm{O}$ atom to NBOHC peroxy radical (POR) can be created. Self-trapped holes $(\mathrm{STH})_{1}$ would be a small polaron consisting of a hole trapped at a normal bridging oxygen in silicon dioxide network, and the $\mathrm{STH}_{2}$ might be an Anderson localized state where a hole is trapped on two normal oxygens [72]. E'-center, POR, and NBOHC are three fundamental centers which can be detected by electron spin resonance.

Laser irradiation can create defects (e.g. oxygen vacancy defect), which leads to photoluminescence (PL) of the sample. The major optical absorption or luminescence bands of defects in non-doped synthetic silica are shown in Appendix VII.

Intrinsic defect PL was firstly observed in an annealed and neutron-irradiated amorphous $a-\mathrm{SiO}_{2}$ [73]. Since then, many investigations have been reported to determine the origins and structure of defects in different types of silica [74]. Details information about silica is shown in Appendix VIII. Gee et al. [75] demonstrated that PL centres are unique bonding configurations which is mainly dependence on the material chemistry rather than on the degree of long range order.

It is worth noting that oriented silicon oxygen deficient center, SiODC(II) defects have been created in silica by IR fs laser irradiation [76]. The SiODC related luminescence (excited at $6.8 \mathrm{eV}$ ) is stronger when the probing polarization is parallel to the laser scanning direction, meaning that the axis passing through the $\mathrm{O}$ atoms is aligned with the laser scanning direction (see details in Figure 12). 
The IR photon energy is below $E_{g}$ of the material but the induced defects are like those observed for UV irradiation. This indicates that the multi-photonic nature of the mechanisms leading to the creation of defects by IR fs laser. The PL of different materials is shown in Appendix IX.

It is worth noting that the laser-induced defects can decrease the damage threshold of silica [77], by creation of defect levels in the gap. Those ones are easier to ionize changing incubation period for collective transformation.

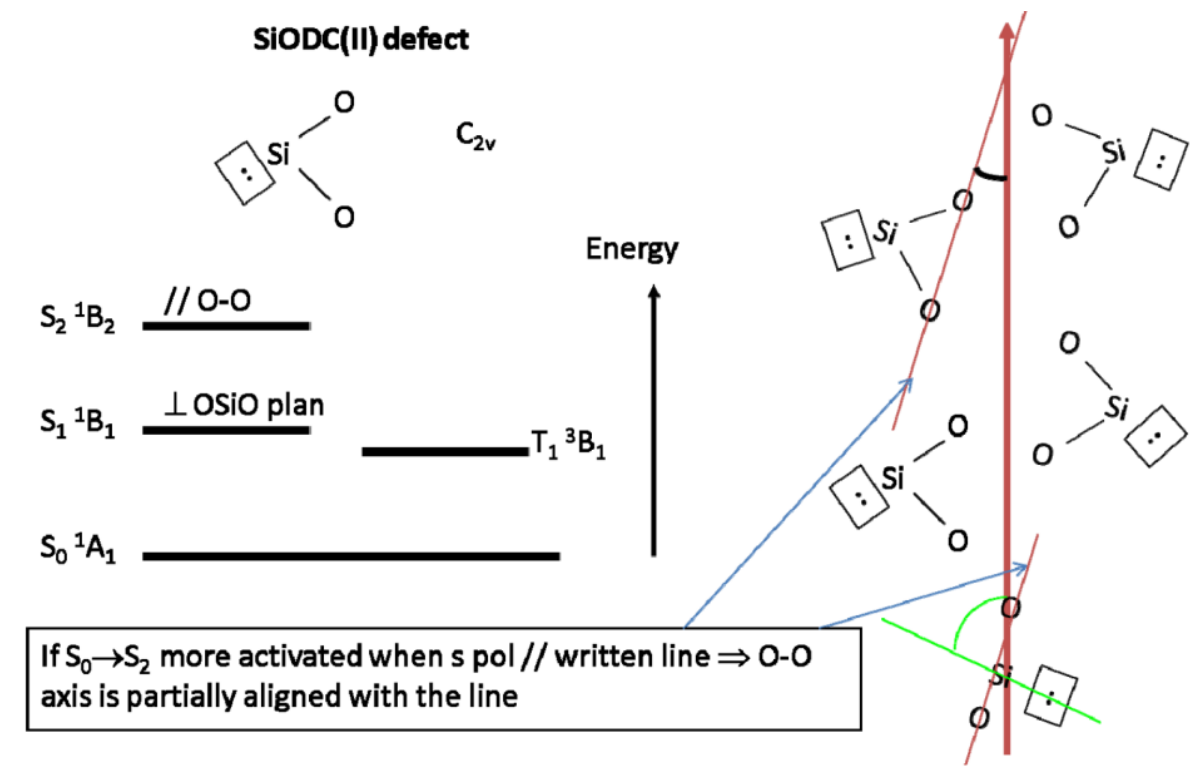

Figure 12 Symmetry of the excited states of $\operatorname{SiODC}(\mathrm{II}):{ }^{1} \mathrm{~A}_{1}$ is the ground state, ${ }^{1} \mathrm{~B}_{1}$ is the first excited singlet, and ${ }^{1} B_{2}$ is the second excited singlet. $S_{1}$ (corresponding to the excitation band at $5.0 \mathrm{eV}$ ) is related to transition from ${ }^{1} A_{1}$ to ${ }^{1} B_{1}$ which is related to an electron transiting from an orbital with symmetry a1 to an orbital with symmetry b1, built mainly on the "non-bonding" $\mathrm{Si}_{3 \mathrm{px}}$ atomic orbital ( $\mathrm{x}$ being perpendicular to the molecular plane). The $S_{0}-S_{1}$ transition is polarized along $x$ direction. $S_{2}$ (corresponding to the excitation band at $6.8 \mathrm{eV}$ ) is related to transition from ${ }^{1} \mathrm{~A}_{1}$ to ${ }^{1} \mathrm{~B}_{2}$ which is related to an electron transiting from a a1 orbital to a b2 orbital, built mainly on the $\mathrm{Si}_{3 \mathrm{p}}-\mathrm{O}_{2 \mathrm{p}}$ anti-bonding molecular orbital in the molecular plan. The transition between $\mathrm{S}_{0}$ and $\mathrm{S}_{2}$ is polarized in the O-Si-O plane (cited from [76])

\subsection{3. fs laser-induced modifications in 3D}

\subsubsection{3D fs laser fabrication set-up}

Modifying glass in 3D with fs laser dates back to 1996, when Davis et al. [51] reported fabricating waveguides inside silica, Ge-doped silica, borate, soda lime silicate, and fluorozirconate bulk glasses by tightly focused fs laser $(810 \mathrm{~nm}, 120 \mathrm{fs}, 200 \mathrm{kHz})$. Since then, fs laser micromachining has received intensive attention in optics [1].

The 3D fabrication set-up is shown in Figure 13. The laser beam was focused by a microscope objective and injected into glass. The glass is fixed in an XYZ stage, being translated at certain speed, either perpendicular or parallel to the incident laser beam, thus fabricating damage lines inside the glasses. 
When laser beam is focused on the near glass surface, machining of the glass, most likely due to thermal shock, occurs at high laser powers [51]. However, when focusing on the interior of the glass, no cracking was detected in any of the glasses regardless of laser power (average power of the laser between 40 and $800 \mathrm{~mW}$ at the glass location). The modification in glass is related to damage thresholds at certain irradiation conditions.

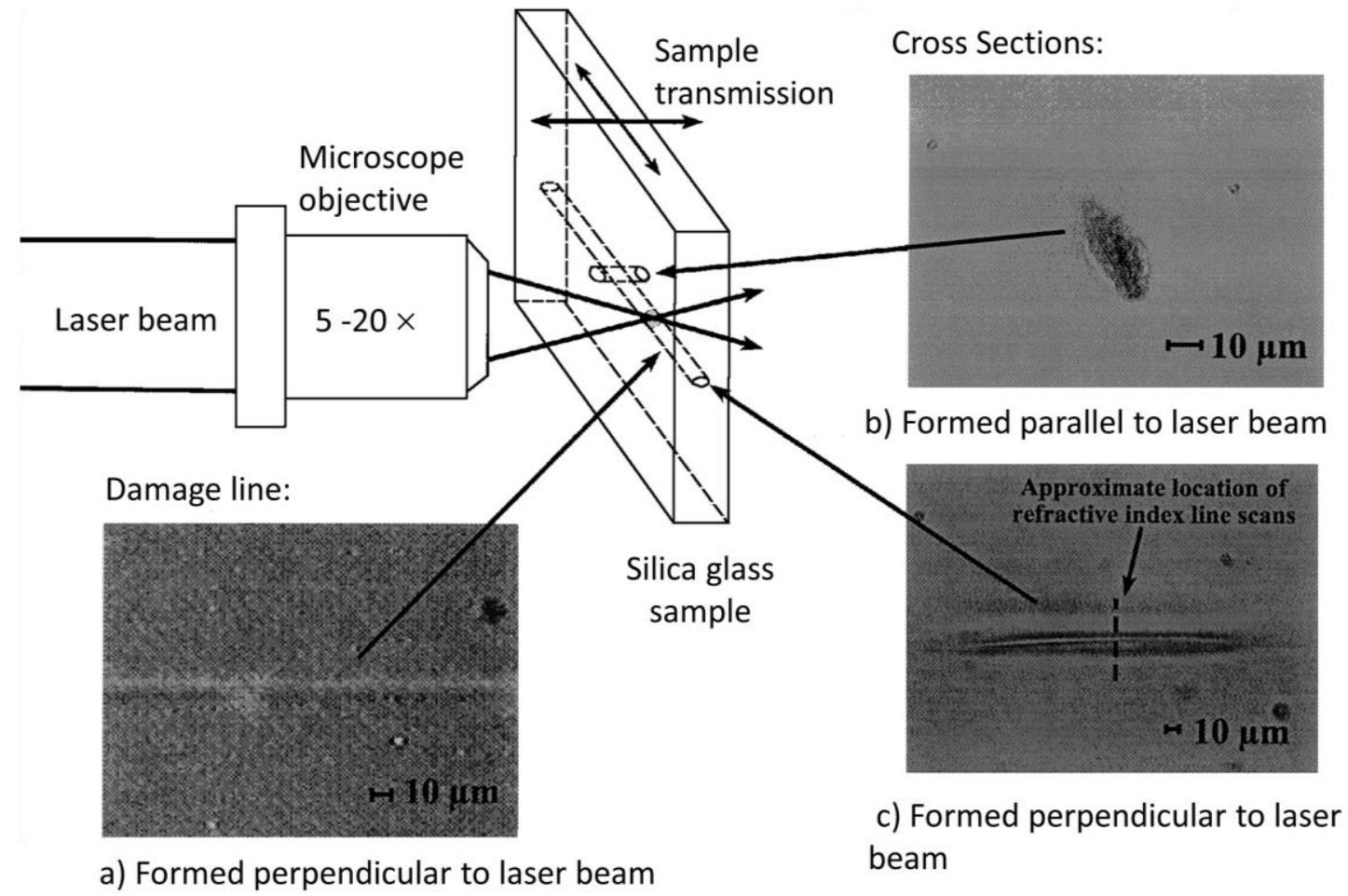

Figure 13 fs laser fabricating in 3D: a) a laser damage line, b) the cross section of a line written by moving the sample parallel to the incident laser beam, and c) the cross section of a line written by moving the sample perpendicular to the incident laser beam (after [51])

\subsubsection{2. fs laser-induced structural changes in glasses}

At low repetition rate, without crystal formation, as shown in Figure 14, depending on the pulse energy delivered to the sample, three regimes with three thresholds have been defined in fused silica with the increase of pulse energy [78].

A first threshold $\mathrm{T} 1$ is the one related to the generation of isotropic refractive index change without obvious structure damage. The contrast of the refractive index change can be either positive or negative dependent on the materials [64]. The refractive index change can reach the maximum at $6 \times 10^{-3}$ in fused silica, which is large compared to the one induced by UV ns laser [76]. This modification can be annealed by heating the sample at certain temperature [79]. The energy operating window for $\mathrm{T} 1$ is narrow which increases in size at the shortest pulse duration [80]. This modification can be used to fabricate low loss optical waveguides [73]. 
Couairon et al. [79] proposed that when some damage is observed, it corresponds for the excited electrons to reach a certain density. However, this proposal is not able to explain the different thresholds from material to material [71]. Poumellec et al. proposed that this modification can be explained by change of the fictive temperature $\left(T_{f}\right)$ of material $[71,82]$. Details of the information of $T_{f}$ will be given in Section 3.3.2.2. Glass modifications are due to the relaxation of STE. The non-radiative relaxation of excited electrons by coupling with the lattice, corresponds to an increase of non-equilibrium temperature of the lattice (several thousand degrees in the illuminated zone) just limited by thermal conduction [71]. The temperature increases during a time long enough, to change the average disorder (i.e. a change of glass $T_{f}$ ) [83]. The laser-induced thermal gradient can increase the $T_{f}$ of material, causing the density changes.

a

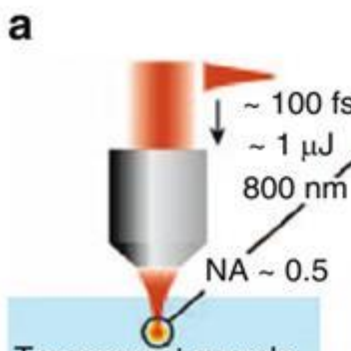

b

Field ionization

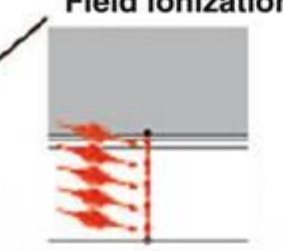

Transparent sample
C

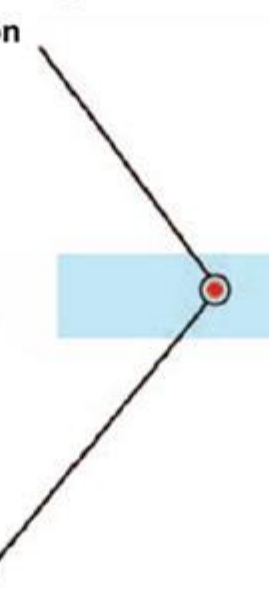

$\overrightarrow{\text { time }}$
High laser intensity in focal volume

$$
\begin{gathered}
\text { Nonlinear } \\
\text { absorption } \\
\text { of laser energy }
\end{gathered}
$$

Hot electron-ion plasma transfers energy to lattice d

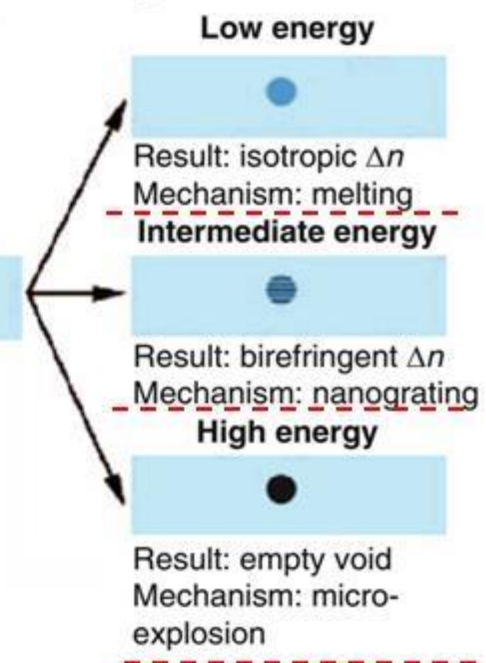

Permanent material change

Figure 14 A schematic showing focused fs laser pulses in bulk fused silica: a) fs laser is focused below the sample surface resulting in a high intensity in the focal volume, b) the energy is nonlinearly absorbed and a free electron plasma is created by multi-photon/tunneling and avalanche photoionization, c) the plasma transfers its energy to the lattice on a 10 ps time scale resulting in one of three types of permanent modification, and d) fs laser-induced various modifications in glass as a function of pulse energy (after [81])

A second threshold $\mathrm{T} 2$ is the generation of form birefringence. The fs laser-induced birefringence can be explained by laser-induced stress [84] or a form birefringence due to self-organized periodic nanostructure formation (i.e. nanograting), oriented perpendicular to laser polarization direction [85]. This modification has the applications such as polarization converters, microfluidic, and nanofluidic channels [4]. 
This anisotropic index change magnitude can reach as large as $10^{-2}$ [32]. Lancry et al. [86] shown that the index contrast is because the nanoplanes are nanoporous, produced by a glass decomposition leading to generation of molecular oxygen. In addition, this type structure shows extraordinary thermal stability and the photo-induced refractive index change (i.e. the absolute value of the ordinary and extraordinary refractive index) increases with the annealing temperature [87].

fs laser-induced oriented nanostructure (i.e. sub wavelength structure induced by fs laser irradiation) has been observed inside silica glass in static mode (i.e. irradiated without moving the sample) [31]. For a long time, this sub wavelength structure was only observed in silica glass. But recently, fs laser-induced nanostructure in other glasses have been reported even at the moving mode (i.e. continuous moving the sample during laser irradiation): firstly, closely to $\mathrm{SiO}_{2}$ (e.g. doped silica [88]), $\mathrm{GeO}_{2}[34,89]$ (a glass with an atomic arrangement close to $\mathrm{SiO}_{2}$ ), $\mathrm{TiO}_{2}$ containing silicate glasses [7, 35], and two multicomponent borosilicate glasses (with a few mol\% of $\mathrm{Na}_{2} \mathrm{O}$ and $\mathrm{Al}_{2} \mathrm{O}_{3}, \mathrm{BK} 7$ (Schott) [7], and Borofloat 33 (Schott) [90], or alkali-free aluminoborosilicate glass [36]). For all of them, a nanostructure similar to the one observed in $\mathrm{SiO}_{2}$ is described. For glasses mentioned above, the form birefringence appears to be related to self-organized density and nanostructure modulation due to a silica decomposition within nanoplanes into understoichiometric silica and oxygen nanobubbles [4]. In addition, narrow range of pulse durations (150-200 fs) is required to observe the birefringence in some multicomponent glasses (e.g. Borofloat 33 glass) [36].

The underlying mechanism of this phenomenon is not well understood. Zimmermann et al. [90] reported that the irradiation produces small nanopores (10-20 nm thick) which start to selforganize in parallel nanoplanes as well as elongated sheets of up to $400 \mathrm{~nm}$ length along the pulse accumulation. The average nanoplane distance changes from $250 \mathrm{~nm}$ to $60 \mathrm{~nm}$ [7], but it would be necessary to compare with the same laser parameters because this quantity depends on them $[80,91,92]$.

As shown in Figure 15, Zimmermann et al. [92] demonstrate that nanostructure formation is beginning with spherical voids after the first incident fs laser pulse which begin to elongate perpendicular to the scanning direction with further irradiation and the appearance of cracks along the scanning direction. With the increase of pulse overlap, these cracks and randomly aligned voids build the template for the subsequent pore growth perpendicular to the laser 
polarization. The nanostructure formation process and the following period reduction occur by increasing sheet number and mutual realignment forming a more porous glass matrix.
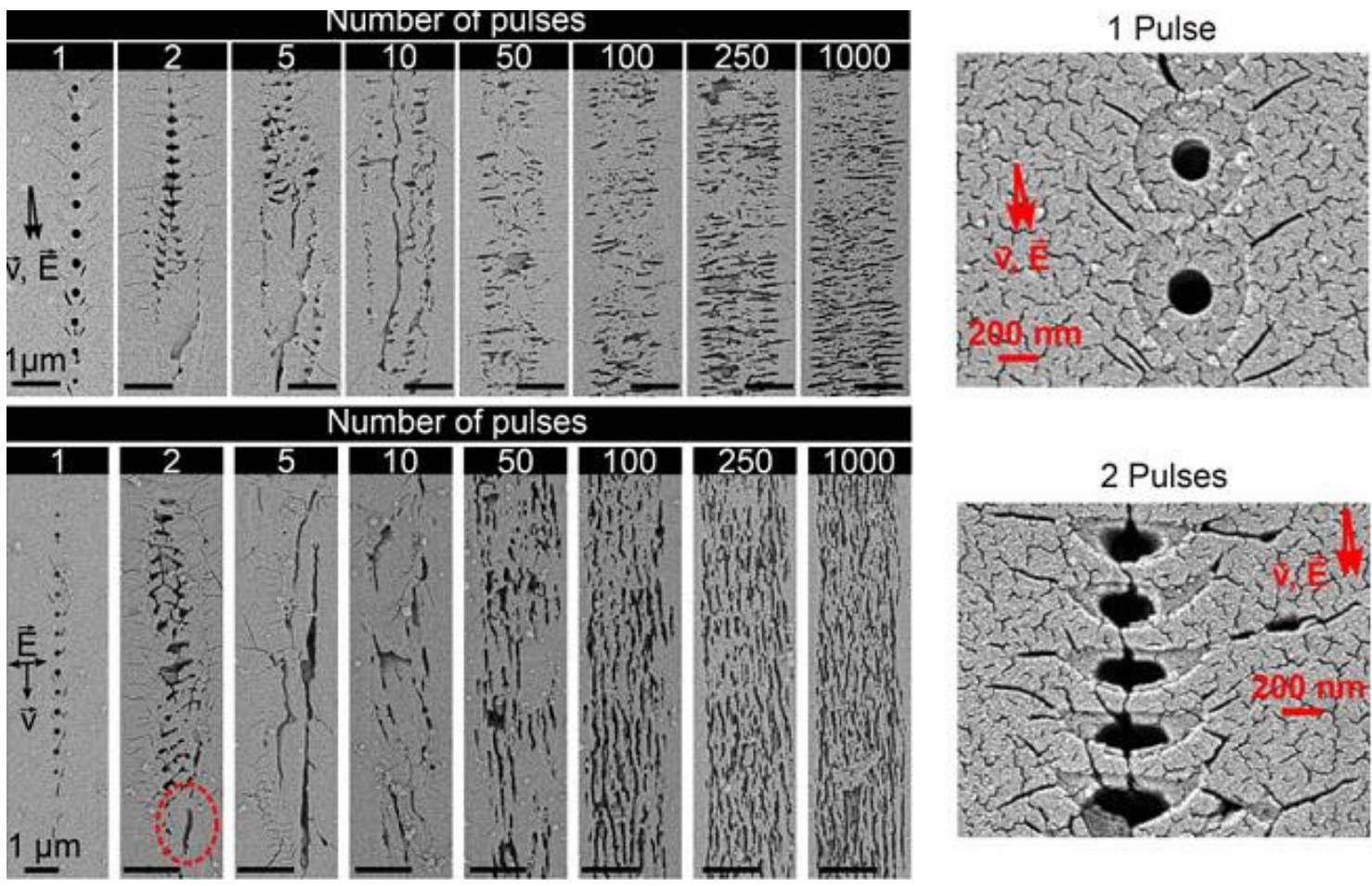

Figure 15 SEM images of nanostructure for parallel scanning (top) and perpendicular scanning (bottom) after polishing and etching (fused silica). The speed of the sample movement was $6.97,2.98,1.25,0.6,0.125,0.06$, 0.023 , and $0.006 \mathrm{~mm} / \mathrm{s}$ from left to right, the right two images are the zoom of SEM images (cited from [92])

A third threshold T3 is the voids formation (disruptive modification). At high pulse energy, a void surrounded by a high refractive index crust occurs. This phenomenon can be explained by the Coulomb repulsion between the ions, which generates pressures larger than the material's Yong's modulus. This leads to a shockwave, resulting in a less dense or hollow core in the focal volume. This kind of modification has potential applications in 3D optical storage [93] and photonic crystal [94].

The domains of these fs laser-induced thresholds in glasses are dependent on laser parameters. As illustrated in Figure 16a, at low pulse duration (45-60 fs), all four regions occur. T1 threshold decreases on with the increase of NA. Figure $16 \mathrm{~b}$ displays that at $160 \mathrm{fs}$, T3 moving down and becoming dependent on NA at strong focusing. Figure 16c shows that at $200 \mathrm{fs}, \mathrm{T} 3$ moving a little bit lower. It is worth noting that at this pulse duration, $\mathrm{T} 1$ is coincident with $\mathrm{T} 2$ at strong focusing. As illustrated in Figure $16 \mathrm{~d}$, at $250 \mathrm{fs}$ pulse duration, T3 is down-shifting and there is no observation of isotropic index zone. It is worth to point out that at this repletion with strong focusing, and pulse energy below self-focusing (SF), it is suitable for transversal microfluidic 
writing, due to the vanish of filamentation. At larger pulse duration, there will be no big change of this diagram because the threshold becomes pulse independent [82].

In addition, Lancry et al. [71] demonstrated that T1 is not dependent on doping and T2 decreases with the Ge, P doping, but increases on $\mathrm{F}$ doping.
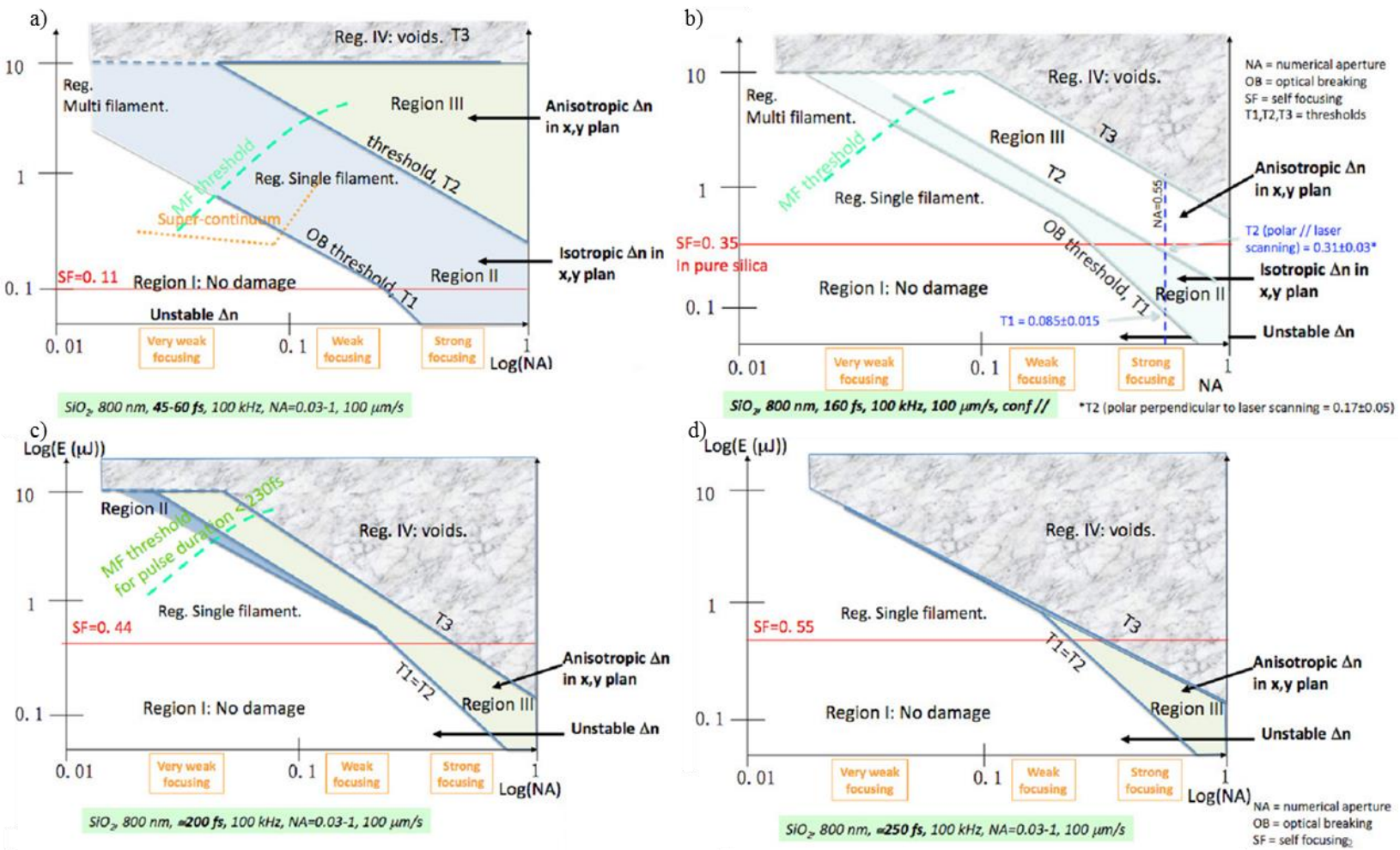

Figure 16 Pulse energy versus numerical aperture (NA) and pulse duration: synthetic diagram for a) small pulse duration, b) $160 \mathrm{fs}, \mathrm{c}) 200 \mathrm{fs}$, and d) $250 \mathrm{fs}$ (region Il disappears) with silica (cited from [82])

At high repetition rate, due to the heat accumulation, causing glass local melting, nanostructures can be erased. This can affect the properties of the fs laser-modified glass. The retardance of fs laser-induced nanostructures in Ge-doped glasses decreases with the increase of repetition rate. The relative retardance decays for large doping concentrations (i.e. 12.5 or 19 wt\% in Ge) is higher. This can be explained by the increasing glass density from pure silica $\left(\sim 2.2 \mathrm{~g} / \mathrm{cm}^{3}\right)$ to 19 wt\% Ge doping $\left(\sim 2.45 \mathrm{~g} / \mathrm{cm}^{3}\right)$ and slight increase $<2 \%$ in both heat capacity and thermal conductivity, which can be neglected, resulting in smaller heat diffusivity and hence the pronounced accumulation of heat between two consecutive pulses [95].

fs laser irradiation can create periodic bubbles (pearl-chains) along the laser propagation direction [96] or scanning direction [97]. As shown in Figure 17a, the thermally affected zones surrounding bubbles are clearly visible, especially at low pulse energies. Because of heat accumulation, if the glass temperature reaches a critical temperature, rapid bubble nucleation is 
triggered. Bubble formation, however, distorts the laser beam at the focus, effectively switching off the heat source and stopping further bubble nucleation. When the laser beam moves out of the modified region, the process of glass heating and bubble formation starts again. The formation of the first "seed bubbles" inherently modifies the local heat transfer conditions and may substantially increase the material heating rate (Figure 17b). This observation coupled with the random nature of the bubble nucleation process can be used to explain why discontinuous patterns may form at very low-pulse energy close to the threshold for bubble formation. This process is similar to a siphon (Figure 17c). When the water level in the vessel fills the siphon elbow (i.e high $W_{c r}$ ), auto-siphoning is triggered. For high elbows, (i.e. high $W_{c r}$ ), the autosiphoning purge of the system is triggered. For high elbows, random stationary fluctuations in the faucet flow are smoothed out due to the law of large numbers. This mechanism can be used to explain the transition from erratic to organized process [2].

a)

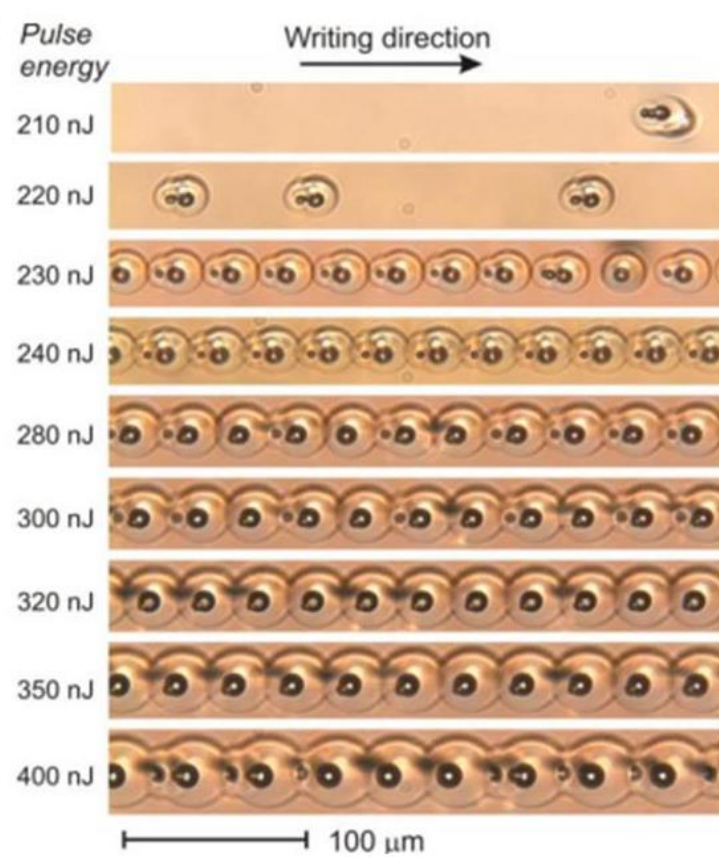

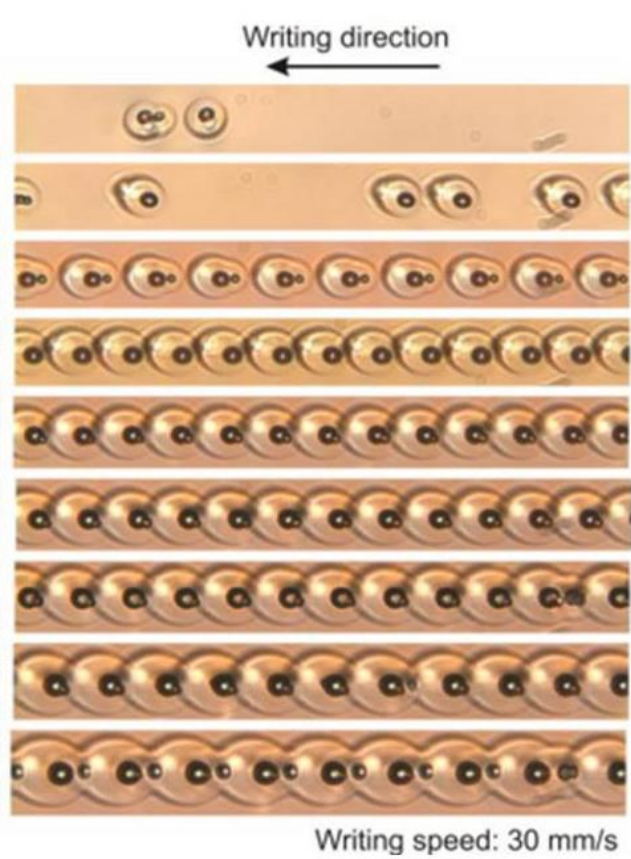

c) b)

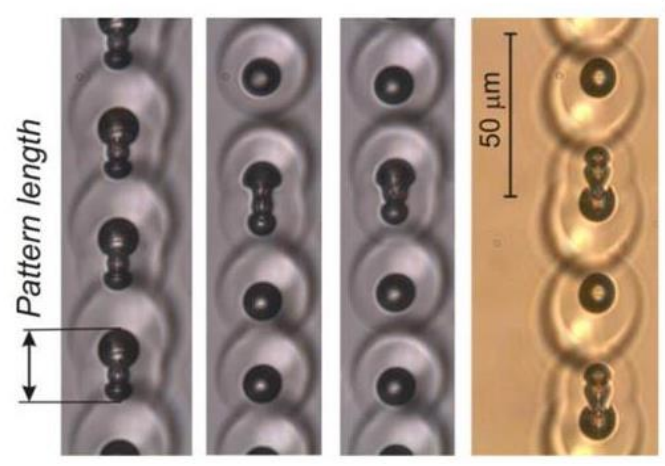

Laser source

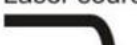

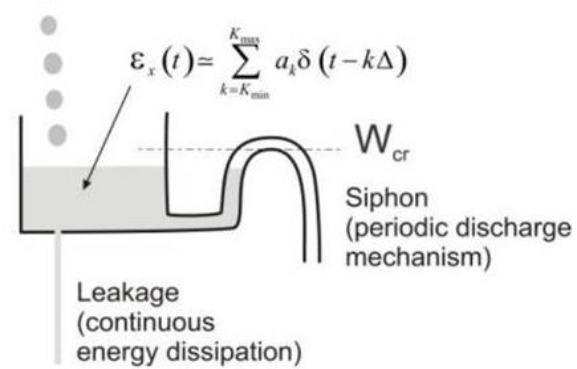

Figure 17 a) Effect of increasing the pulse energy at $30 \mathrm{~mm} / \mathrm{s}$ in fused silica. The patterns were written approximately $200 \mu \mathrm{m}$ below the surface, b) evidences of "frozen-in" bubble coalescence events, and c) qualitative fluid analogy of the laser energy accumulation-discharge process (cited from [97]) 
Another important material for 3D laser fabricating is photo-polymerizable resin, in which twophoton photo polymerization via a pulsed NIR laser [98]. When a high intensity NIR laser beam passes through a molecule, a virtual state is formed when the first photon is absorbed. The lifetime of this virtual state is very short (several fs). Absorption of the second photon before the decay of this virtual state is needed. For single-photon photo-polymerization, the modifications are achieved in the surface and 3D modifications are done by a layer-by-layer technique. For two-photon photo-polymerization, it is negligible linear absorption for common polymers in the laser wavelength region, intrinsic 3D spatial fabricating, and being higher accuracy (120 nm) [99]. This technique has potential applications in 3D printing, photonic crystals, and optical data storage [41].

\subsubsection{Nonlinear effects}

At low light intensity, the property of matter is independent on the light intensity, in which the superposition principle works. However, at high light intensity (i.e. laser), the property of the matter can be modified. As shown in Figure 3b, with the increase of laser intensity, the energy of electrons accelerated in the laser field increases. The regime of conventional nonlinear optics with electrons bound to atoms is replaced by the one of relativistic nonlinear optics with free electrons in relativistic plasmas [100]. At the highest intensities, even protons become relativistic (i.e. nuclear optics), in which various nuclear processes (e.g. fusion) occur.

Ponderomotive energy $\left(U_{p}\right)$ describes the average oscillation energy that is needed by a free electron in the laser radiation field, which is given as

$$
U_{p}=\frac{e^{2} E_{a}^{2}}{4 m_{e} \omega^{2}}=\frac{e^{2} I}{2 c \varepsilon_{0} m_{e} \omega^{2}} \quad \text { with } \quad I=\frac{1}{2} c \varepsilon_{0} E_{a}^{2}
$$

Where e is electron charge $\left(1.60 \times 10^{-19} \mathrm{C}\right), \mathrm{E}_{\mathrm{a}}$ is the linearly polarised electric field amplitude, $\mathrm{m}_{\mathrm{e}}$ is the electron mass $\left(9.11 \times 10^{-31} \mathrm{~kg}\right), \varepsilon_{0}$ is the permittivity in free space $\left(8.85 \times 10^{-12} \mathrm{~F} / \mathrm{m}\right), \mathrm{c}$ is the light speed in vacuum $\left(3 \times 10^{8} \mathrm{~m} / \mathrm{s}\right), \omega$ is the angular frequency of the laser, and I is the laser intensity. Some remarks about the units are shown in Appendix X.

The above formula can be numerically given as [62]

$$
\mathrm{U}_{\mathrm{p}}[\mathrm{eV}]=9.34 \times 10^{-20} \times(\lambda[\mathrm{nm}])^{2} \times \mathrm{I}\left[\mathrm{W} / \mathrm{cm}^{2}\right]
$$

$U_{p}$ can be employed to determine if a laser filed is strong enough by comparison of the characteristic ionisation energy $\left(E_{1}\right)$ [62]. For the ionisation process the strong field regime occurs when $U_{p}>E_{1}$. 
The investigation of laser peak power from $10^{6} \mathrm{~W} / \mathrm{cm}^{2}$ (or $\mathrm{MW} / \mathrm{cm}^{2}$ ) to $10^{9} \mathrm{~W} / \mathrm{cm}^{2}$ (or $\mathrm{GW} / \mathrm{cm}^{2}$ ) scale opens the era of discovering nonlinear effects (e.g. harmonics generation and selffocusing). Because in this power range, the applied laser electric field (typically $10^{5}-10^{8} \mathrm{~V} / \mathrm{m}$ ) is comparable to $E_{a}$ (e.g. classical hydrogen atom is around $5.1 \times 10^{11} \mathrm{~V} / \mathrm{m}$, corresponding to intensity of $3.5 \times 10^{16} \mathrm{~W} / \mathrm{cm}^{2}$ for linear polarization and $7.0 \times 10^{16} \mathrm{~W} / \mathrm{cm}^{2}$ for circular polarization [64]). The susceptibility of material is dependent on the laser electric field. In that case, the refraction index of the material is not a constant but dependent on the laser intensity [101]. Therefore, the response of the material to the laser field is nonlinearly dependent on laser intensity.

When high intensity laser pulses (typical $>10^{18} \mathrm{~W} / \mathrm{cm}^{2}$ ) interact with a target matter, the atoms of the material are ionized, forming plasma. Electrons are preferentially accelerated forward, towards rear side of the target (i.e. creating fast forward electron stream), followed by ion acceleration by the electric charge-separation field (i.e. ion acceleration). The relativistic plasma emits high-energy electrons, ions, incoherent X-rays, coherent high-order harmonics up to the Xray spectral region, and generates secondary particles and radiation [102].

\subsubsection{Optical breakdown}

Optical breakdown is the main nonlinear electronic excitation mechanisms leading to laserinduced modifications in glass (e.g. refractive index changes). It is characterized by the localized dense plasma formation around the geometrical focus, due to the initial multi-photon excitation of electrons, followed by inverse Bremsstrahlung, impact ionization, and electron avalanche processes [9].

The photo-generated plasma due to the processes of optical breakdown transfers its energy to the glass, leading to local heating and melting, in which the material undergoes a structural modification, which may cause a localized permanent change. For example, the molten glass is rapidly cooled, leading to re-solidification of the silica glass, thus and increasing the density and refractive index in the irradiated region. 


\subsubsection{Second harmonic generation}

\subsection{Definition}

As shown in Figure 18a, second harmonic generation (SHG) is a nonlinear optical effect, in which the frequency of incident light is doubled. For comparison, two-photon excited fluorescence (Figure 18b) and coherent anti-Stokes Raman scattering (Figure 18c) are also presented.

As discussed above, the response of the matter to a high intense applied electromagnetic field (ca. $>10^{9} \mathrm{~V} / \mathrm{m}$ ) is nonlinear in the amplitude of the field. The relevant response is described by the polarization induced by the field. The dipolar polarization ( $\mathbf{P}$ ) in given in the form of power series as

$$
\mathbf{P}=\mathbf{P}_{\mathrm{L}}+\mathbf{P}_{\mathrm{NL}}=\mathbf{P}^{(1)}+\mathbf{P}^{(2)}+\mathbf{P}^{(3)}+\ldots=\varepsilon_{0}\left(\chi^{(1)} \mathbf{E}+\chi^{(2)} \mathrm{EE}+\chi^{(3)} \mathrm{EEE}+\ldots\right)
$$

Where $\varepsilon_{0}$ is the free-space permittivity, $\mathbf{P}_{\mathrm{L}}$ is the linear part, $\mathbf{P}_{\mathrm{NL}}$ is the nonlinear part, $\mathbf{P}^{(n)}$ is the $\mathrm{n}^{\text {th }}$ order electric dipole, and $\chi^{(\mathrm{n})}$ is the $\mathrm{n}^{\text {th }}$ dipolar susceptibility, a tensor of rank $\mathrm{n}+1$, which is used to characterize the nonlinear optical response of the matter. Typical values of $\chi^{(2)}$ for dielectric crystals, semiconductors, and organic materials lie in the range from $10^{-24}$ to $10^{-21} \mathrm{~m} / \mathrm{V}$, and the typical values of $\chi^{(3)}$ for glasses, crystals, semiconductors, semiconductor-doped glasses, and organic materials are $10^{-34}$ to $10^{-29}(\mathrm{~m} / \mathrm{V})^{2}[103]$.
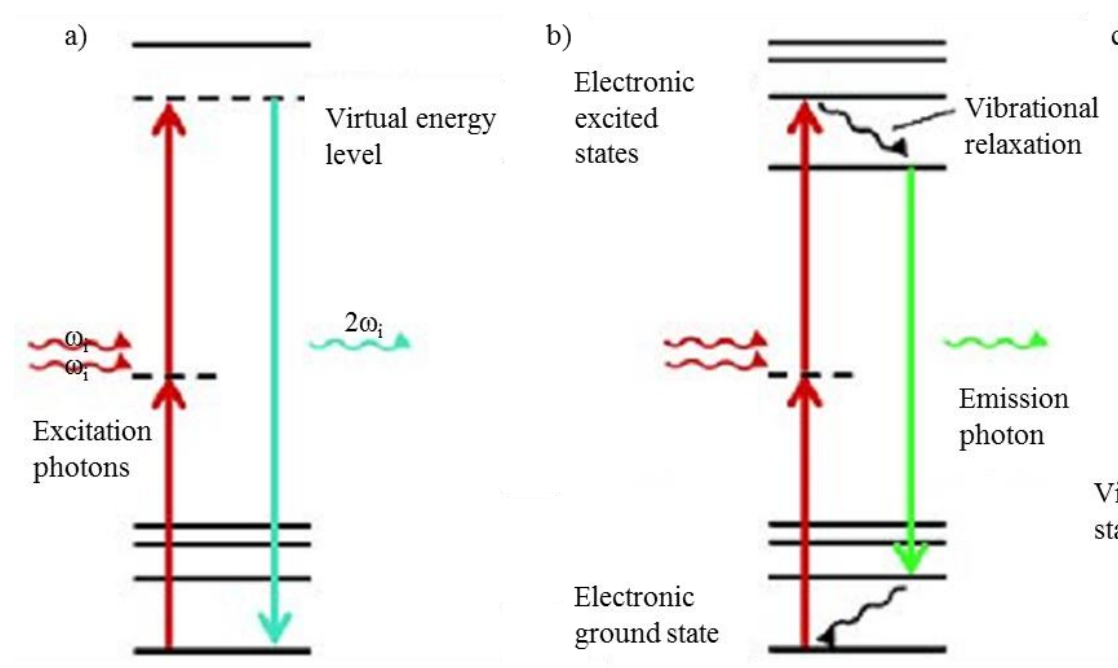

Figure 18 Energy level diagrams of a) second harmonic generation, b) two-photon excited fluorescence, and c) coherent anti-Stokes Raman scattering (after [104])

As shown in Figure 18a, when an intense light (i.e. laser) interacts with a matter (exhibiting noncentrosymmetric structure), the vibrating electric field of the incident beam leads to the polarization of the matter, reemitting light at $\omega_{i}$ (the original frequency) and $2 \omega_{i}$ (i.e. doubled 
frequency or half the wavelength). SHG does not involve the absorption of the photons but involve virtual energy transition and the energy is conserved.

For two-photon excited fluorescence, as shown in Figure 18b, a fluorophore molecule is excited by the simultaneous absorption of two photons (in IR spectral range). It occurs through the absorption of two photons by intermediate virtual energy levels (i.e. real energy transition of electrons). In that case, using ultra-fast (i.e. fs) pulsed lasers, the response time of SHG is at the fs level, which is about several orders of magnitude faster than the ns response of fluorescence, which is suitable for fast and sensitive detection [105].

As illustrated in Figure 18c, coherent anti-Stokes Raman scattering is a third-order nonlinear optical process (i.e. four-wave mixing). The signal is originating from the vibrational motion of the molecules of the sample (typical the nuclear vibrations of the chemical bonds). Because this process produces a coherent signal, this leads to orders magnitude larger than spontaneous Raman emission. The frequency difference between the two input beams is selected to match the frequency of a vibrational energy state of the molecule. If this resonant condition is fulfilled, these stimulating beams will enhance the selected vibrational mode. Using the third beam, this motion can be probed. The resulting coherent anti-Stokes Raman scattering signal (with frequency of $\omega_{p r}+\omega_{p}-\omega_{s}$ ) is generated at the blue shifted (antiStokes) spectral range compared to the input beams [104].

\subsection{The propagation of SHG}

Maxwell's equations are useful for problems involving wave propagation, energy transfer, and boundary conditions. Macroscopic differential form is given as

$$
\begin{aligned}
& \nabla \times \mathbf{E}=-\frac{\partial \mathbf{B}}{\partial t} \\
& \nabla \times \mathbf{H}=\mathbf{J}+\frac{\partial \mathbf{D}}{\partial t} \\
& \nabla \cdot \mathbf{D}=\rho \\
& \nabla \cdot \mathbf{B}=0
\end{aligned}
$$

Where $\mathbf{E}$ is the electric field vector, $\mathbf{B}$ is the magnetic flux density, $\mathbf{H}$ is the magnetic field vector, $\mathbf{J}$ is the current density vector, $\mathbf{D}$ is the electric flux density, and $\rho$ is the sources for the electromagnetic field (volume charge density). For the source free region $\mathbf{J}=0$ and $\rho=0$.

The constitutive relations are the following:

$$
\begin{aligned}
& \mathbf{D}=\varepsilon_{0} \mathbf{E}+\mathbf{P} \\
& \mathbf{E}=\mu_{0} \mathbf{H}+\mathbf{M}
\end{aligned}
$$


Where $\varepsilon_{0}$ is the vacuum permittivity, $\mathbf{P}$ is the induced electric polarization, $\mu_{0}$ is the vacuum permeability, and $\mathbf{M}$ is the induced magnetic polarization. For nonmagnetic matter $\mathbf{M}=0$.

Based on the above equations, we get

$$
\nabla \times \nabla \times \mathbf{E}=-\mu_{0} \varepsilon_{0} \frac{\partial^{2} \mathbf{E}}{\partial \mathrm{t}^{2}}-\mu_{0} \frac{\partial^{2} \mathbf{P}}{\partial \mathrm{t}^{2}}
$$

Where $c$ is the speed of light in a vacuum, $\mu_{0} \varepsilon_{0}=1 / c^{2}$.

To solve the above equation, several simplifications are assumed:

1) the $\mathbf{P}_{\mathrm{NL}}$ is treated as a small perturbation to the total induced polarization, considering the nonlinear effects are relatively weak in matter,

2) $\quad \nabla \times \nabla \times \mathbf{E}=\nabla(\nabla \cdot \mathbf{E})-\nabla^{2} \mathbf{E}=-\nabla^{2} \mathbf{E}$

So we get

$$
\nabla^{2} \mathbf{E}=\frac{1}{c^{2}} \frac{\partial^{2} \mathbf{E}}{\partial t^{2}}+\mu_{0} \frac{\partial^{2} \mathbf{P}}{\partial t^{2}}
$$

This equation can be translated in the Fourier domain as

$$
\nabla^{2} \tilde{\mathbf{E}}(\omega)=\frac{\omega q(\omega)}{c^{2}} \tilde{\mathbf{E}}(\omega)+\frac{\omega^{2}}{c^{2}} \tilde{\mathbf{P}}_{\mathrm{NL}}(\omega)
$$

Where $\varepsilon$ is the dielectric constant.

The temporal variation of electric field at $\omega$ and $2 \omega$ are neglected, and the wave at second harmonic frequency will follow the wave equation as

$$
\nabla^{2} \tilde{\mathbf{E}}(2 \omega)=\frac{\omega^{2} \varepsilon(2 \omega)}{c^{2}} \tilde{\mathbf{E}}(2 \omega)+\frac{\omega^{2}}{c^{2}} \tilde{\mathbf{P}}^{(2)}(2 \omega)
$$

Considering low conversion efficiency, the amplitude of $\mathbf{E}_{\omega}$ remains essentially constant over the interaction length, L, the intensity of SHG is given as

$$
\begin{aligned}
& \mathrm{I}_{2 \omega}(\mathrm{L})=\frac{(2 \omega)^{2}}{8 \varepsilon_{0} \mathrm{c}^{3}} \frac{\left|\chi^{(2)}\right|^{2}}{\mathrm{n}_{\omega}^{2} \mathrm{n}_{2 \omega}} \mathrm{L}^{2}\left(\frac{\sin (\Delta \mathrm{kL} / 2)}{\Delta \mathrm{kL} / 2}\right)^{2} \mathrm{I}_{\omega}^{2} \\
& \text { with } \mathrm{I}_{\omega}^{2}=\frac{1}{2} \varepsilon_{0} \mathrm{cn} \mathrm{n}_{\omega}\left|\mathrm{E}_{\omega}\right|^{2}, \quad \Delta \mathrm{k}=\mathrm{k}_{2 \omega}-2 \mathrm{k}_{\omega}=\frac{2 \omega\left(\mathrm{n}_{\omega}-\mathrm{n}_{2 \omega}\right)}{\mathrm{c}}, \quad \mathrm{L}_{\mathrm{c}}=\frac{\pi}{\Delta \mathrm{k}}
\end{aligned}
$$

Where $L$ is the nonlinear matter length, $\Delta k$ is the wave vector mismatch, $k$ is the wave vector of the electric field, and $L_{c}$ is the coherence length where the SHG efficiency reaches the maximum (SHG intensity oscillates as a function $L$, with a period of $L_{c}$ ). 


\subsection{Phase Matching}

Based on the above analysis, decreasing $\Delta \mathrm{k}$ is important for efficient SHG in homogeneous media. Phase matching happens when a constant phase relationship is maintained between the generated and propagating waves.

There are many methods to achieve phase matching. For birefringent phase matching (BPM), at certain angle $\theta$, the ordinary polarized fundamental and the extraordinary polarised second harmonic own the same refractive index, achieving $\Delta \mathrm{k}=0$. In that case, BPM is sensitive to the propagation angle and this kind phase matching is known as critical phase matching. Another technique, noncritical phase matching can be used, in which the beams propagate down one of the axes of the nonlinear crystal and the temperature of the crystal is adjusted (generally, ne is much more temperature sensitive than no, indicating a phase matching condition can be achieved). Instead of a homogeneous nonlinear crystal material, a material with spatially modulated nonlinear properties can be used [106]. This is the basis quasi-phase matching, which can be achieved by periodic poling of nonlinear crystal materials.

SHG is forbidden in the bulk of centro-symmetric matter within the dipolar approximation. However, this symmetry is broken at the interface between two centro-symmetric media, allowing for SHG [107]. SHG can also be obtained from the random distributed nano-crystals because the emission from a nano-crystal can lose its phase relation with the other nano-crystals due to multiple scattering [30]. Vigouroux et al. [29] have observed isotropic bulk SHG signals in lithium niobium silicate glass ceramics. It can be explained by the radial distribution of the $\mathrm{LiNbO}_{3}$ crystalline in spherulite domains: a macroscopic 10-15 $\mu \mathrm{m}$ c-axis oriented poly-crystallite with consecutive domains spatially radiating incoherent SHG signal. Because spherulites are sparsely distributed in the matrix with distances larger than the coherence length (i.e. 8-10 $\mu \mathrm{m}$ ), they radiate uncorrelated SHG fields. The total SHG intensity is the sum of the individual contribution.

In this thesis, the absolute SHG intensity cannot be compared because of different scattering from place to place. In a disordered material, we could expect a centre-symmetric material and from that the SHG is forbidden. This is obviously experimentally not the case and it is always the case in glass-ceramics (a mixing between glass and nano-crystals). So, we can collect the sum of the intensity coming from each nano-crystal whatever their orientation. 


\subsection{SHG observation and the related applications}

SHG is discovered by Franken et al. [108] in 1961 by projecting a ruby laser (6943 $⿱$ ) through crystalline quartz, generating a SHG (at $\sim 3472 \AA$ ). Then it is described by Bloembergen and Pershan rules for the interaction of light in non-linear medium.

SHG can be obtained by using nonlinear crystal. Figure 19 displays the crystallographic interrelationships between physical properties and noncentrosymmetric crystal. For example, $\mathrm{LiNbO}_{3}$ belongs to $3 \mathrm{~m}\left(\mathrm{C}_{3 \mathrm{v}}\right)$, exhibiting SHG properties (marked by red circle in Figure 19). The nonlinear optical coefficients of common materials are shown in Appendix XI.

Table 2 lists the applications and proposed nonlinear materials. We can see that lithium niobate $\left(\mathrm{LiNbO}_{3}\right)$ has a wide ragne of applications in modulation and switching, directional couplers, single mode fiber optic sensors, and Storage media.

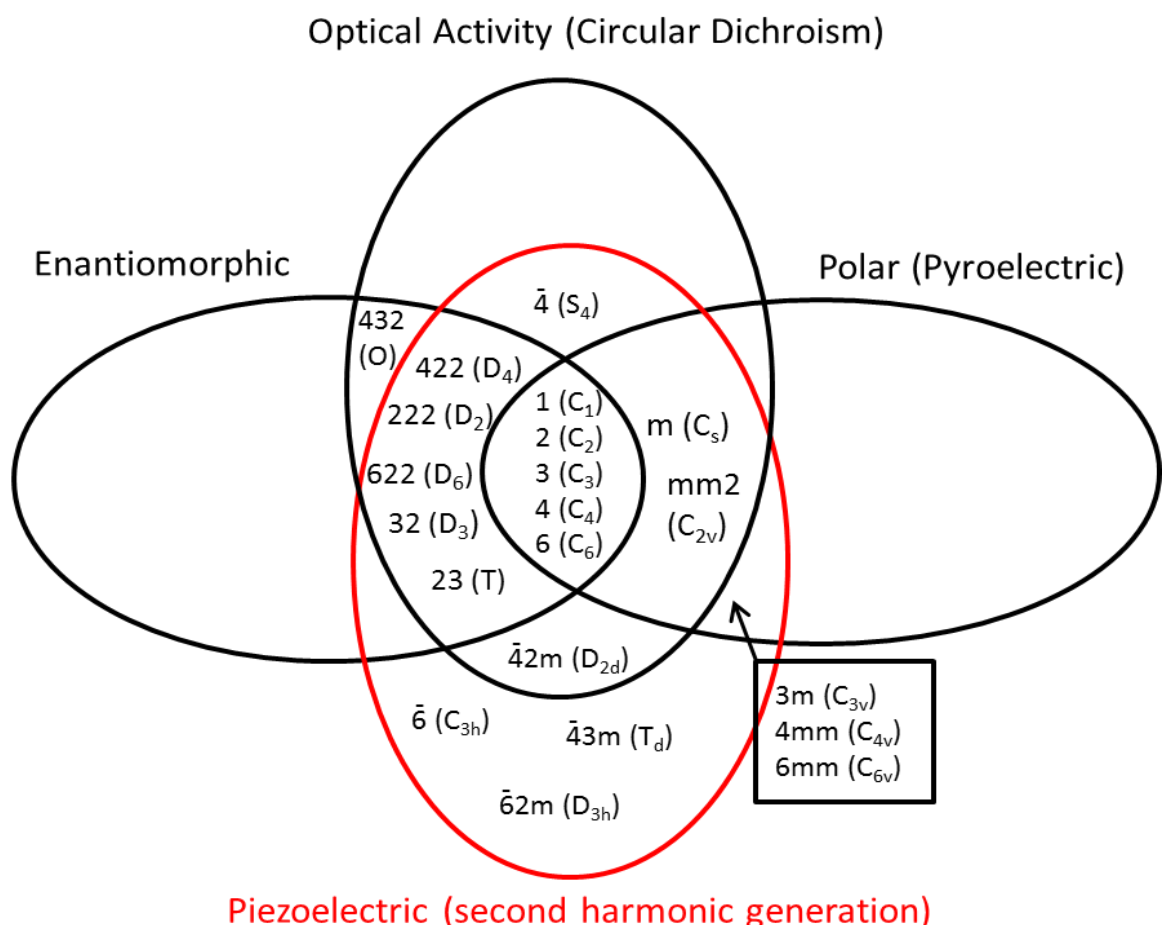

Figure 19 Interrelationships of non centro-symmetric crystal classes in both Hermann-Mauguin and Schoenflies symbols (after [109])

At a surface or interface, the bulk symmetry is broken, allowing the second order nonlinear process even in a centrosymmetric matter. This could be used to investigate the surface information. In addition, artificial SHG can also be obtained by electric field poling, thermal poling or optical poling (see details in Appendix XII). This technique is widely used in photonic device fabrication. 
Table 2 Applications and proposed nonlinear materials

\begin{tabular}{|c|c|}
\hline Application & Material \\
\hline \multicolumn{2}{|l|}{ Communications } \\
\hline Modulation and switching & $\mathrm{LiNbO}_{3}, \mathrm{KTiOPO}_{4}, \mathrm{II}-\mathrm{V}$-semiconductor, multiquantum well's \\
\hline Directional couplers & $\mathrm{LiNbO}_{3}$ \\
\hline Lenses & $\mathrm{LiNbO}_{3}$ with $\mathrm{As}_{2} \mathrm{~S}_{3}$-glass \\
\hline EO Grating modulator & $\mathrm{Ti}: \mathrm{LiNbO}_{3}$ \\
\hline \multicolumn{2}{|l|}{ Sensors } \\
\hline Imaging/processing & $\mathrm{KTiOPO}_{4}, \mathrm{BaTiO}_{3}, \mathrm{KNBO}_{3}$ \\
\hline Single mode fiber optic sensors & $\mathrm{LiNbO}_{3}$ \\
\hline \multicolumn{2}{|l|}{ Instrumentation } \\
\hline Fabry-Perot interferometers & $\mathrm{LiNbO}_{3}$ \\
\hline Mach-Zehnder interferometers & $\mathrm{LiNbO}_{3}$ \\
\hline Autocorrelators & $\mathrm{KH}_{2} \mathrm{PO}_{4}$ \\
\hline Two photon spectroscopy & $\mathrm{KTiOPO}_{4}, \mathrm{KH}_{2} \mathrm{PO}_{4}$ \\
\hline Holographic interferometry & $\mathrm{LiNbO}_{3}, \mathrm{KTiOPO}_{4}, \mathrm{BaTiO}_{3}$ \\
\hline \multicolumn{2}{|l|}{ Computing } \\
\hline Holographic gratings & $\mathrm{BaTiO}_{3}, \mathrm{KTiOPO}_{4}, \beta-\mathrm{BaB}_{2} \mathrm{O}_{4}$ \\
\hline Storage media & $\mathrm{LiNbO}_{3}$ \\
\hline Laser frequency doubling & $\mathrm{SrBaNbO}_{3}, \mathrm{Fe}: \mathrm{LiNbO}_{3}, \mathrm{Bi}: \mathrm{SiO}_{2}$, III-V-and II-VI-semiconductor \\
\hline
\end{tabular}

\subsubsection{Self-focusing and plasma defocusing}

For isotropic material like glass, the one with inversion symmetry, the second order response vanished. Considering the order higher than third is negligible, only the $1^{\text {st }}$ and the $3^{\text {rd }}$ in dipolar polarization are approximation considered as

$$
\mathbf{P}=\mathbf{P}^{(1)}+\mathbf{P}^{(3)}=\varepsilon_{0}\left(\chi^{(1)}+\frac{3}{4} \chi^{(3)}|\mathbf{E}|^{2}\right) \mathbf{E}
$$

Considering

$$
\begin{gathered}
\mathrm{n}=\sqrt{1+\chi}=\sqrt{1+\chi_{\mathrm{L}}+\chi_{\mathrm{NL}}} \approx \mathrm{n}_{0}\left(1+\frac{1}{2 \mathrm{n}_{0}^{2}} \chi_{\mathrm{NL}}\right) \\
\text { with } \mathrm{n}_{0}=\sqrt{1+\chi^{(1)}}
\end{gathered}
$$

Using a Taylor expansion, because $\chi_{\mathrm{NL}} \ll \mathrm{n}_{0}^{2}$,

$$
\mathrm{n}=\mathrm{n}_{0}+\frac{3 \chi^{(3)}}{8 \mathrm{n}_{0}}\left|\mathrm{E}_{\omega}\right|^{2}=\mathrm{n}_{0}+\mathrm{n}_{2} \mid
$$

Where $\mathrm{I}$ is the laser intensity, $\mathrm{n}_{0}$ is the linear refractive index component, and $\mathrm{n}_{2}$ is the nonlinear refractive index, which is usually determined by the polarizability of the ionic components of the 
material. In conventional glass systems, $\mathrm{n}_{2}$ is determined by the amount of nonbridging oxygens and thus on the concentration of glass modifiers [103].

The third order nonlinearity (or Kerr-type nonlinear susceptibility) leads to the refractive index being dependent on laser intensity (i.e. Kerr effect). This formula describes many nonlinear effects in ultra-fast pulse laser, which will be discussed as following.

1) The spatial dependence results in a laser intensity dependent focusing lens for laser beams.

For Gaussian beam profile, the laser intensity is larger at the centre than the edge. For fused silica, $\mathrm{n}_{2}$ is $2.1-3.54 \times 10^{-16} \mathrm{~cm}^{2} / \mathrm{W}$. In that case, the refractive index is larger at the centre that the one in the edge. This spatial distribution of refractive index leads to a lens-like effect (i.e. Kerr-lens).

For a CW Gaussian beam, catastrophic self-focusing occurs when the power is larger than the critical value [110]

$$
\mathrm{P}_{\mathrm{cr}}=3.77 \lambda^{2} / 8 \mathrm{n}_{0} \mathrm{n}_{2}
$$

where $\lambda$ is the laser wavelength, $n_{0}$ and $n_{2}$ are the refractive index $\left(n=n_{0}+n_{2} l\right)$ mentioned above.

The Gaussian beam then collapses to a singularity at a distance given as [110]

$$
\mathrm{Z}_{f}(\mathrm{P})=\frac{0.367 \mathrm{ka}^{2}}{\left[\left(\sqrt{\mathrm{P} / \mathrm{P}_{\mathrm{cr}}}-0.852\right)^{2}-0.0219\right]^{0.5}}
$$

Where $a$ is the Gaussian beam radius (at the $1 / e^{2}$ laser intensity), $k=2 \pi / \lambda$ is the wave number, $\mathrm{ka}^{2}$ is the beam Rayleigh length, $\mathrm{P}$ is the laser power, and $\mathrm{P}_{\mathrm{cr}}$ is the critical power for selffocusing.

For a convergent beam, with the external objective (focal length is $f$ ), the position of the collapse (or the beginning of filament) is given as [111]

$$
z_{f}^{\prime}=\frac{z_{f} f}{Z_{f}+f}
$$

It is like the case with two lenses with the focus depths of $Z_{f}$ and $f$, respectively. For positive focusing, $Z_{f}^{\prime} \leq f$, the self-focusing occurs before the geometric al focus. 
For pulsed laser, P varies with time. With some approximation (e.g. dispersionless medium), selffocusing can be described by a moving focus mode [110].

When the laser beam power is larger than $\mathrm{P}_{\mathrm{cr}}$ (e.g. 4.3 MW for fused silica [112]), self-focusing prevails over diffraction leading to the collapse of the beam on itself, without considering other nonlinear effects. Due to the self-focusing effect, the laser peak intensity $\left(I_{s f}\right)$ will be increased to

$$
\mathrm{I}_{\mathrm{sf}}=\frac{\mathrm{I}}{1-\mathrm{P} / \mathrm{P}_{\mathrm{cr}}}
$$

when $P<0.25 P_{c r}[113]$, where $P$ is the real laser peak power.

When $\mathrm{P}>0.25 \mathrm{P}_{\mathrm{cr}}$ the laser beam can be balanced by electron plasma defocusing, another kind of nonlinear effect. At high laser intensity, the material is highly ionized, generating electronhole plasma. The ionization of the matter initially occurs in the centre of the laser beam, in which the intensity is most significant. Creating under-dense plasmas decreases the local index of the medium, leading to beam defocusing [111]. The real part of the refractive index can be given as (when $\left.\omega_{p} / \omega<n_{0}\right)$

$$
\begin{aligned}
& \mathrm{n}_{0}-N / 2 \mathrm{n}_{0} \mathrm{~N}_{\mathrm{c}} \text { with } \mathrm{N}_{\mathrm{c}}=\omega^{2} \varepsilon_{0} \mathrm{~m}^{*} / \mathrm{e}^{2} \\
& \omega_{\mathrm{p}}=\sqrt{\frac{\rho \mathrm{e}^{2}}{\varepsilon_{0} \mathrm{~m}_{\mathrm{e}}}}
\end{aligned}
$$

Where $\omega_{p}$ is plasma frequency, $\omega$ is driving frequency, $N$ is the characteristic plasma density when $\omega_{p}=\omega, N$ is the density of exited electron in the CB.

Because of the Gaussian spatial intensity distribution, the electron density is higher in the centre than the one in the edge, leading the refractive index in smaller along the light axis and the light is defocusing due to the formation of plasma. So, the refractive index due to the above two nonlinear processes can be written as

$$
\mathrm{n}_{0}-\mathrm{N} / 2 \mathrm{n}_{0} \mathrm{~N}_{\mathrm{c}}+\mathrm{n}_{2} \mathrm{I}
$$

2) The temporal dependence change in the refractive index leads to new frequencies through self-phase modulation (SPM).

The refractive index changes by optical Kerr effect and plasma formation cause a SPM, leading to a phase shift in the pulse, thus resulting in a change of the pulse's frequency spectrum (i.e. spectral broadening). This intensity dependent phase shift of a pulse at $L$ is given as

$$
\omega / \mathrm{c}_{2} l(t) L
$$


SPM together with the Kerr-lens provide a short-pulse generation mechanism: Kerr lens mode locking (KLM).

The dynamical balance between self-focusing and defocusing due to the plasma formation can cause a filamentary propagation, characterized by a near constant beam waist over several Reyleigh lengths [114]. Typically, tight focusing can lead to a shorter filament. The length of filament increases with the pulse energy and the filament onset moves towards the lens direction [9].

When $P<P_{c r}$, the laser beam mainly follows linear propagation dynamics. When $P>P_{c r}$, selffocusing will play an important part in laser propagation: with the increase of laser power, the place of the self-focus and the starting position of the filament will shift from the geometrical focus towards the objective (i.e. towards the glass surface). At high power (i.e. $100 \mathrm{P}_{\mathrm{cr}}$ ), due to the modulational instabilities, the beam can break up into multiple filaments.

These nonlinear effects could be used to explain the morphology of fs laser-induced laser track in LNS glass. 


\subsection{Glass}

This section is to provide the basic knowledge of thermal properties of glasses. The first part is the basic concepts about the glass (e.g. the definition of glass and glass transition). The second part is the crystallization mechanism and examples the nonlinear optical crystallization in oxide glasses. The third part is the summary of the methods to obtain oriented crystals. Finally, is the part about the privious work on fs laser-induced chemical process in glasses.

\subsubsection{Basic knowledge on glass}

\subsubsection{Definition of glass}

Glass is a disordered material, lacking the periodicity of crystal, but behaves as solid below $T_{f}$ [115]. Amorphous, glassy, and vitreous describe the same thing.

a)

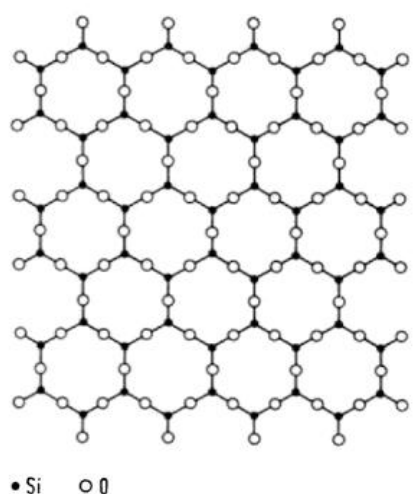

b)

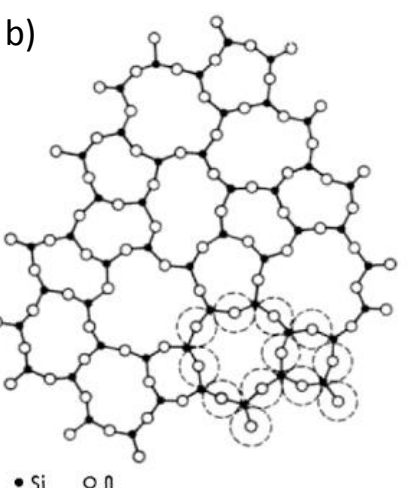

Figure 20 Two-dimensional schematic of a) an ordered $\mathrm{SiO}_{2}$ (the forth bonds of the Si project upward or downward from the plane of the illustration) and b) random $\mathrm{SiO}_{2}$ network, the actual relative surface area requirement of the Oxygens is indicated by dashed lines (cited from [116])

Figure 20a displays the schematically the structure of ordered $\mathrm{SiO}_{2}$ network. It shows an ordered structure with $\left[\mathrm{SiO}_{4}\right]$ tetrahedron unit, in which a central $\mathrm{sp}^{3}$ hybridized $\mathrm{Si}$ atom coordinates four $\mathrm{O}$ atoms with $\mathrm{O}-\mathrm{Si}-\mathrm{O}$ angle of $109.5^{\circ}$ and $\mathrm{Si}-\mathrm{O}$ distance of $1.62 \AA$. For vitreous silica (Figure 20b), the short range order is dominated by this tetrahedron unit, similar to the ordered one. The silica glass is constituted by the network of these $\left[\mathrm{SiO}_{4}\right]$ linked by Si-O-Si bridges.

As illustrated in Figure 21a, the stable form of silica at normal ambient condition is $\alpha$-quartz. It can transform to $\beta$-quartz at $573^{\circ} \mathrm{C}$ (Figure 21b). With the further heating, there will be a transformation to $\beta$-tridymite at $870^{\circ} \mathrm{C}$, and then into $\beta$-cristobalite at $1470^{\circ} \mathrm{C}$, before melting at $1705^{\circ} \mathrm{C}[117]$. Sometimes, the $\beta$-tridymite phase can skip.

If the temperature changes very slowly, this whole process can be reversible. However, if $\alpha$ quartz is heated quickly, it will exhibit another phase transition (Figure 21c), and this process is 
not reversible. As shown in Figure 21d, if we cool silica melt quickly, it can turn into silica glass, an amorphous one. Interestingly, if the silica glass is heated up, it will turn into $\beta$-cristobalite (Figure 21e). So, the phase transformation exhibits its own characteristic kinetics, which is dependent on heating and cooling rates.

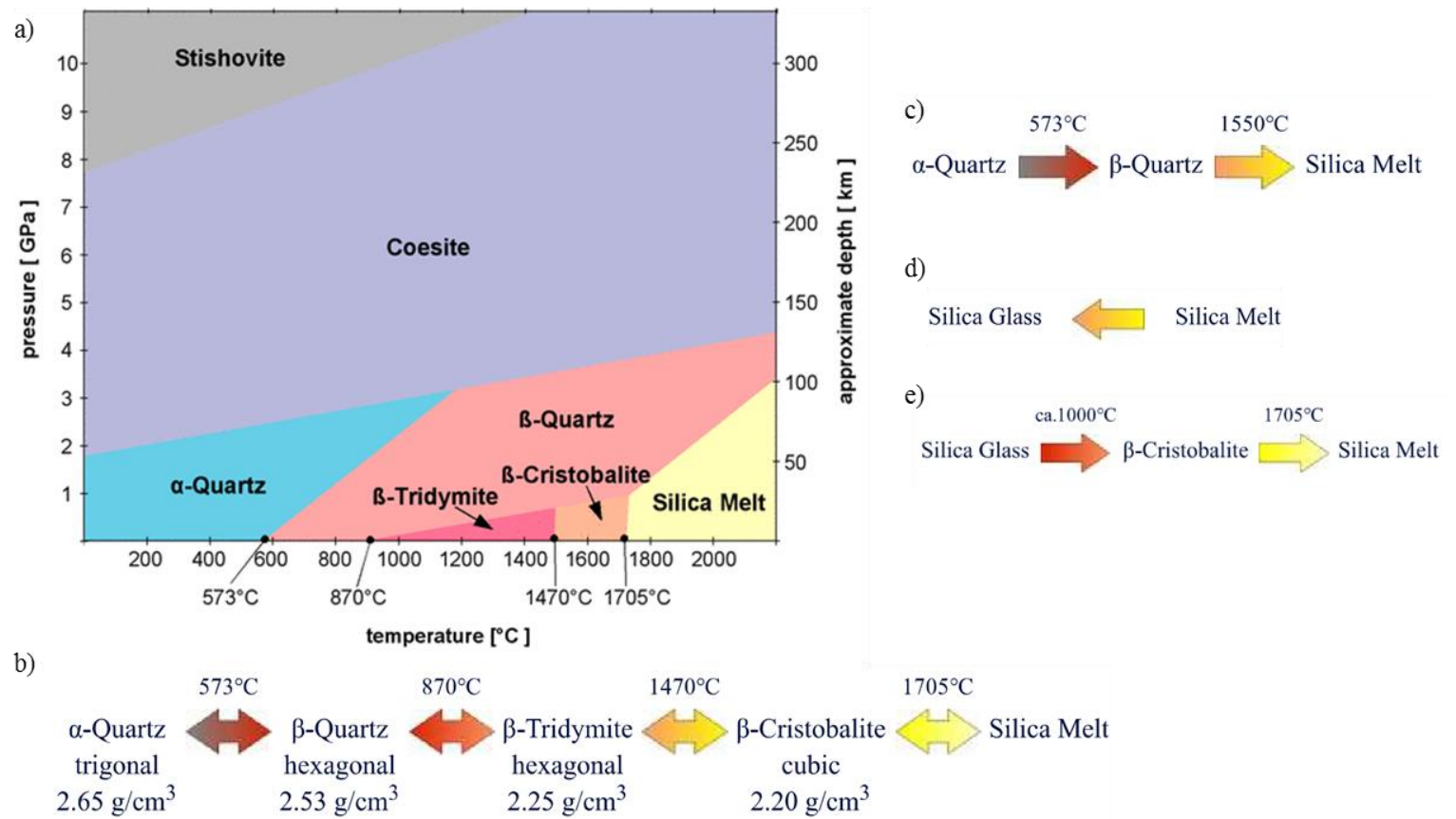

Figure 21 a) Phase diagram of silica and b-e) dependence of silica structure on temperature (cited from [117])

Figure 22 shows the various routes to form glass, the metastable material. Generally, it is formed by rapid cooling of liquid silica. Just as discussed above, when quartz melts, it becomes viscous liquid. Many Si-O bonds are broken, reducing the rigid order of the quartz. If this melt is rapidly cooled, the liquid structure will be preserved and the molecules in the melt to rearrange into crystal structures are prevented. In that case, controlling cooling rate of melt will have a significant effect on the formation of metastable phases.

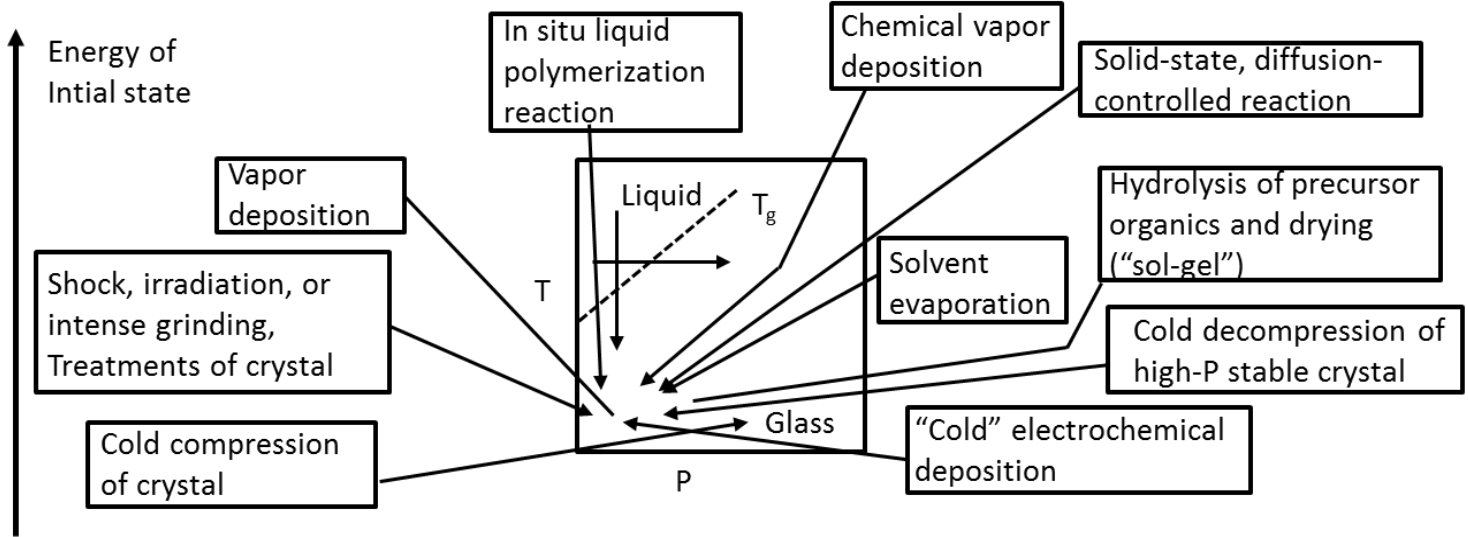

Figure 22 Various routes to the glassy state, roughly indicating the energies of the initial states relative to the final glassy state. The route of crystal compression below $T_{g}$ may yield glasses that are thermodynamically distinct from those obtained by the other routes but which may transform to them by way of non-equilibrium first-order transitions (after [118]) 


\subsubsection{Glass transition phenomenon}

The glass viscosity $(\eta)$ is dependent on the temperature $(T)$. The comparison of the viscosity of different kinds of glass versus temperature is shown in Figure 23. Some important technological points in glass manufacture industry are listed with the decrease of $\eta$.

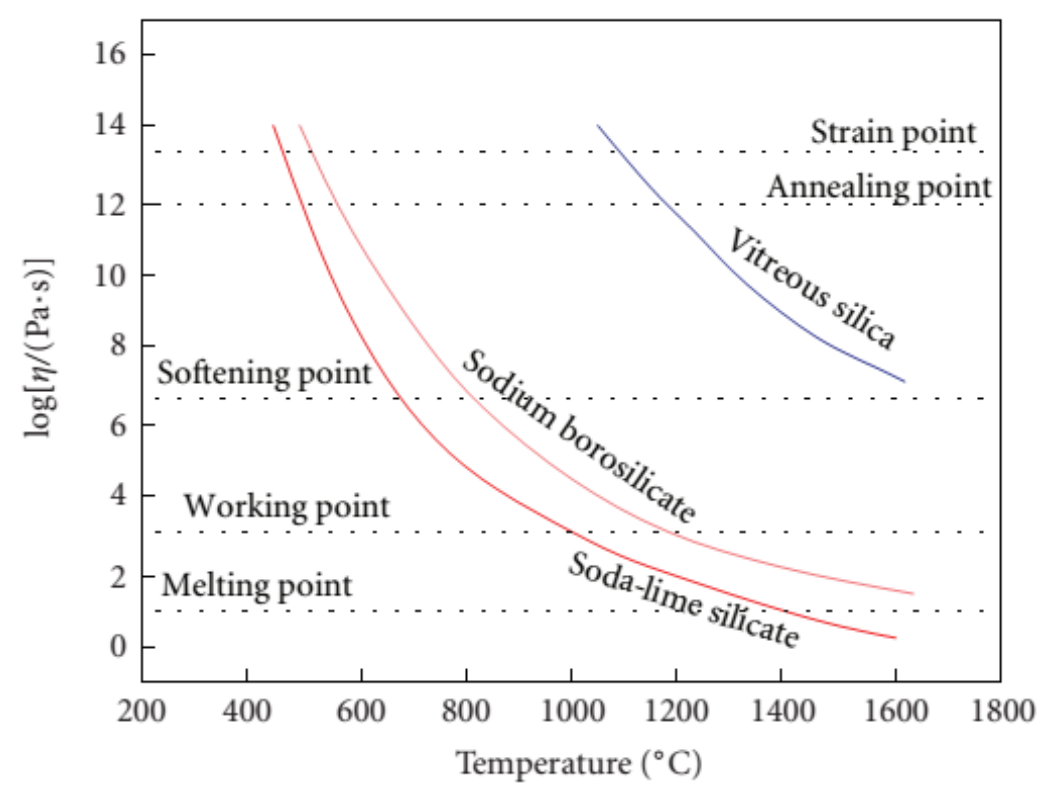

Figure 23 Comparison of the viscosity of several kinds of glasses versus temperature, in silicate systems viscosity attains the largest values for vitreous silica (cited from [119])

Strain point: below this temperature, brittle behaviour (or fracture) occurs, no plastic deformation. Annealing point: the temperature at which internal stress in a glass is safely relieved (i.e. by solid diffusion). Softening point: corresponds to the temperature at a glass does not flow under its own load. Working point: the temperature the glass is sufficiently soft for the shaping in a glass forming process. Working range is the range from the working point to the softening point, in which glass is easily deformed and glass blowers are in this range. Melting temperature $\left(T_{m}\right)$, below this temperature glass is in liquid state.

For molten inorganic glasses at temperature with low viscosity $(<1000 \mathrm{~Pa} \cdot \mathrm{s})$ the relationship $\eta$ and $T$ can be expressed by Arrhenius function [120]. With the decrease of $T$, the structural changes increasingly affect $\eta$. This structural change is a kinetic process. Glass transition temperature $\left(T_{g}\right)$ is the transition from super-cooled liquid to solid glass. When $T>T_{g}$, glass structure reaches equilibrium fast enough, allowing the kinetic effects to be omitted for moderate rates of deformation. The memory effects due to the glass structure change rate should be considered within and below the glass-transition interval [121]. 
The glass transformation process can be clearly seen from the volume as a function of temperature graph. As discussed above, when temperature is larger than $T_{m}$, it is in liquid glass state. With the decrease of temperature, the viscosity of liquid glass increases. In that case, the crystallization process is prevented due to the kinetic barrier to atomic rearrangement. Supercooled liquid occurs if the liquid is cooled below $T_{m}$ without crystallization. This super-cooled liquid is continued to be cooled, at the same time, the viscosity is increasing. This leads to the volume versus temperature deviating from the equilibrium curve, but the one with decreasing slope, until it becomes a frozen solid. The glass transformation region (departure from equilibrium) is the one between liquid and frozen solid. Generally, the final glass density is dependent on the cooling rate: slow cooling process causes a higher density glass than the fast cooling one for the normal glass transition.

Fictive temperature $\left(T_{f}\right)$ is the temperature at which the equilibrium melt structure is the same to that currently existing in the glass. In the plot of molar volume (or enthalpy) as a function of temperature, $T_{f}$ can be obtained from extrapolation along the cooling curve of the glass intersecting the equilibrium liquid line. It is an imprint of thermal history of glass preparation. Generally, at slow cooling rate, denser glass is obtained, with lower $T_{f}$. However, vitreous silica has an anomalous $T_{f}$ behavior (i. e. causing denser phase at a large cooling rate). Denser vitreous silica is formed at fast cooling rate and higher $\mathrm{T}_{\mathrm{f}}[122]$.

Optical glasses with strong thermal expansion (e.g. borosilicate BK7) usually exhibits negative refractive index changes under ultra-fast laser exposure because of volume expansion and mechanical rarefaction. However, Mermillod-Blondin et al. [123] shown that temporally shaped laser excitation on ps scales and at high repetition rates can invert it from negative to positive index variation of BK7, which can be explained by the matter momentum relaxation, producing directional on-axis material compaction. Figure 24a shows the qualitative description of thermo mechanical processes induced by ps laser irradiation. Laser pulse can create a hot pressurized zone. Thermal expansion begins and, in the first ns, an elastoplastic wave is launched. Figure 24a shows the formation of the plastic shell because of the elastoplastic wave. The heat wave follows on a $\mu$ s scale. Figure $24 \mathrm{~b}$ presents the subsequent softening due to the heat wave. Because of the elongated shape of the heat source, elastoplastic and heat waves dissipate faster on axis direction, than that of the radial one. Figure 24c shows the release of stress from the elastically compressed zone with density redistribution. The positive value of the hoop stress is in favour of inward relaxation. Figure $24 d$ displays the qualitative indication of this process. The 
matter is compressed with each heating cycle in the laser pulse direction, creating extended onaxis zone of pressurized matter.

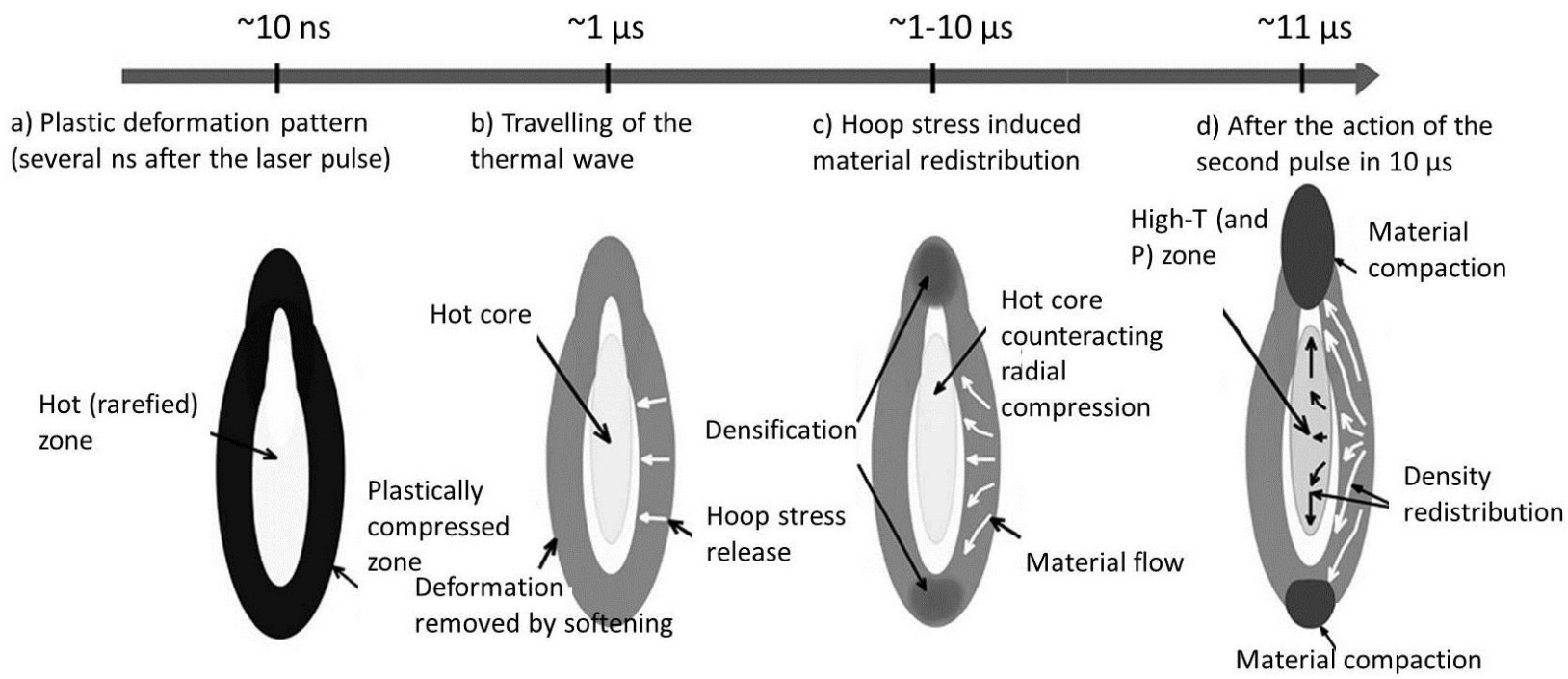

Figure 24 ps laser-induced thermo-mechanical processes: a) the formation of the plastic layer due to the elastoplastic wave, b) softening of the plastic region upon heating, c) stress release and density redistribution, and $d$ ) the potential effect of a subsequent pulse arriving on $\sim 10 \mu$ s is represented (after [123])

So we can see that ultra-fast laser-induced refractive index changes in glasses is a complex process, which is related to the glass properties (e.g. thermal diffusivity and thermal capacity), thermal diffusion, and stress release.

\subsubsection{Crystallization in glass}

\subsubsection{Mechanism of crystallization in glass}

Glass is thermodynamically unstable (non-equilibrium). However, due to the kinetic inhibition (i.e. high viscosity inhibits structural rearrangements), the glass is stable and does not go over into a metastable state at low temperature. If some process (e.g. heating) is performed, relaxation of the glass structure occurs and the glass can reach a metastable liquid state and then evolve to crystalline state.

Crystallization is a two step-process: nucleation and crystal growth. Classical nucleation theory (CNT) is a theoretical model proposed by Gibbs. It is a simple theory for understanding nucleation of a new thermodynamic phase (e.g. liquid or crystal).

For homogeneous nucleation, nucleation occurs in the liquid phase or glass because of the thermal fluctuation of the chemical composition. The nucleation is homogeneous. 
For the one forming a spherical nucleus, change in free energy can be calculated as the sum of surface term (positive) and bulk term (negative) as

$$
\Delta \mathrm{G}_{\mathrm{T}}=4 \pi \mathrm{r}^{2} \gamma+\frac{4}{3} \pi \mathrm{r}^{3} \Delta \mathrm{G}_{\mathrm{V}}
$$

Where $\Delta G_{\mathrm{T}}$ is the total free energy change, $r$ is the radius of the nucleus, $\gamma$ is the specific surface free energy of the critical nucleus-melt interface, and $\Delta G_{V}$ is the difference between the free energies of crystal and liquid per unit volume.

In super-cooled liquid, $\Delta \mathrm{G}_{\mathrm{V}}$ is associated with the liquid-crystal transformation, given as

$$
\Delta G_{V}=\Delta H_{V}-T \Delta S_{V}, \quad \Delta H_{V}=-L_{V}
$$

Where $\Delta H_{v}(<0)$ is the change of volume enthalpy, $L_{v}$ is latent heat, $T$ is the temperature, and $\Delta S_{v}$ is the change of entropy.

Assuming $\Delta \mathrm{H}_{\mathrm{v}}$ is constant, corresponding to the release heat during the crystallization. At $T_{m}$, $\Delta G_{V}=0$. We get

$$
\Delta G_{V}=\frac{\Delta H_{v} \Delta T}{T_{m}}, \quad \Delta T=T_{m}-T
$$

At critical nucleus radius $\left(r^{*}\right)$, the total free energy reaches the maximum and the one with the highest value of $\Delta \mathrm{G}^{*}$.

When $\frac{d \Delta G_{T}}{d r}=0$ we get

$$
r^{*}=\frac{-2 \gamma}{\Delta G_{v}}, \quad \Delta G^{*}=\frac{16 \pi \gamma^{3}}{3\left(\Delta G_{v}\right)^{2}}
$$

When $r<r^{*}$, the nuclei are unstable, when $r>r^{*}$, nuclei are stale and can continue to grow. To have a crystallization, the nuclei have to overcome the thermodynamic barrier for nucleation (i.e. $\left.\Delta G^{*}\right)$.

a)

$\Delta \mathrm{G}$

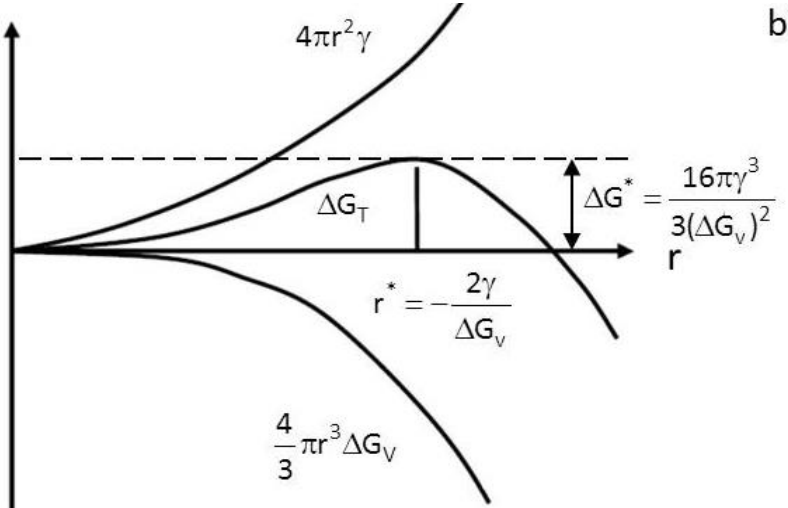

b)

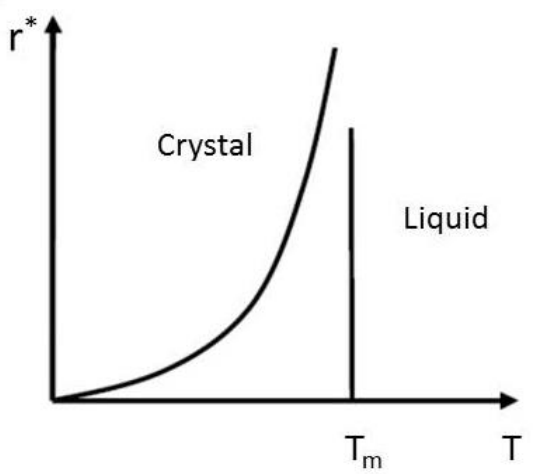

Figure 25 a) The free energy change associated with homogeneous nucleation of a sphere of radius $r\left(T<T_{m}\right)$, and $b$ ) change of $r^{*}$ with the under-cooling of the melt, the asterisk defines critical values (after [124]) 
The key concern of classical homogeneous nucleation theory is a thermodynamic description of initial stage of nucleation from embryo to nucleus with a little larger size than $r^{*}$ [125]. The change of parent phase in the system is assumed to be negligible due to the largeness in volume and mass comparing that of nuclei. So, the nucleation curve (shown in Figure 25a) passes a single maximum point at $r^{*}$.

The number of embryo with radius $r^{*}$ per volume (i.e. $N^{*}$ ) has a relationship with the total number of molecules per volume (i.e. $N$ ) as

$$
N^{*}=N \exp \left(-\frac{\Delta G^{*}}{k_{B} T}\right)
$$

These germs' formation rate can be defined by $\mathrm{N}^{*}$ and the agglomeration rate of molecules on the seed. The atoms diffusion is conditioned by the germ-matrix interface crossing, regarded as a diffusion layer. So, the rate of forming nuclei at $r^{*}$ size is written as

$$
\frac{\mathrm{dn}}{\mathrm{dt}}=\mathrm{N}^{*} \operatorname{Kexp}\left(-\frac{\Delta \mathrm{G}_{\mathrm{D}}}{\mathrm{k}_{\mathrm{B}} \mathrm{T}}\right) \propto \exp \left(-\frac{\Delta \mathrm{G}_{\mathrm{D}}+\Delta \mathrm{G}^{*}}{\mathrm{k}_{\mathrm{B}} \mathrm{T}}\right)
$$

Where $\Delta G_{D}$ is the activation energy to cross the glass-crystal interface (kinetic barrier), $\Delta G^{*}$ is the energy barrier to form a nucleus stable to grow, and $\mathrm{k}_{\mathrm{B}}$ is Boltzmann's constant $\left(1.38 \times 10^{-23} \mathrm{~J} / \mathrm{K}\right)$.

The size of $r^{*}$ is dependent of $T$. Because $\Delta G_{v}$ is as function of $\Delta T$, we get

$$
r^{*} \propto(\Delta T)^{-1}, \Delta G^{*} \propto(\Delta T)^{-2}
$$

So, when $T<T_{m}, r^{*}$ increases with the $T$ (illustrated in Figure $25 b$ ). When $T$ is close to $T_{m}, \Delta G^{*}$ is high, which will play a dominant role in the nucleation rate (i.e. very low). But the diffusion term is high at this temperature. When $T$ decreases, the possibility of forming seeds increases $\left(\Delta \mathrm{G}^{*}\right.$ decreases). However, meanwhile, the viscosity of material increases sharply, restricted atom movement and inhibited the ordered crystal structures formation, thus decreasing the nucleation rate. So there is an optimal T for the maximum nucleation rate. When the $\mathrm{T}$ is close to $\mathrm{T}_{\mathrm{g}}, \Delta \mathrm{G}^{*}$ decreases and nucleation rate increases sharply, which might achieve a maximum nucleation rate when $\Delta \mathrm{G}^{*}$ and $\Delta \mathrm{G}_{\mathrm{D}}$ are at same magnitude order.

Classical homogeneous nucleation theory has been employed to determine the crystallization process of glasses. However, this method exhibits large discrepancy between the calculated and the experimental results, which may be explained that the real practice of solidification of glassy alloys often involves heterogeneous nucleation [126]. 
For heterogeneous nucleation, nucleation occurs at some preferred sites is much greater than that at other sites. Compared to the homogeneous nucleation, there is an effect mainly because of the diminished thermodynamic barrier, leading to a decrease of the contribution of the effective surface energy to the work of critical cluster formation [127].

a)

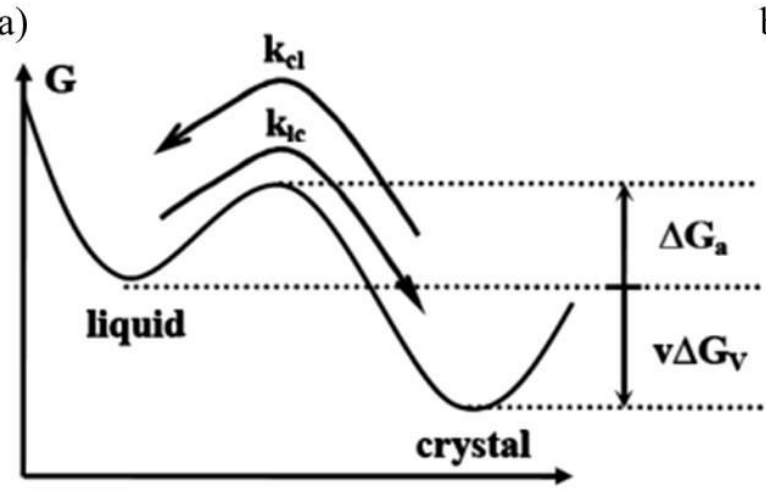

Configuration parameter b) $\quad \mathbf{d n} / \mathbf{d t}$

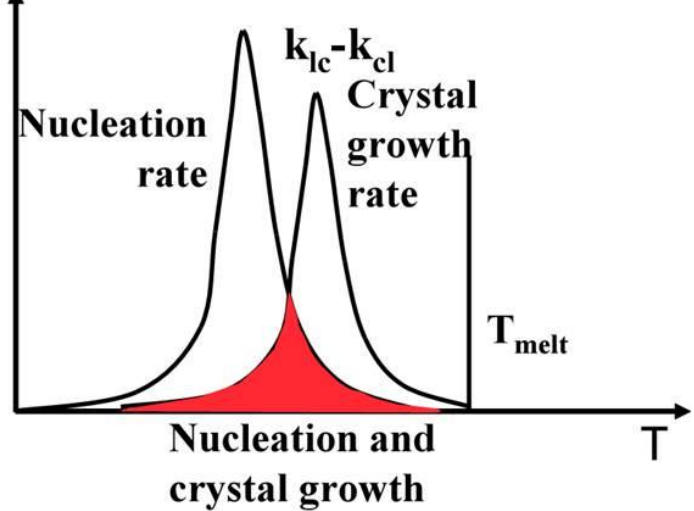

Figure 26 a) Sketch of free enthalpy according to configurational parameter and b) a superimposition of the nucleation and growth rates (after [124])

As shown in Figure 26a, the growth process can be represented by a reversible reaction and the growth rate is thus given as [124]

$$
k_{l c}-k_{c l}=k_{0} \exp \left(-\frac{\Delta G_{a}}{k_{B} T}\right)-k_{0}\left[\exp \left(-\frac{\Delta G_{a}-v \Delta G_{v}}{k_{B} T}\right)\right]=k_{0} \exp \left(-\frac{\Delta G_{a}}{k_{B} T}\right)\left[1-\exp \left(-\frac{1}{k_{B} T} \frac{v L_{v}\left(T_{m}-T\right)}{T_{m}}\right)\right]
$$

As illustrated in Figure $26 \mathrm{~b}$, when temperature is at or large than $T_{m}$, the growth rate is 0 . Below $\mathrm{T}_{\mathrm{m}}$, at higher temperature, crystal growth rates exhibit higher than nucleation rates. The nucleation happens at lower temperature than crystal growth. So, cooling a melt will meet the growth regime before the formation of nuclei. Considering crysal growth occurs after nuclei, cystallization on cooling occurs in the overlap zone (illustrated by red zone in Figure 26b). However, if heating the glass, the crystallization is not limited to the above overlap zone anymore. In addition, chemical composition can also have an influence on crystal growth [128].

So, to obtain nano sized crystal in glass, we need a temperature low enough to prevent growth (i.e. staying in the nucleation zone). To obtain a volume crystallization in glass, we need to stay a time long enough at nucleation temperature and then at a given temperature for growing.

\subsubsection{TTT and CCT diagrams}

Investigation on crystallization during solidification is significant in understanding the phase transformation behavior during non-equilibrium processing. The influence of cooling rate on 
crystallization can be detected from isothermal time temperature transformation (TTT) or continuous cooling transformation (CCT) diagrams [126].

TTT diagram displays the kinetics of isothermal transformations. It can be obtained by cooling the material to a transformation temperature, holding the temperature for certain period, and cooling to room temperature. In CCT diagram, there is continuous cooling. The components are cooled at constant or varying rates. The end products are depending on the material of components and the cooling process.

CCT curve can be got by shifting the TTT curve a little to the downward right. This is because the time required for a reaction to begin and end is delayed, thus the TTT curves are moved to longer times and lower temperatures.

Figure 27 displays a TTT and CCT diagrams and cooling curves. We can see that it needs a lapse to start the crystallization (typically in the second scale). With the decrease of $T$, the increased viscosity inhibits the normal tendency to regular ions and atoms arrangment for inducing crystal nucleation and growth. For faster cooling rate, the crystallization is impossible. When cooling curve tangent to the "nose" of CCT curve, it is named as the critical cooling rate (CCR). So, rapid cooling favors the transition to the glass while minimizing the creation of nucleus and subsequent growth.

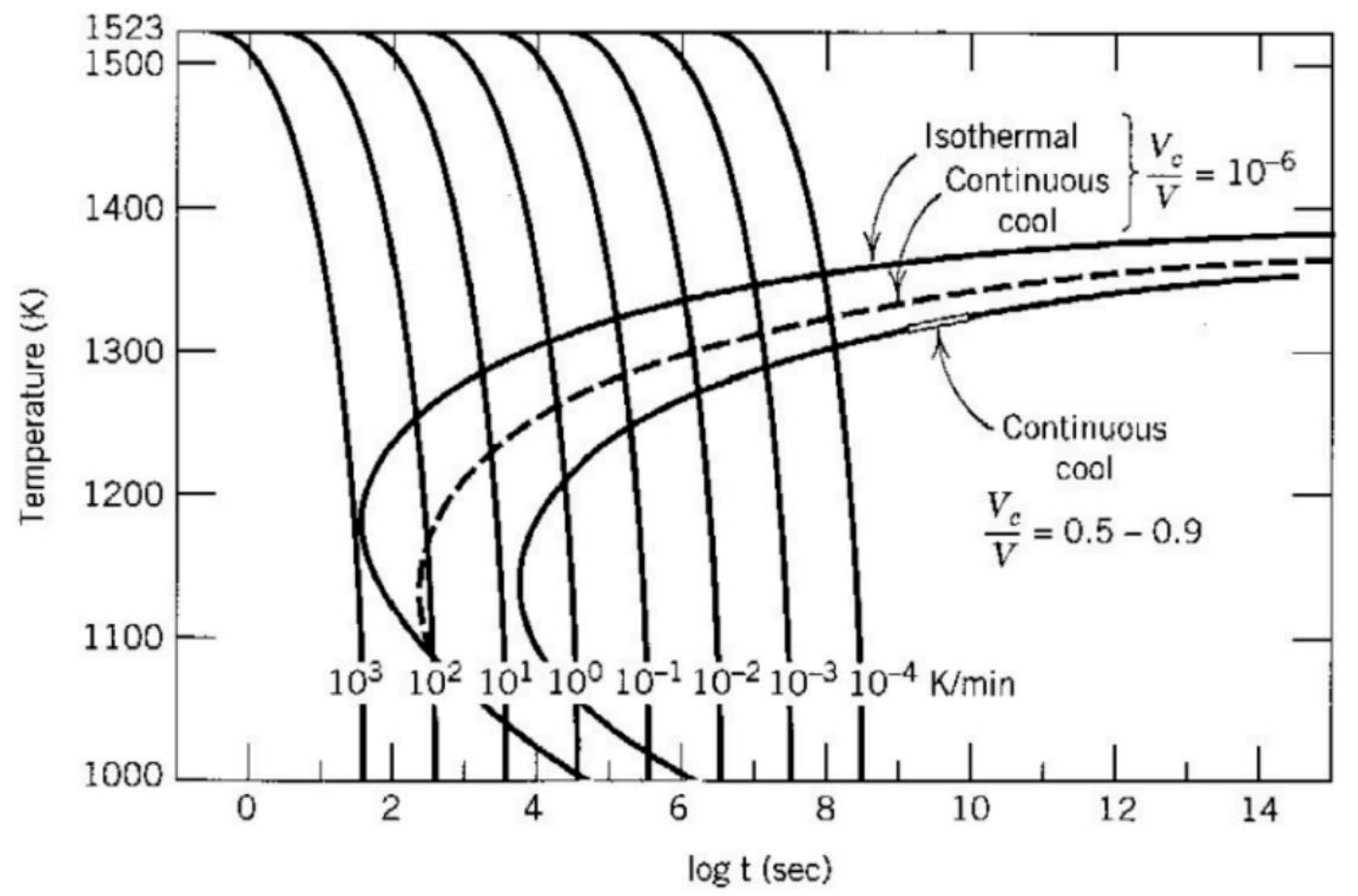

Figure 27 Time temperature transformation curve and continuously cooling transformation curves compared to cooling curves with different cooling rates (cited from [124, 129]) 


\subsubsection{Phase diagrams related to LNS glass}

Phase diagram is obtained under slow cooling (equilibrium) conditions. This diagram can represent the conditions (e.g. composition, temperature, and pressure) at which thermodynamically distinct phases occur and coexist at equilibrium. Phase diagrams can show the information for the controlling of phase and microstructure in materials. It is widely used in mineralogy and materials science.

$\mathrm{Li}_{2} \mathrm{O}-\mathrm{Nb}_{2} \mathrm{O}_{5}-\mathrm{SiO}_{2}$ (LNS) glasses are used in this thesis. Three binary systems comprise the boundaries of this main ternary system: $\mathrm{Li}_{2} \mathrm{O}-\mathrm{SiO}_{2}, \mathrm{Nb}_{2} \mathrm{O}_{5}-\mathrm{SiO}_{2}$, and $\mathrm{Li}_{2} \mathrm{O}-\mathrm{Nb}_{2} \mathrm{O}_{5}$. The former two are listed in this section. The last one will be discussed in Section 2.3.1.1. The phase-equilibrium diagram $\mathrm{Li}_{2} \mathrm{O}-\mathrm{Nb}_{2} \mathrm{O}_{5}-\mathrm{SiO}_{2}$ will be shown in Section 3.1.1.

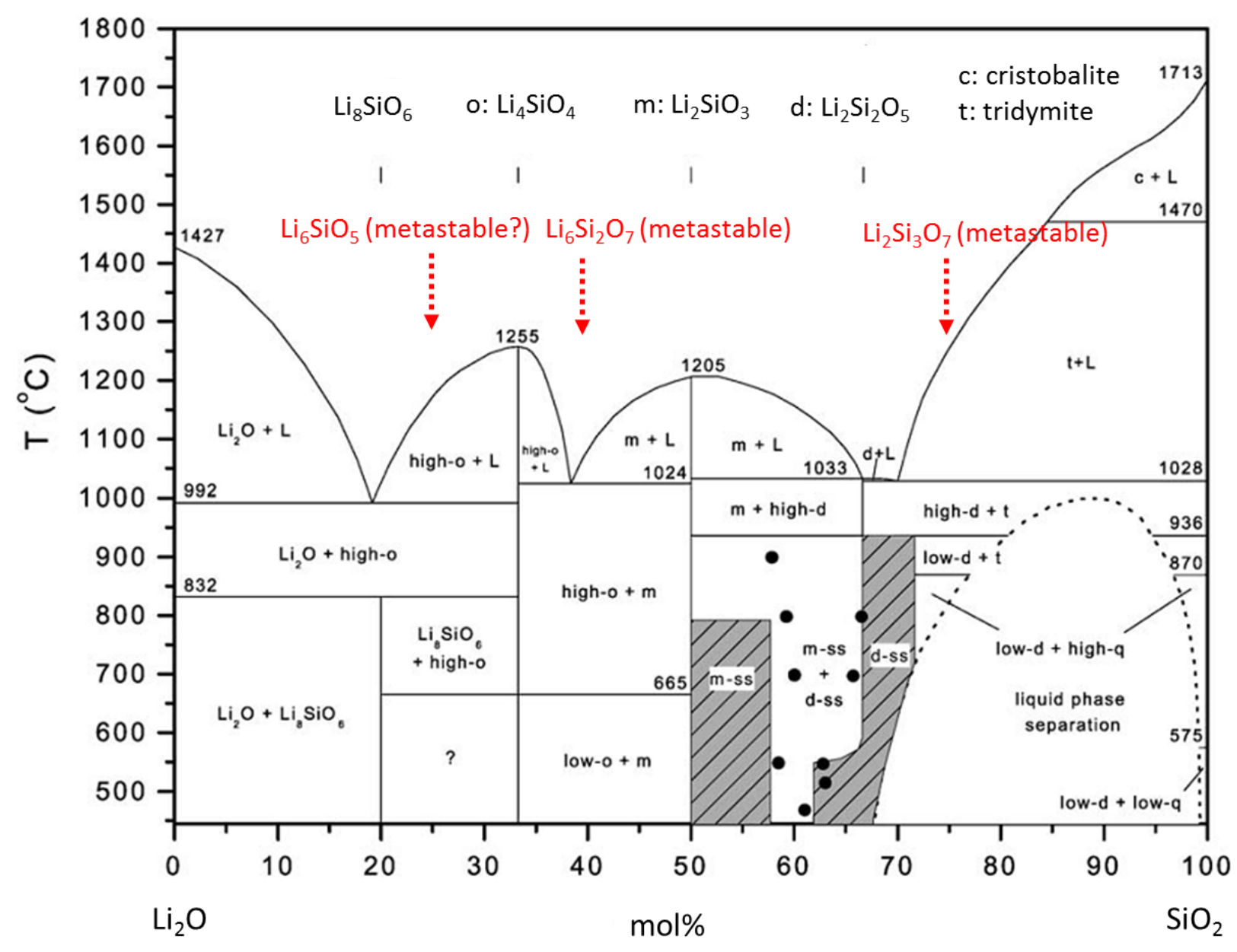

Figure 28 Phase-equilibrium diagram of $\mathrm{Li}_{2} \mathrm{O}-\mathrm{SiO}_{2}$ (after [130]). Tridymite and cristobalite are high temperature poly morphs of silica (see details in Appendix VIII)

Figure 28 displays the phase-equilibrium diagram of binary system $\mathrm{Li}_{2} \mathrm{O}-\mathrm{SiO}_{2}$. Liquid phase of $\mathrm{Li}_{2} \mathrm{O}$ is shown when $\mathrm{SiO}_{2}<20 \mathrm{~mol} \%$ and $\mathrm{T}>992^{\circ} \mathrm{C}$. Li can form highly stable metasilicate, with higher $\mathrm{Tm}$ than that of any other alkali metasilicate. Lithium orthosilicate $\left(\mathrm{Li}_{4} \mathrm{SiO}_{4}\right)$ has $\mathrm{T}_{\mathrm{m}}=1255^{\circ} \mathrm{C}$. The 
eutectic point between $\mathrm{Li}_{4} \mathrm{SiO}_{4}$ and lithium metasilicate $\left(\mathrm{Li}_{2} \mathrm{SiO}_{3}\right)$ is at $1024^{\circ} \mathrm{C}$, $38 \mathrm{~mol} \% \mathrm{SiO}_{2}$. Lithium oxosilicate $\left(\mathrm{Li}_{8} \mathrm{SiO}_{6}\right)$ is the $\mathrm{Li}$ richest phase, and it decomposes into $\mathrm{Li}_{2} \mathrm{O}$ and $\mathrm{Li}_{4} \mathrm{SiO}_{4}$ at $832^{\circ} \mathrm{C}$. The stability of $\mathrm{Li}_{6} \mathrm{SiO}_{5}$ is not clear. $\mathrm{Li}_{6} \mathrm{Si}_{2} \mathrm{O}_{7}$, an intermediate high temperature phase, forms peritectically at $1030^{\circ} \mathrm{C}$ and decomposes eutectoidally at $1020^{\circ} \mathrm{C}$ [131] (see details in Appendix XIII). A eutectic sub-system exists between $\mathrm{Li}_{4} \mathrm{SiO}_{4}$ and $\mathrm{Li}_{6} \mathrm{Si}_{2} \mathrm{O}_{7}$ with the eutectic temperature of $1025^{\circ} \mathrm{C}$ and $\mathrm{SiO}_{2}$ of $38.3 \mathrm{~mol} \%$. Lithium disilicate $\left(\mathrm{Li}_{2} \mathrm{Si}_{2} \mathrm{O}_{5}\right)$ is stable over a narrow region of composition in contact with the liquidus [132]. $\mathrm{Li}_{2} \mathrm{Si}_{3} \mathrm{O}_{7}$ can be fabricated from a range of melt compositions of $23-30$ mol\% of $\mathrm{Li}_{2} \mathrm{O}$, usually with best yields at 25 mol\% [133]. See details in Appendix XIII. In addition, metastable $\mathrm{Li}_{2} \mathrm{Si}_{2} \mathrm{O}_{5}$ can be prepared by ion exchange of $\alpha$ $\mathrm{Na}_{2} \mathrm{Si}_{2} \mathrm{O}_{5}$, retaining the silicate sheet configuration of the parent $\alpha-\mathrm{Na}_{2} \mathrm{Si}_{2} \mathrm{O}_{5}$ and is different from that in stable $\mathrm{Li}_{2} \mathrm{Si}_{2} \mathrm{O}_{5}$ [134]. The experimental crystal structure constants of lithium silicates are shown in Appendix XIII.

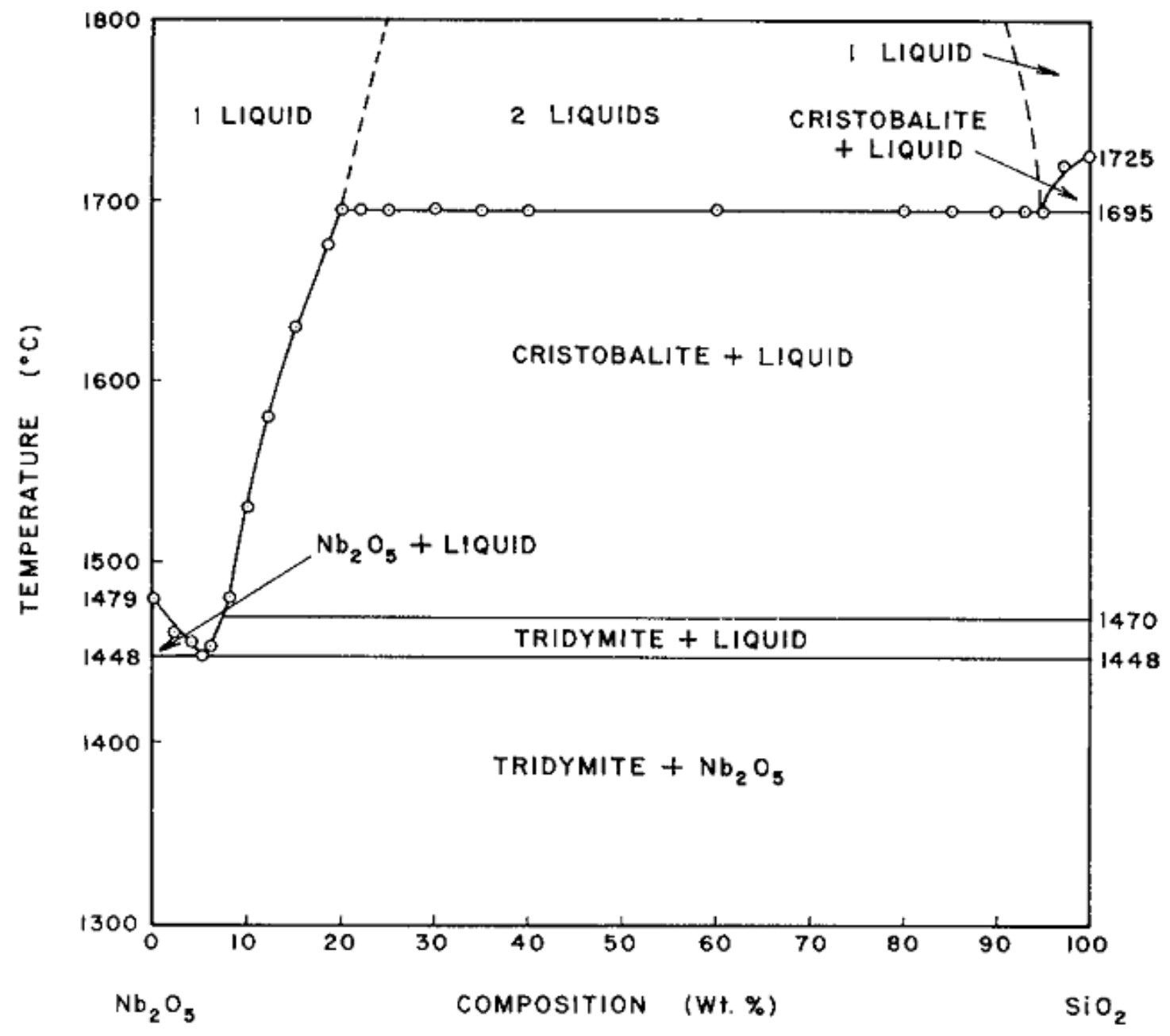

Figure 29 Phase-equilibrium diagram of $\mathrm{Nb}_{2} \mathrm{O}_{5}-\mathrm{SiO}_{2}$ (cited from [135]) 
Lithium-silicon oxide can be crystalline or amorphous. Fast quenching is an effective way for developing new glassy materials and for extending the composition range of glass formation [136]. See details in Appendix XIII.

The phase-equilibrium diagram of binary system $\mathrm{Nb}_{2} \mathrm{O}_{5}-\mathrm{SiO}_{2}$ is shown in Figure 29. There is no phase containing $\mathrm{Nb}$ and $\mathrm{Si}$. One eutectic composition occurs at $95 \% \mathrm{Nb}_{2} \mathrm{O}_{5}$ at $1448^{\circ} \mathrm{C}$ and another at $\sim 5 \% \mathrm{Nb}_{2} \mathrm{O}_{5}$ at $1695^{\circ} \mathrm{C}$. There is an extensive two-liquid region over the range 5 to $80 \%$ $\mathrm{Nb}_{2} \mathrm{O}_{5}$. The minimum temperature of the two-liquid area is $1695^{\circ} \mathrm{C}$.

\subsubsection{Nonlinear optical crystallization in oxide glasses}

Oxide glasses contain network formers (e.g. $\mathrm{SiO}_{2}, \mathrm{~B}_{2} \mathrm{O}_{3}$, and $\mathrm{P}_{2} \mathrm{O}_{5}$ ) and modifiers (e.g. alkaline earth, alkali, and transition metals). It has been reported that the excellent glass formers have electro-negativity values of 1.7-2.1 on the Pauling scale: $\mathrm{Si}, \mathrm{B}$, and $\mathrm{P}$ have values of $1.8,2.0$, and 2.1 for borate, silicate, and phosphate glasses, respectively [137].

Generally, cation-oxygen bonds are the basic structural unit. These cations are separated by oxygen anions and the oxygen anions are in contact with each other, leading to a 3D crosslinking of units (short-range order).

These oxide glasses have advantages of changeable chemical composition, optical transparency, shape forming ability, flexible design and control in chemical/physical properties, and high chemical durability [138].

Because of the poor ability of $\mathrm{LiNbO}_{3}$ for glass forming, it is necessary to add glass formers (e.g. $\mathrm{SiO}_{2}, \mathrm{~B}_{2} \mathrm{O}_{3}, \mathrm{TeO}_{2}$, and $\mathrm{P}_{2} \mathrm{O}_{5}$ ) [139]. In this thesis, $\mathrm{SiO}_{2}$ is chosen as the glass former due to its physical properties. It is desirable that this glass former does not have cations, modifying the $\mathrm{LiNbO}_{3}$ lattice because of silica's small ionic radius and coordination number [139]. In addition, transparent and homogeneous $\mathrm{Li}_{2} \mathrm{O}-\mathrm{Nb}_{2} \mathrm{O}_{5}-\mathrm{SiO}_{2}$ (LNS) glasses can be fabricated in large range chemical composition [140].

Crystallization can be achieved by laser irradiation in glass in static mode (i.e. crystalline dots, irradiated glass without moving the sample) [18] or in writing mode (e.g. crystalline lines) [23]. Once crystals are precipitated in the irradiated dots as discussed above, the crystalline phase will play the role of seed, which is similar to the melting-zone method for single-crystal growth. There are two kinds of writing in the bulk of materials: longitudinal and transverse geometries. For the former one, the crystal can grow from the glass surface to the interior (i.e. long 
crystallization structure) towards the laser movement direction when the laser focus was continuously moved [21]. It has been reported that even glass and crystal having the same chemical composition (i.e. $25 \mathrm{La}_{2} \mathrm{O}_{3}-25 \mathrm{~B}_{2} \mathrm{O}_{3}-50 \mathrm{GeO}_{2}$, mol\%), an unidentified off-stoichiometric phase occurs before $\mathrm{LaBGeO}_{5}$ when focusing deep inside the glass [141]. The transverse geometry is the one the sample is scanned transverse to the writing laser $[23,25]$.

Crystal growth can be controlled by adjusting the writing speed. With the decrease of writing speed, the heated region caused the formation of a stable structure with an layer at the solidliquid interface between a part of the poly-crystal and the heated zone [24], which is suitable for single crystal or single-crystal-like structure formation.

Crystalline lines can be obtained in glass by introducing transition metal ions (e.g. $\mathrm{Cu}^{2+}, \mathrm{Ni}^{2+}, \mathrm{Fe}^{2+}$, and $\mathrm{V}^{2+}$, with small amount, i.e. $>1 \mathrm{~mol} \%$ ) or rare-earth ions (e.g. $\mathrm{Sm}^{3+}, \mathrm{Dy}^{3+}$, with larger amount, i.e. $>8 \mathrm{~mol} \%)[142,143]$. The CW laser energy (e.g. Nd:YAG laser, $1064 \mathrm{~nm}$ ) can be absorbed linearly by doped ions, transferring to the lattice system by a non-radiative relaxation process (electron-phonon coupling) [144]. So, the surrounding of the doped ions is heated and if the temperature of the laser-irradiated zone is high enough, structural modifications (e.g. crystallization) occur [145]. Generally, transition metal ions in oxide glasses have broad $d-d$ band transitions compared to rare-earth ions $f$ - $f$ transitions, the doping amount is larger for rare-earth than transition metal ions [143]. However, the glass may be no more colorless with the doping ions (e.g. being green when LNS glass with CuO addition [144]).

Table 3 lists the precipitation of nonlinear optical crystal inside oxide glasses and the corresponding methods.

Table 3 Glass compositions and the formed nonlinear optical crystalline phases by laser irradiation

\begin{tabular}{|c|c|c|c|}
\hline Glass composition (mol\%) & Crystalline phase & Irradiation conditions & Refs \\
\hline $15 \mathrm{~K}_{2} \mathrm{O}-15 \mathrm{Sm}_{2} \mathrm{O}_{3}-70 \mathrm{P}_{2} \mathrm{O}_{5}{ }^{\circledR}$ & $\mathrm{KSm}\left(\mathrm{PO}_{3}\right)_{4}$ & $\begin{array}{l}\text { heat-assisted CW Nd:YAG laser, } \\
1064 \mathrm{~nm}, 0.8 \mathrm{~W}^{*}\end{array}$ & [146] \\
\hline $\begin{array}{l}10 \mathrm{BaO}-\mathrm{x} \mathrm{Ln}_{2} \mathrm{O}_{3}-(10-\mathrm{x}) \mathrm{Sm}_{2} \mathrm{O}_{3}-80 \mathrm{TeO}_{2} \\
(\mathrm{Ln}: \mathrm{La}, \mathrm{Er})^{\mathrm{m}}\end{array}$ & $\mathrm{Sm}_{2} \mathrm{Te}_{6} \mathrm{O}_{15}$ & $\begin{array}{l}\text { CW Nd:YAG laser, } 1064 \mathrm{~nm}, 0.5-0.7 \\
\text { W }\end{array}$ & [147] \\
\hline $\begin{array}{l}10 \mathrm{Sm}_{2} \mathrm{O}_{3}-10 \mathrm{SrO}-10 \mathrm{BaO}-20 \mathrm{Nb}_{2} \mathrm{O}_{5}- \\
50 \mathrm{~B}_{2} \mathrm{O}_{3} \text { or } 8 \mathrm{Sm}_{2} \mathrm{O}_{3}-10 \mathrm{SrO}-10 \mathrm{BaO}- \\
20 \mathrm{Nb}_{2} \mathrm{O}_{5}-52 \mathrm{~B}_{2} \mathrm{O}_{3}\end{array}$ & $\begin{array}{l}\mathrm{Sm}^{3+} \text {-doped } \mathrm{Sr}_{x} \mathrm{Ba}_{1-} \\
{ }_{x} \mathrm{Nb}_{2} \mathrm{O}_{6}\end{array}$ & $\begin{array}{l}\text { heat-assisted CW Nd:YAG laser" }{ }^{\text {I }} \\
1064 \mathrm{~nm}, 1 \mathrm{~W}\end{array}$ & {$[148]$} \\
\hline $\begin{array}{l}x \mathrm{Xr}_{2} \mathrm{O}_{3}-(18.25-\mathrm{x}) \mathrm{Gd}_{2} \mathrm{O}_{3}-3 \mathrm{Sm}_{2} \mathrm{O}_{3}- \\
63.75 \mathrm{MoO}_{3}-15 \mathrm{~B}_{2} \mathrm{O}_{3}{ }^{\delta}\end{array}$ & $\begin{array}{l}\mathrm{Er}^{3+} \text {-doped } \beta^{\prime}- \\
(\mathrm{Sm}, \mathrm{Gd})_{2}\left(\mathrm{MoO}_{4}\right)_{3}\end{array}$ & CW Yb:YVO 4 laser, $1080 \mathrm{~nm}$ & {$[149]$} \\
\hline $21.25 \mathrm{Sm}_{2} \mathrm{O}_{3}-63.75 \mathrm{MoO}_{3}-15 \mathrm{~B}_{2} \mathrm{O}_{3}$ & $\beta^{\prime}-\mathrm{Sm}_{2}\left(\mathrm{MoO}_{4}\right)_{3}$ & CW Nd:YAG laser, 1064 nm, 0.4 W & {$[150]$} \\
\hline
\end{tabular}




\begin{tabular}{|c|c|c|c|}
\hline $8 \mathrm{Sm}_{2} \mathrm{O}_{3}-37 \mathrm{Bi}_{2} \mathrm{O}_{3}-55 \mathrm{~B}_{2} \mathrm{O}_{3}$ & $\mathrm{Sm}_{x} \mathrm{Bi}_{1-\mathrm{x}} \mathrm{BO}_{3}$ & CW Nd:YAG laser, 1064 nm, 0.9 W & {$[145]$} \\
\hline $10 \mathrm{Sm}_{2} \mathrm{O}_{3}-35 \mathrm{Bi}_{2} \mathrm{O}_{3}-55 \mathrm{~B}_{2} \mathrm{O}_{3}$ & $\mathrm{Sm}_{\mathrm{x}} \mathrm{Bi}_{1-\mathrm{x}} \mathrm{BO}_{3}$ & CW Nd:YAG laser, 1064 nm, 0.66 W & {$[142]$} \\
\hline $10 \mathrm{Sm}_{2} \mathrm{O}_{3}-40 \mathrm{BaO}-50 \mathrm{~B}_{2} \mathrm{O}_{3}$ & $\beta-\mathrm{BaB}_{2} \mathrm{O}_{4}$ & $\begin{array}{l}\text { heat-assisted CW Nd:YAG laser *, } \\
1064 \mathrm{~nm}, 0.6 \mathrm{~W}\end{array}$ & {$[151]$} \\
\hline $10 \mathrm{Dy}_{2} \mathrm{O}_{3}-45 \mathrm{BaO}-45 \mathrm{~B}_{2} \mathrm{O}_{3} \diamond$ & $\beta-\mathrm{BaB}_{2} \mathrm{O}_{4}$ & $\begin{array}{l}\text { heat-assisted CW Nd:YAG laser *, } \\
1064 \mathrm{~nm}, 0.9 \mathrm{~W}\end{array}$ & {$[151]$} \\
\hline $11 \mathrm{TiO}_{2}-63 \mathrm{BaO}-26 \mathrm{~B}_{2} \mathrm{O}_{3}(\mathrm{wt} \%)^{\mathrm{s}}$ & $\beta-\mathrm{BaB}_{2} \mathrm{O}_{4}$ & $\mathrm{CO}_{2}$ laser $10.6 \mu \mathrm{m}, \sim 1.7 \mathrm{~W}$ & {$[152]$} \\
\hline $47.5 \mathrm{BaO}-5.0 \mathrm{Al}_{2} \mathrm{O}_{3}-47.5 \mathrm{~B}_{2} \mathrm{O}_{3}$ & $\beta-\mathrm{BaB}_{2} \mathrm{O}_{4}$ & $\begin{array}{l}800 \mathrm{~nm} \text { Ti-sapphire laser, } 130 \mathrm{fs} \text {, } \\
200 \mathrm{kHz}, 600 \mathrm{~mW}\end{array}$ & {$[24]$} \\
\hline $10 \mathrm{BaO}-5 \mathrm{Na}_{2} \mathrm{O}-45 \mathrm{~B}_{2} \mathrm{O}_{3}$ & $\mathrm{BaNaB}_{9} \mathrm{O}_{15}$ & $\begin{array}{l}\text { heat treatment then being exposed } \\
\text { to laser irradiation (1064 } \mathrm{nm})^{m}\end{array}$ & [153] \\
\hline $10 \mathrm{SrO}-5 \mathrm{Li}_{2} \mathrm{O}-45 \mathrm{~B}_{2} \mathrm{O}_{3}$ & $\mathrm{SrLiB}_{9} \mathrm{O}_{15}$ & $\begin{array}{l}\text { heat treatment then being exposed } \\
\text { to laser irradiation (1064 } \mathrm{nm})^{\text {m }}\end{array}$ & [153] \\
\hline $25 \mathrm{La}_{2} \mathrm{O}_{3}-25 \mathrm{~B}_{2} \mathrm{O}_{3}-50 \mathrm{GeO}_{2}$ & $\mathrm{LaBGeO}_{5}$ & $800 \mathrm{~nm}, 70 \mathrm{fs}, 250 \mathrm{kHz}$ & {$[25]$} \\
\hline $\mathrm{Sm}_{0.5} \mathrm{La}_{0.5} \mathrm{BGeO}_{5}$ & $\mathrm{LaBGeO}_{5}$ & $\begin{array}{l}\text { heat-assisted Nd:YAG CW laser }{ }^{\ddagger} \text {, } \\
1064 \mathrm{~nm}\end{array}$ & [154] \\
\hline $\mathrm{Nd}_{0.2} \mathrm{La}_{0.8} \mathrm{BGeO}_{5}{ }^{\mathrm{J}}$ & $\mathrm{LaBGeO}_{5}$ & $\begin{array}{l}\text { heat-assisted Ti-Sapphire CW laser, } \\
800 \mathrm{~nm}\end{array}$ & {$[155]$} \\
\hline $33.3 \mathrm{BaO}-16.7 \mathrm{TiO}_{2}-50 \mathrm{GeO}_{2}$ & $\mathrm{Ba}_{2} \mathrm{TiGe}_{2} \mathrm{O}_{8}$ & $800 \mathrm{~nm}, 150 \mathrm{fs}, 250 \mathrm{kHz}$ & {$[21]$} \\
\hline $\begin{array}{l}\mathrm{NiO}-, \mathrm{Fe}_{2} \mathrm{O}_{3-} \text {, and } \mathrm{V}_{2} \mathrm{O}_{5} \text {-doped }(0.3- \\
1 \%) 33.3 \mathrm{BaO}-16.7 \mathrm{TiO}_{2}-50 \mathrm{GeO}_{2}\end{array}$ & $\mathrm{Ba}_{2} \mathrm{TiGe}_{2} \mathrm{O}_{8}$ & CW Nd:YAG laser, 1064 nm, $1.0 \mathrm{~W}$ & [143] \\
\hline $33.3 \mathrm{BaO}-16.7 \mathrm{TiO}_{2}-50 \mathrm{SiO}_{2}$ & $\mathrm{Ba}_{2} \mathrm{TiSi}_{2} \mathrm{O}_{8}$ & $800 \mathrm{~nm}, 150 \mathrm{fs}, 250 \mathrm{kHz}$ & {$[10]$} \\
\hline $32.5 \mathrm{BaO}-32.5 \mathrm{TiO}_{2}-35.0 \mathrm{SiO}_{2}$ & $\mathrm{BaTiO}_{3}, \mathrm{Ba}_{2} \mathrm{TiSi}_{2} \mathrm{O}_{8}$ & $800 \mathrm{~nm}, \sim 120 \mathrm{fs}, 200 \mathrm{kHz}$ & {$[18]$} \\
\hline $\begin{array}{l}5.0 \mathrm{Na}_{2} \mathrm{O}-36.0 \mathrm{BaO}-39.0 \mathrm{TiO}_{2}-20.0 \mathrm{SiO}_{2} \\
\Rightarrow\end{array}$ & $\mathrm{BaTiO}_{3}, \mathrm{Ba}_{2} \mathrm{TiSi}_{2} \mathrm{O}_{8}$ & $800 \mathrm{~nm}, \sim 120 \mathrm{fs}, 200 \mathrm{kHz}$ & {$[18]$} \\
\hline $33.3 \mathrm{BaO}-16.7 \mathrm{TiO}_{2}-50 \mathrm{SiO}_{2}-0.5 \mathrm{Er}_{2} \mathrm{O}_{3}{ }^{25}$ & $\mathrm{Ba}_{2} \mathrm{TiSi}_{2} \mathrm{O}_{8}$ & $800 \mathrm{~nm}, 150 \mathrm{fs}, 250 \mathrm{kHz}$ & [156] \\
\hline $\begin{array}{l}\mathrm{NiO}-, \mathrm{Fe}_{2} \mathrm{O}_{3-} \text {, and } \mathrm{V}_{2} \mathrm{O}_{5} \text {-doped (0.3- } \\
1 \%) 33.3 \mathrm{BaO}-16.7 \mathrm{TiO}_{2}-50 \mathrm{SiO}_{2}\end{array}$ & $\mathrm{Ba}_{2} \mathrm{TiSi}_{2} \mathrm{O}_{8}$ & CW Nd:YAG laser, 1064 nm & [143] \\
\hline $40 \mathrm{SrO}-20 \mathrm{TiO}_{2}-40 \mathrm{SiO}_{2}$ & $\mathrm{Sr}_{2} \mathrm{TiSi}_{2} \mathrm{O}_{8}$ & $800 \mathrm{~nm}, 150 \mathrm{fs}, 250 \mathrm{kHz}$ & [157] \\
\hline $33.3 \mathrm{SrO}-16.7 \mathrm{TiO}_{2}-50 \mathrm{SiO}_{2}$ & $\mathrm{Sr}_{2} \mathrm{TiSi}_{2} \mathrm{O}_{8}$ & $1030 \mathrm{~nm}, 300 \mathrm{fs}, 300 \mathrm{kHz}$ & {$[26]$} \\
\hline $\begin{array}{l}\mathrm{CuO}(1 \%) \text {-doped } 25 \mathrm{Li}_{2} \mathrm{O}-25 \mathrm{Nb}_{2} \mathrm{O}_{5}- \\
50 \mathrm{TeO}_{2}\end{array}$ & $\mathrm{LiNbO}_{3}$ & $\begin{array}{l}\text { heat-assisted CW Nd:YAG laser }{ }^{\circ} \text {, } \\
1064 \mathrm{~nm}, 0.59 \mathrm{~W}\end{array}$ & {$[158]$} \\
\hline$(100-x) \mathrm{LiBO}_{2}-\mathrm{xNb}_{2} \mathrm{O}_{5}(5 \leq \mathrm{x} \leq 20)^{\text {๑ }}$ & $\mathrm{LiNbO}_{3}$ & $\begin{array}{l}\text { heat treatment then being exposed } \\
\text { to laser irradiation }(1064 \mathrm{~nm})^{m}\end{array}$ & {$[159]$} \\
\hline
\end{tabular}




\begin{tabular}{|c|c|c|c|}
\hline $32.5 \mathrm{Li}_{2} \mathrm{O}-27.5 \mathrm{Nb}_{2} \mathrm{O}_{5}-40.0 \mathrm{SiO}_{2}$ & $\mathrm{LiNbO}_{3}$ & $800 \mathrm{~nm}, \sim 120 \mathrm{fs}, 200 \mathrm{kHz}$ & {$[18]$} \\
\hline $30.0 \mathrm{Li}_{2} \mathrm{O}-10.0 \mathrm{Nb}_{2} \mathrm{O}_{5}-40.0 \mathrm{SiO}_{2}$ & $\mathrm{LiNbO}_{3}$ & $800 \mathrm{~nm}, \sim 120 \mathrm{fs}, 200 \mathrm{kHz}$ & {$[18]$} \\
\hline $33 \mathrm{Li}_{2} \mathrm{O}-33 \mathrm{Nb}_{2} \mathrm{O}_{5}-34 \mathrm{SiO}_{2}$ & $\mathrm{LiNbO}_{3}$ & $1030 \mathrm{~nm}, 300$ fs, $300 \mathrm{kHz}$ & {$[23]$} \\
\hline $1 \mathrm{CuO}-40 \mathrm{Li}_{2} \mathrm{O}-32 \mathrm{Nb}_{2} \mathrm{O}_{5}-28 \mathrm{SiO}_{2}{ }^{\mathrm{m}}$ & $\mathrm{LiNbO}_{3}$ & $\begin{array}{l}\text { CW lasers, } 795 \mathrm{~nm}, 1064 \mathrm{~nm} \text {, and } \\
1080 \mathrm{~nm}\end{array}$ & [144] \\
\hline $0.5 \mathrm{CuO}-35 \mathrm{Li}_{2} \mathrm{O}-30 \mathrm{Nb}_{2} \mathrm{O}_{5}-35 \mathrm{SiO}_{2}^{\curlyvee}$ & $\mathrm{LiNbO}_{3}$ & $\begin{array}{l}\text { CW Yb-fiber laser laser, } 1080 \mathrm{~nm} \text {, } \\
1.3 \mathrm{~W}\end{array}$ & {$[160]$} \\
\hline $0.5 \mathrm{CuO}-40 \mathrm{Li}_{2} \mathrm{O}-32 \mathrm{Nb}_{2} \mathrm{O}_{5}-28 \mathrm{SiO}_{2}{ }^{\mathrm{g}}$ & $\mathrm{LiNbO}_{3}$ & $\begin{array}{l}\text { CW Yb-fiber laser laser, } 1080 \mathrm{~nm} \text {, } \\
1.3 \mathrm{~W}\end{array}$ & {$[160]$} \\
\hline $\begin{array}{l}0.5 \mathrm{CuO}-0.5 \mathrm{Er}_{2} \mathrm{O}_{3}-40 \mathrm{Li}_{2} \mathrm{O}-32 \mathrm{Nb}_{2} \mathrm{O}_{5^{-}} \\
28 \mathrm{SiO}_{2}{ }^{8, \Omega},\end{array}$ & $\mathrm{LiNbO}_{3}$ & CW Yb-fiber laser laser, $1080 \mathrm{~nm}$ & {$[160]$} \\
\hline $\begin{array}{l}5 \mathrm{Sm}_{2} \mathrm{O}_{3}-35 \mathrm{Li}_{2} \mathrm{O}-30 \mathrm{Nb}_{2} \mathrm{O}_{5}-2 \mathrm{SiO}_{2}- \\
15 \mathrm{~B}_{2} \mathrm{O}_{3}{ }^{8, \Omega}\end{array}$ & $\mathrm{LiNbO}_{3}$ & $\begin{array}{l}\text { CW Yb-fiber laser laser, } 1080 \mathrm{~nm} \text {, } \\
2.4 \mathrm{~W}\end{array}$ & {$[160]$} \\
\hline
\end{tabular}

${ }^{8}$ With the substitution of $\mathrm{K}_{2} \mathrm{O}$ for $\mathrm{P}_{2} \mathrm{O}_{5}$, glass transition and crystallization temperatures decrease. ${ }^{*}$ Lines were not constructed at scanning speed of $1 \mu \mathrm{m} / \mathrm{s}$ but $0.5 \mu \mathrm{m} / \mathrm{s}$. ${ }^{m} \mathrm{x}=0,2$, or 5 for La and $\mathrm{x}=2,5$ for Er; $\mathrm{TeO}_{2}$-based glasses containing a large amount of $\mathrm{Sm}_{2} \mathrm{O}_{3}$ tend to crystallize easily; the glasses containing $\mathrm{Er}_{2} \mathrm{O}_{3}$ show more low thermal stabilities against crystallization compared with other glasses containing $\mathrm{Sm}_{2} \mathrm{O}_{3}$ or $\mathrm{La}_{2} \mathrm{O}_{3}$; the growth rate of $\mathrm{Sm}_{2} \mathrm{Te}_{6} \mathrm{O}_{15}$ depends strongly on the amount of $\mathrm{Sm}_{2} \mathrm{O}_{3}$ content. "During irradiation, the glass plates were heated $\left(\sim 450^{\circ} \mathrm{C}\right.$ and $T_{g}$ is $\left.626^{\circ} \mathrm{C}\right)$, because it was difficult to induce crystallization by laser irradiations without this process. ${ }^{\circ} \mathrm{x}=3$ or 7.625 ; the periodicity of domain structures changes largely depending on $\mathrm{Er}_{2} \mathrm{O}_{3}$ content; with no $\mathrm{Gd}_{2} \mathrm{O}_{3}$, homogeneous crystal lines with no periodic domain structures; the formed periodic domain structure is due to spontaneous strains in $\beta^{\prime}-(\mathrm{Sm}, \mathrm{Gd})_{2}\left(\mathrm{MoO}_{4}\right)_{3}$. ${ }^{b}$ For $0.66 \mathrm{~W}$ one, a homogeneous crystal line (width of $\sim 5 \mathrm{~mm}$ ) is obtained but for $>0.8 \mathrm{~W}$, crystals with a rough morphology grow occured. "The glasses were heated at $\sim 150^{\circ} \mathrm{C}$ to avoid the fracture of glasses; crystal grains (on the glass surface) with a diameter of $5^{\sim 30} \mu \mathrm{m}$ were firstly prepared by a sintering method; then laser was used to irradiate the crystal grains on the surface and grains were dissolved into the interior of the glasses, froming crystalline dots. ${ }^{\circ}$ Crystal lines are $\beta-\mathrm{BaB}_{2} \mathrm{O}_{4}$ single crystals with the c-axis orientation along the laser scanning direction. The $\beta-\mathrm{BaB}_{2} \mathrm{O}_{4}$ phase is easily obtained from a glass when adding $\mathrm{TiO}_{2}$ or $\mathrm{SiO}_{2}$ to the $\mathrm{BaO}^{-} \mathrm{B}_{2} \mathrm{O}_{3}$ glass system; laser-incuced crystallites shown the same morphology as that of ones crystallized by conventional thermal treatment; the crystal growth rate exhibted higher than that the one obtained by heating in an electric furnace. The amorphous glass is heated in the vicinity of $\mathrm{T}_{\mathrm{g}}$, to induce nano-sized crystallites, and then exposed to laser. "Under optimal condition, one of the grains acting as the seed for further growth as a single crystal; devitrifies congruently into a ferroelectric phase of the same composition as the parent glass. "Sometimes, the glass was heated during the laser irradiation; the external sample heating decreased the cooling rate of the laser-irradiated zones, helping in crystallization and preventing cracking. "Polycrystalline lines and dots are produced and the crystallization occurs congruently similar to the one of devitrification through furnace heating. ${ }^{\diamond}$ The irradiation time needed for the crystallization can be drastically decreases from a few minutes to $1 \mathrm{~s}$ or so by adding $\mathrm{Na}_{2} \mathrm{O}$ to the ternary system. ${ }^{25}$ The irradiation time for crystallization in $\mathrm{Er}^{3+}$ doped $\mathrm{BaO}-\mathrm{TiO}_{2}-\mathrm{SiO}_{2}$ (BTS) glasses is longer than that in $\mathrm{BTS}$ glasses under the same irradiation conditions due to the occurrence of upconversion luminescence from $\mathrm{Er}^{3+}$-ions. ${ }^{00} 150^{\circ} \mathrm{C}$; ${ }^{3} \mathrm{LiNb}_{3} \mathrm{O}_{8}$ phase formation increases with the increase of $\mathrm{Nb}_{2} \mathrm{O}_{5}(x \geq 20)$; $\mathrm{LiBO}_{2}$ or $\mathrm{Li}_{2} \mathrm{~B}_{4} \mathrm{O}_{7}$ phases formation when $\mathrm{x} \geq 5$. ${ }^{\mathrm{m}}$ The absorption coefficients at the wavelengths of $795 \mathrm{~nm}$ (LD laser), $1064 \mathrm{~nm}$ (Nd:YAG laser), and $1080 \mathrm{~nm}$ (Yb:YVO laser) for this glass were evaluated to be $7.19,2.91$, and $2.72 \mathrm{~cm}^{-1}$, respectively. ${ }^{\curlyvee}$ Distorted cristalline line was observed. ${ }^{8}$ Uniform 
crystalline lines are formed on the glass surface. ${ }^{\Omega} \mathrm{LiNbO}_{3}$ can be used as hosts for doping with optically active rare earth ions, becoming luminescent materials.

Based on the above discussion, $\mathrm{Li}_{2} \mathrm{O}-\mathrm{Nb}_{2} \mathrm{O}_{5}-\mathrm{SiO}_{2}$ (LNS) glass system was chosen in this thesis because of advantages such as optical transparency [144], wide range of chemical compositions [140], and favorable fs laser-induced crystallization [18]. Furthermore, the laser-induced crystal, $\mathrm{LiNbO}_{3}$, shows excellent nonlinear properties which will be discussed in details in Section 2.3.

\subsubsection{How to orient crystals}

The crystal orientation can be achieved from two aspects: anisotropy in the free enthalpy (i.e. the minimum one) or gradients in kinetics (i.e. the maximum one) as a function of orientation [124]. The anisotropy can be created by external field (e.g. electric field). In that case, by controlling the external field orientation (e.g. temperature gradient), the crystal orientation can be mastered.

Table 4 Methods for glass-ceramics with oriented crystals

\begin{tabular}{|c|c|}
\hline Method & Comments \\
\hline Controlled heat treatment & $\begin{array}{l}\text { Using electric furnace or hot stage, the use of surface discontinuity } \\
\text { or/and a thermal gradient for orientation, time consuming, unable to } \\
\text { locate precipitated zones precisely }\end{array}$ \\
\hline $\begin{array}{l}\text { Ultrasonic surface } \\
\text { treatment }\end{array}$ & $\begin{array}{l}\text { Exposing the samples to ultrasonic energy transmitted through } \\
\text { separate aqueous suspensions of corresponding particles, rinsing, and } \\
\text { then the samples are dried and subjected to a heat treatment }\end{array}$ \\
\hline $\begin{array}{l}\text { Mechanical hot extrusion } \\
\text { (effect of a stress field) }\end{array}$ & $\begin{array}{l}\text { Highly complicated, applied extensional stress field forces the } \\
\text { precipitation in the direction of the main stress. }\end{array}$ \\
\hline $\begin{array}{l}\text { Electrochemical synthesis } \\
\text { (effect of an electric and of } \\
\text { concentration field) }\end{array}$ & $\begin{array}{l}\text { Dendritic growth in the direction of crystallographic c-axis } \\
\text { perpendicular to the electrode surface, a decrease in viscosity in the } \\
\text { vicinity of the cathode. A concentration gradient and a conductivity } \\
\text { gradient and thus an internal electric field in the opposite direction } \\
\text { than the external one is developed, the orientation of the growth is } \\
\text { thus enabled }\end{array}$ \\
\hline Magnetic field effect & $\begin{array}{l}\text { Well-polished glass plates are placed in a superconducting magnet, } \\
\text { and submitted to a heat-treatment, the orientation of the c-axis to the } \\
\text { direction of a high magnetic field ( } 10 \text { Tesla) }\end{array}$ \\
\hline $\begin{array}{l}\text { fs laser-induced } \\
\text { crystallization }\end{array}$ & $\begin{array}{l}\text { Spatially selected structural modifications in transparent materials, } \\
\text { various kinds of lasers can be used }\end{array}$ \\
\hline
\end{tabular}


So far, various methods have been employed to obtain glass-ceramics with oriented crystals, which are listed in Table 4. Heat treatment is a traditional technique, this process is time consuming and the spatially precipitation of oriented crystal is limited [161]. Ferreira et al. [162] have compared the $\mathrm{CO}_{2}$ laser radiation and heat treatment for crystallization in $92 \mathrm{SiO}_{2}-4 \mathrm{Nb}_{2} \mathrm{O}_{5}-$ $4 \mathrm{Li}_{2} \mathrm{O}$ (mol\%) glass. Through laser radiation, the treatment processing time can be reduced more than 28 times than the heat treatment. Appling electric field, the spatial crystal orientation can be improved but it commonly needs complicated facilities and electric energy [163]. Compared with the above two methods, fs laser-induced crystallization in glasses is a promising tool in achieving nonlinear optical crystal orientation with 3D, sub-micrometer spatial resolution [1].

\subsubsection{Privious work on fs laser-induced chemical process in glasses}

As discussed in Section 2.1.2.2, for high repetition rate (ca. $>100 \mathrm{kHz}$ ) in fs laser irradiation, the time between successive laser pulses is shorter than the one for heat to diffuse away (typical at $\mu s$ scale). A thermal accumulation in the focal volume occurs, which can be used to modify glass local structures and properties (e.g. local compositional changes).

There is an opposite migration behavior of glass formers and network modifiers. Shimizu et al. [128] demonstrated that under a thermal gradient by fs laser irradiation ( $80 \mathrm{fs}, 800 \mathrm{~nm}, 250$ $\mathrm{kHz}$ ), in $50 \mathrm{CaO}-50 \mathrm{SiO}_{2}$ (mol\%) glass, the glass former (i.e. Si) strongly bonded with oxygen have a tendency to migrate to the hot region (i.e. the focal region). In contrast, the network modifier (i.e. Ca) showed a migration behavoir in the reverse way. This element redistribution can be explained by temperature-gradient-driven diffusions. It is worth noting that, even with congruent glass system, a destabilizing chemical composition gradient can be created. Similarly phenomena of chemical migration was observed for precipitation of $\mathrm{Sr}_{2} \mathrm{TiSi}_{2} \mathrm{O}_{8}$ from 33.3SrO$16.7 \mathrm{TiO}_{2}-50 \mathrm{SiO}_{2}$ (mol\%) glass after fs laser irradiation (300 fs, $1030 \mathrm{~nm}, 300 \mathrm{kHz}$ ) [26]. Sometimes there is a different distribution of relative concentration between the network modifiers. Luo et al. [164] reported the elemental migration in $60 \mathrm{SiO}_{2}-20 \mathrm{Al}_{2} \mathrm{O}_{3}-20 \mathrm{CaF}_{2}-3 \mathrm{Bi}_{2} \mathrm{O}_{3}(\mathrm{~mol} \%)$ glasses after fs laser irradiation (150 fs, $250 \mathrm{kHz}$ ). They found that the relative concentration of $\mathrm{Si}, \mathrm{Al}$, and $\mathrm{O}$ ions (i.e. the network formers) is larger in the irradiated area and surrounded by a lower concentration area around the laser modified region. Yet, the relative concentration of $\mathrm{Ca}$ ions (i.e. the network modifier) is lower in the modified zone than that in the unmodified zones. However, the relative concentration of $\mathrm{Bi}$ ions (the other network modifier) is opposed to that of 
Ca ions. This discrepancy of the network modifiers may be due to $\mathrm{Bi}$ ions' reduction properties (i.e. $\mathrm{Bi}$ ions are reduced to $\mathrm{Bi}$ ) and their travelling through the glass matrix.

Usually monovalent ions migrate to the low index zone, while multivalent ions concentrate at the high index region. It can be used to improve the refractive index change, thus controlling the waveguide properties. Hoyo et al. [165] shown that high repetition rate fs laser irradiation (1030 $\mathrm{nm}, 500 \mathrm{kHz}, 400 \mathrm{fs}$ ) can result in the local chemical compositional changes, producing high performance optical waveguides in phosphate-based glasses, $\sim 65 \mathrm{P}_{2} \mathrm{O}_{5}-10 \mathrm{La}_{2} \mathrm{O}_{3}-10 \mathrm{Al}_{2} \mathrm{O}_{3}-10 \mathrm{~K}_{2} \mathrm{O}$, mol \%) doped with $\mathrm{Er}_{2} \mathrm{O}_{3}(2 \mathrm{wt} \%)$ and $\mathrm{Yb}_{2} \mathrm{O}_{3}$ (4 wt\%). They found that the waveguide refractive index contrast is mainly due to the cross migration of La ions, enriched in the guiding region. In contrast, $\mathrm{K}$ ions migrate to the low index zone. More importantly, by using a slit shaping technique with moderate NA (NA $=0.68$ ) writing optics, the cross section and refractive index of the guiding structures can be precisely controlled. Similar cross migration of Te ions to the guiding zone was observed in phosphate modified tellurite glass $\left(50 \mathrm{TeO}_{2}-2 \mathrm{P}_{2} \mathrm{O}_{5}-20 \mathrm{Na}_{2} \mathrm{O}-5 \mathrm{ZnO}-\right.$ $5 \mathrm{ZnF}_{2}$ with $1 \mathrm{wt} \% \mathrm{Er}_{2} \mathrm{O}_{3}, 1 \mathrm{wt} \% \mathrm{CeO}_{2}$, and $2 \mathrm{wt} \% \mathrm{Yb}_{2} \mathrm{O}_{3}$ ) by high repetition rate fs laser irradiation (1040 nm, $1 \mathrm{MHz}, 400$ fs) [166]. The authors demonstrated an increase of $\mathrm{TeO}_{3}$ units and a reduction of $\mathrm{TeO}_{4}$ units in the $\mathrm{Te}$ rich regions, increasing the packing fraction to help densification.

The elemental migration can also affect the final shape of crystalline phase. Yonesaki et al. [18] shown that after fs laser irradiation ( $120 \mathrm{fs}, 800 \mathrm{~nm}, 200 \mathrm{kHz}, \sim 600 \mathrm{~mW}$ ), the Si concentration increased in the center of the irradiated region and decreased at the surrounding zone, while there is a depression of $\mathrm{Nb}$ concentration in the focal area center and an increase outside of the center, resulting in a precipitation of ring-shaped crystalline phase in $32.5 \mathrm{Li}_{2} \mathrm{O}-27.5 \mathrm{Nb}_{2} \mathrm{O}_{5}-$ $40.0 \mathrm{SiO}_{2}$ glass. Ring-shaped crystalline phase (i.e. $\beta-\mathrm{BaB}_{2} \mathrm{O}_{4}$ crystal) was also observed in 47.5BaO-5.0Al $\mathrm{O}_{3}-47.5 \mathrm{~B}_{2} \mathrm{O}_{3}$ glass, in which the concentration of $\mathrm{Al}$ increased at the focal area centre after the fs laser irradiation.

It is worth noting that because of the isotropic diffusion of photo-thermal energy, the shape of the elemental spatial distribution is axially symmetric about the laser axis. However, Sakakura et al. [167] showed a method for controlling the shape of the elemental distribution (i.e. squareshaped) in an alumina-borosilicate glass at $\mu \mathrm{m}$ scale by simultaneous irradiation at multiple spots with 250 and $1 \mathrm{kHz}$ laser pulses. 


\subsection{Lithium niobate}

Lithium niobate $\left(\mathrm{LiNbO}_{3}\right)$ is an interesting and multifunctional human-made ferroelectric material. Matthias et al. [168] firstly reported it is ferroelectric in 1949. So far, it has received intensive attention because of its unique electro-optic, non-linear, piezoelectric properties, and the potential applications in acoustic-optics and holographic data processing (see details in Table 2). $\mathrm{LiNbO}_{3}$ was chosen as the material system in this thesis. So, a summary of the general properties of $\mathrm{LiNbO}_{3}$ is outlined in this section.

\subsubsection{Crystallographic properties of $\mathrm{LiNbO}_{3}$ crystal}

\subsubsection{1 $\mathrm{Li}_{2} \mathrm{O}-\mathrm{Nb}_{2} \mathrm{O}_{5}$ phase-equilibrium diagram}

The phase-equilibrium diagram of $\mathrm{LiNbO}_{3}$ is shown in Figure 30a. It can be seen that the phase exists over a large range of solid solution: from Li-poor compositions (i.e. $\sim 45 \mathrm{~mol} \% \mathrm{Li}_{2} \mathrm{O}$ at $1200^{\circ} \mathrm{C}$ ) to the one near to stoichiometric value [169]. This nonstoichiometry is correspond to the mechanism $5 \mathrm{Li}^{+} \Leftrightarrow \mathrm{Nb}^{5+}$ which leads to cation vacancies according to the general formula $\mathrm{Li}_{1-5 x}(\mathrm{Nb})_{1+x} \square_{4 \times} \mathrm{O}_{3}$, where $\square$ indicates a vacancy [40]. $\mathrm{T}_{m}$ is around $1257^{\circ} \mathrm{C}$. Stoichiometric $\mathrm{LiNbO}_{3}$ means that the ratio of $\mathrm{Li} /(\mathrm{Li}+\mathrm{Nb})$ is 0.50 and congruent $\mathrm{LiNbO}_{3}$ means that the ratio $\mathrm{Li} /(\mathrm{Li}+$ $\mathrm{Nb}$ ) is 0.485 . The congruent point is the one where a solid phase transforms directly into a liquid.
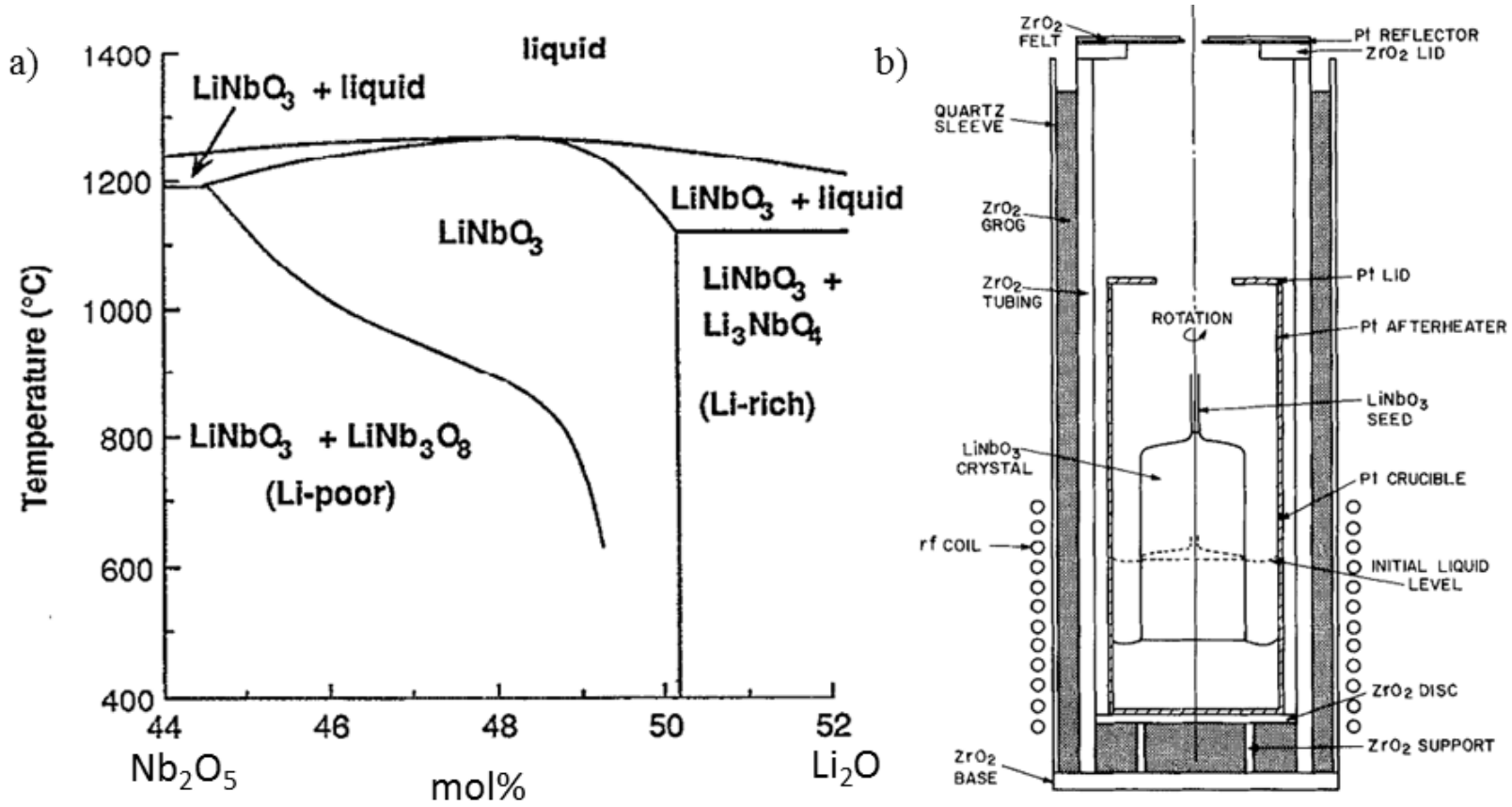

Figure 30 a) Phase-equilibrium diagram for $\mathrm{LiNbO}_{3}$ (cited from $[169,170]$ ) and b) schematic of the furnace for Czochralski growth at $\mathrm{LiNbO}_{3}$ (cited from [171]) 
$\mathrm{LiNbO}_{3}$ is commonly grown by a Czochralski technique with a composition near the congruently melting value ( 48.5 mol\% $\left.\mathrm{Li}_{2} \mathrm{O}\right)$; where high quality single crystal and uniform composition can be grown. Crystals grown from other Li-deficient, Li-surplus, or stoichiometric melts own compositions differing from that of the melt and the composition changing strongly along the growth direction [172].

The schematic of the furnace for Czochralski growth is illustrated in Figure 30b. The starting materials (i.e. $\mathrm{Li}_{2} \mathrm{CO}_{3}$ and $\mathrm{Nb}_{2} \mathrm{O}_{5}$ ) were melted after the complete decomposition of $\mathrm{Li}_{2} \mathrm{CO}_{3}$. By introducing a crystal seed, then the crystal growth began during the pulling process. Finally, the single crystal was formed and taken out. The single crystal is growing along c axis direction, which is a method to control the crystal orientation. However, for these methods, there are some disadvantages such as crack forming, some coloration creation, and segregation of constituent ions.

\begin{tabular}{|l|l|l|}
\hline Phase: $\mathrm{LiNb}_{3} \mathrm{O}_{8}$ & $\mathrm{LiNbO}_{3}$ & $\mathrm{Li}_{3} \mathrm{NbO}_{4}$ \\
\hline Space group: $\mathrm{P}_{1} / \mathrm{a}$ & $\mathrm{R3c}$ & $\mathrm{I}-43 \mathrm{~m}$ \\
\hline & & \\
\hline
\end{tabular}

Figure 31 Space group and structure in $\mathrm{LiNb}_{3} \mathrm{O}_{8}, \mathrm{LiNbO}_{3}$, and $\mathrm{Li}_{3} \mathrm{NbO}_{4}$ (cited from [173])

The phase diagram reveals that for $\mathrm{Li}$ deficient composition, there is separation into $\mathrm{LiNbO}_{3}$ and $\mathrm{LiNb}_{3} \mathrm{O}_{8}$. As shown in Figure 31 left, the symmetry of $\mathrm{LiNb}_{3} \mathrm{O}_{8}$ phase is monoclinic (space group $\mathrm{P} 2_{1} / \mathrm{a}$ ): the oxygen atoms are approximately hexagonally close-packed with the metal atoms in octahedral interstices; the occupied octahedral from zig-zag strings of two types in the $b$ axis direction, with one type containing $\mathrm{Nb}$ atoms and the other a regular alternation of $\mathrm{Nb}$ and $\mathrm{Li}$ atoms [174]. This phase does not contribute to ferroelectric or electro-optic properties. So, for the optical applications, $\mathrm{LiNbO}_{3}$ phase is preferred. H. Akazawa et al. [175] demonstrates that the volume fraction of $\mathrm{LiNb}_{3} \mathrm{O}_{8}$ with respect to $\mathrm{LiNbO}_{3}$ becomes larger at longer annealing periods and at higher annealing temperatures due to the $\mathrm{Li}_{2} \mathrm{O}$ evaporation. Fast crystallization in a vacuum suppresses the formation of the $\mathrm{LiNb}_{3} \mathrm{O}_{8}$ phase. So a rapid thermal annealing of 
oxygen-rich and void-free $\mathrm{LiNbO}_{3}$ film in a vacuum is a solution to obtaining transparent, single $\mathrm{LiNbO}_{3}$ phase film.

For the one with excess $\mathrm{Li}$ composition, it is separated into $\mathrm{LiNbO}_{3}$ and $\mathrm{Li}_{3} \mathrm{NbO}_{4}$. Even when the temperature is up to $1327^{\circ} \mathrm{C}$, no mutual solubility between $\mathrm{Li}_{2} \mathrm{O}$ and $\mathrm{Li}_{3} \mathrm{NbO}_{4}$ was illustrated in the phase diagram of $\mathrm{Li}_{2} \mathrm{O}-\mathrm{Nb}_{2} \mathrm{O}_{5}$ [176]. $\mathrm{Li}_{3} \mathrm{NbO}_{4}$ is a cation-disordered rock salt-type phase. Lithium ions can quickly migrate in percolative network in bulk without a sacrifice in kinetics and $\mathrm{Li}_{3} \mathrm{NbO}_{4}$-based materials can be used for high-capacity electrode materials for rechargeable lithium batteries [177]. As shown in Figure 31 right, the structure is builted of $\mathrm{Nb}_{4} \mathrm{O}_{16}$ clusters, forming a body-centered cubic lattice; lithium ions locate along $\mathrm{Nb}_{4} \mathrm{O}_{16}$ clusters; oxygen ions coordinate to niobium ions and lithium ions octahedrally [178]. For compassion, Figure 31 lists all the three phases. The structure of $\mathrm{LiNbO}_{3}$ will be discussed in details the following section. Only their structure is not centro-symmetric.

$\mathrm{LiNbO}_{3}$ glass can be formed by using the twin-roller quenching technique [179]. However, in most cases, the glass flakes contains crystalline traces. Addition of $\mathrm{SiO}_{2}$ [140], alkaline earth oxides (e.g. $\mathrm{BaO}$ ) [180], or $\mathrm{Al}_{2} \mathrm{O}_{3}$ [181] to $\mathrm{Li}_{2} \mathrm{O}-\mathrm{Nb}_{2} \mathrm{O}_{5}$ system facilitates glass formation (see details in Appendix XIV).

\subsubsection{Crystal structure of $\mathrm{LiNbO}_{3}$}

Curie temperature $\left(T_{c}\right)$ is the temperature above which certain material loses its ferromagnetism and becomes paramagnetic. $\mathrm{LiNbO}_{3}$ has high $\mathrm{T}_{\mathrm{c}}\left(\sim 1210^{\circ} \mathrm{C}\right)$ [38], and this value varied with the crystal composition [169].

a)

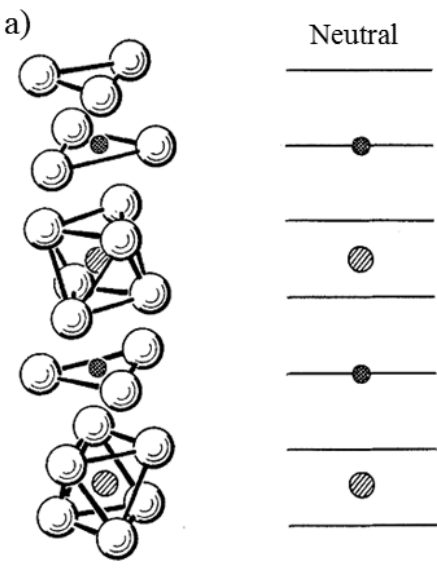

Paraelectric b)

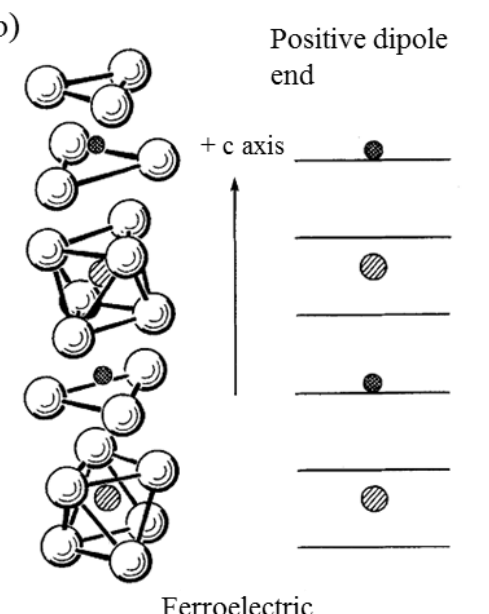

c)

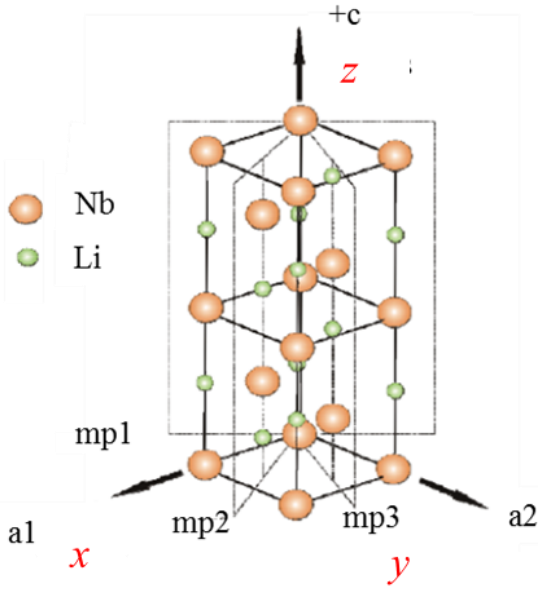

Figure 32 Positions of the lithium atoms (double cross-hatched circles) and the niobium atoms (single crosshatched circles) with respect to the oxygen octahedra in the a) paraelectric phase and b) ferroelectric phase of lithium niobate, the horizontal lines on the right side stands for the oxygen layers (after [38]); c) hexagonal unit cell of $\mathrm{LiNbO}_{3}$, the Cartesian $\mathrm{x}, \mathrm{y}, \mathrm{z}$ system is used to describe the physical tensor properties of lithium niobate (after [124]) 
$\mathrm{LiNbO}_{3}$ is formed by distorted niobium-oxygen octahedron. Chains of the distorted oxygen octahedra with the common faces, aligned along the polar axis. When $\mathrm{T}_{\mathrm{c}}<\mathrm{T}<\mathrm{T}_{\mathrm{m}}, \mathrm{LiNbO}_{3}$ is in paraelectric phases. As shown in Figure 32a, the $\mathrm{Li}^{+}$ions are localized within the oxygen planes, where the $\mathrm{Nb}^{5+}$ ions are in the centre of the oxygen octahedron. It is centro-symmetric.

When $\mathrm{T}<\mathrm{T}_{c}, \mathrm{LiNbO}_{3}$ is in the ferroelectric phase. As shown in Figure 32b, the structure is made of hexagonal close-packed oxide ions approximately where two-thirds of the octahedral sites are occupied by cations: the cations are stacked in such a manner that the sequence $\mathrm{Li}^{+}, \mathrm{Nb}^{5+}$, and cation vacancy repeats every third layer [40]. Ferroelectric behavior occurs when $\mathrm{Nb}^{5+}$ and $\mathrm{Li}^{+}$are displaced from their octahedral centres in opposite directions along the c-axis toward adjacent empty octahedra. Ferroelectric $\mathrm{LiNbO}_{3}$ shows spontaneous polarization (direction along the $\mathrm{c}$ axis, from $-c$ face to $+c$ faces). The corresponding XRD pattern is shown in Appendix XV and the crystallographic parameters are listed in Table 5.

Table 5 Crystallographic parameters of $\mathrm{LiNbO}_{3}$ crystal

\begin{tabular}{ll}
\hline Items & Value \\
\hline Crystal Symmetry & trigonal \\
Point Group & $3 \mathrm{~m}$ \\
Space group & $\mathrm{R} 3 \mathrm{c}($ no. 161) \\
Lattice Constants (hexagonal) & $\mathrm{a}=5.148 \AA$ \\
& $\mathrm{C}=13.863 \AA$ \\
\hline
\end{tabular}

As shown in Figure 32c, ferroelectric phase of $\mathrm{LiNbO}_{3}$ has conventional hexagonal unit cell (point group $3 \mathrm{~m}$, space group $R 3 c)$ with hexagonal axes $\left(a_{1} a_{2} a_{3} c\right)$. The $c$ axis corresponds to the polar axis (or optical axis). When the crystal is cooled from the melt, the face positive is the one defined as $+c$ face [124]. $a_{1} a_{2} a_{3}$ are $120^{\circ}$ apart and lie in the plane normal to the direction of $c$ axis (three-fold rotation symmetry about $c$ axis). A notation scheme of four Miller indices was used to describe direction $\left[a_{1} a_{2} a_{3} c\right]$ or plane $\left(a_{1} a_{2} a_{3} c\right)$ for hexagonal system.

The conventional choice of Cartesian coordinate for $\mathrm{LiNbO}_{3}$ is a right-handed system with the $\mathrm{z}$ axis along polar axis (the sense of $z$ axis is the same as $c$ axis), the $x$ axis is along one of the hexagonal a axis, and $y$ axis is along the direction of $z \wedge x$. In that case, $y$ axes are along the mirror plane. This Cartesian system is used to describe the physical properties of $\mathrm{LiNbO}_{3}$.

In addition, $\mathrm{LiNbO}_{3}$ has very flexible host structure toward dopants and the commonly formation of extensive solid solution areas. Remarkably, the physical properties of $\mathrm{LiNbO}_{3}$ depend on the defects in the structure and can be improved by doping with some cations (e.g. $\mathrm{Co}^{2+}$ and $\mathrm{Ni}^{2+}$ ). In the principal doping mechanism, the total number of cations remains constant [40]. 


\subsubsection{Optical properties of $\mathrm{LiNbO}_{3}$ crystal}

\subsubsection{Absorption characteristic}

The transparency range of $\mathrm{LiNbO}_{3}$ is $420-5200 \mathrm{~nm}$. $\mathrm{LiNbO}_{3}$ is optically homogeneous $\left(\sim 5 \times 10^{-5}\right.$ $/ \mathrm{cm})$, with a damage threshold of $250 \mathrm{MW} / \mathrm{cm}^{2}$ (1064 nm, $\left.10 \mathrm{~ns}\right)$ [182]. The position of the fundamental absorption edge is very sensitive to $\mathrm{LiNbO}_{3}$ composition, which can be used to characterise the composition and homogeneity of $\mathrm{LiNbO}_{3}$ single crystals [172]. The band edge position is defined as the wavelength with certain absorption coefficient (e.g. 15 or $20 \mathrm{~cm}^{-1}$ ) [172].

\subsubsection{Refractive index}

For optically anisotropic material, the electrical excitation is depending on the direction of electric field. In a linear, homogeneous, anisotropic dielectric, D is related to E as

$$
\mathrm{D}=\boldsymbol{\varepsilon} \mathrm{E}
$$

Where $\varepsilon$ is the permittivity of material, expressed as a second-order tensor ( $3 \times 3$ matrix)

$$
\varepsilon_{0}\left[\begin{array}{lll}
\varepsilon_{11} & \varepsilon_{12} & \varepsilon_{13} \\
\varepsilon_{21} & \varepsilon_{22} & \varepsilon_{23} \\
\varepsilon_{31} & \varepsilon_{32} & \varepsilon_{33}
\end{array}\right]
$$

Where $\varepsilon_{0}$ is the vacuum permittivity. Considering the conservation of electromagnetic field energy $\left(\varepsilon_{\mathrm{ij}}=\varepsilon_{\mathrm{ji}}\right)$ and the properties of crystal (symmetry), the number of independent elements could be reduced. The seven crystal classes are shown in Appendix XVI.

When the crystal oriented with the principal axes along the axes of a coordinate system the permittivity tensor could be written as (dielectric matrix)

$$
\varepsilon_{0}\left[\begin{array}{ccc}
\varepsilon_{11} & 0 & 0 \\
0 & \varepsilon_{22} & 0 \\
0 & 0 & \varepsilon_{33}
\end{array}\right]
$$

It can be seen that $\mathbf{D}$ and $\mathbf{E}$ will have the same direction when $\mathbf{E}$ coincides with the direction of the principal axis of the dielectric constants.

The principal refractive index $n_{k k}(k=1,2,3)$ have relationship with $\varepsilon_{\mathrm{kk}}$ with formula of $\mathrm{n}_{\mathrm{kk}}=\sqrt{\varepsilon_{\mathrm{kk}}}$. For uniaxial crystal (e.g. $\mathrm{LiNbO}_{3}$ ), $\varepsilon_{11}=\varepsilon_{22}=\mathrm{n}_{\mathrm{o}}{ }^{2} \neq \varepsilon_{33}=\mathrm{n}_{\mathrm{e}}{ }^{2}$ where $\mathrm{n}_{\mathrm{o}}$ and $\mathrm{n}_{\mathrm{e}}$ is known as ordinary and extraordinary indexes respectively, and the $z$ axis of the uniaxial crystal denoted the optic axis (in the direction of the optic axis). So, the refractive index matrix of $\mathrm{LiNbO}_{3}$ can be written as 


$$
\left[\begin{array}{ccc}
\mathrm{n}_{\mathrm{o}} & 0 & 0 \\
0 & \mathrm{n}_{\mathrm{o}} & 0 \\
0 & 0 & \mathrm{n}_{\mathrm{e}}
\end{array}\right]
$$

$\mathrm{LiNbO}_{3}$ is a negative uniaxial crystal. As shown in Figure $33, \mathrm{n}_{\mathrm{o}}$ is larger than $\mathrm{n}_{\mathrm{e}}$ in the transparent range.

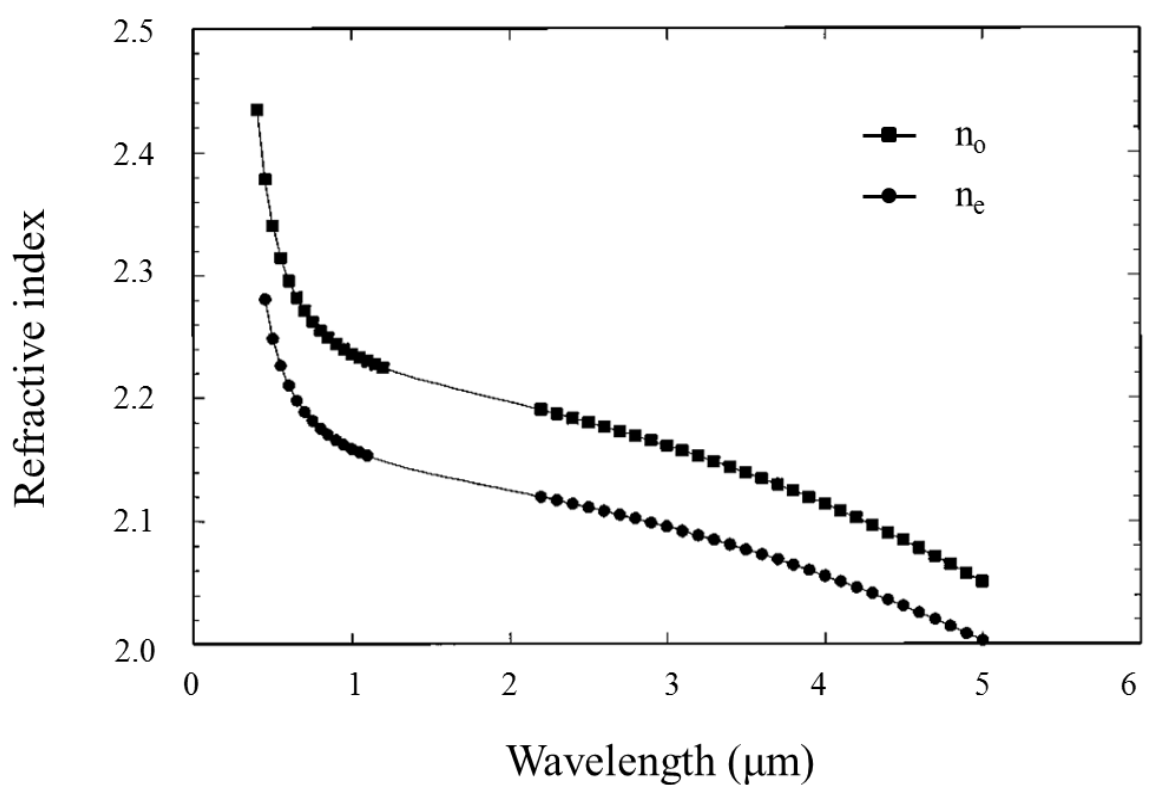

Figure 33 Refractive indices of congruently grown $\mathrm{LiNbO}_{3}$ versus wavelength. The solid curves represent the three-oscillator Sellmeier fitting the data, the measured temperature was $21^{\circ} \mathrm{C}$ (after [183])

It is common to describe the dispersion of the refractive index as a Sellmeier equation

$$
n^{2}(\lambda)=1+\sum_{j} \frac{A_{j} \lambda^{2}}{\lambda^{2}-B_{j}}
$$

Where $A_{j}$ is the absorption resonance of strength at a wavelength $\sqrt{B_{j}}$. The three-oscillator Sellmerier equation of $\mathrm{LiNbO}_{3}$ is given as [183]

$$
\begin{aligned}
& n_{\mathrm{o}}^{2}(\lambda)=1+\frac{2.6734 \lambda^{2}}{\lambda^{2}-0.01764}+\frac{1.2290 \lambda^{2}}{\lambda^{2}-0.05914}+\frac{12.614 \lambda^{2}}{\lambda^{2}-474.6} \\
& n_{\mathrm{e}}^{2}(\lambda)=1+\frac{2.9804 \lambda^{2}}{\lambda^{2}-0.02047}+\frac{0.5981 \lambda^{2}}{\lambda^{2}-0.0666}+\frac{8.9543 \lambda^{2}}{\lambda^{2}-416.08}
\end{aligned}
$$

Where $\lambda$ is the wavelength (unit is $\mu \mathrm{m}$ ).

Besides wavelength, the refractive index of $\mathrm{LiNbO}_{3}$ is also a function of temperature and the chemical composition [184].

The relationship between electric susceptibility and refractive index of crystal is described in [185] for details. In the electric dipole approximation, the induced polarization is given by 


$$
\mathbf{P}_{j}^{(1)}(\omega)=\varepsilon_{0} \mathbf{X}_{\mathrm{jk}}^{(1)}(\omega) \mathbf{E}_{\mathrm{k}}(\omega)
$$

Where $\varepsilon_{0}$ is the permittivity of free space; $\boldsymbol{\chi}_{\mathrm{jk}}^{(1)}$ is linear susceptibility tensor, a second rank tensor, a complex quality; and $\mathbf{E}_{k}(\omega)$ is the incident laser electric field.

The dielectric constant $\varepsilon(\omega)$ and refractive index $n(\omega)$ are related in the eigen frame to the real part of the susceptibility as following

$$
\mathrm{n}_{\mathrm{jk}}^{2}(\omega)=\varepsilon_{\mathrm{jk}}(\omega) / \varepsilon_{0}=1+\chi_{\mathrm{jk}}^{\prime(1)}(\omega)
$$

Electric susceptibility of $\mathrm{LiNbO}_{3}$ has two independent elements: one is along the polar axis, $\chi_{z z}$ and the other one is perpendicular to it $\chi_{x x}\left(\chi_{x x}=\chi_{y y}\right)$.

As discussed above, $\mathrm{LiNbO}_{3}$ has negative uniaxial birefringence with no (ordinary refractive index, electric field polarization normal to $\mathrm{c}$ axis) greater than ne (extraordinary refractive index, electric field polarization parallel to c axis), meaning the fast optical axis is along, whereas, the slow optical axis perpendicular to the $c$ axis. So, it is possible to deduce the dielectric susceptibility from the refractive index. For example, at the wavelength of $1030 \mathrm{~nm}, \mathrm{n}_{\mathrm{o}}=2.234$ and $n_{e}=2.157$, i.e. the index normal to polar axis (i.e. $\chi_{x x}$ ) is larger than the one parallel to polar axis (i.e. $\left.\chi_{z z}\right)$.

\subsubsection{Nonlinear optical properties}

The nonlinear coefficient of $\mathrm{LiNbO}_{3}$ is: $d_{33}=34.4 \mathrm{pm} / \mathrm{V}, \mathrm{d}_{22}=3.07 \mathrm{pm} / \mathrm{V}$, and $\mathrm{d}_{31}=\mathrm{d}_{15}=5.95$ $\mathrm{pm} / \mathrm{V}$ [103]. The orientation of the polar axis of $\mathrm{LiNbO}_{3}$ determines the second order nonlinear optical properties (will be discussed in Section 5.1.1). The relevant coefficient for a probe polarization along the polar axis of $\mathrm{LiNbO}_{3}$ is the largest $\left(d_{33}\right)$, compared to the coefficient for a probe polarization perpendicular to the polar axis $\left(d_{22}\right)$.

The description of the SHG in crystal is summarized in [185] for details. For an input monochromatic wave with frequency $\omega$, the induced polarization at the harmonic frequency $2 \omega$ is written as

$$
\mathbf{P}_{2 \omega}=\frac{\varepsilon_{0}}{2} \mathbf{\chi}_{2 \omega}^{(2)} \mathbf{E}_{\omega}^{\mathrm{P}} \mathbf{E}_{\omega}^{\mathrm{P}}
$$

Where $\varepsilon_{0}$ is the permittivity of free space, $\boldsymbol{\chi}_{2 \omega}^{(2)}$ is the second order nonlinear susceptibility tensor, and $\mathbf{E}_{\omega}^{\mathrm{P}}$ is the probing laser electric field. 
Now let us consider the expression of $\chi_{\mathrm{ijk}}^{(2)}$ components. $\chi_{2 \omega}^{(2)}$ is a third order rank tensor with 27 coefficients. In SHG analysis, another tensor is introduced

$$
\mathrm{d}_{\mathrm{ijk}}^{(2)}=\frac{1}{2} \chi_{\mathrm{ijk}}^{(2)}
$$

For convenience, $d_{i j k}^{(2)}$ is written in a contracted form, a $3 \times 6$ matrix, $d_{i l}^{(2)}$. If $j=k, I=j$, otherwise, I $=9-(j+k)$.

For $\mathrm{LiNbO}_{3}$, it is given as (Class $3 \mathrm{~m}-\mathrm{C}_{3 \mathrm{v}}, \mathrm{m} \perp \mathrm{x}$, see details in Figure $32 \mathrm{c}$ )

$$
d_{i l}=\left[\begin{array}{cccccc}
0 & 0 & 0 & 0 & d_{31} & -d_{22} \\
-d_{22} & d_{22} & 0 & d_{31} & 0 & 0 \\
d_{31} & d_{31} & d_{33} & 0 & 0 & 0
\end{array}\right]
$$




\section{Chapter 3. Experimental section}

The investigations of inscription nonlinear optical crystal in LNS glasses were done in four stages. The first part is the preparation of LNS glasses (e.g. glass making and polishing). The second part is about the fs laser direct writing system including the set-up and writing configuration definition. The third part is about the thermal field modelling in LNS glass, showing that it is possible to master the crystallization in this glass by adjusting the laser parameter (e.g. repetition rate and pulse energy). The last part is about the fs laser-induced modification (i.e. morphology, crystallization, and microstructure) observations by the tools such as scanning electron microscopy (SEM), energy-dispersive X-ray spectroscopy coupled to SEM (SEM/EDS), and electron backscattered diffraction (EBSD) measurements.

\subsection{Glass preparation}

\subsubsection{LNS glass making}

Figure 34 illustrates the pseudo-ternary phase diagram of $\mathrm{Li}_{2} \mathrm{O}-\mathrm{Nb}_{2} \mathrm{O}_{5}-\mathrm{SiO}_{2}$. Black and blue dots indicate it is easy to make glass, but red and green dots indicate that it is at least partly opaque after fabrication. $32.5 \mathrm{Li}_{2} \mathrm{O}-27.5 \mathrm{Nb}_{2} \mathrm{O}_{5}-40 \mathrm{SiO}_{2}$ and $33 \mathrm{Li}_{2} \mathrm{O}-33 \mathrm{Nb}_{2} \mathrm{O}_{5}-34 \mathrm{SiO}_{2}$ (mol\%) glasses were used in this thesis because they are on the amorphous side of the boundary between crystalline and amorphous phases. Devitrification of LNS glass into $\mathrm{LiNbO}_{3}$ has been already reported for these $[18,186]$ or close chemical composition $[18,187]$. The heating of LNS glass may lead to Li poor $\mathrm{LiNbO}_{3}$ phase with $\mathrm{Li}$ rich $\mathrm{SiO}_{2}$ phase.

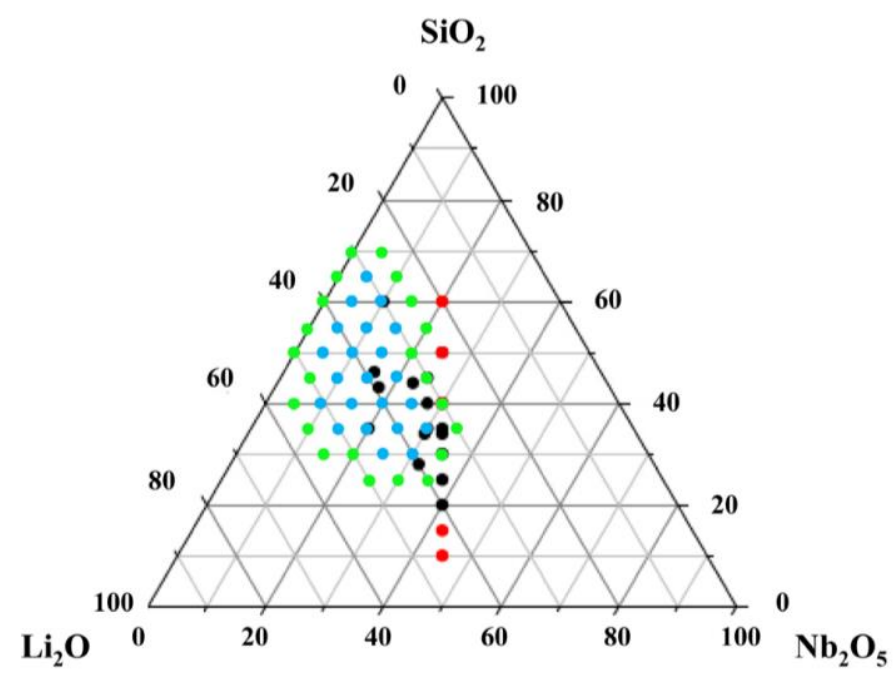

Figure 34 Pseudo-ternary phase diagram of $\mathrm{Li}_{2} \mathrm{O}-\mathrm{Nb}_{2} \mathrm{O}_{5}-\mathrm{SiO}_{2}$. Black and blue dots are glass, while red and green dots represent crystallization (green and blue dots data are redrawn from [140], black and red dots are cited from [188]) 
The annealed treatment of above LNS glasses $\left(680^{\circ} \mathrm{C}, 15 \mathrm{hrs}\right)$ display XRD patterns which can be indexed to $\mathrm{LiNbO}_{3}$ [188], confirming that these two LNS glasses are suitable to achieve $\mathrm{LiNbO}_{3}$ after thermal treatment.

LNS glasses were fabricated by melt-quenching method. $\mathrm{Li}_{2} \mathrm{CO}_{3}, \mathrm{Nb}_{2} \mathrm{O}_{5}$, and $\mathrm{SiO}_{2}$ were melted in a platinum crucible at $1430^{\circ} \mathrm{C}$ for $2 \mathrm{hrs}$. The melt mixture was poured onto a steel plate maintained at $500^{\circ} \mathrm{C}$. Then, the as-quenched glass was annealed at $540^{\circ} \mathrm{C}$ for $6 \mathrm{hrs}$ and cooled at a decreasing rate of $20^{\circ} \mathrm{C} / \mathrm{hr}$ to release the residual stress. Details of the glass preparation process can be found in previous reports $[23,189]$. In some case, $\mathrm{LiNbO}_{3}$ was employed as the starting material but no difference was noted in the properties of the final glasses [190].

The glassy characteristics of these as-quenched glasses were confirmed by XRD and differential thermal analysis (DTA) investigation [188]. The thermal parameters including glass transition temperature $\left(T_{g}\right)$, crystallization onset $\left(T_{x}\right)$, and crystallization peak thermal temperature $\left(T_{p}\right)$ of the LNS glasses are listed in Table 6. It shows that the glass transition temperature of these two LNS glasses is around $566^{\circ} \mathrm{C}$ and the crystallization in LNS glasses occurs at the temperature around $650^{\circ} \mathrm{C}$.

\begin{tabular}{lccc}
\multicolumn{4}{c}{ Table 6 Thermal parameters of LNS glasses } \\
\hline LNS glass composition (mol\%) & $\mathrm{T}_{\mathrm{g}}\left( \pm 2^{\circ} \mathrm{C}\right)$ & $\mathrm{T}_{\mathrm{x}}\left( \pm 2^{\circ} \mathrm{C}\right)$ & $\mathrm{T}_{\mathrm{p}}\left( \pm 2^{\circ} \mathrm{C}\right)$ \\
\hline $32.5 \mathrm{Li}_{2} \mathrm{O}-27.5 \mathrm{Nb}_{2} \mathrm{O}_{5}-40 \mathrm{SiO}_{2}$ & 562 & 651 & 687 \\
$33 \mathrm{Li}_{2} \mathrm{O}-33 \mathrm{Nb}_{2} \mathrm{O}_{5}-34 \mathrm{SiO}_{2}$ & 570 & 648 & 682 \\
\hline cited from $[188]$ & &
\end{tabular}

\subsubsection{LNS glass polishing}

The raw glasses were cut into small pieces by a continuous rim diamond blade (ESCIL, Ref: V1255). Then the glass was mounted to a polishing holder (the precision angle lapper, $\mathrm{PELCO}^{\circledR} \mathrm{PAL}^{\mathrm{TM}}$, product No. 814-600) by epoxy resin, and polished by a polishing machine (ESCIL, ESC $300 \mathrm{GTL}$ ). The polishing disc (PRESI) and the corresponding polishing time are listed in Table 7. Note the choice of polishing paper and polishing time is dependent on the quality of the original glass.

The LNS glasses after the above treatment were used for the following fs laser radiation experiment. It should be mentioned that to have a clearly observation of the fs laser-induced nanostructures in LNS glass by SEM, it is necessary to have fine polishing with colloidal silica (e.g. $0.03 \mu \mathrm{m})$. 
Table 7 Parameters of polishing LNS glasses

\begin{tabular}{ll}
\hline Polishing material & Time/min \\
\hline abrasive paper, P240 & $15-30$ \\
abrasive paper, P600 & 15 \\
abrasive paper, P1200 & 15 \\
diamond disc $15 \mu \mathrm{m}$ & 15 \\
diamond disc $9 \mu \mathrm{m}$ & 15 \\
diamond disc $6 \mu \mathrm{m}$ & 10 \\
diamond disc $3 \mu \mathrm{m}$ & 10 \\
diamond disc $1 \mu \mathrm{m}$ & 10 \\
diamond disc $0.5 \mu \mathrm{m}$ & 10 \\
diamond disc $0.1 \mu \mathrm{m}$ & 10 \\
colloidal silica $0.03 \mu \mathrm{m}$ & 20 \\
\hline
\end{tabular}

Phase shift interferometry (PSI, Phase Shift Technology, Inc.) was used to determine the topography of polished LNS glass. This PSI measurement is based on a amplitude-division interferometry with an objective lens of $40 \times, \mathrm{NA}=0.50$, working distance of $3.9 \mathrm{~mm}$, standard measurement area of $211 \mu \mathrm{m} \times 157 \mu \mathrm{m}$, and wavelength of $550 \mathrm{~nm}$.

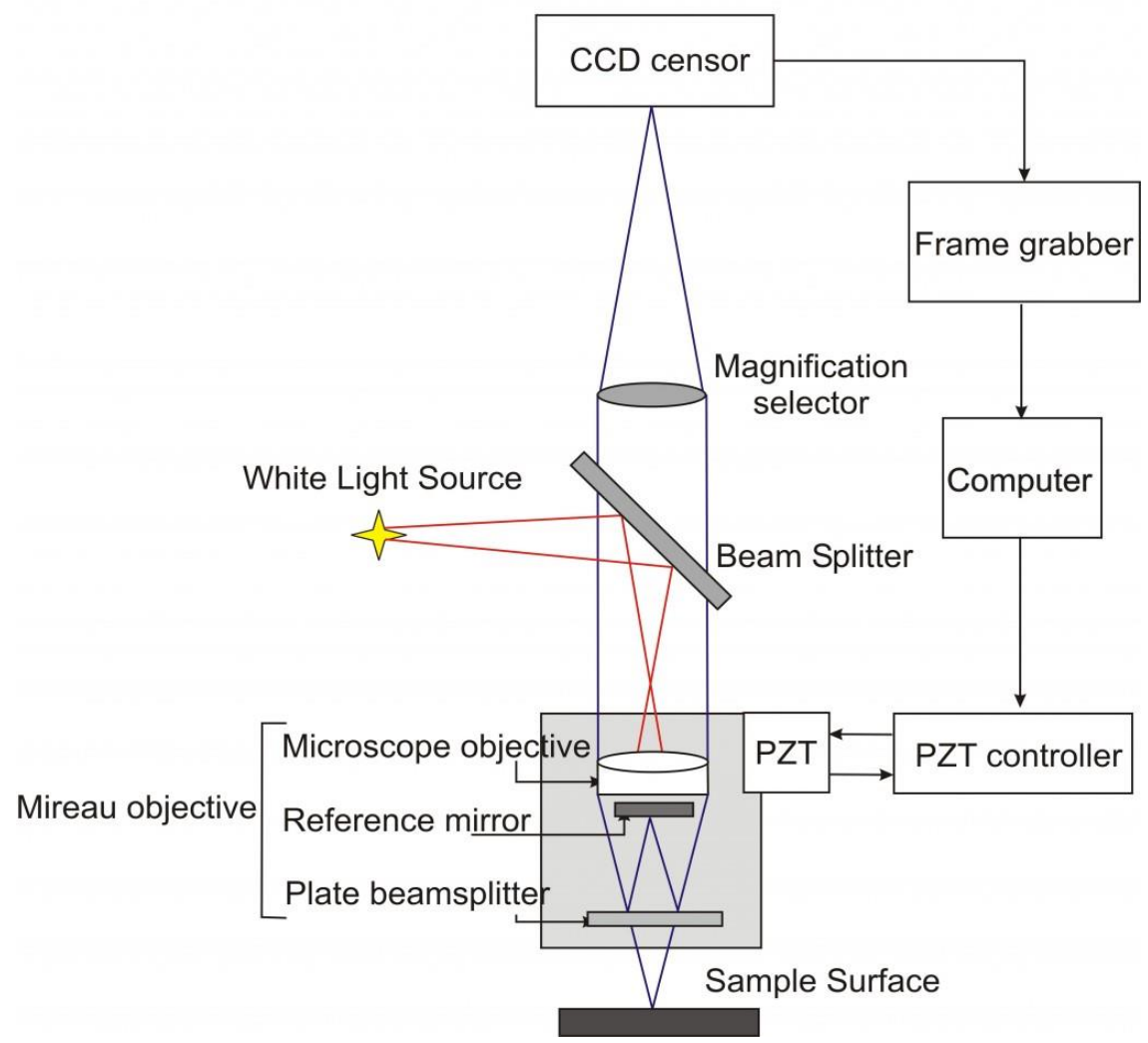

Figure 35 Schematic of interferometer (cited from [191]).

PSI has the advantages such as high sensitivity to the sample surface topography (i.e. sub-nm scale), rapid and flexibility analysis (i.e. compared with atomic force microscopy), no risk of 
destroying the sample surface, and measuring large zones with high lateral resolution, which is limited by the camera pixel numbers and optical diffraction. Recently, laser is used as light source in most interferometry because of the long coherence length, compared with white light source. For white light source, fringes only occur around the best focus position [191].

As illustrated in Figure 35, an interferometer can be used to compare the surface structure of the reference mirror and the sample surface. A beam splitter forms a reference beam and a test beam from the light source. These two beams recombine each other, creating an interference pattern (e.g. constructive or destructive interference), depending on the amplitude and phase of these two beams, thus giving the information of the sample topography information.

In details, it is based on the acquisition of 4 images of the same surface but with a difference of $\lambda / 4$ in the optical path length between two consecutive images, which are recorded by varying the distance between the sample and the interferometric objective[192]. The height of each point $h(x, y)$ in the PSI image is give as

$$
h(x, y)=\frac{\lambda}{4 \pi} \arctan \left[\frac{\left.I_{4}(x, y)-I_{2}(x, y)\right)}{\left.I_{1}(x, y)-I_{3}(x, y)\right)}\right]
$$

Where $I_{i}(i=1,2,3$, and 4$)$ is the intensity at different optical path length $0, \lambda / 4,2 \lambda / 4,3 \lambda / 4$, respectively.

\section{2. fs laser writing system}

\subsection{1. fs laser irradiation system}

Commercial Yb-doped fiber amplifier fs lasers (Satsuma, Amplitude Systèmes Ltd.) were employed as radiation source operating at a wavelength of $1030 \mathrm{~nm}$ at room temperature. One is with pulse duration of $300 \mathrm{fs}$ and the other is the one with $250 \mathrm{fs}$. The frequency can be adjusted form several $\mathrm{kHz}$ to $1 \mathrm{MHz}$. The laser specifications are shown in Appendix V.

As illustrated in Figure 36, the fs laser irradiation process was started on LNS glass. This LNS glass is in amorphous state without observation of crystals (e.g. crystal dots). An aspheric lens (NA = 0.6) focused the laser inside the LNS glasses. Laser propagation was along $+Z$ direction. Irradiated lines were produced continuously in the plane perpendicular to laser propagation direction (i.e. $\mathrm{XY}$ plane) with the scanning speed varied from 1 to $25 \mu \mathrm{m} / \mathrm{s}$. 
The laser polarization is linear varied by rotating a half-wave plate along the light path (marked with red dashed rectangle). Pulse energy was controlled by a combination of a half-wave wave plate and a polarizing beam splitter cube (marked with green dashed rectangle). The pulse energy was measured after the laser beam passing through microscope objective in air. The pulse energy density is measured by a laser power energy meters (Ophir Laser measurement $\mathrm{P} / \mathrm{N}$ 7Z01601) with the formula of pulsed energy = power/laser repetition rate. For example for the measured value $0.300 \mathrm{~W}$ and the laser repetition rate of $300 \mathrm{kHz}$. The pulse energy should be 1.0 $\mu \mathrm{J} /$ pulse.

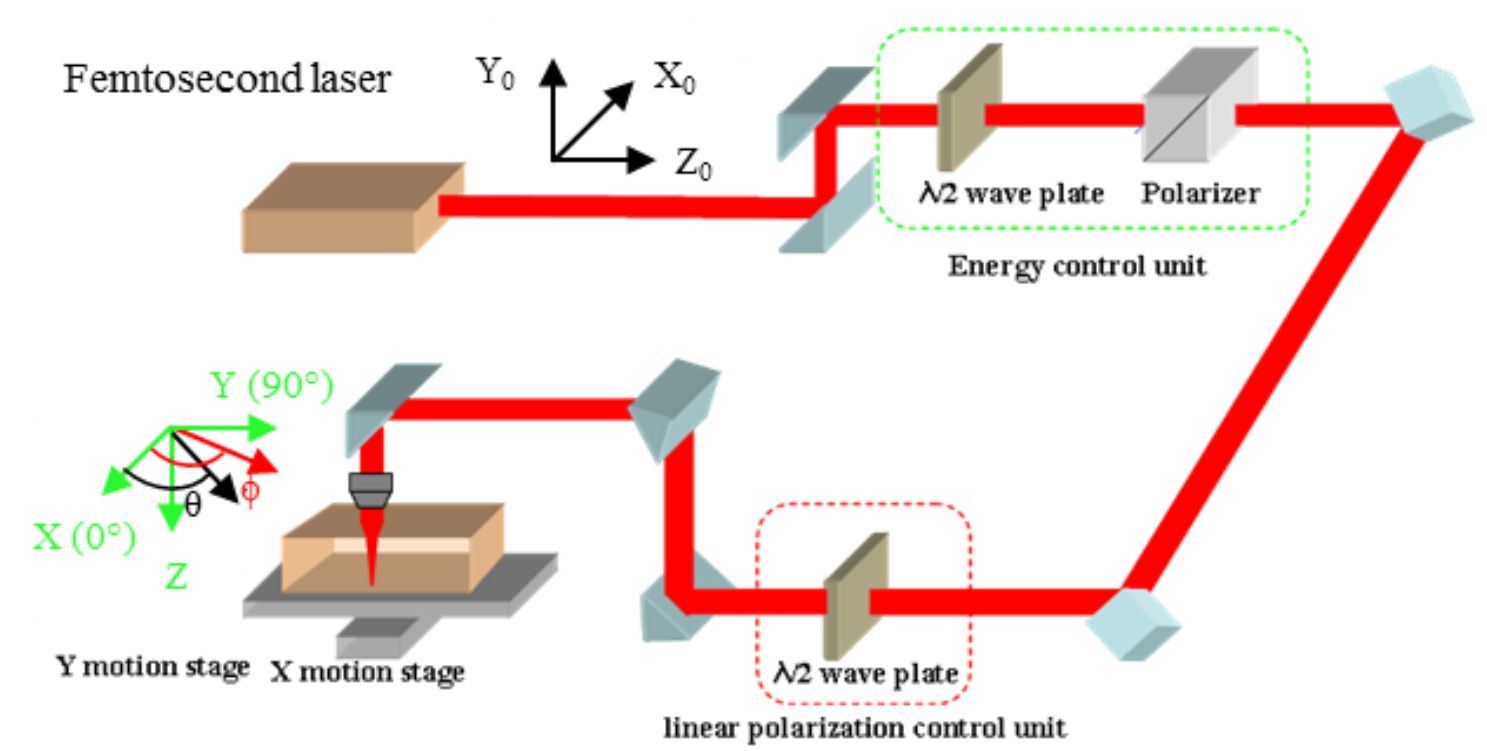

Figure 36 Schematic diagram of the fs laser fabrication system (the reference of writing configuration is based on the beam specificities at the origin shown in black Cartesian coordinate system, and changed to green Cartesian coordinate system when arrived in the sample). N.B.: because the number of mirrors on the optical table is odd, the coordinate system changed from right-handed to left-handed.

\subsubsection{Energy threshold definition}

The test of bulk damage threshold in transparent materials is very difficult. Because of focusing mode of laser beam, together with non-linear effects, the precise measuring of spot size and energy density will be very difficult. Thus, the ISO standards (ISO 11254) for laser-induced damage threshold (LIDT) test did not relate to the test of bulk damage threshold of transparent materials. So, the experimental information on bulk damage test should be clearly depicted.

Herein, the threshold for obtaining crystalline inside LNS glass is defined by listing the irradiation conditions (e.g. laser wavelength, pulse duration, frequency, NA, focus depth, and scanning speed). Notice that this is not a bulk or surface LIDT but a threshold of glass permanent transformations. A more accurate method to determine the threshold of glass transformations under fs laser irradiation has been described in [63]. 
It is worth noting that a static irradiation mode (i.e. irradiating the glass without moving the stage) was needed before the line scanning. If lines are scanned directly without this static mode, it is hard to obtain crystalline lines or even not possible. This is the incubation time for nuclei production in the CCT diagram (see details in Section 2.2.2.2).

\subsubsection{Writing configuration definition}

As matter of fact, from our previous report [189], the angle between incident laser polarization and scanning direction is insufficient to differentiate experiments made with the same angle between these two vectors (such as parallel or perpendicular ones). Therefore, we defined a reference in terms of a Cartesian coordinate system along the laser propagating direction.

Facing the laser beam coming from the laser system, a right-handed Cartesian coordinate system was defined (illustrated in black in Figure 36). $\mathrm{X}_{0}$ : horizontal direction, the right side is declared positive; $\mathrm{Y}_{0}$ : vertical direction, the up side is defined positive; $+\mathrm{Z}_{0}$ : laser propagation direction. This reference changed to another Cartesian coordinate system (shown in green in Figure 36) when it arrived inside the sample due to an odd number of mirrors on the way. The preprogrammed pattern was written in $\mathrm{XY}$ plane by moving the sample stages. Here, writing laser polarization angle $(\phi)$, scanning direction $(\theta)$ (illustrated in Figure 36 ) are referred to $+\mathrm{X}$ direction in $X Y$ plane. The writing configuration is defined as $(\theta, \phi)$.

\subsection{Thermal modelling in the case of fs laser irradiation}

One of our objective is to master the crystallization of $\mathrm{LiNbO}_{3}$ which is classically determined by the local temperature that has to be in a suitable range during time duration large enough for allowing the atoms to move and rearrange themselves (see Section 2.2.2.2). Due to the nonlinear nature of laser-matter interaction, at certain high repetition rate, fs laser is able to drive heat accumulation processes in and around the laser focused zone [22]. In the regions where the temperature is lower than the $T_{m}$ but nevertheless close to $T_{m}$ for a kinetics fast enough, there is formation of crystal nuclei at first, due to statistical atomic association and atomic rearrangement eventually due to atomic diffusion [24]. Then, crystals grow from the nuclei.

By changing the parameters such as the pulse repetition rate [22], the pulse energy [193], the material [194], and the scanning speed [195], the thermal field in material can be controlled. In that case, its modelling provides essential information for optimizing laser irradiation conditions for laser-induced modifications (e.g. crystallization). 
The calculated temperature field (i.e. spatial and temporal variations) in LNS glasses can be found in the literature for different conditions by solving the Fourier heat equation with some assumptions as following:

1) Just heat conduction in glass is considered, neglecting the thermal radiation and convection;

2) The laser light energy is finally transformed into heat, e.g. chemical reaction or phase transformation are neglected;

3) Neglecting nonlinear effects (e.g. self-focusing and plasma defocusing) and spherical aberration, the energy deposition of fs laser beam can be mathematical represented by Gaussian beam profile according to the radius and paraxial wave equation (see details in Section 2.1.1.3);

4) The beam is radially symmetric. It is propagating and converging along $z$ axis $(z=0$ for the laser focal point) and $r$ is the radial distance from the beam axis. Assuming the initial distribution of the heat can be described by a Gaussian shape.

\subsubsection{Heat conduction equation definition}

\subsubsection{Basic heat conduction equations}

Fourier's law of heat conduction, the conduction heat flux $\left(\mathrm{W} \cdot \mathrm{m}^{-2}\right)$ is given as

$$
\vec{j}=-\vec{k} \vec{\nabla} T
$$

Where $\overline{\bar{k}}$ is thermal conductivity $\left(\mathrm{W} \cdot \mathrm{m}^{-1} \cdot \mathrm{K}^{-1}\right)$ and $\vec{\nabla} \mathrm{T}$ is the temperature gradient $\left(\mathrm{K} \cdot \mathrm{m}^{-1}\right)$. The negative sign in this equation shows that the heat flow is in the direction of decreasing temperature, making heat flow positive. The heat transfer direction is perpendicular to the isotherms.

Applying conservation of energy to a differential control volume in which energy transfer is only by conduction and a 3D unsteady-state energy equation for a solid is given as

$$
\vec{\nabla} \mathbf{j}+\frac{\partial \rho_{\mathrm{Q}}}{\partial \mathrm{t}}=\frac{\delta \mathrm{Q}}{\delta \mathrm{t}}, \text { with } \quad \rho_{\mathrm{Q}}=\int_{\mathrm{T}_{\mathrm{a}}}^{T} \rho \mathrm{C}_{\mathrm{p}} \mathrm{dT}
$$

Where $\frac{\delta Q}{\delta t}$ is the rate of the heat source by laser irradiation per unit volume, $\rho_{Q}$ is the energy density $\left(\mathrm{J} \cdot \mathrm{m}^{-3}\right), \rho$ is the material density, $C_{p}$ is the material specific heat, $T_{a}$ is the ambient temperature, and $\mathrm{t}$ is the time.

Considering $\rho$ and $C_{P}$ not temperature dependent, we get 


$$
\vec{\nabla}(-\overline{\bar{k}} \vec{\nabla} \mathrm{T})+\rho c_{\mathrm{p}} \frac{\partial \mathrm{T}}{\partial \mathrm{t}}=\frac{\delta \mathrm{Q}}{\delta \mathrm{t}}
$$

Some simplifying assumptions are made to solve this equation.

Assuming the LNS glass isotropic, the thermal conductivity is reduced to a scalar. If the thermal conductivity is not temperature dependent, we get

$$
-\mathrm{k} \Delta \mathrm{T}+\rho \mathrm{c}_{\mathrm{p}} \frac{\partial \mathrm{T}}{\partial \mathrm{t}}=\frac{\delta \mathrm{Q}}{\delta \mathrm{t}}
$$

Considering thermal diffusivity $D_{\text {th }}=k /\left(\rho C_{p}\right)$, we get

$$
-\mathrm{D}_{\mathrm{th}} \Delta \mathrm{T}+\frac{\partial \mathrm{T}}{\partial \mathrm{t}}=\frac{1}{\rho c_{\mathrm{p}}} \frac{\delta \mathrm{Q}}{\delta \mathrm{t}}
$$

The resolution of this partial differential equation makes possible to obtain the temperature field. To compute the solution, there are two general ways:

One is to say that the deposition of energy has been achieved before a definite time (usually $t=$ 0 ) and that we know the T distribution at that time. Then, the Fourier equation without second member is solved. The second way cannot be avoided when the energy deposition run during the diffusion process. In that case, finite element method has to be performed. It is also the case, if the thermal properties are temperature dependent.

Here, for describing the physics of the process, we will use the first way, considering that the heat from light pulses is deposited in a very short time (actually it is shorter than ps) and then that the heat diffuses afterwards. It is worthwhile to note that typical diffusion length for 1 ps in LNS glass is of the order of $1 \mathrm{~nm}$, so largely below our resolution.

For solving the Fourier equation, we need value of the thermal parameters.

\subsubsection{Thermal parameters of LNS glasses}

Considering chemical reaction occurs during fs laser irradiation in LNS glasses, the thermal parameters of LNS glass are estimated as following.

When considering $32.5 \mathrm{Li}_{2} \mathrm{O}-27.5 \mathrm{Nb}_{2} \mathrm{O}_{5}-40 \mathrm{SiO}_{2}$ glass for example, this LNS glass becomes $55 \mathrm{LiNbO}_{3}-40 \mathrm{SiO}_{2}-5 \mathrm{Li}_{2} \mathrm{O}$ after fs laser irradiation due to $\mathrm{LiNbO}_{3}$ crystallization.

The thermal parameters of this glass are calculated as reported in Table 8. 
Table 8 Thermal properties of LNS glasses for modeling

\begin{tabular}{|c|c|c|c|c|c|}
\hline Reagent & $\begin{array}{l}\text { Density, } \rho \\
\mathrm{kg} \mathrm{m}^{-3}\end{array}$ & $\begin{array}{l}\text { Specific heat, } C_{p} \\
\mathrm{~J} \mathrm{~kg}^{-1} \mathrm{~K}^{-1}\end{array}$ & $\begin{array}{l}\text { Thermal } \\
\text { conductivity, } \mathrm{k} \\
\mathrm{W} \mathrm{m}^{-1} \mathrm{~K}^{-1}\end{array}$ & $\begin{array}{l}\text { Thermal diffusivity, } \\
D_{\text {th }}, m^{2} s^{-1}\end{array}$ & $\begin{array}{l}\text { Melting } \\
\text { point } \\
{ }^{\circ} \mathrm{C}\end{array}$ \\
\hline $\mathrm{Li}_{2} \mathrm{O}$ & $2013\left(25^{\circ} \mathrm{C}\right)$ & $2519.738^{m}$ & $7.532^{m}$ & $1.485 \times 10^{-6}$ & $1432 \pm 6$ \\
\hline $\mathrm{Nb}_{2} \mathrm{O}_{5}$ & - & - & - & - & 1512 \\
\hline $\begin{array}{l}\mathrm{SiO}_{2} \text { (fused } \\
\text { silica) }\end{array}$ & 2200 & $986.6^{\circ 0}$ & $1.9^{\delta}$ & $8.75 \times 10^{-7}$ & 1715 \\
\hline $\mathrm{LiNbO}_{3}$ & $\begin{array}{l}4659 \\
(297.75 K)\end{array}$ & $684.5^{\text {rs }}$ & 2.84 & $0.89 \times 10^{-6}$ @ & 1257 \\
\hline $\begin{array}{l}32.5 \mathrm{Li}_{2} \mathrm{O}- \\
27.5 \mathrm{Nb}_{2} \mathrm{O}_{5-} \\
40 \mathrm{SiO}_{2}\end{array}$ & 3543 & 897.1 & 2.70 & $9.14 \times 10^{-7}$ & \\
\hline $\begin{array}{l}33 \mathrm{Li}_{2} \mathrm{O}- \\
33 \mathrm{Nb}_{2} \mathrm{O}_{5}-34 \mathrm{SiO}_{2}\end{array}$ & 3823 & 787.2 & 2.52 & $8.85 \times 10^{-7}$ & \\
\hline
\end{tabular}

\subsubsection{Laser pulse energy deposition}

As the resolution is conditioned by the shape of the energy deposition, we have to discuss a bit this point.

Neglecting nonlinear effects (e.g. self-focusing and plasma defocusing) and spherical aberration, the energy deposition is defined by the light intensity that is absorbed by the material through mechanisms described above: multi-photon absorption, tunnelling, inverse bremsstrahlung, and avalanche. These are complex processes that are considered in [63], but we will consider here just a simple image.

The expression of the light intensity of one pulse in the case of a converging beam propagating into a non-absorbing material is the following

$$
I(r, z, t)=I_{00}\left(\frac{\omega_{0}}{\omega(z)}\right)^{2} \cdot \exp \left[\frac{-r^{2}}{\omega^{2}(z)}\right] \cdot \exp \left(-4 \ln (2) \frac{t^{2}}{\tau^{2}}\right)
$$

Where $I_{00}$ is the peak intensity, $\omega_{0}$ is the minimum waist at the focus, and $\tau$ is the duration of the pulse. This makes reference to Section 2.1.1.3

Under absorption, the amplitude of the intensity decreases along the average propagation direction ( $\mathrm{z}$ ) 


$$
I_{a}(r, z, t)=I_{0}(r, z)\left(\frac{\omega_{0}}{\omega(z)}\right)^{2} \cdot \exp \left[\frac{-r^{2}}{\omega^{2}(z)}\right] \cdot \exp \left(-4 \ln (2) \frac{t^{2}}{\tau^{2}}\right)
$$

Where $I_{0}$ (for $z$ out of the material) $=I_{00}$.

The function $I_{0}(r, z)$ is defined by the absorption process e.g. a multi-photon ionization. For instance, by solving the following equation

$$
\frac{\partial\left(l_{a}(r, z, t)-I(r, z, t)\right)}{\partial r}+\frac{\partial\left(l_{a}(r, z, t)-I(r, z, t)\right)}{\partial z}=-\alpha_{n} l_{a}^{n}
$$

Where $\alpha_{n}$ is the absorption coefficient for $n$ photons.

The solution is not so simple and usually need to use finite element calculation. Once this one obtained, the rate of deposited energy density is directly computed from the following

$$
\frac{\partial\left(l_{a}(r, z, t)-l(r, z, t)\right)}{\partial r}+\frac{\partial\left(l_{a}(r, z, t)-l(r, z, t)\right)}{\partial z}=\frac{\delta Q}{\delta t}
$$

For sake of simplicity (the physics is conserved) and using results from literature, we will consider

1) that a bell shape along the radius is kept but the width is smaller than $\omega_{0}$ due to the multi-photon absorption;

2) that for the same reason, only around the focus the absorption takes place. In that case, it is usually assumed that $\omega_{z}$ is smaller than $z_{R}$.

Finally, the following expression is the one usually used for a schematic approach.

$$
\frac{\delta \mathrm{Q}}{\delta \mathrm{t}}(\mathrm{r}, \mathrm{z}, \mathrm{t})=\left\{\mathrm{Q}_{0} \exp \left[\frac{-\mathrm{z}^{2}}{\omega_{\mathrm{z}}^{2}}\right]\right\} \cdot\left\{\exp \left[\frac{-\mathrm{r}^{2}}{\omega_{\mathrm{r}}^{2}}\right]\right\} \cdot\left\{\frac{2 \sqrt{\ln (2)}}{\tau \sqrt{\pi}} \exp \left(-4 \ln (2) \frac{\mathrm{t}^{2}}{\tau^{2}}\right)\right\}
$$

For several pulses, the energy deposition is the sum of the various contributions shifted in time.

$$
\frac{\delta Q}{\delta t}(r, z, t)=\left\{Q_{0} \exp \left[\frac{-z^{2}}{\omega_{z}^{2}}\right]\right\} \cdot\left\{\exp \left[\frac{-r^{2}}{\omega_{r}^{2}}\right]\right\} \cdot \sum_{n=0}^{N-1}\left\{\frac{2 \sqrt{\ln (2)}}{\tau \sqrt{\pi}} \exp \left(-4 \ln (2) \frac{(t-n / f)^{2}}{\tau^{2}}\right)\right\}
$$

Where $f$ is the repetition rate. N.B.: due to the short duration of the pulses, usually the Gaussian on time is approximated by a delta function [200]. 


\subsubsection{Solving heat equation}

\subsubsection{One-pulse case}

\subsection{The distribution of the temperature field at $t=0 \mathrm{~s}$}

Because there is no heat diffusion around $t=0 \mathrm{~s}$ during a very short time, we get

$$
\begin{gathered}
\frac{\partial T(r, z)}{\partial t}=\frac{1}{\rho c_{p}} \frac{\delta Q(r, z, 0)}{\delta t} \text { and } \\
\mathrm{T}_{0}(r, z)=\mathrm{T}_{\mathrm{a}}+\frac{1}{\rho c_{\mathrm{p}}} \int_{\text {pulse }} \frac{\delta \mathrm{Q}}{\delta \mathrm{t}} \mathrm{dt}=\mathrm{T}_{\mathrm{a}}+\frac{1}{\rho \mathrm{c}_{\mathrm{p}}}\left\{\mathrm{Q}_{0} \exp \left[\frac{-\mathrm{z}^{2}}{\omega_{\mathrm{z}}{ }^{2}}\right]\right\} \cdot\left\{\exp \left[\frac{-\mathrm{r}^{2}}{\omega_{\mathrm{r}}^{2}}\right]\right\}
\end{gathered}
$$

Where $T_{a}$ is the ambient temperature $\left(25^{\circ} \mathrm{C}\right)$.

$\mathrm{Q}_{0}$ at the focus point can be calculated using the energy conservation of the beam as

$$
\mathrm{Q}_{0}=\frac{2 A \mathrm{E}_{\mathrm{p}}}{\pi^{2} \omega_{\mathrm{r}}^{2} \omega_{\mathrm{z}}}
$$

Where $E_{p}$ is the laser pulse energy for one pulse and $A$ is the fraction of energy absorbed by the glass finally.

\subsection{Thermal diffusion after one pulse}

After the above step, $\frac{\delta \mathrm{Q}}{\delta \mathrm{t}}=0$, the heat diffuses, the evolution of the thermal field is given by the resolution of the homogeneous heat equation as

$$
\frac{\partial \mathrm{T}}{\partial \mathrm{t}}-\mathrm{D}_{\mathrm{th}} \Delta \mathrm{T}=0
$$

The general solution is the following

$$
T(r, z, t)=\frac{a}{\pi^{3 / 2}} \frac{b}{\sqrt{b^{2}+4 D_{t h} t}} \exp \left(-\frac{r^{2}}{b^{2}+4 D_{t h} t}\right) \cdot \frac{c}{\sqrt{c^{2}+4 D_{t h} t}} \exp \left(-\frac{z^{2}}{c^{2}+4 D_{t h} t}\right)+d .
$$

To determine the integration constants (i.e. $a, b, c$ and d), the following boundary condition is applied. At $\mathrm{t}=0 \mathrm{~s}, \mathrm{~T}(\mathrm{r}, \mathrm{z}, \mathrm{t}=0)=\mathrm{T}_{0}(\mathrm{r}, \mathrm{z})$

$$
\mathrm{T}_{0}(r, z)=\frac{a}{\pi^{3 / 2}} \exp \left(-\frac{r^{2}}{b^{2}}\right) \cdot \exp \left(-\frac{z^{2}}{c^{2}}\right)+d=T_{a}+\frac{1}{\rho c_{p}}\left\{Q_{0} \exp \left[\frac{-z^{2}}{\omega_{z}^{2}}\right]\right\} \cdot\left\{\exp \left[\frac{-r^{2}}{\omega_{r}^{2}}\right]\right\}
$$

So, we get $a=\frac{Q_{0} \pi^{3 / 2}}{\rho C_{p}}, b=\omega_{z}, c=\omega_{r}$, and $d=T_{a}$

So, the solution of the temperature field in space (note: the separable variable) is given as [200] 


$$
T(r, z, t)=\frac{Q_{0}}{\rho C_{p}} \frac{\omega_{r}^{2}}{\omega_{r}^{2}+4 D_{t h} t} \frac{\omega_{z}^{2}}{\sqrt{\left(\omega_{z}^{2}+4 D_{t h} t\right)}} \exp \left[-\left(\frac{r^{2}}{\omega_{r}^{2}+4 D_{t h} t}+\frac{z^{2}}{\omega_{z}^{2}+4 D_{t h} t}\right)\right]+T_{a}
$$

With this expression we have the complete distribution of temperature in space and time.

\subsubsection{Application to the change of fictive temperature: definition of the first energy threshold}

The first energy threshold for a modification in glass is defined in a paper from Poumellec et al. $[71,82]$. Some modifications occur if the temperature is high enough during a time long enough. If the cooling time is longer than the time required for the glass structure to change (called relaxation time), the modification will be permanent (i.e. the average disorder of the glass or the fictive temperature will change).

The relaxation time is given as $\eta / G$, where $\eta$ is the glass viscosity and $G$ is the glass shear modulus. These are thermal properties of the glass. This curve should be compared to the cooling curve after a single pulse fs laser. This curve may be deduced from the above resolution of the Fourier equation.

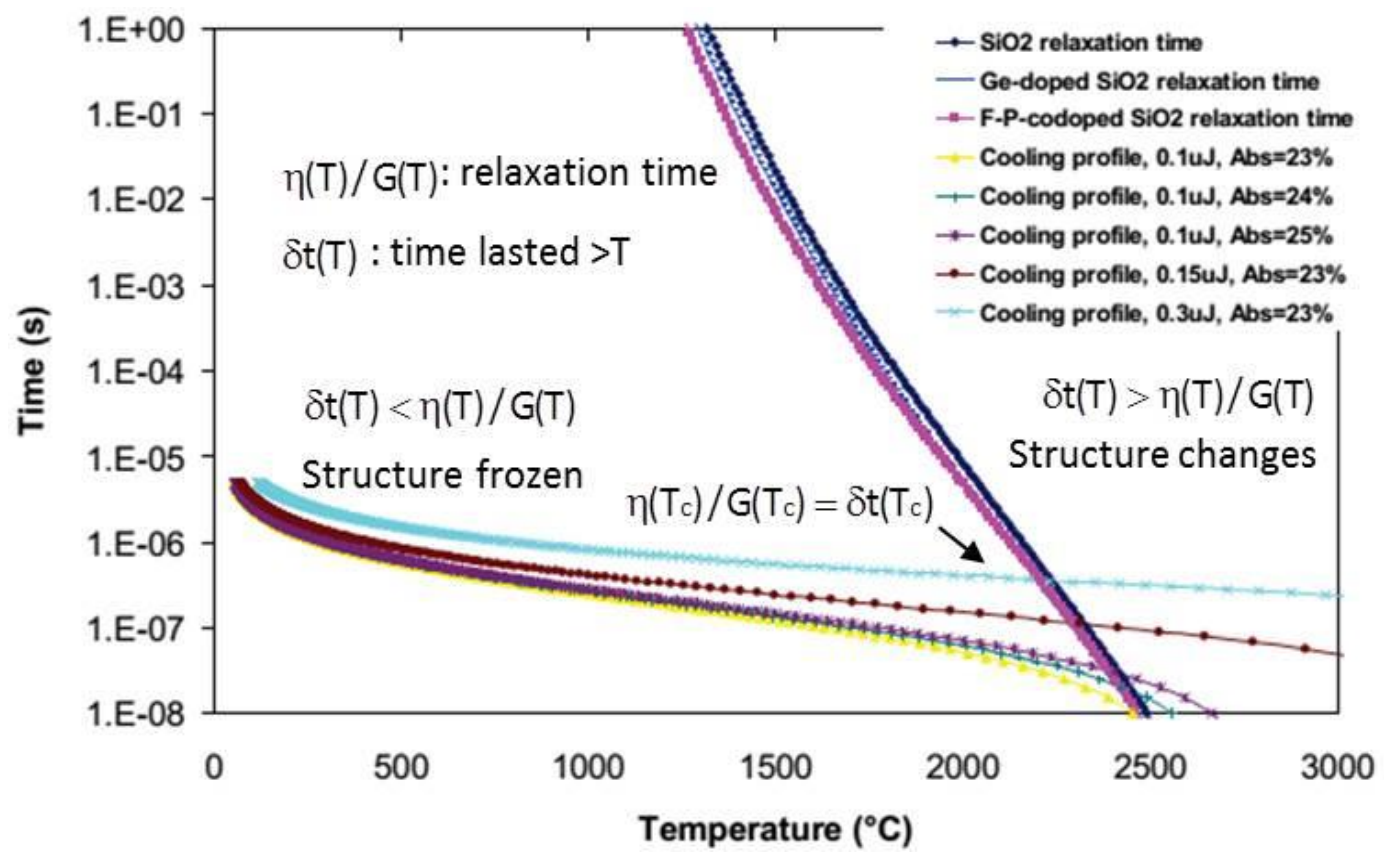

Figure 37 The comparison of the glass relaxation time and the cooling profile after single pulse fs laser irradiation for pure and doped silica. The cooling profiles with varying incident pulse energy are for pure silica only; $T_{c}$ is the new fictive temperature (after [201])

For a pulse energy large enough, these curves intersect (Figure 37) in a point $T_{c}$. For the part of the cooling curve above the relaxation time, the time lasted at $T$ higher than $T_{c}$ is larger than the relaxation time and the material can change its structure in equilibrium with $T$ until $T=T_{c}$. When 
the temperature is lower than $T_{c}$, the glass cannot change its structure. $T_{c}$ is thus a specific temperature characterising the structure of the glass after such a treatment. It is called fictive temperature. Before irradiation, fictive temperature was about $1200^{\circ} \mathrm{C}$, but after irradiation it rises [202].

For pure silica, if we decrease the incident pulse energy, the cooling curve does not intersect the relaxation time curve anymore. This particular energy can define the energy threshold for fictive temperature modification that gives rise also to refractive index change, change of specific volume and so on.

In the same way, we can explain easily that a boundary for a modification (shown in Figure 55a) is clearly appearing. The boundary is the surface for which the cooling curve does not cross the relaxation time anymore.

\subsubsection{Multi-pulse case in static mode}

The thermal conduction model by fs laser pulses may be developed for a series of periodical pulses at a repetition rate of $f=1 / \Delta t_{L}$. The distribution of the temperature after $N$ irradiations can therefore be obtained by summing the solutions for one pulse shifted in time.

The overall solution for taking into account several pulses is given as [200]

$$
T(r, z, t)=\frac{Q_{0}}{\rho C_{p}} \sum_{n=0}^{N-1} \frac{\omega_{r}^{2}}{\omega_{r}^{2}+4 D_{t h}(t-n \Delta t)} \frac{\omega_{z}^{2}}{\sqrt{\left(\omega_{z}^{2}+4 D_{t h}(t-n \Delta t)\right.}} \exp \left[-\left(\frac{r^{2}}{\omega_{r}^{2}+4 D_{t h}(t-n \Delta t)}+\frac{z^{2}}{\omega_{z}^{2}+4 D_{t h}(t-n \Delta t)}\right)\right]+T_{a}
$$

This allows showing two things: a cumulative effect and the control of the heat treatment for the crystallization.

\subsection{Cumulative effect}

Figure 38 displays the temperature in LNS glass at different repetition rate at the center of irradiated zone. At low repetition rate (e.g. $100 \mathrm{kHz}$ ), thermal diffusion has carried the heat away from the focus before arriving the next pulse at a timescale of $10 \mu \mathrm{s}$. So, with one pulse, it is almost impossible to get crystallization. Thermal accumulation occurs when the period of the pulse is smaller than diffusion time (the heating time is of the $1 \mu \mathrm{s}$ ). With large number of pulses, a steady state is reached (larger that a few 10 of pulses). So, with thermal accumulation, we control the heating time properly. 
In LNS glass, the repetition rate minimum is larger than $\sim 100 \mathrm{kHz}$. In the case of heat accumulation, the $T$ distribution is such that as we move further from the focus center, the oscillations are smoothed.

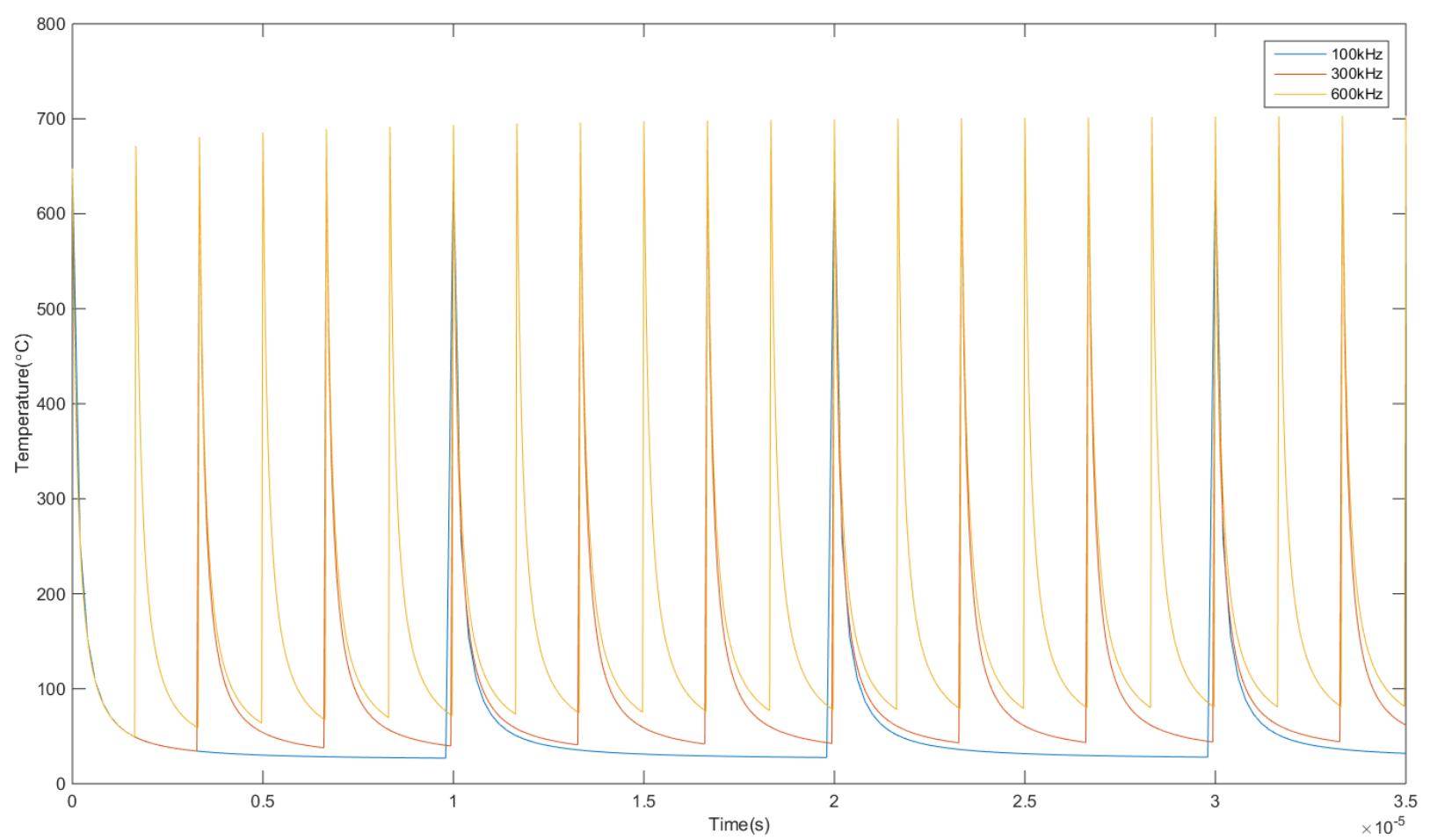

Figure 38 Calculated temperature in LNS glass for multi-pulse case in static mode: varying time and repetition rate at $\mathrm{r}=0$. Other parameters: $33 \mathrm{Li}_{2} \mathrm{O}-33 \mathrm{Nb}_{2} \mathrm{O}_{5}-34 \mathrm{SiO}_{2}(\mathrm{~mol} \%), \mathrm{z}=0,0.5 \mu \mathrm{J} /$ pulse

\subsection{Application to crystallization}

Finally, if we consider a multi-pulse irradiation duration at high repetition rate, the effect is similar to a continuous deposition of energy $[66,67]$. This means that the time variation of the T distribution will become periodic, and the spatial distribution will become steady.

This means that we can control the T distribution in time and in space and use the fs laser like a source of isothermal treatment and especially for controlling the crystallization. But we have to note that the width of the steady state $T$ distribution is larger than the beam size finally due to heat diffusion, so the crystallized volume will be larger than the irradiated one.

In such a case, we can take profit of the theory described in Section 2.2.2.2. With this one, we can build the following schematic (Figure 39). The right hand part of the Figure 39 shows the time average steady state $T$ distribution according to the radius for $z=0$. The left hand part of the picture represents the TTT domain where crystallization occurs. 
For the places in the material for which the temperature is below the melting temperature but nevertheless high enough for a fast kinetics, crystallization occurs. This is the case for the blue curve in Figure 39, from the center and to a radius of the order of three microns. This leads to a disk of crystallization (in fact a rod if we take into acount the $z$ dimension). On the contrary, if the pulse energy is too high, there is a radius range for which the temperature is too high for crystallization (e.g. top of the red or black curves). In that case, the crystallized volume will appear like a crown (in fact a tube with the $z$ dimension). Some features described here can be found experimentally in [26].

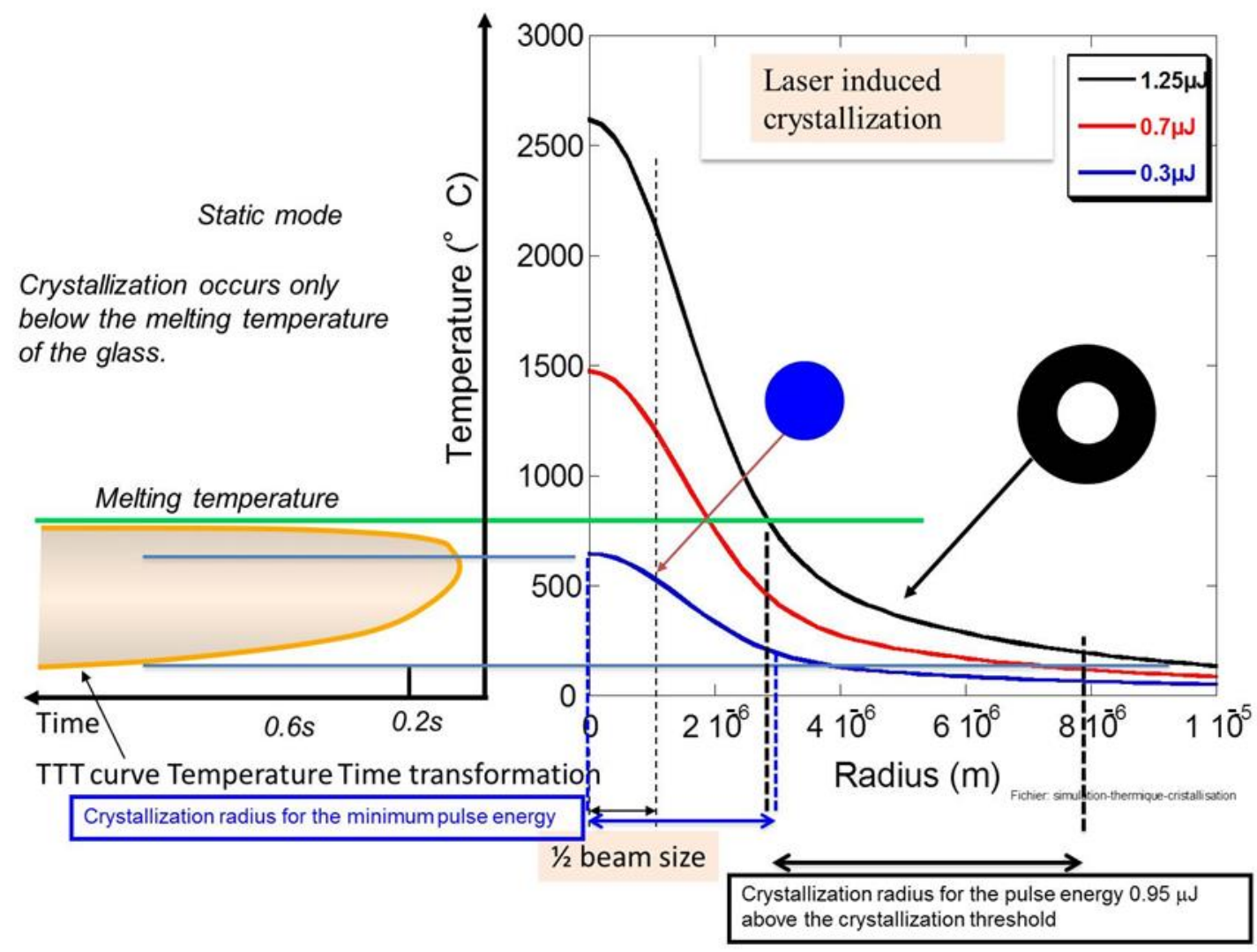

Figure 39 Spatial temperature distribution and TTT curve in static mode (after Poumellec et al. ICMMO, France)

\subsubsection{Multi-pulse case in scanning mode}

One of the obligations in integrated optics is to write circuits and one of the solutions in this direction is to move the beam at a speed such that overlap between two consecutive pulses is very high. This is obviously the case for the speeds we practiced in this thesis (a few microns per $\mathrm{sec})$. The distribution of the temperature after $\mathrm{N}$ irradiation in scanning mode along $\mathrm{x}$ axis can be obtained by summing the solution for a pulse shifted in time space as following 


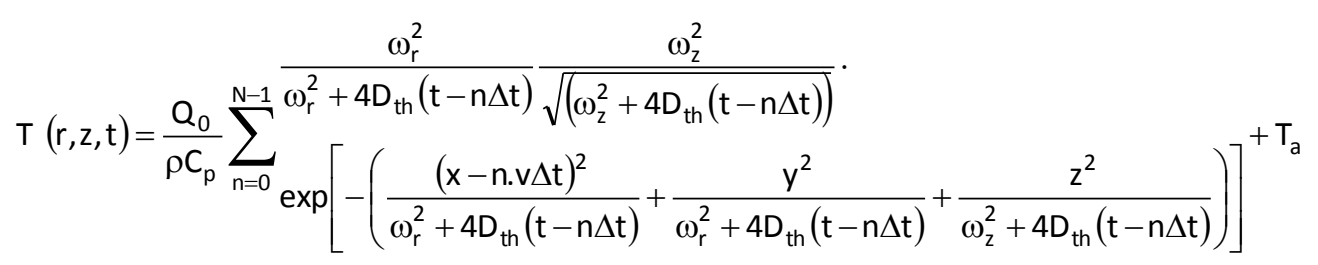

For a given time, and a small speed of the scanning, the spatial T distribution shape remains the same as in static mode. The distribution will change just for a speed such that it is close to or larger than the diffusion speed. This speed may be estimated from the characteristic Fick relation

$$
I^{2}=2 D t
$$

Where $\mathrm{I}$ is of the order of the $\omega_{\mathrm{r}}($ ca. $1 \mu \mathrm{m})$. We get $1 \mathrm{~m} / \mathrm{s}$.

As we use $5 \mu \mathrm{m} / \mathrm{s}$, the shape of the moving $T$ distribution is the same as in static one. Therefore, if we consider a point on the way of the beam, the time evolution of the temperature is obtained by changing the space axis into time axis using the speed of scanning (Figure 40 and Figure 41).

\section{Laser induced crystallization during scanning \\ Dynamic mode}

Crystallization occurs only below the melting temperature from the highest temperature of the glass.

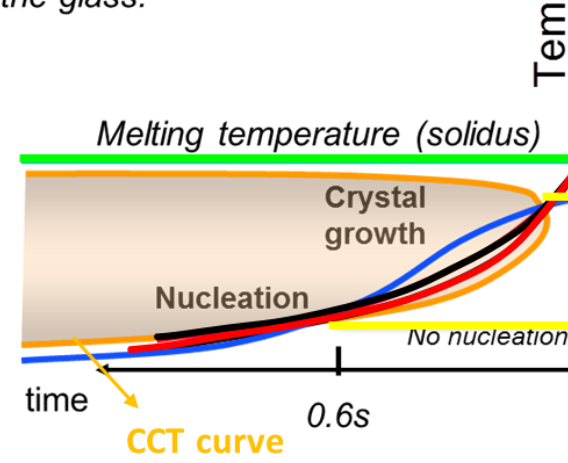

Figure 40

As it has been shown in Section 2.2.2 about the crystallization, the crystallization occurs when the temperature decreases below the melting one but stay at temperature high enough for a fast kinetics. Therefore, for the point considered above. Its temperature rises and then 
decreases. Just the part below the melting temperature and during the decreasing stage has to be considered because higher $T$ erases the past. Therefore, in Figure 40, cooling curves are drawn and compared to the CCT curves for three energies.

In scanning mode (Figure 40), the cooling curve is deduced from the stationary spatial T distribution that is moving at a moderate speed. We have to consider only the part of the decreasing curve that is below the melting temperature.

As illustrated in Figure 41, it is again possible to obtain a crystallized disk (in fact a tube) which transforms into slab under scanning if the pulse energy is not too high, otherwise, the crystallization will be a crown but due to the scanning, will transform into a slab. There is nevertheless a case for which the crystallization will be not completely filled; it is if a chemical migration occurs and that the glass is no more in the condition to crystallize. In that case, the modified volume will be hollow with a peripheral crystallization.

\section{Laser induced crystallization during scanning}

Dynamic mode

Crystallization occurs only below the melting temperature from the highest temperature of the glass.

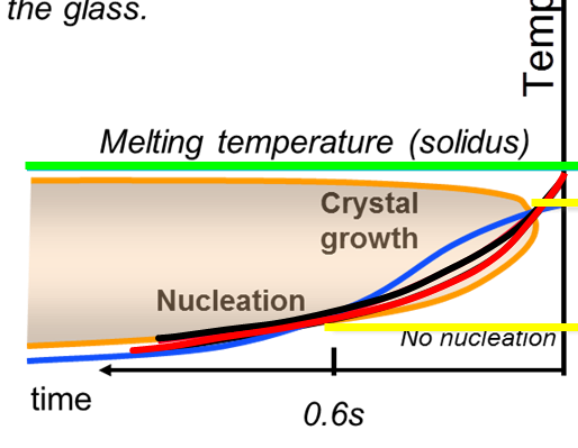

Figure 41 Shape of the crystallized volume according to the pulse energy ampius (m) curves are compared to the CCT domain (after Poumellec et al. ICMMO, France)

In conclusion of this section 3.3, we show that it is possible to master the spatial T distribution playing with the pulse energy for a repetition rate large enough and minimizing the fluctuation. For obtaining crystallization, we need to maintain a temperature close to melting one but below it and during a time large enough. This last parameter is controlled by the speed of beam scanning. 


\subsection{Observation of fs laser-induced modifications}

\subsubsection{Refractive index measurement}

\subsubsection{Brewster's angle method}

The refractive index of LNS glass is measured by the method based on Brewster's angle. As shown in Figure 42a, the incidence plane is defined as the plane containing the incident and reflected $k$ vectors. $p$-polarized wave (or TM, transverse magnetic) is the one with the polarization lying parallel to the plane of incidence (marked with green lines) and s-polarized wave (or TE, transverse electric) the one with polarization sticking out of or into the incidence plane (marked with green dots). The Brewster's angle can be defined as the incident angle at which the reflected beam and the refracted beam makes an angle of $90^{\circ}$. In the case, the incident angle can be calculated as

$$
\theta_{1}=\arctan \left(\frac{\mathrm{n}_{2}}{\mathrm{n}_{1}}\right)
$$

Where $n_{1}$ is the refractive index of the air and (i.e. $\cong 1$ ), $n_{2}$ is the refraction index of sample. So, $\mathrm{n}_{2}$ can be directly calculated through measurement of Brewster's angle.

The Fresnel coefficients for p-polarized and s-polarized light is dependent on the incident angle and refractive index as

$$
\mathrm{r}_{\mathrm{p}}=\frac{\mathrm{n}_{2} \cos \left(\theta_{1}\right)-\mathrm{n}_{1} \cos \left(\theta_{2}\right)}{\mathrm{n}_{2} \cos \left(\theta_{1}\right)+\mathrm{n}_{1} \cos \left(\theta_{2}\right)}, \mathrm{r}_{\mathrm{s}}=\frac{\mathrm{n}_{1} \cos \left(\theta_{1}\right)-\mathrm{n}_{2} \cos \left(\theta_{2}\right)}{\mathrm{n}_{1} \cos \left(\theta_{1}\right)+\mathrm{n}_{2} \cos \left(\theta_{2}\right)}
$$

The reflectance for $\mathrm{p}$-polarized and s-polarized light is calculated as

$$
R_{p}=r_{p}^{2}, R_{s}=r_{s}^{2}
$$

From Figure $42 \mathrm{~b}$, the reflection coefficient in the polarization plane of incidence (i.e. $p$ wave) vanishes at Brewster's angle, or the intensity of this reflected beam is zero. In that case, by measuring the coefficient $r_{p}$ depending on incident angle can give Brewster's angle value.

Herein, we measured the ratio of the reflected intensity to the incident intensity. By changing the incidence angle until total extinction of the reflected light intensity, we record the Brewster's angle. 
Herein, the measured the ratio of the reflected intensity to the incident intensity. By changing the incidence angle, an attenuation of the reflected beam, going up to the total extinction of the light intensity at the Brewster's angle will be recorded.

a) Brewster's angle:

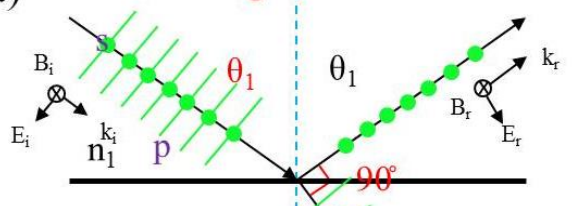

$\mathrm{n}_{2}$

b)

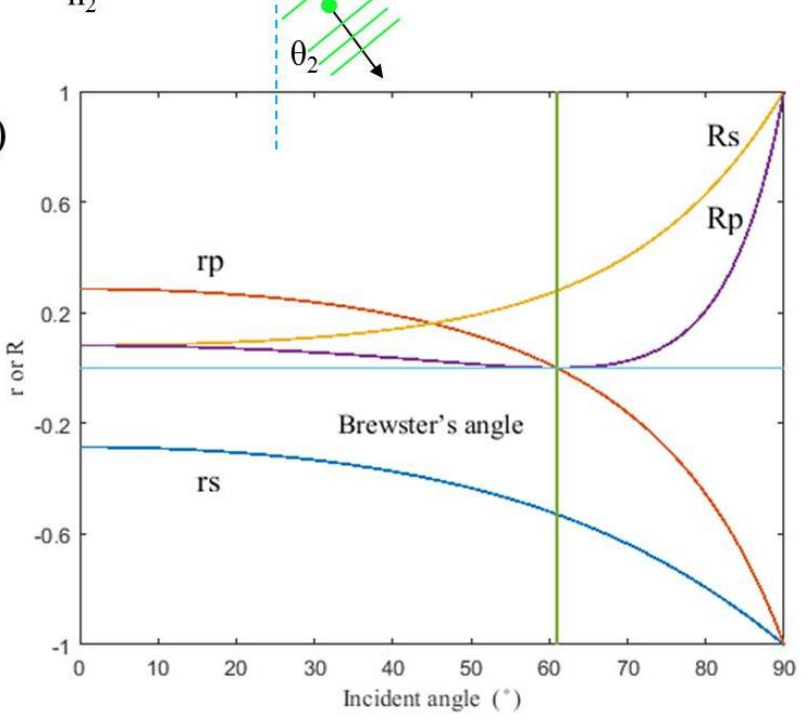

c)

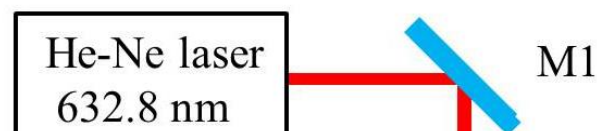

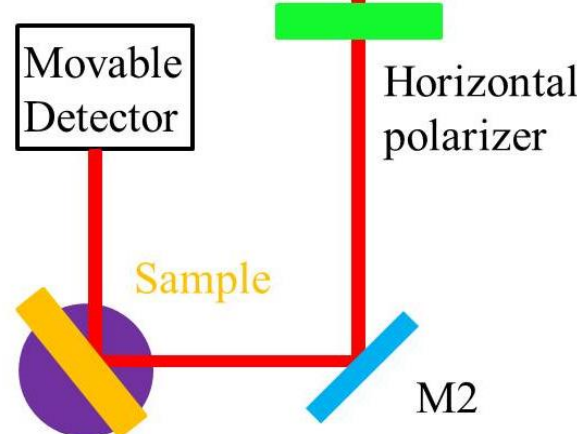

Rotation stage

Figure 42 Schematic diagram of a) Brewster's angle, b) reflection coefficients $(r)$ and reflectance $(R)$ as a function of incidence light, the light polarized in the plane of incidence is marked as $p$ wave, the one polarized perpendicular to the plane is indicated as $s$ wave, $n_{1}=1, n_{2}=1.8$, and $R=r^{2}$, and $c$ ) light path of LNS glass refractive index measurement set-up (M: mirror)

Figure $42 \mathrm{c}$ displays the optical light path for measuring the reflected intensity. A polarizer was used to ensure the light polarization to be in the plane of the incident beam (i.e. p wave). Firstly, the beam is normal to the entry face of the sample. A probing beam is crossing the axis of rotation of the plate that is on the input sample face in order to perform the measurement always at the same sample place whatever the incident angle. The spatial resolution and precision on measuring changes in refractive index was of the order of $\sim 10 \mu \mathrm{m}$. The accuracy of the measurement is $10^{-4}$ on the refractive index. Refractive index of LNS glasses according to chemical composition is shown in Table 9. The refractive index is larger than 1.85. This high refractive index is due to the high content of niobium [203].

Table 9 Refractive index of LNS glasses

\begin{tabular}{ll}
\hline LNS glass composition (mol\%) & Refractive index \\
\hline $32.5 \mathrm{Li}_{2} \mathrm{O}-27.5 \mathrm{Nb}_{2} \mathrm{O}_{5}-40 \mathrm{SiO}_{2}$ & $1.857 \pm 0.004$ \\
$33 \mathrm{Li}_{2} \mathrm{O}-33 \mathrm{Nb}_{2} \mathrm{O}_{5}-34 \mathrm{SiO}_{2}$ & $1.937 \pm 0.004$ \\
\hline
\end{tabular}




\subsubsection{Polarized light microscopy measurement}

The optical anisotropy of the fs laser irradiated lines in LNS glass has been investigated by a polarized light microscope (OLYMPUS BX60). The polarized light microscopy configuration is shown in Figure 43a. It is the conventional microscope equipped with a polarizer (placed before the sample) and an analyzer (positioned before the sample), which is in the crossed position. For details, please refer to [204].

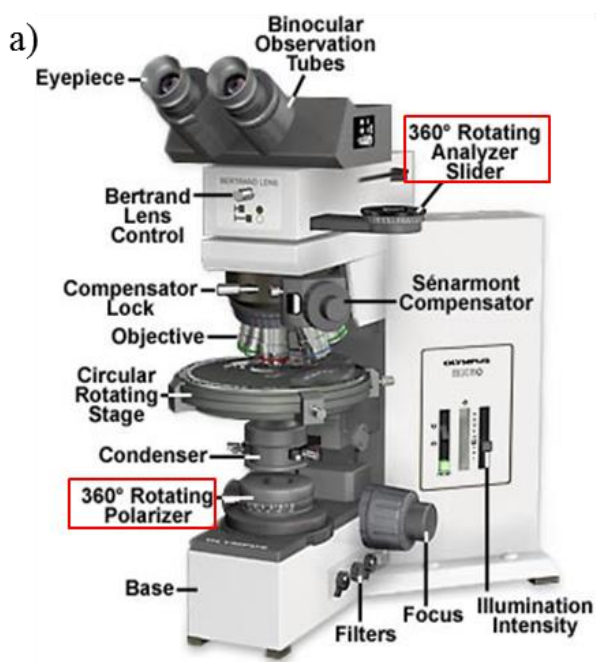

c) Wavefront Propagation in Anisotropic Crystals

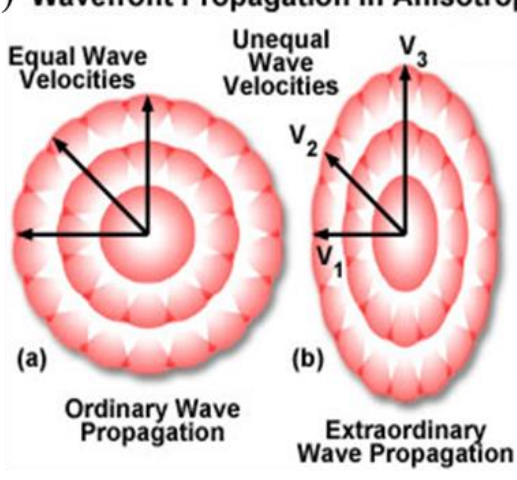

b)

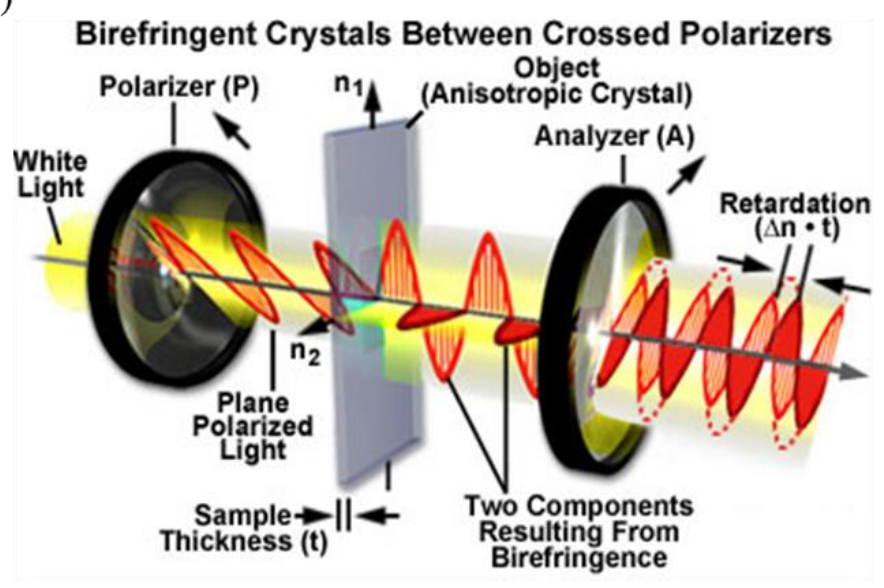

Figure 43 a) Polarized light microscopy configuration, red rectangles mark the polarizer and analyzer along light path (cited from [204]), b) birefringent crystal between crossed polarizers, c) wave front propagation in anisotropic crystal, and d) anisotropic crystals interact with polarized light in an optical microscope (cited from [205])

As illustrated in Figure 43b, when a linearly polarized light (illustrated by a red sinusoidal wave) interacts with a birefringent sample (sample thickness is $t$ ), it has to be separated into two eigen components (polarized in mutually perpendicular directions). The travelling speeds of these two components are different. After passing the sample, the two components will become out of phase with each other, and recombined producing an elliptical wave. 
The difference between the above refractive indices is $\Delta \mathrm{n}$. Optical path difference is defined by the relative phase difference between the two components after passing through the sample, generally given as $\Delta \mathrm{n} \cdot \mathrm{t}$.

Cubic crystal system has one refractive index. Tetragonal or hexagonal crystal systems have two principle refractive indices (i.e. they are uniaxial crystals). Orthorhombic, monoclinic, or triclinic crystal systems have three principle refractive indices (i.e. they are biaxial crystal). The interaction of incident light and sample is dependent on the lattice orientation with the incident light. When an incident light comes along the optical axis of anisotropic material, its polarization is not changed.

In isotropic materials, the index is called ordinary. The light speed is the same in every direction in the sample, obeying the normal refraction laws. For the light point source, travelling in isotropic material, it can be described by a spherical wave front (Figure $43 \mathrm{c} \mathrm{left).} \mathrm{For} \mathrm{a} \mathrm{material}$ with extraordinary component, the speed is dependent on the propagation direction in respect to the sample (Figure 43c middle). As illustrated in Figure 43c right, the fastest speed is achieved when propagating parallel to the shortest axis of the ellipsoid (i.e. fast axis). Similarly, the slowest one is when propagating parallel to the largest axis of the ellipsoid (i.e. slow axis).

As illustrated in Figure 43d, when the long axis (the optical axis is parallel to long axis) of sample and the polarizer axis coincide, a minimum light intensity will be achieved because the wave will be blocked by the analyser. When the polarizer is oriented at $45^{\circ}$ from the optical axis, a maximum intensity ios detected through the analyser. For details, please refer to [205].

When ordinary and extraordinary components pass through the analyzer, because one component is retarded with the other, constructive or destructive wave interference happens depending on the various wavelengths of white light, thus causing a colored spectrum.

The relationship between birefringence, sample thickness and interference colour is shown in Michel-Lévy interference color chart (shown in Appendix XVII), which is used in the field of mineralogy for identifying the mineral in rock. It can be seen from the chart that the most sensitive zone is the first-order red $(\sim 550 \mathrm{~nm})$. Based on this mechanism, a first order red compensator (or full wave retardation plate) can be used to determine the slow axis of certain uniaxial material that having very low order interference colors (i.e. first-order gray-level under crossed polarized illumination). 
The full wave retardation plate is generally made of optically anisotropic thin quartz with a precise thickness. It is located above the nosepiece and between the polarizer and analyzer (diagonally between crossed polarizers). It produces a specific optical path length difference of mutually perpendicular plane-polarized light waves (i.e. entire wavelength for the green one). The slow axis direction of the full wave plate is marked with " $\lambda$ " accompanied with a doubleheaded arrow. It is at $45^{\circ}$ from the analyser or polarizer.

\section{First Order Retardation Plate in White Light}

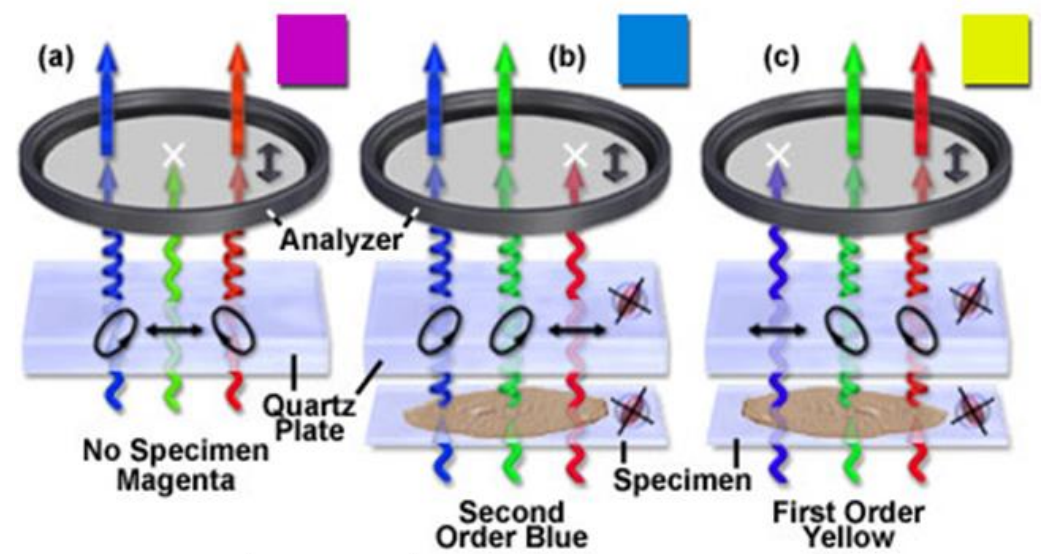

Figure 44 Working mechanism of first order retardation plate measuring slow axis of sample (cited from [206])

The polarized white light is symbolized by three wave fronts: blue, green, and red. As illustrated in Figure 44a, the background is magenta (i.e. the green wavelength is missing) when light passes through the full wave retardation plate and after an isotropic or without sample. When the slow axis of sample is placed parallel to the slow axis of the first order plate, the full wave retardation plate retardance is added to the sample retardance (i.e. the relative retardation of the orthogonal wave fronts increase). In that case, the red light is linear polarized and blocked by the analyser, resulting in second-order blue (Figure 44b). When the fast axis of sample is parallel to the slow axis of the full wave retardation plate, first-order yellow occurs because blue color is blocked (Figure 44c).

In our experiment, the fs laser-induced retardance in LNS glass is less than $90 \mathrm{~nm}$, which is suitable for this method to determine slow or fast axis of irradiated line in XY plane. See details in Section 5.2.1.2. 


\subsubsection{Quantitative birefringence microscopy}

The birefringence of irradiated line (measured in XY plane) was measured quantitatively by quantitative birefringence microscopy (QBM).

QBM is a technique that calculates the retardance magnitude and orientation at every pixel within seconds. The configuration of this system is shown in Figure 45a. A circularly polarized light was used for illuminating the sample. Green band-pass filter was used to make high contrast images in green through the eyepieces. This is necessary for the liquid crystal (LC) polarizing compensator optics. Then the transmitted light was passed through a "universal compensator".
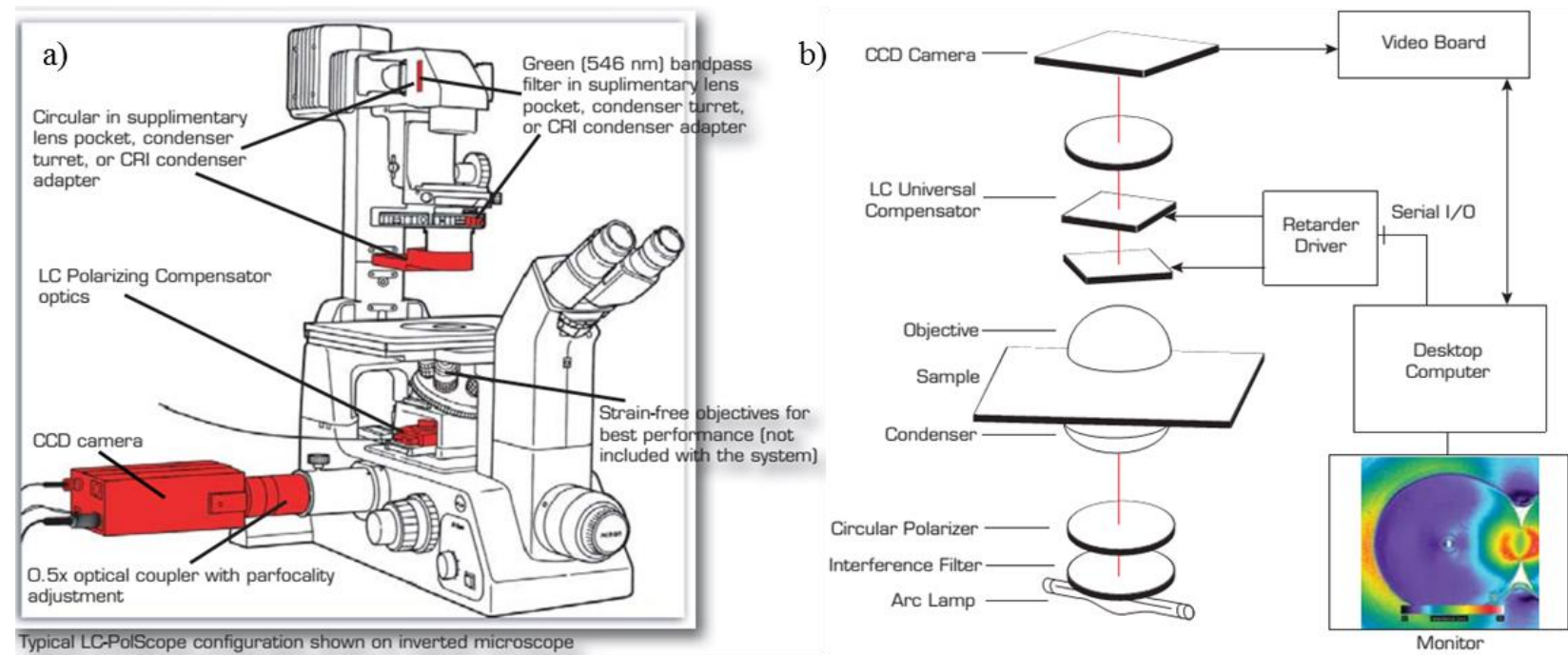

Figure 45 a) Quantitative birefringence microscopy set-up and b) the corresponding schematic diagram (cited from Abrio Product Bulletin, CRI, Inc)

As illustrated in Figure 45b, the "universal compensator" is a polarizing compensator made of two liquid crystal devices, changing the polarization state of the light without moving parts and without image shift. Optical coupler was used to increase the amount of light hitting the CCD, reducing the image capture time. The LC tunable retarder (i.e. universal compensator), electronic image, and digital image analysis are computer-controlled. By analyzing the images recorded under several LC compensator set-ups, 2D distribution of the slower polarization axis direction (large index) and phase differences between the two eigen axis of transmitted light (i.e. retardance proportional to the birefringence) are deduced [207]. 


\subsubsection{Digital holographic microscopy}

Digital holographic microscopy (DHM) records holograms produced by the interference between the beam transmitted through the sample, and a reference beam generated inside of the microscope.

For the irradiated line (measured in XY plane), the orientation averaged refractive index of the irradiated line $(\bar{n})$ can be calculated based on

$$
\Delta \varphi=\frac{2 \pi}{\lambda_{\text {DHM }}} t\left(\bar{n}-n_{b}\right), \quad \bar{n}=\frac{\left(n_{a}+n_{b}\right)}{2}
$$

Where $\Delta \phi$ is the measured phase difference between glass matrix and irradiated line (unit is in $\mathrm{rad}), \lambda_{\mathrm{DHM}}$ is measured wavelength which is $666 \mathrm{~nm}, \mathrm{n}_{\mathrm{b}}$ is refractive index of the background (i.e. glass matrix). $n_{a}$ and $n_{b}$ are the eigen value of the index in the plane perpendicular to the light propagation direction.

\subsubsection{Second harmonic generation measurement}

Second harmonic generation (SHG) microscopy images of irradiated lines were performed below the irradiation energy threshold for glass modification with the same laser [23].

As illustrated in Figure 46, the angular dependence of SHG intensity with the incident light polarization was performed in transmission mode with the fundamental beam (1030 nm) propagating perpendicularly to sample XY plane. The transmitted SHG light $(515 \mathrm{~nm})$ was recorded after passing through a low-pass filter. By rotating a half-wave plate, the polarization azimuth of fundamental beam, $\gamma$, was varied over $360^{\circ}$ (referred to $+\mathrm{X}$ direction in $\mathrm{XY}$ plane as defined in Figure 36). Data were recorded 5 times to deduce the average value and standard error. The SHG intensity for each written line was normalized to the largest value.

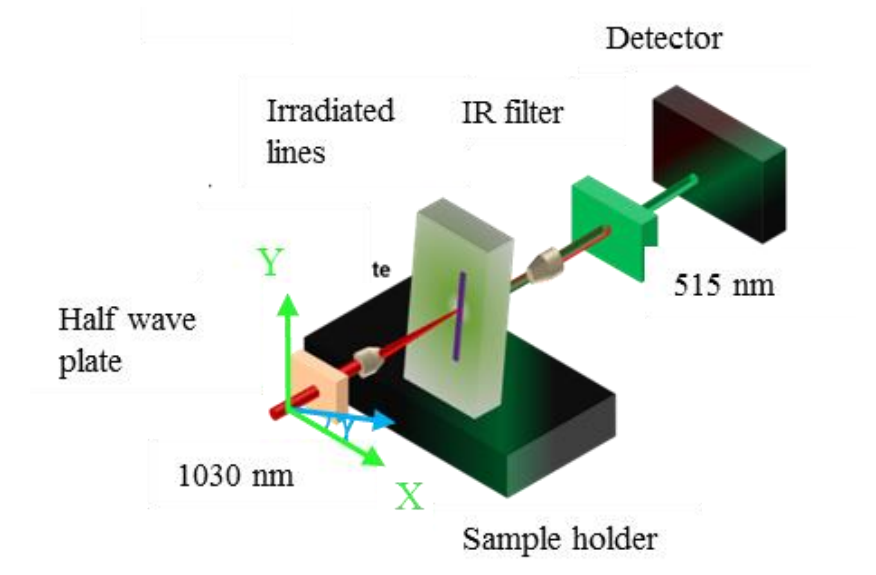

Figure 46 Second harmonic generation measurement set-up 


\subsubsection{Morphology and crystallization measurement}

The resolution of light microscopy (typical $\sim 0.2 \mu \mathrm{m}$ ) is limited by the Fresnel diffraction (i.e. the wavelength used for imaging the sample, in which the shorter the wavelength, the higher the resolution, and resolution $\propto \lambda / \mathrm{NA})$. To have a higher resolution of the $\mathrm{fs}$ laser-induced modifications in LNS glasses, electron microscopy will be employed.

\subsubsection{Scanning electron microscopy}

Scanning electron microscopy (SEM) was used for the observation of microstructure of lasermodified area. SEM measurements were conducted in a field-emission a gun scanning electron microscope (FEG-SEM ZEISS SUPRA 55 VP). Electron backscatter diffraction (EBSD), an accessory of SEM, was performed to determine the microstructure heterogeneities such as grain size, texture of the laser-modified area.

The schematic diagram of a SEM is shown in Figure 47a. The electron source may be from thermionic emission (e.g. $\mathrm{LaB}_{6}$ ) or field emission. The magnetic lenses guide and focus the electron beam (with a spot of few $\mathrm{nm}$ ). By using scanning coils, the focused electron beam scans across the specimen surface.

There is an interaction between the specimen and electron beam. Figure $47 \mathrm{~b}$ illustrates the schematic representation of processes resulting from electron bombardment and interaction with specimen. Electrons penetrate inside and generate various signals [208].

The common used detectors in SEM are the ones for secondary electrons and the backscattered electrons. Secondary electrons are emitted from the top surface, which can be used for topographic imaging. The backscattered electrons are from a deeper zone in the specimen. Their intensity is depending on atomic number $(Z)$, which can be used to provide information about the chemical composition (i.e. the higher the $Z$, the brighter the image contrast). Finally, by the imaging system, image is formed. The resolution of the method is depending on the measured specimen volume (i.e. the detected electron), the accelerating voltage, working distance, and the probe size.

Because the laser-modified area is embedded in the glass matrix (i.e. several hundred $\mu \mathrm{m}$ below the glass surface), it is necessary to expose the irradiated area for morphology, crystallization observations, and chemical analysis by electron microscopy. One way is to cut the irradiated line along the plane perpendicular to the scanning direction, polished. Characterizations were thus 
performed on the cross section of the irradiated lines. The other way is to polish along the plane perpendicular to the laser propagation direction until to have the laser-modified part at the surface. In addition, hydrofluoric acid (HF) was sometimes used to increase the contrast in SEM measurement.

a)

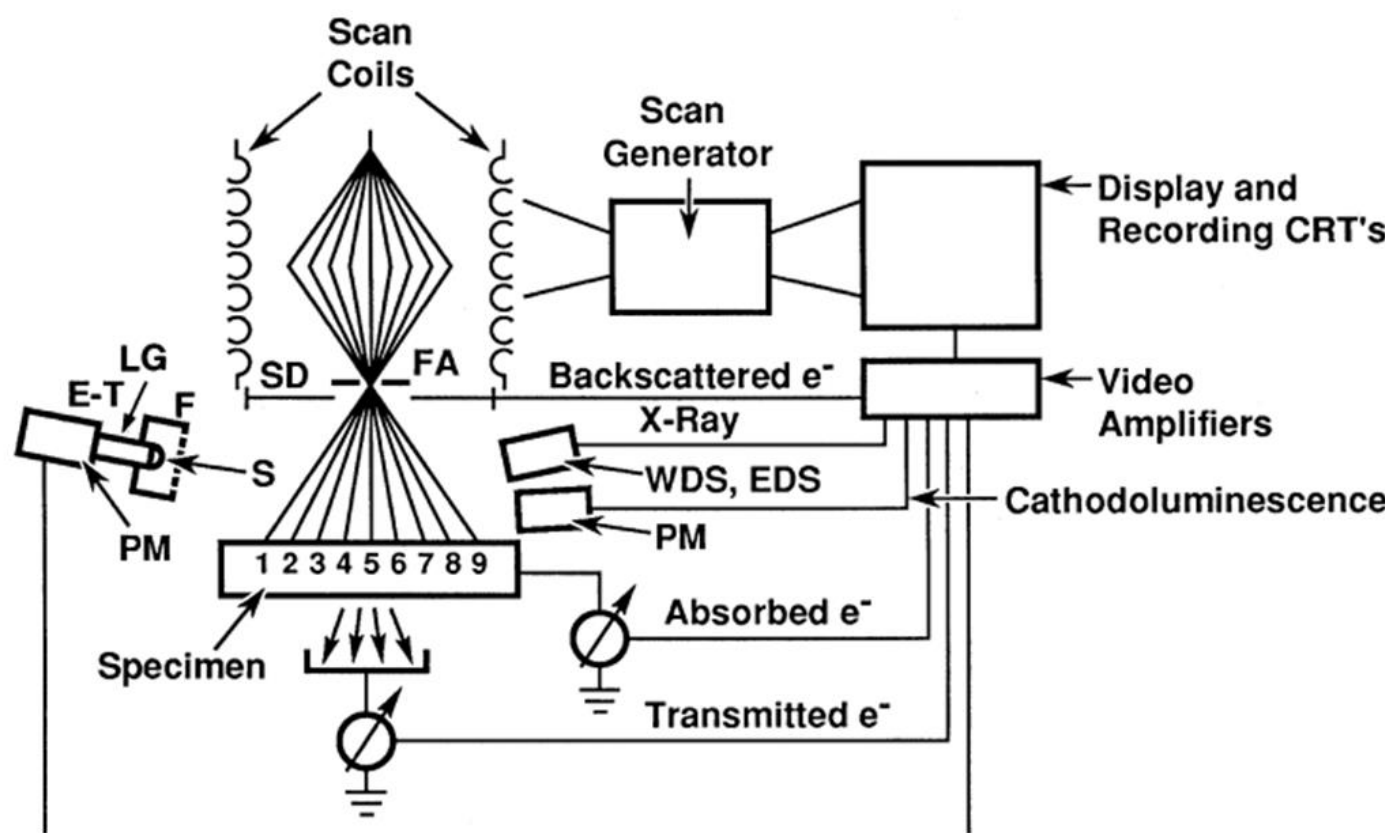

b)

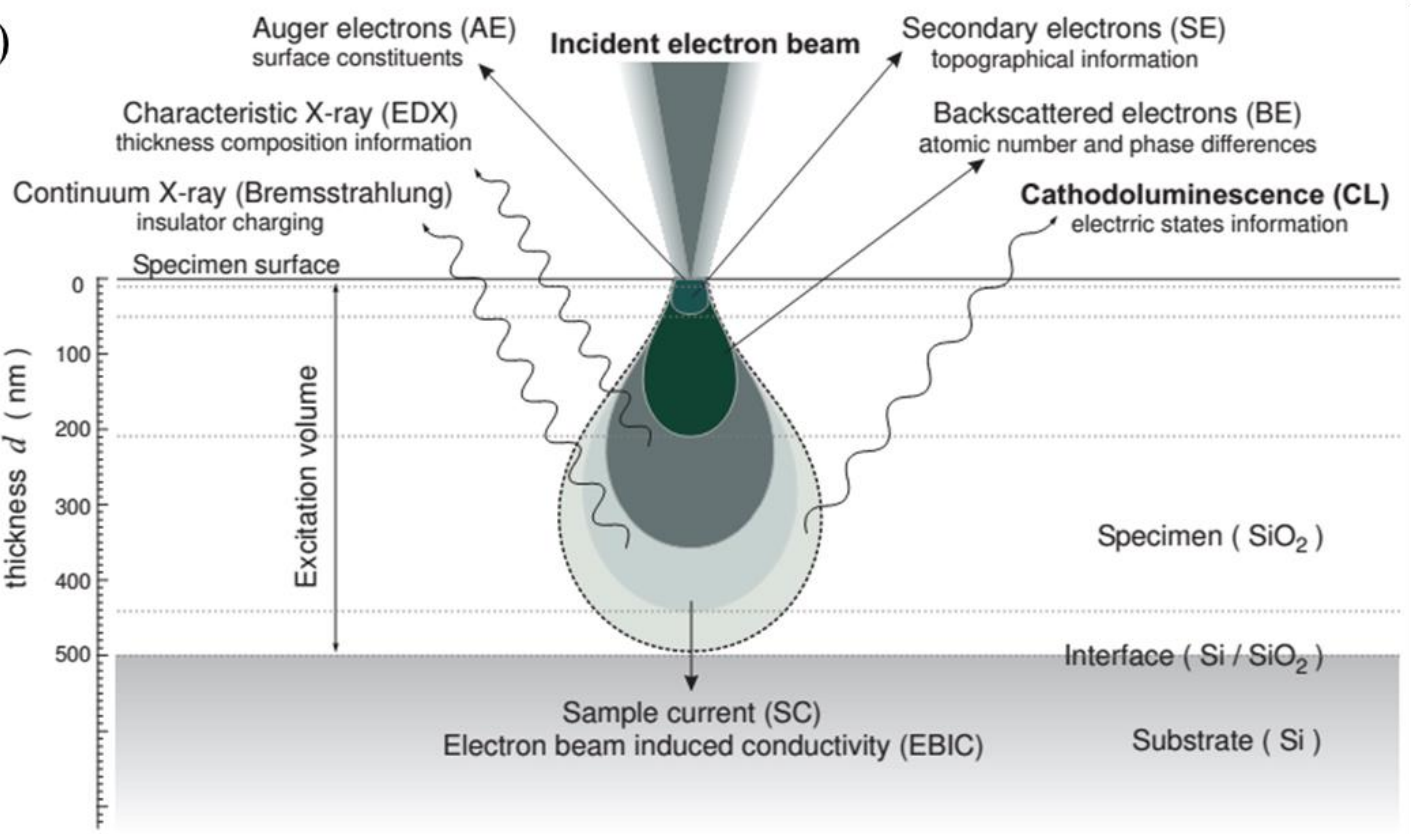

Figure 47 a) Schematic representations of scanning electron microscopy. FA: final aperture; SD: solid state backscattered electron detector; EDS: energy-dispersive X-ray spectrometer; WDS: wavelength-dispersive X-ray spectrometer; CRT: cathode ray tube; E-T: Everhart-Thornley secondary/backscattered electron detector, consisting of F: Faraday cage; S: scintillator; LG: light guide; and PM: photomultiplier (cited from [209]), b) processes resulting from electron bombardment (cited from [208]) 


\subsubsection{Electron backscatter diffraction}

Electron backscatter diffraction (EBSD) is a useful tool to determine the amount of crystallized matter, the size of the crystal, their spatial distribution and their orientation.

EBSD analysing is based on the formation of Kikuchi patterns. As illustrated in Figure 48a-b, they are formed when a beam is converged on a sample whose surface is placed at a highly tilted angle (about $70^{\circ}$ with relative to the incident beam). A small portion of backscattered electrons undergo Bragg reflection on material lattice planes. These electrons are refracted according to a diffraction cone whose apex is located at the impact point of the primary electron beam with the sample (Figure 48c). They are then detected by a charge-coupled device (CCD) sensor behind a phosphorescent screen. The intersection between this screen and the cone form lines called "pseudo-Kikuchi" whose number, arrangement (i.e. symmetry), intensity, and width are characteristics of the crystal lattice and orientation.

a)

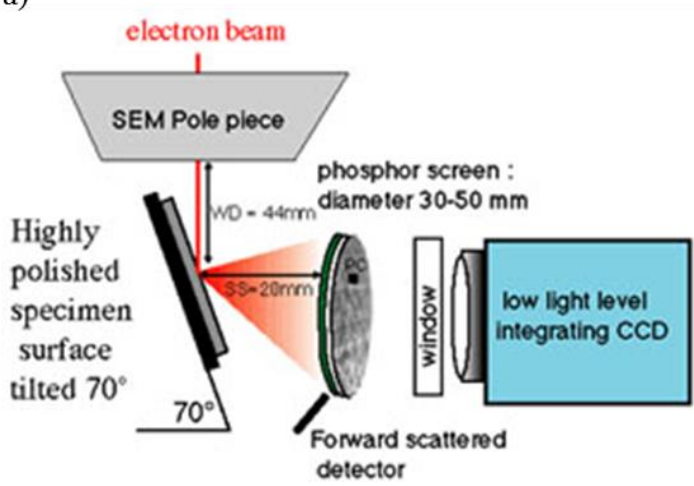

b)

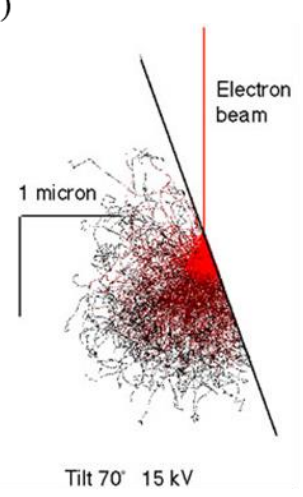

c)

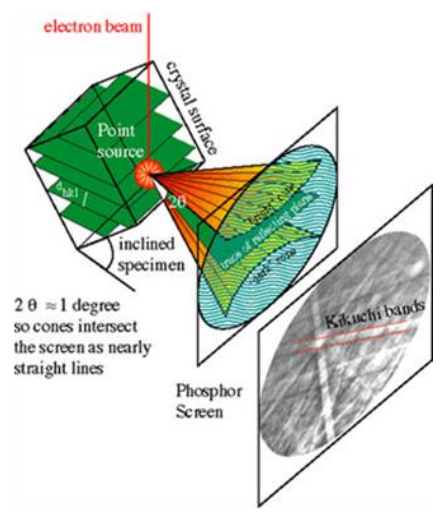

Figure 48 How electron backscatter diffraction measurements work: a) schematic diagram of the EBSD system, b) the beam-specimen interaction, and c) formation of a backscattered Kikuchi band (cited from [210])

Diffraction lines were detected, indicating the crystal property of laser tracks. Then, by entering space group of the expected phase (e.g. in our case, $\mathrm{R} 3 \mathrm{c}$ and the crystal parameters of $\mathrm{LiNbO}_{3}$ ), we can get the indexation of the Kikuchi lines using Orientation Imaging Microscopy $\left(\mathrm{OIM}^{\mathrm{TM}}\right)$ software. The diffraction patterns of laser traces consistent with that of $\mathrm{LiNbO}_{3}$ crystalline phase, indicating that the crystalline nature of the radiations area and the same group of symmetry as $\mathrm{LiNbO}_{3}$ crystals, whereas the surrounding of the traces is proved to be remained glassy. The next step is to examine the Orientation Distribution Function (ODF, the angular density of crystals among the Euler space) for determining if a texture exists. Then, we can plot the Inverse Pole Figure (IPF) for displaying the preferred orientations in choosing a sample direction suitable to show the detected texture. In IPF, the red-green-blue (RGB) based color stands for the crystal direction along the choose sample direction as recorded by EBSD [211]. 
The spatial resolution of EBSD is in the nanometre range but the nature of the phases, the combination of the microstructure/phase mean atomic number $(Z)$ value/high voltage/or the data treatment procedure such as "cleaning up" may decrease the resolution [212]. In the following part we will discuss several important issues in EBSD analysis. For details, please refer to orientation imaging microscopy $\left(\mathrm{OIM}^{\mathrm{TM}}\right)$ analysis manual.

Image quality map: Image quality (IQ) map constructed from EBSD data provides an effective approach to visualize microstructure [213]. It is a map with a grayscale component according to the relative intensity of the diffraction pattern, generally, with the maximum IQ value in white and the minimum one in black. The contrast in this image may due to the phase, stain, and grain boundaries.

Confidence Index: Confidence Index $(\mathrm{Cl})$ indicates the degree of confidence that the orientation calculation is correct, ranging from 0 (good) to 1 (perfect). It is a quantitative measure of the reliably of the indexed pattern. The low $\mathrm{Cl}$ data is the one with very poor pattern quality, which may due to the amorphous phase, cracks, or grain boundary. The $\mathrm{Cl}$ does not have to be 1 to consider the indexing is correct. The $\mathrm{Cl}$ of the correct indexing solution is depending on the material properties [214].

Inverse Pole Figure: Inverse Pole Figure (IPF) displays the position of crystal orientation relative to a sample reference frame. In our case, the colour in IPF codes the crystallographic orientation aligned with the considered sample direction. Spatial distribution information of crystals can also be seen from IPF. In this image, the color in the black background represents the crystalline part that could be detected by EBSD.

"Clean up" process: "Clean up" process is a method in the software (OIM ${ }^{\mathrm{TM}}$ Data Analysis) to treat the points that did not give rise to diffraction or give a too weak diffraction pattern but having neighbouring diffracting points. If the misorientation among the neighbouring points is within the grain tolerance angle, they will belong to the same "grain" after clean up treatment. This means that, if a small amorphous region is located among large crystals that belong to the same "grain", the amorphous region will be considered as crystallized with the orientation of the major "grain", thus hiding the amorphous phase. Generally, one to two times "clean up" process is used in EBSD data analysis. In other words, there is a risk in using "clean up" process in EBSD data treatment exaggeratedly to introduce artifacts [212]. 


\subsubsection{Transmission electron microscopy}

Transmission electron microscopy (TEM) was used to observe the nanostructure and to determine the crystalline property of laser-modified area. It was conducted on a TOPCON 002B electron microscope (200 kV with resolution of $0.18 \mathrm{~nm}$ ).

There are two modes for the observations: bright field (BF) mode and dark field (DF) modes of TEM measurement. For BF mode, as shown in Figure 49b, an aperture is placed in the back focal plane of the objective lens, allowing the transmitted beam to pass and the diffracted electrons are stopped by the diaphragm. The image results from a weakening of the direct beam by its interaction with the sample. The image contrast is due to the variation of intensities of transmitted beams. Mass-thickness and diffraction contrast can contribute the formation of image: thick zones, regions with enriched heavy atoms, or crystalline areas occur with dark contrast [215]. For DF mode, as shown in Figure 49c, the direct transmitted beam is blocked by the aperture but one of the diffracted beams can pass the objective aperture. Because diffracted beams have strongly interacted with the sample, some useful information is present in DF images (e.g. about planar defects, stacking faults, or particle size) [215].

a)

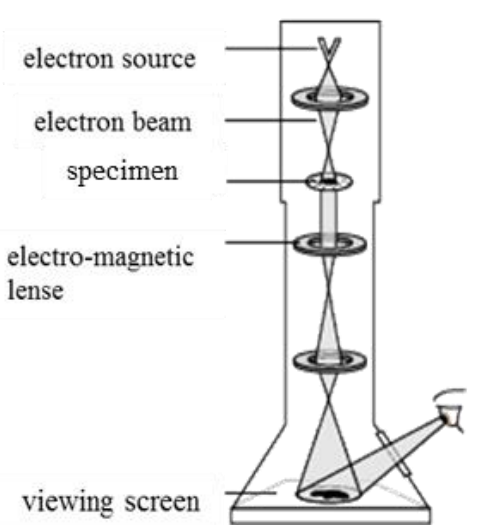

b)

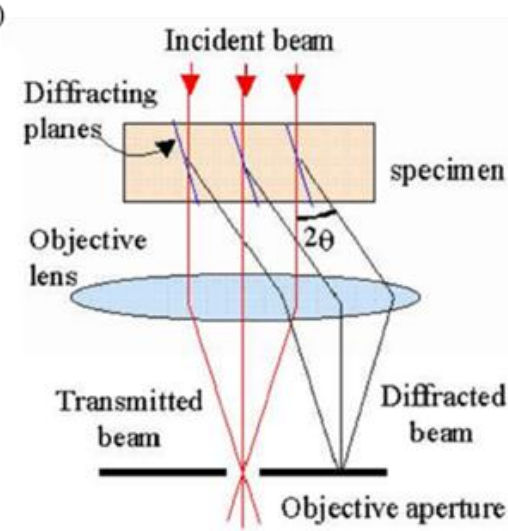

c)

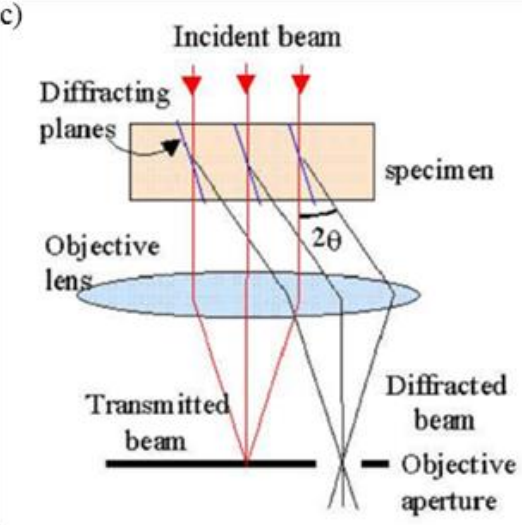

Figure 49 a) Working principle of TEM (cited from [216]), b) bright field, and c) off-axis dark field imaging of TEM (cited from [217])

Inside TEM, selected area electron diffraction (SAED), a crystallographic experimental technique can be conducted. Parallel electron illumination is used to obtain diffraction pattern. An aperture in the image plane is employed to select the diffracted region of the sample, leading to siteselective diffraction analysis. SAED patterns are a projection of the reciprocal lattice, with lattice reflections showing as sharp diffraction spots.

Using a focused ion beam (e.g. Ga ${ }^{+}$), the material can be precisely removed at a submicron scale [218]. Herein, focused ion beam (FIB) was used for TEM-based observation sample preparation 
[219]. A two-step FIB technique was used to cut the sample and then to transform it carefully into super thinner slice for TEM-based observation and the corresponding process is illustrated in Figure 50.

a)

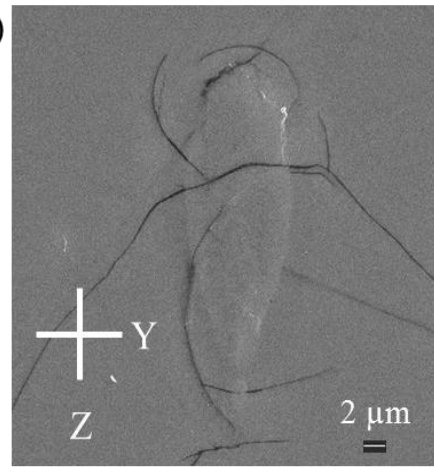

c)

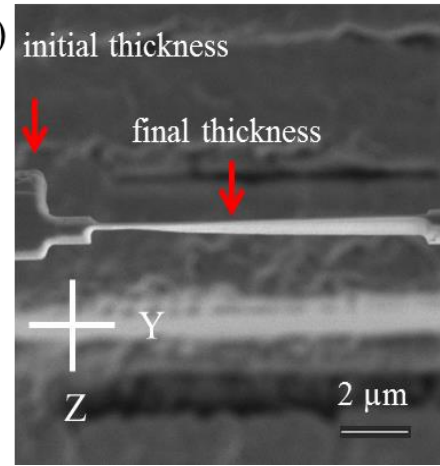

b)

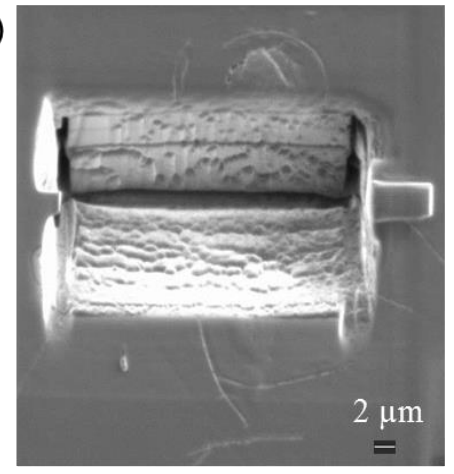

d)

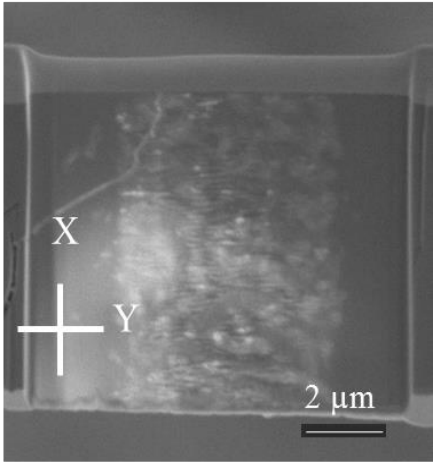

Figure 50 Illustration of sample dissecting process by FIB: a) the sample was cut along the plane perpendicular to the scanning direction (i.e. $X$ direction) and polished, $b$ ) a volume of irradiated part was picked up from the irradiated line, c) the "picked-up" part was dissected along laser propagation direction (i.e. $Z$ direction) by FIB, and d) image of the irradiated line after FIB treatment

\subsubsection{Chemical analysis}

\subsubsection{SEM coupled to energy dispersive X-ray spectroscopy}

SEM coupled to energy dispersive $X$-ray spectroscopy (SEM/EDS) is used to determine the distribution of $\mathrm{Nb}$ and $\mathrm{Si}$ of in and around laser-modified area. It was conducted in a fieldemission a gun scanning electron microscope (FEG-SEM ZEISS SUPRA 55 VP).

EDS is a kind of X-ray spectrometer. The interaction of incident electron beam with atoms in the sample can cause inter shell electron transitions, resulting in X-ray emission. The incident electrons kick out an electron located around the atomic nucleus. Then, an electron from an outer shell comes down to take the place of the previously ejected electron. Each element produces a unique set of characteristics $\mathrm{X}$-rays (i.e. an energy and wavelength characteristic of the element). The energy-dispersive software sorts the $X$-rays depending on their energy, producing an output in the profile of a plot of intensity as a function of X-ray photon energy. 


\subsubsection{Wavelength dispersive spectroscopy microprobe}

To have a more quantitative profile and measure 0 , wavelength dispersive spectroscopy (WDS) microprobe was conducted in Institute de Chimie et des Matériaux Paris-Est.

WDS is another kind of X-ray spectrometer. The sample surface, analyzing crystal, and X-ray detector are positioned on a circle (i.e. Rowland Circle) to satisfy Bragg's law ( $n \lambda=2 d \sin \theta$ ). At angle $\theta$, strong scattering of $\mathrm{X}$-rays with wavelength of $\mathrm{n} \lambda$ occurs. For all other angles, the scattering is weak. In that case, only X-rays with certain wavelength can enter the detector at one time. So, several crystals with different inter-planar space are necessary to cover the required wavelength range. The comparison of WDS with conventional EDS is shown in Appendix XVIII.

\subsubsection{Ion beam analyses}

Ion beam analyses were conducted with protons (beam size $2 \times 2 \mu \mathrm{m}^{2}$ ) in the Alternative Energies and Atomic Energy Commission (CEA). The schematic of lon beam analyses is shown in Figure 51. Proton-induced X-ray emission (PIXE): it can provide the information on "light" elements $(Z>15)$ : trace element/dopant, the limit concentration is $10 \mathrm{ppm}$.

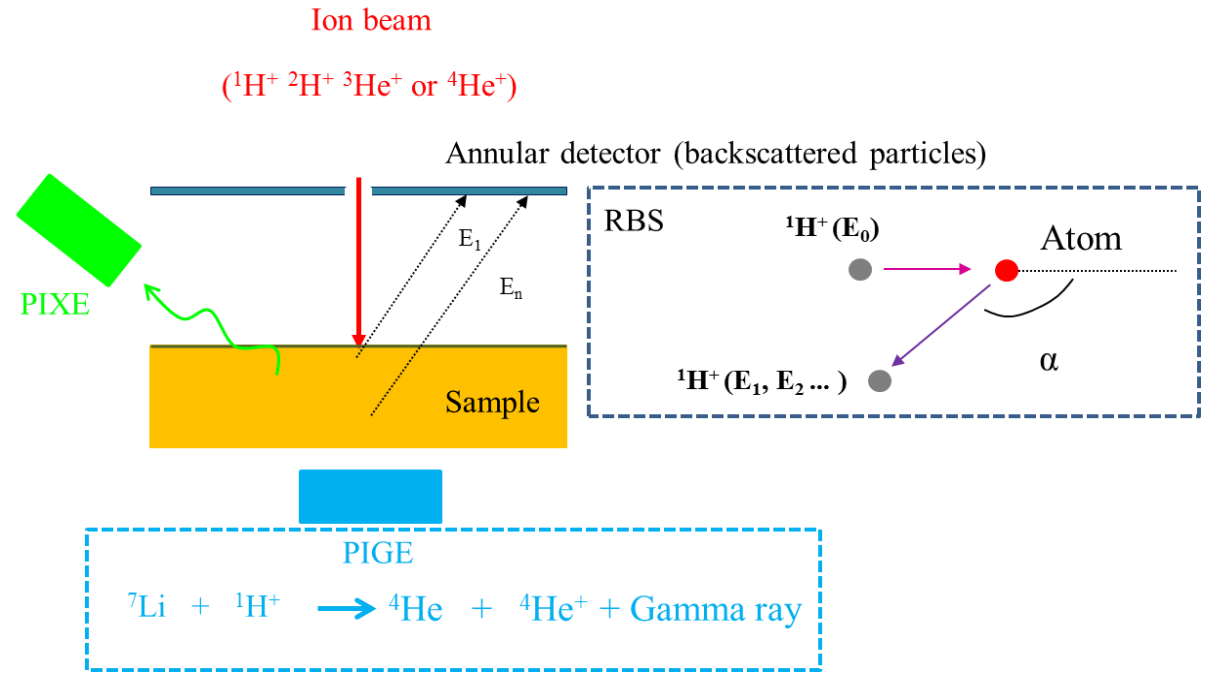

Figure 51 Schematic of Ion beam analyses: proton-induced X-ray emission (PIXE), Rutherford backscattering spectrometry (RBS), and proton-induced Gamma-ray emission (PIGE)

Rutherford backscattering spectrometry (RBS): Rutherford backscattering is an elastic collision between a high kinetic energy particle (e.g. protons) and a stationary particle located in the sample. So it is also named as high energy ion scattering spectrometry. By recording the backscattering of a beam of high energy ions, which are impinged on the sample, RBS can be used to investigate the composition of sample. 
Proton-induced Gamma-ray emission (PIGE): gamma-ray emission occurs due to excitation of the end core of a nuclear reaction. The detection elements are $3<Z<30$.

In our case, PIXE was applied to measure $\mathrm{Si}$, RBS was used to measure Nb content, and PIGE was employed to analyze Li.

\subsubsection{X-ray absorption spectroscopy}

X-ray absorption spectroscopy (XAS) was used to determine the local environment of $\mathrm{Nb}$ and $\mathrm{Si}$. It was recorded at the Synchrotron Radiation Facility SOLEIL, with the beam line "LUCIA" (line for ultimate characterization by imaging and absorption) in total electron yield (TEY) mode using an InSb (111) monochromator. "LUCIA" is characterized by the production of a micro-focused beam in the energy range of $0.8-8 \mathrm{keV}$. It allows the possibility to investigate heterogeneous samples at a micrometer scale [220].

\subsubsection{STEM-HAADF}

Scanning transmission electron microscopy-high angle annular dark-field (STEM-HAADF) studies and STEM/EDS analysis were performed on a Tecnai FEG electron microscope.

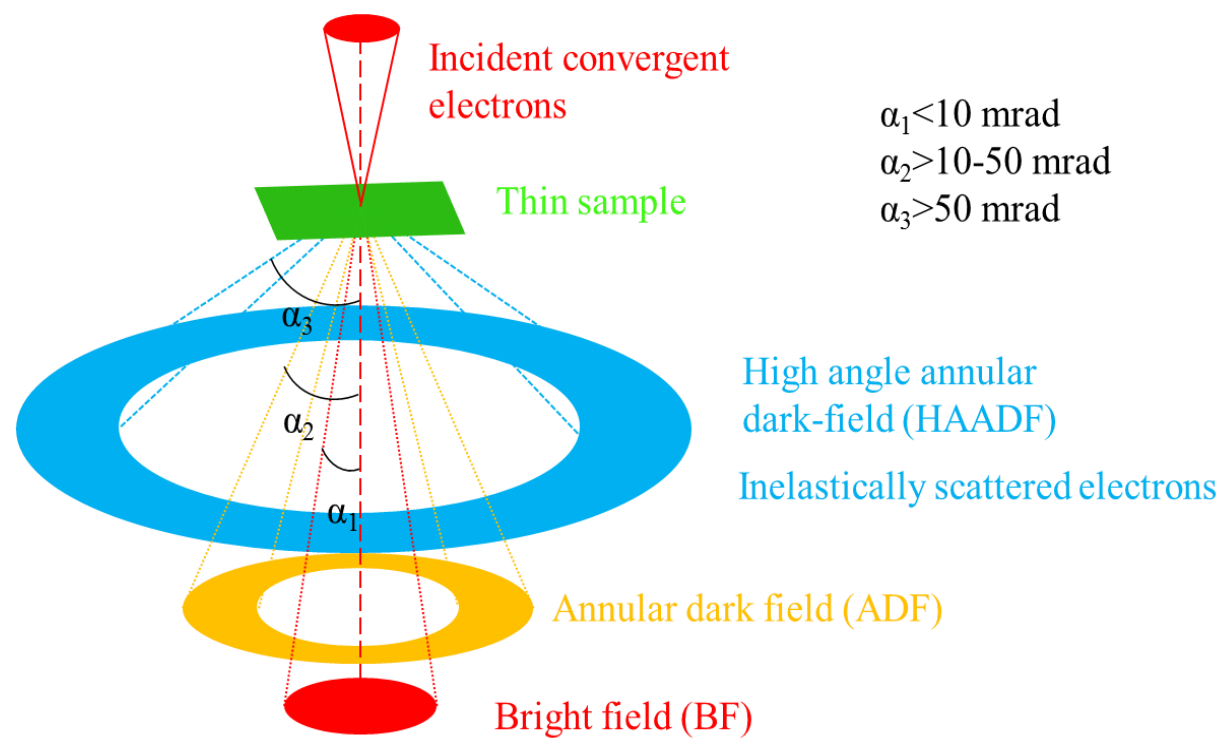

Figure $\mathbf{5 2}$ Schematic of different domains of the electron diffusion angle after passing the sample (after [221])

STEM-HAADF is a technique strongly sensitive to variations in the atomic number of the sample [222]. A STEM image is acquired by displaying the integrated intensities of the electrons in synchronism with the incident probe position. As shown in Figure 52, STEM-HAADF records inelastically scattered electrons or thermal diffuse scattering at high angles by an annular darkfield (ADF) detector $\left(1 \mathrm{mrad}=0.057^{\circ}\right)$. 
Figure 53 displays the sample's excited volumes induced by electron and proton beams. In bulk sample, for SEM, electrons are extensively scattered in depth, thus inducing thus a "pear"-like excited volume (Figure 53a). For STEM, due to the thinner samples and higher accelerating voltage, the resolution can reach nanoscale (Figure 53b). Protons experience minor deflection and the path of protons is longer than the electrons ones (Figure 53c).

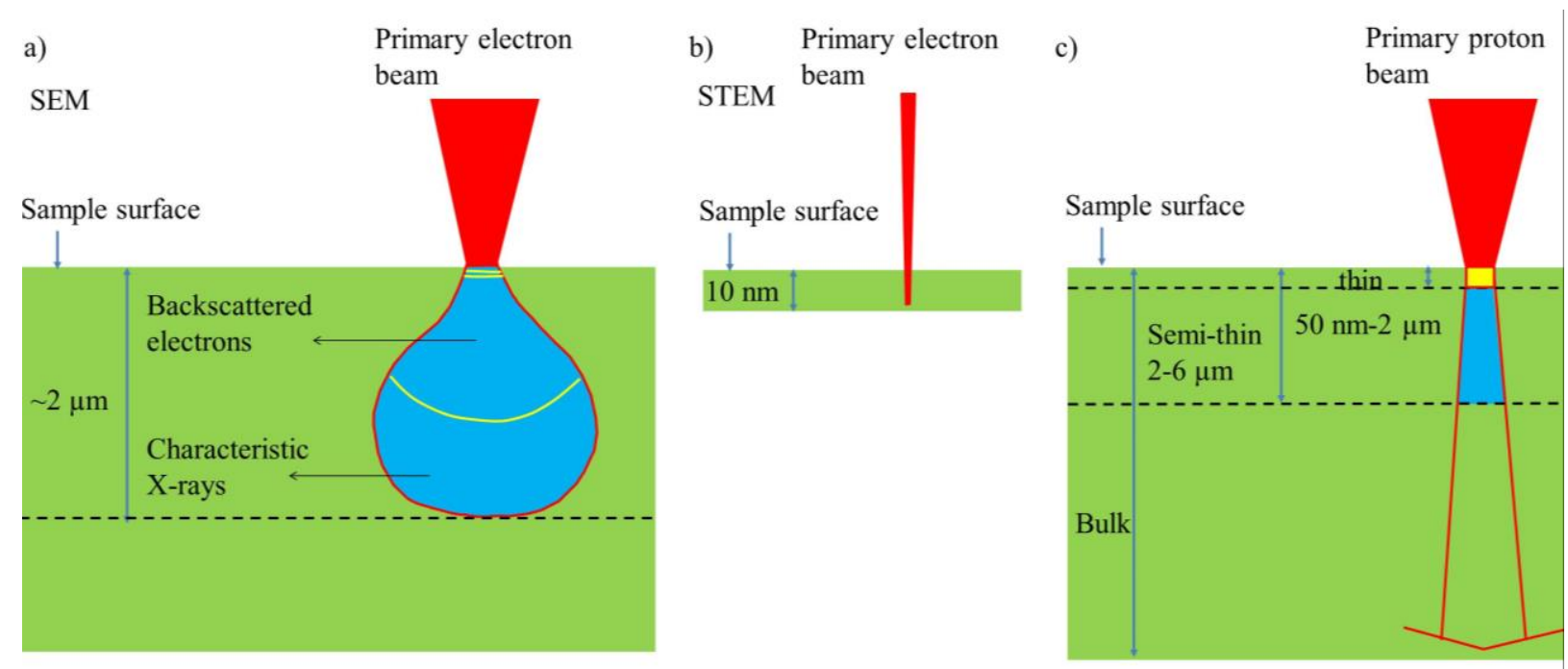

Figure 53 Schematic illustration of the comparison of excited sample volumes from a) electrons in a SEM, b) electrons in a STEM, and c) from proton beams (after [223, 224])

\subsubsection{X-ray photoelectron spectroscopy}

X-ray photoelectron spectroscopy (XPS) or electron spectroscopy for chemical analysis is a surface analysis method, which can provide elemental and chemical state information from the outer 5 to $10 \mathrm{~nm}$ of a sample surface. It is based on the detection of the photoelectrons' energies, emitting from atoms when irradiated by soft X-ray photons (1000-2000 eV).

The XPS experiment was performed in ICMMO with ThermoFisher, operated on CAE 5Constant Analyser Energy mode. Pass energies: $200 \mathrm{eV}$ for the acquisition of surveys, $50 \mathrm{eV}$ for the acquisition of high-resolution spectra. Energy step: $1 \mathrm{eV}$ for the acquisition of surveys, $0.1 \mathrm{eV}$ for the acquisition of high-resolution spectra. The X-ray source is Al Ka monochromatic. Spot sizes: initially $30 \mu \mathrm{m}$ (not enough emission to detect $\mathrm{Li}_{1 \mathrm{~s}}$ ), then $200 \mu \mathrm{m}$. Angle Analyser/Source $=56^{\circ}$. Analysis chamber pressure $=2 \times 10^{-9} \mathrm{mbar}$ at the beginning of analysis, $2 \times 10^{-7} \mathrm{mbar}$ during acquisition (due to the Flood Gun which uses Ar gas), $5 \times 10^{-7}$ mbar during etching (as a higher $\mathrm{Ar}$ pressure is required). Samples were cleaned thoroughly with isopropanol before mounting. Carbon tape was used the fix the samples on the sample holder. 


\subsubsection{Laser-induced breakdown spectroscopy}

Laser-induced breakdown spectroscopy (LIBS) phenomenon is due to the plasma formation accompanied by emission which usually lasts longer than the initiating laser pulse (generally the plasma duration is several $\mu \mathrm{s})$. Emission spectroscopy can be used for elemental analysis of the targets from which the luminous plasma is generated [225]. LIBS measurement was performed in Commissariat à l'énergie atomique (CEA) by a UV beam with wavelength of $266 \mathrm{~nm}$ (the size for emission is around $100 \mu \mathrm{m})$.

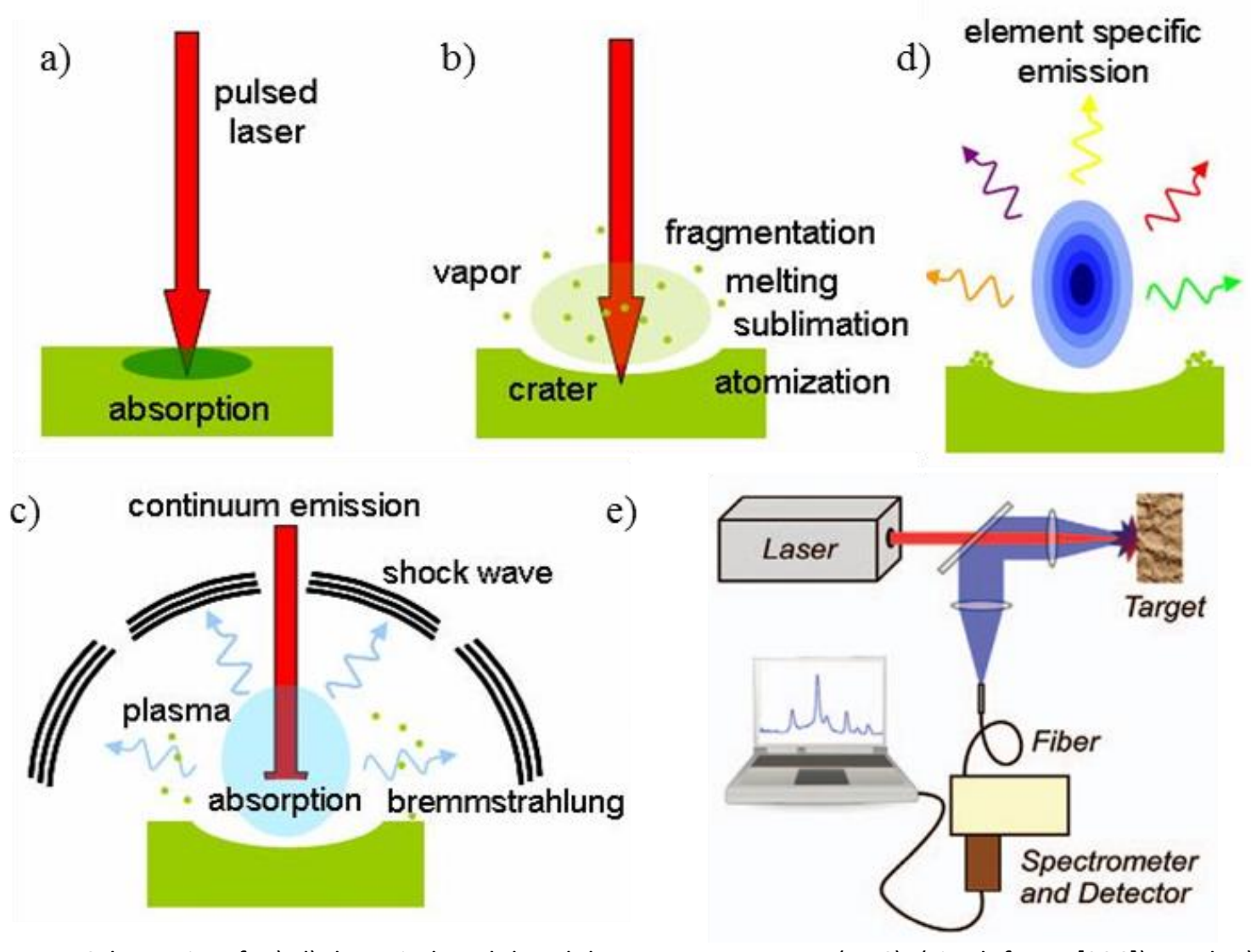

Figure 54 Schematic of a)-d) laser-induced breakdown spectroscopy (LIBS) (cited from [226]), and e) a "traditional" LIBS set-up (cited from [227]).

As shown in Figure 54a, a pulsed laser is used as excitation source, focused onto the material surface. The absorbed energy can be converted into heating rapidly. The temperature can reach the material boiling point, resulting in a fraction of material $(\sim \mu \mathrm{g})$ ejected from the surface (i.e. laser ablation). The removal material causes a formation of a vapour (Figure 54b). The laser pulse continues to illuminate the vapor plume and the vapor tends to condense into sub- $\mu \mathrm{m}$ droplets, resulting in the absorption and scattering of the laser beam (Figure 54c) [226]. The dynamical evolution of the plasma plume is then characterized by a fast expansion (supersonic speeds) and subsequent cooling. During this time the excited ions and atoms can emit 
characteristic radiation due to the lower energy states reverting (Figure 54d). Analysis such optical radiation can give information of the material elemental composition. A "traditional" LIBS set-up (Figure 54e), is operates by focusing a single laser (by a spherical lens) close to the target position and collects the plasma radiation axially with a pierced mirror and a spherical lens to create an image of the plasma on the entrance of an optical fiber, which is optically coupled with a monochromator [227]. The ideal requirements of a spectroscopic method are listed in Appendix XIX. 


\section{Chapter 4. fs laser-induced modifications in LNS glasses}

In this section, the fs laser-induced modifications (i.e. morphology, crystal texture, and nanostructure) in LNS glasses were explored to get insight on the ultra-fast laser-matter interaction according to the parameters such as pulse energy, writing laser polarization, and writing configuration (laser polarization direction versus scanning one), which may guide users to the optimal parameters for the applications in optics.

\section{1. fs laser-induced crystallization}

To control the phase transformation in glass and the morphology of related nanostructure after fs laser irradiation is of great importance for fabricating functional optics, in which glass crystallization is involved to obtain nonlinear optical properties.

In this section, the crystallization inside LNS glass induced by fs laser irradiation according to the parameters (e.g. pulse energy and laser repetition rate) was investigated systematically. Electron backscatter diffraction (EBSD) is a useful technique for analysing the morphology, texture of materials. In the following part, EBSD was used to investigate the morphology, orientation, and texture of laser tracks. Chemical analysis characterizations (e.g. SEM/EDS and STEM/EDS) were also measured to explore the element distribution (e.g. $\mathrm{Li}, \mathrm{Si}$, and $\mathrm{Nb}$ ) of irradiated LNS glass. To comparison, other method for obtaining crystal (i.e. floating zone method) was also performed.

\subsubsection{Nonlinear optical crystal formation}

\subsubsection{Three regimes of modification according to pulse energy}

Irradiated lines were cut and polished along the plane perpendicular to the scanning direction, and observations were performed perpendicular to scanning direction. The crystalline nature of the interaction volume is proved by the diffraction patterns of laser traces (Kikuchi lines). Diffraction lines have been indexed according to group of symmetry of $\mathrm{LiNbO}_{3}$. The energy threshold for fs laser-induced crystalline line is around $0.5 \mu \mathrm{J} /$ pulse at $1030 \mathrm{~nm}, 300 \mathrm{kHz}, 300 \mathrm{fs}$, $\mathrm{NA}=0.6$, focal depth $\sim 350 \mu \mathrm{m}$, and scanning speed $5 \mu \mathrm{m} / \mathrm{s}$. Three regimes of fs laser-induced structural changes were defined varying the pulse energies. 
Figure 55 presents the SEM images of the cross sections of irradiated lines in LNS glass (after HF treatment) with the increase of pulse energy.

Regime 1: When pulse energy is lower than the threshold for obtaining crystalline line in LNS glass (i.e. 0.3-0.4 $\mu /$ pulse), a needle-shaped over etched area (by HF) is observed in SEM image (zone B, illustrated in Figure 55a). The aspect is the same as the unmodified glass matrix (i.e. vitreous) but it is more sensitive to the chemical etching than glass matrix (zone $A$, marked in Figure 55a). In addition, a clear boundary between this over etched area and the glass matrix is detected (illustrated by red arrows in Figure 55a). No crystallization is detected in this lasermodified area by EBSD measurement. This regime is probably similar to the one described in [201]. See details in Section 3.3.2.2.

Regime 2: When increasing pulse energies slightly above this energy threshold (i.e. 0.5-0.9 $\mu \mathrm{J} /$ pulse), three zones with two boundaries are obtained (illustrated by red arrows in Figure 55b left). Besides the surrounding glass matrix and the over etched zone, a rough structure is detected at the center of laser track.
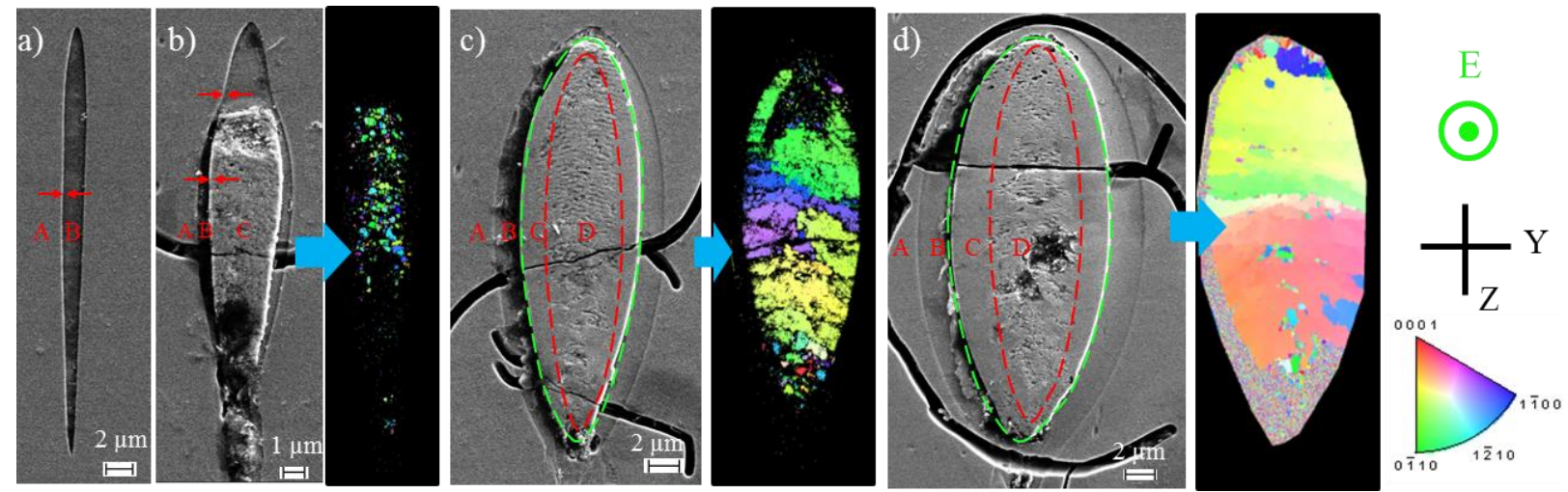

Figure 55 SEM pictures according to the pulse energy defining three types of material modifications obtained in the plane perpendicular to scanning direction (after HF treatment): a) regime 1, b) regime 2, and c-d) regime 3. The color in IPFs (b, c, and d right) is based on $\mathrm{R} 3 \mathrm{c}$ space group, $\mathrm{LiNbO}_{3}$, coding the crystal axis along laser polarization direction. Other parameters: $33 \mathrm{Li}_{2} \mathrm{O}-33 \mathrm{Nb}_{2} \mathrm{O}_{5}-34 \mathrm{SiO}_{2}(\mathrm{~mol} \%), 1030 \mathrm{~nm}, 300 \mathrm{fs}, 300 \mathrm{kHz}, \mathrm{NA}=0.6$, focus depth $350 \mu \mathrm{m}$ in glass, $5 \mu \mathrm{m} / \mathrm{s}$, pulse energy of a) 0.4 , b) 0.7, c) 1.3 , and d) $1.8 \mu \mathrm{J} /$ pulse, laser polarization (illustrated by $\mathrm{E}$ ) is parallel to scanning direction (i.e. $\mathrm{X}$ direction).

In Inverse Pole Figure (IPF), the colour in the black background represents the crystalline part that could be detected by EBSD. As shown in Figure 55b right, the corresponding IPF reveals the formation of crystals in the rough zone. In the colour coding, basic red is used for polar axis of

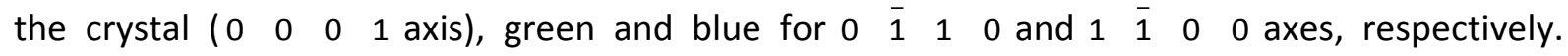
The inter-mediate orientations are colored by a RGB mixture of the primary components. The IPF is almost green or blue but not red when coding along laser polarization direction, indicating that 
polar axis of crystal are distributed along the plan perpendicular to laser polarization direction. In other word, a texture is achieved.

0001-PF (Figure 56) clearly displays blue region (no density) around laser polarization direction ( $X$ direction) and accumulates (red color) around the plane perpendicular to laser polarization direction thus confirming that polar axes of nano-crystals avoid the direction of laser polarization.

Regime 3: When glass was exposed to higher pulse energy (1.0-2.2 $\mu \mathrm{J} /$ pulse), as shown in Figure $55 \mathrm{c}$ left, a teardrop-shaped laser-induced modification with four zones and three clear boundaries was revealed (illustrated by dashed line).

We quoted them from the glass matrix to the centre of the laser track as $A, B, C$, and D. Zone $A$ is the unmodified glass matrix; Zone B is the over etched amorphous region (like in regime 1); Zones $C$ and $D$ are the parts containing nonlinear optical crystals (IPF, Figure $55 \mathrm{c} \mathrm{right).} \mathrm{It} \mathrm{is} \mathrm{thus}$ the volume where the thermal condition for crystallization are gathered [17]. Zone $C$ is a smooth part bordering the zone $\mathrm{D}$, which is a rough part at the center of laser-modified area. The IPF displays a texture (i.e. preferred crystal orientation) in zone $D$, with orientation similar to the neighboring zone $\mathrm{C}$ when laser polarization is parallel to scanning direction. The similarity is so tight that it is not possible to distinguish zone $C$ and $D$ anymore from IPF.

Zone $C$ could be observed from $1.0 \mu \mathrm{J} /$ pulse, and the width of this zone increase with the increase of pulse energy (illustrated in Figure $55 \mathrm{c}$ and d). In addition, IPF displays that the crystal orientation of Zone $\mathrm{C}$ has similar orientation as the neighbouring Zone $\mathrm{D}$ when laser polarization is parallel to scanning direction (illustrated in Figure $55 \mathrm{c}$ and d).

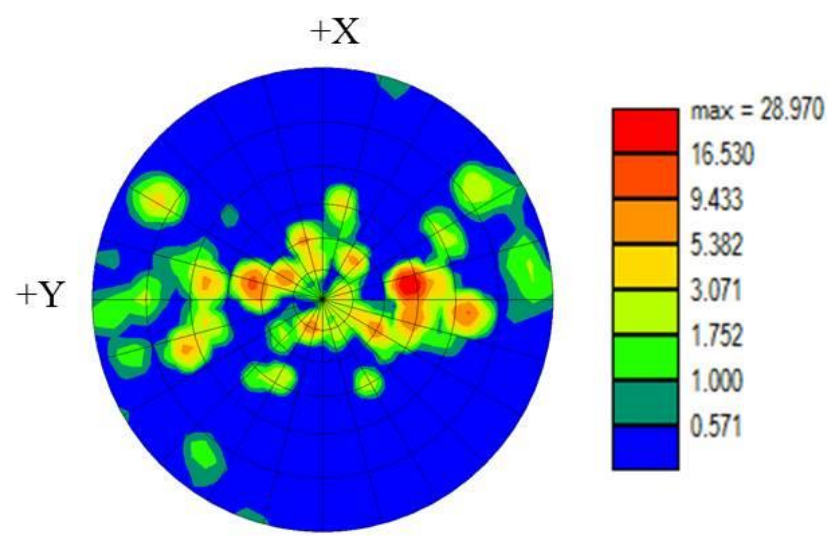

Figure 56 0001-Polar Figure. The color scale refers to multiple of the pole density for random distribution (quoted 1 and green). Other parameters: $33 \mathrm{Li}_{2} \mathrm{O}-33 \mathrm{Nb}_{2} \mathrm{O}_{5}-34 \mathrm{SiO}_{2}$ (mol\%), $1030 \mathrm{~nm}, 300 \mathrm{fs}, 300 \mathrm{kHz}, 0.7$ $\mu \mathrm{J} /$ pulse, $N A=0.6$, focus depth $350 \mu \mathrm{m}$ in glass, $5 \mu \mathrm{m} / \mathrm{s}$, writing laser polarization direction is parallel to $X$ direction and laser propagation direction is along $\mathrm{Z}$ direction. 
Based on the above observation, it can be concluded that

1) Crystalline lines can be patterned in LNS glass above certain energy threshold (e.g. 0.5 $\mu \mathrm{J} /$ pulse at $1030 \mathrm{~nm}, 300 \mathrm{kHz}, 300 \mathrm{fs}, \mathrm{NA}=0.6$, focal depth around $350 \mu \mathrm{m}$, and scanning speed $5 \mu \mathrm{m} / \mathrm{s})$;

2) The crystal size can be adjusted by varying pulse energy: the size of crystal increase of pulse energy when laser polarization direction is parallel to writing direction (0.5-2.2 $\mu \mathrm{J} /$ pulse at $1030 \mathrm{~nm}, 300 \mathrm{kHz}, 300 \mathrm{fs}, \mathrm{NA}=0.6$, focal depth around $350 \mu \mathrm{m}$, and scanning speed $5 \mu \mathrm{m} / \mathrm{s}$ ). The nano-sized crystal is obtained in regime 2;

3) There is a change of texture with the increase of pulse energy: the polar axis of crystal changes from a direction avoiding being along the writing laser polarization direction (regime 2) to a direction to be along laser polarization (regime 3).

It is worth noting that for regime 3, when laser polarization is perpendicular to scanning direction, as shown in Figure 57a-b, the zone C exhibits larger crystal size than zone D. Both of them have disordered crystal orientation.
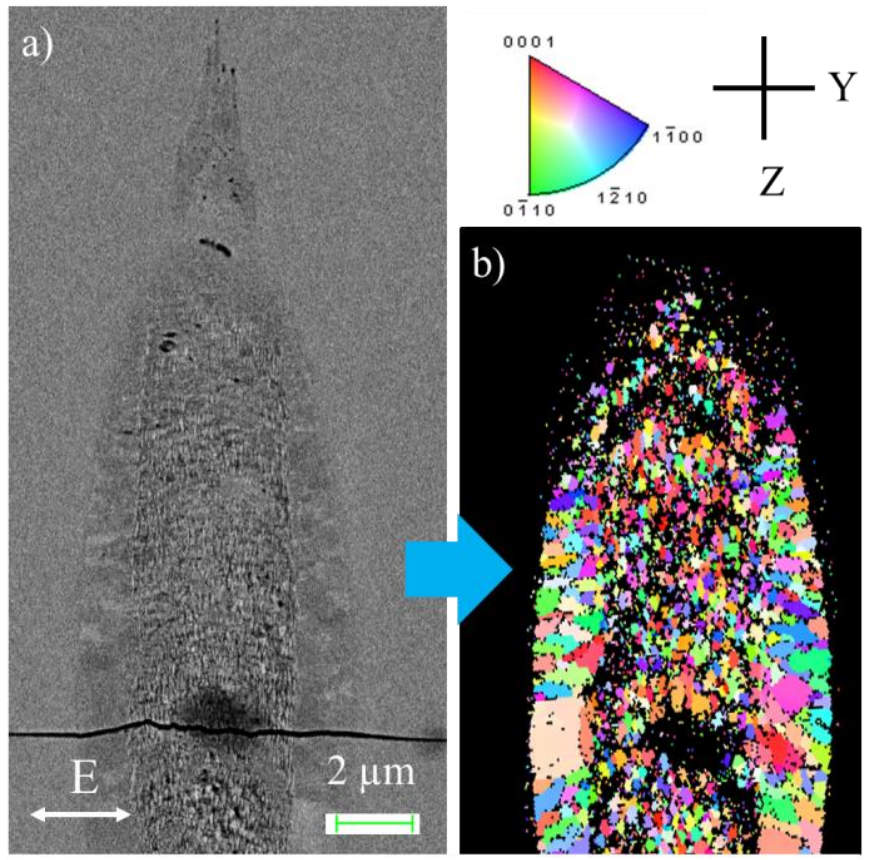

c)
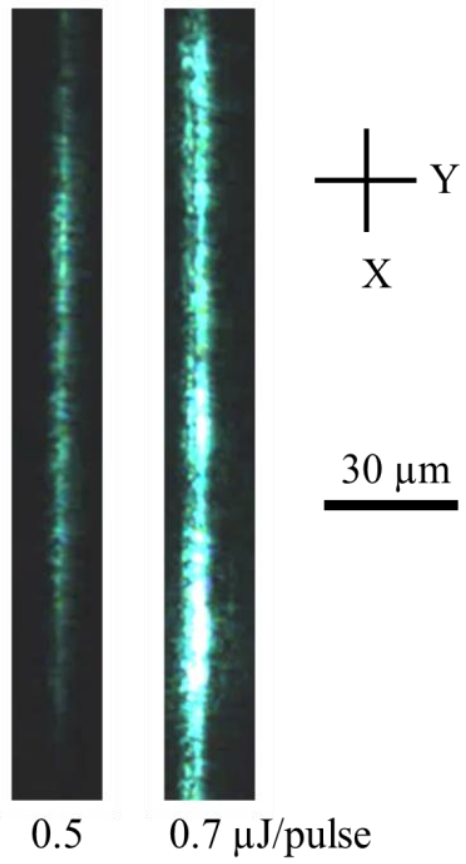

Figure 57 a) SEM image in regime 3 and the corresponding and b) IPF of laser track (1.8 $\mu /$ pulse). The color in IPF is based on $\mathrm{R} 3 \mathrm{c}$ space group, $\mathrm{LiNbO}_{3}$. Laser polarization is perpendicular to scanning direction. IPF is coding along scanning direction. $E$ indicates the laser polarization direction. c) SHG microscopy images of irradiated lines at different pulse energies. Other parameters: $33 \mathrm{Li}_{2} \mathrm{O}-33 \mathrm{Nb}_{2} \mathrm{O}_{5}-34 \mathrm{SiO}_{2}$ (mol\%), $1030 \mathrm{~nm}, 300 \mathrm{fs}, 300 \mathrm{kHz}$, $\mathrm{NA}=0.6$, focus depth $350 \mu \mathrm{m}$ in glass, $5 \mu \mathrm{m} / \mathrm{s}$, wring direction is along $\mathrm{X}$ direction 
Green light was observed in irradiated LNS glass from the pulse energy of $0.5 \mu \mathrm{J} /$ pulse (1030 nm, $300 \mathrm{kHz}, 300 \mathrm{fs}, \mathrm{NA}=0.6$, focal depth around $350 \mu \mathrm{m}$, and scanning speed $5 \mu \mathrm{m} / \mathrm{s}$ ). As the pulse energy (0.5-2.2 $\mu \mathrm{J} /$ pulse) increased, the green light became stronger. SHG microscopy images of irradiated lines were taken (Figure $57 \mathrm{c}$ ), confirming the presence of fs laser-induced nonlinear optical crystals according to pulse energy (> $0.5 \mu \mathrm{J} /$ pulse). Taking into account the laser wavelength $(1030 \mathrm{~nm})$, the green irradiation results from the precipitation of nonlinear optical crystals ( $\mathrm{LiNbO}_{3}$ ), which convert the wavelength from $1030 \mathrm{~nm}$ to $515 \mathrm{~nm}$ [109]. In other words, SHG was observed during fabrication for regime 2 and regime 3 but not for regime 1, which is in agreement with the observation from EBSD.

\subsubsection{Texture analysis}

Based on the above discussion, fs laser can induce textured crystals in LNS glass at certain pulse energy and laser polarization. To go a step further, in this section, one low pulse energy $(0.8$ $\mu \mathrm{J} /$ pulse) another high pulse energy $(1.7 \mu \mathrm{J} /$ pulse) with different laser polarization directions and writing directions (i.e. along $X$ or $Y$ directions, see details in Figure 36) was performed to have a clear picture of the fs laser-induced crystal orientation in LNS glass. Writing configuration considering laser scanning direction and incident laser polarization angle is defined as $(\theta, \phi)$, displayed for EBSD pictures as following.

\subsection{Crystallization information at low energy writing}

As presented in Figure 58 and Figure 60, structure modifications of teardrop-shaped laser traces were clearly observed in the laser-irradiated region at low pulse energy (i.e. $0.8 \mu \mathrm{J} /$ pulse). Herein, for each laser track, with the help of EBSD, we coded crystal orientation along X (left), Y (middle), and $Z$ direction (right), respectively (the $X, Y$, and $Z$ correspond to the directions as described in Figure 36).

Let's consider the writing configuration of $\left(180^{\circ}, 0^{\circ}\right)$ in Figure 58a. More red (the direction of the

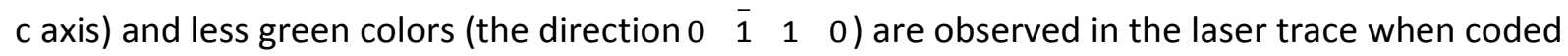
direction is changed from laser polarization (i.e. $X$ direction) to laser propagation direction ( $Z$ direction) or $\mathrm{Y}$ direction, indicating that, polar axis of $\mathrm{LiNbO}_{3}$ crystals are not oriented along the laser polarization direction (i.e. $X$ direction) but rather to $Y$ or $Z$ ones. On the contrary, $\begin{array}{llllll}0 & \overline{1} & 1 & 0\end{array}$ axis is more frequent in the $X$ direction. This observation can be made also using the Figure 59a. Such a figure displays the texture completely. We can see that most of the crystals have 


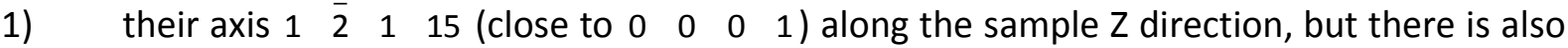

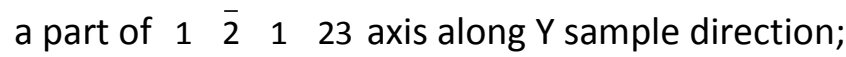

2) their axis $\begin{array}{lllllll}0 & \overline{3} & 3 & 2\end{array}$ (close to $\left.0 \begin{array}{llll}0 & \overline{1} & 1 & 0\end{array}\right)$ is along $X$ sample direction. We observe the same things if we change the orientation of writing (i.e. $\left(0^{\circ}, 0^{\circ}\right)$ writing configuration in Figure $58 b$ and Figure 59b).

a) $\left(180^{\circ}, 0^{\circ}\right)$

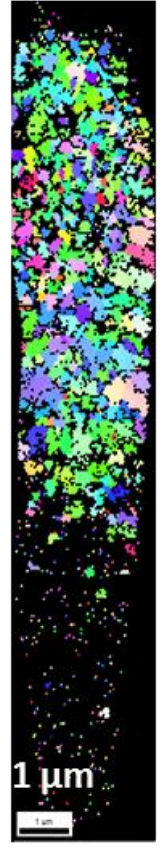

c) $\left(270^{\circ}, 90^{\circ}\right)$

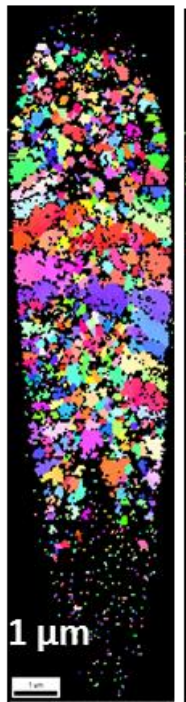

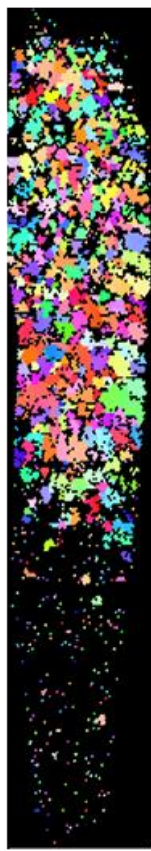
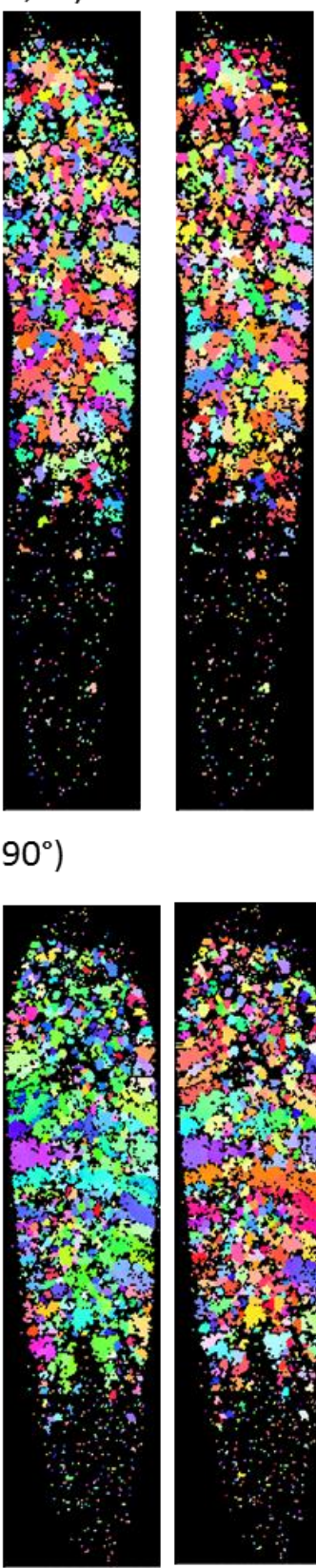

b) $\left(0^{\circ}, 0^{\circ}\right)$
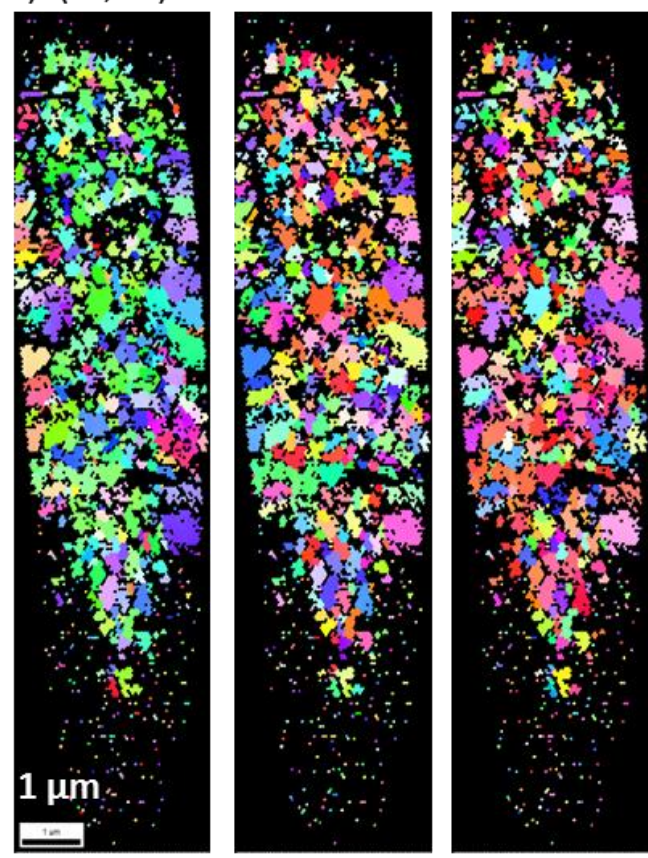

d) $\left(90^{\circ}, 90^{\circ}\right)$
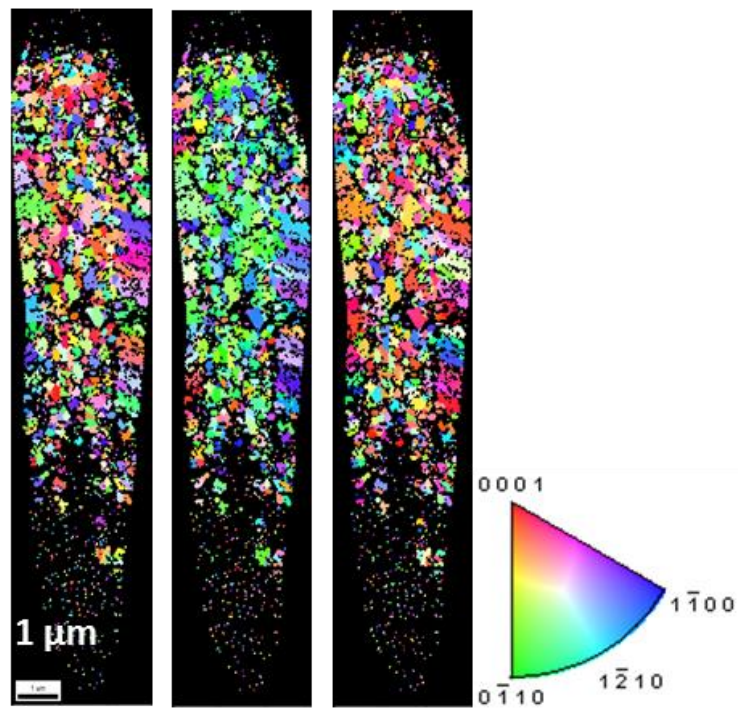

Figure 58 EBSD maps of cross section of laser tracks for parallel writing configuration, coding the crystal orientation along the directions parallel to $X$ (left), $Y$ (middle), and Z direction (right), respectively (see details in Figure 36). The color in EBSD map is based on $\mathrm{R} 3 \mathrm{c}$ space group, $\mathrm{LiNbO}_{3}$ (inset at the right bottom). Writing configuration considering laser scanning direction and incident laser polarization angle is defined as $(\theta, \phi)$, displayed for each picture. Other parameters: $33 \mathrm{Li}_{2} \mathrm{O}-33 \mathrm{Nb}_{2} \mathrm{O}_{5}-34 \mathrm{SiO}_{2}(\mathrm{~mol} \%), 1030 \mathrm{~nm}, 300 \mathrm{fs}, 500 \mathrm{kHz}, 5$ $\mu \mathrm{m} / \mathrm{s}, \mathrm{NA}=0.6$, focal depth $610 \mu \mathrm{m}$ in glass, $0.8 \mu \mathrm{J} / \mathrm{pulse}$. The laser came from the top. 
a) $\left(180^{\circ}, 0^{\circ}\right)$

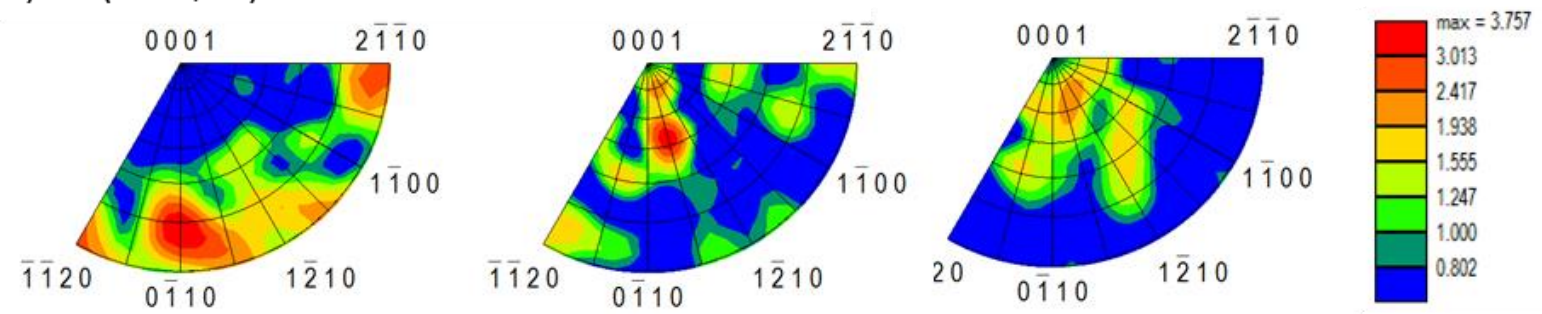

b) $\left(0^{\circ}, 0^{\circ}\right)$
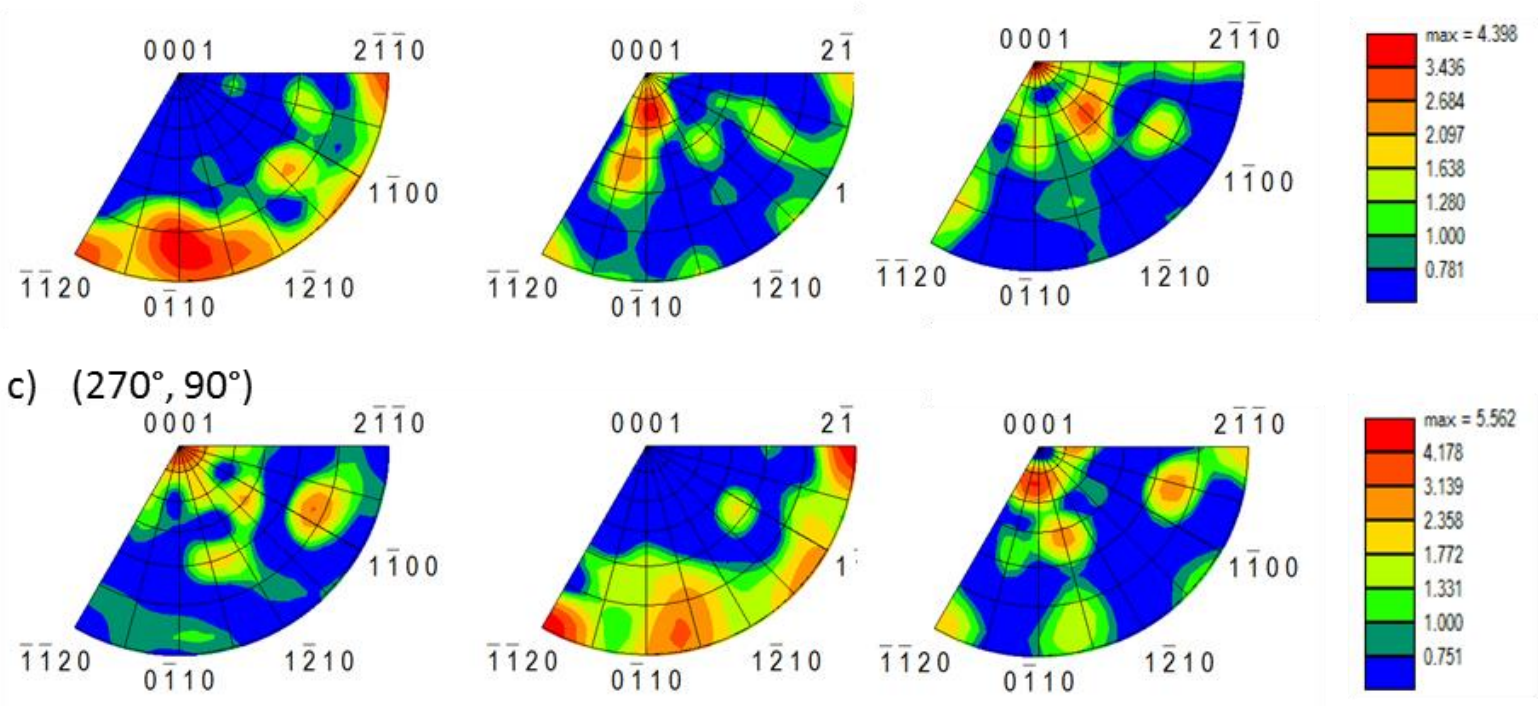

d) $\left(90^{\circ}, 90^{\circ}\right)$
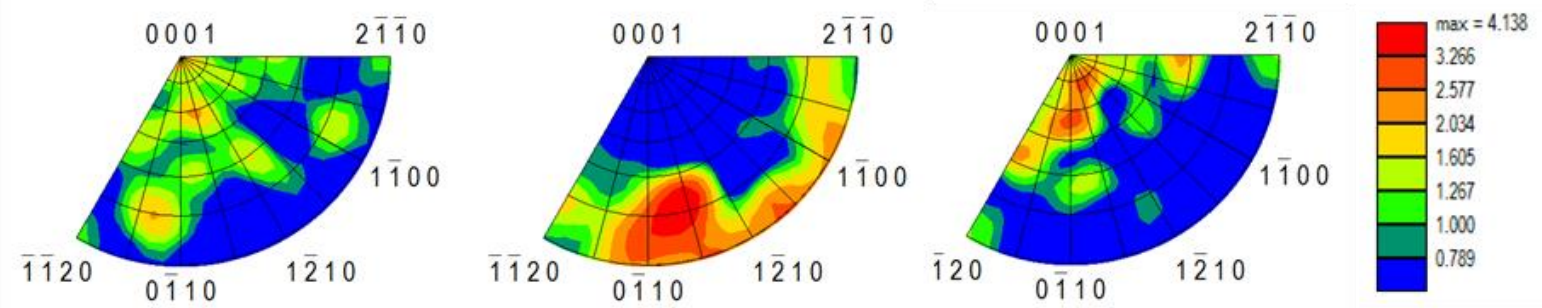

Figure 59 Polar Figures of cross section of laser tracks for parallel writing configuration, coding the crystal orientation along the directions parallel to $X$ (left), $Y$ (middle), and $Z$ direction (right), respectively (see details in Figure 36). Writing configuration considering laser scanning direction and incident laser polarization angle is defined as $(\theta, \phi)$, displayed for each picture. Other parameters: $33 \mathrm{Li}_{2} \mathrm{O}-33 \mathrm{Nb}_{2} \mathrm{O}_{5}-34 \mathrm{SiO}_{2}(\mathrm{~mol} \%), 1030 \mathrm{~nm}, 300$ fs, $500 \mathrm{kHz}, 5 \mu \mathrm{m} / \mathrm{s}, \mathrm{NA}=0.6$, focal depth $610 \mu \mathrm{m}$ in glass, $0.8 \mu \mathrm{J} / \mathrm{pulse}$

Now let us consider the other direction of writing (i.e. along $Y$ direction, defined in Figure 36) with the laser polarization parallel to scanning direction: writing configuration $\left(270^{\circ}, 90^{\circ}\right)$ (Figure $58 \mathrm{c}$ and Figure 59c). The conclusion is almost the same. Most of the crystals have

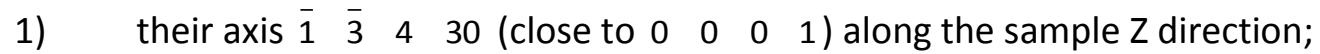

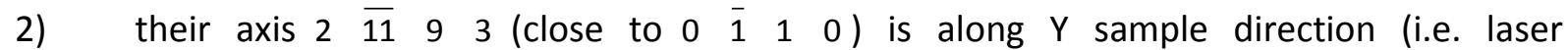
polarization direction). This does not change if we change the orientation of writing (i.e. writing configuration $\left(90^{\circ}, 90^{\circ}\right)$ in Figure 58d and Figure 59d). 
a) $\left(180^{\circ}, 90^{\circ}\right)$
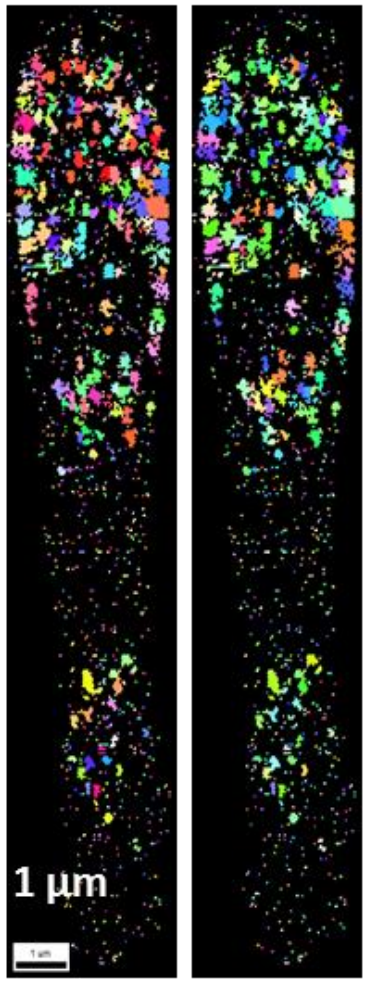

c) $\left(270^{\circ}, 0^{\circ}\right)$
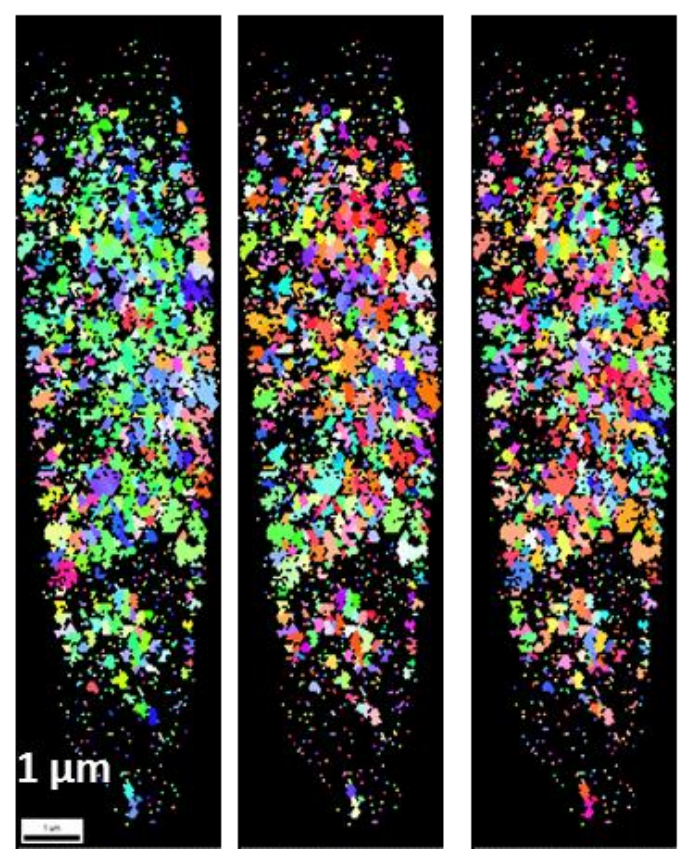

d) $\left(90^{\circ}, 0^{\circ}\right)$

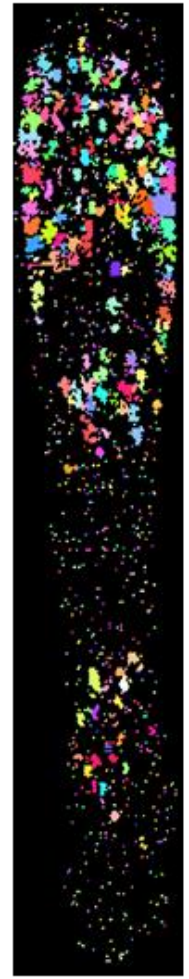

b) $\left(0^{\circ}, 90^{\circ}\right)$
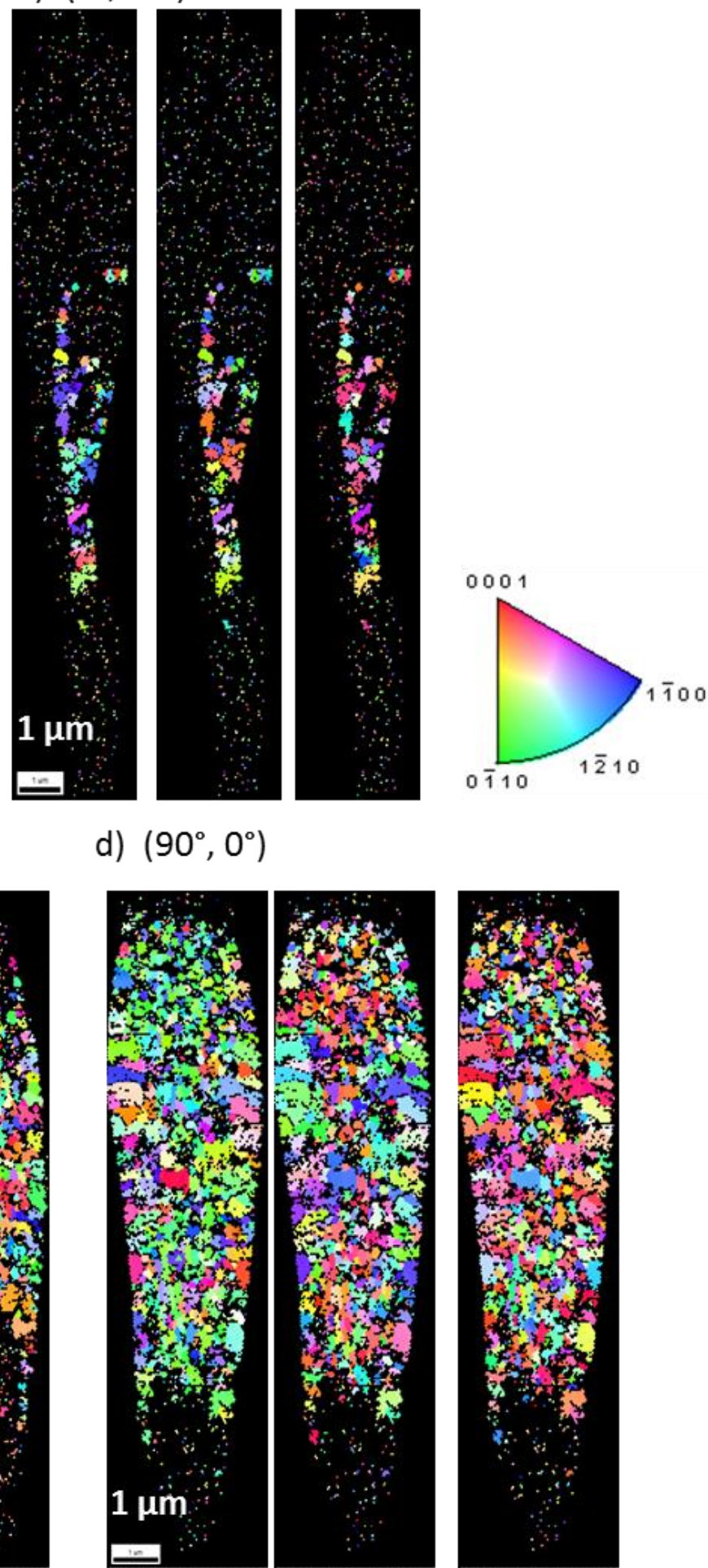

Figure 60 EBSD maps of cross section of laser tracks for perpendicular writing configuration, coding the crystal orientation along the directions parallel to $X$ (left), $Y$ (middle), and $Z$ direction (right), respectively (see details in Figure 36). The color in EBSD map is based on $\mathrm{R3}$ c space group, $\mathrm{LiNbO}_{3}$ (inset at the right top). Writing configuration considering laser scanning direction and incident laser polarization angle is defined as $(\theta, \phi)$, displayed for each picture. Other parameters: $33 \mathrm{Li}_{2} \mathrm{O}-33 \mathrm{Nb}_{2} \mathrm{O}_{5}-34 \mathrm{SiO}_{2}$ (mol\%), $1030 \mathrm{~nm}, 300 \mathrm{fs}, 500 \mathrm{kHz}, 5$ $\mu \mathrm{m} / \mathrm{s}, \mathrm{NA}=0.6$, focal depth $610 \mu \mathrm{m}$ in glass, $0.8 \mu \mathrm{J} / \mathrm{pulse}$. The laser came from the top. 
When the laser polarization is no more parallel to the direction of writing, there are some differences. Figure $60 \mathrm{a}$ and Figure 61 a (for writing configuration $\left(180^{\circ}, 90^{\circ}\right)$ ) show that polar axis are not along $Y$ direction (i.e. laser polarization direction). Figure $60 \mathrm{c}$ and Figure $61 \mathrm{c}$ (for $\left(270^{\circ}\right.$, $\left.0^{\circ}\right)$ writing configuration) or Figure $60 \mathrm{~d}$ and Figure $61 \mathrm{~d}$ (for $\left(90^{\circ}, 0^{\circ}\right)$ writing configuration) display the similar information. Texturation for the other sample direction is not so clear. Lastly, for reversing the orientation of writing seems not changing the previous observation. It is worth noting that $\left(0^{\circ}, 90^{\circ}\right)$ writing configuration does not lead to crystallization large enough for getting reliable observation (Figure 60b and Figure 61b).

So, we can conclude that axes close to 0 0 - 01 avoid being in the direction of laser polarization. This is clearer when writing laser polarization is parallel to the direction of writing, which is in agreement with the observation of regime 2, discussed in Section 4.1.1.1.

It is worth noting that the interaction areas were different, varying with writing configuration. The statistics of widths and lengths of corresponding laser tracks were shown in Table 10. When writing is along $X$ and laser polarization is parallel to writing direction, $\left(0^{\circ}, 0^{\circ}\right)$ configurations, the interaction area are larger than in the case of $\left(0^{\circ}, 90^{\circ}\right)$ configurations. When writing is along $Y$, we observe a larger interaction volume for parallel configurations, $\left(90^{\circ}, 90^{\circ}\right)$ or $\left(270^{\circ}, 90^{\circ}\right)$, than for perpendicular ones $\left(90^{\circ}, 0^{\circ}\right)$ or $\left(270^{\circ}, 0^{\circ}\right)$, respectively.

Interestingly, asymmetric orientational writing effects were also observed, especially when the laser linear polarization direction is parallel to writing direction. For $\mathrm{Xx}$ writing configuration, $\left(0^{\circ}\right.$, $\left.0^{\circ}\right)$ exhibits more crystals with polar axis closed to $Z$ direction than $\left(180^{\circ}, 0^{\circ}\right)$ configuration and $\left(90^{\circ}, 90^{\circ}\right)$ owns more crystals with polar axis closed to $Z$ direction than $\left(270^{\circ}, 90^{\circ}\right)$ configuration, indicating that there is an angle of the orientation of polar axis between different orientational writings, which proves that it is a parameter for controlling the orientation of crystals for the further fs laser fabrication.

Table 10 Statistics of the widths and the lengths of laser tracks for the different writing configurations at low pulse energy

\begin{tabular}{ccccccccc}
\hline $\begin{array}{c}\text { Writing } \\
\text { configuration }\end{array}$ & $\left(180^{\circ}, 0^{\circ}\right)$ & $\left(0^{\circ}, 0^{\circ}\right)$ & $\left(180^{\circ}, 90^{\circ}\right)$ & $\left(0^{\circ}, 90^{\circ}\right)$ & $\left(270^{\circ}, 90^{\circ}\right)$ & $\left(90^{\circ}, 90^{\circ}\right)$ & $\left(270^{\circ}, 0^{\circ}\right)$ & $\left(90^{\circ}, 0^{\circ}\right)$ \\
\hline Width/ $\mu \mathrm{m}$ & 2.6 & 3.9 & 3.2 & 2.1 & 3.7 & 3.5 & 3.5 & 3.4 \\
Length/ $\mu \mathrm{m}$ & 16.1 & 13.5 & 17.5 & 8.7 & 14.9 & 16.8 & 13.2 & 13.3 \\
\hline
\end{tabular}

Writing configuration considering laser scanning direction and incident laser polarization angle is defined as $(\theta$, $\phi)$. Other parameters: $33 \mathrm{Li}_{2} \mathrm{O}-33 \mathrm{Nb}_{2} \mathrm{O}_{5}-34 \mathrm{SiO}_{2}$ (mol\%), $1030 \mathrm{~nm}, 300 \mathrm{fs}, 500 \mathrm{kHz}, 5 \mu \mathrm{m} / \mathrm{s}, \mathrm{NA}=0.6$, focal depth $610 \mu \mathrm{m}$ in glass, $0.8 \mu \mathrm{J} /$ pulse. 
a)
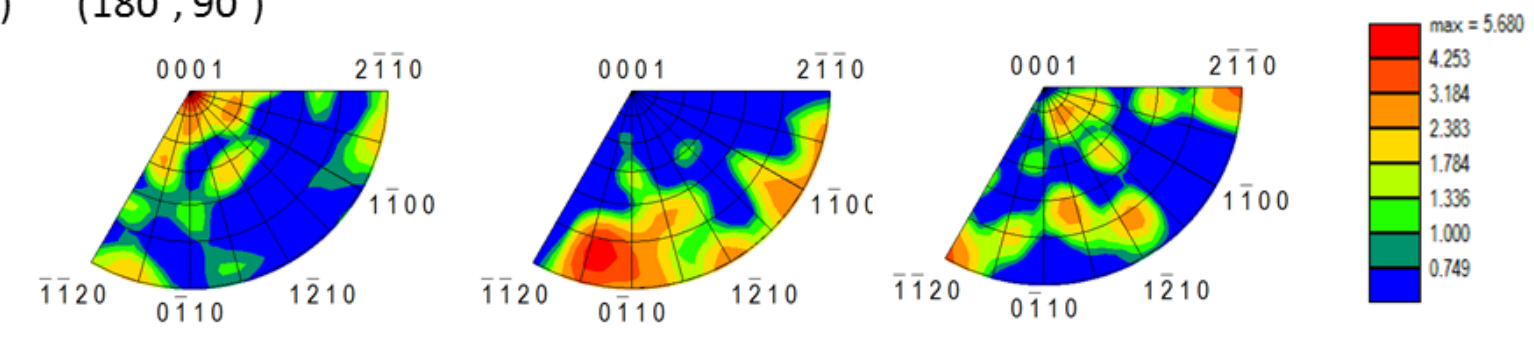

b) $\left(0^{\circ}, 90^{\circ}\right)$
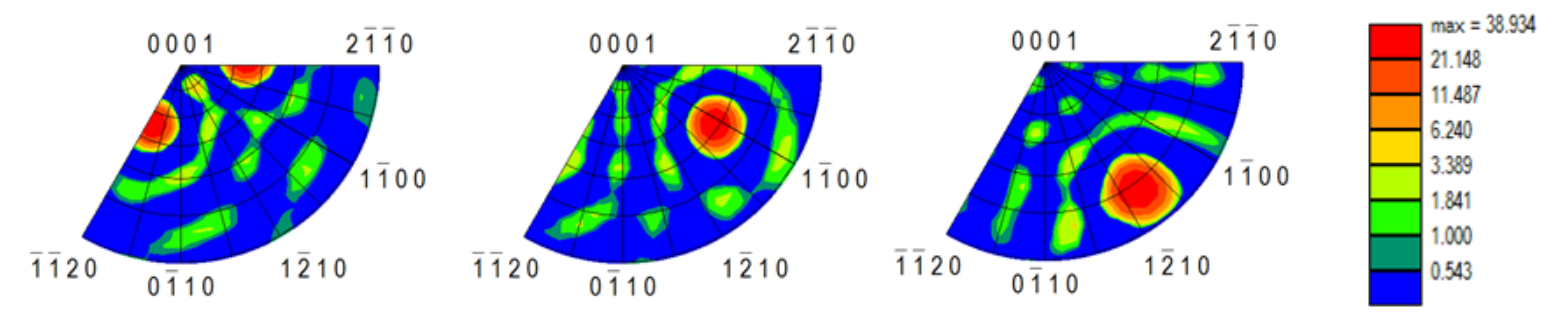

c) $\left(270^{\circ}, 0^{\circ}\right)$
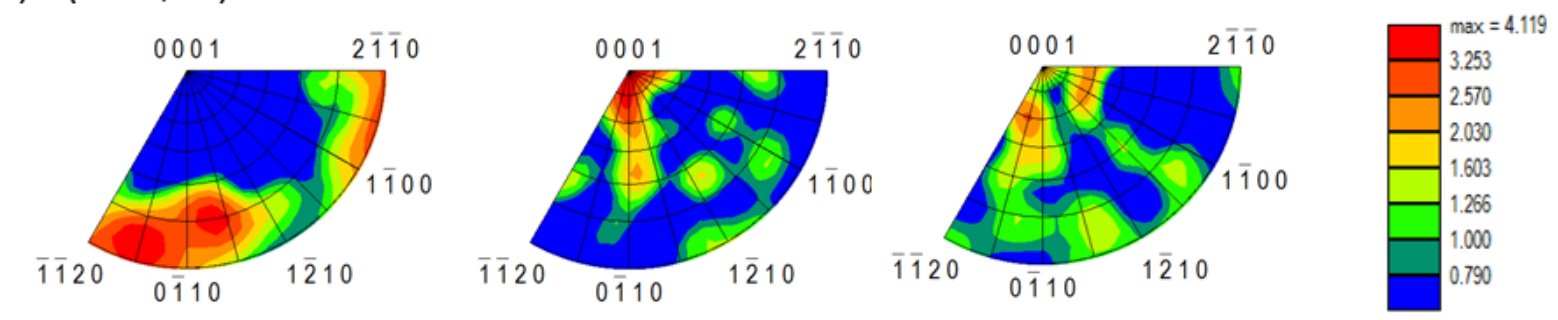

d) $\left(90^{\circ}, 0^{\circ}\right)$
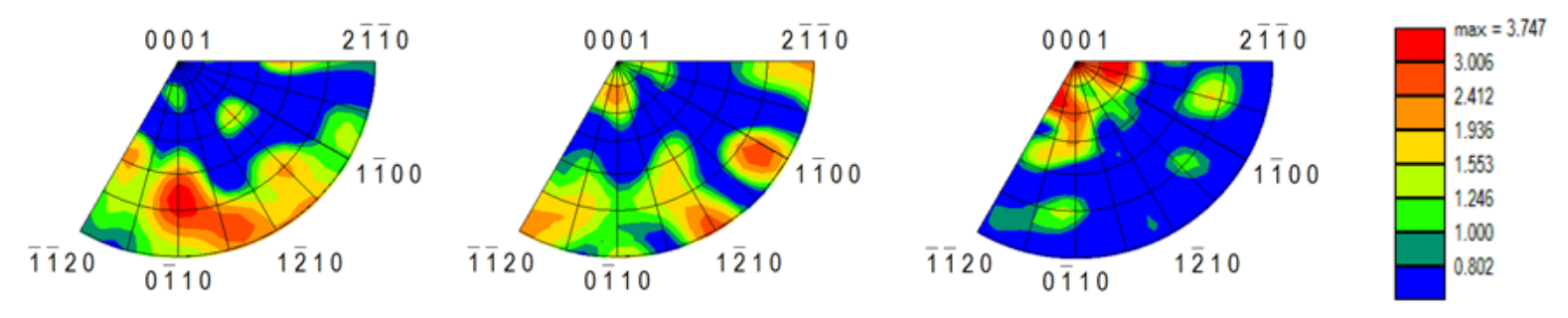

Figure 61 Polar Figures of cross section of laser tracks for perpendicular writing configuration, coding the crystal orientation along the directions parallel to $X$ (left), $Y$ (middle), and $Z$ direction (right), respectively (see details in Figure 36). Writing configuration considering laser scanning direction and incident laser polarization angle is defined as $(\theta, \phi)$, displayed for each picture. Other parameters: $33 \mathrm{Li}_{2} \mathrm{O}-33 \mathrm{Nb}_{2} \mathrm{O}_{5}-34 \mathrm{SiO}_{2}\left(\mathrm{~mol}^{2}\right), 1030$ $\mathrm{nm}, 300 \mathrm{fs}, 500 \mathrm{kHz}, 5 \mu \mathrm{m} / \mathrm{s}, \mathrm{NA}=0.6$, focal depth $610 \mu \mathrm{m}$ in glass, $0.8 \mu \mathrm{J} /$ pulse

\subsection{Crystallization information at high energy writing}

To go a step further, another pulse energy (1.7 $\mu \mathrm{J} /$ pulse) was used to investigate the laser polarization dependence of spatial orientation of $\mathrm{LiNbO}_{3}$ crystals. The orientation of crystals was gradually destroyed, yet local micro-scale orientated crystals are observed (marked with red circle in Figure 62), more complex structures, including core-shell structure in the head or along the laser trace body (marked with yellow circles in Figure 62 and Figure 63), secondary focus (marked with green circle in Figure 62 and Figure 63), are observed. 


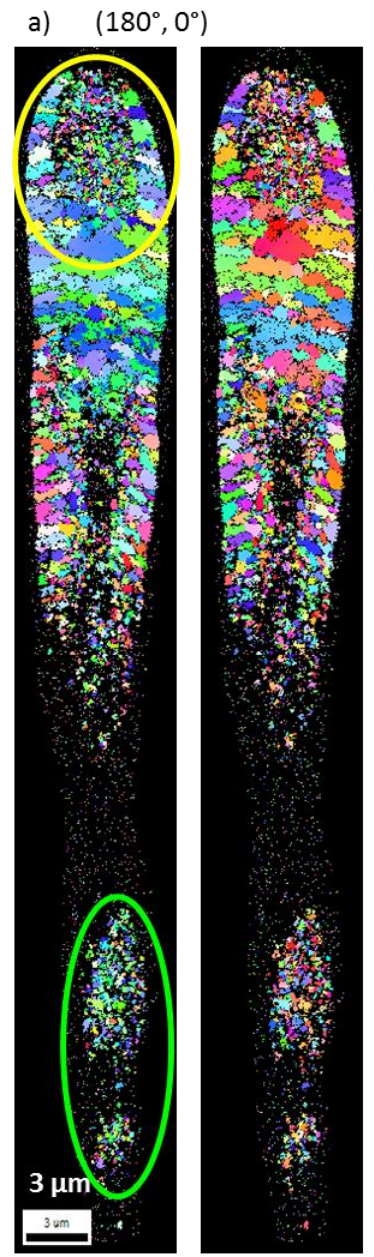

b) $\left(0^{\circ}, 0^{\circ}\right)$

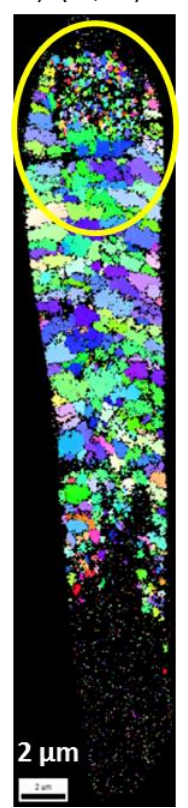

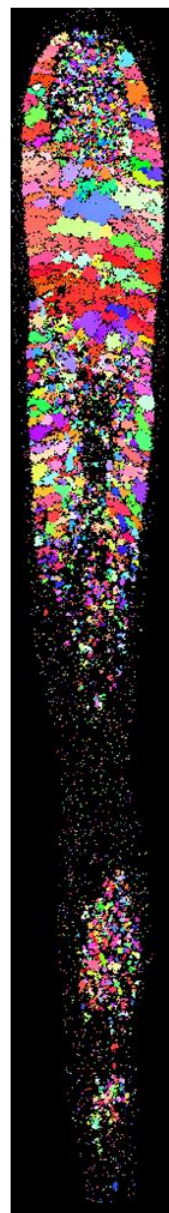

c) $\left(270^{\circ}, 90^{\circ}\right)$
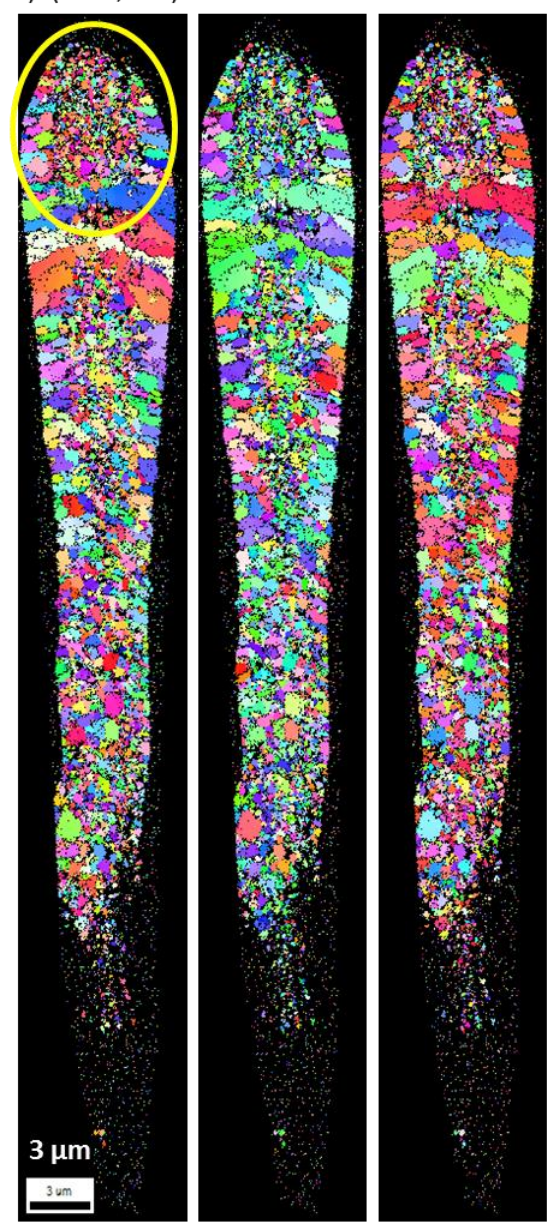

d) $\left(90^{\circ}, 90^{\circ}\right)$
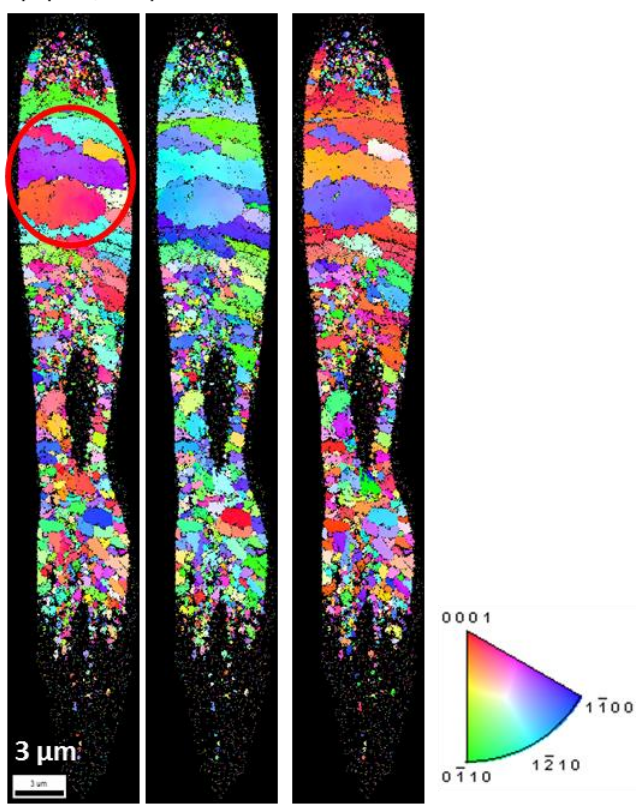

Figure 62 EBSD maps of cross section of laser tracks for parallel writing configuration, coding the crystal orientation along the directions parallel to $X$ (left), $Y$ (middle), and $Z$ direction (right), respectively (see details in Figure 36). The color in EBSD map is based on $\mathrm{R3c}$ space group, $\mathrm{LiNbO}_{3}$ (inset at the right bottom). Writing configuration considering laser scanning direction and incident laser polarization angle is defined as $(\theta, \phi)$, displayed for each picture. Other parameters: $33 \mathrm{Li}_{2} \mathrm{O}-33 \mathrm{Nb}_{2} \mathrm{O}_{5}-34 \mathrm{SiO}_{2}(\mathrm{~mol} \%), 1030 \mathrm{~nm}, 300 \mathrm{fs}, 500 \mathrm{kHz}, 5$ $\mu \mathrm{m} / \mathrm{s}, \mathrm{NA}=0.6$, focal depth $610 \mu \mathrm{m}$ in glass, $1.7 \mu \mathrm{J} /$ pulse. The laser came from the top. 
For a further comment, polar figure maps (Figure 64 and Figure 65) were employed to study the texture of $\mathrm{LiNbO}_{3}$ crystals as mentioned above. Comparing with low energy (i.e. $0.8 \mu \mathrm{J} / \mathrm{pulse}$ ), when increased pulse energy, the orientation of crystals are destroyed. When laser polarization are parallel to writing direction (Xx or Yy writing configuration), there is still a texture of polar axis, avoid, $0 \quad \overline{1} 10$ axis intend to be oriented along laser polarization direction (shown in Figure 64).

The ability of controlling the orientation of polar axis was more achievable when laser linear polarization direction is parallel rather than perpendicular to writing direction. Different distributions of polar axis of crystals varying with orientational writing are also obtained when the laser polarization direction is parallel to writing direction, which is consistent with the low pulse energy results.

Meanwhile, at high pulse energy, the differences of the interaction area between writing configurations are more observed, especially for the orientational writing (as show in Table 11). To be specific, the interaction area of $\left(0^{\circ}, 0^{\circ}\right)$ configuration is larger than that of $\left(0^{\circ}, 90^{\circ}\right)$ one, and $\left(90^{\circ}, 90^{\circ}\right)$ configuration is larger than that of $\left(90^{\circ}, 0^{\circ}\right)$ one, which is similar to the one observed at low pulse energy.

From these results discussed above, it is demonstrated that laser polarization plays an important role in the orientation of polar axis of crystals, at low pulse energy, polar axis of crystals have local texture that avoids orientation along laser polarization direction. By changing the parameters of laser polarization, writing direction, and pulse energy the orientation of crystals induced in glass-matrix could be controlled.

Table 11 Statistics of the widths and the lengths of laser tracks for the different writing configurations at high pulse energy

\begin{tabular}{ccccccccc}
\hline $\begin{array}{c}\text { Writing } \\
\text { configuration }\end{array}$ & $\left(180^{\circ}, 0^{\circ}\right)$ & $\left(0^{\circ}, 0^{\circ}\right)$ & $\left(180^{\circ}, 90^{\circ}\right)$ & $\left(0^{\circ}, 90^{\circ}\right)$ & $\left(270^{\circ}, 90^{\circ}\right)$ & $\left(90^{\circ}, 90^{\circ}\right)$ & $\left(270^{\circ}, 0^{\circ}\right)$ & $\left(90^{\circ}, 0^{\circ}\right)$ \\
\hline Width/ $\mu \mathrm{m}$ & 7.2 & 6.1 & 6 & 6.1 & 7.7 & 7.2 & 5.7 & 5.7 \\
Length $/ \mu \mathrm{m}$ & 57.6 & 32.3 & 59.3 & 51.3 & 55.7 & 47.3 & 40 & 45 \\
\hline
\end{tabular}

Writing configuration considering laser scanning direction and incident laser polarization angle is defined as $(\theta$, $\phi)$. Other parameters: $33 \mathrm{Li}_{2} \mathrm{O}-33 \mathrm{Nb}_{2} \mathrm{O}_{5}-34 \mathrm{SiO}_{2}$ (mol\%), $1030 \mathrm{~nm}, 300 \mathrm{fs}, 500 \mathrm{kHz}, 5 \mu \mathrm{m} / \mathrm{s}, \mathrm{NA}=0.6$, focal depth $610 \mu \mathrm{m}$ in glass, $1.7 \mu \mathrm{J} /$ pulse. 
a) $\left(180^{\circ}, 90^{\circ}\right)$

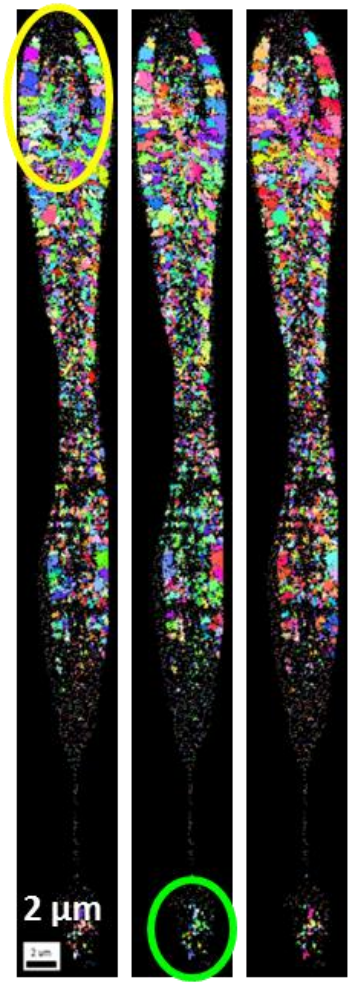

c) $\left(270^{\circ}, 0^{\circ}\right)$
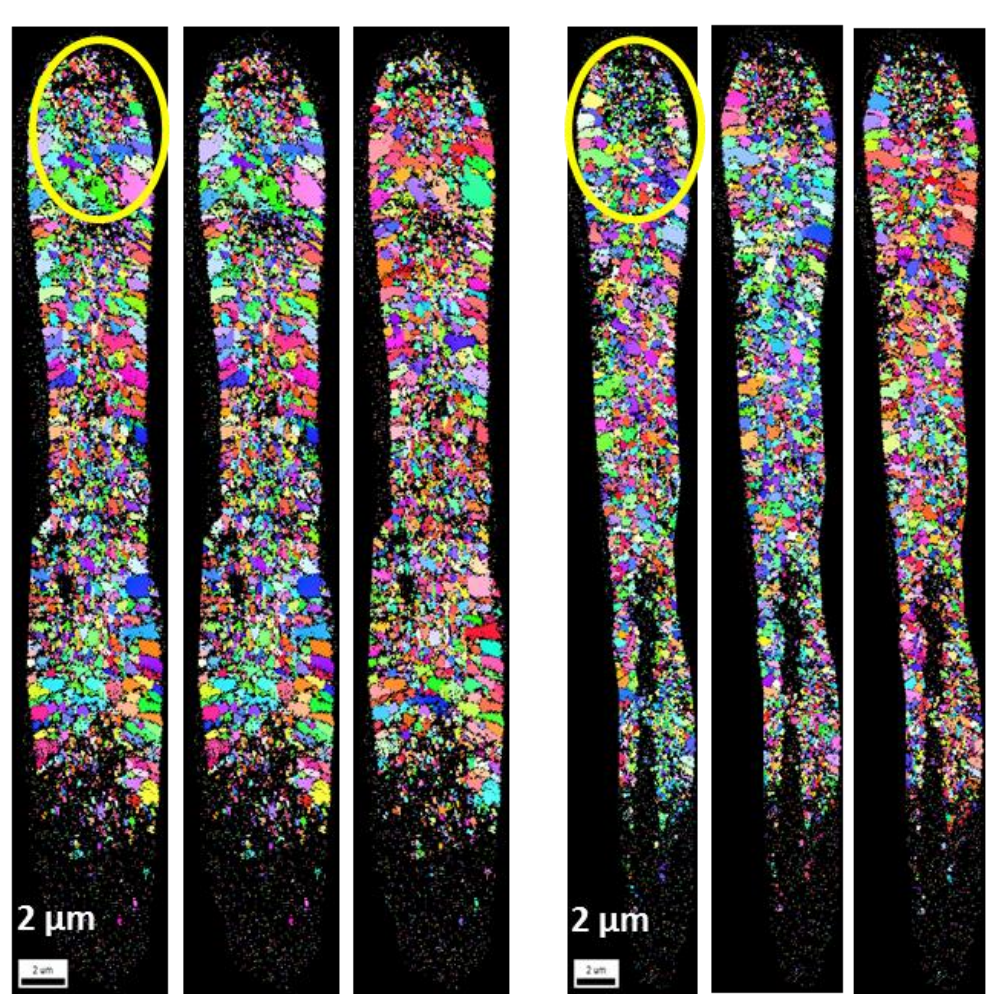

Figure 63 EBSD maps of cross section of laser tracks for perpendicular writing configuration, coding the crystal orientation along the directions parallel to $X$ (left), $Y$ (middle), and $Z$ direction (right), respectively (see details in Figure 36). The color in EBSD map is based on $\mathrm{R3c}$ space group, $\mathrm{LiNbO}_{3}$ (inset at the right top). Writing configuration considering laser scanning direction and incident laser polarization angle is defined as $(\theta, \phi)$, displayed for each picture. Other parameters: $33 \mathrm{Li}_{2} \mathrm{O}-33 \mathrm{Nb}_{2} \mathrm{O}_{5}-34 \mathrm{SiO}_{2}(\mathrm{~mol} \%), 1030 \mathrm{~nm}, 300 \mathrm{fs}, 500 \mathrm{kHz}, 5$ $\mu \mathrm{m} / \mathrm{s}, \mathrm{NA}=0.6$, focal depth $610 \mu \mathrm{m}, 1.7 \mu \mathrm{J} /$ pulse. The laser came from the top. 
a) $\left(180^{\circ}, 0^{\circ}\right)$
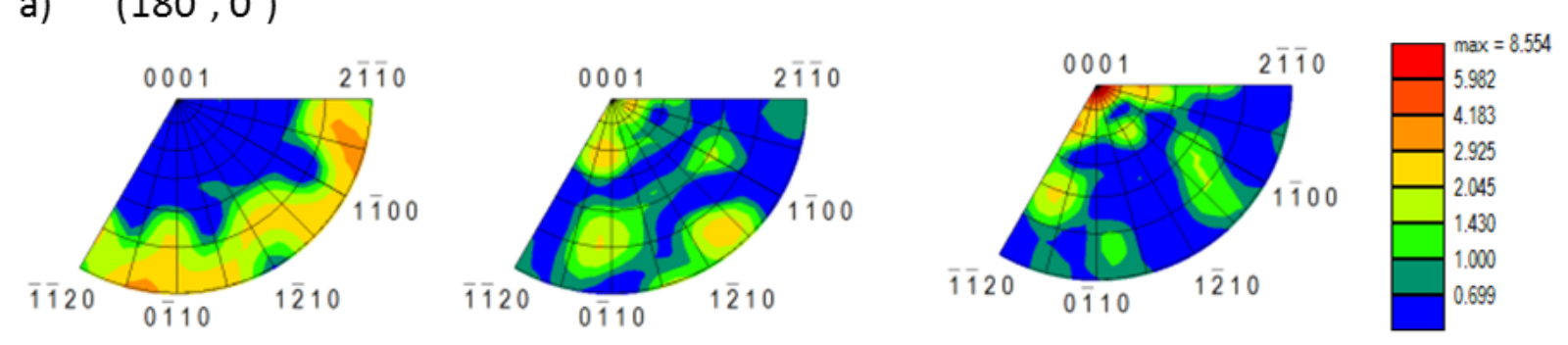

b) $\left(0^{\circ}, 0^{\circ}\right)$
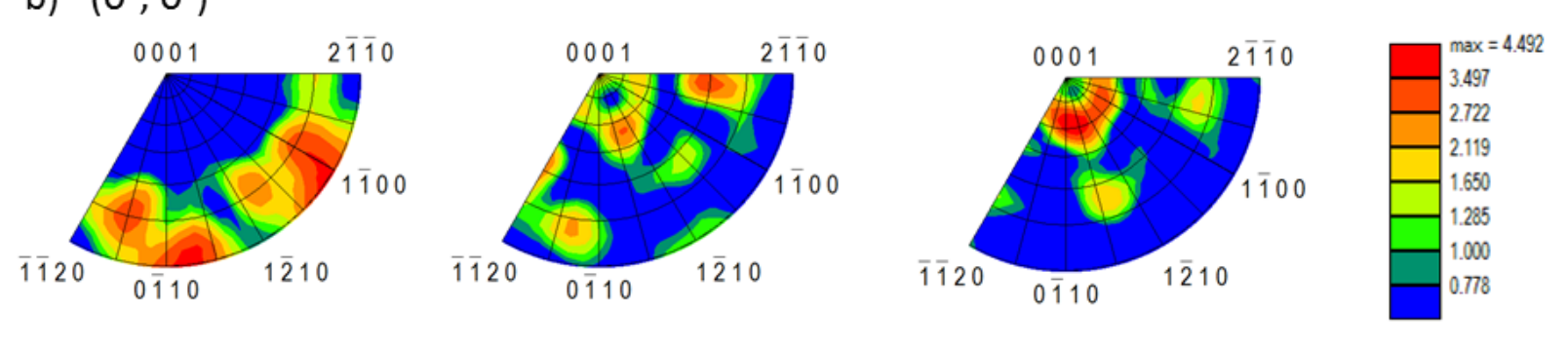

\section{c) $\left(270^{\circ}, 90^{\circ}\right)$}
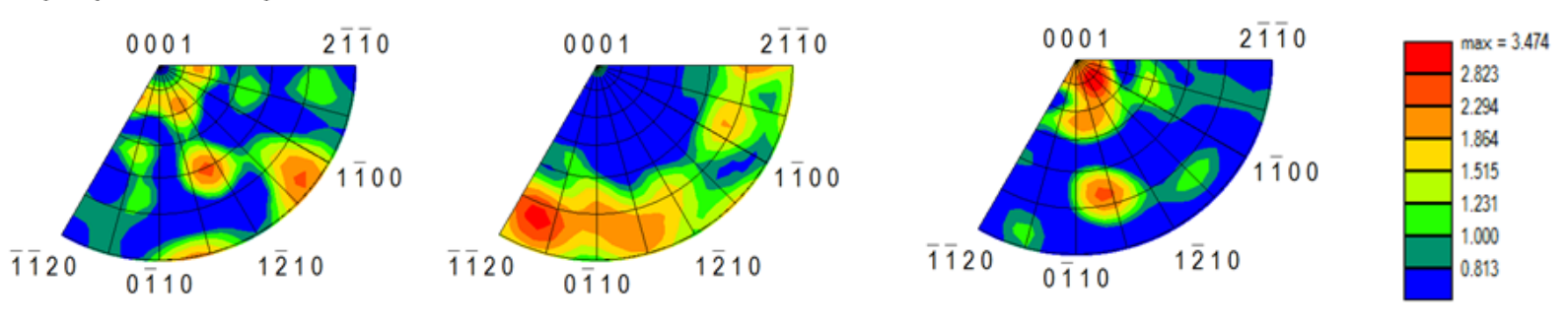

d) $\left(90^{\circ}, 90^{\circ}\right)$
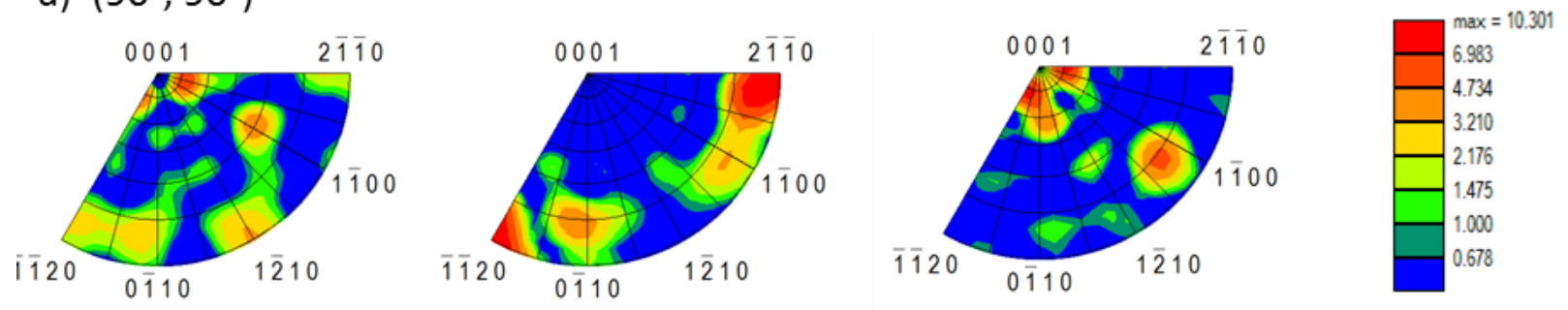

Figure 64 Polar Figures of cross section of laser tracks for parallel writing configuration, coding the crystal orientation along the directions parallel to $X$ (left), $Y$ (middle), and $Z$ direction (right), respectively (see details in Figure 36). Writing configuration considering laser scanning direction and incident laser polarization angle is defined as $(\theta, \phi)$, displayed for each picture. Other parameters: $33 \mathrm{Li}_{2} \mathrm{O}-33 \mathrm{Nb}_{2} \mathrm{O}_{5}-34 \mathrm{SiO}_{2}(\mathrm{~mol} \%), 1030 \mathrm{~nm}, 300$ fs, $500 \mathrm{kHz}, 5 \mu \mathrm{m} / \mathrm{s}, \mathrm{NA}=0.6$, focal depth $610 \mu \mathrm{m}$ in glass, $1.7 \mu \mathrm{J} /$ pulse. 
a)
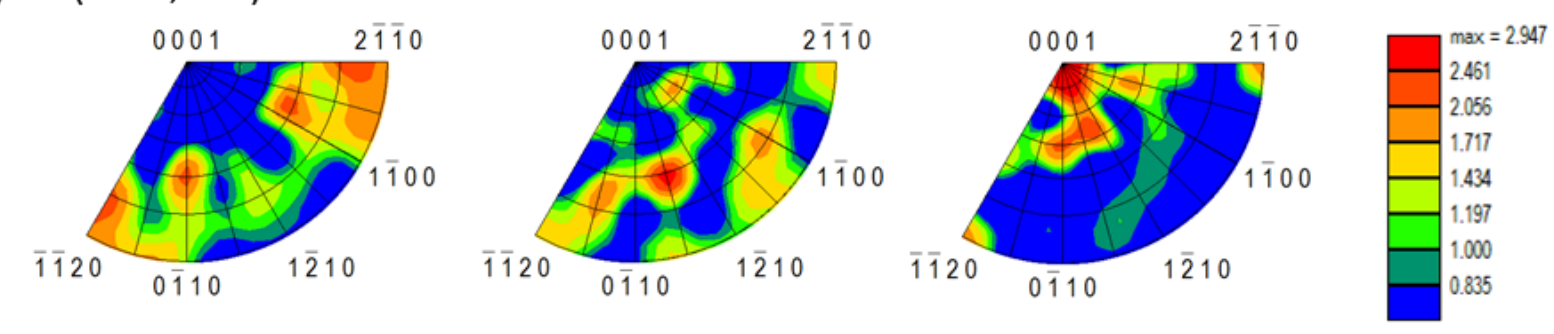

b) $\left(0^{\circ}, 90^{\circ}\right)$
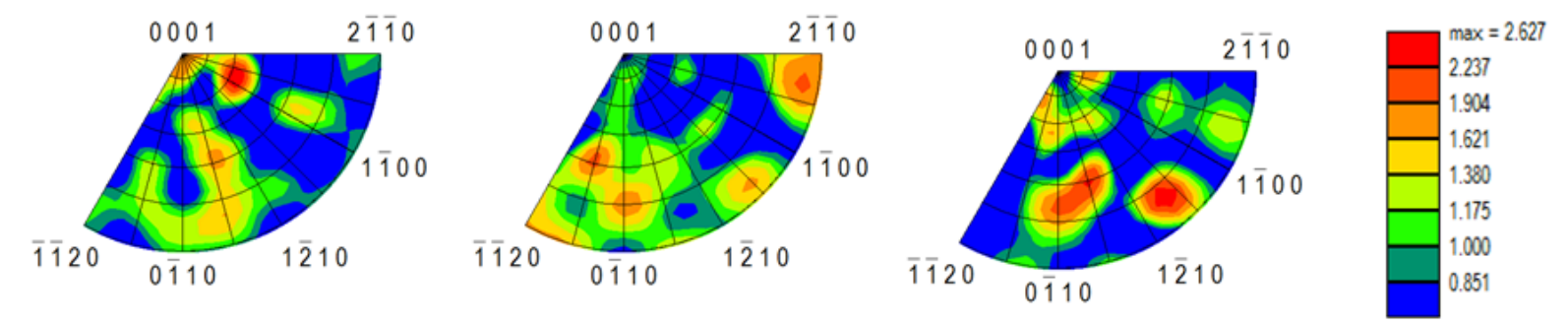

c) $\left(270^{\circ}, 0^{\circ}\right)$
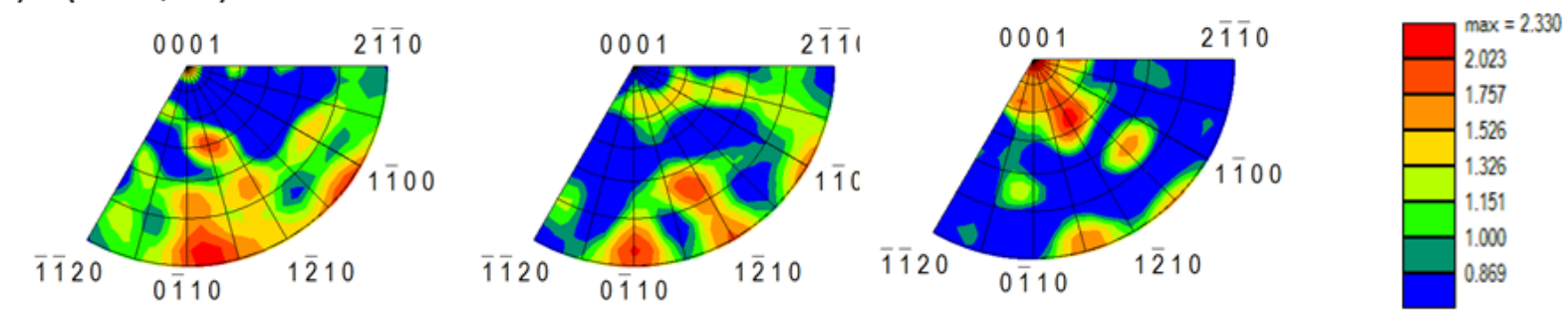

d) $\left(90^{\circ}, 0^{\circ}\right)$
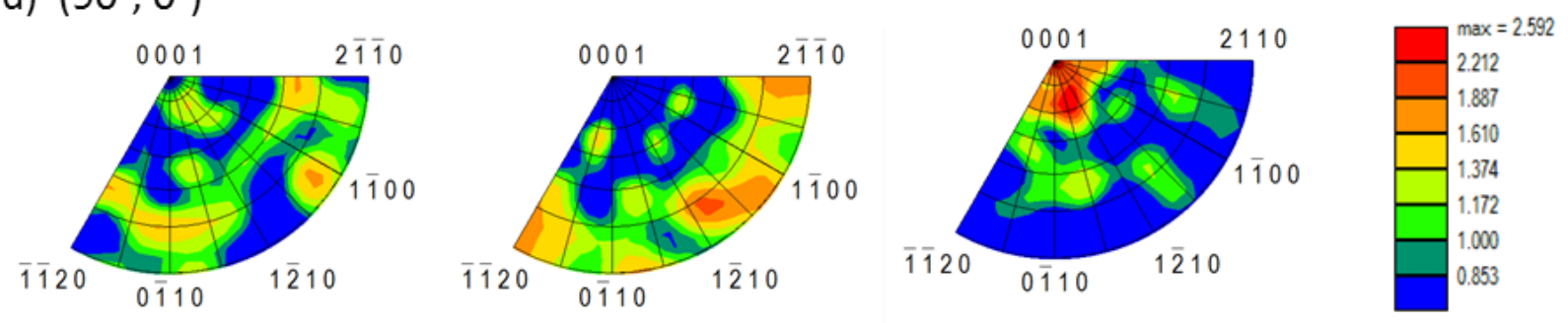

Figure 65 Polar Figures of cross section of laser tracks for perpendicular writing configuration, coding the crystal orientation along the directions parallel to $X$ (left), $Y$ (middle), and $Z$ direction (right), respectively (see details in Figure 36). Writing configuration considering laser scanning direction and incident laser polarization angle is defined as $(\theta, \phi)$, displayed for each picture. Other parameters: $33 \mathrm{Li}_{2} \mathrm{O}-33 \mathrm{Nb}_{2} \mathrm{O}_{5}-34 \mathrm{SiO}_{2}(\mathrm{~mol} \%), 1030$ $\mathrm{nm}, 300 \mathrm{fs}, 500 \mathrm{kHz}, 5 \mu \mathrm{m} / \mathrm{s}, \mathrm{NA}=0.6$, focal depth $610 \mu \mathrm{m}$ in glass, $1.7 \mu \mathrm{J} /$ pulse. 


\subsection{2. fs laser-controlled crystallization}

Based on the above analysis, it is possible to obtain nonlinear optical crystals in LNS glass at certain fs laser energies (i.e. the pulse energy range above the threshold to obtain crystalline line). In this section, extended investigation of fs laser-induced crystallization is presented according to the laser parameters such as laser repetition rate, to show how to control the crystallization in LNS glasses.

\subsubsection{Pulse energy effect at high repetition rate}

The energy threshold for fs laser-induced crystalline line is around $0.6 \mu \mathrm{J} /$ pulse at $1030 \mathrm{~nm}, 250$ $\mathrm{kHz}, 300 \mathrm{fs}, \mathrm{NA}=0.6$, focal depth around $300 \mu \mathrm{m}$ in air, and $5 \mu \mathrm{m} / \mathrm{s}$ in $32.5 \mathrm{Li}_{2} \mathrm{O}-27.5 \mathrm{Nb}_{2} \mathrm{O}_{5}-40 \mathrm{SiO}_{2}$ (mol\%) glass.

Irradiated lines were cut along the direction perpendicular to writing direction. The morphology of the cross section was determined by scanning electron microscopy (SEM, illustrated in Figure $66 a-c)$.

a)

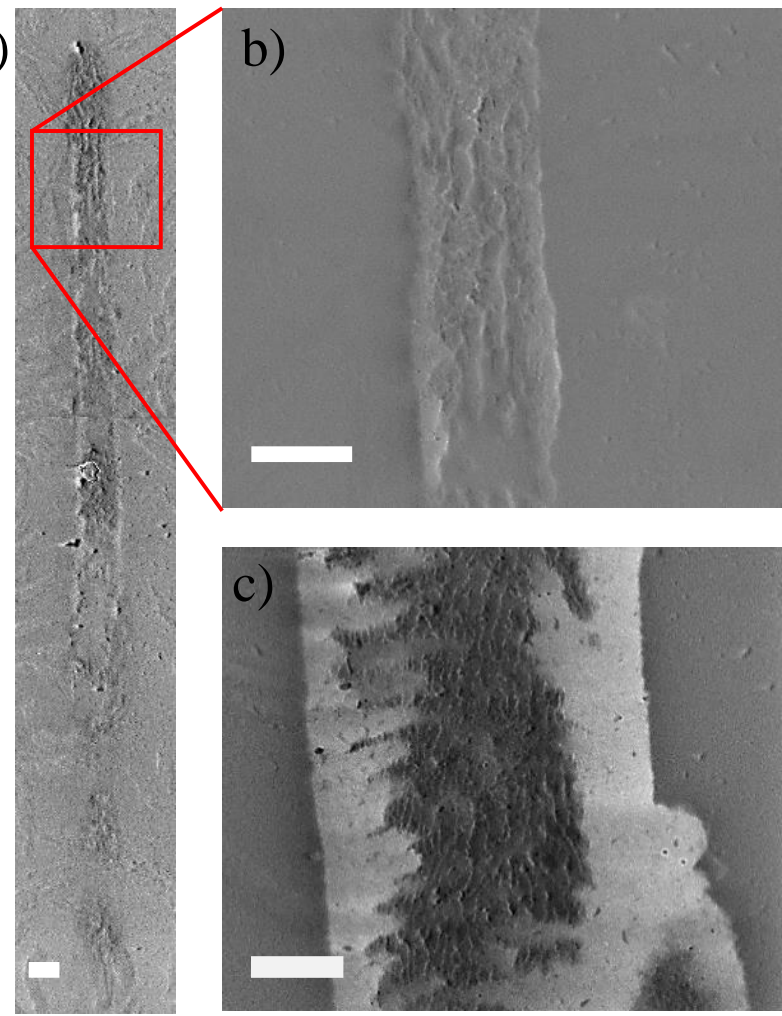

d)

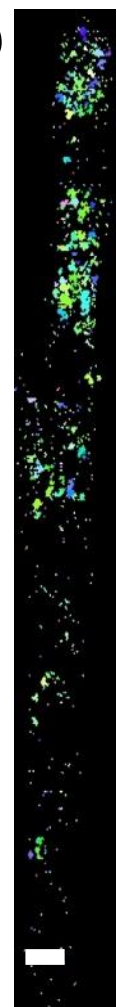

e)

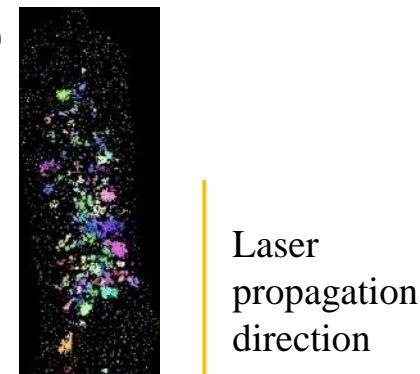

Figure 66 Structure of the laser trace by scanning electron microscopy: a) SEM image for $0.8 \mu \mathrm{J} /$ pulse, b) the magnification of the part framed in red in a). c) A similar part of the laser track but for $1.4 \mu \mathrm{J} / \mathrm{pulse}$; d) the corresponding IPFs coding crystal direction along the writing laser polarization direction for $0.8 \mu \mathrm{J} / \mathrm{pulse}$; e) similar information for $1.4 \mu \mathrm{J} /$ pulse. The color in IPF maps (inset at the right bottom) is based on R3c space group, $\mathrm{LiNbO}_{3}$. All scale bars are $1 \mu \mathrm{m}$. Other parameters: $32.5 \mathrm{Li}_{2} \mathrm{O}-27.5 \mathrm{Nb}_{2} \mathrm{O}_{5}-40 \mathrm{SiO}_{2}(\mathrm{~mol} \%), 1030 \mathrm{~nm}, 300 \mathrm{fs}$, $250 \mathrm{kHz}, 5 \mu \mathrm{m} / \mathrm{s}$, focus depth $300 \mu \mathrm{m}$ in air, writing direction along $45^{\circ}$ and laser polarization direction parallel to $90^{\circ}$ 
At regime 2 (i.e. $0.8 \mu \mathrm{J} /$ pulse), a ginseng like shape laser track with a width of $1.4 \mu \mathrm{m}$ of and length of $30 \mu \mathrm{m}$ is obtained (Figure 66a). Figure 66b, the magnification of the previous figure, displays a rough structure with ribbon-like shape. With the increase of the pulse energy (1.4 $\mu \mathrm{J} /$ pulse, regime 3), as illustrated in Figure $66 \mathrm{c}$, the width of the laser trace increased to about 4 $\mu \mathrm{m}$, another part appearing in white in Figure $66 \mathrm{c}$ is obtained around a rough structure. This is in agreement with the modifications at high repetition rate, varying with pulse energy observed in Section 4.1.1.1.

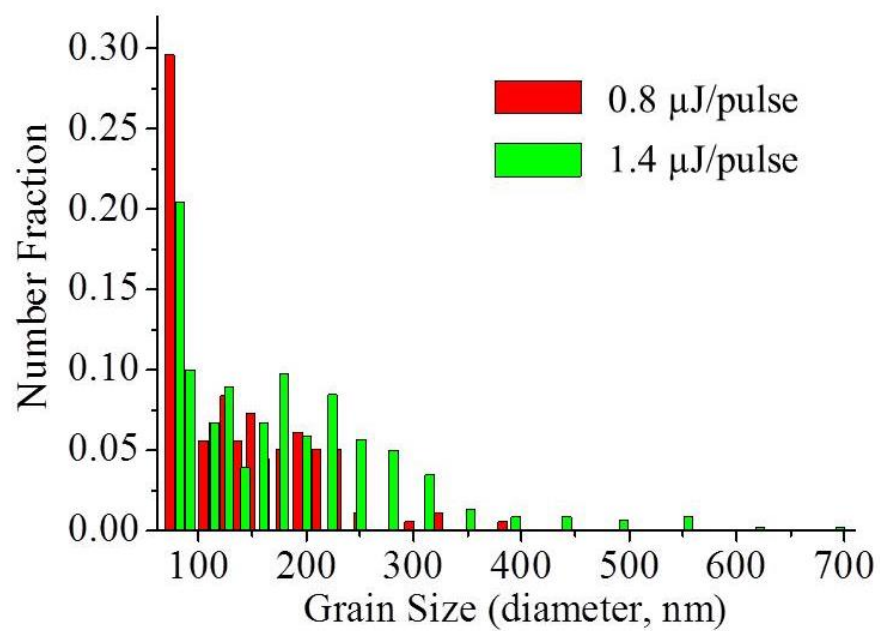

Figure 67 Apparent grain size (diameter) distribution of laser tracks varying pulse energy. Other parameters: $32.5 \mathrm{Li}_{2} \mathrm{O}-27.5 \mathrm{Nb}_{2} \mathrm{O}_{5}-40 \mathrm{SiO}_{2}(\mathrm{~mol} \%), 1030 \mathrm{~nm}, 300 \mathrm{fs}, 250 \mathrm{kHz}, 5 \mu \mathrm{m} / \mathrm{s}$, writing direction along $45^{\circ}$ and laser polarization direction parallel to $90^{\circ}$.

IPFs show that along laser propagation direction (Figure 66d and e), crystals showed higher density and larger grains in the head rather than in the tail. At $0.8 \mu \mathrm{J} /$ pulse, the IPF is completely green and blue, indicating that $\begin{array}{lllllllll}0 & \overline{1} & 0 & 0\end{array}$ and $1 \quad \overline{1} \quad 0 \quad 0$ axes are parallel to the writing laser

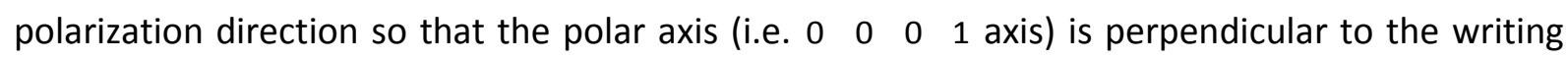
laser polarization direction. This is in agreement with the regime 2, defined in Section 4.1.1.1. Figure 66e shows that sub-micro sized crystals, with nano-crystals obtained in the core of laser track or in the tail and micro-sized crystals in the head part, especially outside of the core. Additionally, with the increase of pulse energy, the color of IPF changes from completely green to various color, indicating that other orientations occur. This is in agreement with the regime 3 , defined in Section 4.1.1.1.

As illustrated in Figure 67 (shown by red columns), at $0.8 \mu \mathrm{J} /$ pulse, grain sizes are ranging from 77 to $387 \mathrm{~nm}$, with an average grain size of $134 \mathrm{~nm}$, indicating nano-sized crystals have been produced for these conditions. The grain size increases with pulse energy (illustrated by green columns in Figure 67). 
To have a clear picture of the distribution of polar axis, 0001-Pole Figure (PF) map was plotted. As shown in Figure 68a, at $0.8 \mu \mathrm{J} /$ pulse (i.e. regime 2 ), the polar axis of the crystal $\left(\begin{array}{llll}0 & 0 & 0 & 1\end{array}\right)$, is distributed perpendicular to the laser polarization direction (i.e. $90^{\circ}$ ) like a fiber texture. Figure $68 \mathrm{~b}$ and $\mathrm{c}$ show two textures: one with the polar axis perpendicular to writing laser polarization direction (i.e. $90^{\circ}$ ) like the one for low energy and another one for $1.4 \mu \mathrm{J} /$ pulse and $1.8 \mu \mathrm{J} /$ pulse.

a)

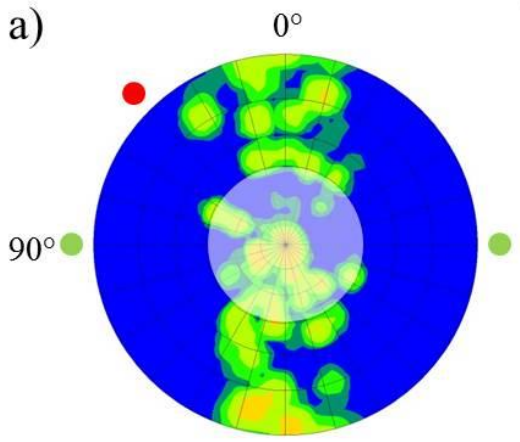

c)

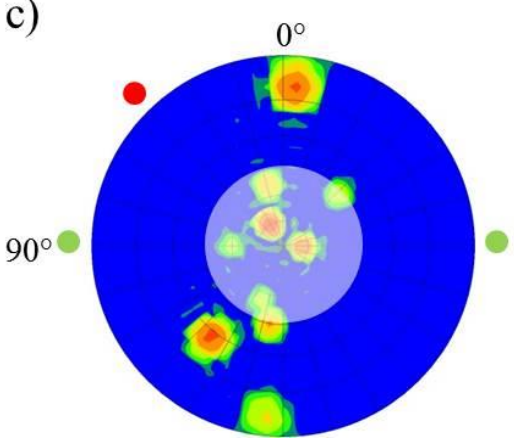

b)

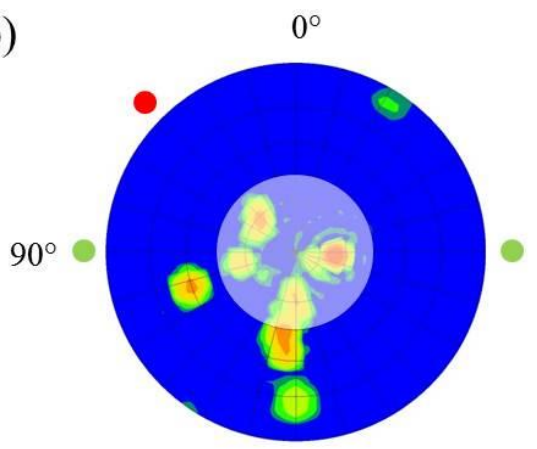

Figure 68 0001-Pole Figure of texture calculated from EBSD-scans i.e. the polar axis from EBSD scans: at pulse energy of a) 0.8, b) 1.4 , and c) $1.8 \mu \mathrm{J} /$ pulse. The milky disks at the centre of the pole figures indicate the part of the distribution that should not be taken into account for SHG interpretation with probe polarization in XY plane (will be used in Section 5.1.3.1). Green dots illustrate the writing laser polarization and red dots illustrate the writing direction.

\subsubsection{Writing laser polarization dependent nano-crystal orientation}

To confirm the crystal texture observed at regime 2 (i.e. $0.8 \mu \mathrm{J} /$ pulse), we fixed the pulse energy but using another writing configuration (i.e. different writing direction or writing laser polarization direction).

The scanning direction was along $45^{\circ}$ and the writing laser polarization angle was at $135^{\circ}$. A laser trace width of about $1.4 \mu \mathrm{m}$ and length of about $18 \mu \mathrm{m}$ was observed from the cross section of irradiated line (Figure 69a). The magnification image (the part marked with red rectangle in Figure 69a) illustrates the laser irradiated part appearing rougher than the glass matrix (Figure 69b). 
Spatial distribution information of crystals can be seen from IPF map (Figure 69c-e). Along laser propagation direction, crystals showed higher density and larger grains in the head rather than in the tail, which is consistent with the above observations.

Grain size distributions of induced crystals were determined (Figure 69f), ranging from 76 to 228 $\mathrm{nm}$, with an average grain size of $135 \mathrm{~nm}$, indicating that nano-crystals were achieved in the present glass matrix after fs laser irradiation under our experimental conditions.

The color in IPF indicates the crystal direction aligned with the coding sample direction. For example, the red in IPF means that polar asxis of crystal direction is along the coding sample direction. As can be seen from Figure 69c, no red grain appears in IPF map upon coding along incident laser polarization direction. But when changing coding direction to other directions, such as scanning orientation (Figure 69d) or laser propagation direction (Figure 69e), pink or red were observed in IPFs, indicating that polar axes of nano-crystals tend to be perpendicular to writing laser polarization direction.

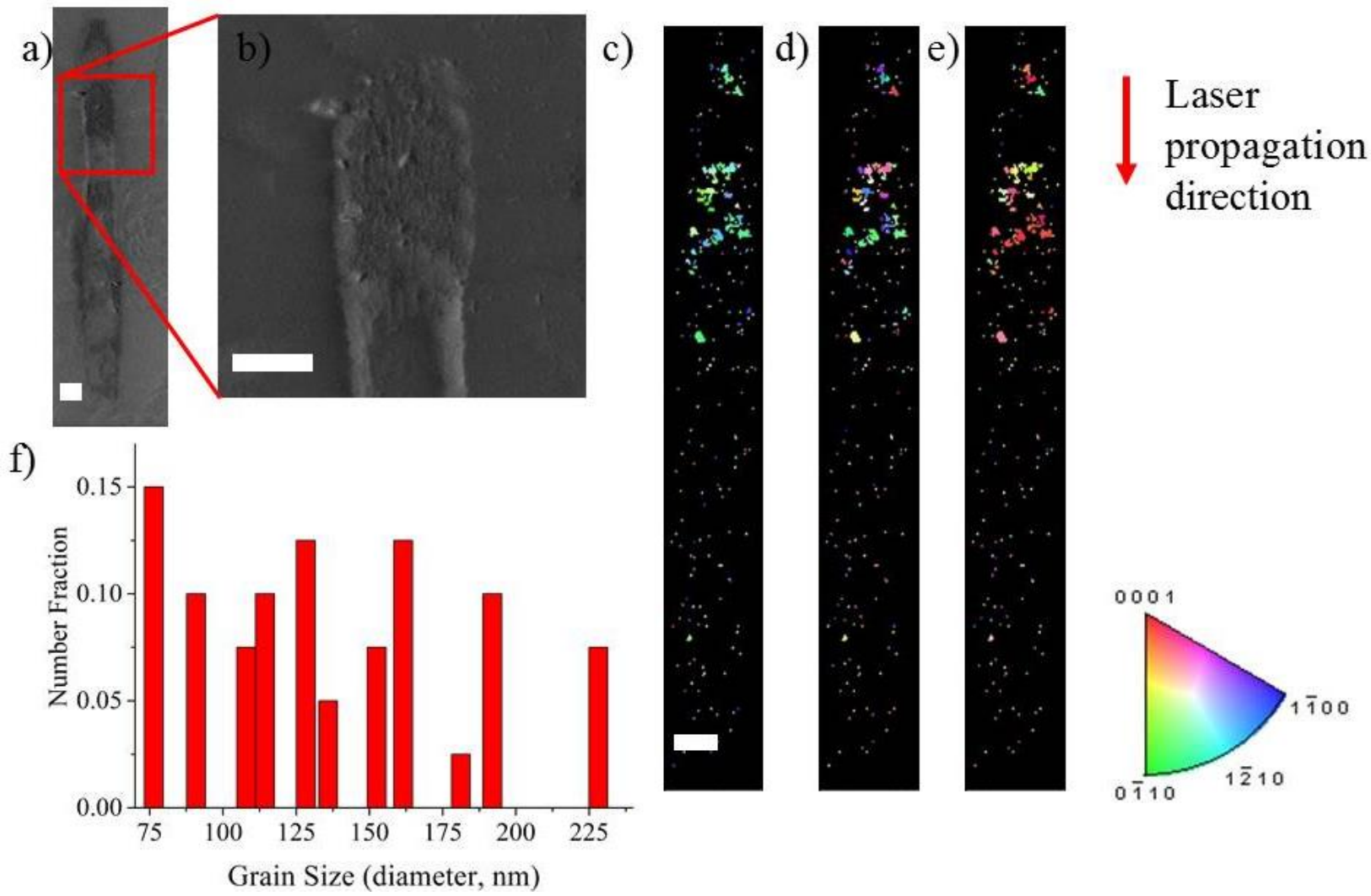

Figure 69 Structure of the laser trace investigated by SEM. The section was achieved perpendicularly to the scanning direction: a-b) SEM images of glass after irradiation; Inverse pole figure (IPF), coding the crystal orientation along c) writing laser polarization direction, d) scanning direction, and e) along the laser propagation direction; f) apparent grain size (diameter) distribution. The colors in the IPF map are based on R3c space group, $\mathrm{LiNbO}_{3}$ (inset at the right bottom). Scanning direction is along $45^{\circ}$ and incident laser polarization angle is $135^{\circ}$. All scale bars are $1 \mu \mathrm{m}$. 
To be more quantitative, IPF of texture was employed to quantitatively display the density of crystal orientations along the chosen coding sample directions. Polar axis of crystal avoids to have a component on incident laser polarization direction (i.e. tends to be perpendicular), because no accumulation of polar axis in texture map for this direction (red arrow in Figure 70a) is detected. It accumulates instead, along scanning direction (Figure 70b) and laser polarization direction (Figure 70c). On the contrary, axes close to $\begin{array}{lllllll}0 & \overline{1} & 1 & 0\end{array}$ axes accumulate along incident laser polarization (white arrow in Figure 70a).

As illustrated in Figure 71, polar axes are distributed around a plane perpendicular to the writing laser polarization direction at regime 2 . Sometimes it is quite well defined e.g. $\left(\theta=45^{\circ}, \phi=90^{\circ}\right)$ and sometimes less organized e.g. $\left(45^{\circ}, 0^{\circ}\right)$. So, at regime 2 , even the scanning direction or laser polarization is varied, the distribution of polar axis is the same: distributed in the plan perpendicular to writing laser polarization direction.

a) writing laser polarization direction

b) scanning direction

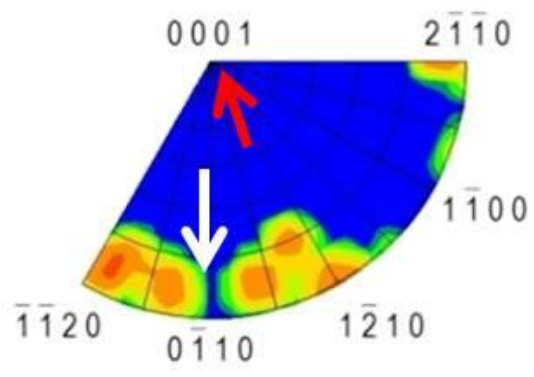

c) laser propagation direction

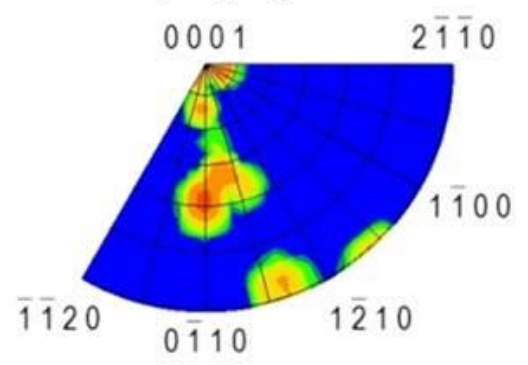

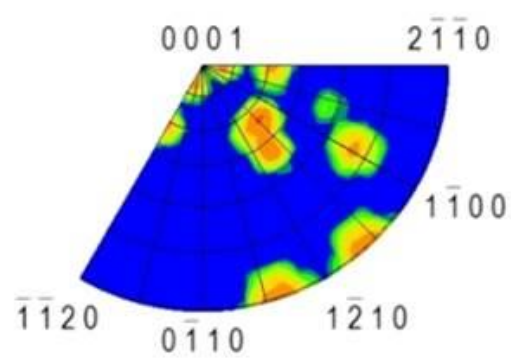

25.000

13.133

6.899

3.624

1.904

1.000

0.525

Figure 70 IPFs of texture calculated from EBSD-scans for configuration $\left(45^{\circ}, 135^{\circ}\right)$ coding the crystal orientation along a) incident laser polarization direction, b) scanning direction, and c) laser propagation direction. The red arrow indicates the absence of crystal with polar axis parallel to incident laser polarization direction. The white arrow indicates the crystals with an accumulation of axes close to $0 \quad \overline{1} 10$ axis, in the direction of the incident laser polarization. The color scale refers to multiple of the pole density in the case of random distribution (quoted 1 and green). Laser irradiation-configuration considering scanning direction and incident laser polarization angle is defined as $(\theta, \phi)$. 
a)

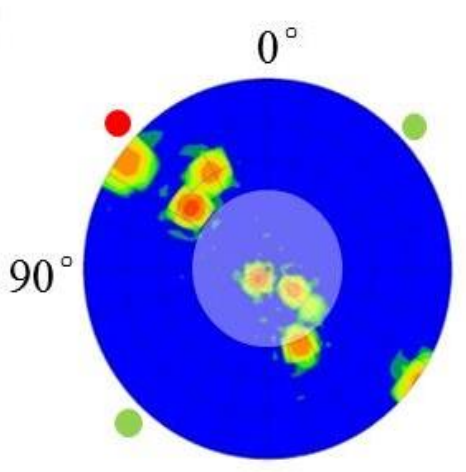

c)

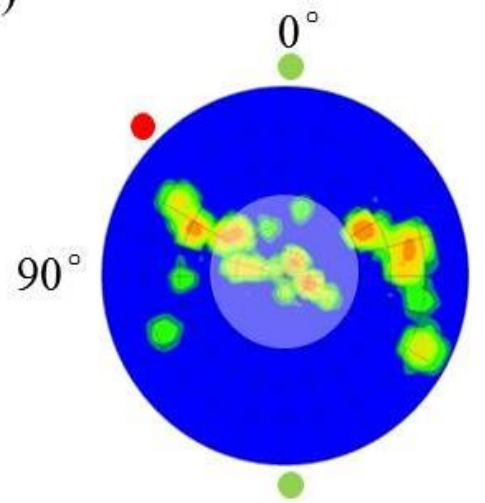

b)

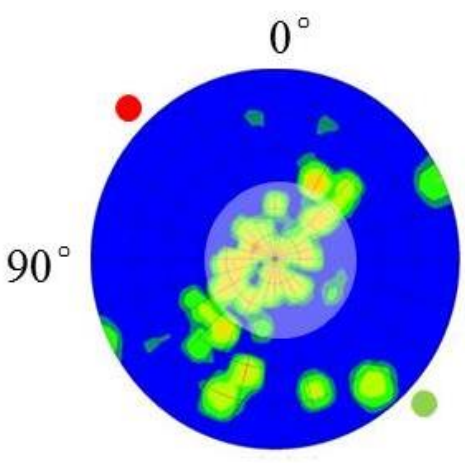

d)

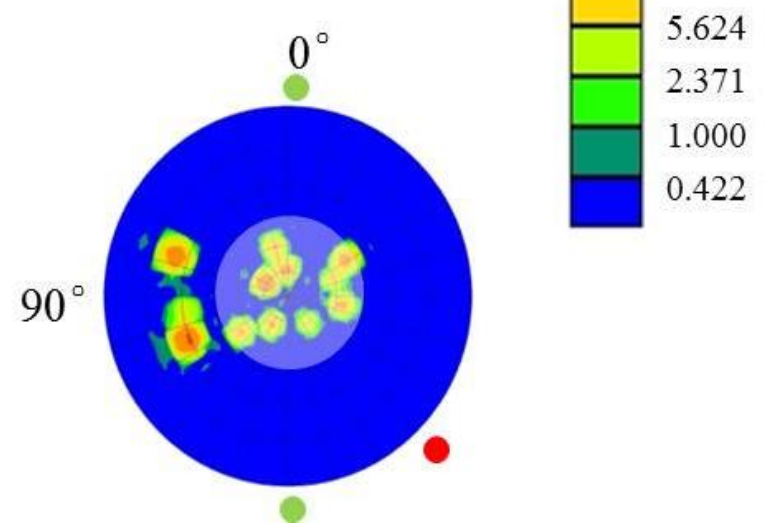

75.000 31.626

13.336

5.624

2.371

1.000

0.422

Figure 71 0001-Pole Figure of texture calculated from electron backscatter diffraction (EBSD)-scans i.e. the polar axis from EBSD scans for laser radiation-configurations $\left(45^{\circ}, 135^{\circ}\right)$ for a), $\left(45^{\circ}, 45^{\circ}\right)$ for b), $\left(45^{\circ}, 0^{\circ}\right)$ for $\left.\mathrm{c}\right)$, and $\left(225^{\circ}, 0^{\circ}\right)$ for $\left.\mathrm{d}\right)$. The milky disks at the centre of Pole Figures indicate the part of the distribution that has not to be taken into account for interpretation of SHG angular dependence with polarization in XY plane (will be discussed in Section 5.1.3.1). The color scale refers to multiple of the pole density for random distribution (quoted 1 and green). Laser radiation-configuration considering scanning direction and incident laser polarization angle are defined as $(\theta, \phi)$. Green dots illustrate the writing laser polarization and red dots illustrate the writing direction.

Based on the above discussion, the fs laser-induced crystallization leads to crystals not randomly oriented but with textures, indicating some forces are active for orienting the crystals during their formation.

\subsubsection{Repetition rate dependent crystallization}

To go a step further, another low repetition rate (i.e. $100 \mathrm{kHz}$ ) and high repetition rate (i.e. 500 $\mathrm{kHz}$ ) were performed to have a clear picture of fs laser-induced crystalline in LNS glass. The results are shown in Figure 72. Some remarks are listed as following.

1) The static irradiation mode means irradiating the glass without moving the stage. The crystallization-threshold is defined as the lowest pulse energy to obtain green irradiation in this mode. Generally, it takes some time (e.g. $80 \mathrm{~s}$ ) of static irradiation, and then the sample translation stage was moved to write crystalline lines at certain writing velocity (e.g. $5 \mu \mathrm{m} / \mathrm{s}$ ). If 
lines are scanned directly without this static irradiation, it is hard to obtain continue or not possible to obtain green irradiation during fs laser fabrication;

2) At $250 \mathrm{kHz}$ or $500 \mathrm{kHz}$, the static mode crystallization-threshold is around $0.4 \mu \mathrm{J} /$ pulse, but the difference is that it takes few seconds for $500 \mathrm{kHz}$, but few minutes for $250 \mathrm{kHz}$ to obtain green irradiation. In other words, it takes less time for higher repetition rate than for lower repetition rates to induced crystalline onset. This indicates the existence of an incubation state which is far beyond the waiting time reported in TTT domain (see Section 2.2.2.2). This could be an indication that some chemical diffusion should take place by cumulative effect;

3) The threshold for obtaining crystalline line in LNS glass (i.e. scanning mode) for $250 \mathrm{kHz}$ is around $0.6 \mu \mathrm{J} /$ pulse at $5 \mu \mathrm{m} / \mathrm{s}$, which is larger than the static mode crystallization-threshold (i.e. around $0.4 \mu \mathrm{J} /$ pulse). It indicates that the writing velocity can affect the outcome of crystal at certain repetition rate. It indicates a thermal accumulation, which is playing an important role in this crystallization process, which has been discussed in details in Section 3.3.2.3.2;

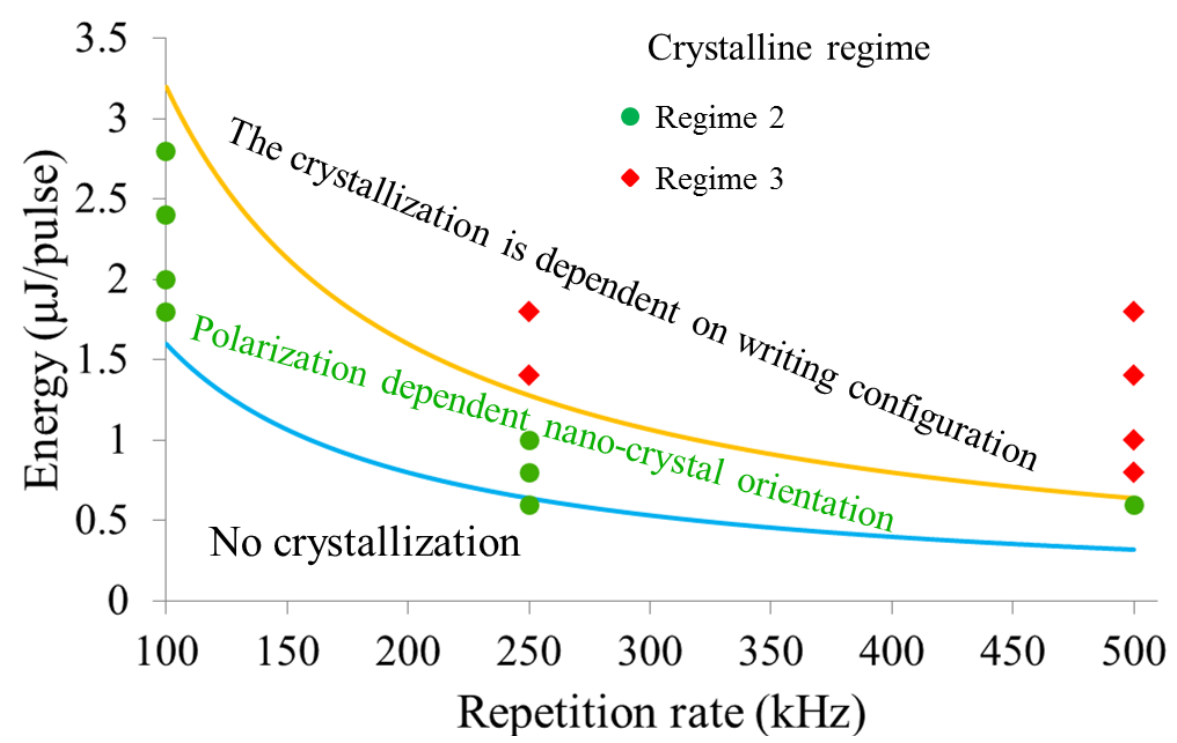

Figure 72 Domain of fs laser-induced crystallization in LNS glass varying pulse energy and repetition rate. Other parameters: $32.5 \mathrm{Li}_{2} \mathrm{O}-27.5 \mathrm{Nb}_{2} \mathrm{O}_{5}-40 \mathrm{SiO}_{2}$ (mol\%), $1030 \mathrm{~nm}, 300 \mathrm{fs}, \mathrm{NA}=0.6$, focus depth $300 \mu \mathrm{m}$ in air, $5 \mu \mathrm{m} / \mathrm{s}$ (the dots are the measured data)

4) At the same pulse energy (e.g. $1.4 \mu \mathrm{J} /$ pulse), with the increase of pulse energy form 250 $\mathrm{kHz}$ (Figure 66c) to $500 \mathrm{kHz}$ (Figure 73a), the width of zone $\mathrm{C}$ of regime 3 (the smooth crystalline part which is around the central one) is increasing. In addition, there are many cracks around the laser track (black curves in Figure 73a);

5) At low repetition rate $(100 \mathrm{kHz})$, the threshold for obtaining crystalline line in LNS glass is $\sim 1.8 \mu \mathrm{J} /$ pulse. Below this pulse energy, no matter how long time of irradiation is performed in 
static mode (> $3 \mathrm{~min}$ ), no green light is detected. Even the pulse energy is as high as $2.8 \mu \mathrm{J} / \mathrm{pulse}$, only a rough structure was obtained at the centre without the surrounding smooth part (Figure 73b);

6) We found that the boundary of neighbour regimes followed the equaition: $E=$ a/repetition rate, in which a is the deposited average power. For the boundary between regimes 1 and 2 , illustrated by blue line in Figure $72, a_{1}$ is around $0.16 \mathrm{~W}$ and for the boudndary between regimes 2 and 3 , marked by orange line in Figure $72, a_{2}$ is around $0.32 \mathrm{~W}$.

7) There is always an HF over etched part surrounding the central crystalline part (illustrated in Figure 73).

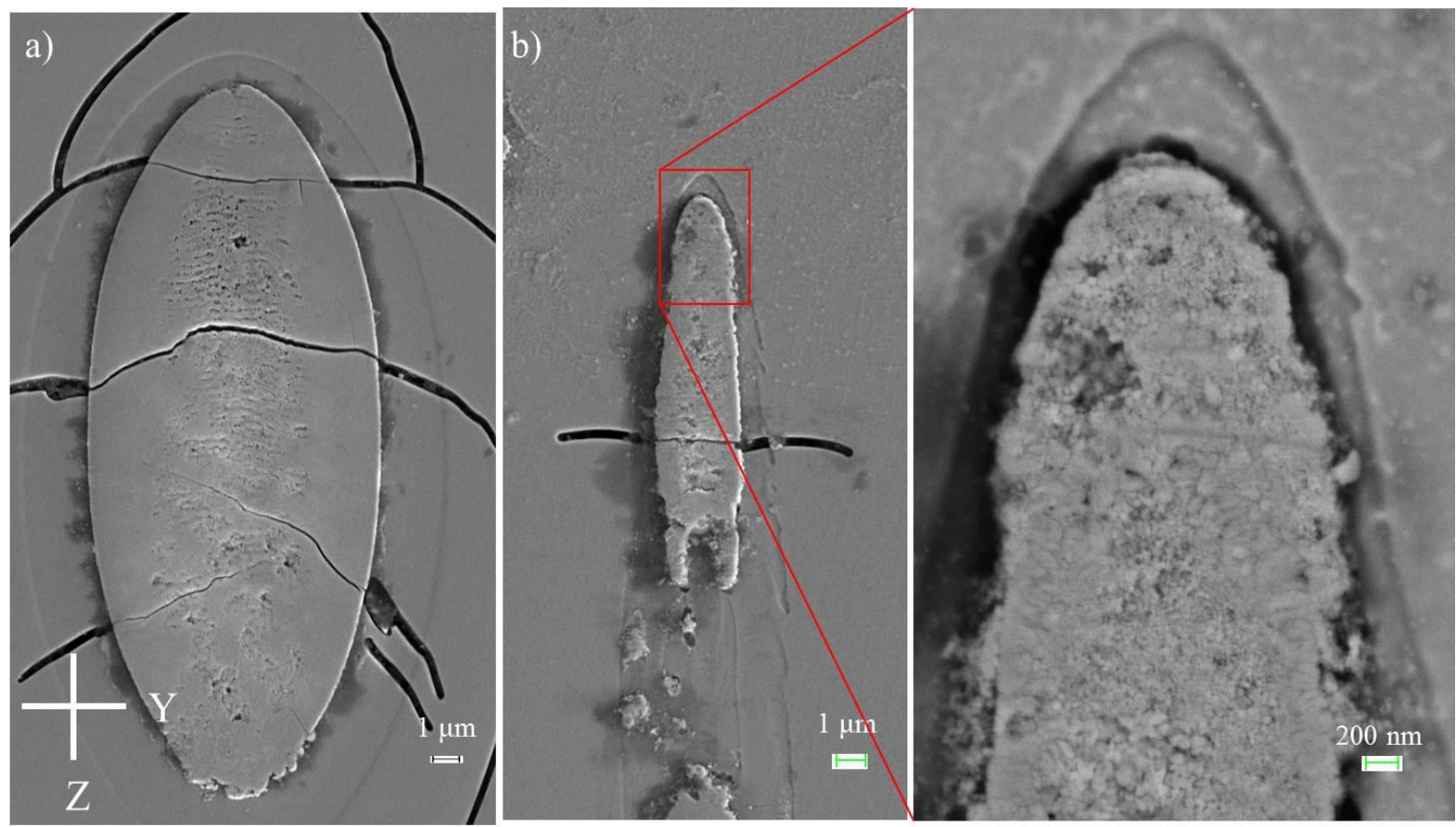

Figure 73 SEM images of laser tracks: a) $500 \mathrm{kHz}, 1.4 \mu \mathrm{J} /$ pulse and b) $100 \mathrm{kHz}, 2.8 \mu \mathrm{J} /$ pulse (after HF treatment). Other parameters: $32.5 \mathrm{Li}_{2} \mathrm{O}-27.5 \mathrm{Nb}_{2} \mathrm{O}_{5}-40 \mathrm{SiO}_{2}$ (mol\%), $1030 \mathrm{~nm}, 300 \mathrm{fs}$, NA = 0.6, focus depth $300 \mu \mathrm{m}$ in air, 5 $\mu \mathrm{m} / \mathrm{s}$ 


\subsubsection{Interpretation of fs laser polarization controlled nano- crystal orientation}

Classically, crystallization is determined by the local temperature that has to be in a suitable range during a time duration large enough for allowing the atoms to move and rearrange themselves (see Section 2.2.2.1). If the temperature is not uniform, the temperature gradient is related to a force that leads to orient the crystal nuclei. This is when no other force is considered, but when an effect of incident laser polarization is detected like in our case and other ones [23], there is obviously an effect of light forces.

The first idea is a torque exerted on the electric dipole of a nano-crystal during the nucleation. Assuming that the nuclei have already the structure of the final crystals, we can notice that $\mathrm{LiNbO}_{3}$ may have a spontaneous electric dipole, it has also an induced electric dipole, like any crystal, but with an anisotropic susceptibility the induced dipole is not always parallel to the applied electric field. For such crystal orientation, a torque is therefore resulting.

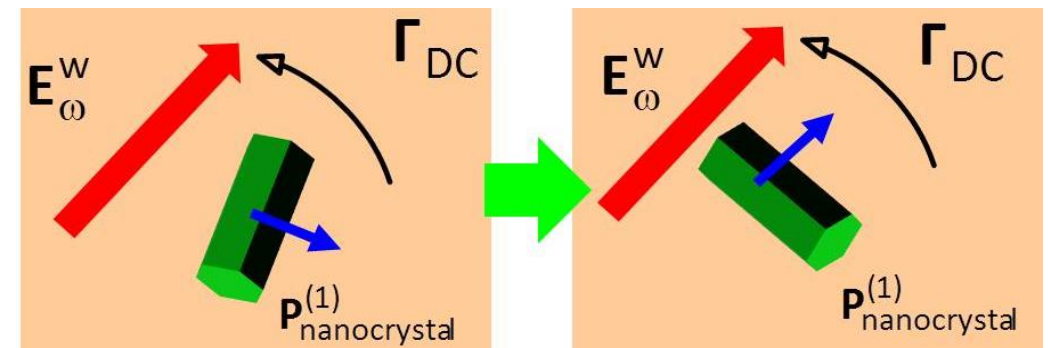

Figure 74 Schematic of non-oscillating torque acting on crystal orientation

Figure 74 illustrates a non-oscillating torque, suggested to be applied on the nucleus when they are at the state of its birth and during its moderate growth compared to radiation wavelength. There may be several types of torques (in volume), but the most relevant here, and likely the largest, is the one deduced from the electromagnetic wave:

$$
\Gamma=\operatorname{DCpart}\left(\mathbf{P}^{(1)} \wedge \mathbf{E}_{\omega}^{\mathrm{w}}\right)=\operatorname{DCpart}\left(\varepsilon_{0} \boldsymbol{\chi}_{\omega}^{(1)} \mathbf{E}_{\omega}^{\mathrm{w}} \wedge \mathbf{E}_{\omega}^{\mathrm{w}}\right)
$$

Where $\mathbf{E}_{\omega}^{\mathrm{w}}$ is the incident laser electric field, $\mathbf{P}^{(1)}$ is the induced dipole at the laser frequency during laser radiation, and $\boldsymbol{\chi}_{\omega}^{(1)}$ is the first order susceptibility tensor.

For hexagonal crystal class, $\boldsymbol{\chi}_{\omega}^{(1)}$ could be written as (reference is based on its principal axes, the $z$ axis, see details in Figure 32c).

$$
\boldsymbol{x}_{\omega}^{(1)}=\left[\begin{array}{ccc}
\chi_{x x} & 0 & 0 \\
0 & \chi_{x x} & 0 \\
0 & 0 & \chi_{z z}
\end{array}\right]
$$


Because $\chi_{x x}>\chi_{z z}$, the stable equilibrium position for the crystal is the isotropic plane (xy plane, see details in Figure 32c) containing the incident laser polarization, i.e. polar axis tend to be perpendicular to the incident laser polarization as it is experimentally observed.

Meanwhile, it is possible to achieve crystal rotation during the pulse duration, which is very short as a matter of fact (300 fs) but with the possibility to cumulate the very small actions as in a ratcheting effect.

Based on above discussion (Section 4.1.2.2), the nano-crystal orientation would be dependent on the polarization of the laser. There is a theory for which during the interaction mechanism, a DC field could be formed by movement of the electronic plasma [228]. Therefore a DC external field was applied on LNS glasses to see whether there is some effect or not.

After the application of DC electric field, we found a chemical reaction occurs in LNS glass in short time (few seconds) at high voltage (i.e. $3 \mathrm{kV}$ ). The DC electric field induced black clouds in LNS glasses at the negative electrode. These black clouds can be moved by the application of an opposite electric field: repulsed by the negative electrode and attracted by the positive one. It maybe useful for electric switch or display applications (see details in Appendix XX). 


\section{2. fs laser-induced chemical migration}

Since the glass composition is $33 \mathrm{Li}_{2} \mathrm{O}-33 \mathrm{Nb}_{2} \mathrm{O}_{5}-34 \mathrm{SiO}_{2}$ (mol\%) and if a phase separation occurs into crystalline $\mathrm{LiNbO}_{3}$ and amorphous $\mathrm{SiO}_{2}$ phases, 34\% of the area should be not covered with crystals, and should not be colored in IPF. However, Figure 55c-d show that the laser-modified region is totally colored. What can be the origin of this discrepancy? There are two possibilities for this: either $\mathrm{Si}$ is in the modified volume but hide by the crystallization or Si migrates out of it.

\subsubsection{Nanoscale chemical migration observation}

\subsubsection{Chemical analyses in bulk irradiated LNS glass}

To identify the chemical composition, SEM/EDS measurements were performed on bulk irradiated LNS glass after polishing to expose a section of a written line on the surface. Figure 75 displays almost flat profiles for $\mathrm{Si}$ and $\mathrm{Nb}$ in and around the irradiated volume.

a)

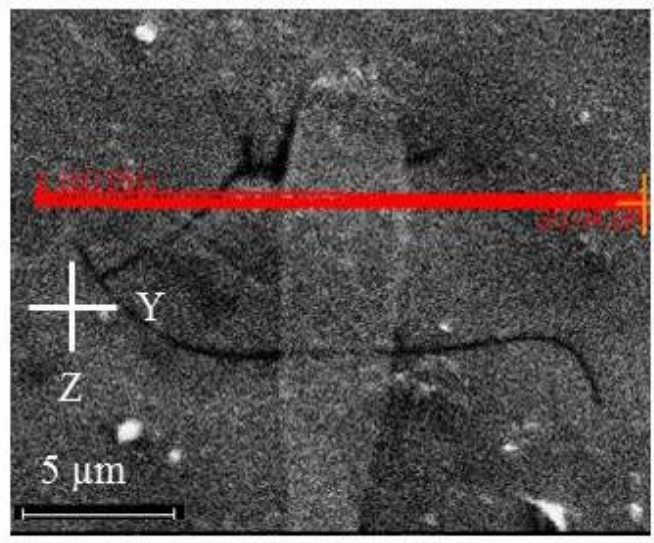

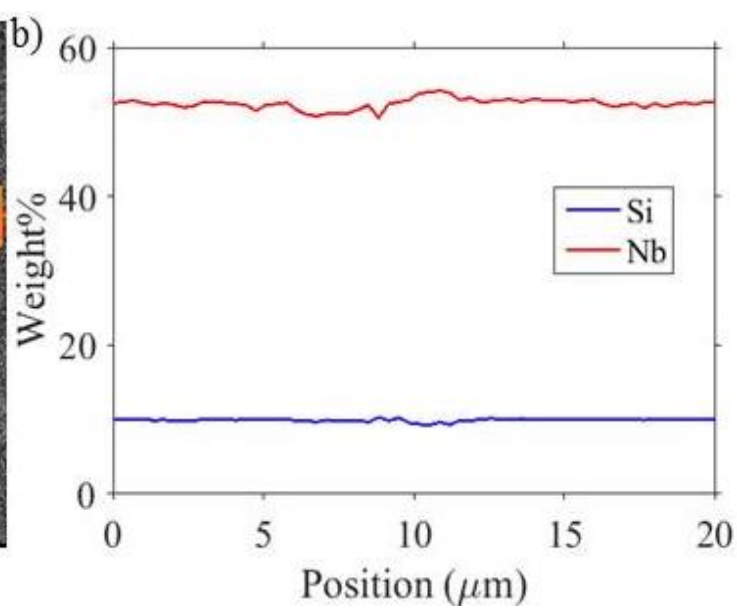

Figure 75 Chemical analysis of irradiated glass by SEM/EDS in bulk sample: a) the SEM image and b) the corresponding chemical profiles across the head of the laser track of Si (blue) and $\mathrm{Nb}$ (red). Other parameters: $33 \mathrm{Li}_{2} \mathrm{O}-33 \mathrm{Nb}_{2} \mathrm{O}_{5}-34 \mathrm{SiO}_{2}$ (mol\%), $1030 \mathrm{~nm}, 300 \mathrm{fs}, 300 \mathrm{kHz}, 0.9 \mu \mathrm{J} /$ pulse, $5 \mu \mathrm{m} / \mathrm{s}, \mathrm{NA}=0.6$, focal depth $350 \mu \mathrm{m}$ in glass

To obtain more quantitative profiles, including oxygen measurements, wavelength dispersive spectroscopy (WDS) microprobe measurements were conducted. As illustrated in Figure 76, no obvious fluctuation in the concentrations of $\mathrm{O}, \mathrm{Si}$, and $\mathrm{Nb}$ were observed, confirming the SEM/EDS analysis. 

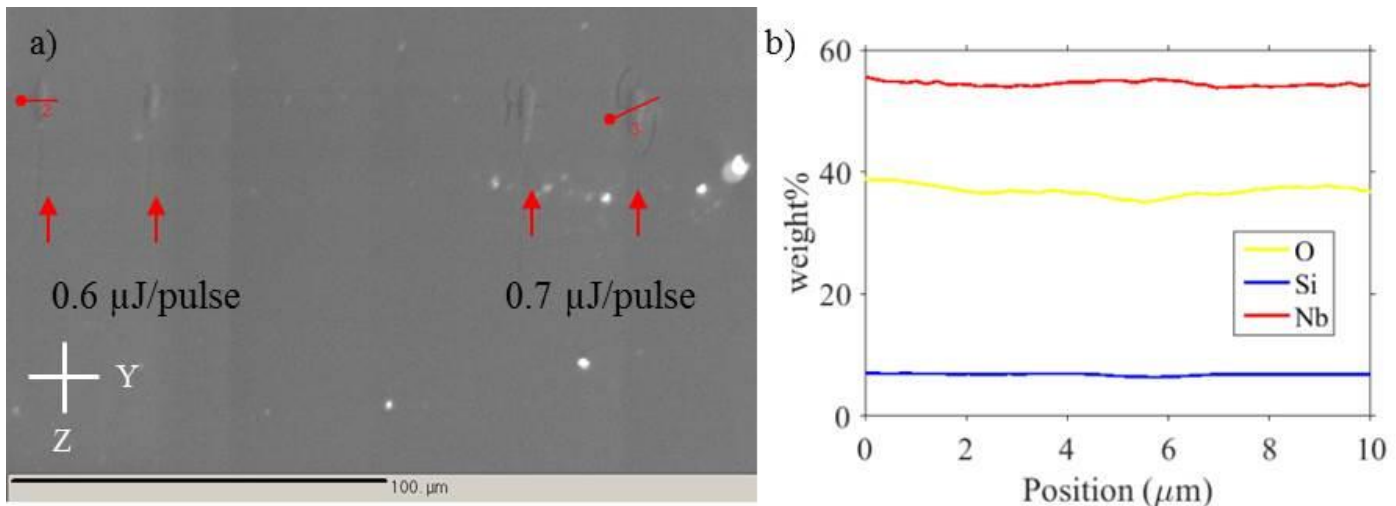

Figure 76 Chemical analysis of irradiated glass by WDS microprobe in bulk sample: a) the secondary electron image of the cross section of laser tracks varying the pulse energy (marked with red arrows), the red lines illustrate the elemental analysis line profiles, starting from the red points and $b$ ) the corresponding chemical profiles across the laser track of $\mathrm{O}$ (yellow), $\mathrm{Si}$ (blue), and $\mathrm{Nb}$ (red). Other parameters: $33 \mathrm{Li}_{2} \mathrm{O}-33 \mathrm{Nb}_{2} \mathrm{O}_{5}-34 \mathrm{SiO}_{2}$ (mol\%), $1030 \mathrm{~nm}, 300 \mathrm{fs}, 300 \mathrm{kHz}, 0.6 \mu \mathrm{J} /$ pulse, $5 \mu \mathrm{m} / \mathrm{s}, \mathrm{NA}=0.6$, focal depth $350 \mu \mathrm{m}$ in glass

Alkaline atoms migrate easily under the laser irradiation [11] and so ion beam analyses were performed to determine the $\mathrm{Li}$ distribution. Ion beam analysis is a powerful tool to simultaneously investigate concentrations and distributions of different constituent elements. lons beam analysis is a powerful tool to simultaneously investigate concentrations and distributions of the different constituent elements. The analyzed depth ranges of these techniques are from a few ten nanometers to a few ten micrometers, depending on the density of the sample. These techniques are also insensitive to the crystallographic structure of the sample. Thus, the same signal will be obtained if at the surface we have for example $\mathrm{Li}_{2} \mathrm{O}$ and in the depth $\mathrm{Nb}_{2} \mathrm{O}_{5}$ or only the composition $\mathrm{LiNbO}_{3}$.

a)

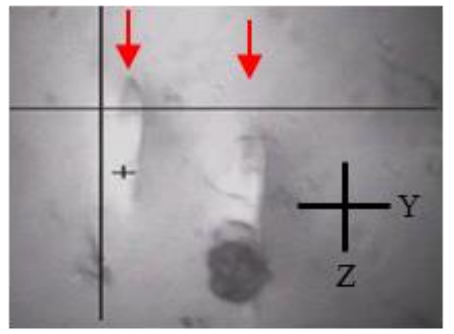

c)

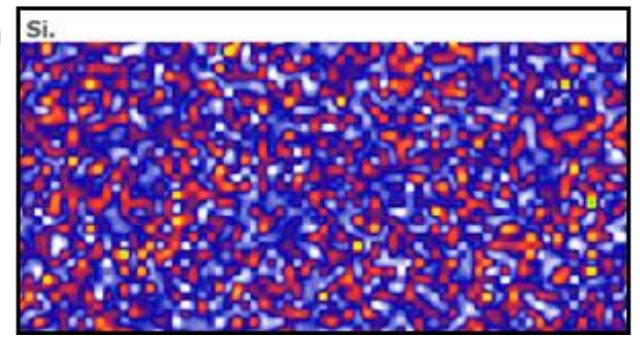

b)

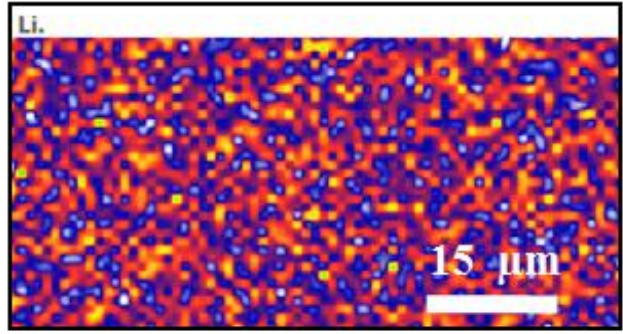

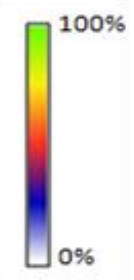

d)

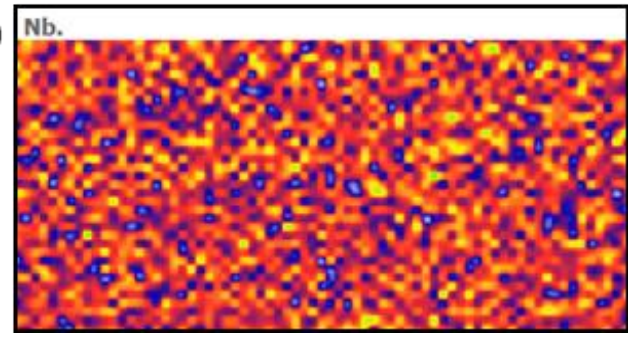

Figure 77 Chemical analysis of irradiated glass by ions beam analysis in bulk sample (beam size $2 \times 2 \mu \mathrm{m}^{2}$ ): a) a camera photo of the cross section of two laser tracks (marked with red arrows, with distance of $30 \mu \mathrm{m}$, map size $70 \times 34 \mu^{2}$ ) and the corresponding elemental distribution mappings of b) $\left.\mathrm{Li}, \mathrm{C}\right) \mathrm{Si}$, and d) $\mathrm{Nb}$ of the irradiated glass. Color bar: proportional of the number of counts on $\mathrm{Li}, \mathrm{Si}$ or $\mathrm{Nb}$ atoms. Other parameters: $33 \mathrm{Li}_{2} \mathrm{O}-33 \mathrm{Nb}_{2} \mathrm{O}_{5}-34 \mathrm{SiO}_{2}$ (mol\%), $1030 \mathrm{~nm}, 300 \mathrm{fs}, 300 \mathrm{kHz}, 2.2 \mu \mathrm{J} /$ pulse, $5 \mu \mathrm{m} / \mathrm{s}, \mathrm{NA}=0.6$, focal depth $350 \mu \mathrm{m}$ in glass 
We performed the same experiment with a grazing angle and the same results were obtained: no obvious fluctuation in the concentrations of $\mathrm{Li}, \mathrm{Si}$, and $\mathrm{Nb}$ in and around the laser tracks (Figure 77). This is in accordance with the results from the SEM/EDS and WDS microprobe.

The chemical analysis of the irradiated glass is thoroughly investigated by a series of the above characterization techniques in bulk sample. A series of laser traces were measured varying the pulse energies (0.5-2.2 $\mu \mathrm{J} /$ pulse), writing configuration (i.e. laser polarization is perpendicular or parallel to scanning direction), scanning speed ( $5 \mu \mathrm{m} / \mathrm{s}$ or $10 \mu \mathrm{m} / \mathrm{s}$ ), or even the same laser trace with different positions along laser propagation direction. Similar results were obtained. The above observation implies that no chemical migration occurs in and around the laser track in bulk irradiated LNS glass at our irradiated conditions.

\subsubsection{X-ray absorption spectroscopy analysis}

X-ray absorption spectroscopy (XAS) was used to determine the environment around $\mathrm{Nb}$ and $\mathrm{Si}$. XAS using synchrotron radiation is a versatile technique to obtain direct structural information on materials [229]. This method is suitable for following local coordination change. This is achieved by recording any changes on the $\mathrm{X}$-ray absorption edges at Si K and $\mathrm{Nb} \mathrm{L}$ edges.

Some preliminary XAS raw data of the cross section of laser tracks in glass are shown in Figure 78a. Si K edge, $\mathrm{Nb} L_{\|}$and $\mathrm{Nb} L_{\| I}$ edges are clearly observed. However, there are some artifacts after the Si K edge, appearing on a steep background that renders the EXAFS analysis not achievable. $\mathrm{Nb} \mathrm{L}_{\text {III }}$ edge XANES spectrum consists in an intense double-featured resonance followed by a weak structure.

This double-featured intense resonance probes the $4 d$ unoccupied states, because the $2 p \rightarrow 4 d$ transitions (such as peak $D$ and $E$ in in Figure $78 \mathrm{~b}$ ) are partly dipole allowed in centered symmetry groups compared to $4 \mathrm{~d}$ levels dipole forbidden at the $\mathrm{K}$ edge [221]. The observed D-E separation corresponds to the ligand-field splitting of the $4 \mathrm{~d}$ state of $\mathrm{Nb}^{5+}$. The following weak structure at around $2385 \mathrm{eV}$ is the allowed transition to the empty molecular orbital $\mathrm{Nb}_{5 \mathrm{~s}}$ with symmetry $A_{1 g}$ in $O_{h}$ ) [230]. The point group symmetry character tables of $C_{3 v}$ and $O_{h}$ are shown in Appendix XXI.

For $\mathrm{LiNbO}_{3}$, the local symmetry around $\mathrm{Nb}$ is $\mathrm{C}_{3 \mathrm{v}}$. In such a group, there are two processes: a $\mathrm{C}_{3}$ distortion of the octahedron coordination shell and a symmetry lowering compared to regular octahedron. The first process leads to degeneracy lift off which is shown to be modest in [231]. 
The second leads to a larger intensity of the $D$ and $E$ transitions as we can show that the molecular orbital levels, concerned in these transitions is larger than that in a regular octahedron. For the following, we can also mention that a complete symmetry distortion will leads to intensity much larger than in $C_{3 v}$. At the same time, the gap between the eg and the $t_{2 g}$ levels is filled and the splitting between D and E structure would become less contrasted.

The normalized intensity of the D-E structure is slightly higher in the glass matrix than within the irradiated areas. Meanwhile, the contrast defined by the amplitude of the modulation divided by the average intensity between the $D$ and $E$ structures increases from the glass matrix to the irradiated areas. If we make reference to the remark above, we deduce that the $\mathrm{Nb}$ environment order increases after irradiation. This is consistent with crystallization around $\mathrm{Nb}$ atoms.
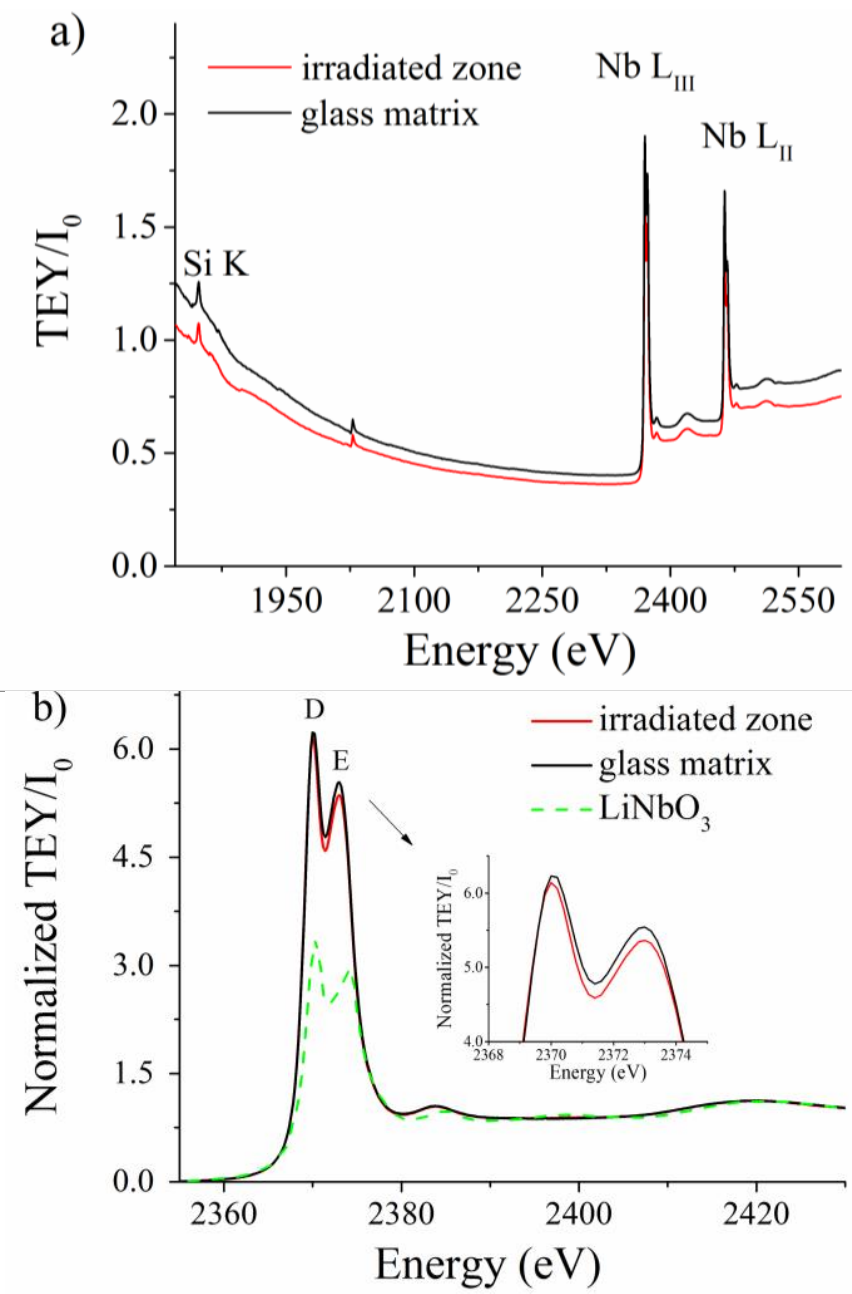

Figure 78 a) X-ray absorption spectra (XAS) and b) X-ray absorption near edge structure (XANES) of Nb $\mathrm{L}_{\text {|II }}$ edge for glass matrix (black curve), for irradiated area (red curve) and $\mathrm{LiNbO}_{3}$ (green curve). For glass matrix and irradiated area, XANES is recorded from normalized total electron yield (TEY) mode (TEY/I 0 ) with InSb (111) monochromator. For crystal $\mathrm{LiNbO}_{3}$, XANES is from X-ray absorption spectrum. TEY/I for irradiated area has been recorded in the cross section of the laser track, perpendicular to scanning direction; other parameters: $33 \mathrm{Li}_{2} \mathrm{O}-33 \mathrm{Nb}_{2} \mathrm{O}_{5}-34 \mathrm{SiO}_{2}$ (mol\%), $1030 \mathrm{~nm}, 300 \mathrm{fs}, 300 \mathrm{kHz}, 1.8 \mu \mathrm{J} /$ pulse, $5 \mu \mathrm{m} / \mathrm{s}$, laser polarization is parallel to scanning direction. The insert is the magnification of irradiated zone (red curve) and glass matrix (black curve). The green dashed line is redrawn from previous work [231], left shift $1.4 \mathrm{eV}$. 
Based on the above discussion, it is necessary to obtain direct observation of the distribution of crystals at smaller scale (i.e. nanoscale). For bulk sample, SEM/EDS, WDS microprobe, and ions beam analysis have a resolution at micrometre scale (see details in Section 3.4.4.5). For STEM/EDS, due to the thinner samples and higher accelerating voltage, nano or even angstrom spatial resolution can be obtained [223].

\subsubsection{Chemical analysis of irradiated glass at nanoscale}

Results of STEM-HAADF observations (Figure 79a) point out a lamella-like nanostructure with alternated bright and dark regions, occurring at the center of the laser-modified area. The magnified image in Figure 79b presents bright and dark zones according to the volume-averaged atomic number. The bright zones have a width of $\sim 100 \mathrm{~nm}$ and the dark zones have a width of $\sim 20 \mathrm{~nm}$. Chemical analysis (mapping mode, measured step $\sim 8 \mathrm{~nm}$, marked with a rectangle in Figure 79b) reveals that the bright zones in the STEM-HAADF image are rich in Nb (Figure 79c) and the black zones are rich in Si (Figure 79d). Deduced chemical profiles (marked with dashed lines in the mapping images) shown in Figure 79e, reveal that $\mathrm{Si}$ and $\mathrm{Nb}$ contents vary inversely.

STEM/EDS results revealed separated regions: one with a large Si content and another one with a large $\mathrm{Nb}$ content (Figure 79). It provides the proof of chemical migration and $\mathrm{LiNbO}_{3}$ phase separation from $\mathrm{SiO}_{2}$ at the nanoscale. TEM was conducted to obtain crystallographic information about the nanostructure. The contrast difference in TEM images may come from the variable ability of the materials to diffract electrons. Specifically, in bright field mode, the crystalline phase appears with darker contrast than amorphous phase.

Figure 80a displays the lamella-like nanostructure shown in Figure 79a. The bright walls are amorphous phases. The dark regions are diffracting, corresponding to crystalline phases. The thicknesses of the amorphous wall and the crystalline wall are $\sim 10$ and $\sim 100 \mathrm{~nm}$, respectively (Figure 80b). This is in agreement with STEM-HAADF observations. Therefore, we identify a nanoscale chemical migration of $\mathrm{Si}$ and $\mathrm{Nb}$ and a phase separation into $\mathrm{SiO}_{2}$ amorphous phase and $\mathrm{LiNbO}_{3}$ crystalline phase. 
a)
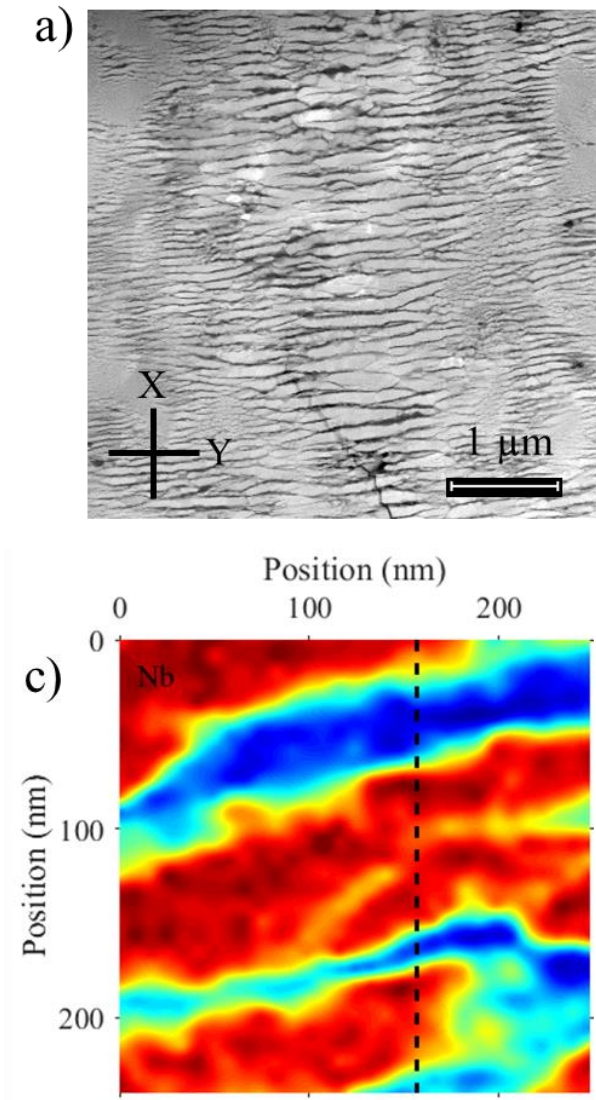

b)
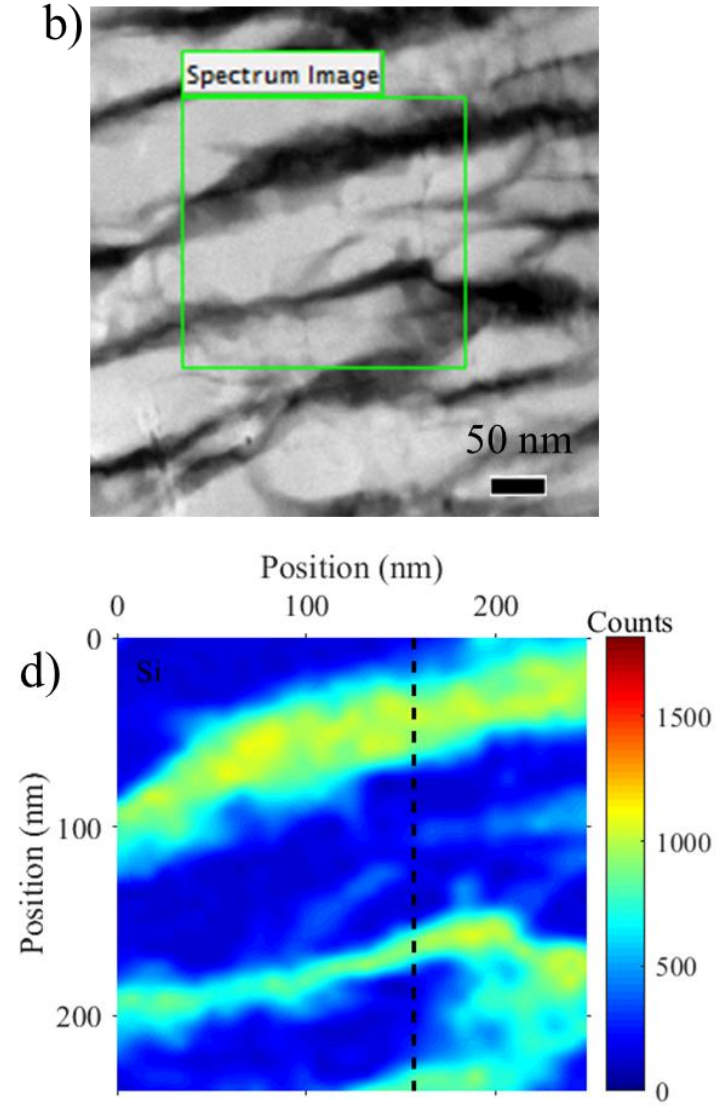

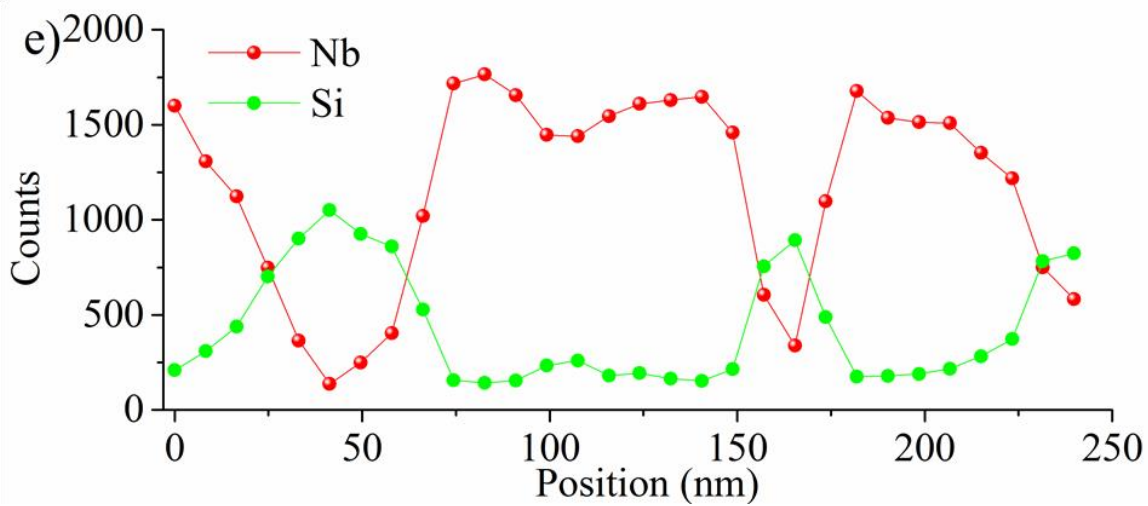

Figure 79 Chemical analysis of irradiated glass at the nanoscale. a) An overview of the laser-modified; b) the magnification image (green rectangle marks the area for $\mathrm{Nb}$ and Si mappings). STEM/EDS characterization of irradiated lithium niobium silicate glass in the mapping mode for $\mathrm{c}$ ) $\mathrm{Nb}$ and d) $\mathrm{Si}$; and e) the line profile crossing alternated bright and dark regions illustrated by dashed lines in the mapping images (from top to bottom). Pulse energy $1.3 \mu \mathrm{J} /$ pulse. Other parameters: $33 \mathrm{Li}_{2} \mathrm{O}-33 \mathrm{Nb}_{2} \mathrm{O}_{5}-34 \mathrm{SiO}_{2}$ (mol\%), $1030 \mathrm{~nm}, 300 \mathrm{fs}, 300 \mathrm{kHz}, 1.3$ $\mu \mathrm{J} /$ pulse, $5 \mu \mathrm{m} / \mathrm{s}, \mathrm{NA}=0.6$, focal depth $350 \mu \mathrm{m}$ in glass, and laser polarization is parallel to scanning direction (i.e. $\mathrm{X}$ direction).

For comparison, lines irradiated with 1.8 and $0.7 \mu \mathrm{J} /$ pulse were recorded. As illustrated in Figure $80 c-d$, similar nanoscale phase separation was observed. For the amorphous wall, whether at high (1.8 or $1.3 \mu \mathrm{J} /$ pulse) or moderate $(0.7 \mu \mathrm{J} /$ pulse) pulse energies:

1) There is amorphous lamella oriented perpendicular to the laser polarization direction (i.e. X direction); 
2) The thickness of the amorphous wall is $\sim 10 \mathrm{~nm}$; the distance between the neighbouring vitreous walls is $\sim 100 \mathrm{~nm}$ from the center of the laser-modified area;

3) The contrast between the amorphous and the crystalline phases becomes clearer with increasing pulse energy.

In conclusion, STEM/EDS and TEM confirm a nanoscale phase separation whereby $\mathrm{LiNbO}_{3}$ crystals are embedded in lamella-shaped frames of amorphous $\mathrm{SiO}_{2}$. The control of the fs laser parameters (e.g. laser polarization) allows the properties of nonlinear optical nano-crystals (e.g. geometries, morphology, and size) to be adjusted, thus increasing the structure diversity of fs laser-modified material, providing an ideal platform for exploring fs laser parameters dependent functional 3D device properties. Because the chemical migrations in our irradiated glasses are at nanoscale, it can explain why chemical migrations can be observed by STEM/EDS, but not in bulk sample by SEM/EDS, WDS microprobe, and ions beam analysis (see details in Section 3.4.4.5).
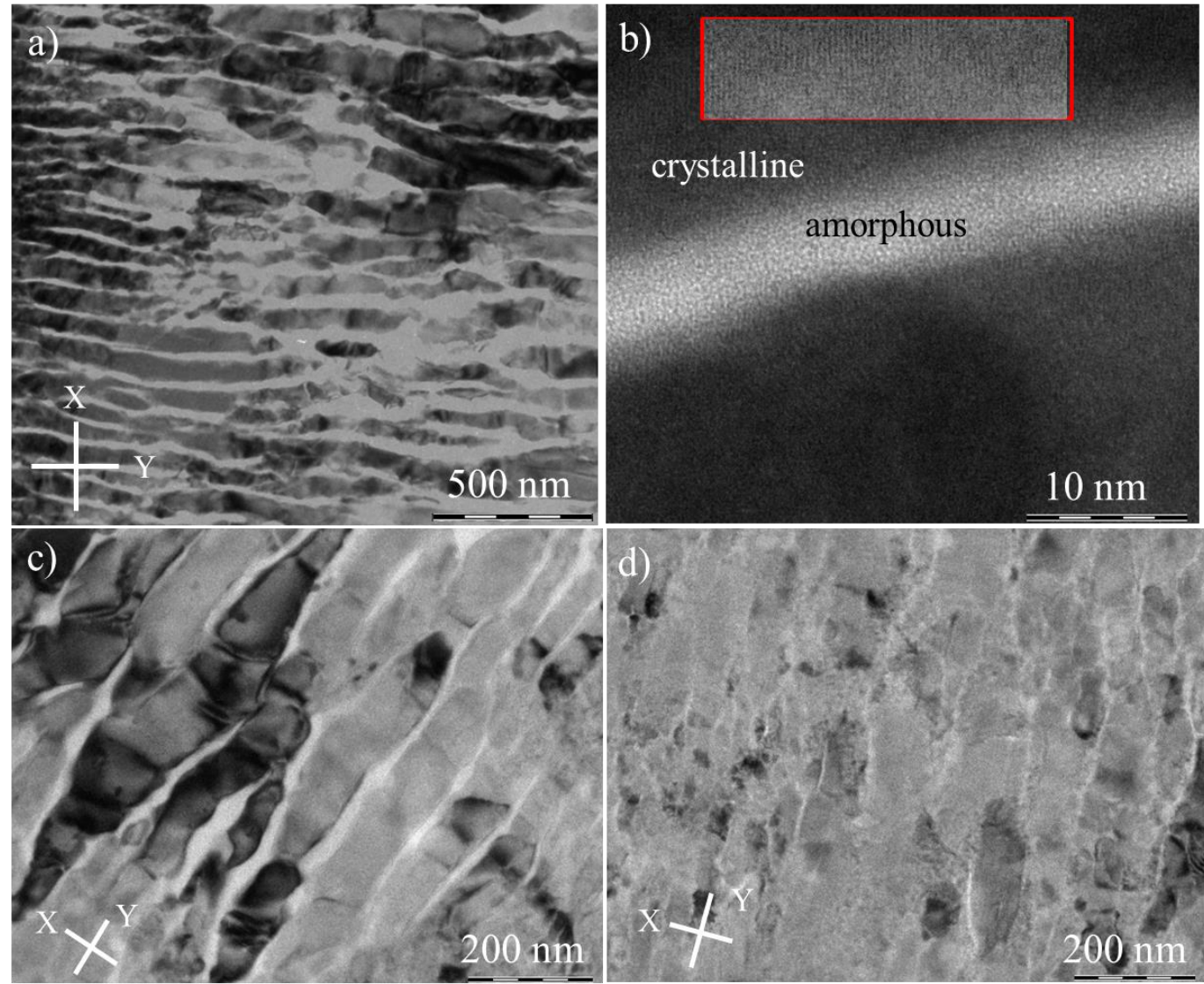

Figure $\mathbf{8 0}$ Nanostructure properties of irradiated glass investigated by TEM (bright field mode): a) TEM image of the laser-modified area and b) high resolution TEM showing the diffracting properties of the two zones (the zone in the red rectangle is the one with high contrast to show the crystal lattice) for $1.3, \mathrm{c}) 1.8$ and d) 0.7 $\mu \mathrm{J} /$ pulse. Other parameters: $33 \mathrm{Li}_{2} \mathrm{O}-33 \mathrm{Nb}_{2} \mathrm{O}_{5}-34 \mathrm{SiO}_{2}$ (mol\%), $1030 \mathrm{~nm}, 300 \mathrm{fs}, 300 \mathrm{kHz}, 5 \mu \mathrm{m} / \mathrm{s}, \mathrm{NA}=0.6$, focal depth $350 \mu \mathrm{m}$ in glass, and laser polarization is parallel to scanning direction (i.e. X direction). 


\subsubsection{Selective chemical etching}

Due to the different $\mathrm{HF}$ etching rates of $\mathrm{LiNbO}_{3}$ and $\mathrm{SiO}_{2}$ (i.e. the $\mathrm{HF}$ sensitivity of $\mathrm{SiO}_{2}$ is larger than $\mathrm{LiNbO}_{3}$ [232]), a $\mathrm{HF}$ etching of irradiated lines was conducted to check whether there is a selective chemical etching effect.

Figure 81 gives the PSI images of the polished irradiated LNS glass before and after HF treatment. This sample is polished along XY plane (the plane perpendicular to laser propagation direction). Before HF treatment (Figure 81a), the height of irradiated line is larger than the background (i.e. ca. $20 \mathrm{~nm}$ ), indicating different mechanical hardness pointed out by polishing compared with the glass matrix. After HF treatment (Figure 81b), the difference between the irradiated line and the background increases to around $49 \mathrm{~nm}$ (some part is even as high as 110 $\mathrm{nm})$. In addition, the fluctuations along the irradiated lines increase compared with the one without HF treatment. This indicates a different chemical sensitivity that can be accounted for by periodic distribution of amorphous $\mathrm{SiO}_{2}$ phase and crystalline $\mathrm{LiNbO}_{3}$ phase pointed out from STEM and TEM.
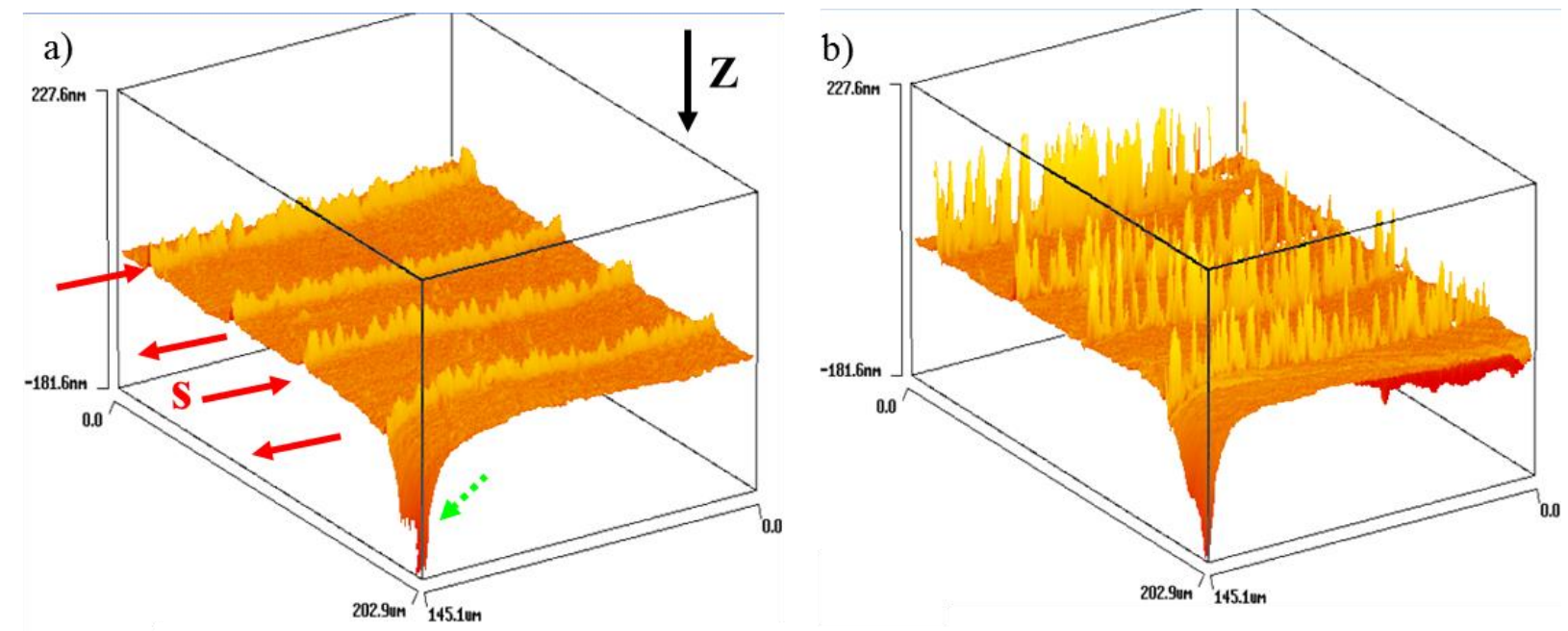

Figure 81 Phase shift interferometry (PSI) images of the polished glass a) before and b) after HF treatment (the right bottom part is a broken edge, used for marking, illustrated by green dashed arrow; the red arrows illustrate the scanning direction ( $\mathrm{S}$ ); $\mathrm{Z}$ is the laser propagation direction; the distance between each line is 50 $\mu \mathrm{m}$. Other parameters: $32.5 \mathrm{Li}_{2} \mathrm{O}-27.5 \mathrm{Nb}_{2} \mathrm{O}_{5}-40 \mathrm{SiO}_{2}$ (mol\%), $1030 \mathrm{~nm}, 300 \mathrm{fs}, 250 \mathrm{kHz}, 0.8 \mu \mathrm{J} / \mathrm{pulse}$, and $5 \mu \mathrm{m} / \mathrm{s}$, $\mathrm{NA}=0.6$, focal depth $300 \mu \mathrm{m}$ in air (regime 2)

\subsubsection{Interpretation of nanoscale chemical migration}

In previous report [18], a crystalline phase was observed in irradiated LNS glass in static mode (i.e. irradiation without moving the glass). The shape of this phase appeared as a ring centered around the irradiated phase. But the author did not use EBSD for location of the crystal directly. The $\mathrm{LiNbO}_{3}$ phase was confirmed by X-ray diffraction, and the crystal distribution was deduced 
from the SHG observation (in the vicinity of the focal spot) and the elemental analysis was conducted with electron probe microanalyzer [18].

He. et al. [26] also observed such a shape of crystallization region. In that case, the temperature at the center in the heating accumulation regime remains too high, above the melting temperature. Crystallization is only induced at the periphery where the temperature is high enough, without being higher than the melting temperature. Under these conditions, the amount of $\mathrm{Si}$ increases at the center of the irradiated area and decreases outside from the center; $\mathrm{Nb}$ migrates outward from the center. Therefore, in this case, temperature and its associated gradient may induce chemical migration. This is an effect that has nothing to do with chemical separation from phase stability but moves the chemical composition of the glass into the $\mathrm{LiNbO}_{3}$ crystallization domain.

However, under our irradiation condition, no ring-shaped crystalline phase was observed and the crystallization occurs at the center of laser-modified area, indicating that the pulse energy is suitable. But we have also to take into account that we used moving mode. Even if the pulse energy is high enough for melting, the movement will leave a crystallized zone in the back [17] and the center of the line will be finally crystallized if chemical migration has not changed the chemical formula too largely and thus has not changed the chemical system under consideration.

According to previous report [187], the $\mathrm{LiNbO}_{3}$ phase appears after demixing of LNS glass containing less than 25 mol\% of $\mathrm{Nb}_{2} \mathrm{O}_{5}$. Zeng et al. [233] demonstratedd that the crystallized glass of $25 \mathrm{Li}_{2} \mathrm{O}-25 \mathrm{Nb}_{2} \mathrm{O}_{5}-50 \mathrm{SiO}_{2}$ (mol\%) consists of crystalline phase $\mathrm{LiNbO}_{3}$ and non-crystalline phase $\mathrm{SiO}_{2}$. Ferreira et al. [162] reported that the glass $92 \mathrm{SiO}_{2}-4 \mathrm{Li}_{2} \mathrm{O}-4 \mathrm{Nb}_{2} \mathrm{O}_{5}(\mathrm{~mol} \%)$ decomposes into $\mathrm{LiNbO}_{3}, \alpha$-quartz, and $\beta$-cristobalite by $\mathrm{CO}_{2}$ laser irradiation. Thus, a phase separation under the thermal equilibrium conditions can be expected. Vigouroux [234] shown that the chemical composition plays an important role in crystallization or amorphous state of prepared LNS glass: totally opaque for $30 \mathrm{Li}_{2} \mathrm{O}-25 \mathrm{Nb}_{2} \mathrm{O}_{5}-45 \mathrm{SiO}_{2}$ (mol\%), partial crystallization for $32 \mathrm{Li}_{2} \mathrm{O}-33 \mathrm{Nb}_{2} \mathrm{O}_{5}-$ $36 \mathrm{SiO}_{2}$ (mol\%), and transparent glass for $35 \mathrm{Li}_{2} \mathrm{O}-25 \mathrm{Nb}_{2} \mathrm{O}_{5}-40 \mathrm{SiO}_{2}$ (mol\%). We succeeded in preparing the transparent glass $33 \mathrm{Li}_{2} \mathrm{O}-33 \mathrm{Nb}_{2} \mathrm{O}_{5}-34 \mathrm{SiO}_{2}$ (mol\%) following the pseudo-ternary phase diagram of $\mathrm{Li}_{2} \mathrm{O}-\mathrm{Nb}_{2} \mathrm{O}_{5}-\mathrm{SiO}_{2}$ (see details in Figure 34). 
Based on the above discussion, we deduce that the LNS glass used here is close to a thermodynamical product. This may explain why a phase separation occurs in this irradiated LNS glass and not a crystal with a chemical composition close to the initial formulation of the glass.

\section{3. fs laser-induced nanostructures}

fs laser-modified glass composed of nanostructure represents a significant route to design and fabricate new architectures in optics (e.g. such a nanostructure is useful for a 3D control of the birefringence) [235]. So far, such structures have been found exclusively in fused silica [85] and are based on planar modifications [4] including nanopores [5]. Therefore, the materials for obtaining such a useful nanostructure for the optical applications (point modification of the refractive index) are limited.

Figure 80 displays a similar nanostructure in Zone D of regime 3 or Zone $C$ of regime 2. In this section, the fs laser-induced structure is investigated in 3D by SEM and TEM. We describe the nanostructure and crystal texture in details when this one is available, for the three perpendicular planes: $\mathrm{YZ}, \mathrm{XY}$, and $\mathrm{XZ}$ (scanning direction is along $\mathrm{X}$ and laser propagation direction is along $+Z$ ). The comparison between this nanostructure and the reported fs laserinduced nanostructures is presented.

\subsubsection{Observation of nanostructures in 3D}

\subsubsection{Self-organized nanostructures and textures observed in the plane perpendicular to scanning direction (YZ plane)}

Firstly, the fs laser-induced structure was investigated by SEM in the plane perpendicular to writing direction. For zone D of regime 3, self-organized nanostructure can be clearly observed from SEM. It is discussed below according to the direction of the modulations (i.e. along the laser propagation direction or along laser polarization direction).

In the case of the laser polarization parallel to scanning direction (i.e. $\mathrm{X}$ direction), as shown in the Figure $82 a-b$, in region $D$ of regime 3 , we observed a periodic structure along laser propagation direction. The distance between these structure lines is about $280 \mathrm{~nm}$ around the head of the laser track $\left(\Lambda_{z}\right.$, shown in Figure $\left.82 c\right)$. The thickness of the structure line is about 190 $\mathrm{nm}\left(\mathrm{d}_{z}\right.$, illustrated in Figure $82 \mathrm{c}$ ). The magnified SEM image (left bottom in Figure 82b) shows a smooth morphology of the structure line (illustrated by blue lines in Figure 82d) for this writing configuration. 
Considering that the laser polarization is a key parameter controlling the orientation of nanostructure in silica glass [5], we changed the laser polarization (i.e. the laser polarization is rotated in order to be perpendicular to scanning direction).

When laser polarization is perpendicular to scanning direction, as shown in Figure 83a, four regions with three clear boundaries are detected (marked with A-D). Another quasi-periodic nanostructure perpendicular to the propagation direction is detected in Zone $D$, with a modulation in the direction of the polarization vector (Figure 83b). Such a nanostructure has been already reported in fused silica, and its orientation is found adjustable by polarization direction [236].
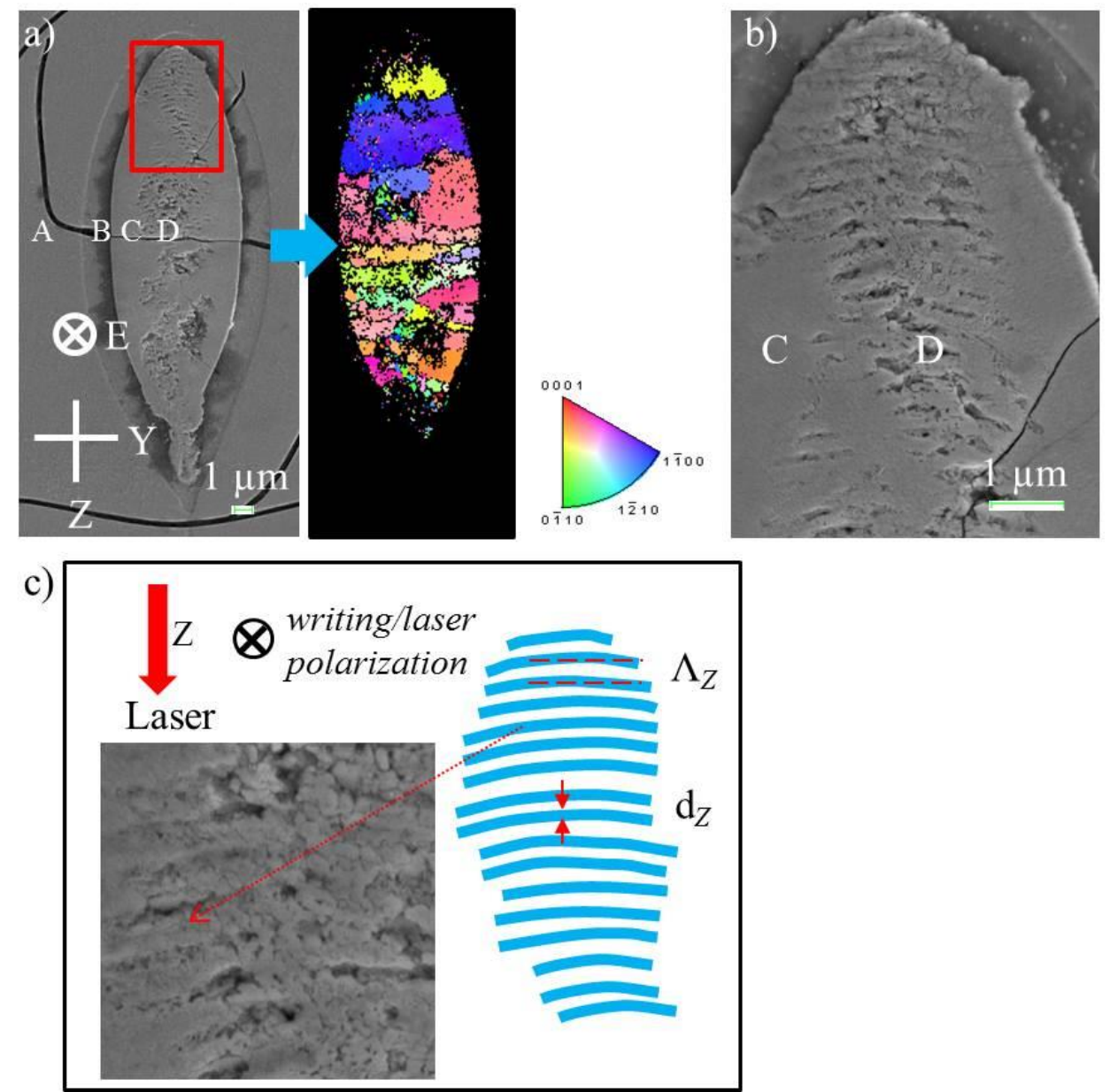

Figure 82 a) fs laser-induced four regions (quoted with A-D) of regime 3 with three clear boundaries (left) and the corresponding IPF showing crystallization and crystal orientation, coding along laser polarization direction (right), b) the magnification of Figure 82a marked with red rectangle, and c) schematic of the laser-induced modulated structures along the propagation direction (the magnified SEM showing the smooth structure line). Other parameters: $32.5 \mathrm{Li}_{2} \mathrm{O}-27.5 \mathrm{Nb}_{2} \mathrm{O}_{5}-40 \mathrm{SiO}_{2}$ (mol\%), $1030 \mathrm{~nm}, 500 \mathrm{kHz}, 300 \mathrm{fs}, 1.0 \mu \mathrm{J} / \mathrm{pulse}, 5 \mu \mathrm{m} / \mathrm{s}, \mathrm{NA}=$ 0.6 , focal depth $300 \mu \mathrm{m}$ in air, and laser polarization is parallel to scanning direction (i.e. X direction).

In the head of the laser track, this nanostructure is along the lines and along the laser polarization direction. It consists of dashes with a thickness around $50 \mathrm{~nm}\left(\mathrm{~d}_{E}\right.$ illustrated in Figure 
83c). These dashes are patterned parallel to each other. Along the polarization direction, the distance between these dashes around $100 \mathrm{~nm}\left(\Lambda_{E}\right.$, shown in Figure $\left.83 \mathrm{c}\right)$. Within region $D$, we also observed crystallization with nanostructure but the crystals are smaller and their orientations are more disordered, compared in the surrounding region C.

Furthermore, the fs laser-induced nanostructures were investigated varying pulse energies. Similar kind of dashes parallel to each other, aligned along laser polarization direction is observed in region D of regime 3 (Figure 83d-e). Their distance to each other seems not to vary significantly. The width of region $D$ increases with the increase of pulse energy $(\sim 3.2 \mu \mathrm{m}$ at 0.8 $\mu \mathrm{J} /$ pulse and $\sim 6.2 \mu \mathrm{m}$ at $1.4 \mu \mathrm{J} /$ pulse).
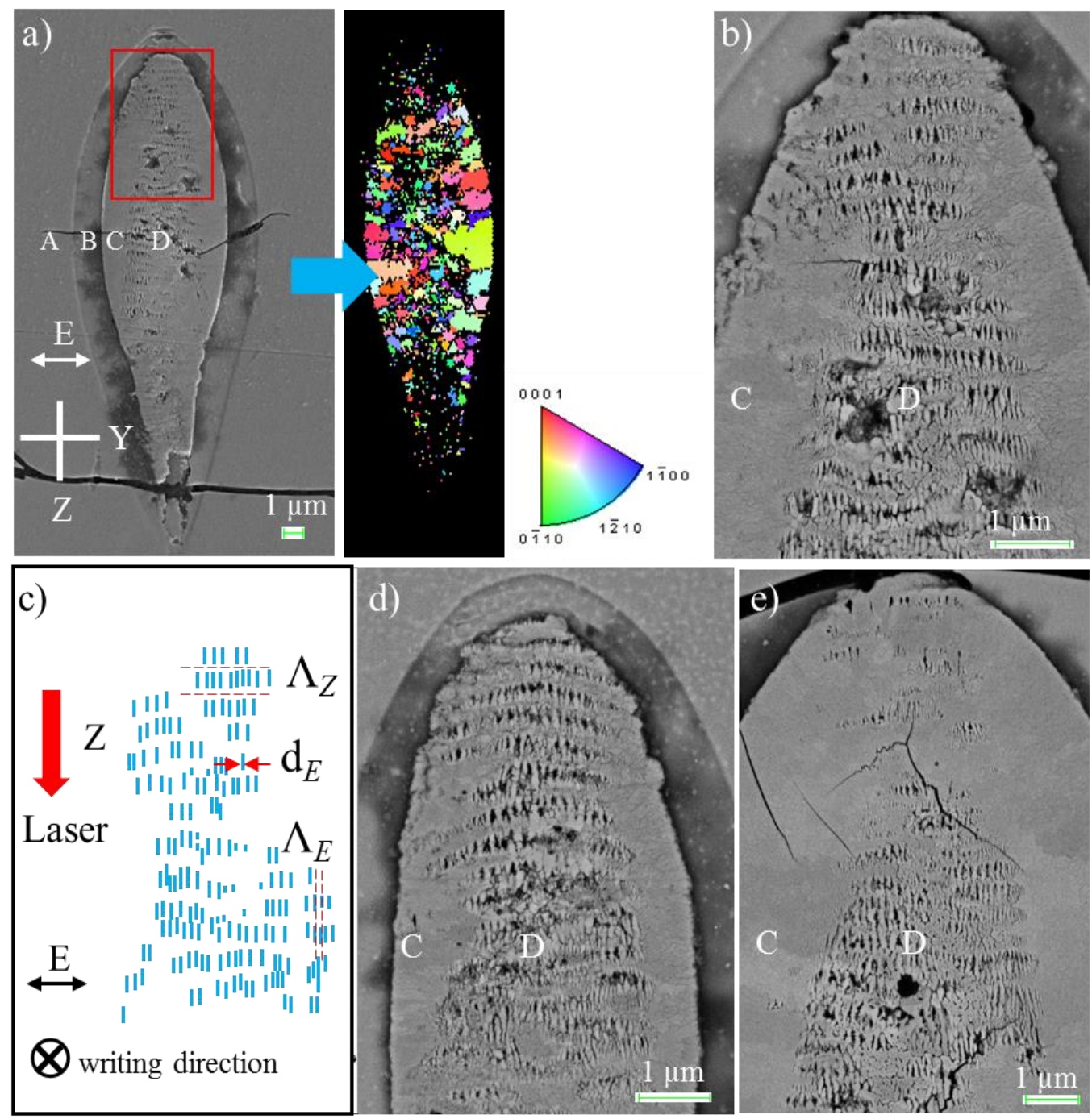

Figure 83 Morphology of the fs laser track, when laser polarization (i.e. Y direction) is perpendicular to scanning direction (i.e. $X$ direction): a) SEM image (left) and the corresponding IPF coding along laser polarization direction (right) for $1.0 \mu \mathrm{J} /$ pulse, b) the magnification of Figure 83a marked with red rectangle, and c) the schematic of the laser-induced structures. Morphology of the laser track varying the pulse energy: d) 0.8 and e) $1.4 \mu \mathrm{J} /$ pulse. Other parameters: $32.5 \mathrm{Li}_{2} \mathrm{O}-27.5 \mathrm{Nb}_{2} \mathrm{O}_{5}-40 \mathrm{SiO}_{2}(\mathrm{~mol} \%), 1030 \mathrm{~nm}, 500 \mathrm{kHz}, 300 \mathrm{fs}, 5 \mu \mathrm{m} / \mathrm{s}, \mathrm{NA}=$ 0.6 , focal depth $300 \mu \mathrm{m}$ in air 
In addition, Figure 84 displays the SEM images of irradiated glass before and after HF treatment. It can be seen that after HF treatment: the contrast in SEM becomes stronger; zone B (the one sensitive to HF etching) is revealed; the self-organized nanostructure in zone D of regime 3 can be clearly observed.

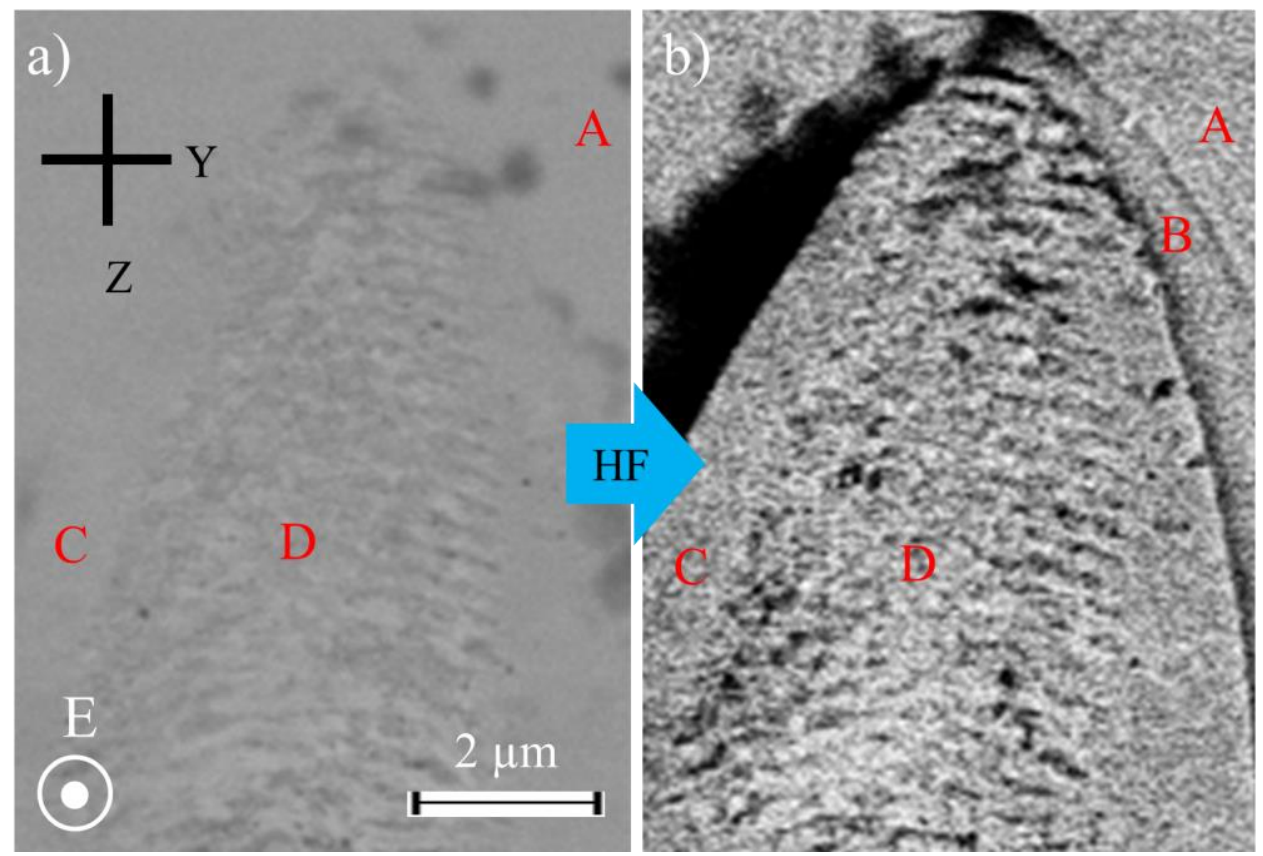

Figure 84 Comparisons of SEM images of irradiated LNS glass a) before and b) after HF treatment. It was observed in the plane perpendicular to scanning direction, E indicates the laser polarization direction, and laser polarization is parallel to scanning direction, laser propagation is along $\mathrm{Z}$ direction. Other parameters: $33 \mathrm{Li}{ }_{2} \mathrm{O}-$ $33 \mathrm{Nb}_{2} \mathrm{O}_{5}-34 \mathrm{SiO}_{2}$ (mol\%), $1030 \mathrm{~nm}, 300 \mathrm{fs}, 300 \mathrm{kHz}, 1.3 \mu \mathrm{J} / \mathrm{pulse}, 5 \mu \mathrm{m} / \mathrm{s}$, and $\mathrm{NA}=0.6$, focal depth $350 \mu \mathrm{m}$ in glass, laser polarization direction is parallel to writing direction.

\subsubsection{Self-organized nanostructure investigated in the direction perpendicular to laser propagation direction (XY plane)}

TEM was conducted in order to get a high resolution of the above nanostructure. Focused ion beam was used to dissect the irradiated lines to intercept the nanostructure in the plane perpendicular to laser propagation direction (i.e. XY plane).

As shown in Figure 85a (TEM, bright field), a lamella-shaped nanostructure, oriented perpendicular to laser polarization, was observed in zone $\mathrm{C}$ of regime 2 (defined in Figure 55b). The clear boundary (marked with red arrows) between the glass matrix and the modified amorphous zone can be clearly observed from TEM image. The fs laser-modified amorphous part (left part in Figure 85b) shows less density fluctuation than the glass matrix (right part in Figure 85b). With irradiations, it is recognized that the Rayleigh scattering increases [237, 238]. Recently, Zimmermann et al. [202] shown that the fictive temperature increases a lot after irradiation as we have suggested in previous work [201]. The Rayleigh scattering is related to 
density fluctuations as it was shown by Lines [239]. From the above work, it can be deduced that fs irradiation can modify the homogeneity of the glass as shown in Figure 85b.

The crystallization is not always together with lamella-shaped nanostructure formation. For regime 3, oriented lamella-shaped nanostructures were detected only in zone D but not in zone C.
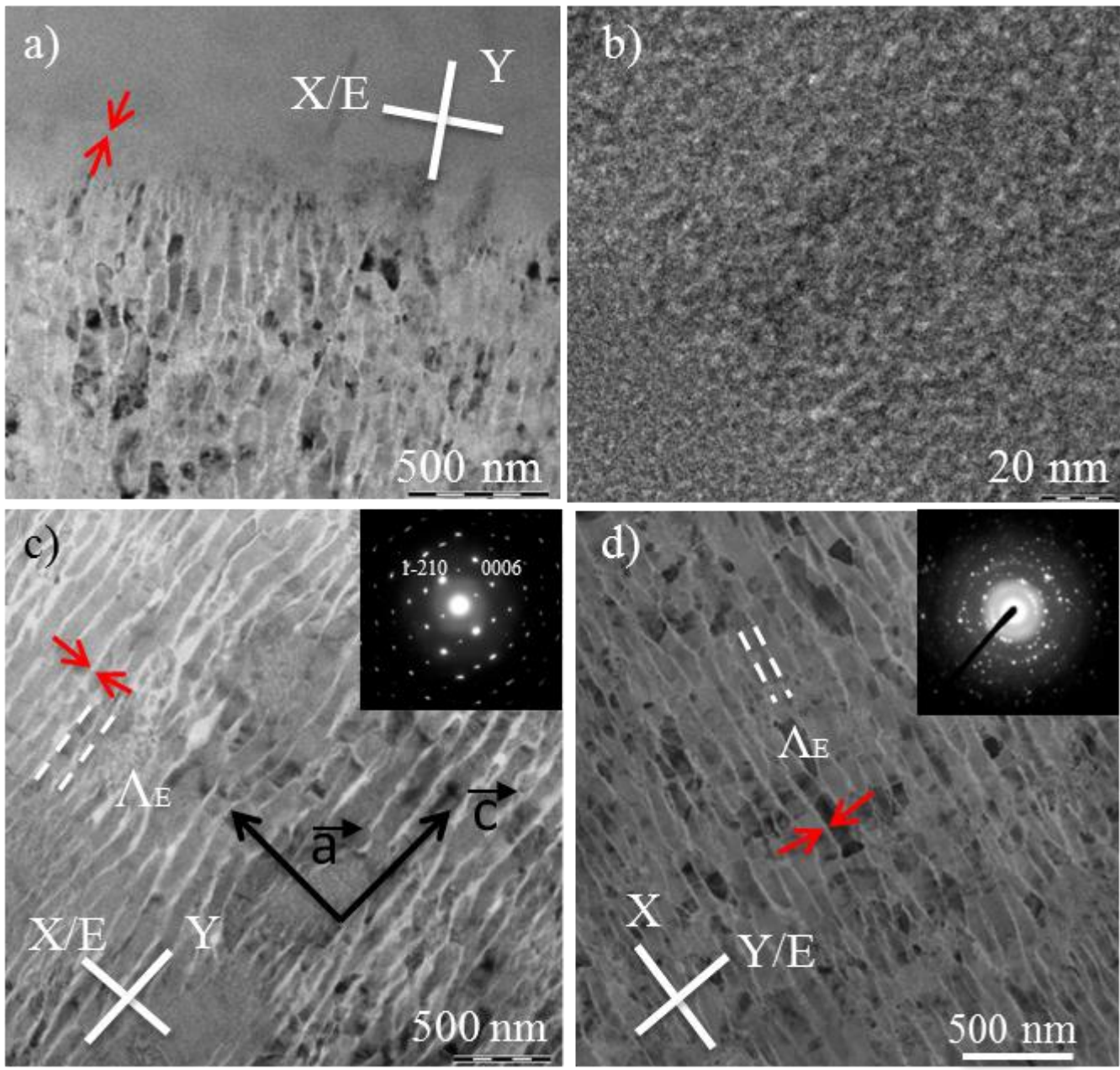

Figure 85 Nanostructure formation in LNS glass in the plane perpendicular to laser propagation direction: TEM images of the laser modified area (bright field). a-b) $0.7, c-d) 1.8 \mu \mathrm{J} /$ pulse. a-c) laser polarization is parallel to scanning direction and d) laser polarization is perpendicular to scanning direction. The insets of a, c-d) are the corresponding SAED patterns. In the c) case for which the texture is well defined, we can see that the crystalline phase produced is $\mathrm{LiNbO}_{3}$. E indicates the laser polarization direction. Other parameters: $33 \mathrm{Li}_{2} \mathrm{O}-$ $33 \mathrm{Nb}_{2} \mathrm{O}_{5}-34 \mathrm{SiO}_{2}$ (mol\%), $1030 \mathrm{~nm}, 300 \mathrm{fs}, 300 \mathrm{kHz}, 5 \mu \mathrm{m} / \mathrm{s}, \mathrm{NA}=0.6$, focal depth $350 \mu \mathrm{m}$ in glass.

When laser polarization is parallel to scanning direction, as illustrated in Figure 85c (TEM, bright field), a lamella-shaped nanostructure oriented perpendicular to $X$ (the direction of laser polarization) is revealed. Black parts are embedded in white lamella (about $24 \mathrm{~nm}$ in width, illustrated by red arrows in Figure 85c). The quasi-period of this oriented nanostructure is about $117 \mathrm{~nm}\left(\Lambda_{\mathrm{E}}\right.$, shown in Figure $\left.85 \mathrm{c}\right)$. The white part is amorphous and the black part is crystalline. 
Selected area electron diffraction (SAED), a technique similar to X-ray diffraction but with a better resolution than the conventional one, can be used to identify crystal structures. The SAED image (inset in Figure 85c) reveals the single crystalline pattern of the black part in TEM image (bright field). It is indexed with the hexagonal structure of $\mathrm{LiNbO}_{3}(\mathrm{a}=0.515 \mathrm{~nm}, \mathrm{c}=0.1386 \mathrm{~nm}$, R3c). On this projection, lamellas are elongated along the c-axis of the hexagonal structure.

When laser polarization is perpendicular to scanning direction, similar lamella-shaped nanostructure, which is oriented perpendicular to laser polarization direction is observed when changed the laser polarization. The quasi-period of this nanostructure $\left(\Lambda_{E}\right.$, shown in Figure $\left.85 \mathrm{~d}\right)$ is the same as the one when laser polarization is parallel to scanning direction (presented in Figure 85c). But the SAED pattern of the black part (inset in Figure 85d) appears polycrystalline. The electron diffraction pattern does not reveal any common crystallographic axis between nano-crystals embedded in the self-organized nanostructure.

\subsubsection{Self-organized nanostructure investigated in XZ plane}

To have a clear picture of the self-organized nanostructure in 3D, the nanostructure was investigated by TEM in XZ plane.

When laser polarization is perpendicular to scanning direction, as illustrated in Figure 86a,

1) A lamella-shaped nanostructure is oriented perpendicular to laser polarization ( $\sim 19 \mathrm{~nm}$ in width, marked by red arrows in Figure 86a) with periodicity of about $101 \mathrm{~nm}\left(\Lambda_{E}\right.$, shown in Figure 86a);

2) The major axis of the white lamella is oriented along laser propagation direction;

3) The density of this oriented nanostructure decreases along laser propagation direction.

4) The SAED image (inset in Figure 86a) reveals the single crystalline pattern when laser polarization is parallel to scanning direction. As previously, for the XY plane (Figure 85c), the diffraction diagram corresponds to the hexagonal structure of $\mathrm{LiNbO}_{3}$.

When laser polarization is perpendicular to scanning direction, in contrast to the lamella-shaped nanostructure observed above, randomly oriented crystals are detected (Figure 86b).

Based on the above discussion, the schematic of the fs laser-induced 3D structures when laser polarization is parallel to scanning direction is presented in Figure 86c. The one when laser polarization is perpendicular to scanning direction is illustrated in Figure $86 \mathrm{~d}$. The lamella- 
shaped nanostructure was illustrated in red and the random distributed crystal is shown in purple.
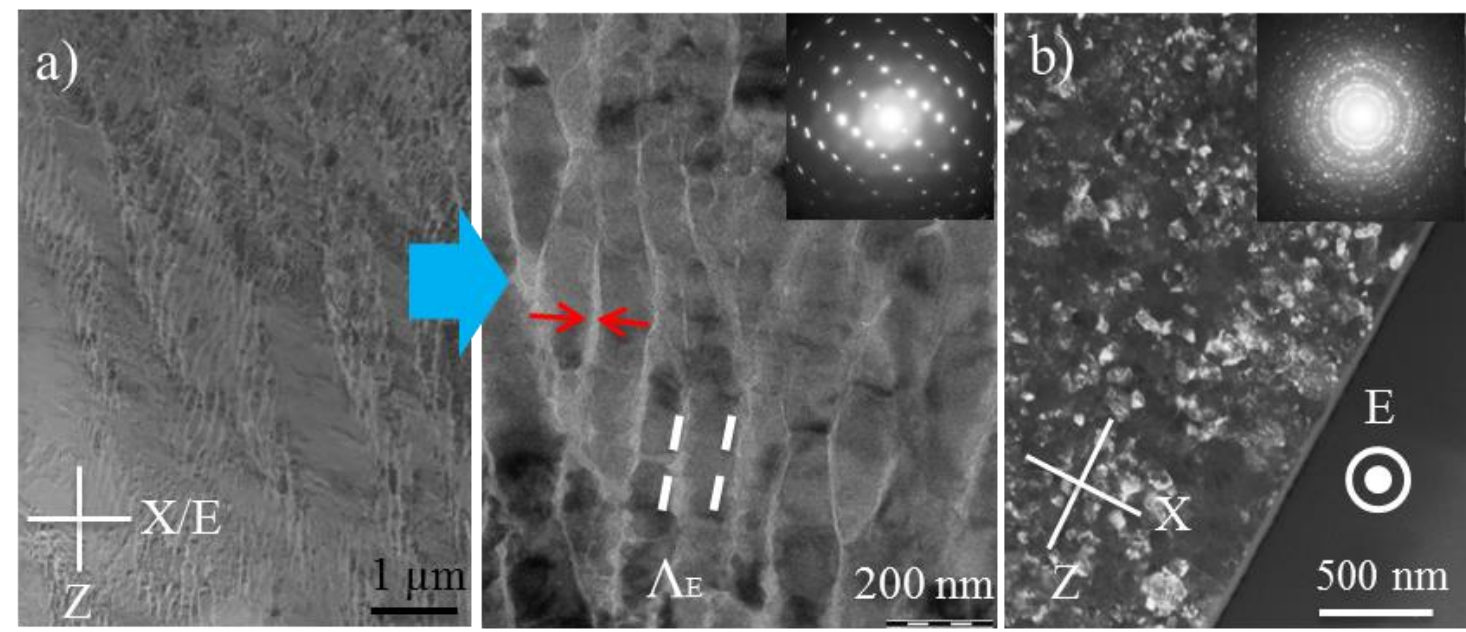

c)
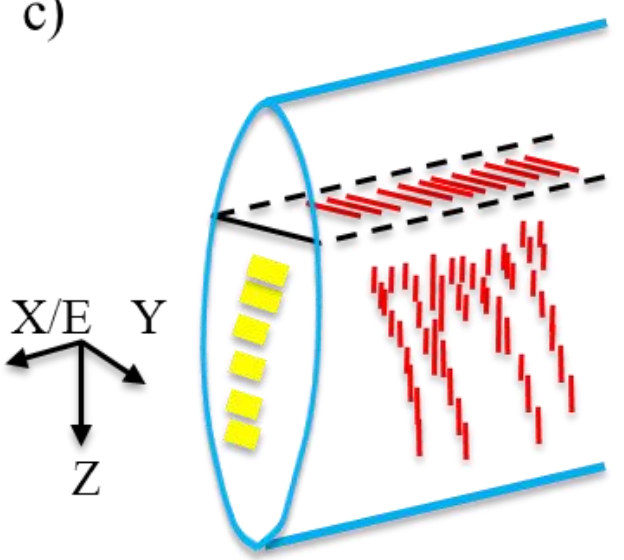
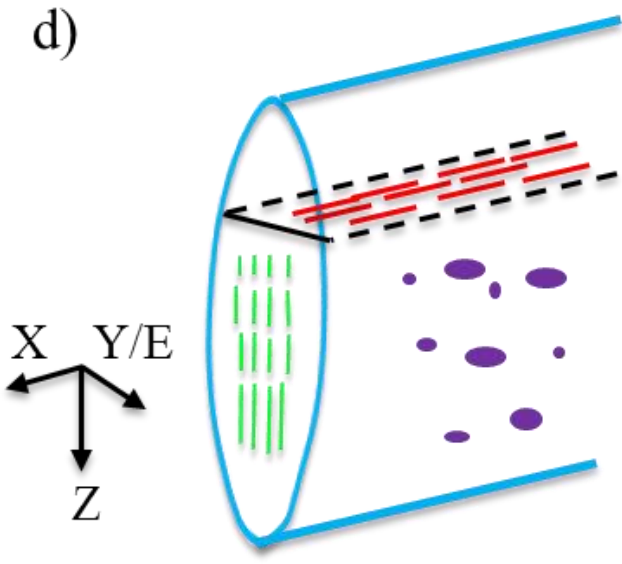

Figure 86 fs laser-induced nanostructures investigated in XZ-plane: a) TEM images of the laser trace (the inset is the SAED) for laser polarization direction is parallel to writing direction. b) The similar information for the one when laser polarization is perpendicular to writing direction. In the a) case for which the texture is well defined, we can see that the crystalline phase produced is $\mathrm{LiNbO}_{3}$. Other parameters: $33 \mathrm{Li}_{2} \mathrm{O}-33 \mathrm{Nb}_{2} \mathrm{O}_{5}-34 \mathrm{SiO}_{2}(\mathrm{~mol} \%)$, $1030 \mathrm{~nm}, 300 \mathrm{fs}, 300 \mathrm{kHz}, 1.8 \mu \mathrm{J} /$ pulse, $5 \mu \mathrm{m} / \mathrm{s}, \mathrm{NA}=0.6$, focal depth $350 \mu \mathrm{m}$ in glass. Schemes of fs laserinduced nanostructure in $3 \mathrm{D}$ for laser polarization is $\mathrm{c}$ ) parallel and d) perpendicular to writing direction.

\subsubsection{Distribution of crystalline and amorphous phases}

To have a clear picture of this fs laser-induced nanostructure, chemical analyses of $\mathrm{Nb}$ and $\mathrm{Si}$ were conducted by STEM/EDS in another case with different pulse energy and writing configuration (1.8 $\mu \mathrm{J} /$ pulse, laser polarization is perpendicular to scanning direction) compared with the one measured in Figure $79(1.3 \mu \mathrm{J} /$ pulse, laser polarization is parallel to scanning direction). The STEM-HAADF image (Figure 87a), displays alternated white region ( $100 \mathrm{~nm}$ in width) and black region ( $25 \mathrm{~nm}$ in width) at the center of laser-modified area. Considering that STEM-HAADF is a method sensitive to variations in the atomic number of the material [222], the contrast in this image is due to the separation of $\mathrm{Nb}$ and $\mathrm{Si}$. The white regions in STEM image 
(dark field) are rich in $\mathrm{Nb}$ and the black ones are rich in Si. The element mappings of these two elements are shown in Figure 87b and c. As shown in Figure 87d, the concentration of $\mathrm{Nb}$ and $\mathrm{Si}$ vary reversely (profile mode, illustrated by the dashed line marked in Figure 87a-c). In other words, a nanoscale chemical migration of $\mathrm{Nb}$ and $\mathrm{Si}$ is revealed, even when changed the writing configuration or pulse energy (comparing with Figure 79).

Furthermore, EBSD was used to determine the crystalline and amorphous phase distribution. Image quality (IQ) map constructed from EBSD data provides an effective approach to visualize microstructure [213]. As shown in Figure 88a, an alternation of bright and dark zones are observed, indicating the periodic phases. The IPF (Figure 88b) demonstrates that the bright zones of IQ image are crystalline whereas dark zones are amorphous, revealing a phase separation at the nanoscale.

To go a step further, a composite image of IQ and IPF is shown in Figure 88c. It is interesting to point out that a periodic nanostructure can be obtained from IQ but not from IPF (marked with red arrows). This is due to the EBSD date treatment, such as "clean up" process [212]. "Clean up" process is a method in the software (OIM ${ }^{\mathrm{TM}}$ Data Analysis) to treat the points that did not give rise to diffraction or to yield too weak diffraction pattern but having neighboring diffracting points. If the misorientation among the neighboring points is within the grain tolerance angle, they will belong to the same "grain" after clean up treatment. This means that, if a small amorphous region is located among large crystals that belong to the same "grain", the amorphous region will be considered as crystallized with the orientation of the major "grain", thus hiding the amorphous phase. Generally, one to two times "clean up" process is used in EBSD data analysis. In other words, there is a risk in using "clean up" process in EBSD data treatment exaggeratedly to introduce artifacts, especially in our case with nanoscale phase separation of crystalline and amorphous phases.

The combination of TEM, STEM/EDS, and EBSD observations demonstrate a fs laser-induced phase separation into $\mathrm{LiNbO}_{3}$ and $\mathrm{SiO}_{2}$ together with the self-organized nanostructure in zone $\mathrm{C}$ of regime 2 or zone D of regime 3 in LNS glass. 

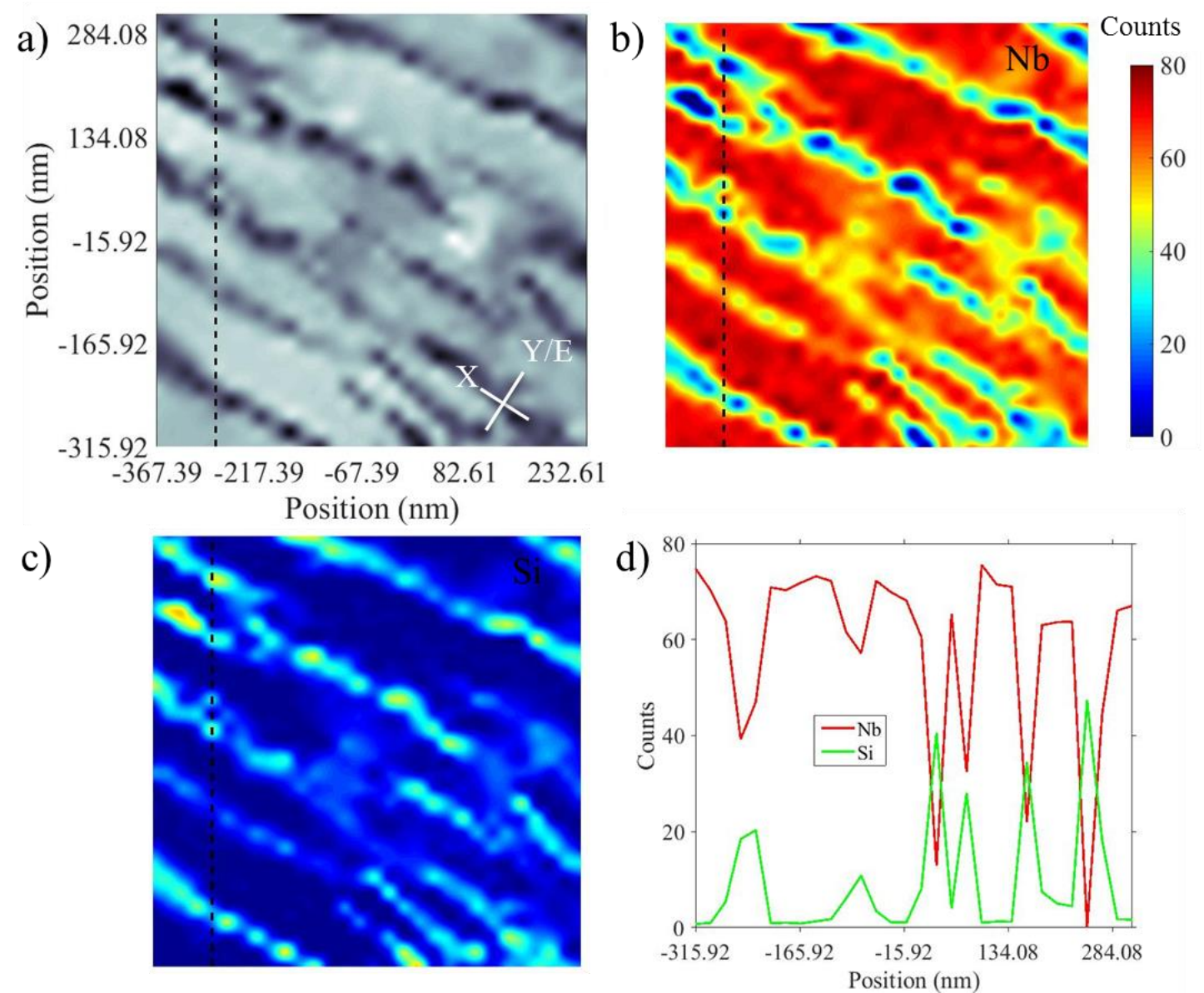

Figure $87 \mathrm{fs}$ laser-induced nanoscale chemical migration in LNS glass: a) STEM-HAADF image, elemental mappings of b) $\mathrm{Nb}, \mathrm{c}$ ) $\mathrm{Si}$, and line profile of $\mathrm{Nb}$ (red line) and $\mathrm{Si}$ (green line); $\mathrm{E}$ indicates the laser polarization direction. Other parameters: $33 \mathrm{Li}_{2} \mathrm{O}-33 \mathrm{Nb}_{2} \mathrm{O}_{5}-34 \mathrm{SiO}_{2}$ (mol\%), $1030 \mathrm{~nm}, 300 \mathrm{fs}, 300 \mathrm{kHz}, 1.8 \mu \mathrm{J} /$ pulse, $5 \mu \mathrm{m} / \mathrm{s}$, $\mathrm{NA}=0.6$, and focal depth $350 \mu \mathrm{m}$ in glass.
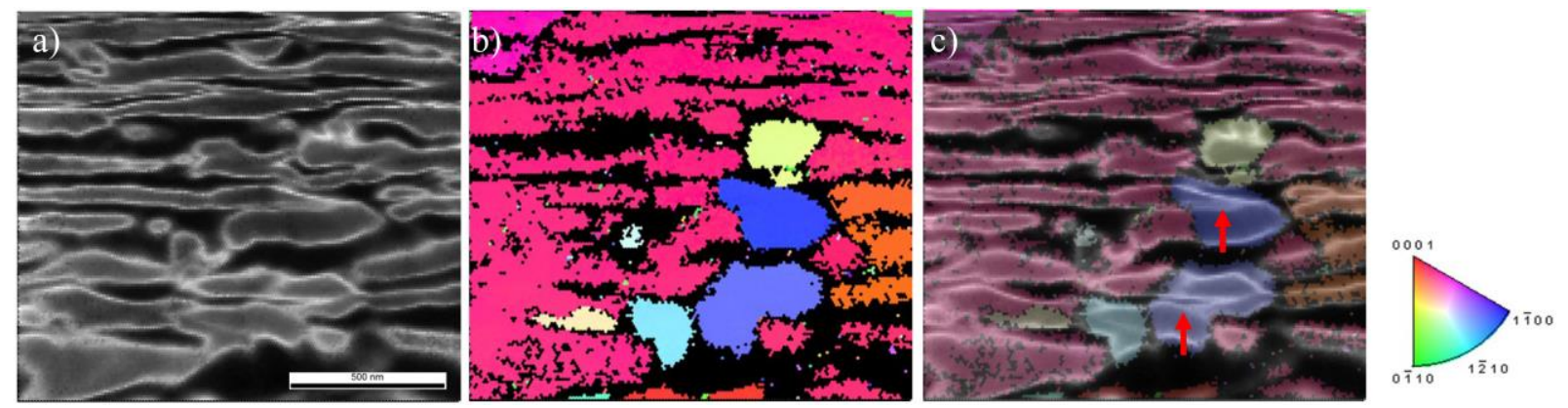

Figure $88 \mathrm{fs}$ laser-induced nanoscale phase separation in LNS glass: a) quality image (IQ), b) IPF (colors are based on $\mathrm{R} 3 \mathrm{C}$ group, $\mathrm{LiNbO}_{3}$, coding along laser propagation direction), and c) composite image: IQ+IPF. Other parameters: $33 \mathrm{Li}_{2} \mathrm{O}-33 \mathrm{Nb}_{2} \mathrm{O}_{5}-34 \mathrm{SiO}_{2}$ (mol\%), $1030 \mathrm{~nm}, 300 \mathrm{fs}, 300 \mathrm{kHz}, 1.3 \mu \mathrm{J} /$ pulse, $5 \mu \mathrm{m} / \mathrm{s}$, NA = 0.6, and focal depth $350 \mu \mathrm{m}$ in glass. 


\subsubsection{Relation with known similar structures}

It is worth noting that the crystallization is not always accompanying a nanostructure (i.e. region C of regime 3). The crystallization/nanostructures system (i.e. region D of regime 3 ) is discussed below according to the direction of the modulations (i.e. along the laser propagation direction or along laser polarization direction).

\subsubsection{Nanostructure aligned along the laser propagation direction}

Kazansky et al. [235] have observed a periodic structure (period close to $\lambda / n$ ) in the head of laser track and the period increases as a square root of the distance from the sample edge. They proposed an exciton-polariton (interference between exciton and light) leading to an aperiodic polarisation grating in the direction of light propagation. However, as shown in Figure $82 \mathrm{c}$, a period of $\sim 280 \mathrm{~nm}$ is around $\lambda / 2 \mathrm{n}\left(\lambda=1030 \mathrm{~nm}\right.$, and $\mathrm{n}$ is around 1.8 for $32.5 \mathrm{Li}_{2} \mathrm{O}-27.5 \mathrm{Nb}_{2} \mathrm{O}_{5^{-}}$ $40 \mathrm{SiO}_{2}$, mol\% glass) and no obvious increase of the period is detected in the direction of laser propagation, which cannot be explained by the above mechanism.

\subsubsection{Nanostructure aligned along the laser polarization direction}

Shimotsuma et al. [31] who firstly published on nanostructure in silica, have proposed that interference between the incident laser field and electron plasma waves (plasmon) leads to a periodic modulation of the excited electron plasma concentration and thus to a periodic modulation of the permanent structural changes in silica glass after electron trapping in the lattice. Bhardwaj et al. [33] suggested another explanation based on inhomogeneous plasma density: multi-photon ionization can produce series of nano-plasma that can evolve into arrayed planes recorded in the modified material with their normal parallel to the laser polarization direction. Under some special conditions, they can self-organize in quasi periodicity leading to period around $\lambda / 2 \mathrm{n}$ [33]. Moreover, Liao et al. [240] have shown that the constructive interference of the scattering light from nanostructures formed in the early stage, can create an intensity maximum located between the two adjacent nanoplanes, thus leading to the shortening of the nanograting period by half $(\lambda / 4 n)$.

Quite recently, Rudenko et al. [241] have reported simulations that show that conduction band electron density can form nanoplasmas elongated into nanoplanes perpendicular to the laser polarization. They confirm the role played by interfering scattering waves but they show that the quasi-periodicity $(\lambda / p n$ with $p=2,3,4)$ is determined by the chemical inhomogeneity density. In our case, as illustrated in FIG. 2b, the periodicity along laser polarization is around 50-100 nm 
which is close to $\lambda / 5 n-\lambda / 11 n$. This can be explained by the mechanism proposed by Rudenko et al. [241] taking into account that the glass we used is likely not to be so homogeneous as industrial silica glass.

Yang et al. [91] have demonstrated a period of 240-450 $\mathrm{nm}$ for $\lambda=1045 \mathrm{~nm}$ and $\mathrm{n}=1.45$ in fused silica. Richter et al. [7] have pointed out a period of $250 \mathrm{~nm}(\lambda=800 \mathrm{~nm})$ in ULE ${ }^{\mathrm{TM}}$, which is corresponding to $\lambda / 2 n(n=1.48)$. But Zimmermann et al. [90] have reported a period down to 60 $\mathrm{nm}(\lambda=800 \mathrm{~nm})$ in borosilicate glass and this corresponds to $>\lambda / 9 n$ with $\mathrm{n}=1.511$ [242]. However, very often, if the nanoplanes are structured with their normal parallel to the laser polarization, they are not well ordered and a periodicity is hardly defined like we can see in Figure 83.

Looking at the effect of the pulse energy, Yang et al. [91] have reported that for pure silica, the period along laser polarization decreases by $20 \%$ about from 0.8 to $1.4 \mu \mathrm{J} /$ pulse. However, in our case, no variation is detected (illustrated in Figure $83 \mathrm{~b}, \mathrm{~d}$ and e).

The final question is if the crystallization can play a role in the organization of the nanostructure. Nanostructure occurs without crystallization in some glasses, but when crystallization appears, are the specificities of the nanostructure the same (e.g. the interplane distance, the amplitude modulation)? There is a lack of information to conclude on that point. Reversely, is there an effect of the nanostructure on the crystallization? It is worth noting that no bubble was observed in our case. Is the texture influenced by the existence of the self-organized nanostructure? It is likely not as the same texture is measured in Figure $82 \mathrm{a}$ in zone $\mathrm{C}$ and D. This leads to consider that light is acting directly on the crystallization process through a non-thermal process.

\subsubsection{Formation of oriented nanostructure interpretation}

No one in the domain of $\mathrm{fs}$ laser irradiation mentioned a crystallization coexisting with a nanostructure. But they investigated the birefringence magnitude that is produced by nanostructure including self-organized bubbles. Zimmermann et al. [90] reported that the radiation produces small nanopores (10-20 nm thick) which start to self-organize in parallel nanoplanes as well as elongated sheets of up to $400 \mathrm{~nm}$ length along the pulse accumulation (shown in Figure 89). 
In the previous cases, the bubbles form first in the electric hot region, and the nanostructure forms afterwards by alignment [2]. Here, we did not detect any bubble even at high resolution during TEM investigation. Thus, we can thus deduce that the mechanism is different.

For irradiated LNS glass, it may be important to determine which occurs first between the nanostructure and the crystallization. If we consider a thermal process, Sigaev et al. [187] mentioned a glass demixing with the formation of nanoheterogeneities that crystallize into $\mathrm{LiNbO}_{3}$ afterwards upon continuous heating. As the migration of these heterogeneities is not possible (contrary to nanobubbles), we deduce that the driving force or the canvas of the nanostructure exists before and drives the chemical separation. The $\mathrm{SiO}_{2}$ content would be concentrated in the equivalent of nanoplanes, where we assume that the local excitation is very high and $\mathrm{LiNbO}_{3}$ would stay between colder regions. The nanostructure, which is dependent on laser polarization (i.e. oriented perpendicular to laser polarization direction) might be affected by the heterogeneity density as described in previous work [241].

a)

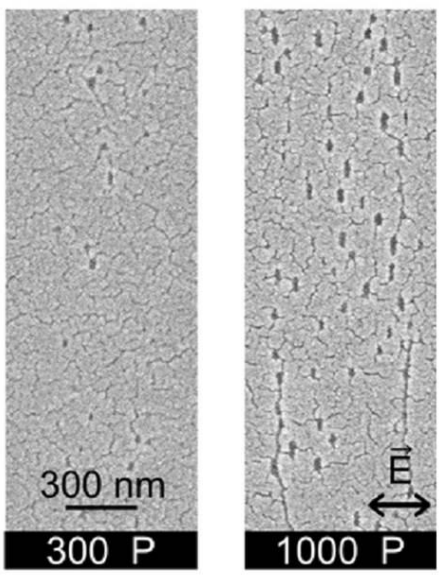

b)

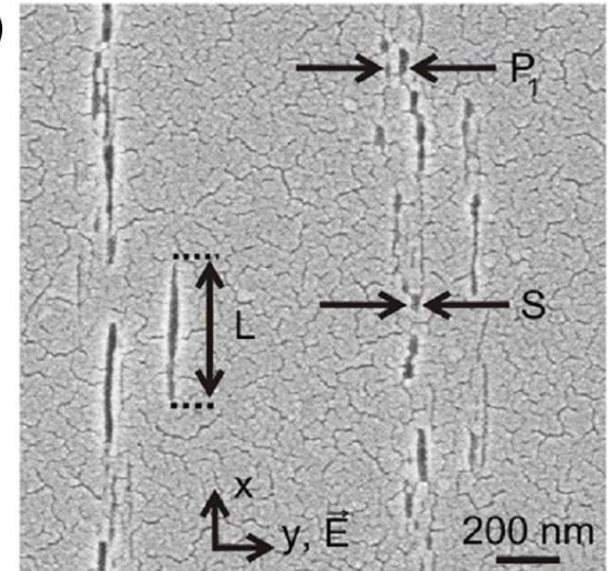

Figure $89 \mathrm{fs}$ laser-induced nanogratings in borosilicate glass: the SEM images indicate laser written traces ( $x-y$ plane) after polishing and etching, a) 300 P, 1000 P respectively, and b) 2000 P, $130 \mathrm{~nJ}, \mathrm{P}$ denotes the incident pulse number (cited from [90])

It is worth nothing that ordered structures could be obtained by cooling eutectic compositions: decomposition of the liquid into two solid phases by an eutectic reaction, which is limited by diffusion and reaction $[243,244]$. However, in this case, the light has no effect on the crystal orientation, contrary to the results shown in Section 4.1.2.2.

To better understand crystalline process and phase separation in LNS glass under irradiation, it was heated following thermo-dynamical equilibrium by floating zone method.

The process of crystal growth by optical floating-zone method is illustrated in Figure 90a. Two pieces of glass (in yellow) was fixed in vertical position. The optical floating zone furnace is 
equipped with several lamps (in red). The temperature is controlled by ellipsoidal mirrors (in green) focusing the image of the lamps at the common focal point. The temperature of the molten zone was controlled by adjusting the current through the lamps. Once the two pieces of glass is melting and connected, the current are stopped rapidly. The glass after irradiation is shown in Figure 90b. A neck-shaped structure was observed in the molten zone part (marked with red arrow). Then the irradiated glass was polished to expose the melting zone for the following measurements. X-ray Diffraction (XRD) was conducted in ICMMO.

a)

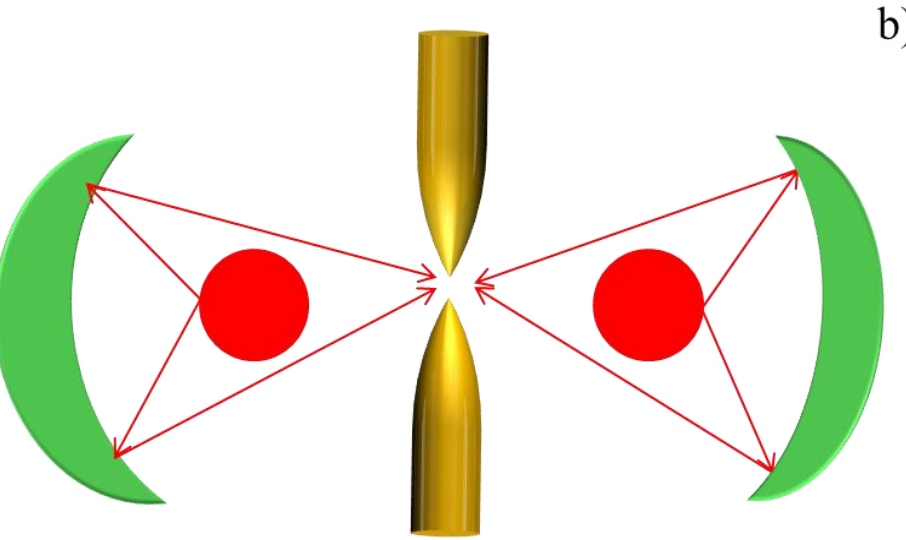

b)
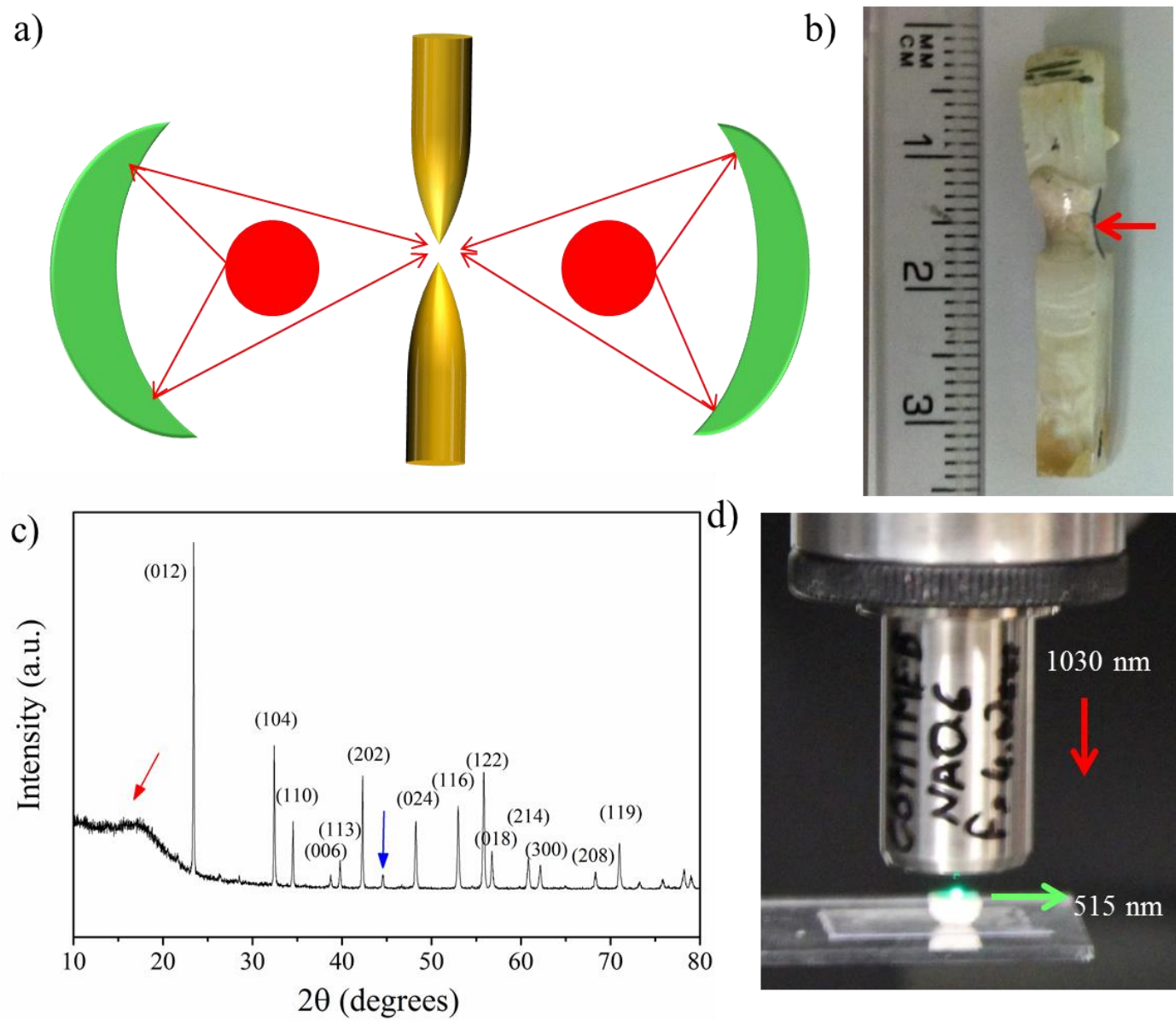

d)

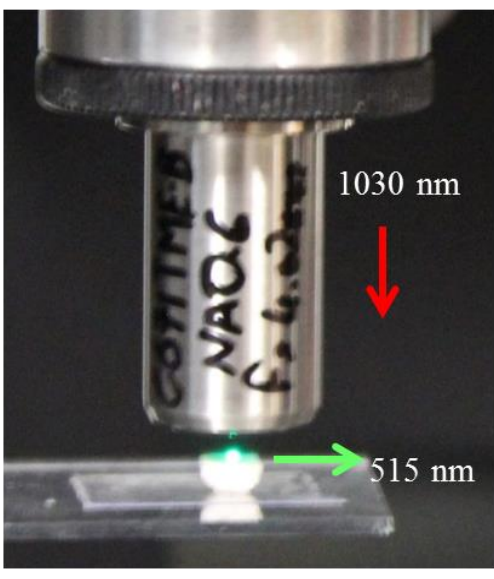

Figure 90 Nonlinear optical crystal precipitation by optical floating-zone method: a) diagram of the optical floating zone method set-up (glasses are marked in yellow, the infrared radiation sources in red, and the ellipsoidal mirrors in green), b) a sample photo, c) XRD pattern, and d) SHG image of irradiated glass.

Firstly, the formation of crystalline phase in molten zone was confirmed by X-ray diffraction (XRD). As illustrated Figure 90c, the main peaks are attributed to lithium niobate $\left(\mathrm{LiNbO}_{3}\right)$. The peak around $44.5^{\circ}$ (marked by blue arrow) is due to aluminum-made sample holder. A broad peak occurs in the range of 10 to $20^{\circ}$, indicating the existence of amorphous in the melted zone. Then, the nonlinear optical property (i.e. second harmonic generation, SHG) of molten zone was investigated in Figure 90d. Considering the fundamental wave $(1030 \mathrm{~nm})$, the observed green 
irradiation $(515 \mathrm{~nm})$ is due to the precipitate of nonlinear optical crystals ( $\left.\mathrm{LiNbO}_{3}\right)$ [109], which is in agreement with the XRD result.

The morphology of irradiated glass from the non-melted zone to the melted one (left to right) is shown in Figure 91a. It changes from small sand-shaped (marked with green rectangle) to polygon (marked with red rectangle) then to star-shaped (marked with yellow rectangle) until a smooth part. The melted zone consists of discrete regions with parallel lines oriented differently (marked with red arrows). The magnified picture (Figure 91b), reveals a second structure between these parallel lines, which can be clearly illustrated in Figure 91c: a lamella-shaped structure embedded in the above parallel lines. Cellular and dendritic microstructures are typical solidified structure in metal alloys [245]. In our case, a new type of solidified microstructure (i.e. a parallel line structure) was observed in glass ceramics.
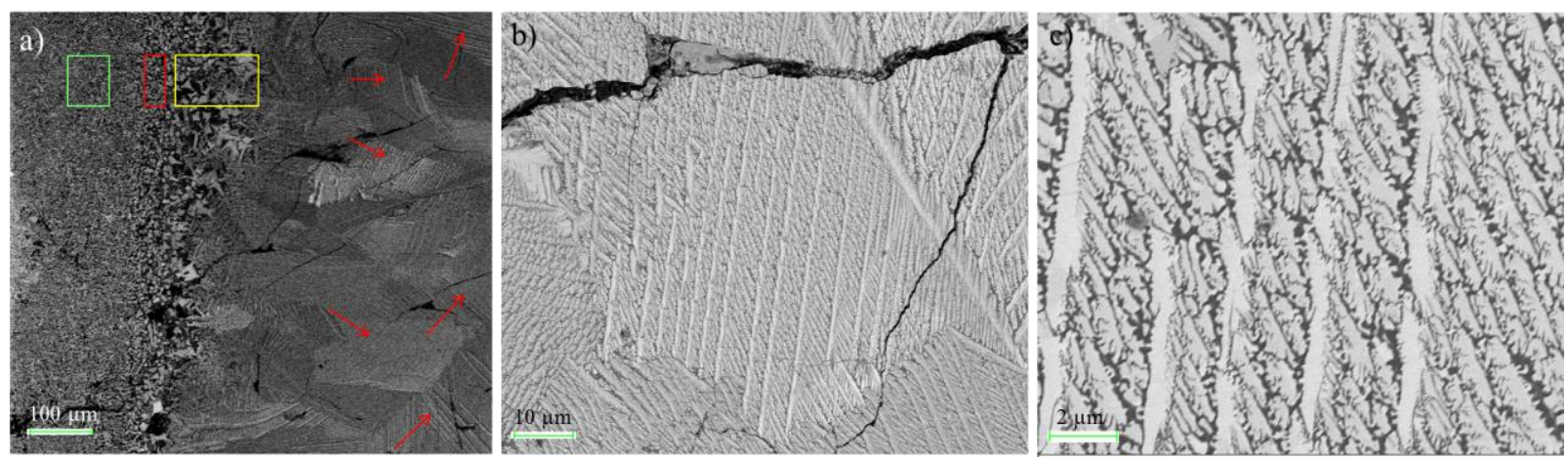

Figure 91 Microstructure in irradiated LNS glass observed by SEM

Furthermore, the spatial nonlinear optical crystal was investigated by EBSD. Image quality (IQ) map constructed from EBSD data provides an effective approach to visualize microstructure [213]. It is a map with a grayscale component according to the relative intensity of the diffraction pattern, generally, with the maximum IQ value in white and the minimum one in black. The contrast in this image may be due to the phase, strain, and grain boundaries.

To have a clear picture of the microstructure observed in Figure 91. IQ map (Figure 92a) was used together with IPF (Figure 92b) to provide more information. We found that from the nonmelted zone to melted zone, the crystal grains grow from random oriented grains to micrometer scale ordered grains. Crystal region as larger as $200 \mu \mathrm{m}$ can be observed in the melted zone (the yellow one in Figure 92b). In the melted zone, the parts with different series of parallel lines own crystal grains with different orientation (i.e. various color in Figure 92b).

As presented in Figure 93a, the chemical analysis including $\mathrm{O}$ (green), Si (red), and $\mathrm{Nb}$ (orange) was recorded by SEM/EDS. Interestingly, no obvious migration of above element was observed 
from the melted zone (left) to the not melted zone (right). The fluctuation of chemical profile is larger in the right than in the left side. It is rougher in the un-melted zone but smoother in the melted zone, especially for the interface, where there is a large quantity of holes and smaller grains.
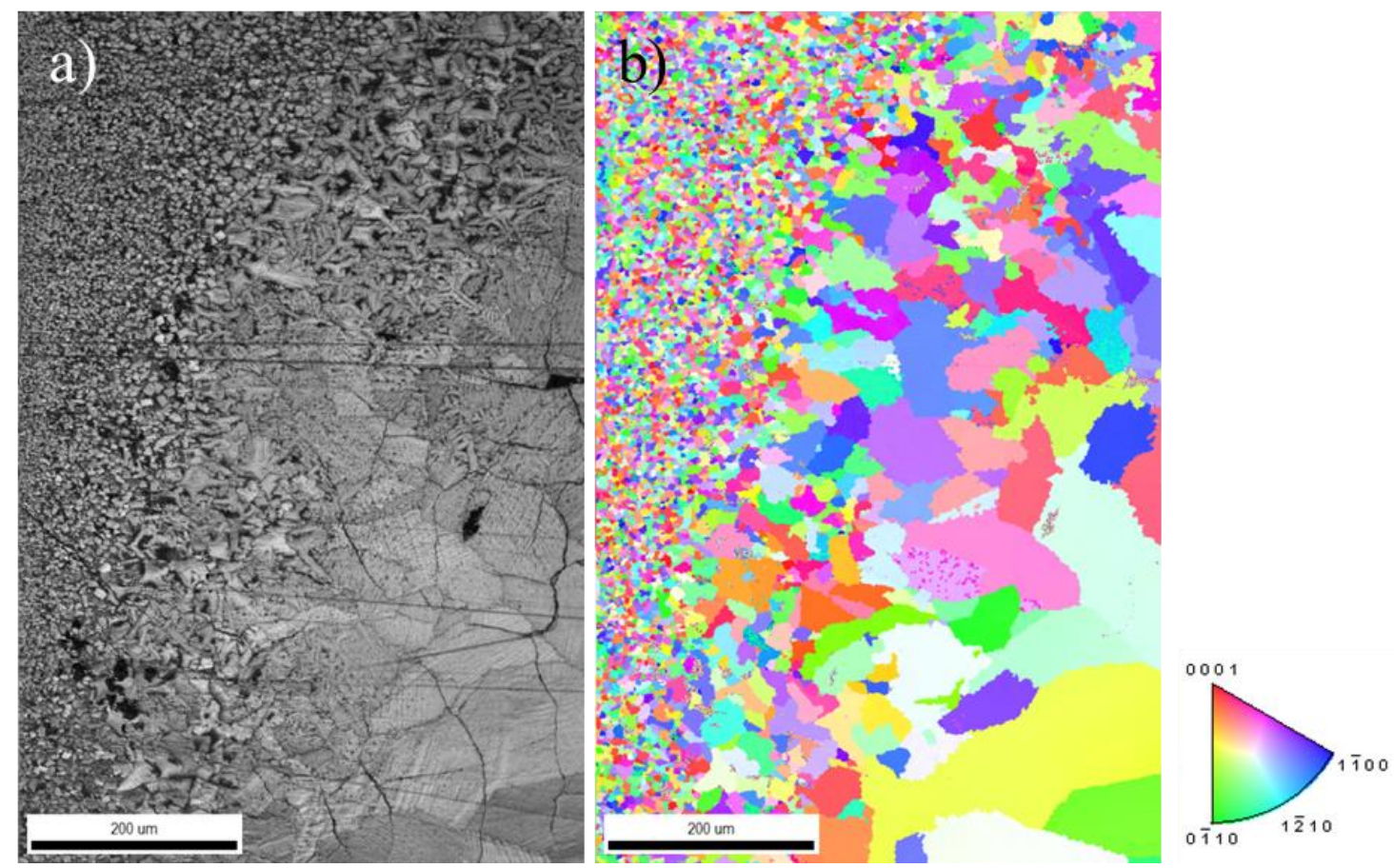

Figure 92 Spatial crystals distribution of irradiated glass investigated by EBSD: a) Image quality map and b) the corresponding IPF of irradiated LNS glass

In addition, laser-induced breakdown spectroscopy (LIBS) was conducted to determine the element distribution in irradiated glass (i.e. Si and $\mathrm{Nb}$ ). As illustrated in Figure 93b top, a measured region cross the non- melted and melted zone was performed (from $A$ to $B$, illustrated by the red rectangle). It is worth to note that the laser points are blurred in the non-melted part but clear in the melted zone (Figure 93b bottom), which may be due to different mechanical behavior of these two zones. Yet, no obvious fluctuation of $\mathrm{Si}$ and $\mathrm{Nb}$ were observed (Figure 93c). This confirmed the results from SEM/EDS.

In conclusion, $\mathrm{LiNbO}_{3}$ has been successfully grown by optical floating-zone method. An oriented structure was observed in melted zone. However, no chemical migration was observed at micrometer scale by SEM/EDS or LIBS like it was the case for fs laser irradiation. This is similar to chemical distribution situation of Zone $\mathrm{C}$ of regime 3 . So, in the thermodynamical equilibrium, is there a phase separation or $\mathrm{Si}$ is contained in $\mathrm{LiNbO}_{3}$ structure? It needs further investigation to address this question. 
a)

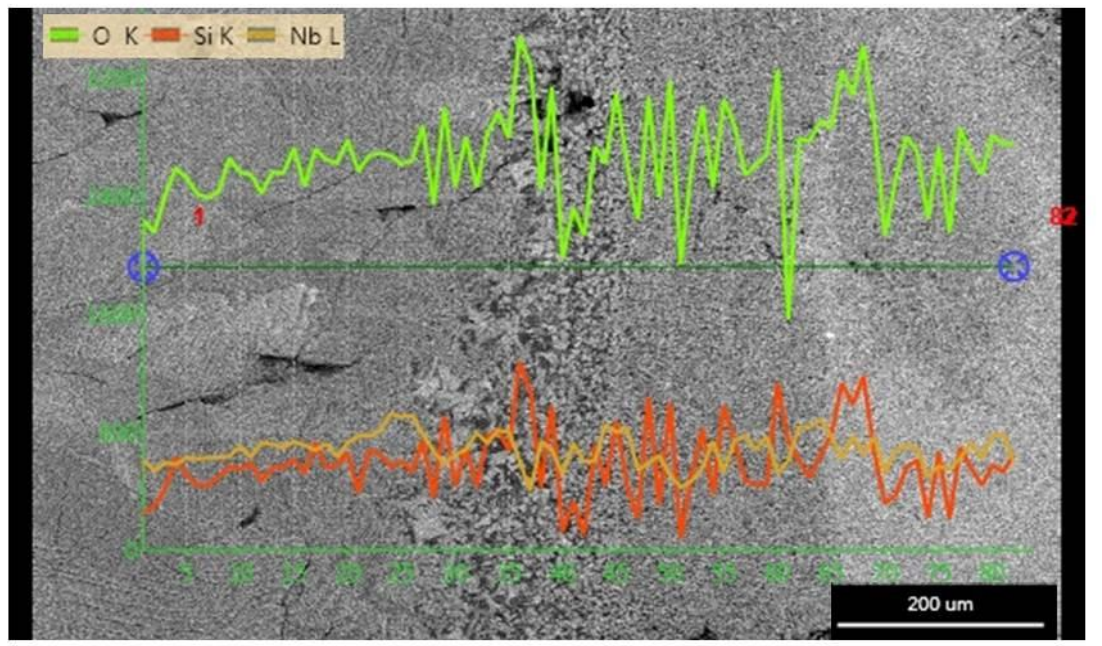

b)
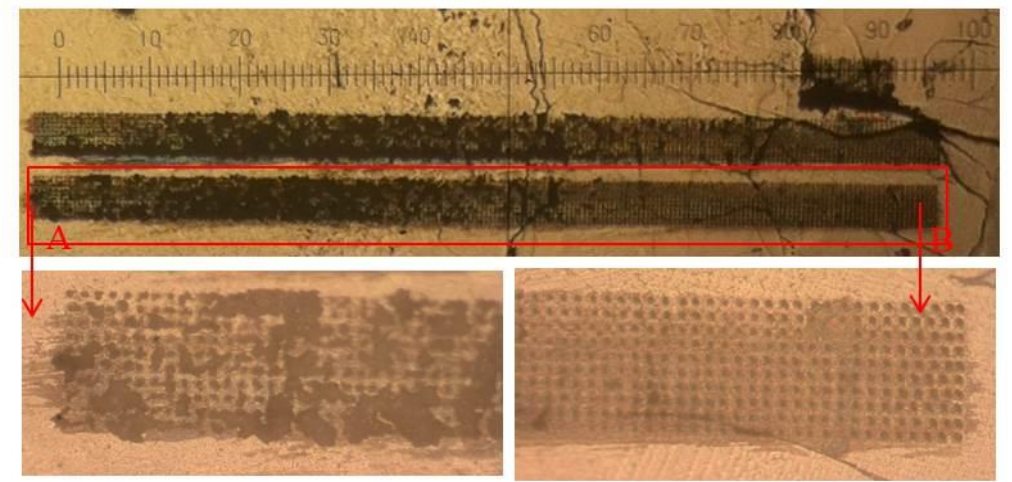

c)

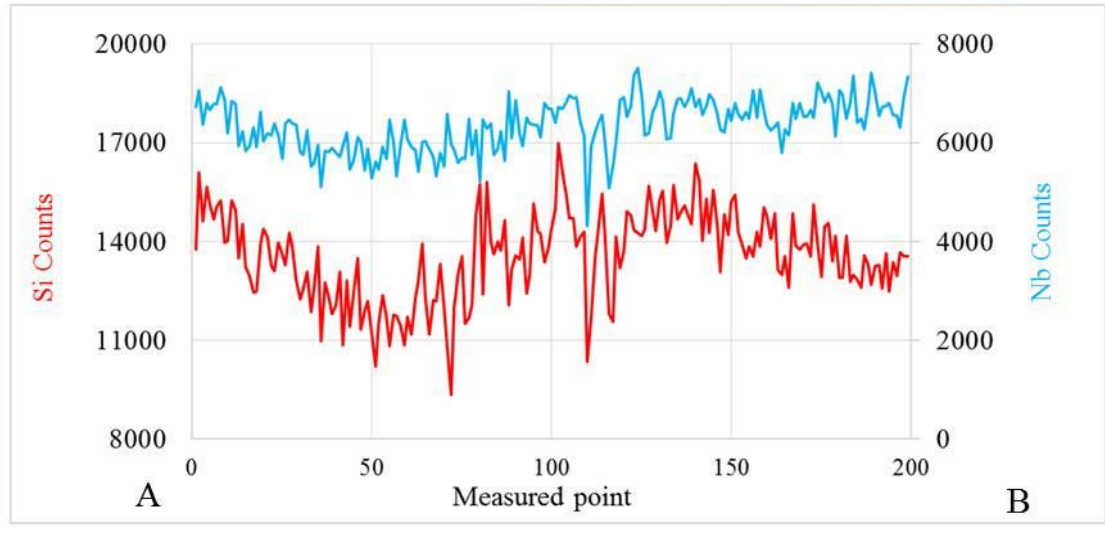

Figure 93 Chemical analysis of irradiated glass: a) SEM/EDS taken across the melted (left) and un-melted zone (right), superimposing the SEM image for O (green), Si (red), and $\mathrm{Nb}$ (orange) chemical profiles (82 points with step $10 \mu \mathrm{m})$, b) a photograph illustrating the region for laser-induced breakdown spectroscopy measurement (marked with red rectangle, the scale bar is $2 \mathrm{~mm}$ ) and c) the element distribution from A (un-melted zone) to $B$ (melted zone). 


\section{Chapter $5 . \quad$ fs laser-induced properties in LNS glasses}

\subsection{Second harmonic generation}

Active optical devices (e.g. frequency converters) require material with second-order nonlinear optical response that can be investigated through SHG. Analysing the angular dependence of SHG response (i.e. SHG intensity of irradiated lines varying with the probing laser polarization angle) is a way to investigate the SHG properties. Herein, this was investigated on fs laser irradiated LNS glasses by changing the parameters such as pulse energy, writing speed, writing orientation, and laser polarization, systematically.

\subsubsection{Second harmonic generation in $\mathrm{LiNbO}_{3}$ crystal}

$\mathrm{LiNbO}_{3}$ nano-crystals are distributed with the constraint that the polar axis remains perpendicular to writing laser polarization direction. So for having a clear picture, the relation between polar axis of $\mathrm{LiNbO}_{3}$ and probing laser polarization is necessary. In the section, we consider three simple cases (the probing laser polarization is in the $x z, x y$, or $y z$ planes of $\left.\mathrm{LiNbO}_{3}\right)$.

The relationship between the probing laser polarization (linear) and $\mathrm{LiNbO}_{3}$ is shown in Figure 94.
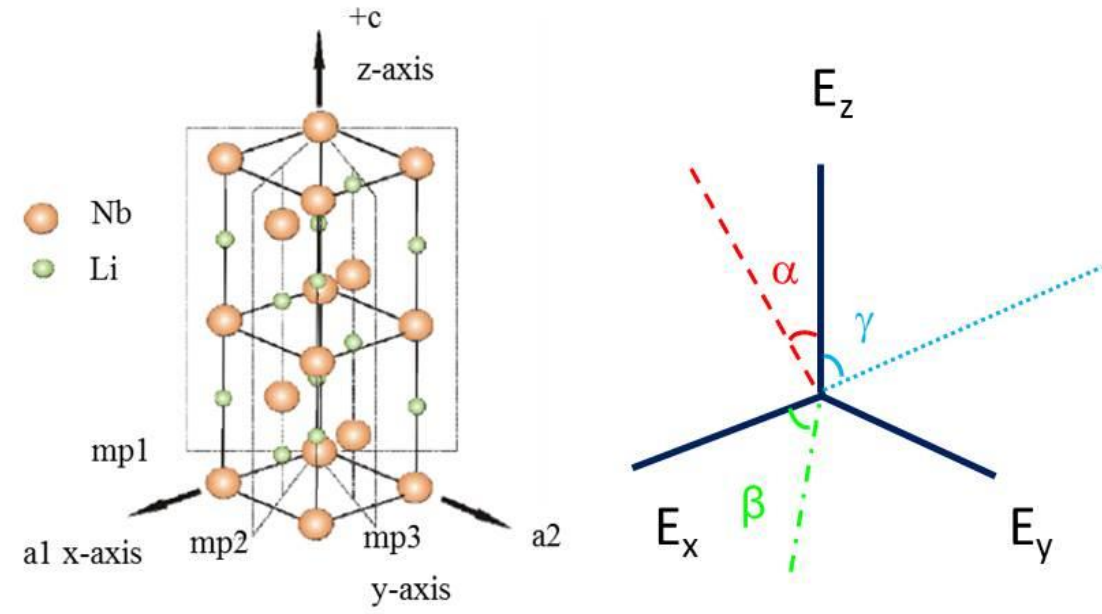

Figure 94 The relationship between the probing laser polarization angle and $\mathrm{LiNbO}_{3}$ cell: the probing laser angle $(\alpha)$ varying in $x z$ plane, the probing laser angle $(\beta)$ varying in xy plane, and the probing laser angle $(\gamma)$ varying in yz plane.

Based on the above discussion, we can simply deduce that

$$
\mathrm{I}_{\mathrm{SHG}} \propto \mathbf{P}_{2 \omega}^{2} \propto\left(\mathbf{X}_{2 \omega} \mathbf{E}_{\omega}^{\mathrm{P}} \mathbf{E}_{\omega}^{\mathrm{P}}\right)^{2}
$$


Or it can be written as

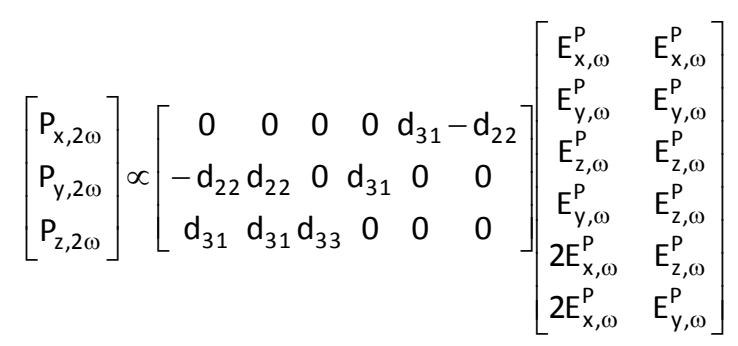

Where $I_{S H G}$ is the detected SHG intensity, $P_{2 \omega}$ is the induced polarization at $2 \omega, \chi_{2}$ is the second order nonlinear susceptibility tensor of $\mathrm{LiNbO}_{3}$ crystal, and $\mathbf{E}_{\omega}^{\mathrm{p}}$ is the probing electric field at $\omega$ frequency.

When probing laser polarization is parallel to polar axis of $\mathrm{LiNbO}_{3}$ (i.e. $E_{3}$ ), we get $d_{33} E_{z, \omega}^{2}$.

When probing laser polarization angle $(\alpha)$ is rotated in $x z$ plane, we get

$$
\left[\begin{array}{c}
2 d_{31} \sin \alpha \cos \alpha \\
-d_{22} \sin ^{2} \alpha \\
d_{31} \sin ^{2} \alpha+d_{33} \cos ^{2} \alpha
\end{array}\right] E_{\alpha, \omega}^{2}
$$

Similarly, when the laser probing angle is rotated in xy plane $(\beta)$ or in yz plane $(\gamma)$, we get the following, respectively

$$
\left[\begin{array}{c}
-2 d_{22} \cos \beta \sin \beta \\
-d_{22} \cos ^{2} \beta+d_{22} \sin ^{2} \beta \\
d_{31}
\end{array}\right] E_{\beta, \omega}^{2} \text { and }\left[\begin{array}{c}
0 \\
d_{22} \sin ^{2} \gamma+2 d_{31} \cos \gamma \sin \gamma \\
d_{31} \sin ^{2} \gamma+d_{33} \cos ^{2} \gamma
\end{array}\right] E_{\gamma, \omega}^{2}
$$

Figure 95 shows the normalized SHG intensity as probing laser angles (normalized at the largest value, i.e. probing laser polarization is parallel to polar axis of $\mathrm{LiNbO}_{3}$ ).

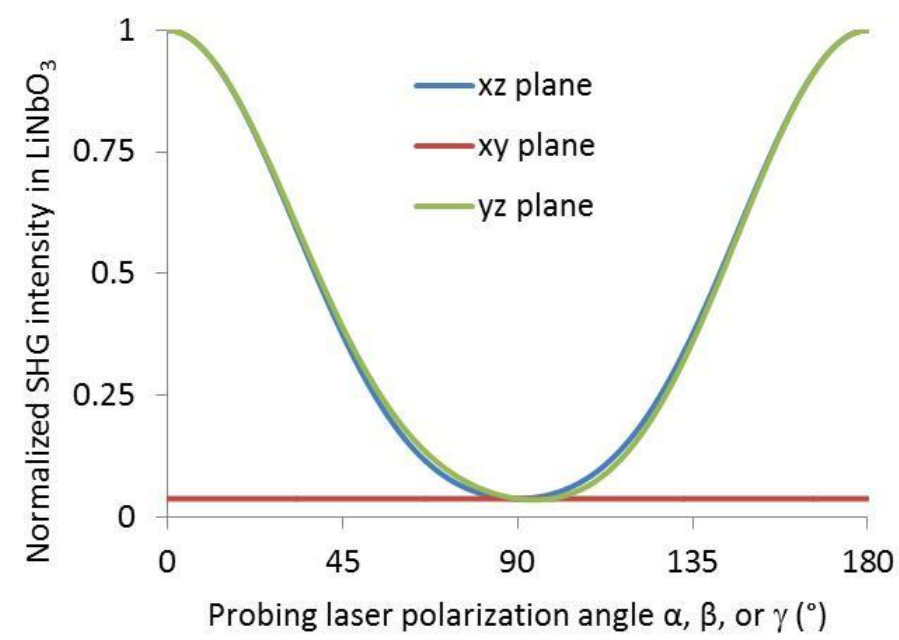

Figure 95 Normalized SHG intensity (normalized at the largest value) as the function of the probing laser polarization angle $(\alpha)$ varying in $x z$ plane, the probing laser angle $(\beta)$ varying in $x y$ plane, and the probing laser angle $(\gamma)$ varying in yz plane. 
For the probing angle in xz plane $(\alpha)$, the maximum SHG intensity is reached when probing laser polarization is parallel to the $\mathrm{LiNbO}_{3}$ crystal polar axis (i.e. $\mathrm{z}$ crystal direction) and the minimum is for probing laser polarization perpendicular to polar axis of $\mathrm{LiNbO}_{3}$ crystal $\left(\alpha=90^{\circ}\right.$, with normalized value of 0.0379 (blue curve in Figure 95). For the probing angle in xy plane ( $\beta$ ), it always has the same SHG intensity (with normalized value of 0.0379 , illustrated by brown curve in Figure 95) whatever $\beta$ is. For the probing angle in yz plane $(\gamma)$, the maximum SHG intensity reaches when probing laser polarization is parallel to polar axis of $\mathrm{LiNbO}_{3}$ crystal (i.e. $z$ direction), but the minimum one when $\gamma=94.35^{\circ}$ (with normalized value of 0.0355 , shown in green curve in Figure 95).

\subsubsection{Second harmonic generation observation}

\subsubsection{SHG images of irradiated lines}

Green irradiation was observed during laser writing process from the pulse energy of 0.8 $\mu \mathrm{J} /$ pulse at $1030 \mathrm{~nm}, 300 \mathrm{fs}, 500 \mathrm{kHz}, \mathrm{NA}=0.6$, focus depth $650 \mu \mathrm{m}$ in glass, $5 \mu \mathrm{m} / \mathrm{s}$. Then SHG images of irradiated lines were taken as shown in Figure 96.

At pulse energy lower than this crystalline line threshold (i.e. regime 1), no matter what the probing laser polarization is, the images are dark and no SHG was observed. At the pulse energy slightly higher than the threshold to obtain crystalline line (i.e. $0.8 \mu \mathrm{J} /$ pulse), as illustrated in Figure 96a, the SHG intensity reaches the minimum when probing laser polarization (marked with white double arrow) is parallel to writing laser polarization direction (marked with red double arrow), but the maximum when probing laser polarization is perpendicular to writing laser polarization direction (Figure 96b).

When increasing the pulse energy to $1.4 \mu \mathrm{J} /$ pulse (Figure $96 \mathrm{c}-\mathrm{d}$ ), the intensity of SHG becomes stronger and stronger. SHG is observed along the edges of the line than the centre one. The distribution of the SHG is not homogenous along writing direction. This phenomenon becomes obvious for the increase of pulse energy (Figure 96e-f).

From the above results, we can deduce that

1) It is possible to observe SHG of the irradiated line at certain pulse energy;

2) At the pulse energy slightly higher than obtaining SHG (shown in Figure 96a and b), the SHG intensity reaches the maximum when probing laser polarization is perpendicular to writing laser polarization direction but minimum for the parallel one. It indicates the texture, which is in 
agreement with the crystal orientation observed from EBSD (i.e. the polar axis of nano-crystal is oriented in the plane perpendicular to writing laser polarization direction). Moreover, this SHG property can be maintained along writing direction;

3) At high pulse energy (illustrated in Figure 96e and f), strong SHG can be observed whatever the direction of probing laser polarization.
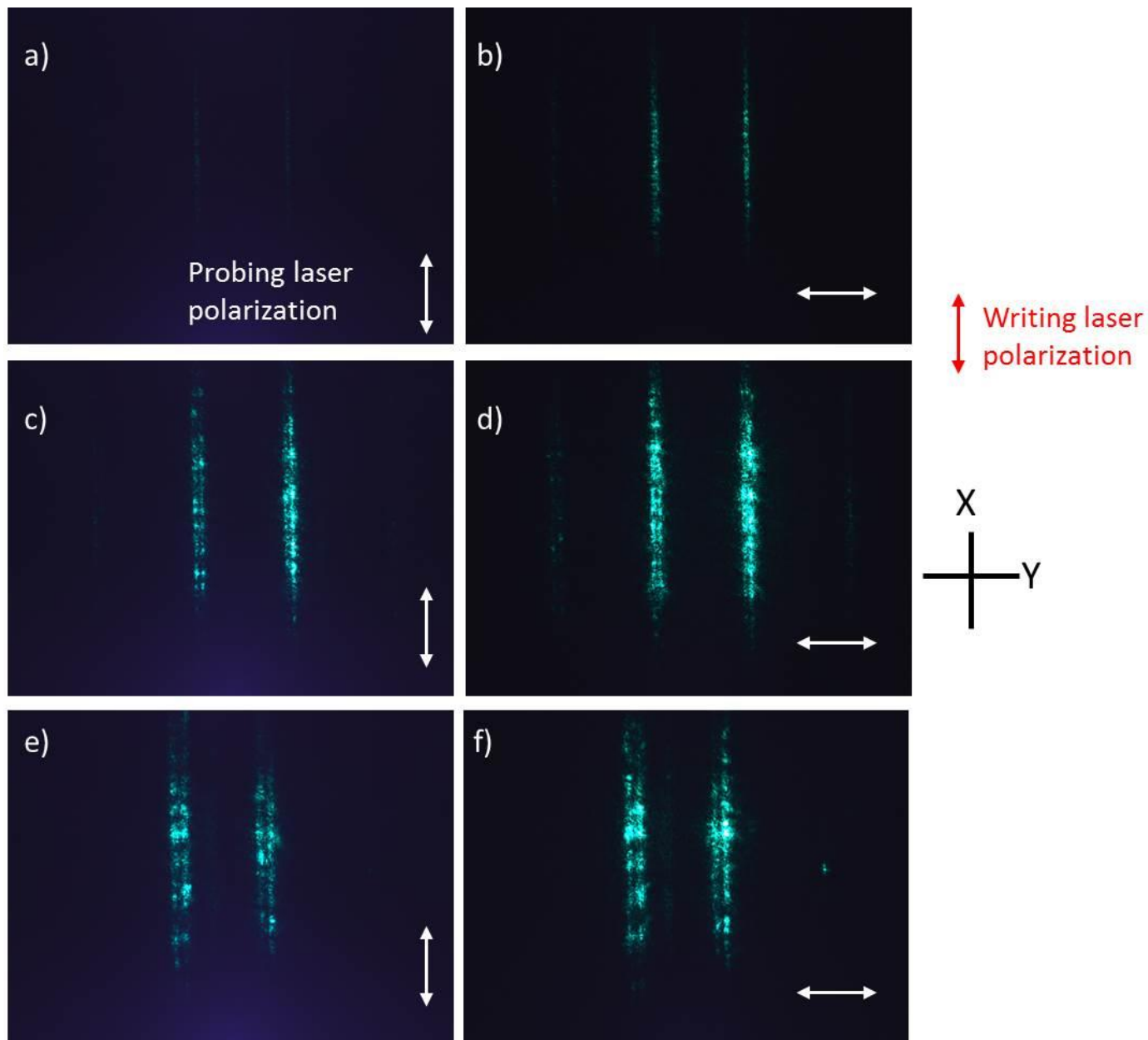

Figure 96 SHG of irradiated lines varying with pulse energy: a-b) $0.8, c-d) 1.4$, and e-f) $2.2 \mu \mathrm{J} /$ pulse. The double arrows mark the probing laser polarization. Other parameters: $33 \mathrm{Li}_{2} \mathrm{O}-33 \mathrm{Nb}_{2} \mathrm{O}_{5}-34 \mathrm{SiO}_{2}(\mathrm{~mol} \%), 1030 \mathrm{~nm}, 300$ fs, $500 \mathrm{kHz}, \mathrm{NA}=0.6$, focus depth $650 \mu \mathrm{m}$ in glass, $5 \mu \mathrm{m} / \mathrm{s}$, laser polarization direction is parallel to writing direction (i.e. $\mathrm{X}$ direction). Two lines in each energy group, the distance between each line is $50 \mu \mathrm{m}$.

To go a step further, the angular dependence of SHG (i.e. SHG intensity of irradiated lines varying with the probing laser polarization angle) were recorded to have a clear picture of the SHG of irradiated line. 


\subsubsection{Angular dependence of SHG of irradiated lines in the XY plane}

\subsection{Pulse energy effect}

As shown by red curve in Figure 97, at $0.8 \mu \mathrm{J} /$ pulse (i.e. regime 2 defined in Section 4.1.1.1), a well-defined cosine-like curve with period of $180^{\circ}$ is observed. The maximum SHG intensity peaks when the probing laser is perpendicular to writing laser polarization direction (i.e. 0 or $180^{\circ}$ ) and the minimum SHG intensity is obtained at an angle close to writing laser polarization direction $\left(90^{\circ}\right)$.

As illustrated by green curve in Figure 97, when increasing the pulse energy is increased to 1.4 $\mu \mathrm{J} /$ pulse (regime 3 defined in Section 4.1.1.1), the cosine-like curve is modified: a second maximum occurs at the place of the minimum on the simple cosine-like curve. This phenomenon becomes obvious when increased the pulse energy to $1.8 \mu \mathrm{J} /$ pulse (blue curve in Figure 97). This pulse energy dependent SHG curve is in agreement with the SHG image observation.

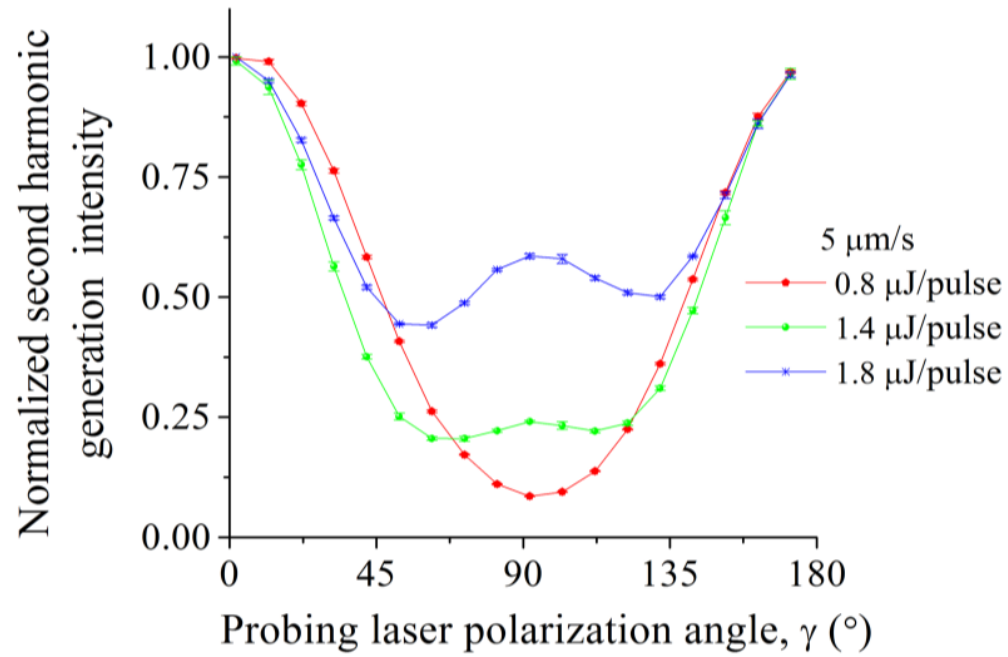

Figure 97 Normalized SHG intensity of irradiated lines as function of probing laser polarization angle, measured in the $\mathrm{XY}$ plane: the variable parameter is the pulse energy. Other parameters: $32.5 \mathrm{Li}_{2} \mathrm{O}-27.5 \mathrm{Nb}_{2} \mathrm{O}_{5}-40 \mathrm{SiO}_{2}$ (mol\%), $1030 \mathrm{~nm}, 300 \mathrm{fs}, 250 \mathrm{kHz}, \mathrm{NA}=0.6$, focus depth $300 \mu \mathrm{m}$ in air, writing direction is along $45^{\circ}$, and laser polarization is parallel to $90^{\circ}$.

\subsection{Writing speed effect}

For investigating the dependence of SHG with the writing speed, the pulse is fixed. As shown in Figure 98a, well defined cosine-like curves are obtained whatever the writing speed (1 to 25 $\mu \mathrm{m} / \mathrm{s}$ ) at $0.8 \mu \mathrm{J} /$ pulse (regime 2, defined in Section 4.1.1).

With the increase of writing speed, the minimum value of SHG shifts from $102^{\circ}$ to $91^{\circ}$, which is closer to the writing polarization angle $\left(90^{\circ}\right)$. In contrast, at $1.4 \mu \mathrm{J} /$ pulse, the result is quite different from the previous ones. With the decrease of the writing speed from 25 to $1 \mu \mathrm{m} / \mathrm{s}$, the 
SHG curve changed from simple cosine-like curve (blue curve in Figure 98b) to the modified ones (green and red curves in Figure 98b). The modified one becomes more obvious with the decrease of writing speed.
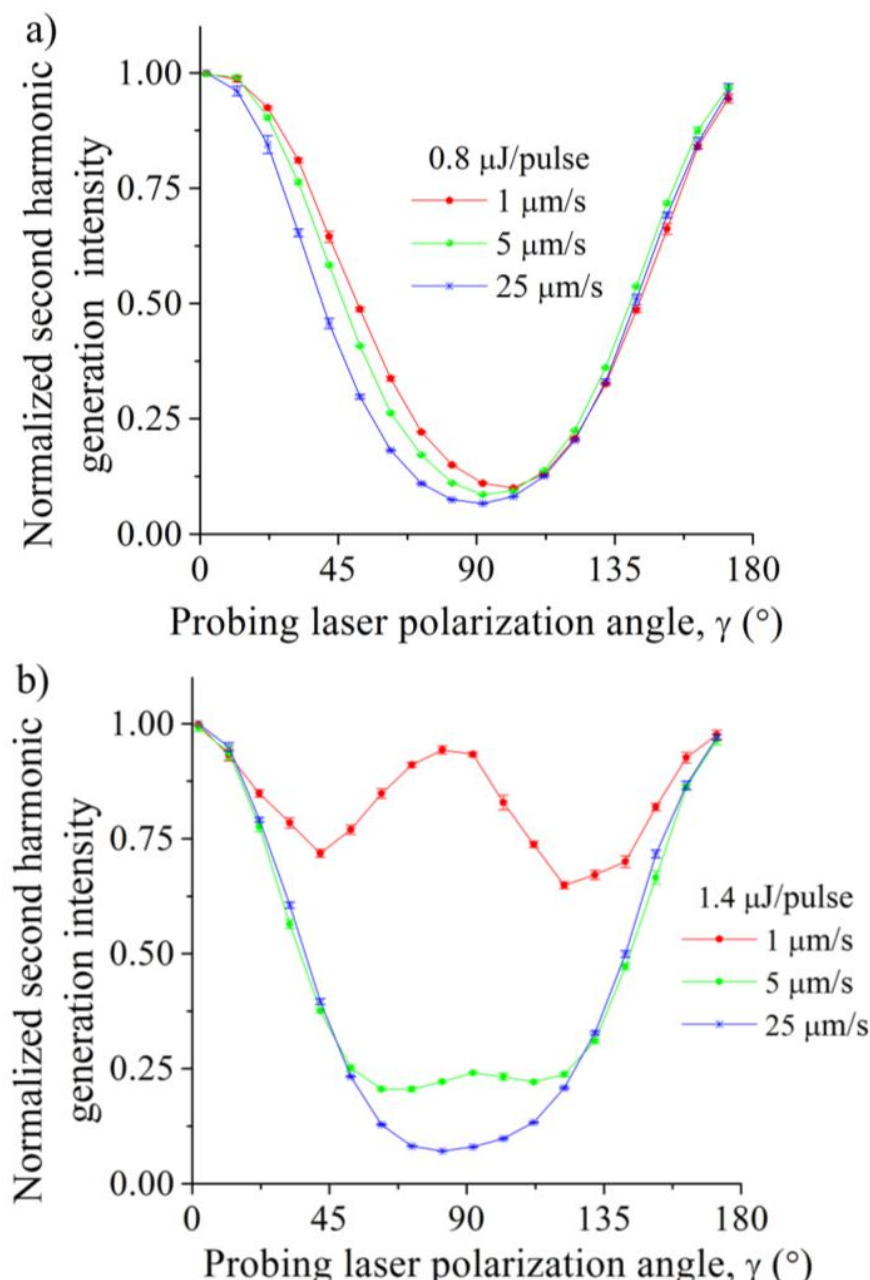

Figure 98 Normalized SHG intensity of irradiated lines as function of probing laser polarization angle, measured in the $X Y$ plane: the variable parameter is the writing speed at pulse energy of a) 0.8 and $b$ ) $1.4 \mu \mathrm{J} /$ pulse. Other parameters: $32.5 \mathrm{Li}_{2} \mathrm{O}-27.5 \mathrm{Nb}_{2} \mathrm{O}_{5}-40 \mathrm{SiO}_{2}$ (mol\%), $1030 \mathrm{~nm}, 300 \mathrm{fs}, 250 \mathrm{kHz}, \mathrm{NA}=0.6$, focus depth $300 \mu \mathrm{m}$ in air, writing direction is along $45^{\circ}$, and laser polarization is parallel to $90^{\circ}$.

\subsection{Writing orientation effect}

Writing orientation here means the movement along a given direction. There are two orientations for a given direction. The orientation effect in writing is peculiar for ultra-brief lasermatter interaction [246].

The glass is centre-symmetric, considering that the laser beam would be axis-symmetric and the experimental geometry is also $\mathrm{XY}$-symmetric; there should be no difference between the orientation of writing i.e. between forward direction (here $45^{\circ}$ ) and backward direction (here $\left.225^{\circ}\right)$. However, experimentally, it is not the case. In both cases, there are two textures: one with 
polar axis of nano-cystals perpendicular to the writing laser polarization, direction and one with the polar axis closely parallel to writing laser polarization. However, the ratio between the two components and the angular position of the maxima seems slightly different. This is particularly clear in Figure 99a, at writing speed of $1 \mu \mathrm{m} / \mathrm{s}(1.4 \mu \mathrm{J} /$ pulse).
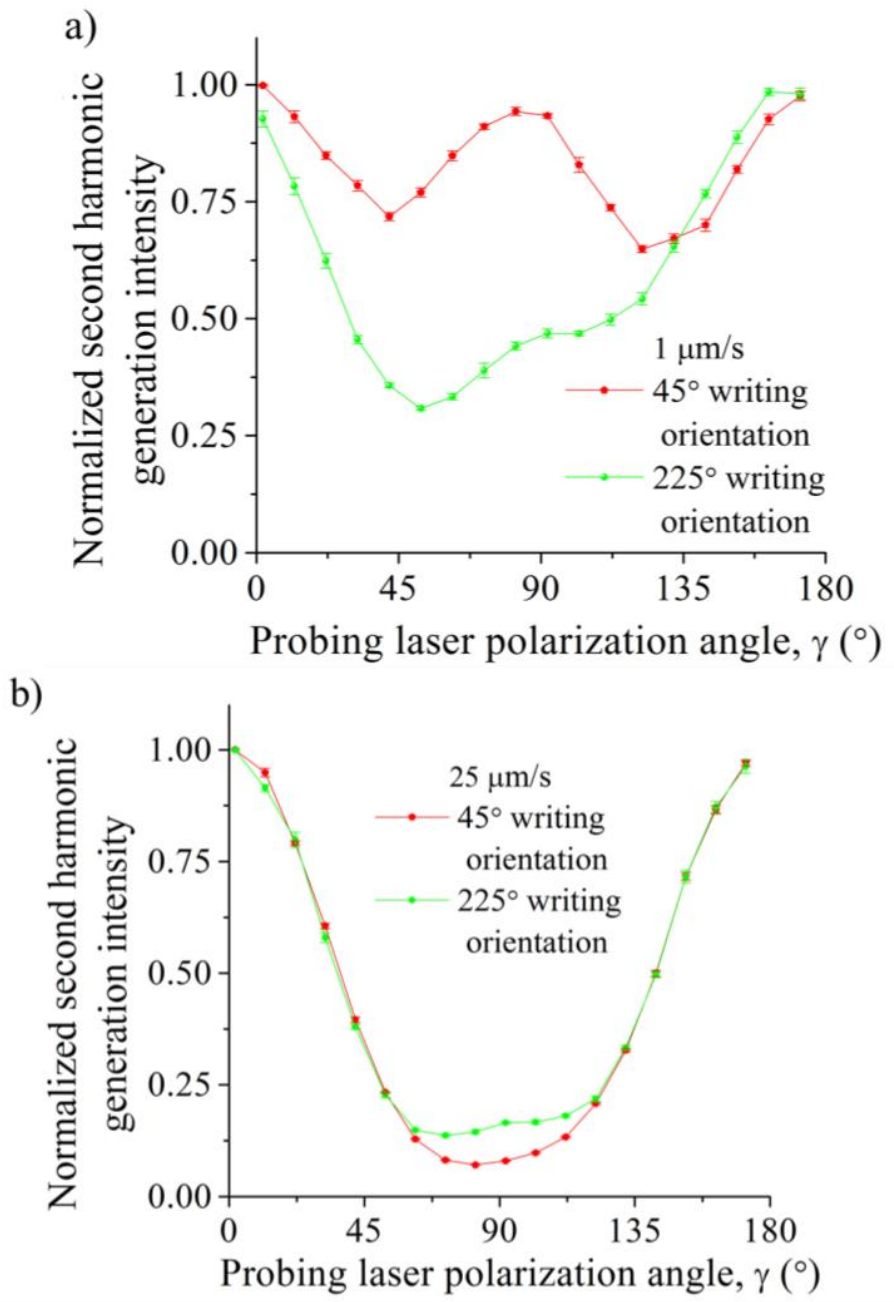

Figure 99 Normalized SHG intensity of irradiated lines as function of probing laser polarization angle, measured in the XY plane: the writing orientation is the variable parameter for writing speeds at a) 1 and b) $25 \mu \mathrm{m} / \mathrm{s}$. Other parameters: $32.5 \mathrm{Li}_{2} \mathrm{O}-27.5 \mathrm{Nb}_{2} \mathrm{O}_{5}-40 \mathrm{SiO}_{2}(\mathrm{~mol} \%), 1030 \mathrm{~nm}, 300 \mathrm{fs}, 250 \mathrm{kHz}, \mathrm{NA}=0.6$, focus depth $300 \mu \mathrm{m}$ in air, $1.4 \mu \mathrm{J} /$ pulse, and laser polarization is parallel to $90^{\circ}$.

This is an Asymmetric orientational writing (AOW). AOW means that different properties (e.g. birefringence, refractive index changes and stress) could be observed between $\theta$ and $\theta+180^{\circ}$ scanning direction in an isotropic media after fs or picosecond laser radiation but not ns laser or continuous ones [228]. As illustrated in Figure 99b, with the increase of writing speed from $1 \mu \mathrm{m} / \mathrm{s}$ to $25 \mu \mathrm{m} / \mathrm{s}$, the curves are cosine-like ones and the AOW effect is reduced. It is the first time that an AOW effect is clearly demonstrated in SHG and related to photo precipitation of crystals in a silica-based glass. 


\subsection{Sum up of angular dependence of SHG in the XY plane}

Herein, a quantity, named anisotropy magnitude, deduced from the angular dependence of the SHG and defined as $\left(\mathrm{SHG}_{\max }-\mathrm{SHG}_{\min }\right) /\left(\mathrm{SHG}_{\max }+\mathrm{SHG}_{\min }\right)$ has been used to characterize the angular contrast.

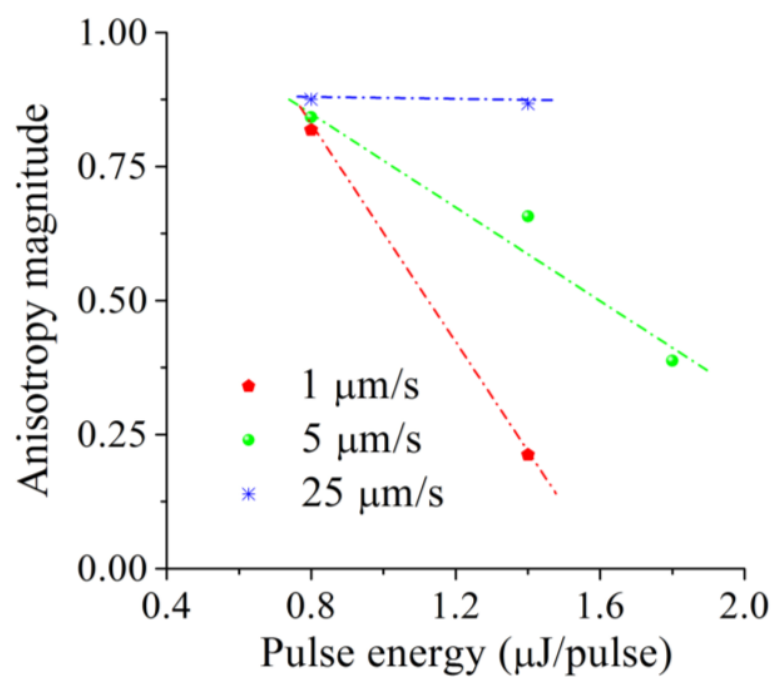

Figure 100 Anisotropy magnitude of irradiated lines. Anisotropy magnitude is defined by the following ratio: $\left(\mathrm{SHG}_{\max }-\mathrm{SHG}_{\min }\right) /\left(\mathrm{SHG}_{\max }+\mathrm{SHG}_{\min }\right)$. The lines are guides for the eyes. Writing direction is along $45^{\circ}$. Other parameters: $32.5 \mathrm{Li}_{2} \mathrm{O}-27.5 \mathrm{Nb}_{2} \mathrm{O}_{5}-40 \mathrm{SiO}_{2}$ (mol\%), $1030 \mathrm{~nm}, 300 \mathrm{fs}, 250 \mathrm{kHz}, \mathrm{NA}=0.6$, focus depth $300 \mu \mathrm{m}$ in air, writing direction is along $45^{\circ}$, and laser polarization is parallel to $90^{\circ}$.

As illustrated in Figure 100, at the fixed writing speed $(5 \mu \mathrm{m} / \mathrm{s})$, the anisotropy magnitude decreases with the increase of pulse energy (green line in Figure 100). At high speed $(25 \mu \mathrm{m} / \mathrm{s})$, the anisotropy magnitude is not dependent on pulse energy (blue line in Figure 100), whereas it is absolutely not the case when the writing speed is decreased, especially at high pulse energy (red line in Figure 100).

\subsection{Writing laser polarization direction effect}

Based on the above discussion, at low pulse energy or at high pulse energy with fast scanning speed, the maximum SHG intensity is obtained at the position when the probing laser angle closely perpendicular to writing laser polarization direction and the minimum SHG intensity is obtained at the position when probing laser writing laser polarization direction. In other words, the SHG maximum intensity position is related to writing laser polarization. To clarify the writing laser polarization effect, the writing direction (e.g. $45^{\circ}$ ) has been fixed, but the writing laser polarization direction has been varied from $0^{\circ}$ to $135^{\circ}$.

As shown in Figure 101a, all irradiated lines exhibited well-defined SHG response $(0.8 \mu \mathrm{J} /$ pulse, 5 $\mu \mathrm{m} / \mathrm{s}$ ) with a period of $180^{\circ}$. SHG curves are probing polarization dependence. The probing laser 
polarization angle for achieving minimum SHG intensity, noted $\gamma_{\min }$ is changing. As shown in Figure $101 \mathrm{~b}, \gamma_{\min }$ was close to $\phi$ that is represented by the red dash line. Similarly, $\gamma_{\max }$ was close to $\phi+90^{\circ}$, illustrated by the green dash line. These results indicates that the writing laser polarization angle $(\phi)$ have an effect on SHG angular dependence of irradiated lines (e.g. adjusting the value of $\gamma_{\min }$ and $\left.\gamma_{\max }\right)$.
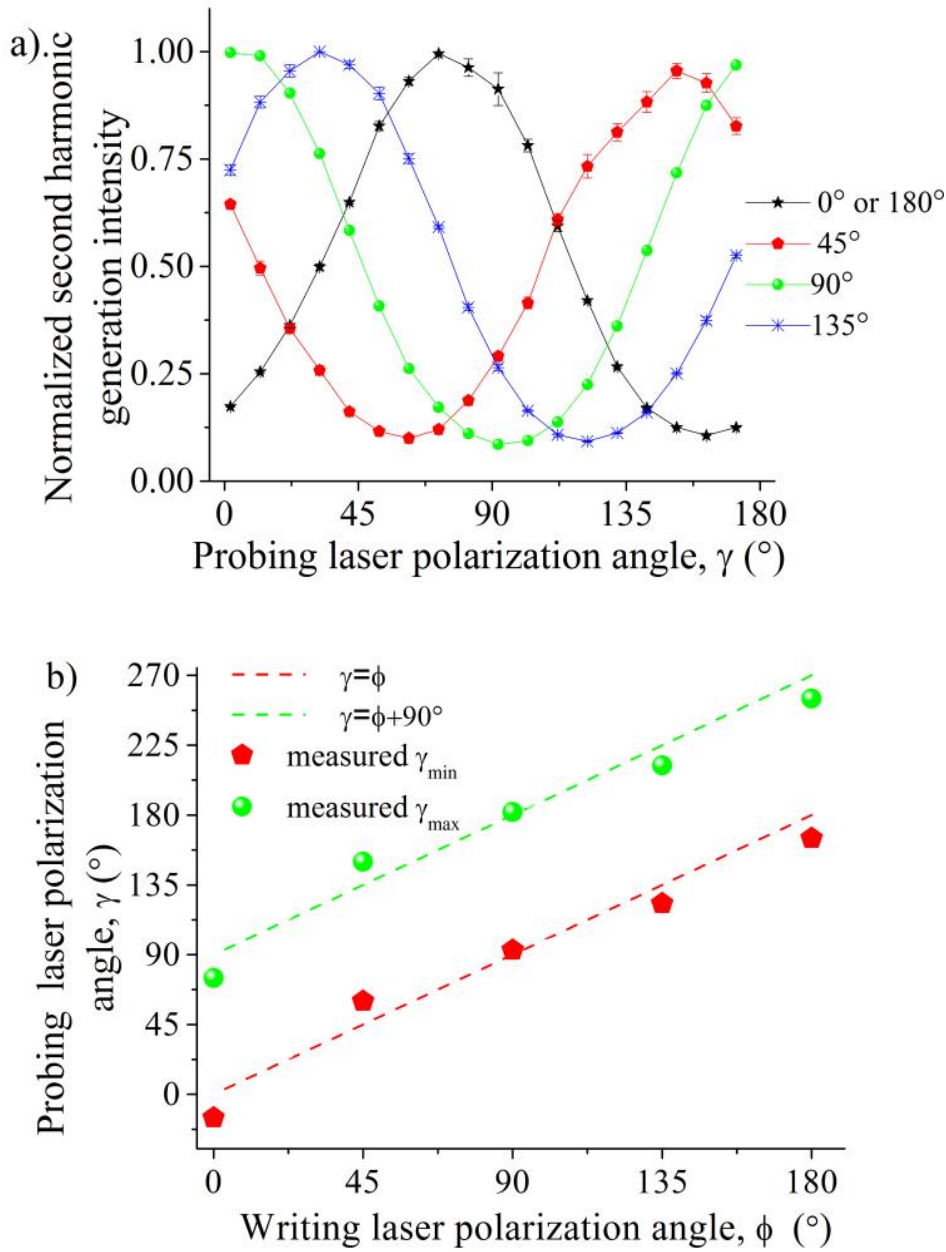

Figure 101 Normalized polarization dependent SHG of irradiated lines: a) normalized SHG intensity as function of probing laser polarization angle $\gamma$, the variable parameter is writing laser polarization angle $\phi$. b) The probing laser polarization angle at the minimum SHG intensity is quoted $\gamma_{\min }$ and maximum SHG intensity is quoted $\gamma_{\max }$ according to $\phi$. Other parameters $32.5 \mathrm{Li}_{2} \mathrm{O}-27.5 \mathrm{Nb}_{2} \mathrm{O}_{5}-40 \mathrm{SiO}_{2}$ (mol\%), $1030 \mathrm{~nm}, 300 \mathrm{fs}, 250 \mathrm{kHz}, 0.8 \mu \mathrm{J} / \mathrm{pulse}, 5$ $\mu \mathrm{m} / \mathrm{s}, \mathrm{NA}=0.6$, focus depth $300 \mu \mathrm{m}$ in air, writing direction is along $45^{\circ}$.

To have a better picture of the effect of the incident laser polarization angle on SHG property of irradiated lines, various lines considering writing direction, $\theta$, and writing laser polarization angle, $\phi$, were performed. Similar cosine-like SHG curves of irradiated lines were obtained.

Writing laser polarization angle $(\phi)$ and writing direction $(\theta)$ are the parameters that can be exactly controlled during laser radiation. In the following experiment, we precise the relationship between $\gamma_{\max }$ and $\phi$ and $\theta$. 

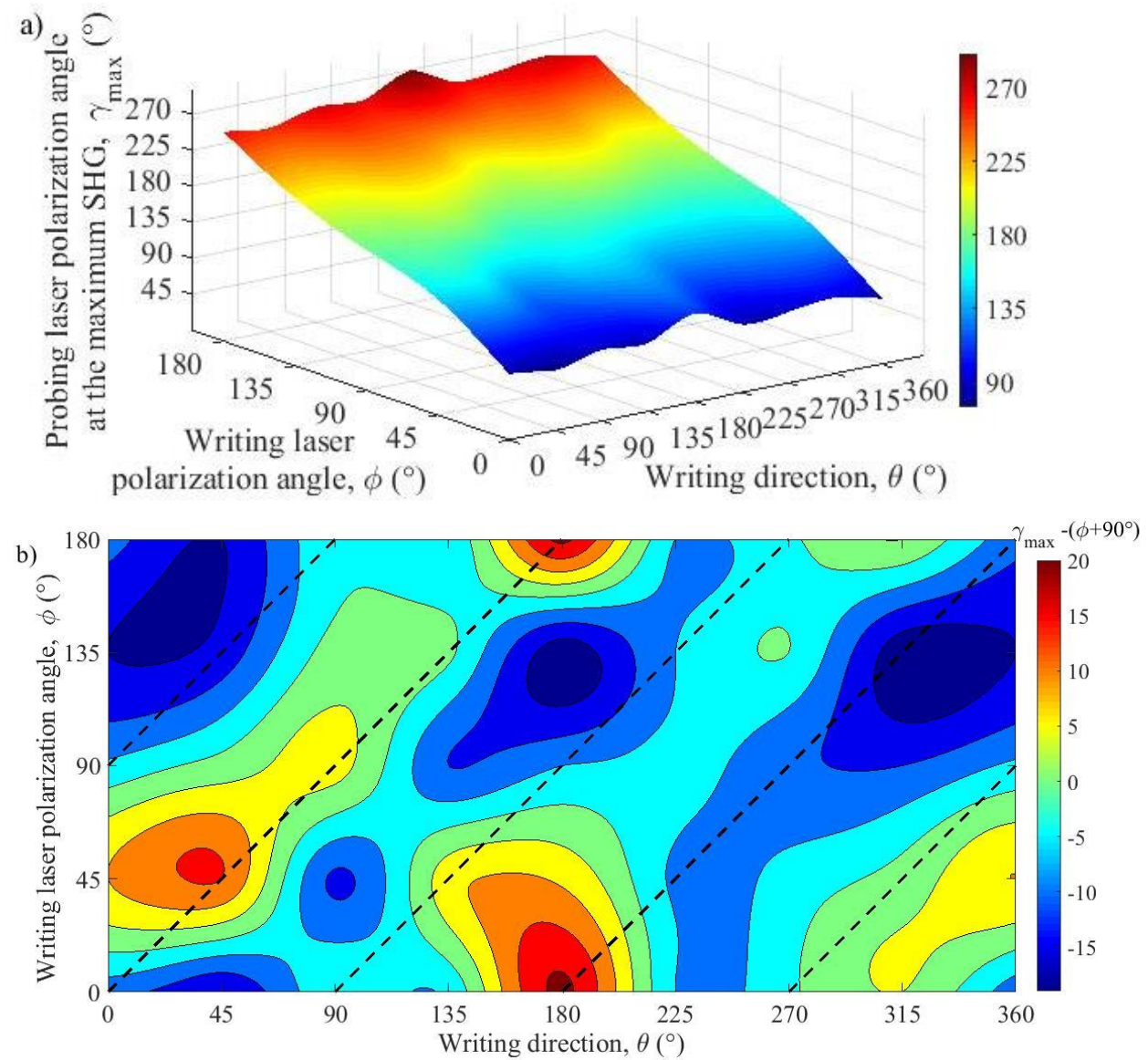

Figure 102 SHG property of irradiated lines varying writing direction $(\theta)$ and writing laser polarization angle $(\phi)$ : a) $\gamma_{\max }$ and b) $\gamma_{\max }-\left(\phi+90^{\circ}\right)$, according to $\theta$ and $\phi$. The probing laser polarization angle at the maximum SHG intensity is quoted $\gamma_{\max }$. Other parameters $32.5 \mathrm{Li}_{2} \mathrm{O}-27.5 \mathrm{Nb}_{2} \mathrm{O}_{5}-40 \mathrm{SiO}_{2}(\mathrm{~mol} \%), 1030 \mathrm{~nm}, 300 \mathrm{fs}, 250 \mathrm{kHz}, 0.8$ $\mu \mathrm{J} /$ pulse, $5 \mu \mathrm{m} / \mathrm{s}, \mathrm{NA}=0.6$, focus depth $300 \mu \mathrm{m}$ in air.

Figure 102a displays the probing laser polarization angle at the maximum SHG intensity, $\gamma_{\max }$ versus $\theta$ and $\phi$. At a fix scanning direction, when varying incident laser polarization angle from $0^{\circ}$ to $180^{\circ}, \gamma_{\max }$ changed from $\sim 90^{\circ}$ (blue) to $\sim 270^{\circ}$ (red). In contrast, at a fixed writing laser polarization angle, when adjusting the scanning direction, $\gamma_{\max }$ is distributed around $\phi+90^{\circ}$. Plotting $\gamma_{\min }$ yields similar results. The above observation indicates that $\gamma_{\max }$ and $\gamma_{\min }$ exhibit a weaker dependence on $\theta$ than on $\phi$. Departures of $\gamma_{\max }$ from $\phi+90^{\circ}$ according to $\theta$ and $\phi$ are shown in Figure 102b. 


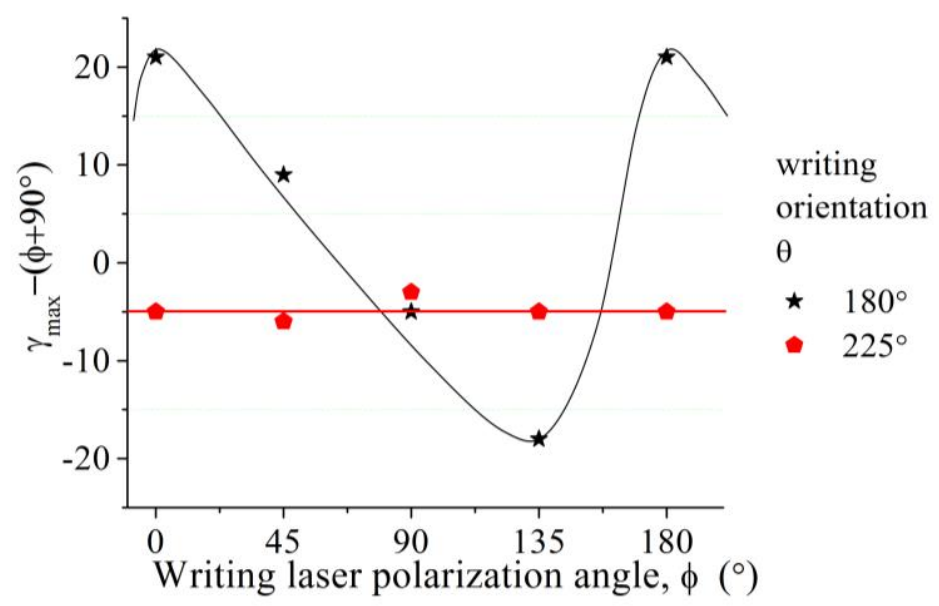

Figure $103 \gamma_{\max }-\left(\phi+90^{\circ}\right)$ as a function of the writing laser polarization $\phi$ for two particular writing orientation (The lines are guides for the eyes, where the red line illustrated a weak, while the black one represented a strong departure amplitude of $\gamma_{\max }$ from $\phi+90^{\circ}$, varying with $\left.\phi\right)$. Other parameters $32.5 \mathrm{Li}_{2} \mathrm{O}-27.5 \mathrm{Nb}_{2} \mathrm{O}_{5}-40 \mathrm{SiO}_{2}$ (mol\%), $1030 \mathrm{~nm}, 300 \mathrm{fs}, 250 \mathrm{kHz}, 0.8 \mu \mathrm{J} /$ pulse, NA = 0.6, focus depth $300 \mu \mathrm{m}$ in air, $5 \mu \mathrm{m} / \mathrm{s}$

At a fixed scanning direction, $\gamma_{\max }-\left(\phi+90^{\circ}\right)$ is varying differently with incident laser polarization direction $(\phi)$.It is worth noting that there are some fluctuations with alternated minimum (i.e. $\gamma_{\max }-\left(\phi+90^{\circ}\right)$ is near $\left.0^{\circ}\right)$ and maximum (i.e. $\gamma_{\max }-\left(\phi+90^{\circ}\right)$ is far from $\left.0^{\circ}\right)$ along diagonal lines (i.e. $\theta-\phi=$ constant, illustrated by dashed lines in Figure 102b). This may reveal a term, dependent on the angle difference, $\theta$ - $\phi$, also acting on SHG properties of irradiated lines. There was a large difference of SHG for $\theta$ between $0^{\circ}$ and $180^{\circ}$ on one hand and $180^{\circ}$ and $360^{\circ}$ on the other hand (Figure 102b). This indicates an AOW effect in SHG.

The maximum and minimum fluctuations are clearly illustrated in Figure 103 . For $\theta=225^{\circ}, \gamma_{\max }-$ $\left(\phi+90^{\circ}\right)$ is stable at about $-5^{\circ}$ but for $\theta=180^{\circ}$, there is a large oscillation with a minimum at $\phi=$ $135^{\circ}$ and a maximum at $0^{\circ}$.

\subsubsection{Modelling/Interpretation}

\subsubsection{1. $S H G$ \& EBSD results}

We have analysed the shape of the curves in Figure 97 on the basis of SHG angular response of a simple texture i.e. all nano-crystals have their polar axis along a given direction. The simulation of the angular dependence of SHG intensity with the probe polarization under some cases (e.g. probing laser polarization is parallel to polar axis of $\mathrm{LiNbO}_{3}$ ) is discussed in Section 5.1.1. The second order nonlinear tensor for the symmetry R3c attached to $\mathrm{LiNbO}_{3}$ crystal is such that the largest SHG coefficient $\left(d_{33}=34.4 \mathrm{pm} / \mathrm{V}\right)$ is supplied along the polar axis direction. The other ones are $d_{31}=5.95 \mathrm{pm} / \mathrm{V}$ and $d_{22}=3.07 \mathrm{pm} / \mathrm{V}$. From this, we built an expression that may 
account for by two populations: one textured (written as the function $\left.f\left(\alpha-\alpha_{1}\right)\right)$ and another one randomly oriented.

$$
\mathrm{ISHG}_{\mathrm{SH}}^{\mathrm{Norm}}=\frac{r_{1}+r_{2} f\left(\alpha-\alpha_{1}\right)}{\left[\mathrm{r}_{1}+r_{2} f\left(\alpha-\alpha_{1}\right)\right]_{\max }}
$$

Where $\alpha_{1}$ is an angle shift from 0 direction (shown in Figure 97); $r_{1}$ and $r_{2}$ the weight of the two populations. We obtained a fit with $r_{1}=0.013, r_{2}=3.2$ and $\alpha_{1}=3.4^{\circ}$. Result shown in Figure 104a for pulse energy of $0.8 \mu \mathrm{J} /$ pulse seems to indicate a large proportion of crystal with the polar axis oriented in the $0^{\circ}$ direction versus non randomly oriented nano-crystals.
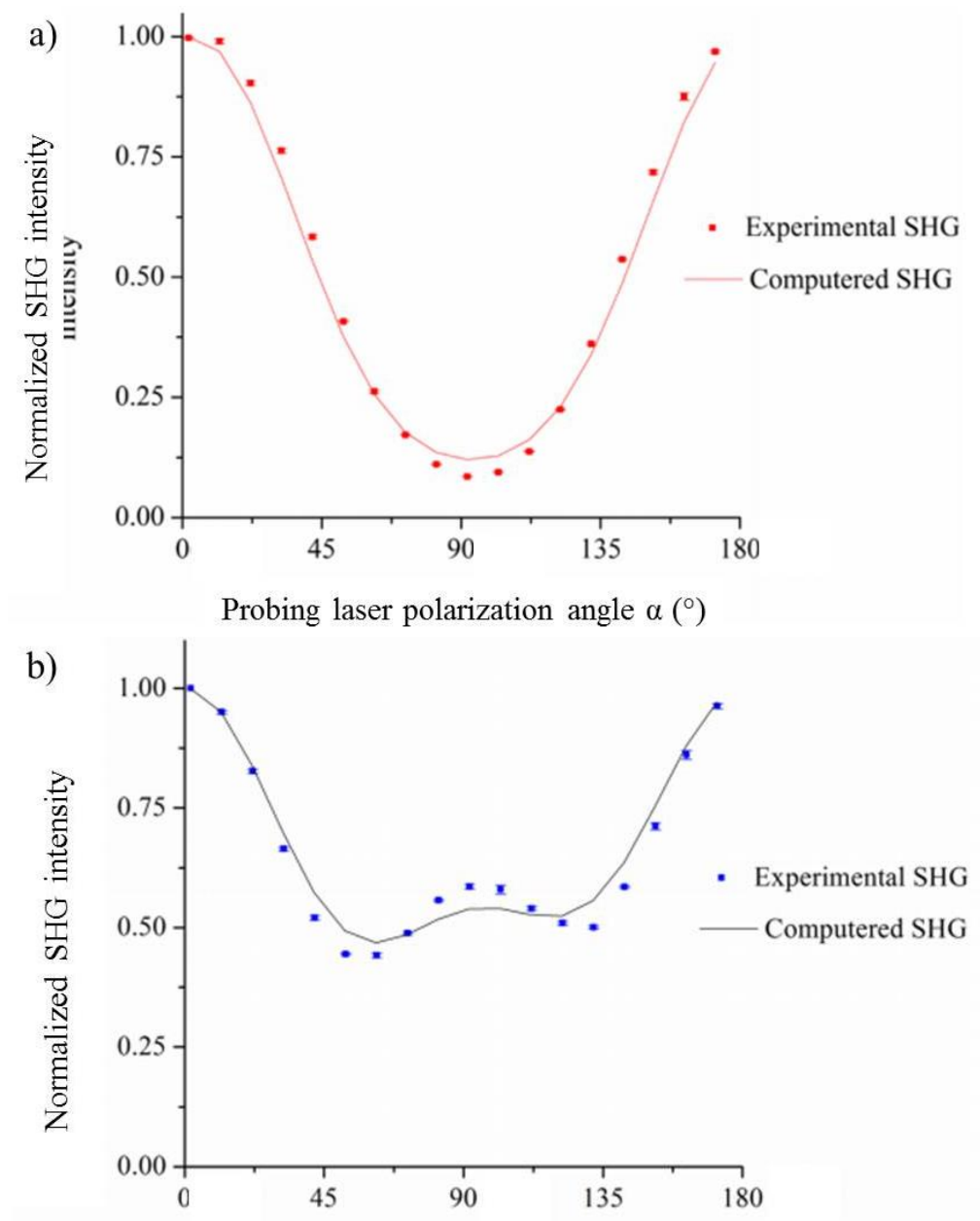

Probing laser polarization angle $\alpha\left({ }^{\circ}\right)$

Figure 104 Experimental data and computered data of a) $0.8 \mu \mathrm{J} /$ pulse and b) $1.8 \mu \mathrm{J} /$ pulse shown in Figure 97

The same procedure has been applied to pulse energy of $1.8 \mu \mathrm{J} /$ pulse considering two oriented distributions. In this case, we adjusted the expression with three populations: one randomly oriented and two textured as

$$
I_{S H G}^{N o r m}=\frac{r_{1}+r_{2} f\left(\alpha-\alpha_{2}\right)+r_{3} f\left(\alpha-\alpha_{3}\right)}{\left[r_{1}+r_{2} f\left(\alpha-\alpha_{2}\right)+r_{3} f\left(\alpha-\alpha_{3}\right)\right]_{\max }}
$$


The result is shown in Figure $104 \mathrm{~b}$ with one distribution with the polar axis oriented along $0^{\circ}$ direction (at the level of $66 \%$ ) and another with the polar axis oriented closely to $90^{\circ}$ direction (a few degrees away actually, at the level of $34 \%)$.

A second texture has actually been detected and is shown in Figure 68 using pole figures. At low pulse energy (Figure 68a), we can see that the polar axis of the crystal, is distributed perpendicular to the laser polarization direction $\left(90^{\circ}\right)$ like a fiber texture. The SHG intensity is mainly defined by the angle between the probe laser polarization direction and the polar axis (discussed in Section 5.1.1). The width of this function at half maximum is just deduced from the equation $f$ and reaches $\pm 45^{\circ}$. This acceptance is represented by the insertion of a milky disk in Figure 68a meaning "not to take into account the distribution $45^{\circ}$ around $\mathrm{Z}$ axis.

The angular dependence plotted in Figure 97-Figure 99 corresponds in these pictures to the arc drawn from $0^{\circ}$ to $180^{\circ}$. The density shows a maximum for $0^{\circ}$ but a minimum for $90^{\circ}$ as it is appearing in Figure 97 red curve.

The same procedure, applied to the curves in Figure 97 green and blue curves (i.e. 1.4 and $1.8 \mu \mathrm{J} /$ pulse). Figure $68 \mathrm{~b}$-c shows two textures: one with the polar axis perpendicular to writing laser polarization (marked by green dots) and another one at another angle from $0^{\circ}: 112^{\circ}$ for $1.4 \mu \mathrm{J} /$ pulse or $140^{\circ}$ for 1.8 $\mu \mathrm{J} /$ pulse. This is not exactly consistent with the fit that we have obtained in angular curve fitting but we have also to note that it is not exactly the same interaction volume considered in SHG analysis (XY plane) and cross section analysis (135, Z plane). In the case of SHG, the measurement is performed through the sample whereas for EBSD it is performed at the surface.

For regime 2 (e.g. $0.8 \mu \mathrm{J} /$ pulse $250 \mathrm{kHz}$ ), EBSD demonstrated that nano-crystal polar axis is perpendicular to the writing laser polarization direction (see details in Figure 71). So SHG intensity of irradiated line reaches the maximum when probing laser polarization direction is perpendicular to incident laser polarization direction.

It is worth pointing out that changing the writing orientation (from $\theta$ to $\theta+180^{\circ}$, e.g. from $45^{\circ}$ to $225^{\circ}$ ) leads to a different distribution of polar axis Figure $71 \mathrm{c}$ and d). This indicates that AOW effect is active during laser induced crystallization process as it was also the case for SHG.

The simple preferential orientation is the one that the polar axis is perpendicular to the writing laser polarization direction, which can be explained by a torque exerted on a dipole of the nanocrystal during the nucleation (see details in Section 4.1.3). 
The existence of a second texture in the SHG angular response is more difficult to explain. There are two possibilities: this last is not at the same location in the laser track than the previous one and at that place the predominant electromagnetic force is of different origin (non-linear), or the force is of different nature and this last is dominant (e.g. thermal but this one is not dependent of the laser polarization). This discussion may become speculative but in previous paper [228], a theoretical approach for trying to understand this effect has been proposed, ariseing from the peculiarity of the fs laser interaction with dielectrics

Briefly, the laser light is absorbed through multi-photon ionization or tunnelling ionization producing a quasi-free electron plasma in the $\mathrm{CB}$. Then, the formed plasma may be further heated by the rest of the laser pulse through free carrier one or several-photon absorption and/or grows through avalanche ionization (see details in Section 2.1.2.1). In a previous publication [228], for explaining the existence of the appearancee of asymmetric effect in writing with the fs laser like it is also described in SHG. Here, we have assumed that a ponderomotive force (the force created by the light on the electrons, see details in Section 2.1.4) increases the plasma density on a side of the beam and then that the trapping afterwards "records" the subsequent space charge in the materials, producing a DC field and a stress field that can act between the pulses (memory effect).

In such a way, a second torque is appearing based on the spontaneous dipole of the nano-crystal that may have a direction parallel to the polar axis. The direction of the polar axis is thus driven by the direction of the induced DC electric field that is discussed in ref [228]. It depends on the orientation of the pulse front tilt, of the writing laser polarization direction and of the direction of writing. The pulse front tilt has been measured here rotated by $142^{\circ}$ around the $Z$ axis from $X$ (pulse front tilt azimuth) and tilted by $67^{\circ}$ from the $Z$ axis (pulse front tilt colatitude). The angle between the writing laser polarization and pulse front tilt makes $55.6^{\circ}$. This means from the theory developed in [228] that the polarization can play a role. On the other hand, it is also noticed in this paper, that the stress field modify the kinetics and the stress field is dependent on the orientation of writing. We can also remark that the angles between the direction of writing and the pulse front tilt one are $83.6^{\circ}$ (for $45^{\circ}$ writing direction) and $96.4^{\circ}$ (for $225^{\circ}$ writing direction). Finally, the combination of a stress field differently oriented compared to the pulse front tilt vector may lead to a variation of the crystallization, here detected on the intensity of the second texture (the one aligned with the laser polarization). 
So the SHG results are in agreement with the EBSD one. In other words, the SHG curve can be explained by the crystal distribution.

\subsubsection{Laser radiation-configuration effect}

Figure 103 shows the $\gamma_{\max }-\left(\phi+90^{\circ}\right)$ as the function of writing laser polarization direction at fixed writing orientations (i.e. along $180^{\circ}$ and $225^{\circ}$ ). These fluctuations are not exactly perpendicular to writing laser polarization direction by $-5^{\circ}$ for writing orientation $225^{\circ}$. For the case of writing orientation $180^{\circ}$ (illustrated by black curve in Figure 103), the fluctuations of probing laser polarization direction is large as $\sim 40^{\circ}$ in amplitude. This likely indicates that other torques were active. In the first case, the torque is not dependent on writing laser polarization direction or on writing orientation but dependent on radiation intensity of laser. It may arise from temperature gradient following a rather classical way. In the case of writing orientation $225^{\circ}$ (illustrated by red curve in Figure 103), the torque is almost not dependent on writing orientations.

In a previous publications [228], an explanation has been suggested based on the creation of a non-oscillating electric field $\left(\mathbf{E}_{D C}^{w}\right)$ by trapping of inhomogeneous plasma and a torque produced by the action of this field on the spontaneous polarization of the nuclei assuming that they have already the structure of the final crystals (ferroelectric phase). This yields $\Gamma_{D C}=\mathbf{P}_{D C}^{w} \wedge \mathbf{E}_{D C}^{w}$. We will not go further in the description of this kind of model that we proposed in [228], but such a term may explain the AOW effect observed from SHG.

The orientation of the non-oscillating electric field is defined mainly by the orientation of the pulse front tilt. On the other hand, a stress field is produced (a shear stress field [13]), which is dependent on the scanning direction.

Because $\mathrm{LiNbO}_{3}$ crystal is piezoelectric, another dielectric polarization contribution may be produced: $P=\delta \tau$, with $\delta$ the third order piezoelectric tensor and $\tau$ the stress field. This term may provide torque contribution that is dependent in a complex way on scanning direction in itself or versus the direction of the pulse front tilt. 


\subsection{Refractive index change}

\subsubsection{Refractive index observation}

\subsubsection{Microscopy observation in natural light}

Firstly, microscopy observation using natural light is conducted to check if there is contrast between written lines and background (the part that has not been irradiated by fs laser). The factors inducing contrast may be due to the index change, absorption, or scattering.
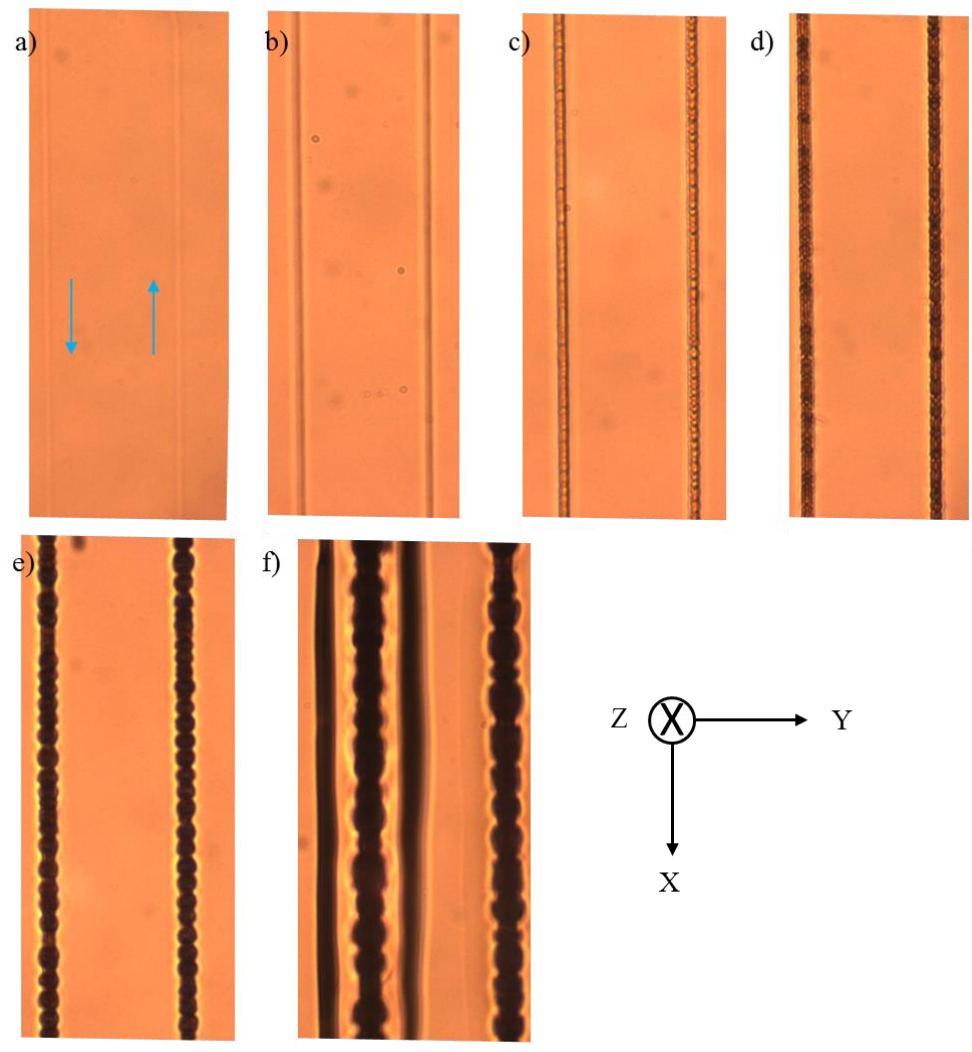

Figure 105 Irradiated lines taken by natural light microscopy (transmission mode) measured in XY plane. Other parameters: $33 \mathrm{Li}_{2} \mathrm{O}-33 \mathrm{Nb}_{2} \mathrm{O}_{5}-34 \mathrm{SiO}_{2}$ (mol\%), $1030 \mathrm{~nm}, 300 \mathrm{fs}, 500 \mathrm{kHz}, \mathrm{NA}=0.6$, focus depth $650 \mu \mathrm{m}$ in glass, 5 $\mu \mathrm{m} / \mathrm{s}$, pulse energy: a) 0.4 , b) 0.6 , c) 0.8, d) 1.0 , e) 1.4 , and f) $2.0 \mu \mathrm{J} /$ pulse (two lines in each energy group, the blue arrows indicate the writing orientations), laser polarization direction is parallel to scanning direction. The distance between each line is $50 \mu \mathrm{m}$.

Figure 105 shows the microscopy images (nature light) of irradiated lines varying pulse energies. As shown in Figure 105a, at $0.4 \mu \mathrm{J} /$ pulse, the irradiated lines are almost transparent. At the energy of 0.6 $\mu \mathrm{J} /$ pulse (Figure 105b), homogeneous thin darker lines are observed. At 0.8-1.2 $\mu \mathrm{J} /$ pulse, there are nearly some half dots or overlapped ones and the lines become darker with the increase of pulse energy (Figure 105c-d). At 1.4-1.6 $\mu /$ pulse, the size of black dots became larger (shown in Figure 105e). At 1.8-2.2 $\mu \mathrm{J} /$ pulse, separated black dot series are obtained and the written lines become darker (illustrated in Figure 105f). 


\subsubsection{Microscopy observation in polarized light}

Then polarized microscopy was used to determine whether there is birefringence of irradiated lines. In this experiment, crossing of the analyzer and polarizer are denoted $A$ and $P$, respectively (illustrated in Figure 106 right bottom). Images are obtained between two polarizers in transmission mode.

A full wave retardation plate was used to check the slow and fast axis of the irradiated lines (this method is mentioned in Section 3.4.1.2). Before attempting an analysis of birefringence, written lines should be oriented with the neutral axis in a diagonal position with respect to the microscope polarizer and analyzer. The full wave retardation plate was inserted the angle of $45^{\circ}$ with respect to the microscope polarizer and analyzer.

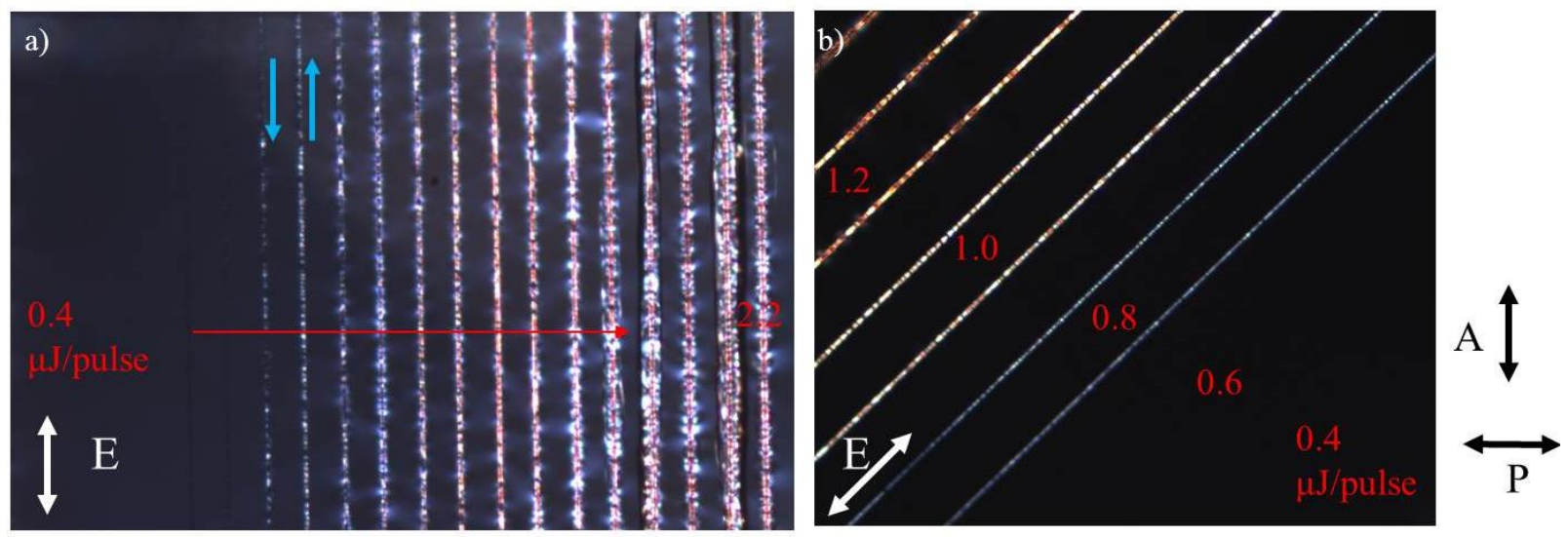

Figure 106 Images of irradiated lines taken between crossed polarizers measured in XY plane: a) parallel or perpendicular and b) diagonal position with respect to the polarizers. Other parameters: $33 \mathrm{Li}_{2} \mathrm{O}-33 \mathrm{Nb}_{2} \mathrm{O}_{5}-$ $34 \mathrm{SiO}_{2}$ (mol\%), $1030 \mathrm{~nm}, 300 \mathrm{fs}, 500 \mathrm{kHz}, \mathrm{NA}=0.6$, focus depth $650 \mu \mathrm{m}$ in glass, $5 \mu \mathrm{m} / \mathrm{s}$, pulse energy: 0.4 to $2.2 \mu \mathrm{J} /$ pulse with a step of $0.2 \mu \mathrm{J} /$ pulse (from left to right, two lines in each energy group, the blue arrows indicate the writing orientations), writing laser polarization direction is illustrated by $\mathrm{E}$. The distance between each line is $50 \mu \mathrm{m}$.

As show in Figure 106, the image of irradiated lines taken between two crossed polarizers (i.e. A and P), no birefringence is obtained for irradiated lines at the pulse energies of 0.4 and 0.6 $\mu \mathrm{J} / \mathrm{pulse}$.

With the increase of pulse energy (i.e. 0.8 to $2.2 \mu \mathrm{J} /$ pulse, regime 2), birefringence of irradiated lines is obtained. When these irradiated lines were rotated around the laser propagate direction, an intensity variation (period of $90^{\circ}$ ) of irradiated line was observed: the intensity of these irradiated lines reaches minimum when laser polarization direction is parallel or perpendicular to the analyser (Figure 106a), but the maximum when the laser polarization direction is oriented to a diagonal position to analyser (Figure 106b). In contrast, the non-irradiated area is stable (totally dark). 
For pulse energies 1.4-2.2 $\mu /$ pulse, strong scattering of irradiated lines is obtained no matter the direction of irradiated lines is in respect to the polarizer and analyser, especially for high pulse energies.

It is worth noting that these irradiated lines are colourful (Figure 106b), which is because of the constructive and destructive wave interference occurring in the different wavelengths of white light (note: white light is used in the polarized microscopy), thus causing a colour spectrum.

In the following experiment, we focus on the pulse energies of 0.8-1.2 $\mu \mathrm{J} /$ pulse (i.e. regime2). A full-wave retardation plate (530 nm, Olympus U-TP530) was used to determine slow axis of irradiated lines at this pulse energy range.
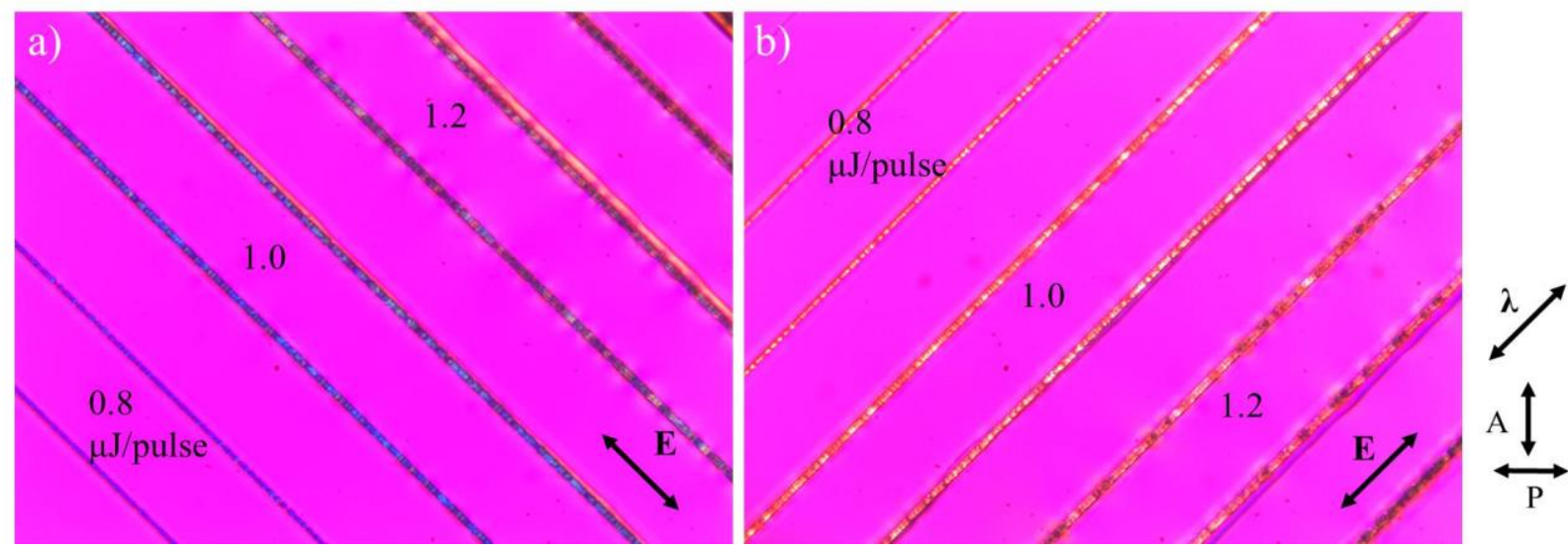

Figure 107 Images taken between crossed polarizers (denoted as A and P, respectively) and full wave retardation plate $\left(\lambda\right.$, double-headed arrow indicates slow optical axis of the retardation plate, $45^{\circ}$ of the polarizer axis) with the irradiated lines oriented at a) $-45^{\circ}$ and b) $45^{\circ}$. Other parameters: $33 \mathrm{Li}_{2} \mathrm{O}-33 \mathrm{Nb}_{2} \mathrm{O}_{5}-34 \mathrm{SiO}_{2}$ (mol\%), $1030 \mathrm{~nm}, 300 \mathrm{fs}, 500 \mathrm{kHz}, \mathrm{NA}=0.6$, focus depth $650 \mu \mathrm{m}$ in glass, $5 \mu \mathrm{m} / \mathrm{s}$, laser polarization direction is illustrated by $\mathrm{E}$. Two lines in each energy group, the distance between each line is $50 \mu \mathrm{m}$.

A full-wave retardation plate (denoted as $\lambda$ in Figure 107) was oriented with the slow axis in a diagonal position with respect to the crossed polarizers (illustrated by $A$ and $P$ in Figure 107). The background is isotropic, appearing magenta. When line appears blue (Figure 107a), it means that the slow axis of irradiated line at that place is parallel to the slow axis of the full-wave retardation plate. In contrast, if yellow (Figure 107b), the slow axis irradiated line at that place is perpendicular to the slow axis of the full-wave retardation plate. This phenomenon is clear for low pulse energy one (i.e. $0.8 \mu \mathrm{J} /$ pulse, shown in Figure 107) than for the higher ones (i.e. 1.0 and $1.2 \mu \mathrm{J} /$ pulse, shown in Figure 107). So, the large refractive index direction (slow axis) of the birefringence region is perpendicular to laser polarization direction (illustrated by $E$ in Figure $107)$ in $X Y$ plane. It is worth mentioning that for the case of $1.2 \mu \mathrm{J} /$ pulse, the line is composed by two sides with opposite birefringence. 
Similar information (i.e. slow axis of irradiated line is perpendicular to laser polarization direction in $\mathrm{XY}$ plane) is obtained whatever the scanning direction or laser polarization for regime 2.

\subsubsection{Amplitude of the mean index change}

Digital holographic microscopy measurement was used to determine the amplitude of the mean index change of irradiated lines.
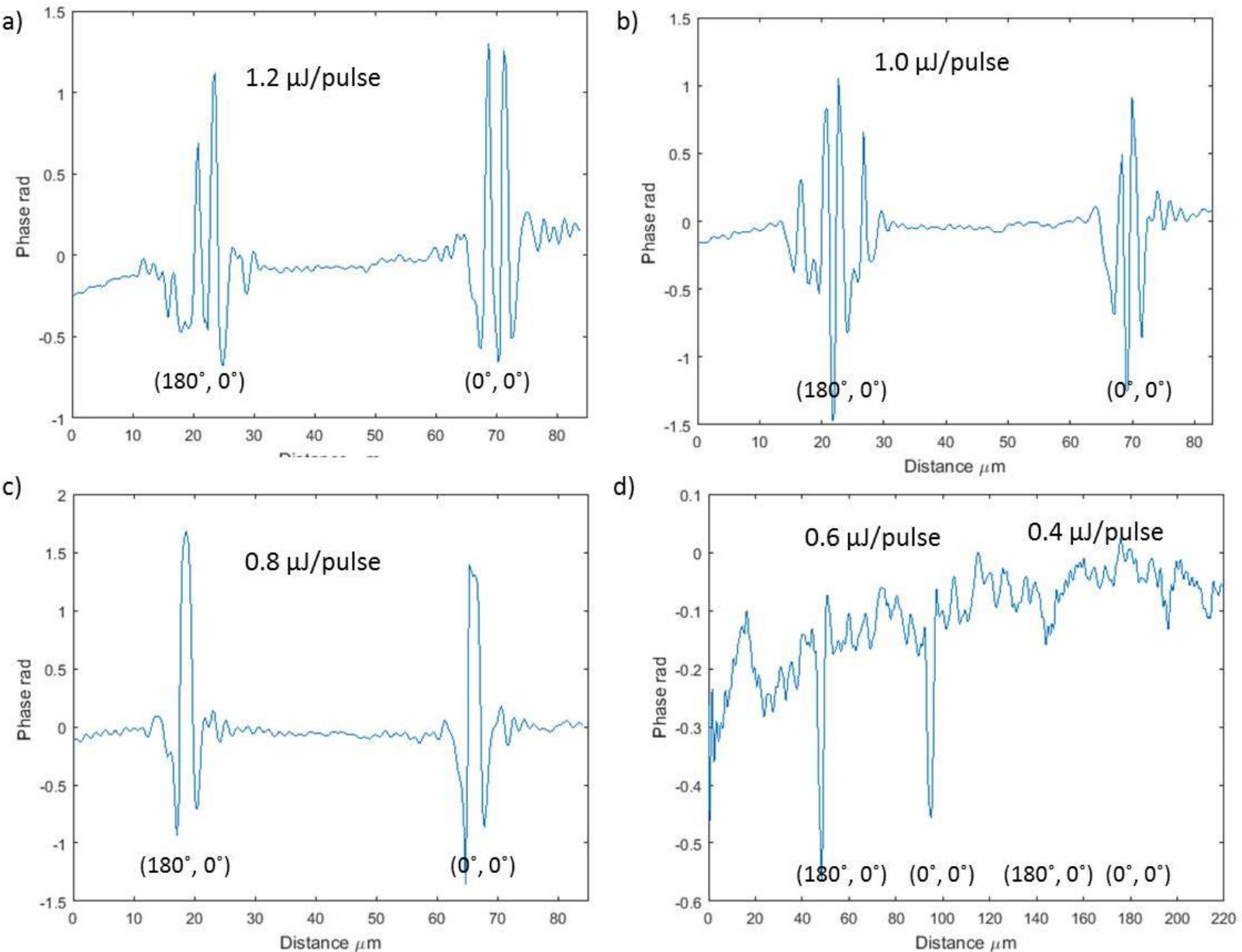

Figure 108 Digital holographic microscopy images of irradiated lines at varying pulse energy. Other parameters: $33 \mathrm{Li}_{2} \mathrm{O}-33 \mathrm{Nb}_{2} \mathrm{O}_{5}-34 \mathrm{SiO}_{2}$ (mol\%), $1030 \mathrm{~nm}, 300 \mathrm{fs}, 500 \mathrm{kHz}, \mathrm{NA}=0.6$, focus depth $650 \mu \mathrm{m}$ in glass, $5 \mu \mathrm{m} / \mathrm{s}$, laser polarization direction is parallel to writing direction. Two lines in each energy group, the distance between each line is $50 \mu \mathrm{m}$. Writing configuration considering laser scanning direction and incident laser polarization angle is defined as $(\theta, \phi)$, displayed for each picture.

At 1.0 to $1.2 \mu \mathrm{J} /$ pulse, the crystallization leads to oscillations of positive and negative index changes in the irradiated area (Figure 108a-b). When decreasing the pulse energy (i.e. 0.8 $\mu \mathrm{J} /$ pulse, Figure 108c), there is one single positive index change oscillation at the center (as large as $1.7 \mathrm{rad}$ ) surrounded by small negative ones. A negative index change is recorded for 0.6 $\mu \mathrm{J} /$ pulse, shown in Figure 108d, almost no crystallization of irradiated line is detected for 0.4 $\mu \mathrm{J} /$ pulse (Figure 108d). 
The index change is thus consistent with fictive temperature change for low pulse energy reaching -1 to $-2.1 \times 10^{-3}$. When increasing the energy, index change become positive and reaches $10^{-2}$ due to crystallization. Then for higher pulse energy, behaviour is complex but remaining at a high positive level.

\subsubsection{Quantitative birefringence microscopy measurements}

Figure 109 shows the birefringence properties of fs laser-irradiated lines varying pulse energy (marked by the white arrow in Figure 109a, from left to right 0.4-2.2 $\mu \mathrm{J} /$ pulse with a step of 0.2 $\mu \mathrm{J} /$ pulse, two lines in each enery group but different writing orientation illustrated by the yellow arrows, the distance between each line is $50 \mu \mathrm{m}$ ). The writing direction is along $X$ direction, the laser propagating direction is paerpendiuclar to the paper plane.
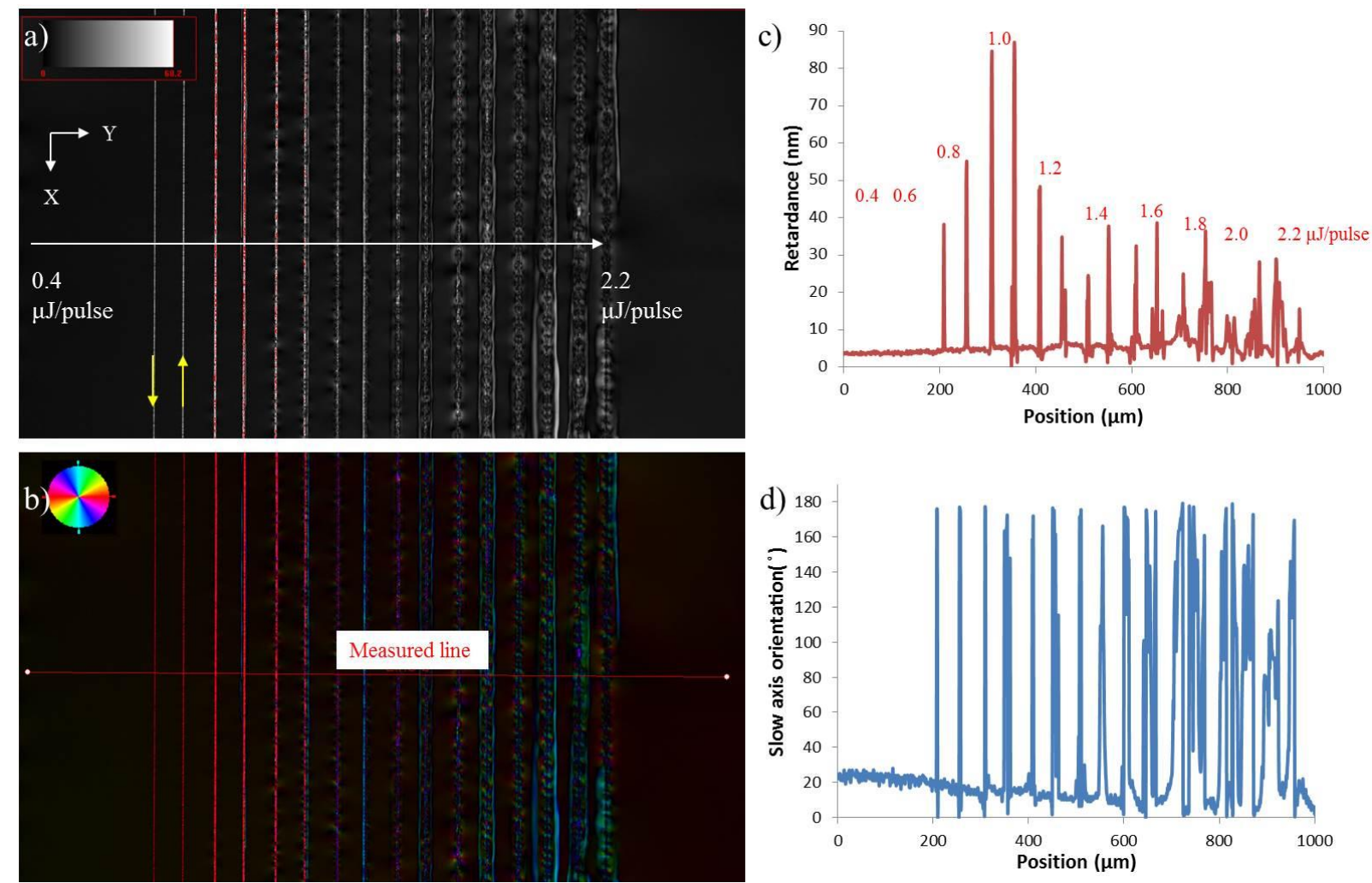

Figure $109 \mathrm{fs}$ laser-induced birefringence of irradiated lines in LNS glass varying pulse energy (0.4-2.2 $\mu \mathrm{J} /$ pulse with a step of $0.2 \mu \mathrm{J} /$ pulse, two lines with different scanning orientations for one pulse energy and the scanning directions are illustrated in yellow arrows): a) retardance amplitude image, b) quantitative birefringence microscopy image, c) profiles of the retardance, and d) the slow axis orientation, respectively. The color in QBM image shows the slow axis direction of the irradiated line and the color of the circular legend displays slow axis direction. Other parameters: $33 \mathrm{Li}_{2} \mathrm{O}-33 \mathrm{Nb}_{2} \mathrm{O}_{5}-34 \mathrm{SiO}_{2}(\mathrm{~mol} \%), 1030 \mathrm{~nm}, 300 \mathrm{fs}, 500 \mathrm{kHz}, \mathrm{NA}=$ 0.6 , focus depth $650 \mu \mathrm{m}$ in glass, $5 \mu \mathrm{m} / \mathrm{s}$, laser polarization direction is parallel to writing direction (i.e. $X$ direction). Two lines in each energy group, the distance between each line is $50 \mu \mathrm{m}$.

As shown in Figure 109a, no retardance was detected at 0.4 and $0.6 \mu \mathrm{J} /$ pulse consistently with the observation made at the Section 5.2.1.2, but from 0.8 to $2.2 \mu \mathrm{J} /$ pulse. 
Figure 109b illustrates the slow axis orientation of the corresponding irradiated lines. At 0.8-1.2 $\mu \mathrm{J} /$ pulse, the irradiated line appears nearly completely red and homogeneous along scanning direction, indicating that the slow axis (i.e. the largest refractive index axis) is oriented perpendicularly to the writing laser polarization direction. This is in agreement with the result measured from full wave retardation plate by polarized light microscopy in Section 5.2.1.2. If we continue to increase the pulse energy, the slow axis orientation becomes chaotic along scanning direction (Figure 109b right part).

Figure $109 \mathrm{c}$ and $\mathrm{d}$ shows the amplitude of the retardance and slow axis orientation of irradiated lines (the measured part of the irradiated lines is illustrated in Figure 109b, from left to right, i.e. low puls enery to high pulse energy), respectively.

It is worth noting that in Figure 109c, at $0.8 \mu \mathrm{J} /$ pulse, the retardance amplitude for $\left(180^{\circ}, 0^{\circ}\right)$ writing configuration is larger than for $\left(0^{\circ}, 0^{\circ}\right)$ one revealing an AOW effect (clearly shown in Figure 110).

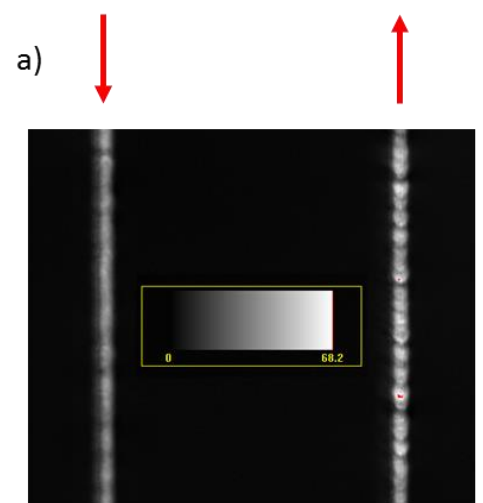

$\left(0^{\circ}, 0^{\circ}\right)$

$\left(180^{\circ}, 0^{\circ}\right)$

b)
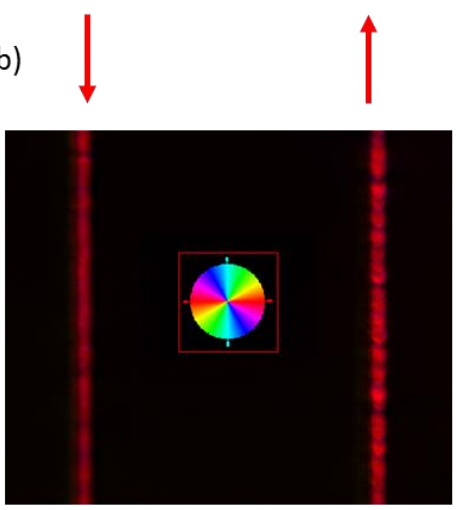

$\left(0^{\circ}, 0^{\circ}\right)$

$\left(180^{\circ}, 0^{\circ}\right)$

Figure 110 AOW effect of $f s$ laser-induced birefringence of irradiated lines in LNS glass: a) retardance amplitude image and b) quantitative birefringence microscopy image. The color in QBM image shows the slow axis of the irradiated line and the color of the circular legend displays slow axis direction. The arrows indicate the writing direction. Writing configuration considering laser scanning direction and incident laser polarization angle is defined as $(\theta, \phi)$, displayed for each picture.Other parameters: $33 \mathrm{Li}_{2} \mathrm{O}-33 \mathrm{Nb}_{2} \mathrm{O}_{5}-34 \mathrm{SiO}_{2}(\mathrm{~mol} \%), 1030 \mathrm{~nm}, 300$ $\mathrm{fs}, 500 \mathrm{kHz}, \mathrm{NA}=0.6$, focus depth $650 \mu \mathrm{m}$ in glass, $5 \mu \mathrm{m} / \mathrm{s}, 0.8 \mu \mathrm{J} /$ pulse, laser polarization direction is parallel to writing direction. Two lines in each energy group, the distance between each line is $50 \mu \mathrm{m}$, red arrows indicate the writing orientation.

\subsubsection{Summary of index change observation}

So, under fs laser irradiation, increasing the pulse energy, we observe three regimes of refractive index changes with three pulse energy thresholds:

1) The first one is defined by the appearance of isotropic negative index change; 
2) The second one is the appearance of crystalline lines with textured crystal orientation. In that case, the polar axis of nano-crystal is distributed in the plane perpendicular to writing laser polarization direction. When the pulse energy is slightly higher than this threshold (regime 2), birefringence with slow axis perpendicular to writing laser polarization direction was detected in $\mathrm{XY}$ plane (i.e. the plane perpendicular to laser propagation direction);

3) The third threshold is the one when it appears smooth crystalline zone surrounding the central rough crystalline zone (i.e. crystals distributed in oriented nanostructure). Above this threshold (i.e. regime 3), strong scattering is observed by polarized microscopy. The birefringence is chaotic along writing direction, indicating a disordered texture at high pulse energy (see details in Section 4.1.1.2.2).

\subsection{2. fs laser-induced form birefringence in LNS glasses}

In Section 5.2.1, we show that it is possible to obtain birefringence in LNS glasses at certain pulse energies. The birefringence orientation is therefore well defined at the regime 2 (e.g. 0.5-0.9 $\mu \mathrm{J} /$ pulse at $300 \mathrm{kHz}$ ), and the nano-crystal orientation is also well defined in this pulse energy range. The idea is, thus, that birefringence originates from nano-crystal texturation. For a semiquantitative explanation, we have, thus, investigated the texture of the laser-induced nanocrystals in in LNS glass.

\subsubsection{Refractive Index modelling of fs laser-induced textured nano- crystals}

The refractive index of textured nano-crystals in regime 2 is calculated in the XY plane based on EBSD data. Refractive Index is derived from the relative permittivity as

$$
\begin{aligned}
& =2 \\
& \mathrm{n}_{\mathrm{c}}=\varepsilon_{\mathrm{r}, \mathrm{c}}
\end{aligned}
$$

For $\mathrm{LiNbO}_{3}$ crystal, the relative permittivity matrix in crystal reference can be written as

$$
{ }_{\varepsilon_{r, c}}=\left[\begin{array}{ccc}
\varepsilon_{\mathrm{o}} & 0 & 0 \\
0 & \varepsilon_{\mathrm{o}} & 0 \\
0 & 0 & \varepsilon_{\mathrm{e}}
\end{array}\right]
$$

Where $\varepsilon_{\mathrm{o}}$ is the relative permittivity perpendicular to polar axis and $\varepsilon_{\mathrm{e}}$ is the one along the polar axis. 
We can deduce the dielectric susceptibility from the refractive index at $515 \mathrm{~nm}, \mathrm{n}_{0}=2.332$ and $\mathrm{n}_{\mathrm{e}}=2.241[183]$.

In the IPF (e.g. Figure 56), each crystal grain has an Euler angle $\left(\begin{array}{lll}\varphi_{1} & \Phi & \varphi_{2}\end{array}\right)$ in sample reference (i.e. XYZ-coordinate shown in Figure 56).

The relationship between the crystal reference and sample reference is given as

$$
\begin{aligned}
& \begin{array}{l}
=\text { sample } \\
\varepsilon_{\mathrm{r}}
\end{array}=\overline{\mathrm{B}}_{\varepsilon_{\mathrm{r}, \mathrm{c}} \mathrm{B}}^{=-1} \\
& \overline{\mathrm{B}}=\left[\begin{array}{ccc}
\cos \varphi_{1} \cos \varphi_{2}-\sin \varphi_{1} \sin \varphi_{2} \cos \Phi & \sin \varphi_{1} \cos \varphi_{2}+\cos \varphi_{1} \sin \varphi_{2} \cos \Phi & \sin \varphi_{2} \sin \Phi \\
-\cos \varphi_{1} \sin \varphi_{2}-\sin \varphi_{1} \cos \varphi_{2} \cos \Phi & -\sin \varphi_{1} \sin \varphi_{2}+\cos \varphi_{1} \cos \varphi_{2} \cos \Phi & \cos \varphi_{2} \sin \Phi \\
\sin \varphi_{1} \sin \Phi & -\cos \varphi_{1} \sin \Phi & \cos \Phi
\end{array}\right]
\end{aligned}
$$

The $B$ matrices are obtained by the EBSD measurements. The relative permittivity tensor of textured crystal assembly can then be obtained by weighted averaging of the crystal grain distribution according to the following formula (ignoring the effect of the grain boundaries).

$$
\varepsilon_{r}=\frac{\sum_{i}^{=\text {texture }} \varepsilon_{r, i} \mathrm{~S}_{i}}{\sum_{\mathrm{i}} \mathrm{S}_{\mathrm{i}}}, \mathrm{i}=1 . . \mathrm{N}
$$

Where $\mathrm{i}$ is relative to a grain (a connex space part with a given crystal direction), $\mathrm{S}_{\mathrm{i}}$ is the number of measured points for this grain, and $\mathrm{N}$ is the number of crystal grains.

Based on the above discussion, we can get for Figure 55b.

$$
\begin{aligned}
& =\text { texture } \\
& \varepsilon_{r}
\end{aligned}=\left[\begin{array}{ccc}
5.3995 & 0.0133 & -0.0032 \\
0.0133 & 5.2646 & 0.0157 \\
-0.0032 & 0.0157 & 5.2344
\end{array}\right]
$$

The diagonalization of this matrix leads to the exact eigen values and exact neutral axis direction but we can see that the off diagonal terms are very small compared to the diagonal ones and thus the neutral axes of the texture appears to coincide with the sample frame. This is due to the particular texture of the nano-crystal assembly.

So, the index in the direction of the laser polarization (i.e. 2.324 in X direction) is larger than the one in the direction perpendicular to the laser propagation and to the laser polarization direction (i.e. 2.294 in $Y$ direction). This is opposite to what we have measured from QBM shown in Figure 111 (i.e. large index along $\mathrm{Y}$ in $\mathrm{XY}$ plane). 
For addressing this discrepancy, we have to go further to take into account the nanostructure of the irradiated area.
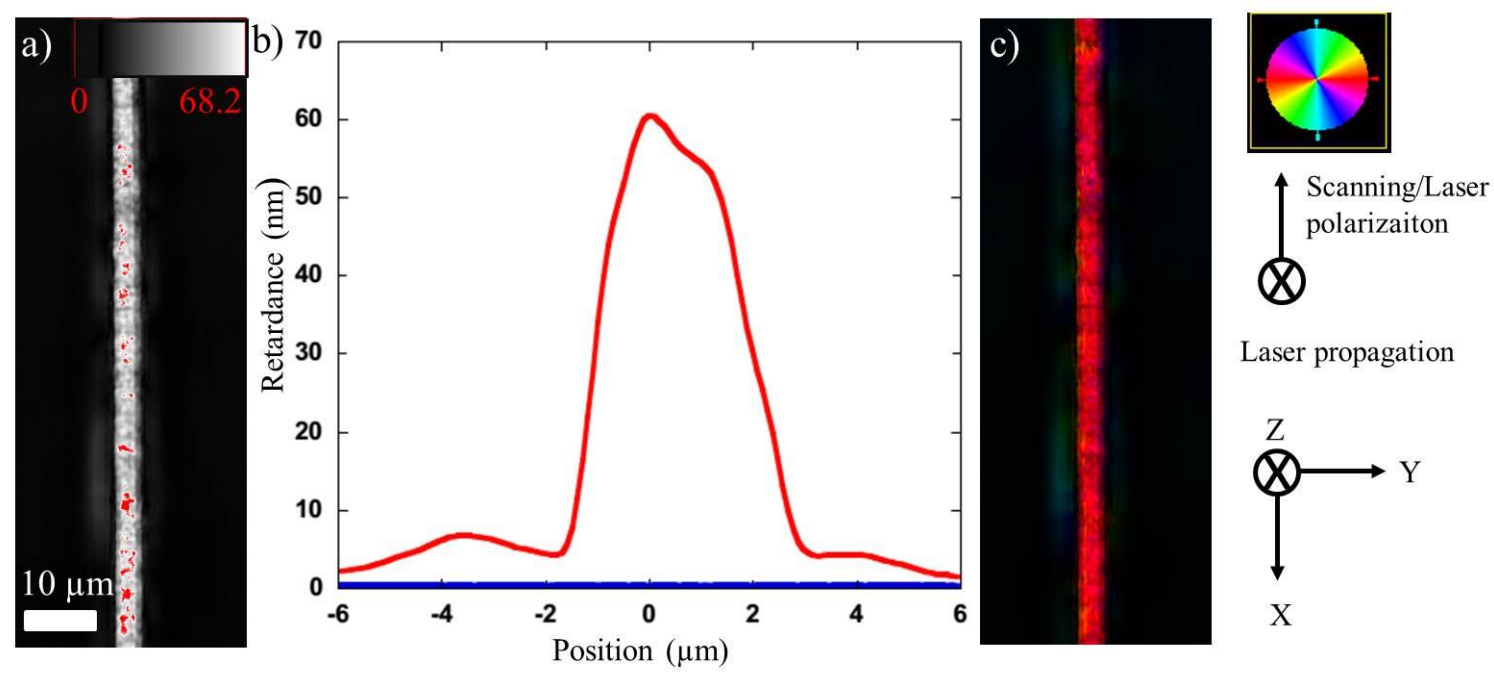

Laser propagation

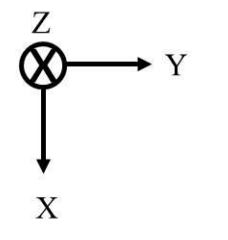

Figure $111 \mathrm{fs}$ laser-induced birefringence of irradiated line: a) retardance image, b) retardance profile of the irradiated line, and c) QBM image. The color in QBM images displays the slow axis of the birefringence zone. The color of the circular legend presents the direction of the slow axis (right top). Other parameters: $33 \mathrm{Li}_{2} \mathrm{O}-$ $33 \mathrm{Nb}_{2} \mathrm{O}_{5}-34 \mathrm{SiO}_{2}$ (mol\%) , $1030 \mathrm{~nm}, 300 \mathrm{fs}, 300 \mathrm{kHz}, 0.7 \mu \mathrm{J} /$ pulse, NA = 0.6, focus depth $350 \mu \mathrm{m}$ in glass, and 5 $\mu \mathrm{m} / \mathrm{s}$.

\subsubsection{Form birefringence in homogenous case (isotropic in YZ plane)}

To compute the birefringence of irradiated lines containing a nanostructure, we will start with the homogenous case (the refractive indices of all plates are isotropic). For details please refer to [247].

As illustrated in Figure 112, we consider an array of parallel plates with a thickness $d_{1}$ and a dielectric constant $\varepsilon_{1}$. The distance between neighbouring plates is called $d_{2}$ and a filling factor $f$ is defined as $d_{1} /\left(d_{1}+d_{2}\right)$. The matter between these plates has a dielectric constant of $\varepsilon_{2} . d_{1}$ and $d_{2}$ are smaller but we assume that the face of the plate (i.e. YZ plane) is larger than the wavelength. The incident monochromatic probing wave is considered to propagate along $Z$ direction.
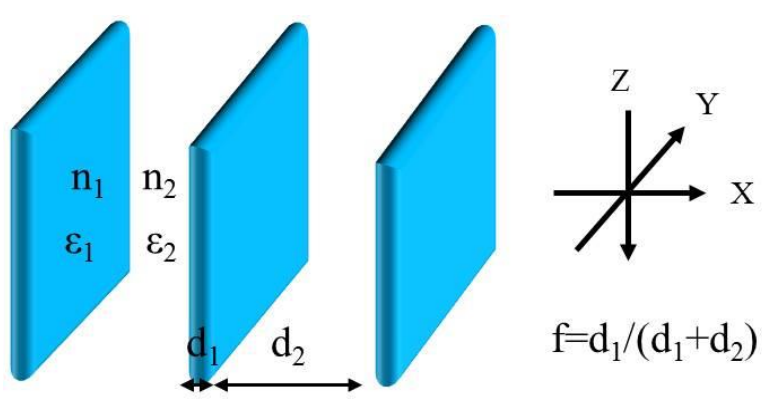

$$
\mathrm{f}=\mathrm{d}_{1} /\left(\mathrm{d}_{1}+\mathrm{d}_{2}\right)
$$

Figure 112 Schematic representation of the nanostructure involved in form birefringence with array of parallel plates 
For the case where electric vector is along $Y$ direction: from the continuity of tangential component of electric field across the surface, the effective dielectric constant $\varepsilon_{Y}$ is given as

$$
\varepsilon_{\mathrm{Y}}=\mathrm{f} \varepsilon_{1}+(1-\mathrm{f}) \varepsilon_{2}
$$

For the case where electric vector is along $X$ direction: since the continuity of normal component of electric displacement across the surface, the effective dielectric constant $\varepsilon_{\mathrm{x}}$ is given as

$$
\varepsilon_{\mathrm{X}}=\frac{\varepsilon_{1} \varepsilon_{2}}{\mathrm{f} \varepsilon_{2}+(1-f) \varepsilon_{1}}
$$

For all directions parallel to the $\mathrm{YZ}$ plane, the effective dielectric constant is the same. This structure is like a uniaxial material with the extraordinary optical axis along $X$ direction. The refractive indices are given as

$$
n_{0}^{z}=f n_{1}^{2}+(1-f) f n_{2}^{2}, \quad n_{e}^{z}=\frac{n_{1}^{2} n_{2}^{2}}{f n_{2}^{2}+(1-f) n_{1}^{2}}
$$

\subsubsection{Form birefringence in the case of a nanostructure containing} textured crystals

After preparation of sample by FIB, we observed the irradiated area at the nanometre-scale by TEM. We found a nanostructure consisting of a modulation of structural phase with alternated chemical compositions. As illustrated in Figure 113a, the nanostructure consists of network of "walls" oriented perpendicular to laser polarization direction (in white in the figure) and material in-between the above "walls" (black or grey regions in the figure). HRTEM image (Figure 113b) confirmed the crystalline property of the black or grey regions, demonstrating that nano-crystals are embedded in an amorphous network.

It is obvious that such a nanostructure behaves optically differently than an isotropic material and due to the oriented nanostructure we can expect a form birefringence.

Let us try to estimate its amplitude. The nanostructure is schemed by the simplified model in Figure 113c, where the blue plates are for the amorphous phase (material quoted 1 ) and the red one stands for the oriented nano-crystal area in-between the amorphous plates (material quoted 2). 
For computing the refractive index anisotropy of this assembling, we used a similar approach than the one discussed in Section 5.2.2.2, but included an anisotropic material between the plates. It is only necessary to say that due to the quasi-diagonal property of $\begin{aligned} & =\text { texture } \\ & \varepsilon_{r}, \text { sample }\end{aligned}$

$$
\begin{aligned}
& n_{x x}^{2}=\frac{n_{1}^{2} n_{2, x x}^{2}}{f n_{2, x x}^{2}+(1-f) n_{1}^{2}} \\
& n_{r y}^{2}=f n_{1}^{2}+(1-f) n_{2, w y}^{2} \\
& n_{2, x x}^{2}=\varepsilon_{r, s a m p l e, x x}^{\text {texture }}, \quad n_{2, w y}^{2}=\varepsilon_{r, s a m p l e, w}^{\text {texture }}
\end{aligned}
$$

Numerically, the refractive index of amorphous phase assumed as fused silica, and thus $\mathrm{n}_{1}$ is taken equal to 1.462 at $515 \mathrm{~nm}$ [248]. The thickness of the plate $\left(d_{1}\right)$ is deduced from the TEM observations to $9 \pm 1 \mathrm{~nm}$. The nano-crystals (in-between the plates) are textured as it is described in Section 4.1.2.2. This area has a thickness $d_{2}$ amounting $92 \pm 7 \mathrm{~nm}$. Refractive index along $X$ (i.e. $n_{2, x x}$ ) is 2.324 but the value for along $Y, n_{2, Y Y}$ is 2.294 . So, we deduce that $n_{X X}=2.180 \pm 0.022$ and $n_{Y Y}=2.232 \pm 0.011$.

This explains why the slow axis (i.e. the largest refractive index axis) of irradiated line (in regime 2 ) is experimentally observed oriented perpendicular to writing laser polarization direction in $X Y$ plane (i.e. QBM measurement, shown in Figure 111c).

Quantitatively, experimental birefringence measurement is deduced from the retardance $(R)$ of the sample by $R / t$ [71], where $t$ is the thickness of the region which produces the birefringence. From Figure 55b, we found that $t$ is around $6.2 \mu \mathrm{m}$. Taking $R$ from Figure $111 \mathrm{~b}$, we get $B$ is 0.0097 , which is a quite large value. Note the calculated one with the idealized model $n_{W}-n_{x x}$ is $0.052 \pm 0.012$. So, our form birefringence modeling in LNS glass account for the correct sign of the experimental birfringence. 

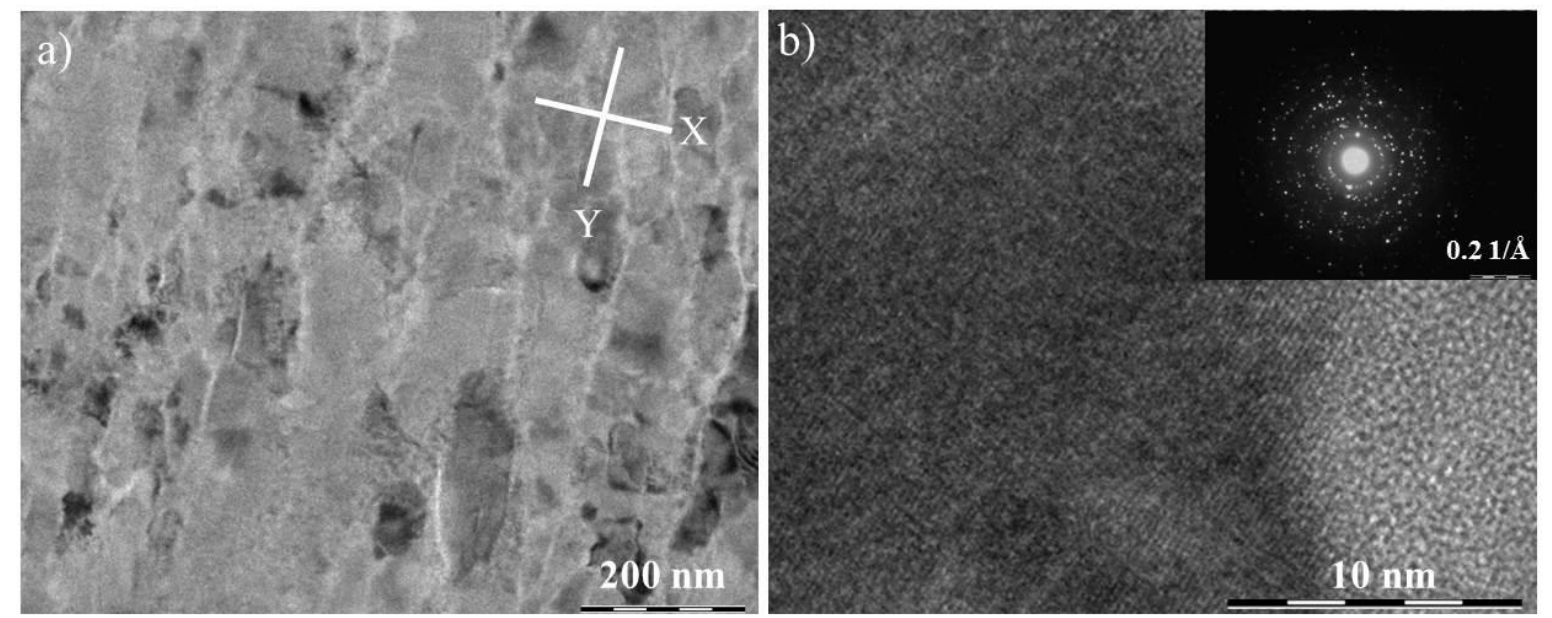

c)
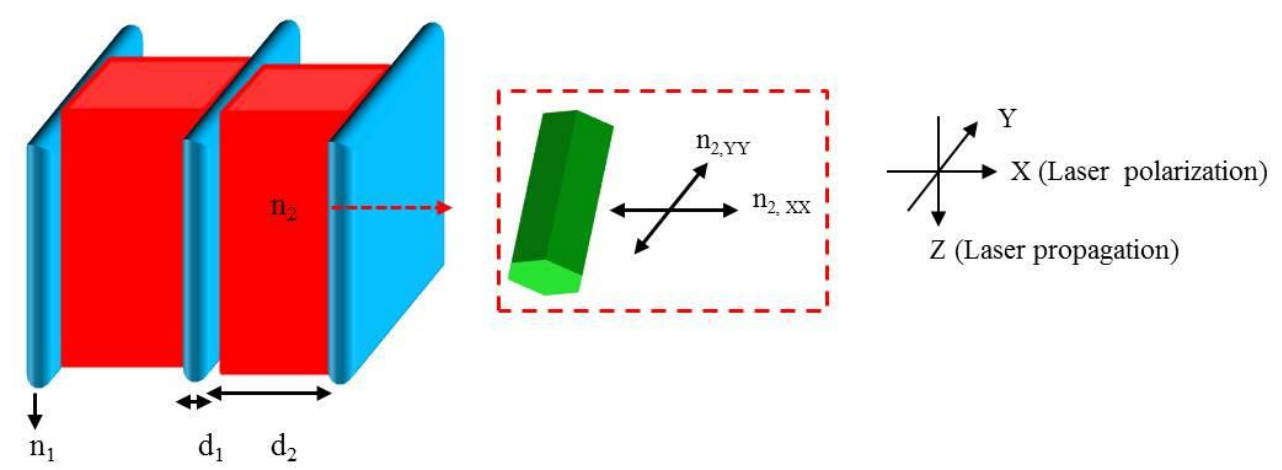

Figure $113 \mathrm{fs}$ laser-induced nanostructures investigated by TEM (bright field mode): a) overview of the FIB slice in the $\mathrm{XY}$ plan and $\mathrm{b}$ ) HRTEM image showing a zoom on a single nano-layer (the inset is the SAED pattern) for $33 \mathrm{Li}_{2} \mathrm{O}-33 \mathrm{Nb}_{2} \mathrm{O}_{5}-34 \mathrm{SiO}_{2}$ (mol\%), $1030 \mathrm{~nm}, 300 \mathrm{fs}, 300 \mathrm{kHz}, 0.7 \mu \mathrm{J} / \mathrm{pulse}, \mathrm{NA}=0.6$, focus depth $350 \mu \mathrm{m}$ in glass, and $5 \mu \mathrm{m} / \mathrm{s}$. c) Schematic illustration of fs laser-induced nanostructures with amorphous "walls" (blue plates, oriented perpendicular to laser polarization direction) and nano-crystals (red plates). The nano-crystal (illustrated by green hexagonal prism) is oriented with polar axis perpendicular to laser polarization direction. 


\section{Chapter 6. Conclusions and future works}

\subsection{Conclusions}

In this thesis, the modifications inside LNS glass by tightly focused fs laser pulses have been investigated systematically according to morphology, crystallization, and nanostructure by electron microscope, chemical analysis, and measurement of optical properties. Novel phenomena (e.g. laser polarization dependent nano-crystal orientation and oriented nanostructure formation) and the excellent properties such as form birefringence and tunable SHG were discovered. These topics may have potential applications in 3D functional optical device fabrications.

\section{Overview of the fs-laser induced modifications in LNS glasses}

Depending on the pulse energy $(0.3-2.2 \mu \mathrm{J} /$ pulse at $1030 \mathrm{~nm}, 300 \mathrm{fs}, 300 \mathrm{kHz}, 5 \mu \mathrm{m} / \mathrm{s}$, focus depth $\sim 350 \mu \mathrm{m}$, and NA $=0.6$ ), the fs laser-induced modifications in LNS glass show three regimes with three thresholds (illustrated in Figure 114).

The first threshold is the occurrence of amorphous state modification (like glass fictive temperature). The second threshold (i.e. $0.5 \mu \mathrm{J} /$ pulse) is the occurrence of $\mathrm{LiNbO}_{3}$ crystals in LNS glass. Below this threshold, the glass fictive temperature is modified and thus some physical properties (regime 1): the glass gets larger sensitivity to the chemical etching (i.e. HF) (see zone B in Figure 55a).

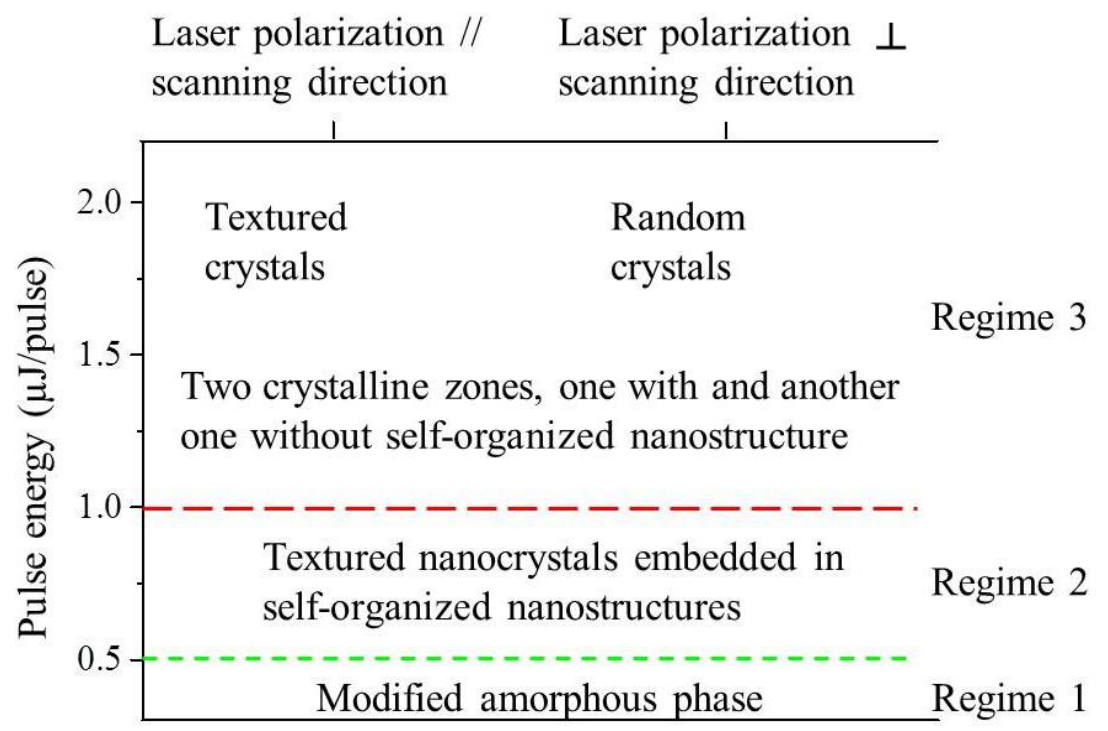

Figure 114 Three regimes of $\mathrm{fs}$ laser-induced modifications of irradiated lines in LNS glass as the function of pulse energy (0.3-2.2 $\mathrm{\mu J} /$ pulse). Other parameters: $33 \mathrm{Li}_{2} \mathrm{O}-33 \mathrm{Nb}_{2} \mathrm{O}_{5}-34 \mathrm{SiO}_{2}(\mathrm{~mol} \%), 1030 \mathrm{~nm}, 300 \mathrm{fs}, 300 \mathrm{kHz}, 5$ $\mu \mathrm{m} / \mathrm{s}, \mathrm{NA}=0.6$, focus depth $350 \mu \mathrm{m}$ in glass 
When pulse energy is slightly higher than the second threshold (i.e. $0.5-0.9 \mu \mathrm{J} /$ pulse, in regime 2), nano-crystals are observed distributed with polar axis perpendicular to laser polarization direction in Zone $\mathrm{C}$ of regime 2 (illustrated in Figure 55b), no matter the laser polarization or scanning direction are. Moreover, these textured nano-crystals are embedded in lamella-like amorphous phases (illustrated in Figure 85a).

This polarization dependent nano-crystal orientation can be explained by fs laser-induced torques on the nano-crystal electric dipole (see details in Section 4.1.3). This self-assembled nanostructure consisting of quasi-periodic phase changes can induce a form birefringence in the irradiated line with slow axis perpendicular to laser polarization direction (see details in Section 5.2.2.3).

The third threshold ( $1.0 \mu \mathrm{J} /$ pulse) is defined by the occurrence of crystallization without selforganized nanostructure (i.e. zone $\mathrm{C}$ of regime 3 shown in Figure $55 \mathrm{c}-\mathrm{d}$ ). This one is surrounding the zone $D$ of regime 3 that contains self-organized nanostructures together with crystals at the centre of irradiated line. When increasing the pulse energy furthermore (i.e. 1.0-2.2 $\mu \mathrm{J}$ ), the quality of crystallization is dependent on the writing configuratio: textured crystals when laser writing polarization is parallel to scanning direction (shown in Figure 55c-d), but random ones when writing laser polarization is perpendicular to scanning direction (Figure 57a). In both cases, the crystals are distributed in lamella-like amorphous phases in Zone D (shown in Figure 85c-d).

In regime 2 or 3 , the orientation of nanostructure can be controlled by laser polarization direction but regime 2 is more adapted if crystal orientation is required for the applications. This topic may have potential applications in fabricating SHG-based devices.

Crystallization: We demonstrate three types of texture/nanostructure in LNS glass by fs laser direct writing. Regime 2: when the pulse energy is slightly higher than the threshold for obtaining crystalline line (e.g. $0.5 \mu \mathrm{J} /$ pulse at $300 \mathrm{kHz}$ or $0.6 \mu \mathrm{J} /$ pulse at $250 \mathrm{kHz}, 300 \mathrm{fs}, 5 \mu \mathrm{m} / \mathrm{s}$ ), textured nano-crystals are distributed in self-organized amorphous nanostructures. Regime 3: two other kinds of nanostructures are detected in crystallization: textured crystals when writing laser polarization is parallel to scanning direction, but random crystals when writing laser polarization is perpendicular to scanning direction. This work indicates that fs laser-induced crystallization is not done only by thermal accumulation but there is an effect of laser polarization. 
Phase separation: We investigated the phase separation in fs laser irradiated LNS glass. Nonlinear optical crystal was confirmed by EBSD and SHG measurements. The first idea was that $\mathrm{LiNbO}_{3}$ crystallization occurs and thus $\mathrm{Si}$ migrates out of the irradiated area. SEM/EDS, WDS microprobe, and ion beam analysis did not reveal significant chemical profiles of $\mathrm{Li}, \mathrm{O}, \mathrm{Si}$, and $\mathrm{Nb}$ in or around the irradiated volume in bulk sample. At this step of the analysis, there were two possibilities: either $\mathrm{LiNbO}_{3}$ phase contains $\mathrm{SiO}_{2}$ in its crystallography structure or there is a phase separation beyond the scale of the resolution of the above chemical analyses. As the "horizontal" resolution in EDS is limited by the sample thickness (for less than $1 / 2$ micron), FIB was used to fabricate a thin sample suitable for TEM-based measurements. A self-organized nanostructure made of lamellas of $\mathrm{SiO}_{2}$ was then observed with embedded $\mathrm{LiNbO}_{3}$ crystalline plates at the center of the laser-modified region.

The phase diagram of $\mathrm{Li}_{2} \mathrm{O}-\mathrm{Nb}_{2} \mathrm{O}_{5}$ [169] reveals that for $\mathrm{Li}$ excess from $\mathrm{LiNbO}_{3}$, there is a phase separation into $\mathrm{LiNbO}_{3}$ and $\mathrm{Li}_{3} \mathrm{NbO}_{4}$. Even when the temperature is above $1127^{\circ} \mathrm{C}$, no solubility exists between $\mathrm{Li}_{2} \mathrm{O}$ and $\mathrm{LiNbO}_{3}$. For $\mathrm{Li}$ deficient composition, there is a phase separation into $\mathrm{LiNbO}_{3}$ and $\mathrm{LiNb}_{3} \mathrm{O}_{8}$ at lower temperature. At high temperature, $\mathrm{LiNbO}_{3}$ can show large departure in $\mathrm{Li}_{2} \mathrm{O}$ (up to 6 mol\%). $\mathrm{LiNb}_{3} \mathrm{O}_{8}$ does not contribute to ferroelectric or electro-optic properties, but a fast crystallization in a vacuum allows suppressing the formation of the $\mathrm{LiNb}_{3} \mathrm{O}_{8}$ phase [175]. On the other hand, the phase diagram of $\mathrm{Li}_{2} \mathrm{O}-\mathrm{SiO}_{2}$, shows that $\mathrm{Li}$ is soluble into $\mathrm{SiO}_{2}$ [132]. Lastly, from the pseudo-ternary phase diagram of $\mathrm{Li}_{2} \mathrm{O}-\mathrm{Nb}_{2} \mathrm{O}_{5}-\mathrm{SiO}_{2}$, the glass composition (i.e. $33 \mathrm{Li}_{2} \mathrm{O}-33 \mathrm{Nb}_{2} \mathrm{O}_{5}-34 \mathrm{SiO}_{2}, \mathrm{~mol} \%$ ) used here is on the amorphous side of the boundary between crystalline and amorphous phases [140]. Devitrification of LNS glass into $\mathrm{LiNbO}_{3}$ has been already reported for this [186] or close chemical composition [18, 187]. So, it is conceivable that a lack of $\mathrm{Li}$ will be replaced by $\mathrm{Si}$. This leads us to various chemical analysis investigations that did not give the answer and to TEM that showed a nanoscale phase separation.

SHG (i.e. nonlinear optical properties) of crystalline line was observed in our case and the crystal can be successfully coded by the lattice parameters of $\mathrm{LiNbO}_{3}$ in EBSD analysis, indicating the formation of $\mathrm{LiNbO}_{3}$ structure only.

In view of the above discussion, we can tentatively explain the observations. By controlling the beam energy, the speed of writing, and the repetition rate for a given NA and focusing depth, we can manage the temperature distribution in the glass and put it in the condition of crystallization. The chemical composition that we used is a composition ready to crystallize by 
simple heating. A phase separation occurs into Li poor crystalline $\mathrm{LiNbO}_{3}$ and $\mathrm{Li}$ rich amorphous $\mathrm{SiO}_{2}$. This is achieved at the nanometer scale. It can occur with or without forming a nanostructure, with or without a texture depending on the polarization/writing direction configuration.

Self-organized nanostructure: Such a nanostructure was first discovered in silica glass in 2003

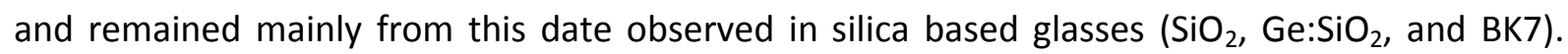
Here we show the ability to generate nanostructures by fs laser irradiation in LNS glass with a composition largely different than silica.

Two types of organizations are pointed out: one is along the laser propagation direction with $\lambda / 2 \mathrm{n}$ periodicity for laser polarization parallel to scanning direction and another one along laser polarization direction.

We extend this possibility to very different glass (i.e. LNS glass) with the originality to create SHG and form birefringence in a glass (i.e. two functions in one-way processing). A relation with known similar structures is proposed for understanding this effect, which is quite unusual in materials science. This finding extends the occurrence of nanostructure formation in glass and shows that it is possible to obtain them in the same time as nonlinear crystals via ultra-fast laser irradiation.

Second harmonic generation: We developed a flexible way for mastering SHG in LNS glass. By adjusting the pulse energy and writing speed, angular dependence of SHG with the probing laser polarization can be obtained with a high contrast. In our experimental conditions:

1) A well-defined cosine-like curve with period of $180^{\circ}$ could be obtained at low pulse energy or high pulse energy with high writing speed; SHG reaches the maximum intensity when the probing laser polarization is closely perpendicular to incident laser polarization.

2) A double cosine-like curve revealing a second texture of the crystals at high pulse energy $(1.4 \mu \mathrm{J} /$ pulse, $250 \mathrm{kHz})$ with low writing speed $(5 \mu \mathrm{m} / \mathrm{s})$ and with the polar axis oriented closer parallel to the writing laser polarization.

3) An asymmetric orientational writing was observed, especially at high pulse energy with low writing speed so when the second texture was active.

EBSD result reveals that nano-crystals produced by the fs laser radiation were oriented under the effect of an incident laser polarization modified by an effect of the scanning direction during 
fs laser radiation process. Polar axes of nano-crystals distributed around a plane perpendicular to the incident laser polarization direction.

The main discovering is the possibility to orient the maximum intensity of SHG. This is achieved by orienting the nano-crystal with the laser polarization. This is the first example of effective effect of laser polarization in material science.

A discussion has been presented, including the mechanism for tentatively explain the above observations. The main force would be the effect of the electromagnetic polarization of the writing laser on the anisotropic induced dipole. This investigation contributes to a better understanding of the mechanism of the SHG orientations and contributes to reveal the technological potential in fabricating 3D nonlinear optical devices.

Form birefringence: So far, form birefringence has been observed in limited materials, such as fused silica [92]. However, none of them have been pointed out nonlinear optical crystal embedded in such nanostructures. Therefore, exploring possibility of fs laser-induced form birefringence in glass, showing nonlinear optical properties may extend the material choice, provide insight into the ultrafast laser-matter interaction, and open new frontiers in fabricating functional devices. Herein, birefringence was achieved in LNS glass by fs laser direct writing.

We found that at regime 2, the slow axis (i.e. the direction for which the refractive index is the largest) of irradiated line is perpendicular to laser polarization direction (e.g. 0.5-0.9 $\mu \mathrm{J} /$ pulse at $300 \mathrm{kHz}$ or $0.8 \mu \mathrm{J} /$ pulse at $250 \mathrm{kHz}$ ). Unlike related results obtained in fused silica [31], we report a new kind of form birefringence in LNS glass by fs laser direct writing, which arises from a selforganized nanostructure: textured nonlinear optical nano-crystals embedded in a self-organized network of vitreous phase.

This opens the possibility to elaborate, for instance, a birefringent waveguide with optically nonlinear properties in a single step. This finding sheds light on comprehension of the formation of nanostructure in glass via ultra-fast laser radiation as well as construction of nonlinear optical nano-devices.

Asymmetric orientational writing: It is the first time that an AOW effect is clearly demonstrated in SHG and related to photo precipitation of crystals in a silica-based glass. This topic may help understand the ultrafast laser-matter interaction. In addition, an AOW effect has also observed in the birefringence (illustrated in Figure 110). 


\subsection{Further works}

Crystallization: The contribution of this work is clearly to show that crystallization by focused fs laser radiation is far beyond to be only a thermal effect. This topic presents the advancement achieved in fs laser micro-machining and the understanding of ultra-fast laser-matter interaction.

The mechanism responsible for the crystal formation together with nanostructure by ultra-fast laser irradiation is still under discussion. The next step is to know if the crystallization can play a role in the organization of the nanostructure and vice-versa:

Nanostructure occurs without crystallization in some glasses, but when crystallization appears, are the specificities of the nanostructure the same (e.g. the inter-plan distance, the amplitude modulation)? There is a lack of information to conclude on that point.

Reversely, is there an effect of the nanostructure on the crystallization? Is the texture (i.e. crystal orientation) influenced by the existence of the self-organized nanostructure? For zone $D$ of regime 3, when laser polarization is parallel to scanning direction, SAED results show crystalline pattern, but polycrystalline pattern when laser polarization is perpendicular to scanning. This leads to consider that light is acting directly on the crystallization process through a non-thermal process.

Lastly, it is interesting to investigate the possibility to rewrite the texture/nanostructure as it has been shown in fused silica by changing the polarization orientation [249].

Chemical migration: it is worth noting that Li distribution at nanoscale is not given yet. Considering $\mathrm{Li}_{2} \mathrm{O}-\mathrm{SiO}_{2}$ system can be in amorphous state, it would be necessary to measure the $\mathrm{Li}$ concentration locally by electron energy loss spectroscopy (EELS) or some other methods.

Modelling: In this thesis, constant thermal parameters of materials are used in the thermal modelling. In addition, other phenomena such as the complex chemical reaction and shock wave (i.e. multi-field coulping) are not considered. So, a completed model of fs laser-induced modifications in needed.

Extend to other material: herein, we master $\mathrm{LiNbO}_{3}$ with controlled orientation and size in LNS glass by fs laser irradiation. The information about how to choose nonlinear optical crystal (e.g. space group) and the glass compositions (see details in 2.2.2.4) has been discussed, which may 
guide the users to choose other materials system such as $\mathrm{Na}_{2} \mathrm{O}-\mathrm{BaO}-\mathrm{TiO}_{2}-\mathrm{SiO}_{2}, \mathrm{BaO}-\mathrm{Al}_{2} \mathrm{O}_{3}-\mathrm{Ba}_{2} \mathrm{O}_{3}$, and $\mathrm{La}_{2} \mathrm{O}_{3}-\mathrm{B}_{2} \mathrm{O}_{3}-\mathrm{GeO}_{2}$ glass systems. In addition, the mechanism and modelling of $\mathrm{fs}$ laser-induced thermal field in LNS glass is given which can be used to determine the irradiation condition (e.g. pulse energy and repetition rate, see details in Section 3.3).

SHG waveguides fabrication: it is necessary to measure the efficiency. For problem of phase matching, for a conventional SHG, there is an optical phase relation between all point of the doubling medium. In that case, there is extinction of the SHG intensity according to the SHG medium thickness. These leads to $n_{2 \omega}=2 n_{\omega}$ condition that is not possible to fulfil in waveguide. But another condition is possible in waveguide if the medium is modulated along the propagation direction: this is the quasi phase matching condition. The medium is alternately doubling and not according to a spatial period. But for nano-crystals, which lose the optical phase relation between them there is no more phase matching condition to fulfil. In that case the intensity maximum is smaller than the one with a single crystal but their use is more flexible. Meanwhile, it is also necessary to measure the loss of this waveguide.

With further improvements in the fabrication techniques, the application of this work is to achieve functional optical devices such as frequency doubling optical waveguide and birefringence-based devices. 


\section{Appendix}

\section{Laser development}

Table A 1 Timeline of laser science and technology

\begin{tabular}{|c|c|c|c|}
\hline Period & Time & Authors & Contribution \\
\hline \multirow[t]{2}{*}{ Theory } & 1900 & M. Planck & quantum theory \\
\hline & 1916 & A. Einstein & stimulated emission \\
\hline \multirow[t]{2}{*}{ Maser } & 1953 & C. H. Townes' group & develop maser \\
\hline & 1958 & $\begin{array}{l}\text { C. H. Townes and A. } \\
\text { Schawlow }\end{array}$ & $\begin{array}{l}\text { "optical maser", masers could be made to operate in the optical } \\
\text { and infrared regions }\end{array}$ \\
\hline \multirow[t]{27}{*}{ Laser } & 1960 & T. H. Maiman & first laser, pulsed ruby laser ("pink" ruby) \\
\hline & 1960 & P. Sorokin and M. Stevenson & $\begin{array}{l}\text { uranium and samarium lasers, four-level laser system, cryogenic } \\
\text { cooling and emitted in the IR }\end{array}$ \\
\hline & 1960 & $\begin{array}{l}\text { A. Javan, W. Bennett Jr. and } \\
\text { D. Herriott }\end{array}$ & first CW and gas laser, He-Ne laser, $1.15 \mu \mathrm{m}$, \\
\hline & 1961 & L. F. Johnson and K. Nassau & first Nd-doped solid state laser \\
\hline & 1961 & E. Snitzer & $\begin{array}{l}\text { first } \mathrm{Nd} \text {-glass laser in a millimeter-scale rod with the Nd glass in a } \\
\text { high-index core, making it essentially for the first fiber laser }\end{array}$ \\
\hline & 1962 & Bell Labs & Yttrium aluminum garnet (YAG) laser \\
\hline & 1962 & A. White and D. Rigden & He-Ne laser, $632.8 \mathrm{~nm}$, first CW laser with a visible beam \\
\hline & 1962 & R. Hall & GaAs laser, first semiconductor laser, cryogenically cooled \\
\hline & 1962 & N. Holonyak Jr. & red-emitting GaAsP laser diode, visible coherent light \\
\hline & 1963 & $\begin{array}{l}\text { L. E. Hargrove, R. L. Fork and } \\
\text { M. A. Pollack }\end{array}$ & $\begin{array}{l}\text { mode-locked laser, fundamental for laser communication and is } \\
\text { the basis for fs lasers }\end{array}$ \\
\hline & 1963 & H. G. Heard & molecular nitrogen lasers, emitting at $337 \mathrm{~nm}$ \\
\hline & 1964 & J. E. Geusic et al. & first Nd:YAG laser \\
\hline & 1964 & K. Patel & $\mathrm{CO}_{2}$ laser, high-energy continuous output \\
\hline & 1964 & W. B. Bridges & $\begin{array}{l}\text { pulsed argon-ion laser, output at several visible and UV } \\
\text { wavelengths }\end{array}$ \\
\hline & 1965 & J.V.V. Kasper and G. Pimentel & first chemical laser, a $3.7 \mu \mathrm{m}$ hydrogen chloride instrument \\
\hline & 1966 & P. Sorokin and J.Lankard & demonstration of first dye laser action \\
\hline & 1967 & B. Soffer and B. B. McFarland & making dye lasers the first broadly tunalbe lasers \\
\hline & 1970 & $\begin{array}{l}\text { Alferov's group, M. Panish, } \\
\text { and I. Hayashi }\end{array}$ & first CW room-temperature semiconductor lasers \\
\hline & 1970 & B. Snavely's group & $\begin{array}{l}\mathrm{CW} \text { dye laser, the only } \mathrm{CW} \text { laser with adequate power at dye } \\
\text { absorption wavelengths }\end{array}$ \\
\hline & 1970 & N. Basov's group & first excimer laser \\
\hline & 1972 & C. H. Henry & quantum well laser \\
\hline & 1975 & J. J.Ewing and C.Brau & first rare-gas halide laser \\
\hline & 1976 & J. M.J. Madey group & free-electron laser \\
\hline & 1982 & P. F. Moulton & Ti:sapphire laser, short pulses in the ps and fs ranges \\
\hline & 1994 & J. Faist et al. & quantum cascade laser \\
\hline & 1996 & W. Ketterle & first pulsed atom laser \\
\hline & 1997 & S. Nakamura et al. & GaN laser that emits brightblue-violet light in pulsed operation \\
\hline
\end{tabular}

after [250, 251] 


\section{Laser safety}

The increasing number of using powerful lasers requires the users to acquire the knowledge laser safety to avoid accidents (e.g. damage to the eyes and skin as illustrated in Table A 2). A laser beam with a few $\mathrm{mW}$ hitting the macula zone of the retina will cause partial or total loss of visual acuity, so eye-protectors is necessary (note: even with goggles, never look along the axis of the laser beam, but keep eyes above the level of the optical table).

Table A 2 Effects of laser radiation as a function of wavelength region in the eye and skin

\begin{tabular}{|c|c|c|}
\hline Wavelength region $(\mathrm{nm})$ & Eye & Skin \\
\hline UV-C: $100 \mathrm{~nm}$ to $280 \mathrm{~nm}$ & $\begin{array}{l}\text { Photokeratitis (corneal } \\
\text { inflammation;cornea surface absorbs } \\
\text { all) Photoconjunctivitis }\end{array}$ & $\begin{array}{l}\text { Erythema (reddening of the } \\
\text { skin) Pre-carcinoma }\end{array}$ \\
\hline UV-B: $280 \mathrm{~nm}$ to $315 \mathrm{~nm}$ & $\begin{array}{l}\text { Photokeratitis Photoconjunctivitis } \\
\text { Cataractogenesis (cataract formation) }\end{array}$ & $\begin{array}{l}\text { Increased pigmentation } \\
\text { Enhanced skinphotoaging } \\
\text { (accelerated skin aging) } \\
\text { Erythema } \\
\text { Oedema } \\
\text { Pre-carcinoma } \\
\text { Carcinoma (skin cancer) }\end{array}$ \\
\hline UV-A: $315 \mathrm{~nm}$ to $400 \mathrm{~nm}$ & $\begin{array}{l}\text { Cataract formation (lens is principal } \\
\text { absorber) }\end{array}$ & $\begin{array}{l}\text { Acute pigmentation } \\
\text { Enhanced skinphotoaging } \\
\text { Skin burns Carcinoma (skin } \\
\text { cancer) }\end{array}$ \\
\hline VIS: $400 \mathrm{~nm}$ to $700 \mathrm{~nm}$ & $\begin{array}{l}\text { Photochemical and photothermal } \\
\text { damage to the retina }\end{array}$ & $\begin{array}{l}\text { Photosensitive reactions } \\
\text { (photosensitization) } \\
\text { Skin burns (reddening of } \\
\text { skin, blisters, charring) }\end{array}$ \\
\hline IR-A: $700 \mathrm{~nm}$ to $1400 \mathrm{~nm}$ & $\begin{array}{l}\text { Cataract formation from heating } \\
\text { proteins in the lens, Photothermal } \\
\text { retinal damage }\end{array}$ & $\begin{array}{l}\text { Skin burns (reddening of } \\
\text { skin, blisters, icharring, } \\
\text { damage to underlying } \\
\text { organs) }\end{array}$ \\
\hline IR-B: $1400 \mathrm{~nm}$ to $3000 \mathrm{~nm}$ & $\begin{array}{l}\text { Cataract formation (corneal or } \\
\text { lenticular opacities) Photothermal } \\
\text { damage (burns) to the cornea }\end{array}$ & $\begin{array}{l}\text { Oedema of the skin, Skin } \\
\text { burns }\end{array}$ \\
\hline IR-C: $3000 \mathrm{~nm}$ to $1 \mathrm{~mm}$ & Photothermal damage of the cornea & Skin burns \\
\hline
\end{tabular}

The definition of laser categories and the summary of the effects of the beam by laser classification are illustrated in Table A 3. For details, please consult the laser safety standard documents. Table A 4 lists the energy lighting on the retina when viewing common luminescent objects. 
Table A 3 Definition of laser categories and the summary of the effects of the beam by laser classification

\begin{tabular}{c|c|c|c|c|c|cc} 
CLASSES & 1 & $1 \mathrm{M}$ & 2 & $2 \mathrm{M}$ & $3 \mathrm{R}$ & $3 \mathrm{~B}$ & 4 \\
\hline $\begin{array}{c}\text { Eye: vision with } \\
\text { optical assistance }\end{array}$ & $\mathrm{X}$ & $*$ & $\mathrm{X}$ & $\mathrm{XX}$ & $\mathrm{XX}$ & $\mathrm{XX}$ \\
\hline $\begin{array}{c}\text { Eye: direct beam } \\
\text { and mirrored } \\
\text { reflections }\end{array}$ & & & $*$ & $*$ & ${ }^{*}, \mathrm{X}$ & $\mathrm{XX}$ & $\mathrm{XX}$ \\
\hline $\begin{array}{c}\text { Eye: diffuse } \\
\text { reflections }\end{array}$ & & & & & & + & $\mathrm{XX}$ \\
\hline Skin & & & & & & $\mathrm{X}$ & $\mathrm{XX}$ \\
\hline Fire & & & & & & & 0 \\
\hline
\end{tabular}

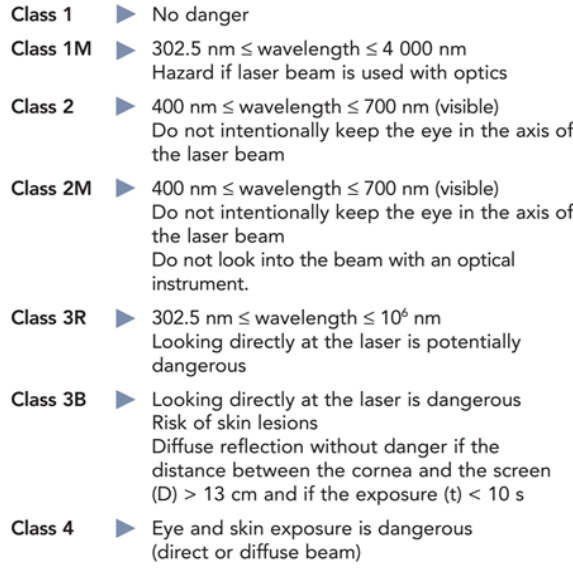

Class 3B Looking directly at the laser is dangerous Risk of skin lesions

Diffuse reflection without danger if the distance between the cornea and the screen (D) $>13 \mathrm{~cm}$ and if the exposure $(\mathrm{t})<10 \mathrm{~s}$

Class 4 Eye and skin exposure is dangerous (direct or diffuse beam)

$X$ can be dangerous

* no danger with palpebral reflex

$O$ can cause fire

cited from "Lunettes de protection laser" Guide, CEA

Table A 4 Energy lighting on the retina when viewing common luminescent objects

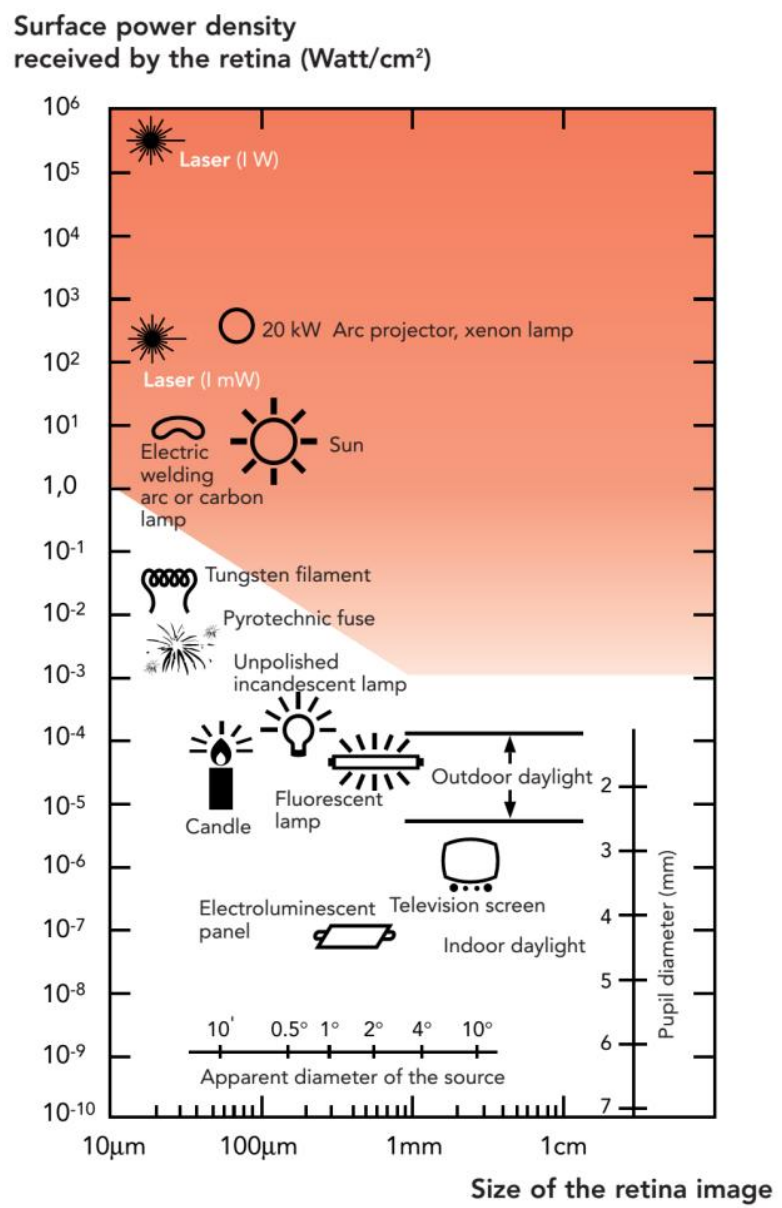

cited from "Lunettes de protection laser" Guide, CEA 


\section{Applications of various types of lasers}

Table A 5 Applications of various types of lasers

\begin{tabular}{|c|c|c|c|}
\hline Laser type & Examples and typical wavelength & Advantages \& disadvantages & Applications \\
\hline \multirow[t]{5}{*}{$\begin{array}{l}\text { Solide state } \\
\text { laser }\end{array}$} & $\begin{array}{l}\text { ruby laser } 694.3 \mathrm{~nm} \\
\text { (three-level system) }\end{array}$ & $\begin{array}{l}\text { high output power, high power } \\
\text { pumping source, low efficiency, } \\
\text { pulsed laser } \mu \text { s duration }\end{array}$ & $\begin{array}{l}\text { holography, material } \\
\text { processing }\end{array}$ \\
\hline & $\begin{array}{l}\mathrm{Nd} \text { :YAG laser } 1064 \mathrm{~nm} \text { (four-level } \\
\text { system) }\end{array}$ & $\begin{array}{l}\text { high power density, small focal } \\
\text { spot size, small Rayleigh length }\end{array}$ & $\begin{array}{l}\text { material processing, hard to } \\
\text { cut thicker material }\end{array}$ \\
\hline & Nd:glass laser $1054 \mu \mathrm{m}$ & $\begin{array}{l}\text { tunable wavelength dependent on } \\
\text { the glass }\end{array}$ & high power, high energy \\
\hline & $\begin{array}{l}\text { fiber laser, optical fiber doped with } \\
\text { rare-earth elements (e.g. Nd, Yb, Er), } \\
1064 \mathrm{~nm}, 532 \mathrm{~nm}\end{array}$ & $\begin{array}{l}\text { compact size, high output power, } \\
\text { easily delivered, unique beam } \\
\text { quality for focusing }\end{array}$ & $\begin{array}{l}\text { material processing, } \\
\text { telecommunications }\end{array}$ \\
\hline & $\begin{array}{l}\text { Ti:sapphire laser } 650-1100 \mathrm{~nm}, \\
\text { operating most efficiently at } \sim 800 \mathrm{~nm}\end{array}$ & wide range of wavelength & mode-locked IR laser \\
\hline \multirow[t]{4}{*}{$\begin{array}{l}\text { Semiconductor } \\
\text { laser }\end{array}$} & GaN 400 nm & $\begin{array}{l}\text { small size, high efficiency, fast } \\
\text { response }\end{array}$ & blue-ray technology \\
\hline & InGaN $400-500 \mathrm{~nm}$ & & projectors \\
\hline & $\begin{array}{l}\text { Quantum cascade laser (unipolar } \\
\text { laser), entire mid IR, far IR }\end{array}$ & $\begin{array}{l}\text { room-temperature operation, long } \\
\text { life tme, wavelength dependent on } \\
\text { layer thickness }\end{array}$ & $\begin{array}{l}\text { remote sensing of gases in } \\
\text { the atmosphere, medical }\end{array}$ \\
\hline & hybrid silicon laser, mid-infrared & low cost & optical communications \\
\hline Chemical laser & $\mathrm{HCl}$ laser, mid IR & high power & military \\
\hline \multirow[t]{4}{*}{ Gas laser } & He-Ne laser, $638.2 \mathrm{~nm}$ & CW, low power & spectroscopy \\
\hline & Argon laser, from blue to green & several wavelength & lithography \\
\hline & Copper vapor laser, $510.6-578.2 \mathrm{~nm}$ & $\begin{array}{l}\text { resonator mirrors are not } \\
\text { necessary }\end{array}$ & \\
\hline & $\mathrm{CO}_{2}$ laser, $10.64 \mu \mathrm{m}$ & $\begin{array}{l}\mathrm{CW} \text {, high power, long Rayleigh } \\
\text { length, low power density }\end{array}$ & $\begin{array}{l}\text { welding, cutting tool in } \\
\text { surgery and industry, cut } \\
\text { thicker material }\end{array}$ \\
\hline Dye laser & stilbene, $390-435 \mathrm{~nm}$ & broadly tunalbe lasers & spectroscopy \\
\hline Excimer laser & $\begin{array}{l}\text { KrF laser (248 nm), ArF laser (193 } \\
\mathrm{nm}), \mathrm{XeCl} \text { laser (308 nm) }\end{array}$ & $\begin{array}{l}\text { high pulse energy } \\
\text { ultraviolet laser } \\
\text { using toxic gases }\end{array}$ & $\begin{array}{l}\text { high-resolution } \\
\text { photolithography, } \\
\text { micromachining organic } \\
\text { material, eye surgery, pump } \\
\text { sources }\end{array}$ \\
\hline $\begin{array}{l}\text { Free-electron } \\
\text { laser }\end{array}$ & $0.1 \mathrm{~nm}$-several mm & $\begin{array}{l}\text { tunable wavelength range, high } \\
\text { power, high efficiency }\end{array}$ & material science \\
\hline
\end{tabular}




\section{Chirped pulse amplification}

Amplifying the short pulses directly in the laser amplifier can lead to damages to it. The capability of laser materials for energy storage, generating high energy pulses pumping, and generating high peak power are shown in Figure A 1.
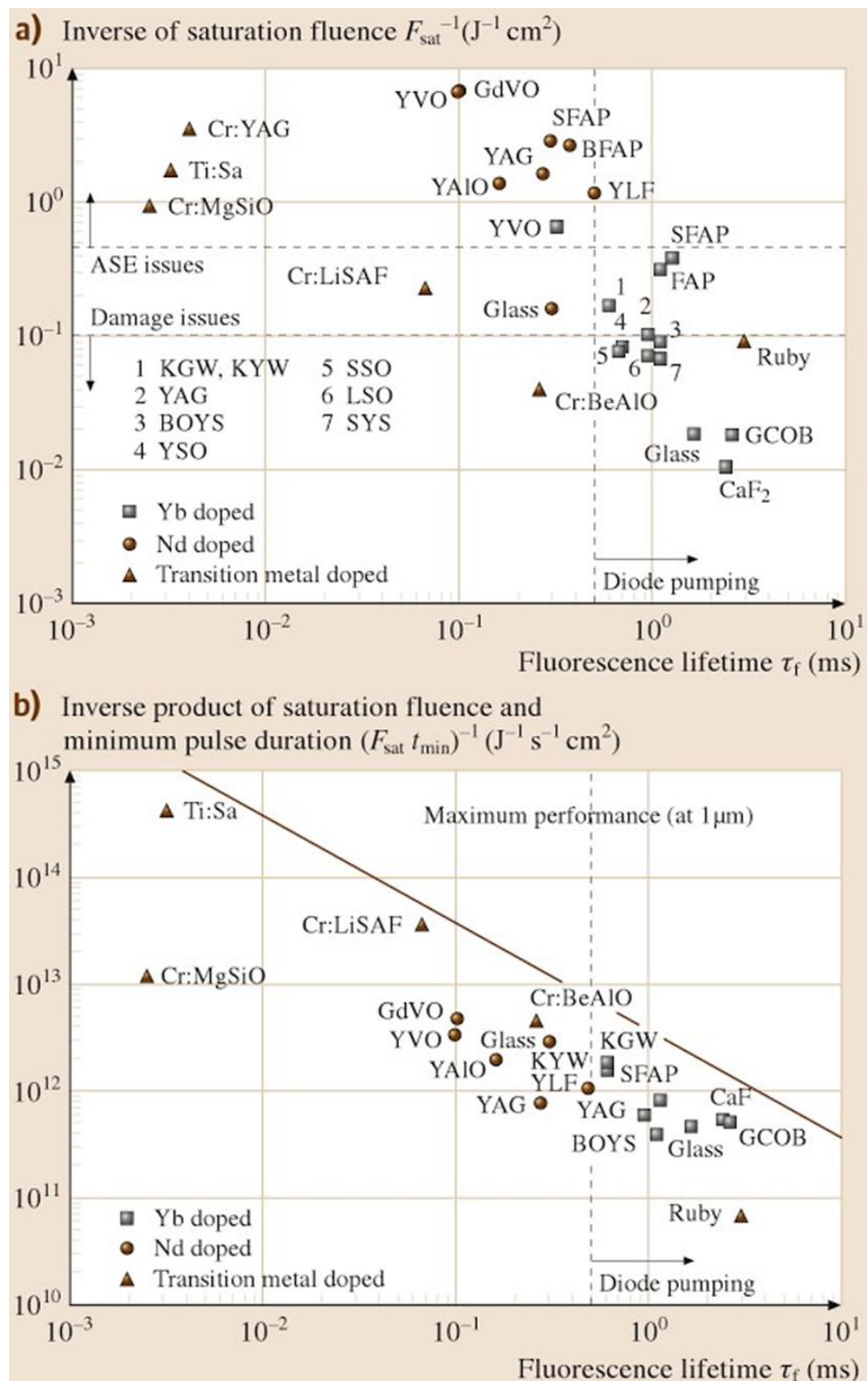

Figure A 1 Capability of laser materials for a) energy storage and generating high energy pulses pumping, b) energy storage and generating high peak power, AES: amplified spontaneous emission (cited from [103])

Chirp of an optical pulse is the time dependence of its instantaneous frequency. When lower frequency light travels ahead of higher frequency one, it is defined as positive and negative if in the opposite way.

The diagrammatic scheme of a CPA laser is shown in Figure A 2. 
Firstly, the oscillator products low energy short pulse (nJ), the original short pulse. Then it is stretched in time, done by stretching out the various colours of the pulse by using grating pair. This pair of gratings is used to delay the blue end of the spectrum stretches original pulse around a factor of 1000 in duration. In other words, there is a chromatically dispersed of the pulse emerging from the dispersive medium. Generally, stretcher introduces positive chirp, providing a long chirped pulse suitable for amplification. Ti:sapphire exhibits a large bandwidth of the laser transition $-230 \mathrm{~nm}$.

Subsequently, this long pulse is travelling through a broadband amplifier amplified to a desired energy level but below the amplifier damage threshold (i.e. amplified stretched pulse). Finally, by recompressing the laser pulse by a second pair of gratings (i.e. bringing the stretched colors back together), a high energy laser pulse output with an ultra-short duration close to the original low energy short pulse is produced.

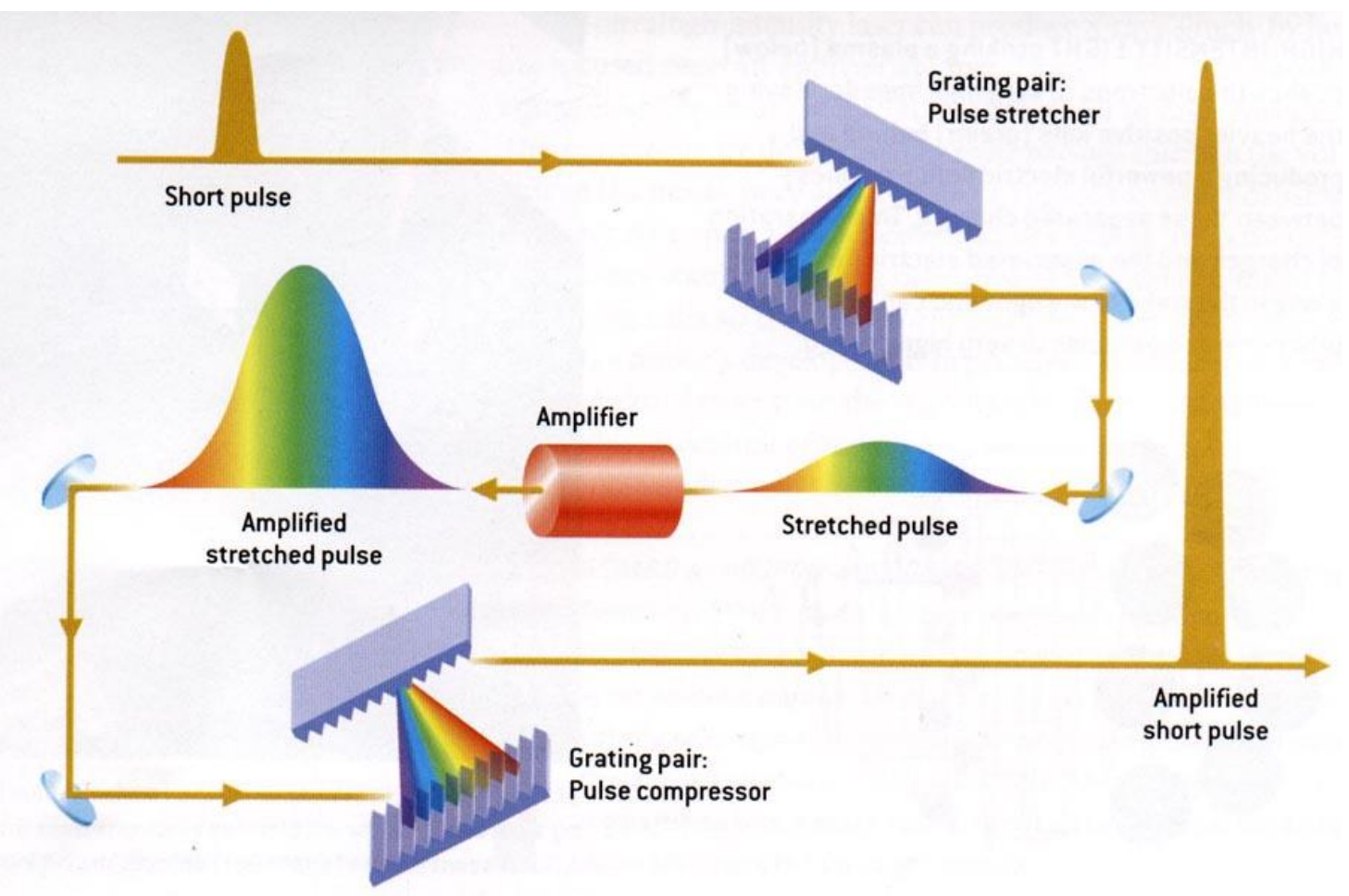

Figure A 2 Diagrammatic scheme of a chirped pulse amplification laser (cited from [46])

For a certain pulse spectrum, the minimum pulse duration is achieved when there is no chirp. $\mathrm{A}$ pulse can exhibit a chirp during travelling a transparent medium because of the effects of chromatic dispersion and nonlinear effect. In semiconductor lasers or amplifiers, chirps are due to the refractive index changes associated with the carrier density changes. The chirp of a pulse can be removed or reversed by propagating it through optical components with suitable chromatic dispersion [252]. 


\section{Laser specifications}

Fiber amplification is an attractive technology to achieve high optical power. Amplitude Systemes provides ultrafast fiber lasers with high average power and high output energy. Satsuma is a compact, high energy ultrafast fiber laser, delivering ultra-short pulse duration, high repetition rate, and high energy in a compact, and highly stable housing. The laser specifications are shown in Table A 6.

Table A 6 Laser specification of fs laser

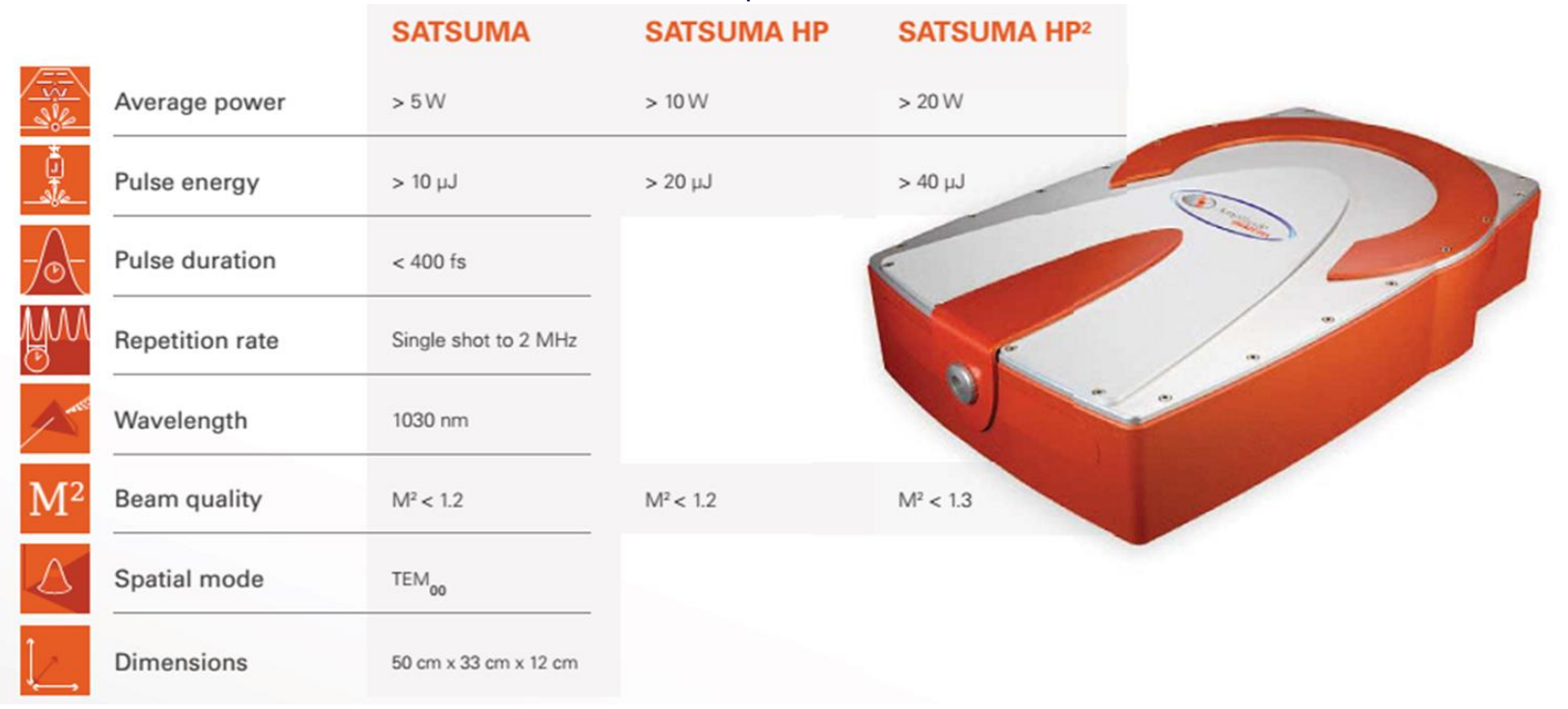

cited from Satsuma Amplitude Systèmes website 


\section{Comparison of a few common micro- and nanofabrication technologies}

Table A 7 Comparison of a few common micro- and nanofabrication technologies

\begin{tabular}{|c|c|}
\hline Technology & Characteristics \\
\hline $\begin{array}{l}\text { Femtosecond laser } \\
\text { direct writing }\end{array}$ & $\begin{array}{l}\text { Patterning in 3D with the sub micrometer scale, rapid, flexible and } \\
\text { clean fabrication, without using toxic photo resist comparing with } \\
\text { photolithography }\end{array}$ \\
\hline $\begin{array}{l}\text { UV photolithography } \\
\text { used with metal } \\
\text { deposition and liftoff } \\
\text { or etching }\end{array}$ & $\begin{array}{l}\text { Can pattern large areas in 2D. Many steps involved in the creation } \\
\text { of a single layer pattern. Multiple layers can be patterned on top } \\
\text { of each other, but difficult to create more than a handful of layers, } \\
\text { or complex 3D structures. Harsh chemicals are required for the } \\
\text { processing steps. Features sizes are on the order of } 500 \mathrm{~nm} \text { and } \\
\text { larger }\end{array}$ \\
\hline $\begin{array}{l}\text { Deep and extreme UV } \\
\text { photolithography } \\
\text { used with metal } \\
\text { deposition and liftoff } \\
\text { or etching }\end{array}$ & $\begin{array}{l}\text { Can achieve much higher resolution than UV photolithography, } \\
\text { but the cost is much higher. Commonly used for applications at } \\
\text { scale }\end{array}$ \\
\hline $\begin{array}{l}\text { Nanoimprint } \\
\text { lithography used with } \\
\text { metal deposition }\end{array}$ & $\begin{array}{l}\text { Can achieve very high resolution with low costs. Can produce 2D } \\
\text { and simple 3D structures (subject to distortion) in a 2D pattern. } \\
\text { Requires multiple steps }\end{array}$ \\
\hline $\begin{array}{l}\text { Electron beam } \\
\text { lithography (EBL) used } \\
\text { with metal deposition } \\
\text { and liftoff or etching }\end{array}$ & $\begin{array}{l}\text { Very high resolution. Can pattern small areas, or stitch together } \\
\text { smaller patterns to create larger samples in 2D. Multiple steps are } \\
\text { involved, and layered fabrication is difficult for 3D }\end{array}$ \\
\hline $\begin{array}{l}\text { Focused ion-beam } \\
\text { (FIB) lithography used } \\
\text { with multiple material } \\
\text { deposition steps. }\end{array}$ & $\begin{array}{l}\text { Very high resolution. To achieve 3D structure, multiple materials } \\
\text { can be layered through deposition steps, and patterned through } \\
\text { material removal with FIB. Many steps are involved, and only } \\
\text { simple 3D patterns can be created. }\end{array}$ \\
\hline $\begin{array}{l}\text { Two-photon } \\
\text { polymerization (TPP) } \\
\text { used with metal } \\
\text { deposition }\end{array}$ & $\begin{array}{l}\text { Can create complex 3D samples. However, to fill voids with metals, } \\
\text { the voids must be accessible-connected such that material can } \\
\text { enter. This precludes the ability to pattern disconnected metal } \\
\text { structures in 3D }\end{array}$ \\
\hline $\begin{array}{l}\text { Direct-write assembly } \\
\text { using conductive ink }\end{array}$ & $\begin{array}{l}\text { Can pattern large areas in 3D, including on curved surfaces. Metal } \\
\text { fabrication is limited to features sizes on order of } 2 \mu \mathrm{m} \text {. Cannot } \\
\text { create disconnected metal structures in 3D }\end{array}$ \\
\hline $\begin{array}{l}\text { Bottom-up methods } \\
\text { (broadly } \\
\text { encompassing } \\
\text { chemical synthesis } \\
\text { methods) }\end{array}$ & $\begin{array}{l}\text { Many chemical methods exist for growing metal particles. They } \\
\text { can be inexpensive, and produce high volumes of material with } \\
\text { high quality (such as single crystal growth). However, the } \\
\text { techniques do not enable arbitrary patterning as top-down } \\
\text { methods do }\end{array}$ \\
\hline
\end{tabular}

This comparison is focused on metal micro- and nanofabrication technologies, after [253] 
VII. Major optical absorption or luminescence bands of defects in non-doped synthetic silica

Figure A 3 shows the major optical absorption or luminescence bands of defects in non-doped synthetic silica.

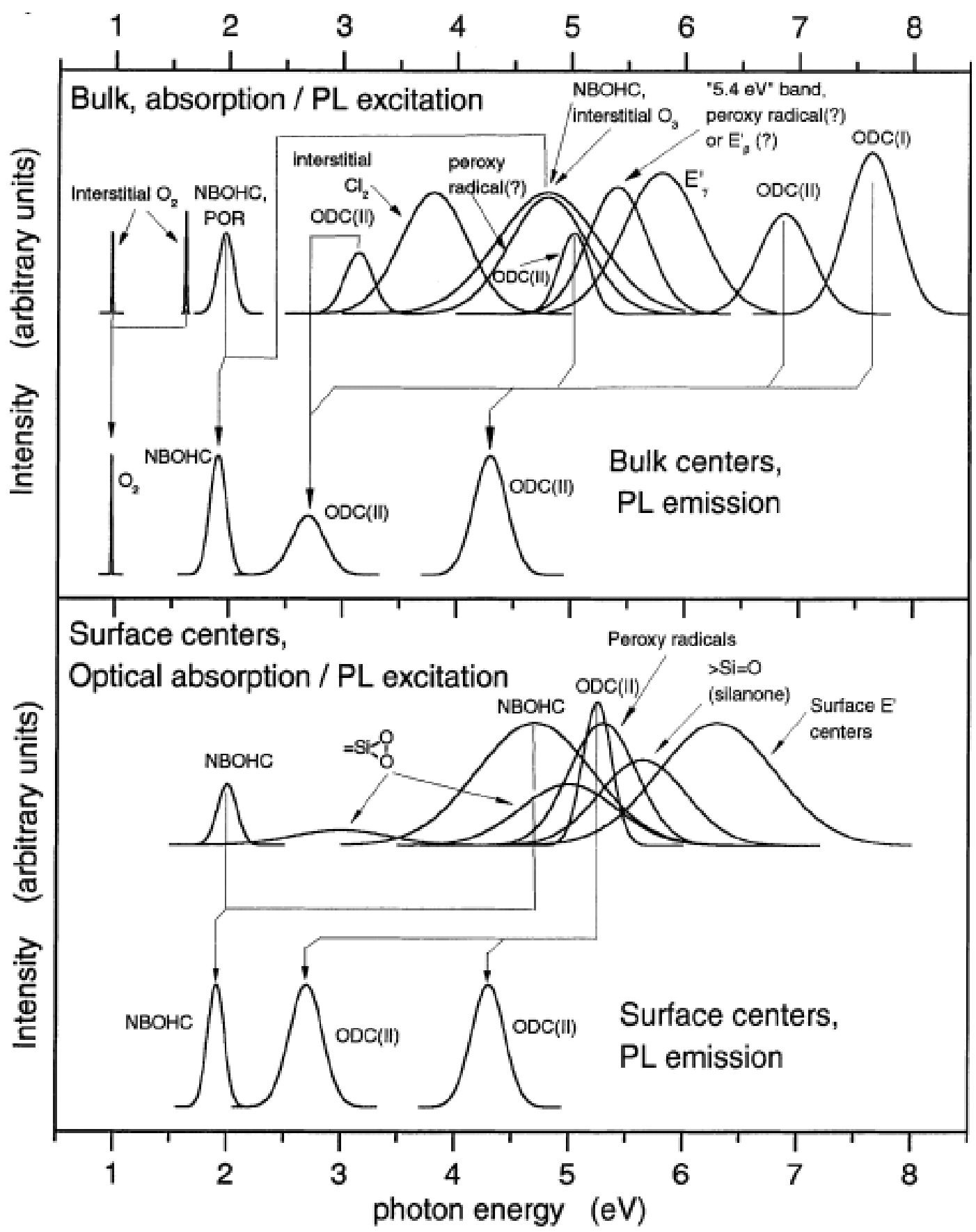

Figure A 3 Major optical absorption/luminescence excitation bands and emission bands of defect centers in synthetic silica (top) and of centers on the surfaces of $\mathrm{SiO}_{2}$ (bottom). The relationship between the emission and excitation bands is indicated. The band intensities shown are arbitrary, relying on the published values of their peak positions and half widths (full width at half maximum, FWHM), assuming Gaussian shape (cited from [254]) 
VIII. Silica

Different kinds of silica glasses are shown in Table A 8. The chemical properties and crystallographic of various $\mathrm{SiO}_{2}$ are shown in Table A 9 and Table A 10.

Table A 8 Different kinds of silica glasses

\begin{tabular}{|c|c|c|c|}
\hline Type & how to produce & properties and structure & Commercial names \\
\hline I & $\begin{array}{l}\text { natural quartz by electrical } \\
\text { fusion under vacuum or inert } \\
\text { gas atmosphere }\end{array}$ & $\begin{array}{l}\text { nearly no } \mathrm{OH} \text {-group }(<5 \mathrm{ppm}) \text {, } \\
\text { relatively high metallic impurities } \\
\text { (e.g. Al, 30-100 ppm, Na } 4 \text { ppm) }\end{array}$ & $\begin{array}{l}\text { Infrasil, IR-Vitreosil, G.E. } \\
\text { 105, 201, } 204\end{array}$ \\
\hline II & $\begin{array}{l}\text { quartz crystal powder by flame } \\
\text { fusion }\end{array}$ & $\begin{array}{l}\text { OH-content (150-400 ppm), } \\
\text { special thermal treatment in } \\
\text { oxygen atmosphere, leading to } \\
\text { good optical transparency in UV } \\
\text { range }\end{array}$ & $\begin{array}{l}\text { Herasil, Homosil, } \\
\text { Optosil, O.G. Vitreosil, } \\
\text { G.E. 104, Ultrasil }\end{array}$ \\
\hline III & $\begin{array}{l}\text { hydroxylation of } \mathrm{SiCl} 4 \text { when } \\
\text { spraying into an oxygen - } \\
\text { hydrogen flame }\end{array}$ & $\begin{array}{l}\text { OH-content (1000 ppm), free from } \\
\text { metallic impurites, } \mathrm{Cl}(100 \mathrm{ppm}), \\
\text { synthetic vitreous silica }\end{array}$ & $\begin{array}{l}\text { Suprasil, Spectrosil, } \\
\text { Corning } 7940\end{array}$ \\
\hline IV & $\begin{array}{l}\mathrm{SiCl} 4 \text { in a water-vapour-free } \\
\text { plasma flame }\end{array}$ & $\begin{array}{l}\text { OH-content ( } 0.4 \mathrm{ppm}), \mathrm{Cl}(200 \\
\mathrm{ppm}), \text { synthetic vitreous silica }\end{array}$ & $\begin{array}{l}\text { Suprasil W, Spectrosil } \\
\text { WF, Corning } 7943\end{array}$ \\
\hline V & $\begin{array}{l}\text { silicon (semiconductor-quality) } \\
\text { oxidized in pure oxygen high- } \\
\text { frequency plasma flame }\end{array}$ & $\mathrm{OH}$-content (extremely low) & $\begin{array}{l}\text { Single case on a } \\
\text { laboratory scale }\end{array}$ \\
\hline
\end{tabular}

after [122], (1 ppm = 1 part per million $\left.=1 \mathrm{mg} \cdot \mathrm{kg}^{-1} \sim 1 \mathrm{mg} \cdot \mathrm{L}^{-1}\right)$

Table A 9 Low Pressure Silica Polymorphs

\begin{tabular}{llll}
\hline high- or $\beta$-polymorph & $\boldsymbol{\beta}$-Quartz & $\boldsymbol{\beta}$-Tridymite & $\boldsymbol{\beta}$-Cristobalite \\
stable at & $573^{\circ} \mathrm{C}-870^{\circ} \mathrm{C}$ & $870^{\circ} \mathrm{C}-1470^{\circ} \mathrm{C}$ & $>1470^{\circ} \mathrm{C}$ \\
metastable at & - & $117^{\circ} \mathrm{C}-870^{\circ} \mathrm{C}$ & $270^{\circ} \mathrm{C}-1470^{\circ} \mathrm{C}$ \\
crystal system & hexagonal & hexagonal & cubic \\
Si-O-Si angle & $153^{\circ}$ & $180^{\circ}$ & $151^{\circ}$ \\
\hline low- or $\alpha$-polymorph & $\boldsymbol{\alpha}$-Quartz & $\boldsymbol{\alpha}$-Tridymite & $\boldsymbol{\alpha}$-Cristobalite \\
stable at & $<573^{\circ} \mathrm{C}$ & - & - \\
metastable at & - & $<117^{\circ} \mathrm{C}$ & $<270^{\circ} \mathrm{C}$ \\
crystal system & trigonal & triclinic & tetragonal \\
Si-O-Si angle & $144^{\circ}$ & $140^{\circ}$ & $147^{\circ}$ \\
\hline
\end{tabular}

The high-temperature silica polymorphs possess a higher symmetry than their low-temperature ones (hexagonal > trigonal > triclinic; cubic > tetragonal), cited from [117]

Table A 10 Chemical properties and crystallographic

\begin{tabular}{|c|c|c|c|c|c|c|}
\hline Name & Formula & Crystal system & $\begin{array}{l}\text { Stability at } \\
\text { Normal }\end{array}$ & $\begin{array}{l}\text { Coordination } \\
\text { Number of Si }\end{array}$ & Si-O Distance & $\begin{array}{l}\text { Si-O- } \\
\text { Si } \\
\text { Angle }\end{array}$ \\
\hline$\alpha$-Quartz & $\mathrm{SiO}_{2}$ & trigonal & stable & 4 - tetrahedral & $0.161 \mathrm{~nm}$ & $144^{\circ}$ \\
\hline
\end{tabular}




\begin{tabular}{|c|c|c|c|c|c|c|}
\hline$\beta$-Quartz & $\mathrm{SiO}_{2}$ & hexagonal & instable & 4 - tetrahedral & $0.162 \mathrm{~nm}$ & $153^{\circ}$ \\
\hline$\alpha$-Tridymite & $\mathrm{SiO}_{2}$ & triclinic & metastable & 4 - tetrahedral & $\begin{array}{l}0.154 \mathrm{~nm},- \\
0.171 \mathrm{~nm}\end{array}$ & $140^{\circ}$ \\
\hline$\beta$-Tridymite & $\mathrm{SiO}_{2}$ & hexagonal & instable & 4 - tetrahedral & $\begin{array}{l}0.153 \mathrm{~nm},- \\
0.155 \mathrm{~nm}\end{array}$ & $180^{\circ}$ \\
\hline$\alpha$-Cristobalite & $\mathrm{SiO}_{2}$ & tetragonal & metastable & 4 - tetrahedral & $\begin{array}{l}0.158 \mathrm{~nm},- \\
0.169 \mathrm{~nm}\end{array}$ & $147^{\circ}$ \\
\hline$\beta$-Cristobalite & $\mathrm{SiO}_{2}$ & cubic & instable & 4 - tetrahedral & $0.151 \mathrm{~nm}$ & $151^{\circ}$ \\
\hline Moganite & $\mathrm{SiO}_{2}$ & monoclinic & $\begin{array}{l}\text { stable - } \\
\text { metastable }\end{array}$ & 4 - tetrahedral & & \\
\hline Coesite & $\mathrm{SiO}_{2}$ & monoclinic & metastable & 4 - tetrahedral & & \\
\hline Stishovite & $\mathrm{SiO}_{2}$ & tetragonal & metastable & 6 - octahedral & & \\
\hline Seifertite & $\mathrm{SiO}_{2}$ & orthorhombic & instable & 6 - octahedral & & \\
\hline Keatite & $\mathrm{SiO}_{2}$ & tetragonal & $?$ & 4 - tetrahedral & & \\
\hline Opal & $\begin{array}{l}\mathrm{SiO}_{2} \bullet \mathrm{n} \\
\mathrm{H}_{2} \mathrm{O}\end{array}$ & $\begin{array}{l}\text { macroscopically } \\
\text { amorphous }\end{array}$ & metastable & 4 - tetrahedral & & \\
\hline Lechatelierite & $\mathrm{SiO}_{2}$ & $\begin{array}{l}\text { amorphous } \\
\text { glass }\end{array}$ & stable & 4 - tetrahedral & & \\
\hline Melanophlogite & $\begin{array}{l}\mathrm{SiO}_{2} \bullet \mathrm{n} \\
(\mathrm{C}, \mathrm{H}, \mathrm{O}, \mathrm{S})\end{array}$ & tetragonal & metastable & 4 - tetrahedral & & \\
\hline Chibaite & $\begin{array}{l}\mathrm{SiO}_{2} \bullet \mathrm{n} \\
\left(\mathrm{CH}_{4}\right. \\
\mathrm{C}_{2} \mathrm{H}_{6} \\
\mathrm{C}_{3} \mathrm{H}_{8}, \\
\left.\mathrm{C}_{4} \mathrm{H}_{10}\right)\end{array}$ & cubic & $?$ & 4 - tetrahedral & & \\
\hline Silhydrite & $\begin{array}{l}3 \mathrm{SiO}_{2} \bullet \\
\mathrm{H}_{2} \mathrm{O}\end{array}$ & orthorhombic & $?$ & 4 - tetrahedral & & \\
\hline
\end{tabular}


IX. Photoluminescence in material due to the laser-induced defects

Table A 11 Photoluminescence in different materials due to the laser-induced defects

\begin{tabular}{|c|c|c|c|}
\hline Material & Irradiation source & Explanation & Refs \\
\hline dry $v-\mathrm{SiO}_{2}$ & $\begin{array}{l}800 \mathrm{~nm}, 130 \mathrm{fs} \text { at } 5 \mathrm{kHz} \text {, } \\
532 \mathrm{~nm}, 30 \text { ps at } 10 \mathrm{~Hz}\end{array}$ & $\begin{array}{l}\text { singlet-singlet transition of } \\
\text { oxygen vacancy for } 283 \mathrm{~nm} \text {, } \\
\text { radiative recombination of } \\
\text { separated carriers on the } \\
\text { vacancy-interstitial pairs }\left(\mathrm{V}_{0} ; \mathrm{O}_{\mathrm{i}}\right) \\
\text { and }\left(\mathrm{V}_{\mathrm{O}} ;\left(\mathrm{O}_{2}\right)_{\mathrm{i}}\right) \text { for } 468 \text { and } 558 \\
\mathrm{~nm} \text {, respectively }\end{array}$ & [74] \\
\hline fused silica & $\begin{array}{l}800 \mathrm{~nm}, 120 \mathrm{fs} \text { at } 1 \mathrm{kHz} \\
\text { or } 160 \mathrm{fs} \text { at } 100 \mathrm{kHz}\end{array}$ & $\begin{array}{l}\text { oriented SiODC(II) defects } \\
\text { depending on the scanning } \\
\text { direction }\end{array}$ & {$[76]$} \\
\hline fused silica & $800 \mathrm{~nm}, 100 \mathrm{fs}, 1 \mathrm{kHz}$ & $\begin{array}{l}650 \mathrm{~nm} \text { is due to the red } \\
\text { luminescence of the NBOHC, } \\
460 \mathrm{~nm} \text {, corresponding to the } \\
\text { twofold coordinated silicon }\end{array}$ & [255] \\
\hline $\begin{array}{l}\alpha-\mathrm{SiO}_{2} \text { glasses with } \\
\text { different impurity levels } \\
\text { (Infrasil 302, Suprasil } \\
312 \text {, and EN1027) }\end{array}$ & $800 \mathrm{~nm}, 100$ fs, 1 kHz & $\begin{array}{l}\text { ODC (II) was correlated to the } \\
\text { onset of visible optical damage } \\
\text { in the form of scattering centers } \\
\text { in bulk sample. Si E' only left the } \\
\text { samples scatter free. threefold } \\
\text { and fourfold rings and revealed } \\
\text { a decrease in the Si-O-Si angle } \\
\text { distribution due to the photo- } \\
\text { induced disruption of strained } \\
\text { bond upon IR fs laser irradiation }\end{array}$ & [256] \\
\hline $\begin{array}{l}\text { Ag nanoparticles in } \\
\text { soda-lime glass }\end{array}$ & $400 \mathrm{~nm}, 150 \mathrm{fs}$ & $\begin{array}{l}\text { broad band centered at } 600 \mathrm{~nm} \\
\text { is due to the small charged } \\
\text { clusters like } \mathrm{Ag}_{2}^{+}, \mathrm{Ag}_{3}{ }^{+} \text {and } \mathrm{Ag}_{3}{ }^{2+}\end{array}$ & [257] \\
\hline cubic-boron nitride & 515 or1030 nm, $230 \mathrm{fs}$ & $\begin{array}{l}546.2 \mathrm{~nm}, 576.7 \mathrm{~nm} \text {, and } 623.1 \\
\mathrm{~nm} \text { due to t the voids made by } \\
\text { fs-laser pulses }\end{array}$ & [258] \\
\hline ZnO nanowires & $\sim 800 \mathrm{~nm}$ & $\begin{array}{l}385 \mathrm{~nm} \text { is attributed to the near } \\
\text { band edge emission, } 515 \mathrm{~nm} \text { is } \\
\text { due to the defect level emission }\end{array}$ & [259] \\
\hline
\end{tabular}




\section{Unit}

The laws of physics are expressed by basic physical quantities (shown in Table A 12). Table A 13 lists some derived physical quantities. Table A 14 displays the SI prefixes.

Table A 12 Base physical quantities

\begin{tabular}{llll}
\hline Base quantity & Symbol & $\begin{array}{l}\text { Symbol for } \\
\text { dimension }\end{array}$ & SI unit \\
\hline length & $\mathrm{I}$ & $\mathrm{L}$ & $\mathrm{m}$ \\
mass & $\mathrm{m}$ & $\mathrm{M}$ & $\mathrm{kg}$ \\
time & $\mathrm{t}$ & $\mathrm{T}$ & $\mathrm{S}$ \\
electric current & $\mathrm{I}$ & $\mathrm{I}$ & $\mathrm{A}$ \\
temperature & $\mathrm{T}$ & $\Theta$ & $\mathrm{K}$ \\
amount of & $\mathrm{n}$ & $\mathrm{N}$ & $\mathrm{mol}$ \\
substance & & & $\mathrm{cd}$ \\
luminous & $\mathrm{L}$ & $\mathrm{J}$ & \\
intensity & & & \\
\hline
\end{tabular}

Table A 13 Some derived physical quantities

\begin{tabular}{|c|c|c|c|}
\hline Derived quantity & Symbol & Dimension & SI units \\
\hline Area & A & $\mathrm{L}^{2}$ & $\mathrm{~m}^{2}$ \\
\hline Area density & $\rho_{\mathrm{A}}$ & $\mathrm{M} \mathrm{L}^{-2}$ & $\mathrm{~kg} \mathrm{~m}^{-2}$ \\
\hline Capacitance & C & $\mathrm{I}^{2} \mathrm{~T}^{4} \mathrm{M}^{-1} \mathrm{~L}^{-2}$ & $\operatorname{farad}\left(F=A^{2} s^{4} k^{-1} m^{-2}\right)$ \\
\hline Catalytic activity & & $\mathrm{N} \mathrm{T}^{-1}$ & katal $\left(\right.$ kat $\left.=\mathrm{mol} \mathrm{s}^{-1}\right)$ \\
\hline Catalytic activity concentration & & $\mathrm{N} \mathrm{L}^{-3} \mathrm{~T}^{-1}$ & kat $\mathrm{m}^{-3}$ \\
\hline Chemical potential & $\mu$ & $\mathrm{M} \mathrm{L}^{2} \mathrm{~T}^{-2} \mathrm{~N}^{-1}$ & $\mathrm{~J} \mathrm{~mol}^{-1}$ \\
\hline Molar concentration & $\mathrm{C}$ & $\mathrm{N} \mathrm{L}^{-3}$ & $\mathrm{~mol} \mathrm{~m}^{-3}$ \\
\hline Current density & $\overrightarrow{\mathbf{J}}$ & $\mathrm{IL}^{-2}$ & $A \mathrm{~m}^{-2}$ \\
\hline Dynamic viscosity & $\eta$ & $\mathrm{M} \mathrm{L}^{-1} \mathrm{~T}^{-1}$ & Pa s \\
\hline Electric charge & Q & $\mathrm{IT}$ & $\mathrm{C}=\mathrm{As}$ \\
\hline Electric charge density & $\rho_{Q}$ & $I \mathrm{~T} \mathrm{~L}^{-3}$ & $\mathrm{C} \mathrm{m}^{-3}$ \\
\hline Electric displacement & $\mathrm{D}$ & $\mathrm{IT} \mathrm{L^{-2 }}$ & $\mathrm{C} \mathrm{m}^{-2}$ \\
\hline Electric field strength & $\overrightarrow{\mathrm{E}}$ & $M L T^{-3} I^{-1}$ & $\mathrm{Vm}^{-1}$ \\
\hline Electrical conductance & $\mathrm{G}$ & $L^{-2} M^{-1} T^{3} I^{2}$ & siemens $\left(S=A^{2} s^{3} \mathrm{~kg}^{-1} \mathrm{~m}^{-2}\right)$ \\
\hline Electric potential & V & $L^{2} M T^{-3} I^{-1}$ & volt $\left(V=k g m^{2} A^{-1} s^{-3}\right)$ \\
\hline Electrical resistance & $\mathrm{R}$ & $\mathrm{L}^{2} \mathrm{M} \mathrm{T}^{-3} \mathrm{I}^{-2}$ & $\operatorname{ohm}\left(\Omega=\mathrm{kg} \mathrm{m}^{2} \mathrm{~A}^{-2} \mathrm{~s}^{-3}\right)$ \\
\hline Energy & $E$ & $\mathrm{M} \mathrm{L}^{2} \mathrm{~T}^{-2}$ & joule $\left(J=k g m^{2} s^{-2}\right)$ \\
\hline Energy density & $\rho_{\mathrm{E}}$ & $\mathrm{M} \mathrm{L}^{-1} \mathrm{~T}^{-2}$ & $\mathrm{~J} \mathrm{~m}^{-3}$ \\
\hline Entropy & S & $\mathrm{M} \mathrm{L}^{2} \mathrm{~T}^{-2} \Theta^{-1}$ & $\mathrm{~J} \mathrm{~K}^{-1}$ \\
\hline Force & $\overrightarrow{\mathrm{F}}$ & $M L T^{-2}$ & newton $\left(\mathrm{N}=\mathrm{kg} \mathrm{m} \mathrm{s}{ }^{-2}\right)$ \\
\hline Fuel efficiency & & $\mathrm{L}^{-2}$ & \\
\hline Impulse & $\overrightarrow{\mathrm{p}}$ & $M L T^{-1}$ & $\mathrm{~kg} \mathrm{~m} \mathrm{~s}^{-1}$ \\
\hline Frequency & $f$ & $\mathrm{~T}^{-1}$ & hertz $\left(H z=s^{-1}\right)$ \\
\hline Heat & $Q$ & $\mathrm{M} \mathrm{L}^{2} \mathrm{~T}^{-2}$ & $J$ \\
\hline Heat capacity & $C_{p}$ & $\mathrm{M} \mathrm{L}^{2} \mathrm{~T}^{-2} \Theta^{-1}$ & $J^{-1}$ \\
\hline
\end{tabular}




\begin{tabular}{|c|c|c|c|}
\hline Heat flux density & $\varphi_{Q}$ & $M T^{-3}$ & $W m^{-2}$ \\
\hline Illuminance & $E_{v}$ & $\mathrm{JL}^{-2}$ & 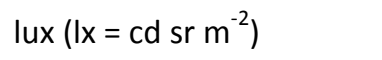 \\
\hline Impedance & Z & $\mathrm{L}^{2} \mathrm{M} \mathrm{T}^{-3} \mathrm{I}^{-2}$ & $\operatorname{ohm}\left(\Omega=\mathrm{kg} \mathrm{m}^{2} \mathrm{~A}^{-2} \mathrm{~s}^{-3}\right)$ \\
\hline Index of refraction & $\mathrm{n}$ & 1 & \\
\hline Inductance & $\mathrm{L}$ & $M L^{2} T^{-2} I^{-2}$ & henry $\left(H=\mathrm{kg} \mathrm{m}^{2} A^{-2} s^{-2}\right)$ \\
\hline Irradiance & $\mathrm{E}$ & $\mathrm{MT}^{-2}$ & $W m^{-2}$ \\
\hline Intensity & । & $\mathrm{MT}^{-3}$ & $W m^{-2}$ \\
\hline Magnetic field strength & $\mathrm{H}$ & $\mathrm{IL}^{-1}$ & $A \mathrm{~m}^{-1}$ \\
\hline Magnetic flux & $\Phi$ & $M L^{2} T^{-2} I^{-1}$ & weber $\left(W b=k g m^{2} A^{-1} s^{-2}\right)$ \\
\hline Magnetization & $M$ & $\mathrm{IL}^{-1}$ & $A \mathrm{~m}^{-1}$ \\
\hline $\begin{array}{l}\text { Mass fraction } \\
\text { (Mass) Density (orvolume density) }\end{array}$ & $\begin{array}{l}x \\
\rho\end{array}$ & $\begin{array}{l}1 \\
\mathrm{ML}^{-3}\end{array}$ & $\begin{array}{l}\mathrm{kg} / \mathrm{kg} \\
\mathrm{kg} \mathrm{m}^{-3}\end{array}$ \\
\hline $\begin{array}{l}\text { Mean lifetime } \\
\text { Molar energy }\end{array}$ & $\tau$ & $\mathrm{T} \mathrm{L} \mathrm{L}^{2} \mathrm{~T}^{-2} \mathrm{~N}^{-1}$ & $\begin{array}{l}\mathrm{S} \\
\mathrm{J} \mathrm{mol}^{-1}\end{array}$ \\
\hline Molar entropy & & $M L^{2} T^{-2} \Theta^{-1} N^{-1}$ & $\mathrm{JK}^{-1} \mathrm{~mol}^{-1}$ \\
\hline Molar heat capacity & c & $\mathrm{ML}^{2} \mathrm{~T}^{-2} \mathrm{~N}^{-1}$ & $\mathrm{JK}^{-1} \mathrm{~mol}^{-1}$ \\
\hline Moment of inertia & 1 & $M L^{2}$ & $\mathrm{~kg} \mathrm{~m}^{2}$ \\
\hline Permeability & $\mu$ & $M L T^{-2} I^{-2}$ & $\mathrm{H} \mathrm{m}^{-1}$ \\
\hline Permittivity & $\varepsilon$ & $I^{2} M^{-1} L^{-3} T^{4}$ & $\mathrm{Fm}^{-1}$ \\
\hline Power & $P$ & $M L^{2} T^{-3}$ & watt (W) \\
\hline Pressure & $\mathrm{p}$ & $\mathrm{M} \mathrm{L}^{-1} \mathrm{~T}^{-2}$ & pascal $\left(\mathrm{Pa}=\mathrm{kg} \mathrm{m}^{-1} \mathrm{~s}^{-2}\right)$ \\
\hline Refractive index & $\mathrm{n}$ & Dimensionless & Unitless \\
\hline Angle & $\theta$ & Dimensionless & $\mathrm{rad} /$ degree \\
\hline Speed & $v$ & $\mathrm{LT}^{-1}$ & $\mathrm{~m} \mathrm{~s}^{-1}$ \\
\hline Specific energy & & $L^{2} T^{-2}$ & $\mathrm{~J} \mathrm{~kg}^{-1}$ \\
\hline Specific heat capacity & c & $L^{2} T^{-2} \Theta^{-1}$ & $\mathrm{~J} \mathrm{~kg}^{-1} \mathrm{~K}^{-1}$ \\
\hline Specific volume & $v$ & $L^{3} M^{-1}$ & $\mathrm{~m}^{3} \mathrm{~kg}^{-1}$ \\
\hline Stress & $\sigma$ & $\mathrm{ML}^{-1} \mathrm{~T}^{-2}$ & $\mathrm{~Pa}$ \\
\hline Surface tension & $\gamma$ & $\mathrm{MT}^{-2}$ & $\mathrm{~N} \mathrm{~m}^{-1}$ or $\mathrm{J} \mathrm{m}^{-2}$ \\
\hline Thermal conductivity & k & $M L T^{-3} \Theta^{-1}$ & $W m^{-1} K^{-1}$ \\
\hline Velocity & $\overrightarrow{\mathrm{v}}$ & $\mathrm{LT}^{-1}$ & $\mathrm{~ms}^{-1}$ \\
\hline Volume & $\mathrm{v}$ & $L^{3}$ & $\mathrm{~m}^{3}$ \\
\hline Wavelength & $\lambda$ & L & $\mathrm{m}$ \\
\hline Wavenumber & k & $L^{-1}$ & $m^{-1}$ \\
\hline Weight & w & $M L T^{-2}$ & newton $\left(\mathrm{N}=\mathrm{kg} \mathrm{m} \mathrm{s}^{-2}\right)$ \\
\hline Work & w & $M L^{2} T^{-2}$ & joule $\left(J=k g m^{2} s^{-2}\right)$ \\
\hline Young's modulus & & $\mathrm{ML}^{-1} \mathrm{~T}^{-2}$ & pascal $\left(\mathrm{Pa}=\mathrm{kg} \mathrm{m}^{-1} \mathrm{~s}^{-2}\right)$ \\
\hline
\end{tabular}

after Wikipedia 
Table A 14 SI prefixes

\begin{tabular}{lll}
\hline Factor & Prefix & Abbreviation \\
\hline $10^{24}$ & yotta & $\mathrm{Y}$ \\
$10^{21}$ & zetta & $\mathrm{Z}$ \\
$10^{18}$ & exa & $\mathrm{E}$ \\
$10^{15}$ & peta & $\mathrm{P}$ \\
$10^{12}$ & tera & $\mathrm{T}$ \\
$10^{9}$ & giga & $\mathrm{G}$ \\
$10^{6}$ & mega & $\mathrm{M}$ \\
$10^{3}$ & kilo & $\mathrm{k}$ \\
$10^{2}$ & hecto & $\mathrm{h}$ \\
$10^{1}$ & deka & $\mathrm{da}$ \\
$10^{-1}$ & deci & $\mathrm{d}$ \\
$10^{-2}$ & centi & $\mathrm{C}$ \\
$10^{-3}$ & milli & $\mathrm{m}$ \\
$10^{-6}$ & micro & $\mathrm{\mu}$ \\
$10^{-9}$ & nano & $\mathrm{n}$ \\
$10^{-12}$ & pico & $\mathrm{p}$ \\
$10^{-15}$ & femto & $\mathrm{f}$ \\
$10^{-18}$ & atto & $\mathrm{a}$ \\
$10^{-21}$ & zepto & $\mathrm{z}$ \\
$10^{-24}$ & yocto & $\mathrm{y}$ \\
\hline & &
\end{tabular}


XI. Nonlinear coefficient

The nonlinear susceptibilities of some crystals are shown in Table A 15.

Table A 15 Nonlinear susceptibilities of exemplary inorganic and organic crystals

\begin{tabular}{|c|c|c|c|c|c|c|c|c|c|c|}
\hline Material & $\begin{array}{l}\text { Crystal } \\
\text { symmetry } \\
\text { neg.: } n_{0}>n_{\mathrm{e}} \\
\text { pos: } n_{\mathrm{e}}>n_{\mathrm{o}}\end{array}$ & $\begin{array}{l}\text { Point } \\
\text { group }\end{array}$ & \multicolumn{2}{|c|}{$\begin{array}{l}\text { Transparency } \\
\text { range }(0.5 \mu \mathrm{m})\end{array}$} & \multicolumn{2}{|c|}{$\begin{array}{l}\text { Breakdown threshold } \\
\left(10^{9} \mathrm{~W} / \mathrm{cm}^{2}\right)\end{array}$} & \multicolumn{2}{|c|}{$\begin{array}{l}\text { NL coefficient } d_{36} \\
\left(10^{-13} \mathrm{~m} / \mathrm{V}\right)\end{array}$} & \multicolumn{2}{|c|}{$\begin{array}{l}\text { Second harmonic } \\
\text { generation: } 1064 \rightarrow 532 \mathrm{~nm}\end{array}$} \\
\hline $\mathrm{KDP}: \mathrm{KH}_{2} \mathrm{PO}_{4}$ & neg. uniaxial & $42 m$ & 0.1765 & 1.7 & 23 & 0.2 & $4.35 / 6.3$ & & 14 & 82 \\
\hline DKDP: $\mathrm{KD}_{2} \mathrm{PO}_{4}$ & neg. uniaxial & $42 m$ & 0.2 & 2.0 & 6 & 0.25 & 4.02 & & 40 & 70 \\
\hline ADP: $\mathrm{NH}_{4} \mathrm{H}_{2} \mathrm{PO}_{4}$ & neg. uniaxial & $42 m$ & 0.184 & 1.5 & 6.4 & 15 & 5.28 & & & \\
\hline DADP: $\mathrm{ND}_{4} \mathrm{D}_{2} \mathrm{PO}_{4}$ & neg. uniaxial & $42 m$ & 0.22 & 1.7 & & & 5.2 & & & \\
\hline RDP: $\mathrm{RbH}_{2} \mathrm{PO}_{4}$ & neg. uniaxial & $42 m$ & 0.22 & 1.5 & $>0.3$ & 12 & 4.02 & & & \\
\hline $\mathrm{CDA}: \mathrm{CsH}_{2} \mathrm{AsO}_{4}$ & neg. uniaxial & $42 m$ & 0.26 & 1.43 & 0.5 & 10 & 4.02 & & 17.5 & 57 \\
\hline $\mathrm{KDA}: \mathrm{KH}_{2} \mathrm{AsO}_{4}$ & neg. uniaxial & $42 m$ & 0.216 & 1.7 & & & 5.2 & & & \\
\hline $\mathrm{LiIO}_{3}$ & neg. uniaxial & 6 & 0.3 & 6 & $3-8$ & 0.12 & $\begin{array}{l}-55.3\left(d_{15}\right) / \\
-72\left(d_{33}\right)\end{array}$ & & 18 & 44 \\
\hline $\mathrm{LiNbO}_{3}$ & neg. uniaxial & $3 m$ & 0.33 & 5.5 & 0.05 & 10 & $\begin{array}{l}-59.5\left(d_{31}\right) / \\
27.6\left(d_{22}\right)\end{array}$ & & 20 & 40 \\
\hline $\mathrm{K}_{3} \mathrm{Li}_{2} \mathrm{Nb}_{5} \mathrm{O}_{15}$ & neg. uniaxial & $4 m m$ & 0.4 & 5 & & & $\begin{array}{l}62\left(d_{15}\right) / \\
127\left(d_{33}\right)\end{array}$ & & & \\
\hline BBO: $\beta-\mathrm{BaB}_{2} \mathrm{O}_{4}$ & neg. uniaxial & $3 m$ & 0.198 & 2.6 & 10 & 0.1 & $\begin{array}{l}22.2\left(d_{22}\right) / \\
1.6\left(d_{31}\right)\end{array}$ & & 6.8 & 70 \\
\hline Proustite: $\mathrm{Ag}_{3} \mathrm{AsS}_{3}$ & neg. uniaxial & $3 m$ & 0.6 & 13 & 0.02 & 20 & & $\begin{array}{l}113\left(d_{15}\right) / \\
180\left(d_{22}\right)\end{array}$ & & \\
\hline Pyrargite: $\mathrm{Ag}_{3} \mathrm{SbS}_{3}$ & neg. uniaxial & $3 m$ & 0.7 & 14 & 0.02 & 17.5 & & $\begin{array}{l}84\left(d_{15}\right) / \\
92\left(d_{22}\right)\end{array}$ & & \\
\hline $\mathrm{AgGaS}_{2}$ & neg. uniaxial & $42 m$ & 0.5 & 13 & 0.025 & 35 & & 134 & & \\
\hline $\mathrm{AgGaSe}_{2}$ & neg. uniaxial & $42 m$ & 0.71 & 18 & 0.03 & 35 & & 330 & & \\
\hline $\mathrm{ZnGeP}_{2}$ & pos. uniaxial & $42 m$ & 0.74 & 12 & 0.003 & 30 & & 754 & & \\
\hline $\mathrm{CdGeAs}_{2}$ & pos. uniaxial & $42 m$ & 2.4 & 18 & & & & 2350 & & \\
\hline GaSe & neg. uniaxial & $62 m$ & 0.65 & 18 & 0.035 & 10 & & $544\left(d_{22}\right)$ & & \\
\hline CdSe & pos. uniaxial & $6 \mathrm{~mm}$ & 0.75 & 20 & $>0.05$ & 10 & & $180\left(d_{15}\right)$ & & \\
\hline $\mathrm{HgS}$ & pos. uniaxial & 32 & 0.63 & 13.5 & 0.04 & 17 & & $502\left(d_{11}\right)$ & & \\
\hline $\mathrm{Se}$ & pos. uniaxial & 32 & 0.7 & 21 & & & & $970\left(d_{11}\right)$ & & \\
\hline $\mathrm{Te}$ & pos. uniaxial & 32 & 3.8 & 32 & & & & $6500\left(d_{11}\right)$ & & \\
\hline Quartz: $\alpha$-SiO & pos. uniaxial & 32 & 0.15 & 4.5 & & & $3.64\left(d_{11}\right)$ & & & \\
\hline $\begin{array}{l}\mathrm{Li} \text {-formate: } \\
\mathrm{LiCOOH} * \mathrm{H}_{2} \mathrm{O}\end{array}$ & neg. biaxial & $m m 2$ & 0.23 & 1.2 & & cw & $\begin{array}{l}1.0\left(d_{31}\right) / \\
-1.16\left(d_{32}\right) / \\
1.68\left(d_{33}\right)\end{array}$ & & & \\
\hline $\begin{array}{l}\text { Ba-Formate: } \\
\mathrm{Ba}(\mathrm{COOH})_{2}\end{array}$ & pos. biaxial & 222 & 0.245 & 2.2 & & & $\begin{array}{l}1.17\left(d_{36}\right) / \\
1.13\left(d_{14}\right) / \\
1.17\left(d_{25}\right)\end{array}$ & & & \\
\hline $\begin{array}{l}\text { Na-formate: } \\
\mathrm{NaCOOH}\end{array}$ & neg. biaxial & $m m 2$ & 0.26 & 1.28 & & & $\begin{array}{l}0.22\left(d_{31}\right) / \\
-2.2\left(d_{32}\right) / \\
3.3\left(d_{33}\right)\end{array}$ & & & \\
\hline $\mathrm{LiB}_{3} \mathrm{O}_{5}$ & neg. biaxial & $m m 2$ & 0.16 & 2.6 & 25 & 0.1 & $\begin{array}{l}10.9\left(d_{31}\right) / \\
11.7\left(d_{32}\right) / \\
0.65\left(d_{33}\right)\end{array}$ & & & \\
\hline KTP: $\mathrm{KTiOPO}_{4}$ & pos. biaxial & $m m 2$ & 0.35 & 4.5 & 15 & 1 & $\begin{array}{l}65\left(d_{31}\right) / \\
50\left(d_{32}\right) / \\
137\left(d_{33}\right)\end{array}$ & & 4 & 60 \\
\hline $\mathrm{Ba}_{2} \mathrm{NaNb}_{5} \mathrm{O}_{15}$ & neg. biaxial & $m m 2$ & 0.37 & 5 & 0.003 & 10 & $\begin{array}{l}-132\left(d_{31}\right) / \\
-182\left(d_{33}\right)\end{array}$ & & 3 & 20 \\
\hline $\mathrm{KNbO}_{3}$ & neg. biaxial & $m m 2$ & 0.4 & 4.5 & 0.012 & 10 & $\begin{array}{l}158\left(d_{31}\right) / \\
-132\left(d_{32}\right) / \\
-201\left(d_{33}\right)\end{array}$ & & & \\
\hline $\mathrm{MgBaF}_{4}$ & neg. biaxial & $m m 2$ & 0.185 & 10 & & & $\begin{array}{l}0.248\left(d_{31}\right) / \\
0.37\left(d_{32}\right) / \\
0.1\left(d_{33}\right)\end{array}$ & & & \\
\hline Urea: $\mathrm{CO}\left(\mathrm{NH}_{2}\right)_{2}$ & pos. uniaxial & $42 m$ & 0.2 & 1.8 & 5 & 10 & 13 & & & \\
\hline $\begin{array}{l}\text { 3-Methyl-4-nitro- } \\
\text { pyridine-1-oxide }\end{array}$ & pos. biaxial & 222 & 0.4 & 3 & 2 & 0.02 & 10 & & & \\
\hline MAP: $\mathrm{C}_{10} \mathrm{H}_{11} \mathrm{~N}_{3} \mathrm{O}_{6}$ & pos. biaxial & 2 & 0.5 & 2 & 3 & 10 & $\begin{array}{l}167\left(d_{21}\right) / \\
184\left(d_{22}\right) / \\
36.8\left(d_{23}\right) / \\
-5.44\end{array}$ & & & \\
\hline $\begin{array}{l}\text { COANP: } \\
\mathrm{C}_{13} \mathrm{H}_{19} \mathrm{~N}_{3} \mathrm{O}_{2}\end{array}$ & pos. biaxial & $m m 2$ & 0.47 & 1.5 & & & $\begin{array}{l}150\left(d_{31}\right) / \\
260\left(d_{32}\right) / \\
100\left(d_{33}\right)\end{array}$ & & & \\
\hline
\end{tabular}

cited from [103] 


\section{Poling of glass}

The electrical poling of glass sheets is shown in Figure A 4. The poling of glass for secondharmonic generation is shown in Table A 16.

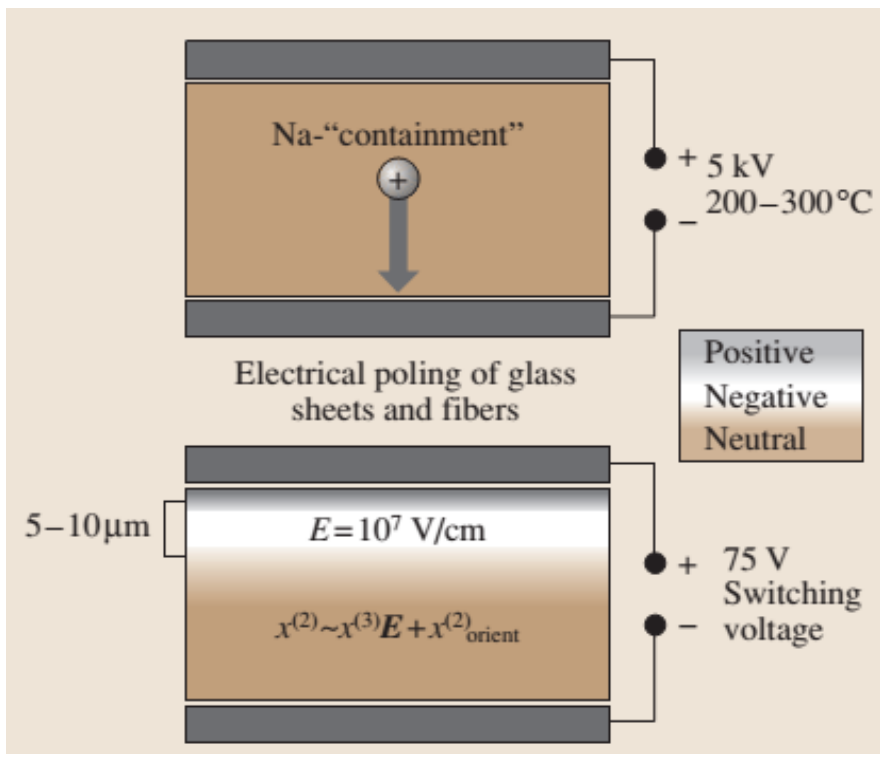

Figure A 4 Schematic of the electrical poling of glass sheets (cited from [103])

Table A 16 Poling of glass for second-harmonic generation

\begin{tabular}{|c|c|c|c|c|}
\hline Glass & Poling & Parameters & & SHG coefficient $d(\mathrm{pm} / \mathrm{V})$ \\
\hline Fused silica, containing $\mathrm{Na}, \mathrm{OH}$ & Thermal & $275^{\circ} \mathrm{C}$ & $5 \mathrm{kV}$ & 1 (in a $10 \mu \mathrm{m}$ layer) \\
\hline $\mathrm{GeO}_{2}-\mathrm{SiO}_{2}$, Herasil & Thermal + electric & $280^{\circ} \mathrm{C}$ & $4.3 \mathrm{kV}$ & 1 \\
\hline Ge-silicate "half"-fiber & Thermal + electric & $275^{\circ} \mathrm{C}$ & $-5 \mathrm{kV}$ & 0.3 \\
\hline Ge-silicate optical fibers & $\begin{array}{l}\text { Electric, thermal } \\
\text { electric and UV }\end{array}$ & & & 0.002 to 6 \\
\hline $\mathrm{GeO}_{2}-\mathrm{SiO}_{2}$ & $\begin{array}{l}\text { Optical + electric } \\
\text { field }\end{array}$ & $\operatorname{ArF}(193 \mathrm{~nm})$ & Electric field & $3.4-5.2$ \\
\hline $\mathrm{GeO}_{2}-\mathrm{SiO}_{2}$ sputtered film & $\begin{array}{l}\text { Optical + electric } \\
\text { field }\end{array}$ & $\begin{array}{l}\operatorname{ArF} \\
100 \mathrm{~mJ} / \mathrm{cm}^{2} / \text { pulse }\end{array}$ & Electric field & up to 12.5 \\
\hline $15.7 \mathrm{GeO}_{2}-84.2 \mathrm{SiO}_{2}(\mathrm{~mol} \%)$ & $\begin{array}{l}\text { Optical + electric } \\
\text { field }\end{array}$ & $\begin{array}{l}\text { ArF: } \\
10-100 \mathrm{~mJ} / \mathrm{cm}^{2} / \text { pulse }\end{array}$ & $3 \times 10^{5} \mathrm{~V} / \mathrm{cm}$ & $7 \times 10^{-3}-2$ \\
\hline Ge-silicate & fs-encoding & $810(960 \mathrm{~mW})$ & $405 \mathrm{~nm}(200 \mathrm{fs}, 200 \mathrm{kHz})$ & \\
\hline $\begin{array}{l}\text { Er-, Tb- and } \mathrm{Sm} \text {-doped } \\
\text { Al-silicate fibers, Er-doped } \\
\mathrm{Ge}: \mathrm{SiO}_{2} \text { fiber }\end{array}$ & Optical & $\begin{array}{l}1065 \mathrm{~nm} \\
\left(30-50 \mathrm{GW} / \mathrm{cm}^{2}\right)\end{array}$ & Seed: $532 \mathrm{~nm}$ & $\begin{array}{l}\text { Conversion effic.: } 10^{-6} \text { to } \\
10^{-3}, 8 \times 10^{-3} \text { in Ge-silicate }\end{array}$ \\
\hline $\begin{array}{l}\text { Ce- and Eu-doped Al-silicate } \\
\text { fibers }\end{array}$ & Optical & Nd:YAG: $1064 \mathrm{~nm}$ & Seed: $532 \mathrm{~nm}$ & $\begin{array}{l}\text { Conversion effic.: } 2 \times 10^{-6} \text { to } \\
1.5 \% \text { for Ce-doped fiber for } \\
200 \mathrm{~W} \text { pump }\end{array}$ \\
\hline Tm-doped aluminosilica fiber & Optical & Nd:YAG: $1064 \mathrm{~nm}$ & Seed: $532 \mathrm{~nm}$ & $\begin{array}{l}\text { SH output e.g. } 4.6 \times 10^{-2} \mathrm{~W} \\
\text { for } 240 \mathrm{~W} \text { pump at } 1064 \mathrm{~nm} \\
\text { and } 4.6 \times 10^{-2} \mathrm{~W} \text { for } 55 \mathrm{~W} \text { at } \\
844 \mathrm{~nm}\end{array}$ \\
\hline Ce-doped lead germanate glass & Optical & 1065 nm Nd:YAG & Seed: $532 \mathrm{~nm}$ KTP & \\
\hline CdSSe-doped: GG495-RG630 & Optical & $1065 \mathrm{~nm}(3 \mathrm{~W})$ & $\begin{array}{l}532 \mathrm{~nm}(1 \mathrm{~W}) \text { pulsed } \\
\text { laser }\end{array}$ & $10^{-4}$ \\
\hline Chalcogenide glasses: GeAsS & Optical & Nd:YAG $1064 \mathrm{~nm}$ & Seed: $532 \mathrm{~nm}$ & $\begin{array}{l}\alpha(532 \mathrm{~nm})=1.99 \mathrm{~cm}^{-1} \text { con- } \\
\text { version effic.: } 10^{4} \mathrm{x} \text { that of } \\
\mathrm{TeO}_{2}-\mathrm{Nb}_{2} \mathrm{O}_{5} \text {-glass }\end{array}$ \\
\hline $\begin{array}{l}\text { Fused silica fibers containing } \\
\text { impurities: } \mathrm{OH}, \mathrm{Na}, \mathrm{Fe}\end{array}$ & Gamma ray & $\begin{array}{l}{ }^{60} \mathrm{Co} \text { source }\left(10^{6} \mathrm{rad} \text { at }\right. \\
400 \mathrm{rad} / \mathrm{s})\end{array}$ & $\begin{array}{l}1064 \mathrm{~nm}(\mathrm{Nd}: \mathrm{YAG}, \\
50 \mathrm{~ns}, 10 \mathrm{~Hz}, 1 \mathrm{~kW}) \text { and } \\
532 \mathrm{~nm} \text { seed }(300 \mathrm{~W}), 1 \mathrm{~h}\end{array}$ & $\begin{array}{l}2 \times 10^{-4}\left(\text { natural } \mathrm{SiO}_{2}\right) \\
9 \times 10^{-3}\left(\mathrm{Ge}: \mathrm{SiO}_{2}+\mathrm{Ce}\right) \\
2 \times 10^{-2}\left(\mathrm{Ge}: \mathrm{SiO}_{2}+\mathrm{Ce}\right)\end{array}$ \\
\hline
\end{tabular}

cited from [103] 


\section{Lithium silicates}

The phase equilibrium of the $\mathrm{Li}_{4} \mathrm{SiO}_{4}-\mathrm{Li}_{2} \mathrm{SiO}_{3}$ sub-system is shown in Figure A 5 .

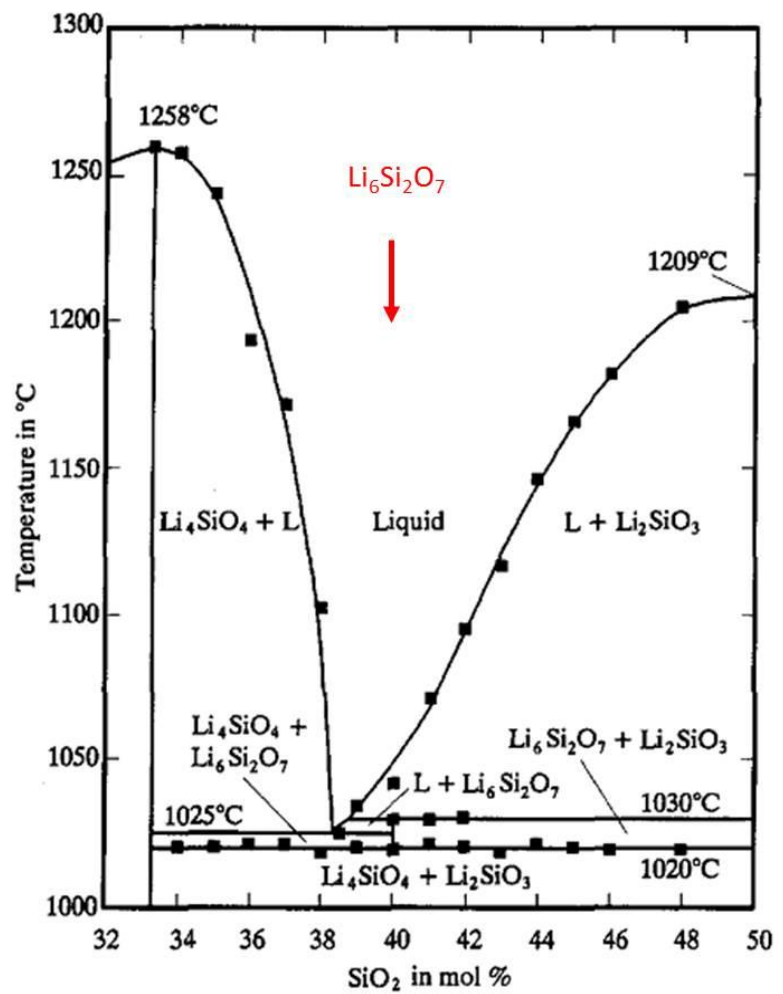

Figure A 5 Phase equilibrium in the $\mathrm{Li}_{4} \mathrm{SiO}_{4}-\mathrm{Li}_{2} \mathrm{SiO}_{3}$ sub-system (after [131])

$\mathrm{Li}_{2} \mathrm{Si}_{3} \mathrm{O}_{7}$ is metastable, persisting at ambient temperature, and can be destroyed by prolonged heating (ca. $12-24 \mathrm{hrs}$ at $\mathrm{T}>650^{\circ} \mathrm{C}$ ).

The relationship between the thermal history of a 75 mol\% $\mathrm{SiO}_{2}$ glass (or melt) and the crystallization products is shown in Figure A 6.

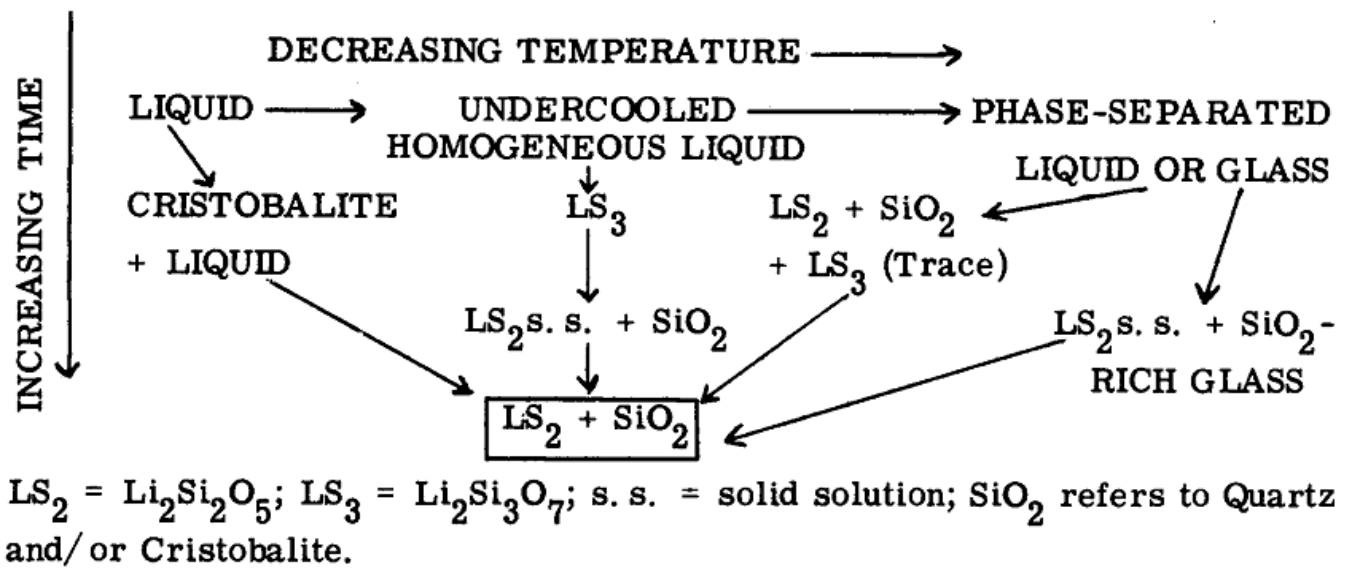

Figure A 6 The relationship between the thermal history of a $75 \mathrm{~mol}^{2} \mathrm{SiO}_{2}$ glass (or melt) and the crystallization products (cited from [133])

The experimental crystal structure constants of lithium silicates are shown in Table A 17 
Table A 17 The experimental crystal structure constants of lithium silicates

\begin{tabular}{|c|c|c|c|}
\hline Crystal & $\begin{array}{l}\text { Lattice } \\
\text { constant }\end{array}$ & Space group & $\mathrm{Li}_{2} \mathrm{O} / \mathrm{SiO}_{2}$ ratio \\
\hline $\mathrm{Li}_{2} \mathrm{O}$ & $a=4.573 \AA$ & $F m-3 m($ no. 225$)$ & $1: 0$ \\
\hline $\mathrm{Li}_{8} \mathrm{SiO}_{6}$ & $\begin{array}{l}a=5.4243 \AA \\
c=10.626 \AA\end{array}$ & $\mathrm{PG}_{3} \mathrm{Cm}$ (no. 185) & $4: 1$ \\
\hline $\mathrm{Li}_{4} \mathrm{SiO}_{4}$ & $\begin{array}{l}a=11.532 \AA \\
b=6.075 \AA \\
c=16.678 \AA \\
b=99.04^{\circ}\end{array}$ & $P 2_{1} / m$ (no. 11$)$ & $2: 1$ \\
\hline $\mathrm{Li}_{6} \mathrm{Si}_{2} \mathrm{O}_{7}$ & $\begin{array}{l}a=7.71 \AA \\
c=4.88 \AA\end{array}$ & $P \overline{4} 2_{1} m$ (o. 113) & $3: 2$ \\
\hline $\mathrm{Li}_{2} \mathrm{SiO}_{3}$ & $\begin{array}{l}a=9.392 \AA \\
b=5.397 \AA \\
c=4.660 \AA\end{array}$ & $\mathrm{Cmc2}_{1}$ (no. 36) & $1: 1$ \\
\hline $\mathrm{Li}_{2} \mathrm{Si}_{2} \mathrm{O}_{5}$ & $\begin{array}{l}a=5.82 \AA \\
b=14.66 \AA \\
c=4.79 \AA \\
B=90^{\circ}\end{array}$ & $\operatorname{Ccc} 2$ (no. 37) & $1: 2$ \\
\hline meta- $\mathrm{Li}_{2} \mathrm{Si}_{2} \mathrm{O}_{5}$ & $\begin{array}{l}a=5.683 \AA \\
b=4.784 \AA \\
c=14.648 \AA\end{array}$ & Pbcn (no. 60) & $1: 2$ \\
\hline $\mathrm{Li}_{2} \mathrm{Si}_{3} \mathrm{O}_{7}$ & $\begin{array}{l}a=19.648 \AA \\
b=5.9969 \AA \\
c=4.8691 \AA\end{array}$ & Pmca (no. 57) & $1: 3$ \\
\hline$\alpha-\mathrm{SiO}_{2}$ & $\begin{array}{l}a=4.8992 \AA \\
c=5.3832 \AA \\
\gamma=120^{\circ}\end{array}$ & $P 3_{2} 21$ (no. 154) & $0: 1$ \\
\hline
\end{tabular}

Table A 18 lists the critical cooling rate of several $\mathrm{Li}_{2} \mathrm{O}-\mathrm{SiO}_{2}$ compositions. Below this cooling rate, detectable crystalline phase can be obtained from the melt. It can be seen that this cooling rate increases with increasing concentration of $\mathrm{Li}_{2} \mathrm{O}$, indicating the glass formation is more difficult at the higher concentration of $\mathrm{Li}_{2} \mathrm{O}$ (i.e. 66.7 mol\%). In addition Tg was observed, implying the flakes prepared are in the glassy state [261].

Table A 18 Chemical compositions, critical cooling rates, and glass transition temperatures in $\mathrm{Li}_{2} \mathrm{O}-\mathrm{SiO}_{2}$

\begin{tabular}{llll}
\hline \multicolumn{2}{l}{ Composition (mol\%) } & Critical cooling rate & $\begin{array}{l}\mathrm{T}_{\mathrm{g}} \\
\mathrm{Li}_{2} \mathrm{O}\end{array}$ \\
\hline 40 & $\mathrm{SiO}_{2}$ & $(\mathrm{~K} / \mathrm{s})$ & $\left({ }^{\circ} \mathrm{C}\right)$ \\
50 & 60 & $4.3 \times 10^{5}$ & 442 \\
60 & 40 & $5.2 \times 10^{6}$ & 414 \\
66.7 & 33.3 & $6.4 \times 10^{7}$ & 270 \\
\hline
\end{tabular}

The critical cooling rate is estimated by the method reported in [262]. $\mathrm{T}_{\mathrm{g}}$ is determined by DSC (cited from [261]) 
XIV. $\mathrm{Li}_{2} \mathrm{O}-\mathrm{Nb}_{2} \mathrm{O}_{5}$ system with $\mathrm{BaO}$ or $\mathrm{Al}_{2} \mathrm{O}_{3}$

Suzuki et al. [263] investigated the glass formation of different binary oxide systems by twinroller quenching. As shown in Figure A 7, they demonstrated that with an increase of the ratio of cation radius in the two oxides, the glass formation can be facilitated. So, it will be effective to put a large cation (e.g. $\mathrm{Ba}^{2+}$ ) into $\mathrm{LiNbO}_{3}$ system (i.e. $\mathrm{Li}_{2} \mathrm{O}-\mathrm{BaO}-\mathrm{Nb}_{2} \mathrm{O}_{5}$ system), where $\mathrm{Li}^{+}$and $\mathrm{Nb}^{5+}$ are small, to more easily obtain glasses containing no crystalline ones.

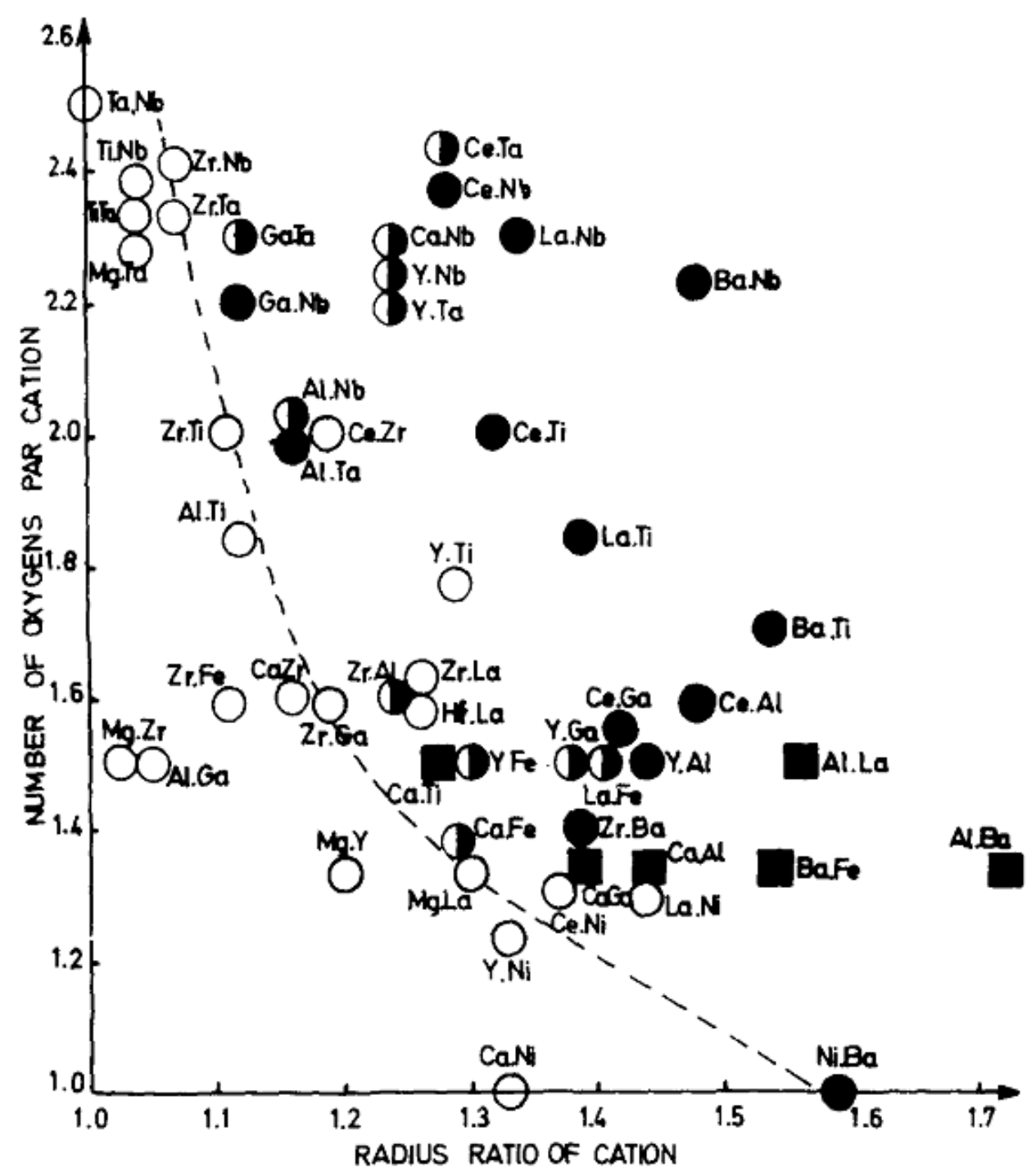

Figure A 7 Radius ratio of cation vs number of oxygens par cation: $\mathbf{Q}=$ = binary oxides which form NCS on rapid quenching (not tested by the twin roller device), obinary oxides which form NCS + crystal on rapid quenching, and $\bigcirc$ binary oxides which do not form NCS on rapid quenching; NCS: non-crystalline solid (cited from [263])

As shown in Figure $\mathrm{A} 8$, in $\mathrm{Li}_{2} \mathrm{O}-\mathrm{BaO}-\mathrm{Nb}_{2} \mathrm{O}_{5}$ system, glasses can be prepared in the large variety of compositions, using a twin-roller quenching technique [180]. The crystallization temperature ranges from $500^{\circ} \mathrm{C}$ to $700^{\circ} \mathrm{C}$ and increases when $\mathrm{Li}_{2} \mathrm{O}$ is replaced by $\mathrm{BaO}$, which can be explained 
by the increase of covalency, promoting glass fromation. It is possible to obtain glass when $\mathrm{Nb}_{2} \mathrm{O}_{5}>35 \mathrm{~mol} \%$.

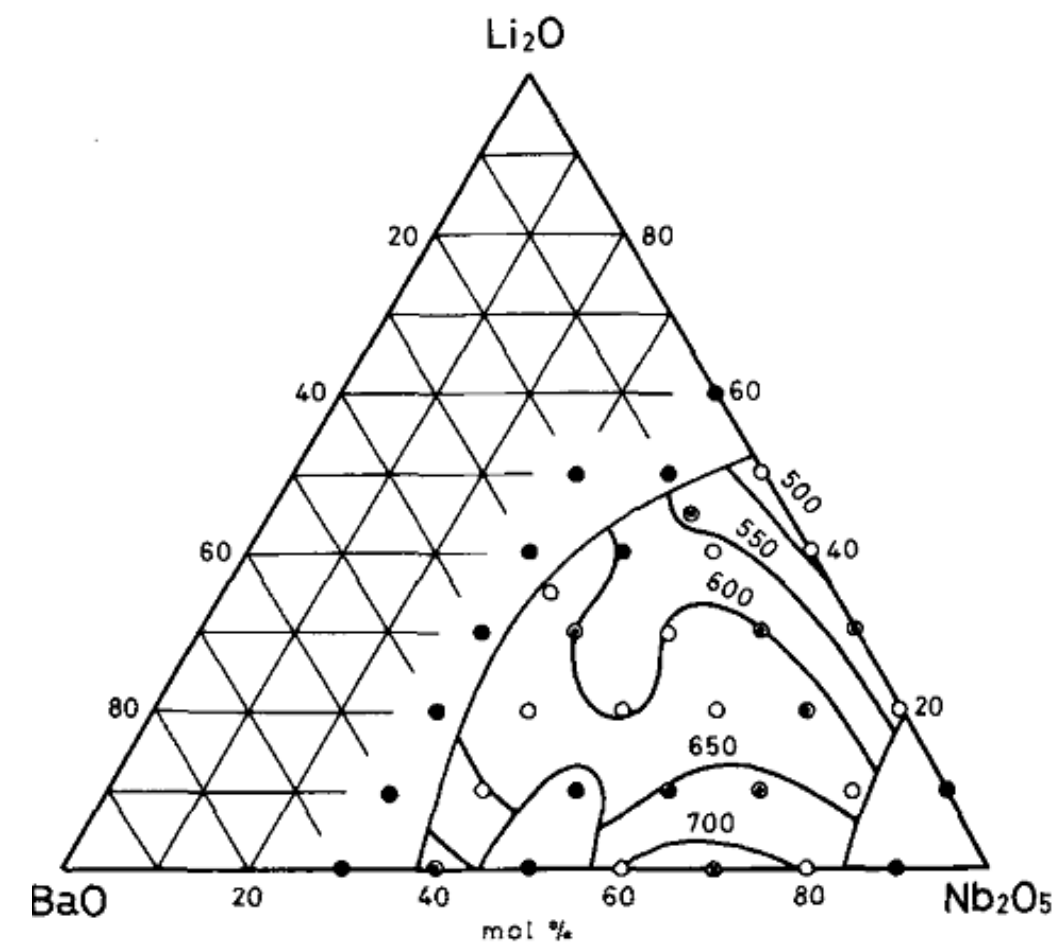

Figure A 8 Glass-forming region and iso- $\mathrm{T}_{\mathrm{c}}\left(\mathrm{in}^{\circ} \mathrm{C}\right)$ curves for the system $\mathrm{Li}_{2} \mathrm{O}-\mathrm{BaO}-\mathrm{Nb}_{2} \mathrm{O}_{5} ;(\mathrm{O})$ glass exhibiting one exotherm in DTA, $(\odot)$ glass exhibiting more than one exotherm in DTA, and $(\bullet)$ crystalline (cited from [180])

Figure A 9 displays the $\mathrm{Li}_{2} \mathrm{O}-\mathrm{Al}_{2} \mathrm{O}_{3}-\mathrm{Nb}_{2} \mathrm{O}_{5}$ system, in which glasses can also be prepared in the large variety of compositions by the twin-roller quenching technique [181]. All the glassy samples were colorless, transparent flakes. The crystallization temperature ranges from $495^{\circ} \mathrm{C}$ to $780^{\circ} \mathrm{C}$ and mainly determined by the concentration of $\mathrm{Li}_{2} \mathrm{O}$ and is not dependent of the concentrations of $\mathrm{Al}_{2} \mathrm{O}_{3}$ and $\mathrm{Nb}_{2} \mathrm{O}_{5}$ when $\mathrm{Li}_{2} \mathrm{O} / \mathrm{Nb}_{2} \mathrm{O}_{5}<1$. But it increases with the replacement of $\mathrm{Nb}_{2} \mathrm{O}_{5}$ by $\mathrm{Al}_{2} \mathrm{O}_{3}$ when $\mathrm{Li}_{2} \mathrm{O} / \mathrm{Nb}_{2} \mathrm{O}_{5}>1 . \mathrm{Al}_{2} \mathrm{O}_{3}$ and $\mathrm{Nb}_{2} \mathrm{O}_{5}$ have high liquidus temperatures (> $1500^{\circ} \mathrm{C}$ ), which make glass formation difficult when $>80 \mathrm{~mol} \%$ of $\mathrm{Al}_{2} \mathrm{O}_{3}$ or $>80$ mol\% $\mathrm{Nb}_{2} \mathrm{O}_{5}$ [181]. 


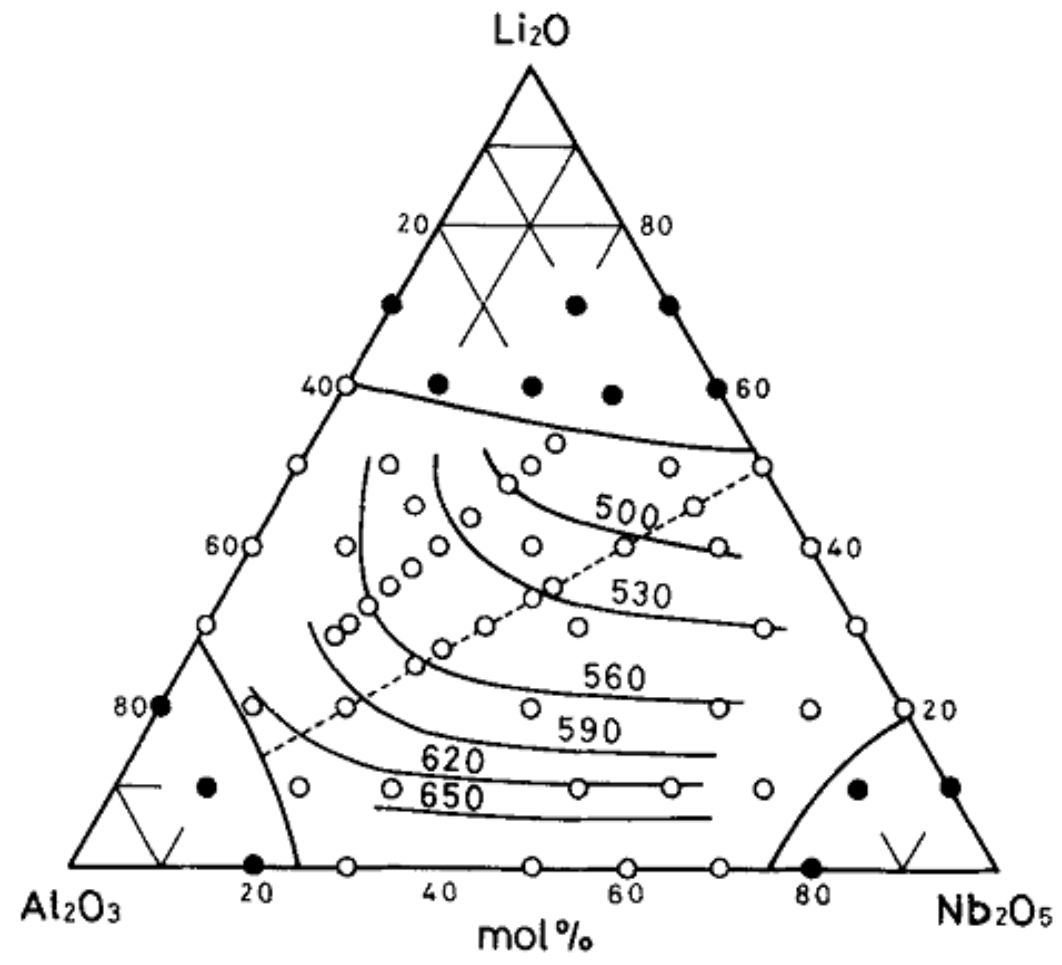

Figure A 9 Glass-forming region and iso- $\mathrm{T}_{\mathrm{C}}\left(\mathrm{in}{ }^{\circ} \mathrm{C}\right)$ curves for the system $\mathrm{Li}_{2} \mathrm{O}-\mathrm{Al}_{2} \mathrm{O}_{3}-\mathrm{Nb}_{2} \mathrm{O}_{5}$ : $(O)$ glass and $(\mathbf{O})$ crystalline (cited from [181]) 
XV. XRD pattern of $\mathrm{LiNbO}_{3}$

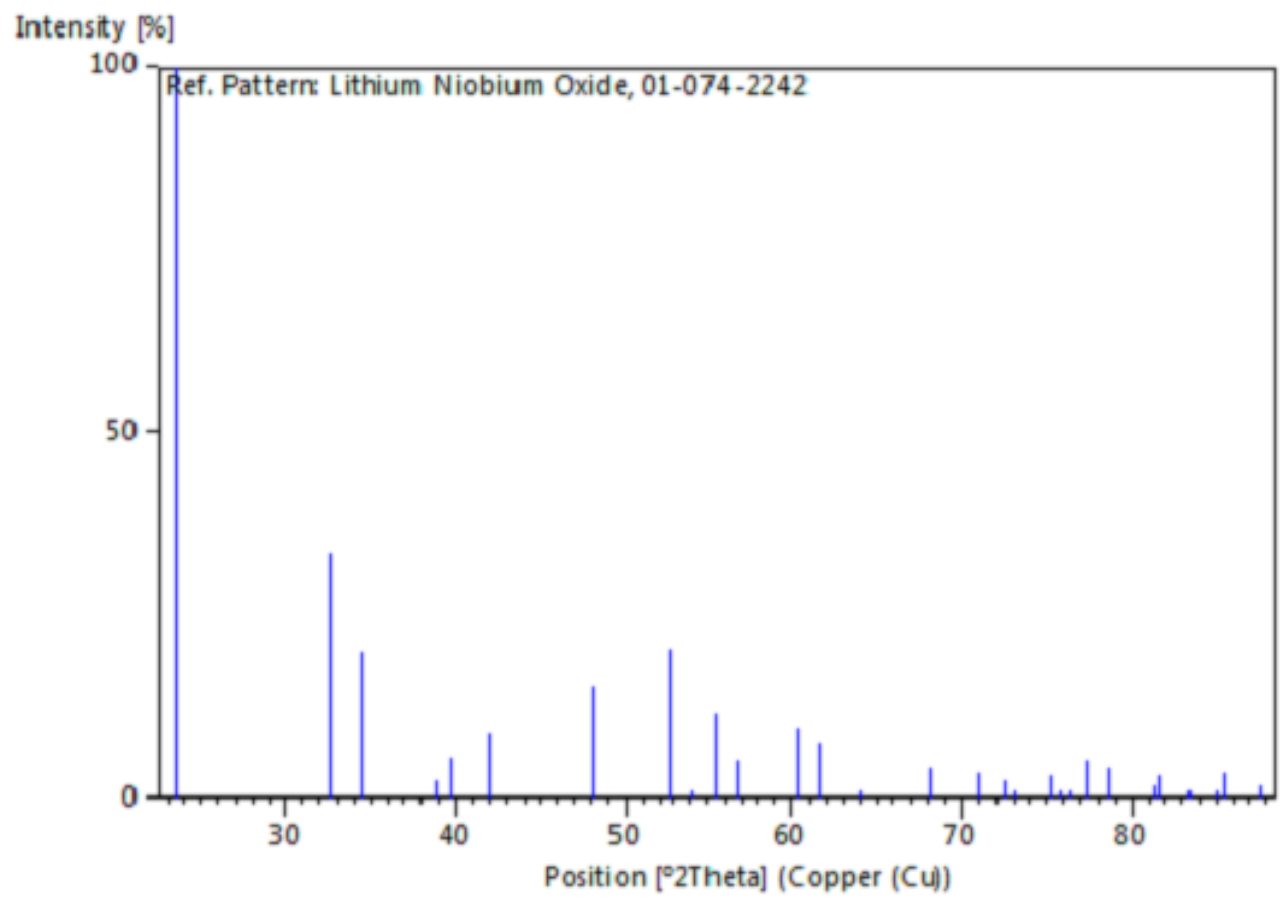

Figure A 10 Stick XRD pattern of $\mathrm{LiNbO}_{3}$

\begin{tabular}{rrrrrrr} 
No. & $\mathrm{h}$ & $\mathrm{k}$ & $\mathrm{l}$ & $\mathrm{d}[\mathrm{A}]$ & 2Theta [deg] & $\mathrm{I}[\mathrm{g}$ ] \\
\hline 1 & 0 & 1 & 2 & 3.78321 & 23.496 & 100.0 \\
2 & 1 & 0 & 4 & 2.75125 & 32.518 & 33.4 \\
3 & 1 & 1 & 0 & 2.60500 & 34.399 & 19.9 \\
4 & 0 & 0 & 6 & 2.31417 & 38.885 & 2.4 \\
5 & 1 & 1 & 3 & 2.27013 & 39.671 & 5.6 \\
6 & 2 & 0 & 2 & 2.14556 & 42.080 & 8.8 \\
7 & 0 & 2 & 4 & 1.89160 & 48.061 & 15.1 \\
8 & 1 & 1 & 6 & 1.73009 & 52.877 & 20.2 \\
9 & 2 & 1 & 1 & 1.69265 & 54.141 & 0.6 \\
10 & 1 & 2 & 2 & 1.65614 & 55.436 & 11.6 \\
11 & 0 & 1 & 8 & 1.61991 & 56.786 & 5.0 \\
12 & 2 & 1 & 4 & 1.53063 & 60.431 & 9.7 \\
13 & 3 & 0 & 0 & 1.50400 & 61.617 & 7.6 \\
14 & 1 & 2 & 5 & 1.45322 & 64.020 & 0.3 \\
15 & 2 & 0 & 8 & 1.37563 & 68.106 & 4.1 \\
16 & 1 & 1 & 9 & 1.32708 & 70.964 & 3.3 \\
17 & 2 & 2 & 0 & 1.30250 & 72.513 & 2.3 \\
18 & 2 & 1 & 7 & 1.29315 & 73.122 & 0.2 \\
19 & 0 & 3 & 6 & 1.26107 & 75.299 & 2.9 \\
20 & 2 & 2 & 3 & 1.25380 & 75.813 & 0.2 \\
21 & 1 & 3 & 1 & 1.24635 & 76.347 & 0.2 \\
22 & 3 & 1 & 2 & 1.23155 & 77.434 & 5.1 \\
23 & 1 & 2 & 8 & 1.21644 & 78.579 & 4.3 \\
24 & 0 & 2 & 10 & 1.18248 & 81.299 & 1.7 \\
25 & 1 & 3 & 4 & 1.17724 & 81.737 & 3.1 \\
26 & 0 & 0 & 12 & 1.15708 & 83.476 & 0.8 \\
27 & 3 & 1 & 5 & 1.14091 & 84.934 & 0.1 \\
28 & 2 & 2 & 6 & 1.13506 & 85.475 & 3.4 \\
29 & 0 & 4 & 2 & 1.11340 & 87.553 & 1.8 \\
& & & & & &
\end{tabular}




\section{Seven crystal classes}

As shown in Table A 19, the 14 Bravais lattices enlarged the seven crystal systems of optical mineralogy by adding centering points to them: body (I), face (F), and base (C) centers. The 14 lattices define 3D distributions of mathematical points such that the environments of all points of the lattice are identical [264].

Combing the 14 Bravais lattices with the point and translational symmetry elements, certain combinations is achieved (i.e. space group). There are 230 possible space groups.

Table A 19 Bravais lattice celles

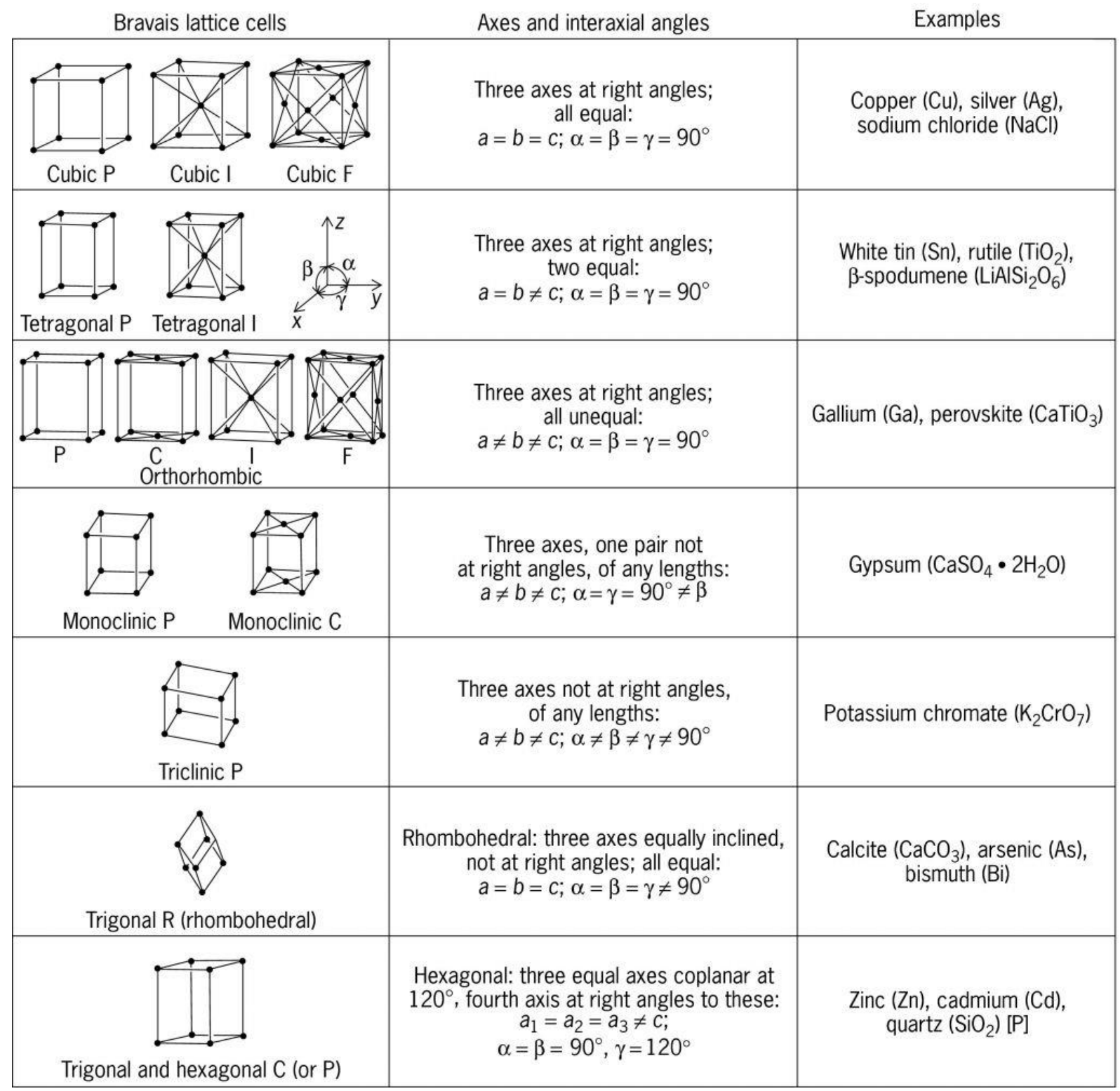

cited from [264] 
Table A 20 Michel-Lévy interference color chart

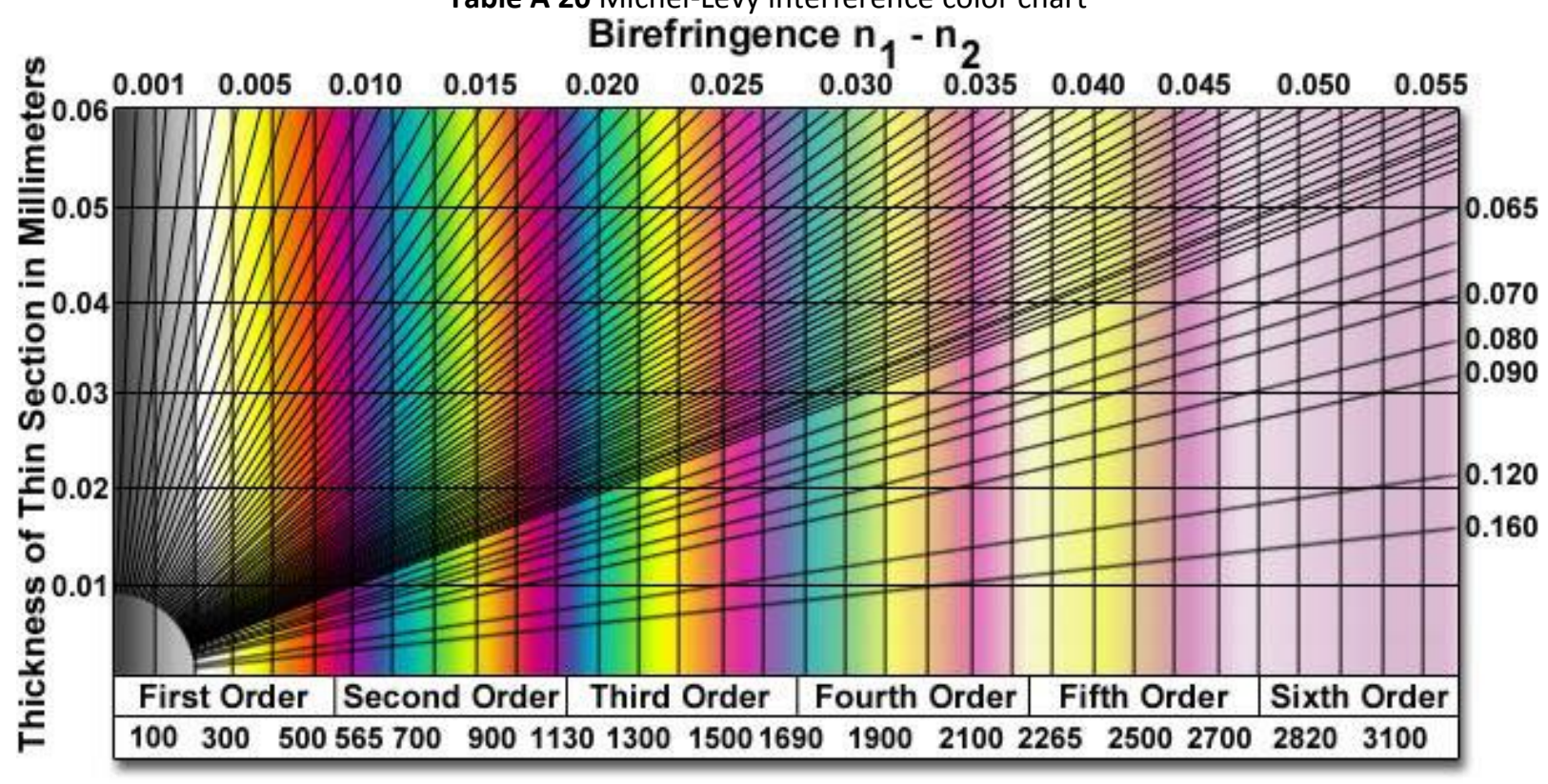

cited from [265]

XVIII. The difference between wavelength-dispersive spectroscopy (WDS) and energydispersive X-ray spectroscopy (EDS)

Table A 21 The difference between WDS and EDS

\begin{tabular}{|c|c|c|}
\hline Operating characteristic & WDS (crystal diffraction) & EDS (silicon, energy-dispersive) \\
\hline $\begin{array}{l}\text { Geometric collection } \\
\text { efficiency }\end{array}$ & Variable, $<0.2 \%$ & $<2 \%$ \\
\hline Overall quantum efficiency & $\begin{array}{l}\text { Variable, }<30 \% \text {, detects } Z \geq \\
4\end{array}$ & $\begin{array}{l}\sim 100 \% \text { for } 2-16 \mathrm{keV} \text {, detects } Z \geq 10 \text { (Be } \\
\text { window); detects } Z \geq 4 \text { (windowless or } \\
\text { thin window) }\end{array}$ \\
\hline Resolution & Crystal-dependent ( $5 \mathrm{eV}$ ) & Energy-dependent (130 eV at $5.9 \mathrm{keV}$ ) \\
\hline $\begin{array}{l}\text { Instantaneous acceptance } \\
\text { range }\end{array}$ & Spectrometer resolution & Entire useful energy range \\
\hline $\begin{array}{l}\text { Maximum count rate } \\
\text { (counts/s) }\end{array}$ & 50000 on an X-ray line & $\begin{array}{l}\text { Resolution-dependent, }<4000, \text { over full } \\
\text { spectrum for best resolution }\end{array}$ \\
\hline $\begin{array}{l}\text { Minimum useful probe size } \\
(\mathrm{nm})\end{array}$ & $\sim 200$ & $\sim 5$ \\
\hline Typical data-collection time & Tens of minutes & Minutes \\
\hline Spectral artifacts & Rare & $\begin{array}{l}\text { Major ones include escape peaks, pulse } \\
\text { pileup, electron-beam scattering, peak } \\
\text { overlap, and window absorption effects }\end{array}$ \\
\hline
\end{tabular}

cited from [209] 


\section{Laser induced breakdown spectroscopy}

Table A 22 Ideal requirements of a spectroscopic method

\begin{tabular}{|c|c|c|}
\hline Characteristic feature & $\begin{array}{l}\text { Is the feature a } \\
\text { LIBS }\end{array}$ & Comments \\
\hline $\begin{array}{l}\text { No sample } \\
\text { preparation }\end{array}$ & Yes & $\begin{array}{l}\text { Indeed, laser-induced plasma can be directly obtained on any sample in any } \\
\text { location. However, that this may limit the accuracy of the technique in } \\
\text { quantitative analysis. }\end{array}$ \\
\hline Liquids, solids, gases & Yes & $\begin{array}{l}\text { This has been demonstrated, although we note the relative difficulties of } \\
\text { both liquids and solids analysis. }\end{array}$ \\
\hline $\begin{array}{l}\text { 3D resolution in solids } \\
\text { (i.e. depth profiling) }\end{array}$ & Yes & $\begin{array}{l}\text { The ability for } 2 \mathrm{D} \text { spatial mapping, as well as depth profiling at a given } \\
\text { location, provides unique 3D capabilities. }\end{array}$ \\
\hline $\begin{array}{l}\text { No spectral } \\
\text { interferences }\end{array}$ & No & $\begin{array}{l}\text { This is strongly related to the available spectral resolution and choice of } \\
\text { analytical lines. }\end{array}$ \\
\hline $\begin{array}{l}\text { No matrix } \\
\text { interferences (perfect } \\
\text { accuracy) }\end{array}$ & No & $\begin{array}{l}\text { In general, LIBS is subject to matrix effects, although as discussed, steps may } \\
\text { be taken to minimize these effects. }\end{array}$ \\
\hline $\begin{array}{l}\text { Absolute } \\
\text { (standardless) }\end{array}$ & Not yet & $\begin{array}{l}\text { Calibration-free (CF) offers an approach for absolute, standardless analysis, } \\
\text { although the CF approach is not perfect. }\end{array}$ \\
\hline $\begin{array}{l}\text { Single-atom and } \\
\text { single molecule } \\
\text { detection limits }\end{array}$ & No & $\begin{array}{l}\text { Laser-based method can reach this limit. However, the overall detection } \\
\text { efficiency of LIBS (as well as of other emission techniques) is too low }\end{array}$ \\
\hline $\begin{array}{l}\text { Precision limited only } \\
\text { by fundamental } \\
\text { counting }\end{array}$ & No & $\begin{array}{l}\text { Emission techniques are limited by extrinsic noise, i.e. by noise that can be } \\
\text { minimized by proper choice of experimental parameters. Intrinsic noise is } \\
\text { not reached }\end{array}$ \\
\hline Stand-off (remote) & Yes & $\begin{array}{l}\text { A capability that is well-suited to LIBS, including single- and double-pulse } \\
\text { configurations. }\end{array}$ \\
\hline $\begin{array}{l}\text { Useful in many } \\
\text { spectral regions }\end{array}$ & Yes & $\begin{array}{l}\text { LIBS is amenable to the UV, visible, and NIR regions. Moreover, spectra in } \\
\text { the mid-IR region can also be used advantageously. }\end{array}$ \\
\hline $\begin{array}{l}\text { Simultaneous all- } \\
\text { channel spectral } \\
\text { acquisition }\end{array}$ & Yes & $\begin{array}{l}\text { The use of CCD and echelle spectrometers allows simultaneous collection of } \\
\text { a broad spectral range. }\end{array}$ \\
\hline $\begin{array}{l}\text { Compatible with } \\
\text { other methods }\end{array}$ & Yes & $\begin{array}{l}\text { Both tandem and hyphenated methodologies provide great diversity to the } \\
\text { LIBS technique. }\end{array}$ \\
\hline $\begin{array}{l}\text { Information-rich } \\
\text { (multivariate) }\end{array}$ & Yes & $\begin{array}{l}\text { LIBS is well-suited to a broad range of multivariate chemometric data } \\
\text { analysis schemes }\end{array}$ \\
\hline $\begin{array}{l}\text { Simple, inexpensive, } \\
\text { compact, portable }\end{array}$ & Yes & $\begin{array}{l}\text { LIBS is relatively simple with regard to implementation and amenable to } \\
\text { low-cost, compact and portable instrument configurations and packages. }\end{array}$ \\
\hline Nondestructive & No & $\begin{array}{l}\text { Strictly speaking, LIBS may be considered a minimally destructive method in } \\
\text { that a small amount of material is ablated. }\end{array}$ \\
\hline
\end{tabular}

cited form [227] 
XX. Electric field treatment of LNS glasses

\section{A 1 Objective}

Herein, a DC external field was applied on LNS glasses to see whether there is some effect or not. The DC field-induced modifications in LNS glasses would have been investigated by the methods of optical microscopy, UV-Vis attenuation spectrum, SEM, EDS, and XPS.

\section{A 2 Experimental details}

The schematic of the electrical field applied on LNS glass is illustrated in Figure A 11. Direct current voltage source is generated by a model C3350 high voltage power supply (HAMAMATSU), which could supply a direct current potential difference $\left(\mathrm{V}_{\mathrm{DC}}\right)$ of $3 \mathrm{kV}$ maximum. A Model 485 autoranging picoammeter is used to measure the current (KEITHLEY). For safety reasons, a $10 \mathrm{M} \Omega$ resistor is used here to limit the current (maximum current $300 \mu \mathrm{A}$, below the safety limit). The electrodes are made of adhesive carbon tape (marked with the black ones in Figure A 11). Two plane carbon electrodes are placed on the opposite sides of sample, one of the electrodes is connected to a direct current voltage source and the other one is grounded. The thickness of the sample (D) is about $1.5 \mathrm{~mm}$, and the gap between to electrodes $(L)$ is about 1 2 $\mathrm{mm}$. The electrical field $E_{D C}=V_{D C} / \sqrt{L^{2}+D^{2}}$ is thus about $1 \mathrm{~V} / \mu \mathrm{m}$ with our voltage source. All experiments are performed in ambient air at atmospheric pressure.

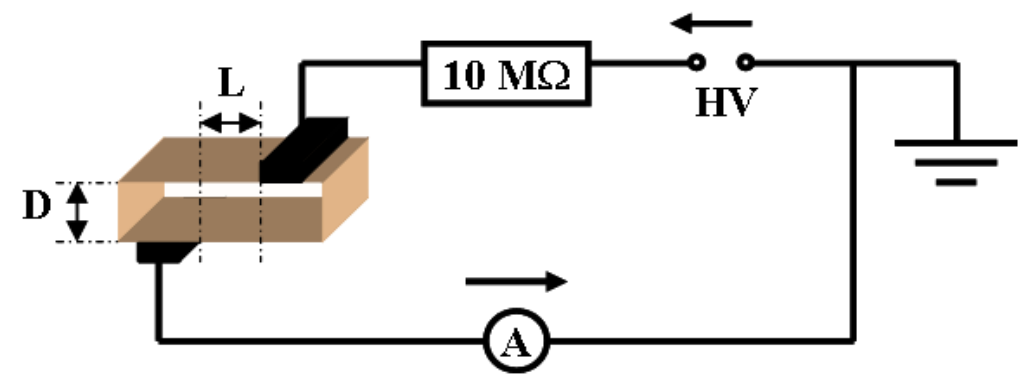

Figure A 11 Schematic of the electric field system (D stands for the thickness of sample and $L$ the gap between two carbon electrodes)

\section{A 3 Results and discussions}

\section{A 3.1 DC electric field applied on LNS glass}

Firstly, a DC voltage was applied on LNS glass $\left(32.5 \mathrm{Li}_{2} \mathrm{O}-27.5 \mathrm{Nb}_{2} \mathrm{O}_{5}-40 \mathrm{SiO}_{2}\right.$, mol\%) from 0 to -3.0 $\mathrm{kV}$ with voltage steps of $0.5 \mathrm{kV}$, and several seconds between each applied voltage. The relationship between voltage and current is shown in Figure A 12. In contrast to silica glass, the 
LNS glass is electrically conducting. The current increases with the increase of applied DC field.

When applying high voltage (i.e. $-3.0 \mathrm{kV}$ ), the current increases quickly.

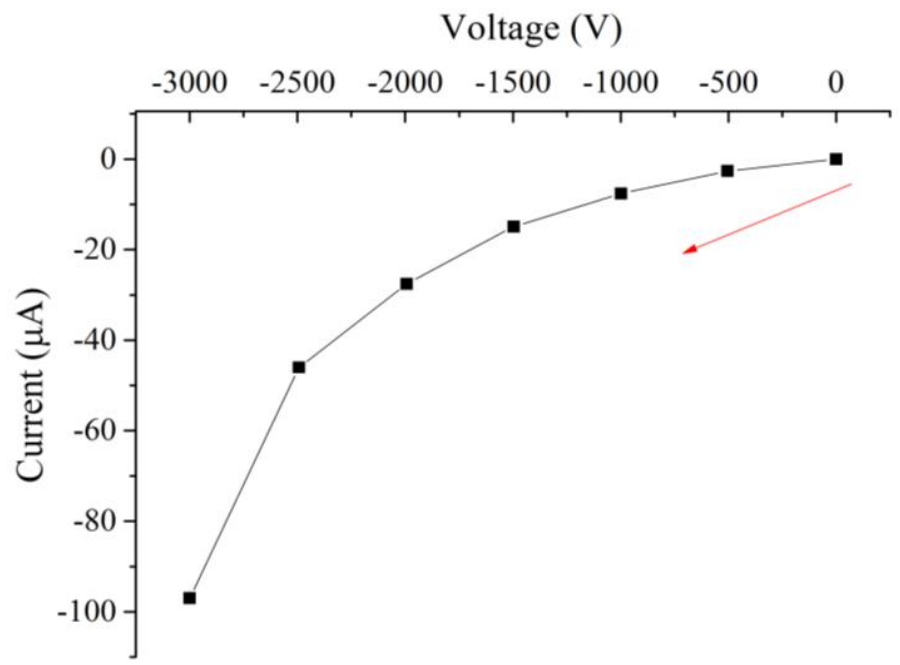

Figure A 12 Relationship between the DC voltage and current on LNS glass $\left(32.5 \mathrm{Li}_{2} \mathrm{O}-27.5 \mathrm{Nb}_{2} \mathrm{O}_{5}-40 \mathrm{SiO}_{2}, \mathrm{~mol} \%\right.$, the red arrow indicates the time direction)

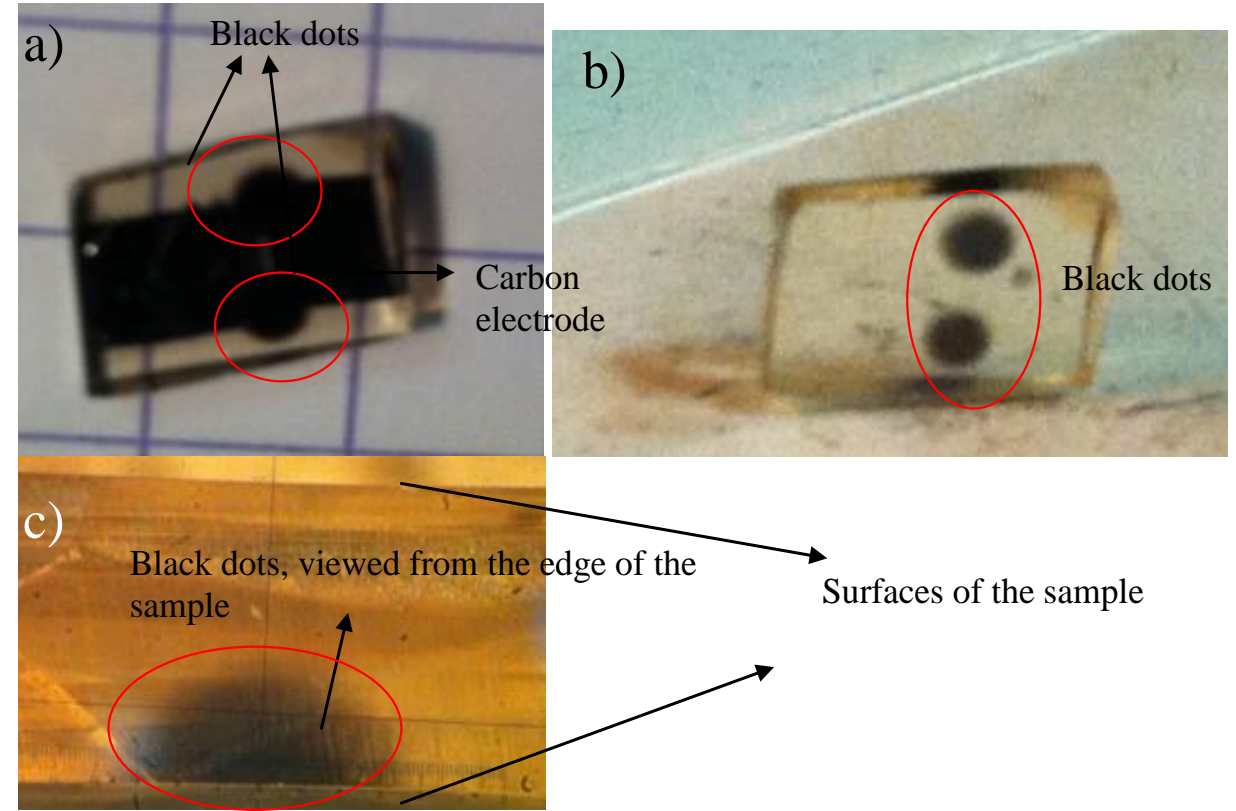

Figure A 13 Photographs of black dots formed in the LNS glass (32.5 $\mathrm{Li}_{2} \mathrm{O}-27.5 \mathrm{Nb}_{2} \mathrm{O}_{5}-40 \mathrm{SiO}_{2}, \mathrm{~mol} \%$.) after DC electrical field treatment a) with carbon electrodes, b) after taking out the carbon electrodes, and c) viewed from the edge of the glass, showing that the black dots are formed from the glass surface to the inside of the sample (black spots are marked in red circles)

Two black dots were clearly observed afterwards close to the negative electrode in LNS glass as shown in Figure A 13a-b. Then optical microscopy was used to check the location of these black clouds in LNS glass. As shown in Figure A 13c, these black clouds are formed from the LNS glass surface, and expanded to the inside of the glass. The size of the black dot is about $1.5 \mathrm{~mm}$ in diameter. 
To characterize the electrical properties of the formed black clouds, we continued with the same sample and made new carbon electrodes (i.e. two black squares in Figure A 14a). The DC voltages were applied from 0 to $-3.0 \mathrm{kV}$. As shown in Figure A 14b, the former black spots changed of shape and was repulsed by the negative electrode and attracted by the positive one (circled in red in Figure A 14b-e). It is worth noting that at high voltage, smell of $\mathrm{O}_{3}$ was detected during the experiment.
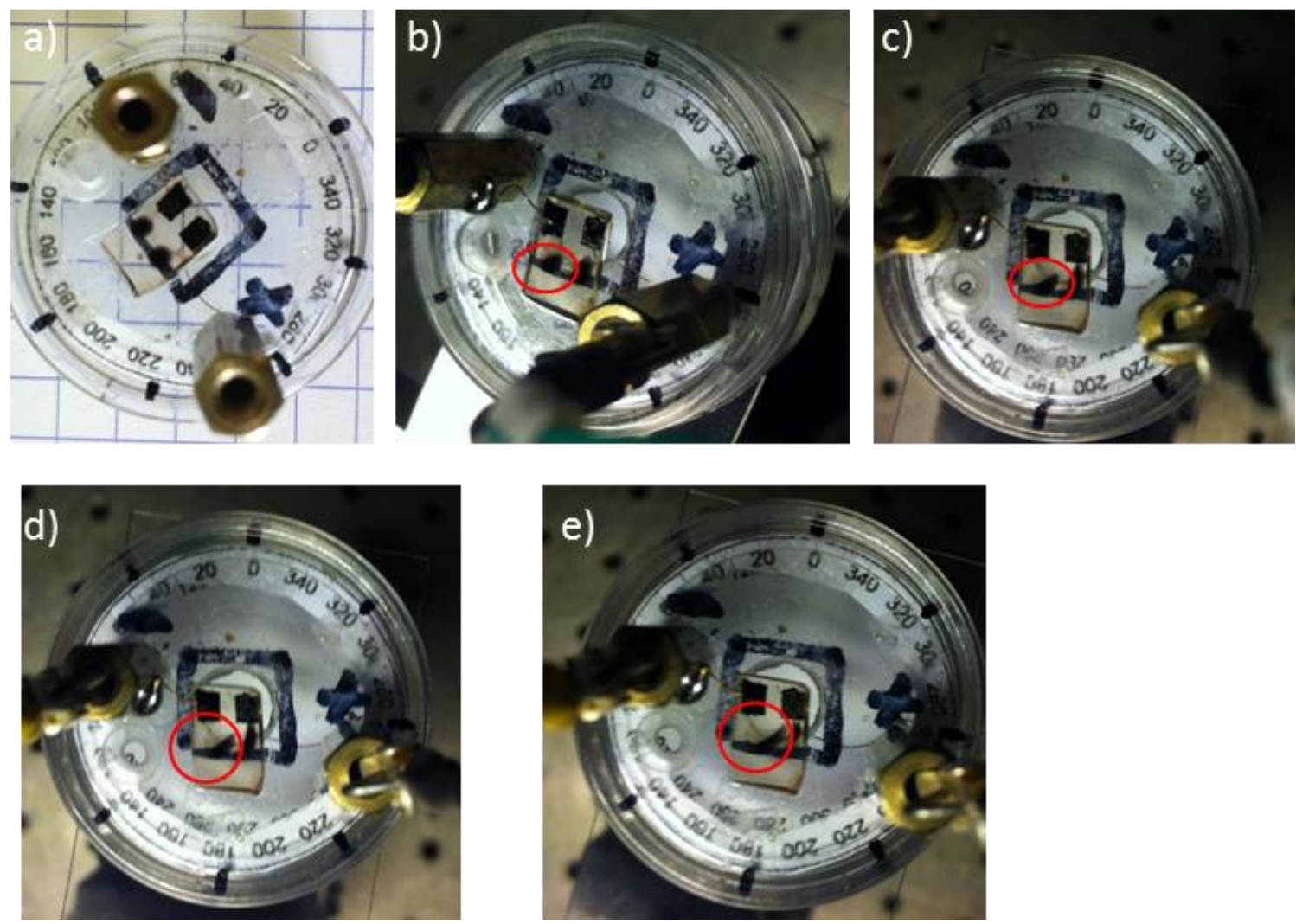

Figure A 14 Photos taken in the course of the experiment a) at the beginning, b) at the end of $-1.5 \mathrm{kV}$ and at the beginning of $-2.0 \mathrm{kV}, \mathrm{c}$ ) at the end of $-2.0 \mathrm{kV}, \mathrm{d}$ ) at the end of $-2.5 \mathrm{kV}$, and e) at the end of $-3.0 \mathrm{kV}$ (NB: the sign of - in the picture stands for negative electrode and + defines the positive electrode). The changes of the former black dots were circled in red. Glass composition: $32.5 \mathrm{Li}_{2} \mathrm{O}-27.5 \mathrm{Nb}_{2} \mathrm{O}_{5}-40 \mathrm{SiO}_{2}, \mathrm{~mol} \%$.

To go a step further, we permuted the positive and negative electrodes as shown in Figure A 15. Voltages were applied from 0 to $-3.0 \mathrm{kV}$. The former black spots changed the shape which was towards the positive electrode and avoid the negative electrode (marked by red circles in Figure A 15), similarly as the results got in Figure A 14 .

A new black spot was observed at the negative electrode (marked by green circles in Figure $A$ 15), which is in agreement with the results observed in Figure $A$ 13. Smell of $\mathrm{O}_{3}$ was also detected, especially from the point at the end of $1.5 \mathrm{kV}$ and at the beginning of $2.0 \mathrm{kV}$. We observed similar results changing the LNS glass composition (i.e. $33 \mathrm{Li}_{2} \mathrm{O}-33 \mathrm{Nb}_{2} \mathrm{O}_{5}-34 \mathrm{SiO}_{2}, \mathrm{~mol} \%$ ). 

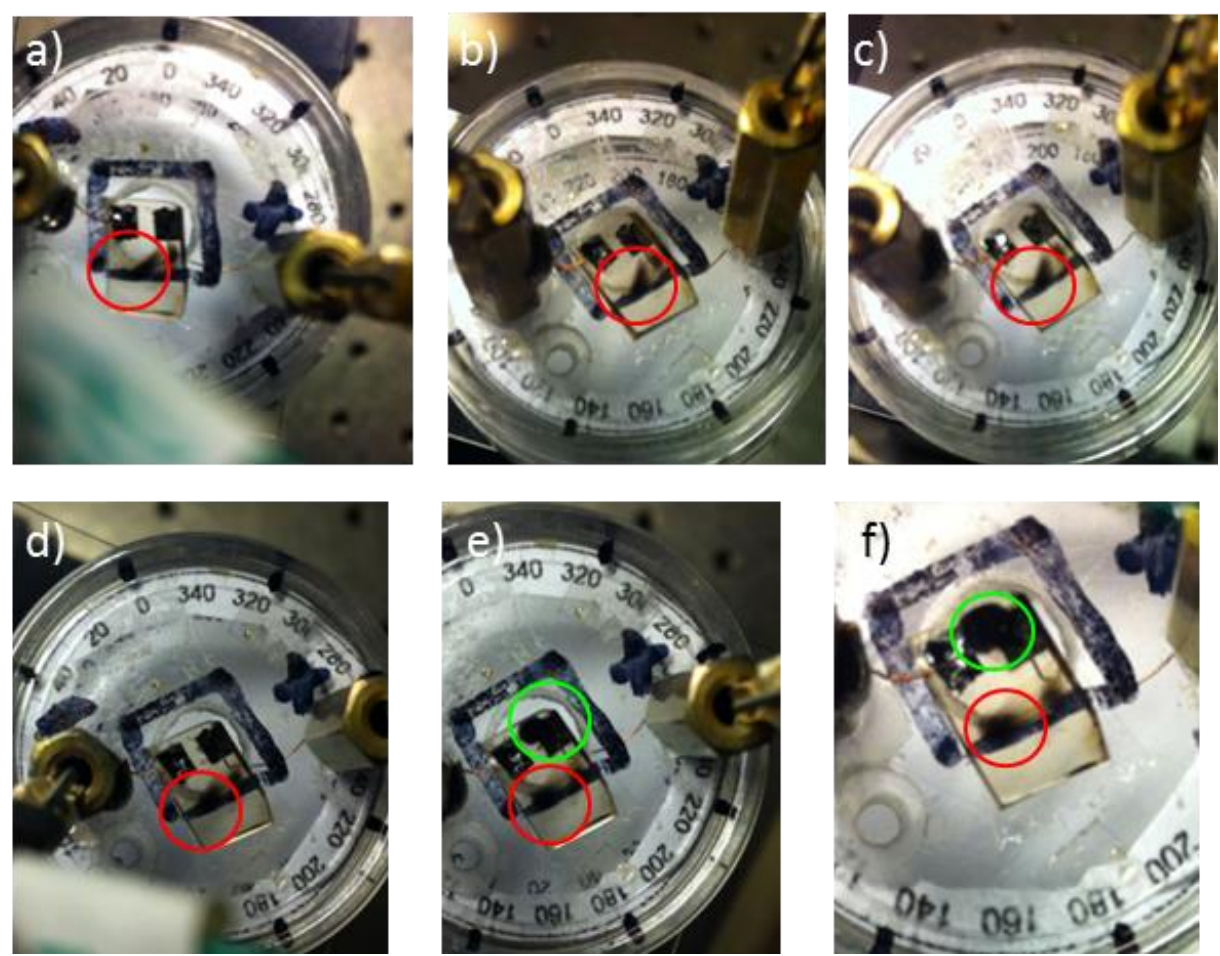

Figure A 15 Photos in the course of the experiment a) at the beginning, b) at $2415 \mathrm{~s}(-0.5 \mathrm{kV}), \mathrm{c})$ at $3795 \mathrm{~s}(-1.0$ $\mathrm{kV}), \mathrm{d})$ at $6078 \mathrm{~s}(-1.5 \mathrm{kV}), \mathrm{e})$ at $6775 \mathrm{~s}(-2.0 \mathrm{kV})$, and f) at $6871 \mathrm{~s}(-2.5 \mathrm{kV})$ (NB: the sign of + stands for negative electrode and - for the positive electrode). Red circles marked the change of the former black dots and green circles marked the newly formed black dot. Glass composition: $32.5 \mathrm{Li}_{2} \mathrm{O}-27.5 \mathrm{Nb}_{2} \mathrm{O}_{5}-40 \mathrm{SiO}_{2}, \mathrm{~mol} \%$.

\section{A 3.2 UV-Vis attenuation measurement}

To get more information on the black clouds induced by DC electric field, UV-Vis attenuation spectrum of the samples were performed. As shown in Figure A 16, comparing with the LNS glass, there is a broad peak $(\sim 473 \mathrm{~nm})$ in the black part after DC field treatment.

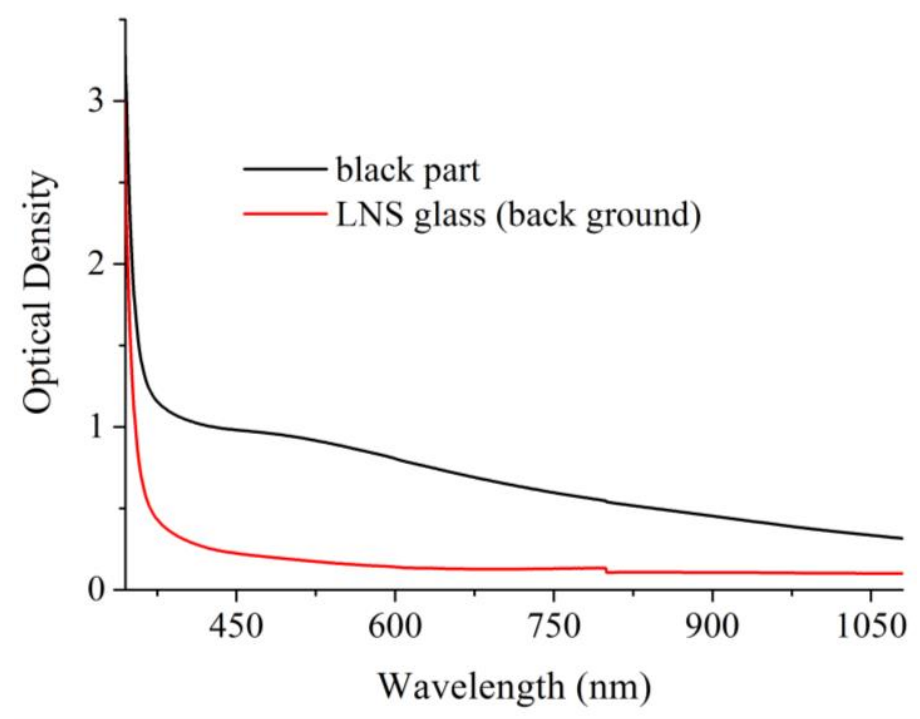

Figure A 16 UV-Vis attenuation spectrums of black spots induced by DC electric field (black curve) and LNS glass (red curve) $\left(32.5 \mathrm{Li}_{2} \mathrm{O}-27.5 \mathrm{Nb}_{2} \mathrm{O}_{5}-40 \mathrm{SiO}_{2}, \mathrm{~mol} \%\right.$, red curve). 


\section{A 3.3 SEM\&EDS}

Then SEM was conducted to check the morphology of the DC field-induced black clouds in LNS glass. As shown in Figure A 17, $\mu \mathrm{m}$-scale dots are obtained in the black part. To compare, not such structure was detected in the LNS glass before the experiment. This indicates that, after the DC field treatment, there is some modifications in the LNS glass.

a)

\section{$2 \mu \mathrm{m}$}

b)

$2 \mu \mathrm{m}$

Figure A 17 SEM images of a) black part induced by DC electric field and b) LNS glass ( $32.5 \mathrm{Li}_{2} \mathrm{O}-27.5 \mathrm{Nb}_{2} \mathrm{O}_{5}-$ $\left.40 \mathrm{SiO}_{2}, \mathrm{~mol} \%\right)$.

To go a step further, EDS was performed to check whether there is some chemical changes after DC field treatment in LNS glass. As shown in Figure A 18, the EDS results show that the carbon peak is higher in the black clouds than out of it. To check whether these black parts are because of carbon tip used for applying electric field, in the following part we applied voltage to LNS glass directly without carbon electrodes.
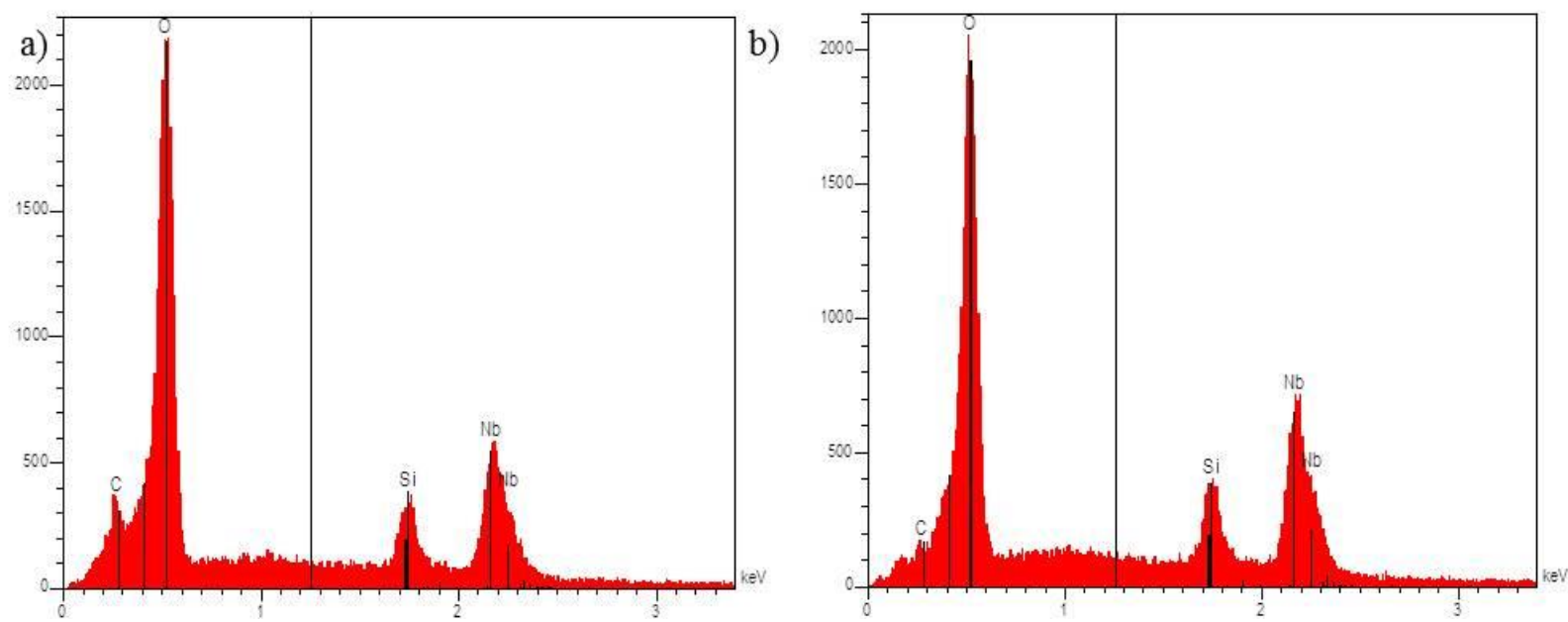

Figure A 18 EDS of a) black part induced by DC electric field (black curve) and b) LNS glass $\left(32.5 \mathrm{Li}_{2} \mathrm{O}-27.5 \mathrm{Nb}_{2} \mathrm{O}_{5}-\right.$ $\left.40 \mathrm{SiO}_{2}, \mathrm{~mol} \%\right)$.

As shown in Figure A 19, we applied the DC voltage directly on LNS glass without carbon electrode. Black clouds are also observed in LNS glass close to the negative electrode side. These 
black clouds expanded on time (marked with green circles in Figure A 19b-d). So even without carbon electrode, the DC field can also induce black clouds in LNS glass.
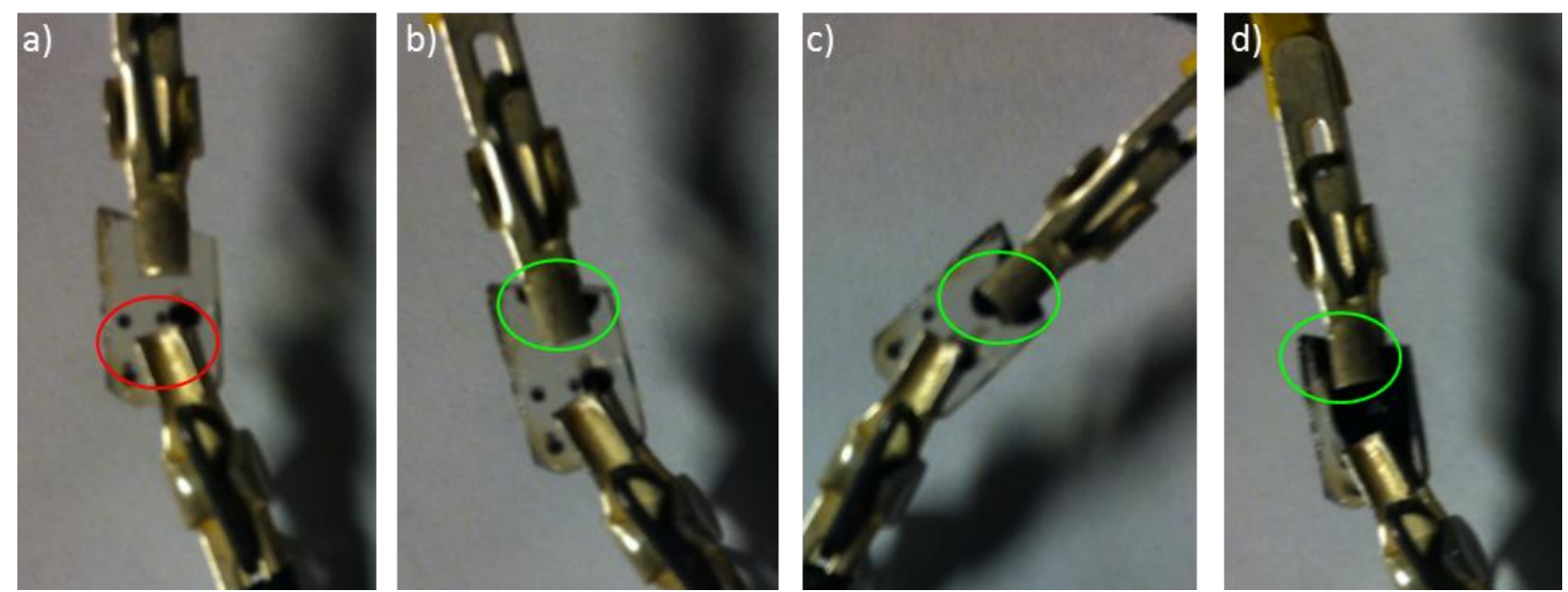

Figure A 19 Photos of LNS glass $\left(33 \mathrm{Li}_{2} \mathrm{O}-33 \mathrm{Nb}_{2} \mathrm{O}_{5}-34 \mathrm{SiO}_{2}, \mathrm{~mol} \%\right)$ in the course of the applied voltage without carbon electrode a) at the beginning (the black parts marked with red circle are from the former experiment), b) at $145 \mathrm{~s}, \mathrm{c}$ ) at $225 \mathrm{~s}$, and d) at $994 \mathrm{~s}$ of $-3.0 \mathrm{kV}$ (the new formed black parts are marked with green circles)

\section{A 3.4 XPS measurement}

To get more chemical information of the DC electric field-induced black clouds in LNS glass, XPS experiment was performed on the LNS glass (as the reference), the black cloud formed with and without carbon electrodes. $\mathrm{O}(531 \mathrm{eV})$ was used to find the sample surface (the maximum) to locate the sample. Some binding energy (BE) values of elements are shown in Table A 23.

Table A 23 Binding energy values of elements in common chemical bonds

\begin{tabular}{|c|c|}
\hline $\mathrm{C}_{1 \mathrm{~s}}$ & $\begin{array}{l}\mathrm{BE}_{\mathrm{C}-\mathrm{C}}=284.8 \mathrm{eV}, \mathrm{BE}_{\mathrm{C}-\mathrm{C}}=284.3 \mathrm{eV} \\
\mathrm{BE}_{\mathrm{C}-\mathrm{O}}=285.8 \pm 0.2 \mathrm{eV} \\
\mathrm{BE}_{\text {metal carbonates }}=289.3 \pm 0.6 \mathrm{eV}\end{array}$ \\
\hline $\mathrm{O}_{1 \mathrm{~s}}$ & $\begin{array}{l}\mathrm{BE}_{\text {carbonates }}=531.3 \pm 0.3 \mathrm{eV} \\
\mathrm{BE}_{\mathrm{Me}-\mathrm{OH}}=531.3 \\
\mathrm{BENb}_{2} \mathrm{O}_{5}=530.4 \pm 0.5 \mathrm{eV}\end{array}$ \\
\hline $\mathrm{Li}_{1 \mathrm{~s}}$ & $\begin{array}{l}\mathrm{BELi}_{2} \mathrm{CO}_{3}=55.2 \pm 0.1 \mathrm{eV} \\
\mathrm{BELiNbO}_{3}=54.8 \pm 54.8 \mathrm{eV}\end{array}$ \\
\hline $\mathrm{Si}_{2 \mathrm{p}}$ & $\begin{array}{l}\mathrm{BESiO}_{2}=103.5 \pm 0.3 \mathrm{eV} \\
\mathrm{BE}_{\text {silicates }} \text { range from } 101.6 \text { to } 103.8 \mathrm{eV}\end{array}$ \\
\hline $\mathrm{Nb}_{3 \mathrm{~d}}$ & $\begin{array}{l}\mathrm{BENbO}_{2}=206.2 \pm 0.1 \mathrm{eV} \\
\mathrm{BENb}_{2} \mathrm{O}_{5}=207.4 \pm 0.8 \mathrm{eV}\end{array}$ \\
\hline
\end{tabular}




\section{A 3.4.1 LNS glass}

The LNS glass surface was etched by Ar ions in order to remove the pollution layer ( 3 etch levels of $5 \mathrm{~s}$ each). As shown in Figure A 20, high-resolution spectra were recorded from of LNS glass before (red curves) and after Ar ions etching (green curves). It can be seen that

1) $\quad \mathrm{Nb}_{3 \mathrm{~d} 5 / 2} \mathrm{BE}$ and shape unchanged (small sub-oxyde contribution at lower BE induced by ion bombardment),

2) $\mathrm{Si}_{2 p}$ doesn't change the $\mathrm{BE}$ or its shape after etching,

3) $\quad \mathrm{Li}_{1 \mathrm{~s}}$ intensity decreases after etching.
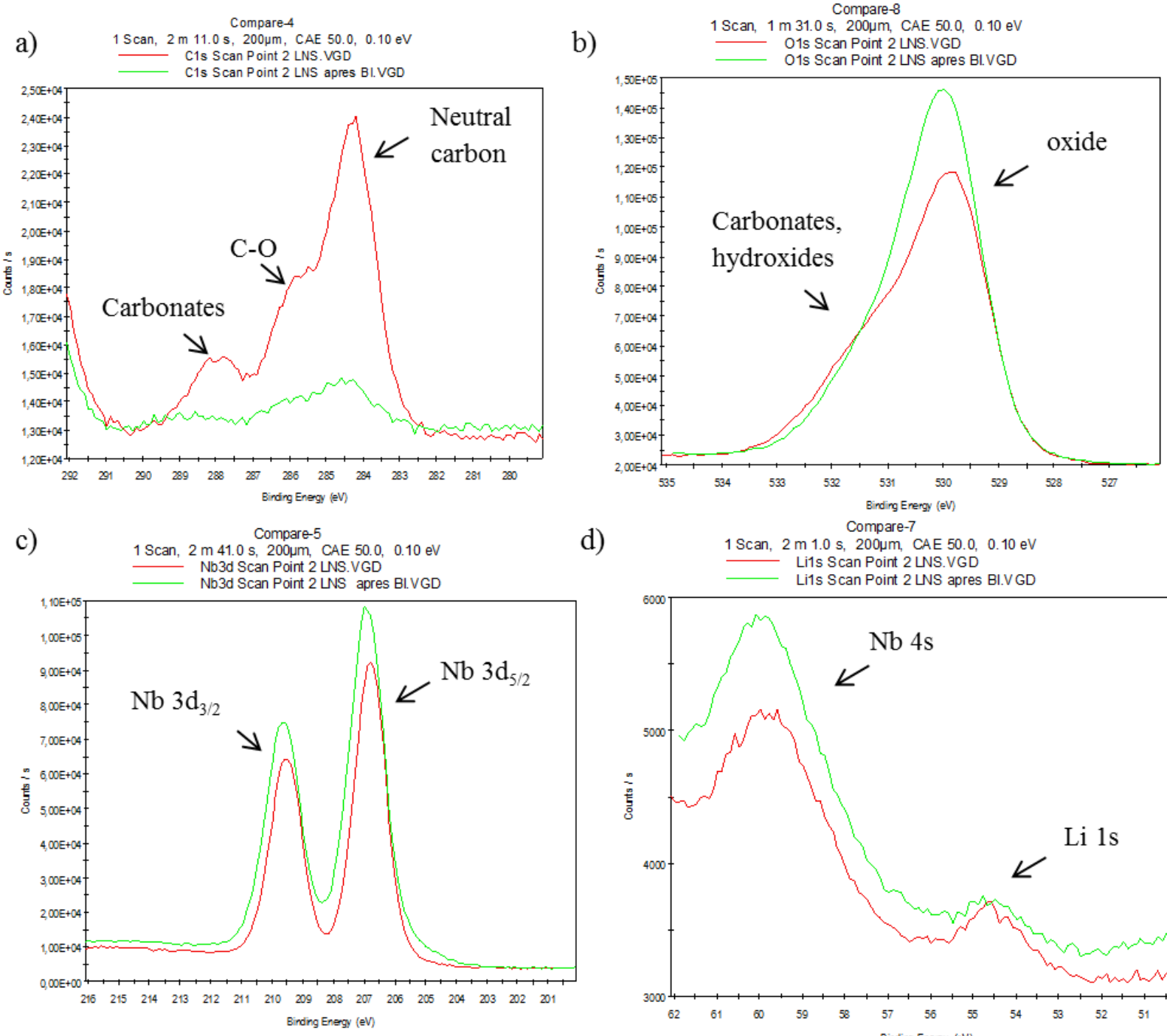

d)

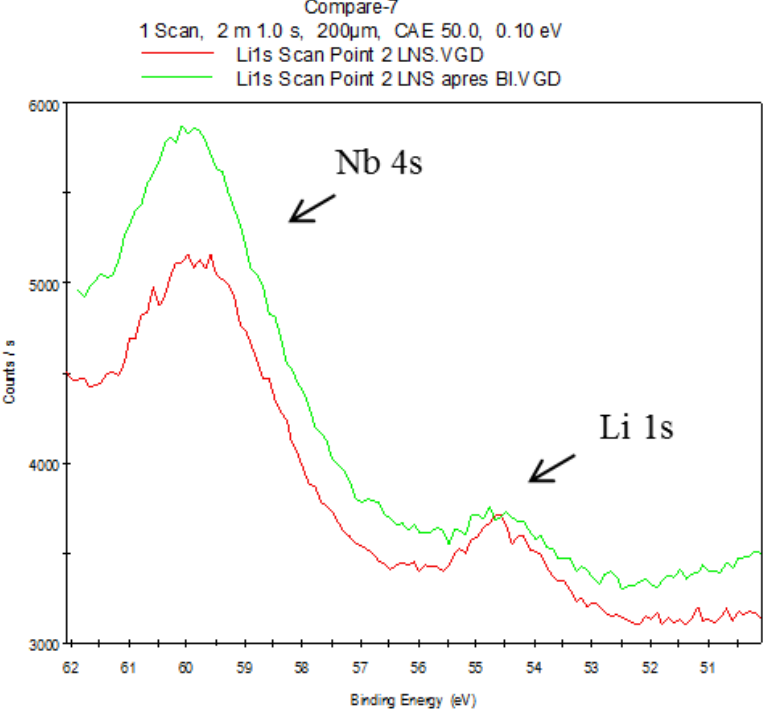

Figure A 20 High-resolution XPS spectra of LNS glass $\left(32.5 \mathrm{Li}_{2} \mathrm{O}-27.5 \mathrm{Nb}_{2} \mathrm{O}_{5}-40 \mathrm{SiO}_{2}\right.$, mol\%) before (red curves) and after (green curves) Ar ion etching 
The atomic percent of $\mathrm{Nb}, \mathrm{Si}$, and $\mathrm{Li}$ of LNS glass before and after Ar ion etching are shown in Table A 24 and Table A 25, respectively. Before Ar ions etching, the ratio between $\mathrm{Nb} / \mathrm{Si}$ is around 1.55, which is not far from the original one 1.38 (i.e. $32.5 \mathrm{Li}_{2} \mathrm{O}-27.5 \mathrm{Nb}_{2} \mathrm{O}_{5}-40 \mathrm{SiO}_{2}, \mathrm{~mol} \%$ ). After Ar ions etching, it increases to 1.69. The Li content decreases from $21 \%$ to $14 \%$. It indicates that the light elements ( $\mathrm{Li}$ or $\mathrm{Si}$ ) are easier to be etched than the heavy one ( $\mathrm{Nb}$ ).

Table A 24 Atomic percent of Nb, Si, and Li of LNS glass before Ar ion etching

\begin{tabular}{ccccccc}
\hline Name & Peak BE & FWHM eV & Area (P) CPS.eV & Atomic\% & Peak Type & SF \\
\hline $\mathrm{Nb}_{3 \mathrm{~d}}$ & 206.58 & 1.22 & 193356.71 & 48 & Standard & 8.21 \\
$\mathrm{Si}_{2 \mathrm{p}}$ & 101.42 & 1.68 & 13097.14 & 31 & Standard & 0.817 \\
$\mathrm{Li}_{1 \mathrm{~s}}$ & 54.38 & 1.47 & 646.91 & 21 & Fitted & 0.057 \\
$\mathrm{Nb}_{4 \mathrm{~s}}$ & 59.35 & 2.29 & 2824.77 & 0.00 & Fitted & 0.402
\end{tabular}

This fit was performed to establish a good baseline for the $\mathrm{Li}_{1 \mathrm{~s}}$ peak. The atomic percentages were calculated from the high-resolution spectra (oxygen was not included). A ratio of these percentages gives the ratio Li:Si:Nb only.

Table A 25 Atomic percent of Nb, Si, and Li of LNS glass after Ar ion etching

\begin{tabular}{lcccccc}
\hline Name & Peak BE & FWHM eV & Area (P) CPS.eV & Atomic\% & Peak Type & SF \\
\hline $\mathrm{Nb}_{3 \mathrm{~d}}$ & 206.9 & 1.36 & 256469.85 & 54 & Standard & 8.21 \\
$\mathrm{Si}_{2 \mathrm{p}}$ & 101.72 & 1.67 & 15985.92 & 32 & Standard & 0.817 \\
$\mathrm{Li}_{1 \mathrm{~s}}$ & 54.64 & 1.58 & 473.54 & 14 & Fitted & 0.057 \\
$\mathrm{Nb}_{4 \mathrm{~s}}$ & 59.61 & 2.55 & 3861.26 & 0.00 & Fitted & 0.402
\end{tabular}

This fit was performed to establish a good baseline for the $\mathrm{Li}_{1 \mathrm{~s}}$ peak. The atomic percentages were calculated from the high-resolution spectra (oxygen was not included). A ratio of these percentages gives the ratio Li:Si:Nb only.

\section{A 3.4.2 DC electric field treated LNS glass with carbon electrodes}

Figure A 21 displays the high-resolution spectra of DC electric field treated LNS glass with carbon electrodes before (red curves) and after Ar ions etching (green curves). It displays that 1 ) Nb $3_{d 5 / 2}$ $\mathrm{BE}$ and shape unchanged (small sub-oxyde contribution at lower BE induced by ion bombardment), 2) $\mathrm{Si}_{2 p}$ doesn't change the $\mathrm{BE}$ or its shape, and 3) $\mathrm{Li}_{1 s}$ intensity does not change (Figure A $21 \mathrm{~d}$ ), which is different than the LNS glass one (Figure A 20d).

The atomic percent of $\mathrm{Nb}, \mathrm{Si}$, and $\mathrm{Li}$ of $\mathrm{DC}$ electric field treated LNS glass with carbon electrodes before and after Ar ion etching are shown in Table A 26 and Table A 27, respectively. The content of $\mathrm{Li}$ is $50 \%$, which is larger than the LNS glass (21\% in Table A 24). Even after Ar ion 
etching, the amount of $\mathrm{Li}$ is still as high as $43 \%$. This result indicates that the content of $\mathrm{Li}$ has increased after the DC electric field treatment.
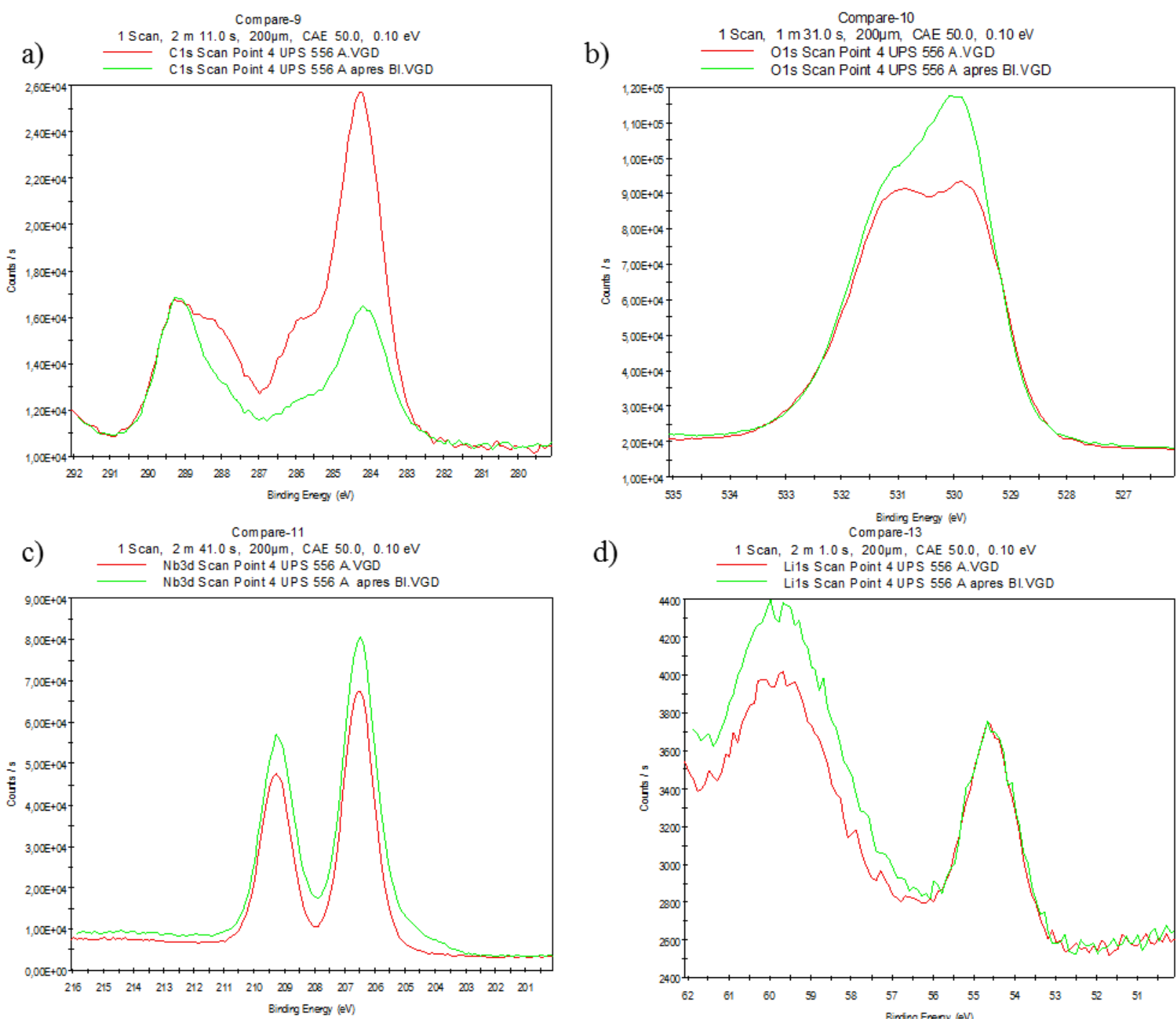

d)

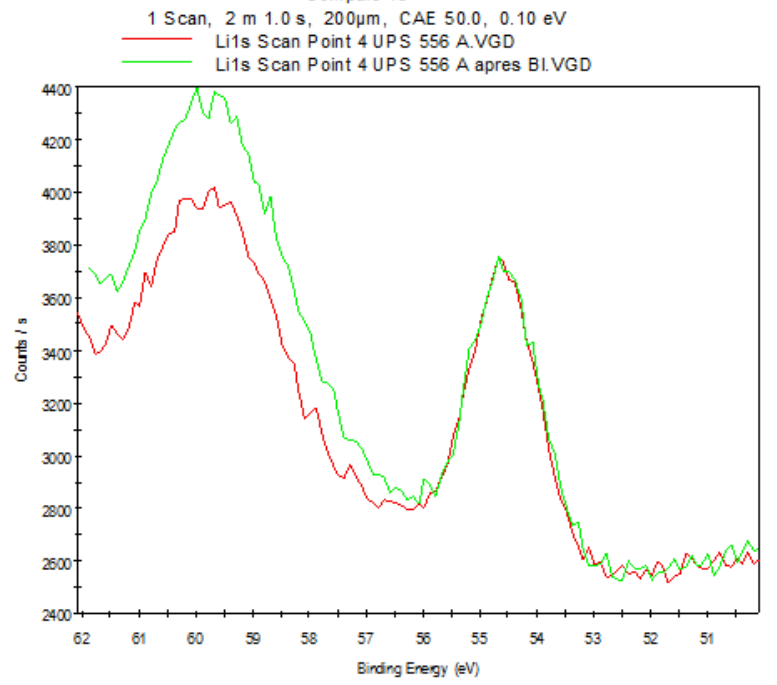

Figure A 21 High-resolution XPS spectra of DC electric field treated LNS glass with carbon electrodes before (red curves) and after (green curves) Ar ion etching

Table A 26 Atomic percent of $\mathrm{Nb}, \mathrm{Si}$, and $\mathrm{Li}$ of DC electric field treated LNS glass with carbon electrodes before Ar ion etching

\begin{tabular}{lllllll}
\hline Name & Peak BE & FWHM eV & Area (P) CPS.eV & Atomic\% & Peak Type & SF \\
\hline $\mathrm{Nb}_{3 \mathrm{~d}}$ & 206.52 & 1.22 & 140295.4 & 34 & Standard & 8.21 \\
$\mathrm{Si}_{2 \mathrm{p}}$ & 101.31 & 1.57 & 7035.1 & 16 & Standard & 0.817 \\
$\mathrm{Li}_{1 \mathrm{~s}}$ & 54.53 & 1.26 & 1557.22 & 50 & Fitted & 0.057 \\
$\mathrm{Nb}_{4 \mathrm{~s}}$ & 59.41 & 2.14 & 2048.46 & 0.00 & Fitted & 0.402
\end{tabular}

This fit was performed to establish a good baseline for the $\mathrm{Li}_{1 \mathrm{~s}}$ peak. The atomic percentages were calculated from the high-resolution spectra (oxygen was not included). A ratio of these percentages gives the ratio Li:Si:Nb only. 
Table A 27 Atomic percent of Nb, Si, and Li of DC electric field treated LNS glass with carbon electrodes after Ar ion etching

\begin{tabular}{ccclclc}
\hline Name & Peak BE & FWHM eV & Area (P) CPS.eV & Atomic\% & Peak Type & SF \\
\hline $\mathrm{Nb}_{3 \mathrm{~d}}$ & 206.69 & 1.31 & 188098.4 & 39 & Standard & 8.21 \\
$\mathrm{Si}_{2 \mathrm{p}}$ & 101.47 & 1.6 & 9197 & 18 & Standard & 0.817 \\
$\mathrm{Li}_{1 \mathrm{~s}}$ & 54.69 & 1.27 & 1522.44 & 43 & Fitted & 0.057 \\
$\mathrm{Nb}_{4 \mathrm{~s}}$ & 59.5 & 2.31 & 2685.44 & 0.00 & Fitted & 0.402
\end{tabular}

This fit was performed to establish a good baseline for the $\mathrm{Li}_{1 \mathrm{~s}}$ peak. The atomic percentages were calculated from the high-resolution spectra (oxygen was not included). A ratio of these percentages gives the ratio Li:Si:Nb only.

Considering that these black clouds are formed from the LNS glass surface, expanded to the inside of the glass (Figure A 13c), a depth analysis (shown in Figure A 22 and Figure A 23) was performed on the black cloud.

a)

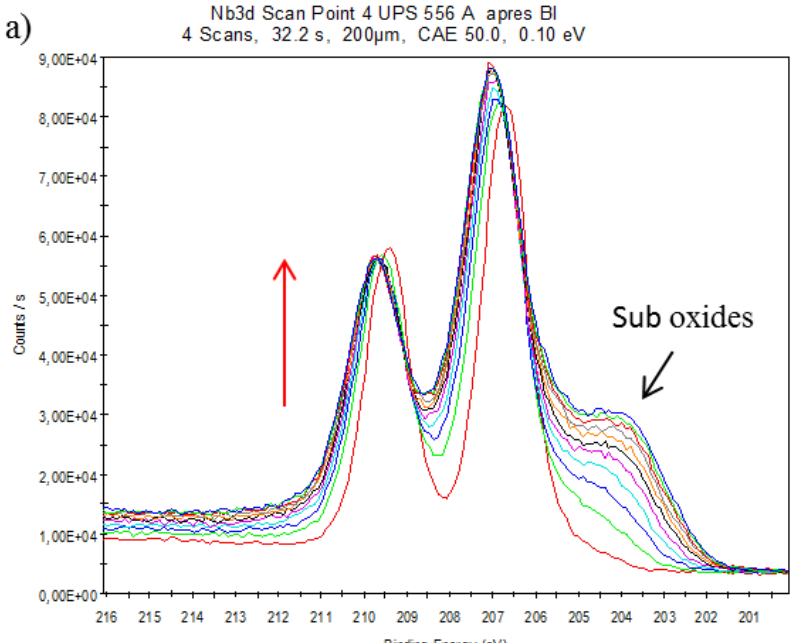

c)

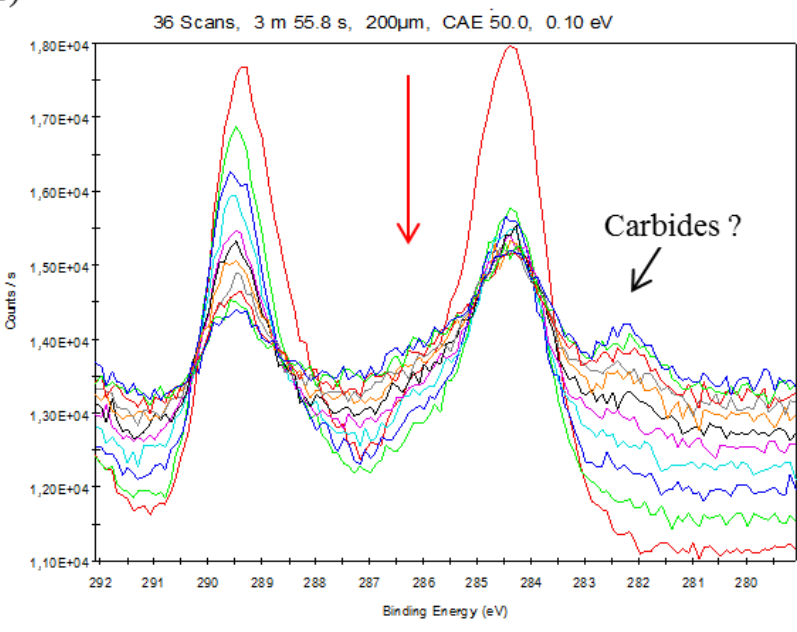

b)

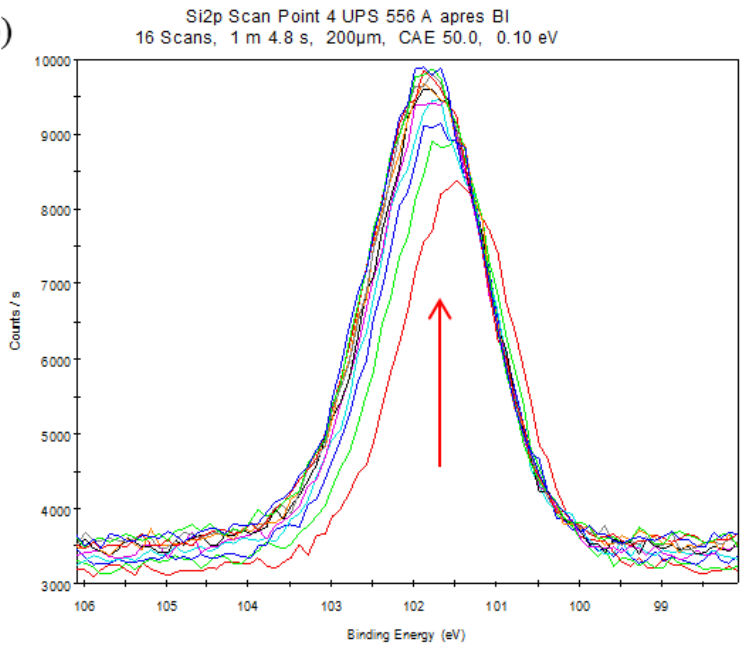

d)

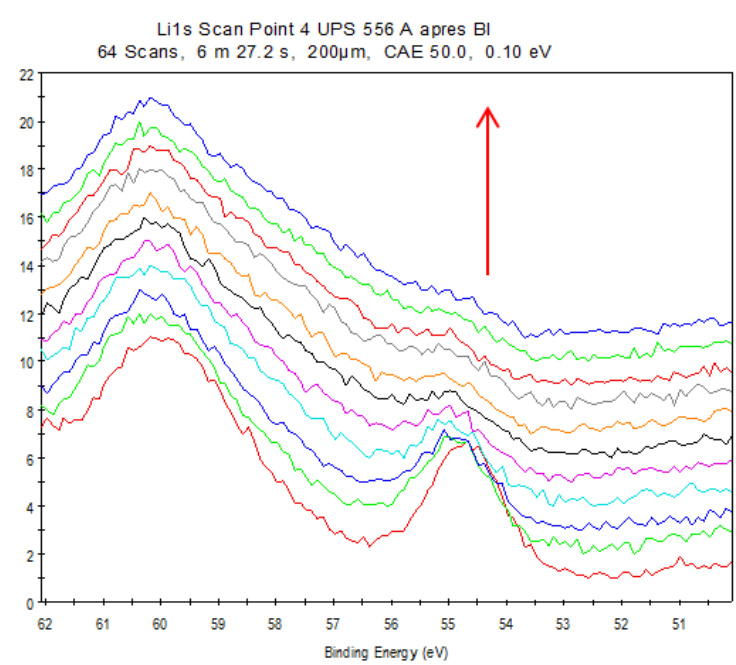

Figure A 22 Depth analysis: high-resolution XPS spectra of DC electric field treated LNS glass with carbon electrodes (the red arrows indicate the direction from surface to the inside). Ar ion bombardment, ion Gun conditions: $\mathrm{E}=200 \mathrm{eV}, \mathrm{I}=$ low Raster size $=1 \mathrm{~mm}$ (the etched area is a rectangle of size $1 \mathrm{~mm} \times 2 \mathrm{~mm}$ ) $20 \mathrm{~s}$ delay after 10 etch levels of $20 \mathrm{~s}$ each. 
The shoulder at about $203.5 \mathrm{eV}$ on the $\mathrm{Nb}_{3 \mathrm{~d}}$ spectrum is due to the sub oxides induced by the ion bombardment (Figure A 22a). Figure A 22c displays that carbon is still present deeper beneath the sample surface; a small peak at around $282-283 \mathrm{eV}$ is appearing, which is usually assigned to carbides. As shown in Figure A 22d, Li concentration is decreasing rapidly with depth (along the red arrow direction). Higher $\mathrm{Li}$ concentration can be correlated with the presence of the carbonates on both $\mathrm{C}_{1 \mathrm{~s}}$ (Figure $\mathrm{A} 22 \mathrm{c}$ ) and $\mathrm{O}_{1 \mathrm{~s}}$ spectra (Figure $\mathrm{A} 23$ ).

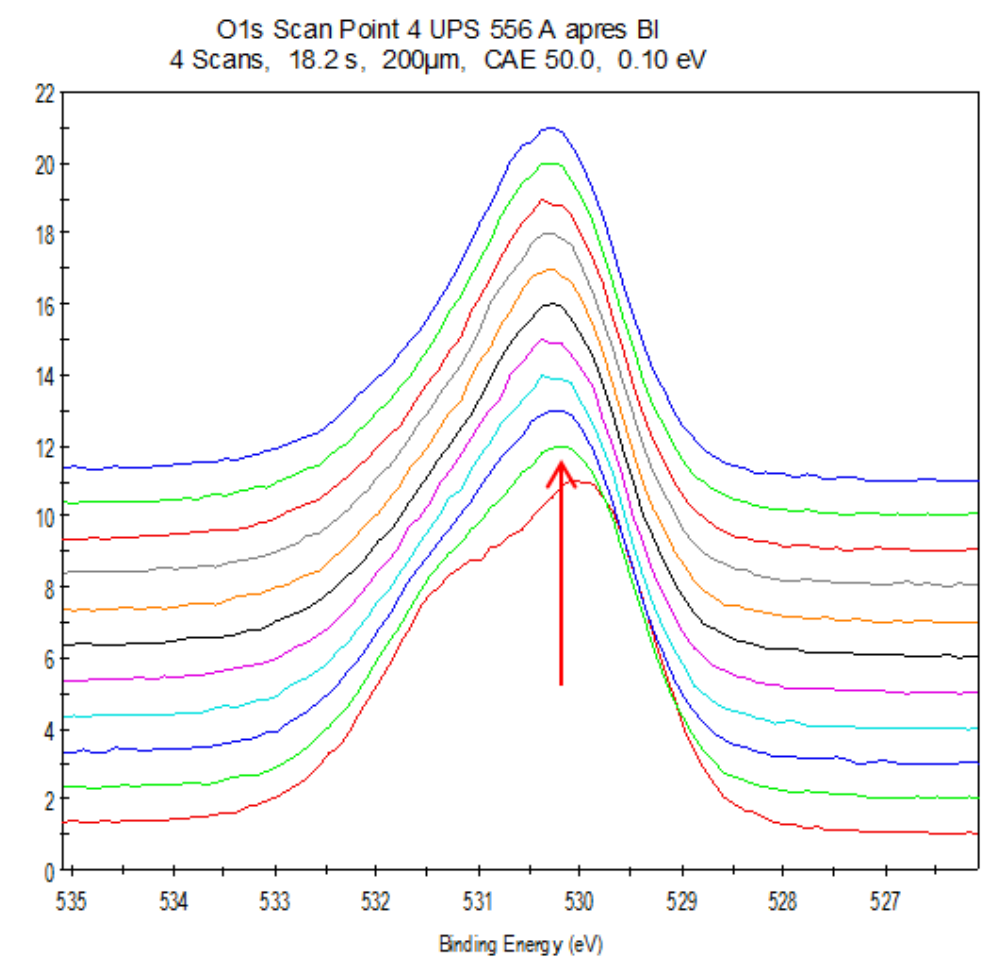

Figure A 23 Depth analysis of $\mathrm{O}_{1 s}$ : high-resolution XPS spectra of DC electric field treated LNS glass with carbon electrodes (the red arrow indicates the direction from surface to the inside). Ar ion bombardment, ion gun conditions: $\mathrm{E}=200 \mathrm{eV}, \mathrm{I}=$ low Raster size $=1 \mathrm{~mm}$ (the etched area is a rectangle of size $1 \mathrm{~mm} \times 2 \mathrm{~mm}$ ) $20 \mathrm{~s}$ delay after etch 10 etch levels of $20 \mathrm{~s}$ each

\section{A 3.4.3 DC electric field treated LNS glass without carbon electrodes}

Figure A 24 displays the high-resolution spectra were measured of DC electric field treated LNS glass without carbon electrodes before (red curves) and after Ar ions etching (green curves). We can see that

1) $\quad \mathrm{Nb}_{3 \mathrm{~d} 5 / 2} \mathrm{BE}$ and shape unchanged (small sub-oxyde contribution at lower $\mathrm{BE}$ induced by ion bombardment),

2) $\mathrm{Si}_{2 p}$ doesn't change the $\mathrm{BE}$ or its shape, and d) the component at higher $\mathrm{BE}$ in the $\mathrm{O}_{1 \mathrm{~s}}$ spectrum, at $531.2 \mathrm{eV}$ (corresponding to carbonate) has become dominant. 
The atomic percent of $\mathrm{Nb}, \mathrm{Si}$, and $\mathrm{Li}$ of $\mathrm{DC}$ electric field treated LNS glass without carbon electrodes before and after Ar ions etching are shown in Table A 28 and Table A 29, respectively. It can be seen that the content of $\mathrm{Li}$ is as high as $78 \%$, which is larger than the DC electric field treated LNS glass with carbon electrodes (50\% in Table A 26). Even after Ar ion etching, the amount of $\mathrm{Li}$ is still as high as $69 \%$.

A line scanning mode analysis of DC electric field treated LNS glass without carbon electrodes was performed from the negative electrode to the positive one. As shown in Figure A 25, Li concentration is higher at the negative electrode (distance set as 0 ) and is decreasing towards the other end (positive pole). It indicates that the high Li concentrations coincide with the coordinates of dark areas.
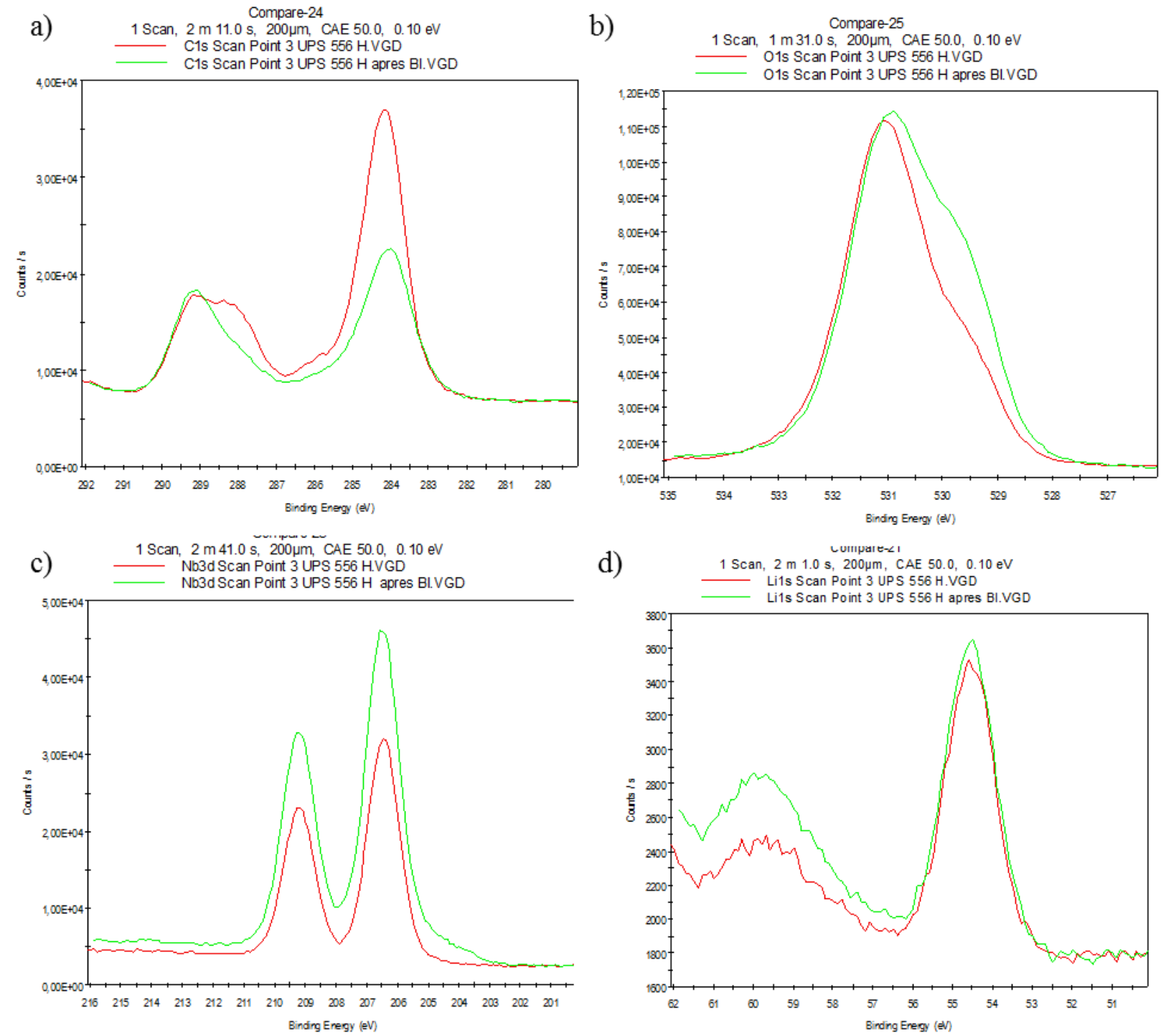

Figure A 24 High-resolution XPS spectra of DC electric field treated LNS glass without carbon electrodes before (red curves) and after (green curves) Ar ion etching 
Table A 28 Atomic percent of Nb, Si, and $\mathrm{Li}$ of DC electric field treated LNS glass without carbon electrodes before Arion etching

\begin{tabular}{lllllll}
\hline Name & Peak BE & FWHM eV & Area (P) CPS.eV & Atomic\% & Peak Type & SF \\
\hline $\mathrm{Nb}_{3 \mathrm{~d}}$ & 206.45 & 1.23 & 64817.16 & 14 & Standard & 8.21 \\
$\mathrm{Si}_{2 \mathrm{p}}$ & 101.26 & 1.61 & 4153.05 & 8 & Standard & 0.817 \\
$\mathrm{Li}_{1 \mathrm{~s}}$ & 54.52 & 1.37 & 2739.54 & 78 & Fitted & 0.057 \\
$\mathrm{Nb}_{4 \mathrm{~s}}$ & 59.35 & 1.98 & 772.1 & 0.00 & Fitted & 0.402
\end{tabular}

This fit was performed to establish a good baseline for the $\mathrm{Li}_{1 \mathrm{~s}}$ peak. The atomic percentages were calculated from the high-resolution spectra (oxygen was not included). A ratio of these percentages gives the ratio Li:Si:Nb only.

Table A 29 Atomic percent of $\mathrm{Nb}, \mathrm{Si}$, and $\mathrm{Li}$ of DC electric field treated LNS glass without carbon electrodes after Ar ion etching

\begin{tabular}{lcccccc}
\hline Name & Peak BE & FWHM eV & Area (P) CPS.eV & Atomic\% & Peak Type & SF \\
\hline $\mathrm{Nb}_{3 \mathrm{~d}}$ & 206.67 & 1.32 & 108059.76 & 19 & Standard & 8.21 \\
$\mathrm{Si}_{2 \mathrm{p}}$ & 101.46 & 1.66 & 6742.64 & 12 & Standard & 0.817 \\
$\mathrm{Li}_{1 \mathrm{~s}}$ & 54.71 & 1.38 & 2869.99 & 69 & Fitted & 0.057 \\
$\mathrm{Nb}_{4 \mathrm{~s}}$ & 59.55 & 2.04 & 1174.29 & 0.00 & Fitted & 0.402 \\
\hline
\end{tabular}

This fit was performed to establish a good baseline for the $\mathrm{Li}_{1 s}$ peak. The atomic percentages were calculated from the high-resolution spectra (oxygen was not included). A ratio of these percentages gives the ratio Li:Si:Nb only.

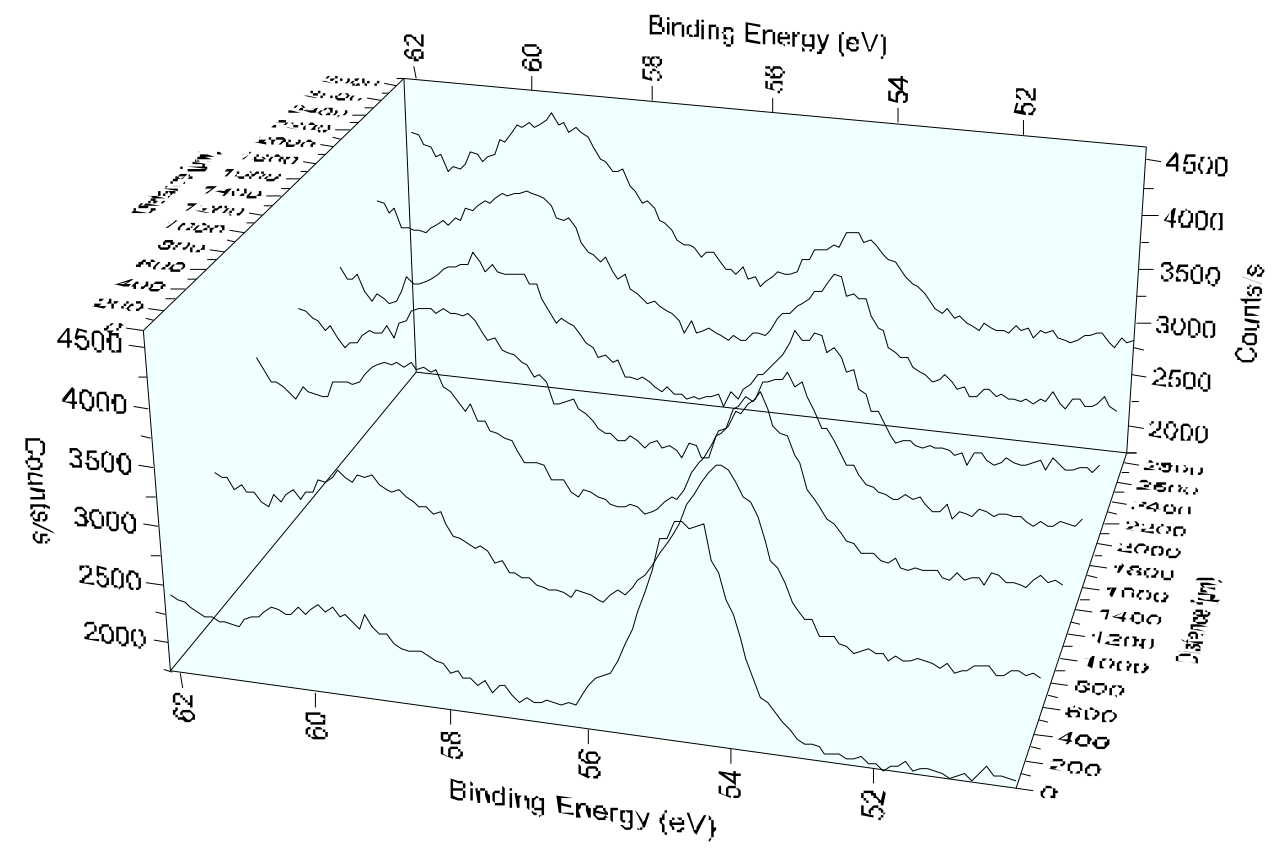

Figure A 25 Line scanning mode analysis: high-resolution XPS spectra of DC electric field treated LNS glass without carbon electrodes (from the negative electrode to the positive one) 


\section{A 4 Conclusion}

After DC electric field treatment, black cloud appears in LNS glass and we found a larger Li concentration than glass matrix. There is a chemical migration of Li:

1) It is higher at the negative electrode and is decreasing towards the other end (positive pole);

2) The Li contents is high in the experiment without carbon electrodes than the one with carbon electrodes;

3) There is a higher Li concentration at the surface at the position of negative electrode and a decrease with the depth;

4) Higher Li concentration can be correlated with the presence of carbonates on both $\mathrm{C}_{1 \mathrm{~s}}$ and $\mathrm{O}_{1 \mathrm{~s}}$ spectra.

Because a chemical reaction occurs in LNS glass in so short a time (few seconds after DC electric field application i.e. $3 \mathrm{kV}$ ), the effect of a DC field under irradiation in the LNS glass cannot be recorded. However, the black clouds induced by DC electric field, can be removed by the application of an opposite electric field (repulsed by the negative electrode and attracted by the positive one). This property maybe useful for electric switch or display applications. 
XXI. Point group symmetry character tables

Group $C_{3 v}$

\begin{tabular}{|c|c|c|c|c|c|}
\hline $\mathrm{C}_{3 \mathrm{v}}$ & $E$ & $2 C_{3}(z)$ & $3 \sigma_{v}$ & linear functions, rotations & $\begin{array}{l}\text { quadratic } \\
\text { functions }\end{array}$ \\
\hline$A_{1}$ & 1 & 1 & 1 & $\mathrm{~T}_{2}$ & $x^{2}+y^{2} ; z^{2}$ \\
\hline$A_{2}$ & 1 & 1 & -1 & $\mathrm{R}_{\mathrm{z}}$ & - \\
\hline $\mathrm{E}$ & 2 & -1 & 0 & $\left(T_{x}, T_{y}\right) ;\left(R_{x}, R_{y}\right)$ & $\left(x^{2}-y^{2}, x y\right) ;(x z, y z)$ \\
\hline
\end{tabular}

Group $\mathrm{O}_{h}$

\begin{tabular}{|c|c|c|c|c|c|c|c|c|c|c|c|c|}
\hline $\mathrm{O}_{\mathrm{h}}$ & $E$ & $8 C_{3}$ & $3 C_{2}=\left(C_{4}\right)^{2}$ & $6 C_{4}$ & $6 C_{2}$ & $\mathrm{i}$ & $8 S_{6}$ & $3 \sigma_{h}$ & $6 S_{4}$ & $6 \sigma_{d}$ & $\begin{array}{l}\text { linear } \\
\text { functions, } \\
\text { rotations }\end{array}$ & $\begin{array}{l}\text { quadratic } \\
\text { functions }\end{array}$ \\
\hline$A_{1 g}$ & 1 & 1 & 1 & 1 & 1 & 1 & 1 & 1 & 1 & 1 & - & $x^{2}+y^{2}+z^{2}$ \\
\hline$A_{2 g}$ & 1 & 1 & 1 & -1 & -1 & 1 & 1 & 1 & -1 & -1 & - & - \\
\hline $\mathrm{E}_{\mathrm{g}}$ & 2 & -1 & 2 & 0 & 0 & 2 & -1 & 2 & 0 & 0 & - & $\left(2 z^{2}-x^{2}-y^{2}, x^{2}-y^{2}\right)$ \\
\hline $\mathrm{T}_{1 \mathrm{~g}}$ & 3 & 0 & -1 & 1 & -1 & 3 & 0 & -1 & 1 & -1 & $\left(R_{x}, R_{y}, R_{z}\right)$ & - \\
\hline $\mathrm{T}_{2 \mathrm{~g}}$ & 3 & 0 & -1 & -1 & 1 & 3 & 0 & -1 & -1 & 1 & - & $(x y, x z, y z)$ \\
\hline$A_{1 u}$ & 1 & 1 & 1 & 1 & 1 & -1 & -1 & -1 & -1 & -1 & - & - \\
\hline $\mathrm{A}_{2 \mathrm{u}}$ & 1 & 1 & 1 & -1 & -1 & -1 & -1 & -1 & 1 & 1 & - & - \\
\hline $\mathrm{E}_{\mathrm{u}}$ & 2 & -1 & 2 & 0 & 0 & -2 & 1 & -2 & 0 & 0 & - & - \\
\hline $\mathrm{T}_{1 \mathrm{u}}$ & 3 & 0 & -1 & 1 & -1 & -3 & 0 & 1 & -1 & 1 & $\left(T_{x}, T_{y}, T_{z}\right)$ & - \\
\hline $\mathrm{T}_{2 \mathrm{u}}$ & 3 & 0 & -1 & -1 & 1 & -3 & 0 & 1 & 1 & -1 & - & - \\
\hline
\end{tabular}




\section{Résumé substantiel en Français}

\section{F 1. Introduction}

L'écriture directe par laser femtoseconde (fs) dans les matériaux transparents revêt désormais un intérêt particulier en raison des propriétés optiques nouvelles et des applications prometteuses dans le domaine de l'optique guidée et des systèmes dédiés à l'opto-fluidique [13]. Il s'agit en particulier d'une excellente méthode pour créer des modifications permanentes des propriétés optiques en trois dimensions (3D), avec une résolution spatiale à l'échelle du micron voire de quelques nanomètres $[4,5]$.

Malheureusement pour certaines applications, le verre présente une symétrie d'inversion, en raison d'un désordre aléatoire, qui conduit à une interdiction de génération de seconde harmonique. Cependant, par irradiation avec un laser fs, il est possible d'induire la précipitation de cristaux présentant des propriétés optiques non-linéaires afin de briser la symétrie d'inversion et de permettre ainsi la réalisation de dispositifs basés sur le doublage de fréquence.

Le but de ces travaux de recherche a donc été d'apporter une contribution à l'élaboration et à l'orientation de ces cristaux non-linéaires avec une résolution spatiale de l'ordre du micron. La famille de verres choisie est $\mathrm{Li}_{2} \mathrm{O}-\mathrm{Nb}_{2} \mathrm{O}_{5}-\mathrm{SiO}_{2}$. Nous avons étudié systématiquement la cristallisation à l'intérieur de cette famille de verres et exploré comment maîtriser les structures induites par irradiation (cristallisation, nanostructures) et les propriétés optiques linéaires et non-linéaires correspondantes.

\section{F 2. Section expérimentale}

La première étape a pour objectif d'ajuster la composition chimique afin d'obtenir un verre qui présente une forte sensibilité au laser fs. Dans un second temps, il s'agit de contrôler les paramètres des écritures laser pour l'obtention de nanocristaux avec une répartition spatiale et une taille correctes.

Nous avons ensuite procédé à l'analyse des nanostructures et des textures à l'aide de la microscopie électronique à balayage (MEB), de la diffraction des électrons rétrodiffusés (EBSD) et de la microscopie électronique à transmission. Par ailleurs des études de génération de seconde harmonique (GSH) et EBSD ont été combinés pour déterminer les distributions spatiales et orientationnelles des nano-cristaux ainsi que leurs propriétés optiques non-linéaires. 


\section{F 3. Résultats et discussion}

Ces études ont permis de mettre en évidence trois régimes de modifications des verres $\mathrm{Li}_{2} \mathrm{O}$ $\mathrm{Nb}_{2} \mathrm{O}_{5}-\mathrm{SiO}_{2}$ en fonction de l'énergie de l'impulsion (0,3-2,2 $\mu \mathrm{J} /$ impulsion à $1030 \mathrm{~nm}, 300 \mathrm{fs}, 300$ $\mathrm{kHz}, 5 \mu \mathrm{m} / \mathrm{s}$, profondeur de focalisation $\sim 350 \mu \mathrm{m}$ et $\mathrm{NA}=0,6)$, avec trois seuils comme cela est illustré sur les Figure F 1 et F2.
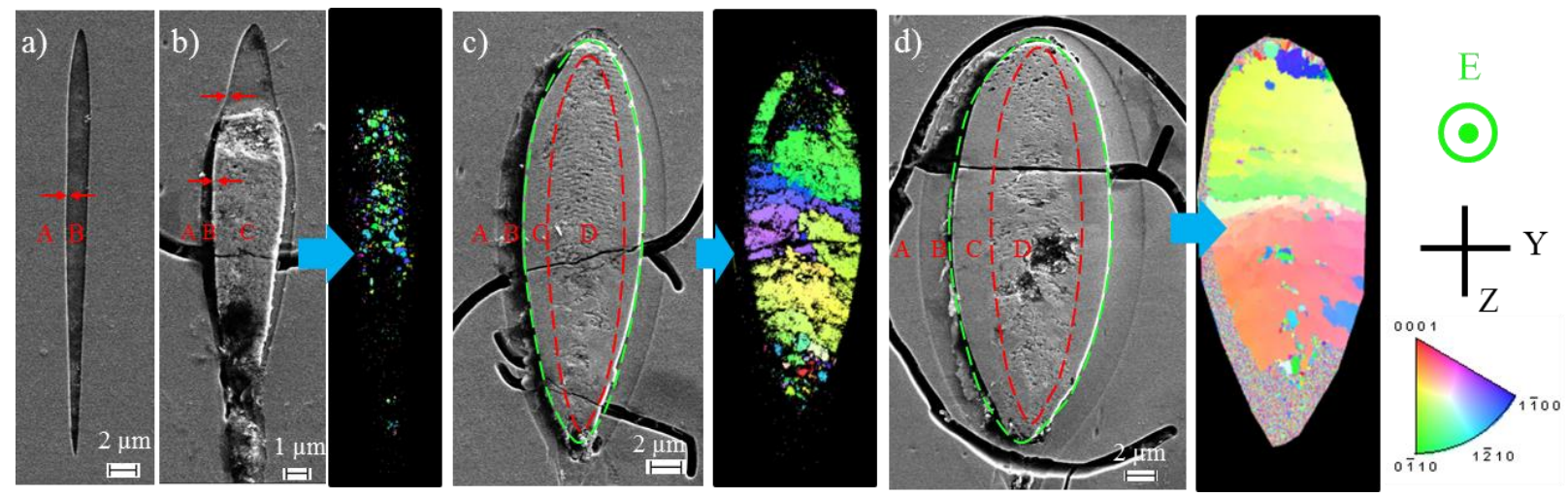

Figure $\mathbf{F}$ 1. Les images MEB en fonction de l'énergie par impulsion montrent les trois régimes de modifications obtenues dans le plan perpendiculaire à la direction de balayage: a) régime $1, b$ ) régime 2 , et $c-d$ ) régime 3 . La couleur dans les figures inverses de pôles (b, droite et $c$, droite, et $d$, droite) est basée sur le groupe d'espace de $\mathrm{LiNbO}_{3}$. Le codage est selon la direction de la polarisation du laser. Les autres paramètres du laser sont: $33 \mathrm{Li}_{2} \mathrm{O}-33 \mathrm{Nb}_{2} \mathrm{O}_{5}-34 \mathrm{SiO}_{2}, 1030 \mathrm{~nm}, 300 \mathrm{fs}, 300 \mathrm{kHz}, 5 \mu \mathrm{m} / \mathrm{s}$, la profondeur de focalisation est $350 \mu \mathrm{m}, \mathrm{NA}=0,6$, a) 0,4 b) 0,7, c) 1,3 et d) $1,8 \mu \mathrm{J} /$ impulsion, la polarisation du laser (illustrée par E) est parallèle à la direction de balayage.

Le premier seuil est mis en évidence par l'observation d'une modification de l'état amorphe qui se traduit par un changement de la température fictive du verre. Le second seuil (ca. 0,5 $\mu \mathrm{J} /$ impulsion) est l'apparition d'une cristallisation le long de la ligne de balayage. En dessous de ce seuil, la température fictive est modifiée et donc la plupart des propriétés physiques (régime 1). On note que le verre présente une plus grande sensibilité à l'attaque chimique HF comme illustré par la zone B sur la Figure F 1a.

Lorsque l'énergie par impulsion est légèrement supérieure au second seuil (0,5-0,9 $\mu \mathrm{J}$ /impulsion, régime 2), on observe une nanostructure en forme de millefeuille dont les "feuilles " fines (autour de 20-50nm nm) sont constituées d'une phase vitreuse riche en $\mathrm{SiO}_{2}$, et la "crème " d'épaisseur plus importante (autour de 100-150 nm), de nano-cristaux texturés. De façon remarquable, les axes polaires des nanocristaux sont perpendiculaires à la direction de la polarisation du laser dans la zone $\mathrm{C}$ du régime 2 comme cela est illustré sur la Figure $\mathrm{F} 1 \mathrm{~b}$. Les "feuilles » sont elles-mêmes orientées perpendiculairement à la direction du laser comme dans le cas des nanoréseaux formées dans la silice pure. Cette orientation nanocristalline dépendante 
de la polarisation peut s'interprêter par des couples induits par les impulsions répétitives du laser femtoseconde sur le dipôle électrique induit des nanocristaux.

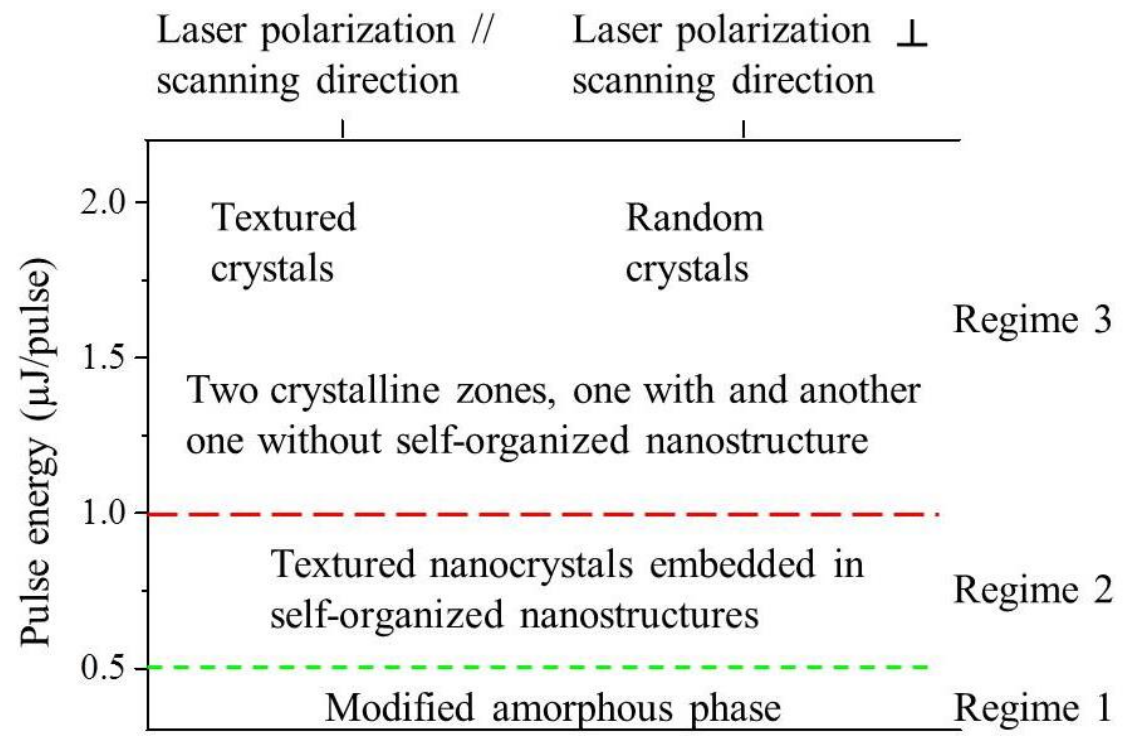

Figure $\mathbf{F}$ 2. Les trois régimes de modifications induites par le laser $\mathrm{fs}$ dans le verre LNS en fonction de l'énergie des impulsions (0,3-2,2 $\mu \mathrm{J} / \mathrm{impulsion})$. Les autres paramètres du laser sont: verre $33 \mathrm{Li}_{2} \mathrm{O}-33 \mathrm{Nb}_{2} \mathrm{O}_{5}-34 \mathrm{SiO}_{2}, 1030$ $\mathrm{nm}, 300 \mathrm{fs}, 300 \mathrm{kHz}, \mathrm{NA}=0,6$, profondeur de focalisation $\sim 350 \mu \mathrm{m}$ et $5 \mu \mathrm{m} / \mathrm{s}$.

Nous montrons que cette nanostructure auto-assemblée et constituée de changements de phase quasi périodiques dont les indices sont différents, conduit donc à l'apparition d'une biréfringence de forme dans la ligne irradiée avec un axe lent qui est perpendiculaire à la direction de polarisation du laser.

Par ailleurs, les nanocristaux texturés conduisent à l'apparition d'une génération de seconde harmonique qui présente une dépendance angulaire avec les paramètres d'écriture. La Figure $F$ 3a illustre cette dépendance angulaire avec la direction de la polarisation du faisceau sonde avec une période de $180^{\circ}$. L'orientation angulaire de la polarisation du faisceau sonde pour obtenir un minimum de génération de second harmonique, notée $\gamma_{\min }$, change de manière cohérente avec la polarisation du faisceau laser d'écriture comme le montre la Figure $F 3 b$. $\gamma_{\min }$ reste proche de la ligne en pointillés rouge. De même, l'analogue $\gamma_{\max }$ reste proche de $\gamma+90^{\circ}$ illustré par la ligne verte. Ce résultat indique que l'angle de polarisation du laser d'écriture $(\theta)$ agit sur la dépendance angulaire de la génération de second harmonique. Cette constatation peut guider les utilisateurs dans des applications en optique.

Le troisième seuil (1,0 $\mu \mathrm{J} /$ impulsion) est défini par l'apparition d'une zone cristallisée sans nanostructure auto-organisée. Cela correspond à la zone $C$ du régime 3 représentée sur les Figure F 1c-d. Dans le domaine d'énergie 1,0-2,2 $\mu$, la morphologie de cristallisation dépend de 
la configuration d'écriture. Les cristaux sont texturés lorsque la polarisation laser est parallèle à la direction de balayage, mais ils sont orientés de manière aléatoire lorsque la polarisation laser est perpendiculaire à la direction de balayage. Dans les deux cas, les cristaux sont répartis au milieu de lamelles vitreuses dans la zone $D$ du régime 3.

Dans les régimes 2 ou 3, l'orientation de la nanostructure peut être contrôlée par la direction de polarisation du laser, mais le régime 2 est plus adapté si une orientation cristalline particulière est requise pour les applications.
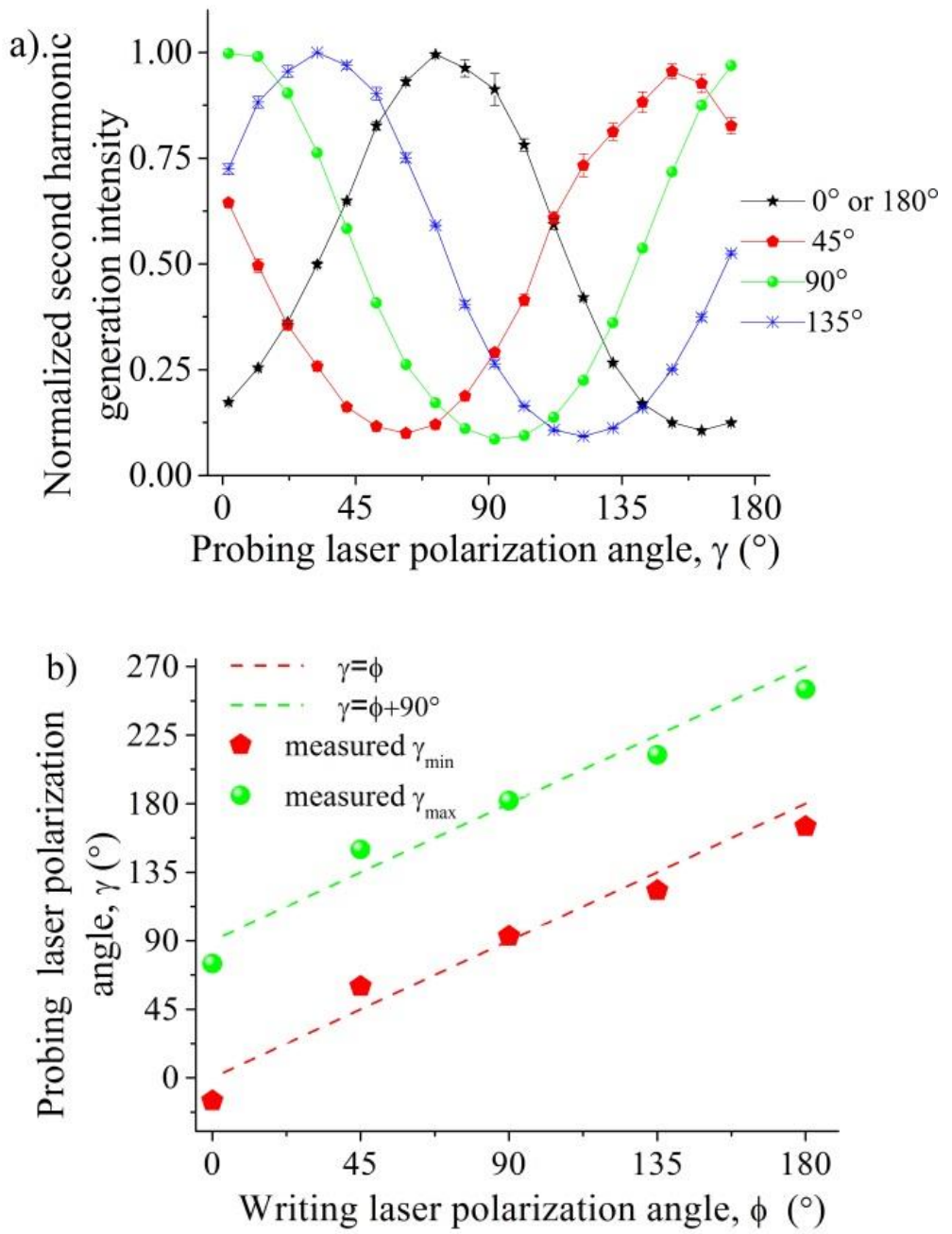

Figure $\mathbf{F}$ 3. Accordabilité angulaire de la génération de seconde harmonique dans le verre LNS. Le contrôle est réalisé par la direction de polarisation du laser fs. Les autres paramètres sont: $32.5 \mathrm{Li}{ }_{2} \mathrm{O}-27.5 \mathrm{Nb}_{2} \mathrm{O}_{5}-40 \mathrm{SiO}_{2}, 1030$ $\mathrm{nm}, 300 \mathrm{fs}, 250 \mathrm{kHz}, 0.8 \mu \mathrm{J} /$ impulsion, $5 \mu \mathrm{m} / \mathrm{s}$, la profondeur de mise au point est de $300 \mu \mathrm{m}, \mathrm{NA}=0,6$, la direction de balayage est le long de $45^{\circ}$.

\section{F 4. Conclusions}

Dans cette thèse, les modifications photo-induites à l'intérieur des verres $\mathrm{Li}_{2} \mathrm{O}-\mathrm{Nb}_{2} \mathrm{O}_{5}-\mathrm{SiO}_{2}$, au moyen d'impulsions femtosecondes fortement focalisées, ont été systématiquement étudiées. 
Des phénomènes nouveaux ont été découverts tels qu'une orientation nanocristalline dépendante de la polarisation du laser ou la formation orientée de nanostructures. Cela a conduit à l'obervation de propriétés optiques innovantes et exploitables telles que de la biréfringence de forme et la possibilité d'accorder la génération de seconde harmonique. Ce travail montre clairement que la cristallisation en volume, induite par une rayonnement laser femtoseconde, implique des phénoménes qui vont est bien au-delà d'un simple effet thermique.

La principale découverte est la possibilité d'orienter l'intensité maximale de la génération de seconde harmonique. Ceci est réalisé en orientant les nanocristaux grâce à la polarisation du laser. C'est le premier exemple d'un effet efficace de la polarisation laser dans le domaine de la science des matériaux. Une discussion a été présentée comprenant le mécanisme permettant d'expliquer les observations ci-dessus: la force principale serait l'effet de la polarisation électromagnétique du laser d'écriture sur le dipôle induit anisotrope de $\mathrm{LiNbO}_{3}$. De plus, I'EBSD couplée à la microscopie électronique à balayage (STEM/EDS) et à la microscopie électronique à transmission a révélé une microstructure orientable similaire à celle appelée "nanoréseaux " apparaissant dans la silice. L'originalité ici est une population de nanocristaux optiques structurés, non linéaires, intégrés dans un réseau de "parois" vitreuse, alignées perpendiculairement à la direction de polarisation du laser. II en résulte que l'orientation de la biréfringence et des propriétés optiques non linéaires du second ordre peut être maîtrisée en même temps. Ces résultats mettent en évidence des modifications spectaculaires du verre par le rayonnement issu d'un laser femtoseconde. Avec d'autres améliorations dans les techniques de fabrication, l'application de ce travail serait de réaliser des guides d'ondes biréfringents etprésentant des propriétés optiques non-linéaires permettant ainsi de réaliser des dispositifs actifs. 


\section{Bibliography}

1. R. R. Gattass and E. Mazur, "Femtosecond laser micromachining in transparent materials," Nat Photon 2, 219-225 (2008).

2. M. Beresna, M. Gecevičius, and P. G. Kazansky, "Ultrafast laser direct writing and nanostructuring in transparent materials," Adv. Opt. Photon. 6, 293-339 (2014).

3. D. Choudhury, J. R. Macdonald, and A. K. Kar, "Ultrafast laser inscription: perspectives on future integrated applications," Laser Photon. Rev. 8, 827-846 (2014).

4. R. Taylor, C. Hnatovsky, and E. Simova, "Applications of femtosecond laser induced self-organized planar nanocracks inside fused silica glass," Laser \& Photon. Rev. 2, 26-46 (2008).

5. M. Lancry, B. Poumellec, J. Canning, K. Cook, J. C. Poulin, and F. Brisset, "Ultrafast nanoporous silica formation driven by femtosecond laser irradiation," Laser \& Photon. Rev. 7, 953-962 (2013).

6. S. Mao, F. Quéré, S. Guizard, X. Mao, R. Russo, G. Petite, and P. Martin, "Dynamics of femtosecond laser interactions with dielectrics," Appl Phys A 79, 1695-1709 (2004).

7. S. Richter, C. Miese, S. Döring, F. Zimmermann, M. J. Withford, A. Tünnermann, and S. Nolte, "Laser induced nanogratings beyond fused silica-periodic nanostructures in borosilicate glasses and ULE ${ }^{\mathrm{TM}}$," Opt. Mater. Express 3, 1161-1166 (2013).

8. F. Chen and J. R. V. de Aldana, "Optical waveguides in crystalline dielectric materials produced by femtosecond-laser micromachining," Laser \& Photon. Rev. 8, 251-275 (2014).

9. A. Saliminia, N. Nguyen, S. Chin, and R. Vallée, "The influence of self-focusing and filamentation on refractive index modifications in fused silica using intense femtosecond pulses," Opt. Commun 241, 529-538 (2004).

10. Y. Dai, B. Zhu, J. Qiu, H. Ma, B. Lu, S. Cao, and B. Yu, "Direct writing three-dimensional $\mathrm{Ba}_{2} \mathrm{TiSi}_{2} \mathrm{O}_{8}$ crystalline pattern in glass with ultrashort pulse laser," Appl. Phys. Lett. 90, 1109 (2007).

11. D. Tan, K. N. Sharafudeen, Y. Yue, and J. Qiu, "Femtosecond laser induced phenomena in transparent solid materials: Fundamentals and applications," Prog. Mater Sci. 76, 154-228 (2016).

12. T. T. Fernandez, J. Siegel, J. Hoyo, B. Sotillo, P. Fernandez, and J. Solis, "Controlling plasma distributions as driving forces for ion migration during fs laser writing," J Phys D Appl Phys 48, 155101 (2015).

13. B. Poumellec, L. Sudrie, M. Franco, B. Prade, and A. Mysyrowicz, "Femtosecond laser irradiation stress induced in pure silica," Opt. Express 11, 1070-1079 (2003).

14. A. Stone, H. Jain, V. Dierolf, M. Sakakura, Y. Shimotsuma, K. Miura, K. Hirao, J. Lapointe, and R. Kashyap, "Direct laser-writing of ferroelectric single-crystal waveguide architectures in glass for 3D integrated optics," Sci Rep 5, 10391 (2015).

15. B. McMillen, B. Zhang, K. P. Chen, A. Benayas, and D. Jaque, "Ultrafast laser fabrication of low-loss waveguides in chalcogenide glass with 0.65 dB/cm loss," Opt. Lett 37, 1418-1420 (2012).

16. E. Cartlidge, "A photonic upgrade for computer memory?," Opt. Photon. News 27, 24-31 (2016). 
17. C. Fan, B. Poumellec, M. Lancry, X. He, H. Zeng, A. Erraji-Chahid, Q. Liu, and G. Chen, "Threedimensional photoprecipitation of oriented $\mathrm{LiNbO}_{3}$-like crystals in silica-based glass with femtosecond laser irradiation," Opt. Lett. 37, 2955-2957 (2012).

18. Y. Yonesaki, K. Miura, R. Araki, K. Fujita, and K. Hirao, "Space-selective precipitation of non-linear optical crystals inside silicate glasses using near-infrared femtosecond laser," J. Non-Cryst. Solids 351, 885-892 (2005).

19. A. Podlipensky, J. Lange, G. Seifert, H. Graener, and I. Cravetchi, "Second-harmonic generation from ellipsoidal silver nanoparticles embedded in silica glass," Opt. Lett 28, 716-718 (2003).

20. J. Choi, M. Bellec, A. Royon, K. Bourhis, G. Papon, T. Cardinal, L. Canioni, and M. Richardson, "Threedimensional direct femtosecond laser writing of second-order nonlinearities in glass," Opt. Lett 37, 1029-1031 (2012).

21. Y. Dai, H. Ma, B. Lu, B. Yu, B. Zhu, and J. Qiu, "Femtosecond laser-induced oriented precipitation of $\mathrm{Ba}_{2} \mathrm{TiGe}_{2} \mathrm{O}_{8}$ crystals in glass," Opt. Express 16, 3912-3917 (2008).

22. S. Eaton, H. Zhang, P. Herman, F. Yoshino, L. Shah, J. Bovatsek, and A. Arai, "Heat accumulation effects in femtosecond laser-written waveguides with variable repetition rate," Opt. Express 13, 4708-4716 (2005).

23. X. He, C. Fan, B. Poumellec, Q. Liu, H. Zeng, F. Brisset, G. Chen, X. Zhao, and M. Lancry, "Size-controlled oriented crystallization in $\mathrm{SiO}_{2}$-based glasses by femtosecond laser irradiation," J. Opt. Soc. Am. B 31, 376-381 (2014).

24. K. Miura, J. Qiu, T. Mitsuyu, and K. Hirao, "Space-selective growth of frequency-conversion crystals in glasses with ultrashort infrared laser pulses," Opt. Lett 25, 408-410 (2000).

25. A. Stone, M. Sakakura, Y. Shimotsuma, G. Stone, P. Gupta, K. Miura, K. Hirao, V. Dierolf, and H. Jain, "Directionally controlled 3D ferroelectric single crystal growth in $\mathrm{LaBGeO}_{5}$ glass by femtosecond laser irradiation," Opt. Express 17, 23284-23289 (2009).

26. X. He, B. Poumellec, Q. Liu, F. Brisset, and M. Lancry, "One-step photoinscription of asymmetrically oriented fresnoite-type crystals in glass by ultrafast laser," Opt. Lett 39, 5423-5426 (2014).

27. G. Beall and D. Duke, "Transparent glass-ceramics," J. Mater. Sci 4, 340-352 (1969).

28. H. Jain, "Transparent ferroelectric glass-ceramics," Ferroelectrics 306, 111-127 (2004).

29. H. Vigouroux, E. Fargin, S. Gomez, B. Le Garrec, G. Mountrichas, E. Kamitsos, F. Adamietz, M. Dussauze, and V. Rodriguez, "Synthesis and multiscale evaluation of $\mathrm{LiNbO}_{3}$-containing silicate glassceramics with efficient isotropic SHG response," Adv. Funct. Mater. 22, 3985-3993 (2012).

30. C. Awada, "Nature cohérente et incohérente de la réponse de second harmonique dans les nanostructures métalliques d'or et d'argent," Doctoral dissertation (Université Claude Bernard-Lyon I, 2009).

31. Y. Shimotsuma, P. G. Kazansky, J. Qiu, and K. Hirao, "Self-organized nanogratings in glass irradiated by ultrashort light pulses," Phys. Rev. Lett. 91, 247405 (2003).

32. E. Bricchi, B. G. Klappauf, and P. G. Kazansky, "Form birefringence and negative index change created by femtosecond direct writing in transparent materials," Opt. Lett. 29, 119-121 (2004). 
33. V. Bhardwaj, E. Simova, P. Rajeev, C. Hnatovsky, R. Taylor, D. Rayner, and P. Corkum, "Optically produced arrays of planar nanostructures inside fused silica," Phys. Rev. Lett. 96, 057404 (2006).

34. F. Zhang, H. Zhang, G. Dong, and J. Qiu, "Embedded nanogratings in germanium dioxide glass induced by femtosecond laser direct writing," J. Opt. Soc. Am. B 31, 860-864 (2014).

35. S. Richter, D. Möncke, F. Zimmermann, E. I. Kamitsos, L. Wondraczek, A. Tünnermann, and S. Nolte, "Ultrashort pulse induced modifications in ULE-from nanograting formation to laser darkening," Opt. Mater. Express 5, 1834-1850 (2015).

36. S. Fedotov, R. Drevinskas, S. Lotarev, A. Lipatiev, M. Beresna, A. Čerkauskaitè, V. Sigaev, and P. Kazansky, "Direct writing of birefringent elements by ultrafast laser nanostructuring in multicomponent glass," Appl. Phys. Lett. 108, 071905 (2016).

37. H. Vigouroux, E. Fargin, A. Fargues, B. L. Garrec, M. Dussauze, V. Rodriguez, F. Adamietz, G. Mountrichas, E. Kamitsos, and S. Lotarev, "Crystallization and second harmonic generation of lithium niobium silicate glass ceramics," J. Am. Ceram. Soc 94, 2080-2086 (2011).

38. R. Weis and T. Gaylord, "Lithium niobate: summary of physical properties and crystal structure," Appl. Phys. A 37, 191-203 (1985).

39. M. M. Choy and R. L. Byer, "Accurate second-order susceptibility measurements of visible and infrared nonlinear crystals," Phys. Rev. B 14, 1693 (1976).

40. M. Paul, M. Tabuchi, and A. R. West, "Defect structure of Ni, co-doped $\mathrm{LiNbO}_{3}$ and $\mathrm{LiTaO}_{3}$," Chem. Mater. 9, 3206-3214 (1997).

41. H.-B. Sun and S. Kawata, "Two-photon photopolymerization and 3D lithographic microfabrication," in NMR 3D Analysis Photopolymerization (Springer, 2004).

42. U. Keller, "Recent developments in compact ultrafast lasers," Nature 424, 831-838 (2003).

43. P. Agostini and L. F. DiMauro, "The physics of attosecond light pulses," Rep. Prog. Phys. 67, 813 (2004).

44. M. Hentschel, R. Kienberger, C. Spielmann, G. A. Reider, N. Milosevic, T. Brabec, P. Corkum, U. Heinzmann, M. Drescher, and F. Krausz, "Attosecond metrology," Nature 414, 509-513 (2001).

45. G. Mourou, C. Labaune, M. Dunne, N. Naumova, and V. Tikhonchuk, "Relativistic laser-matter interaction: from attosecond pulse generation to fast ignition," Plasma Phys Contr F 49, B667 (2007).

46. "Chirped Pulse Amplification," http://cuos.engin.umich.edu/researchgroups/hfs/facilities/chirpedpulse-amplification/, accessed 15 September 2016.

47. D. Strickland and G. Mourou, "Compression of amplified chirped optical pulses," Opt. Commun 56, 219-221 (1985).

48. M. Pessot, P. Maine, and G. Mourou, "1000 times expansion/compression of optical pulses for chirped pulse amplification," Opt. Commun 62, 419-421 (1987).

49. M. Ams, G. D. Marshall, P. Dekker, M. Dubov, V. K. Mezentsev, I. Bennion, and M. J. Withford, "Investigation of ultrafast laser--photonic material interactions: challenges for directly written glass photonics," IEEE J. Sel. Top. Quantum Electron. 14, 1370-1381 (2008).

50. M. Beresna, "Polarization engineering with ultrafast laser writing in transparent media," Doctoral dissertation (University of Southampton, 2012). 
51. K. M. Davis, K. Miura, N. Sugimoto, and K. Hirao, "Writing waveguides in glass with a femtosecond laser," Opt. Lett. 21, 1729-1731 (1996).

52. R. D. Guenther, Modern Optics (John Wiley \& Sons, 1990).

53. "Numerical of aperture a microscope objective," http://www.olympusmicro.com/primer/anatomy/numaperture.html, accessed 24 October 2016.

54. "Gaussian beam optics," https://marketplace.idexop.com/store/SupportDocuments/All_About_Gaussian_Beam_OpticsWEB.pd f, accessed 24 October 2016.

55. L. M. Osterink and J. D. Foster, "Thermal effects and transverse mode control in a Nd:YAG Laser," Appl. Phys. Lett. 12, 128-131 (1968).

56. C. Hnatovsky, R. Taylor, E. Simova, V. Bhardwaj, D. Rayner, and P. Corkum, "High-resolution study of photoinduced modification in fused silica produced by a tightly focused femtosecond laser beam in the presence of aberrations," J. Appl. Phys 98, 013517 (2005).

57. Q. Sun, H. Jiang, Y. Liu, Y. Zhou, H. Yang, and Q. Gong, "Effect of spherical aberration on the propagation of a tightly focused femtosecond laser pulse inside fused silica," J OPT A PURE APPL OP 7, 655 (2005).

58. M. Gu, D. Day, O. Nakamura, and S. Kawata, "Three-dimensional coherent transfer function for reflection confocal microscopy in the presence of refractive-index mismatch," J. Opt. Soc. Am. A 18, 2002-2008 (2001).

59. A. Marcinkevičius, V. Mizeikis, S. Juodkazis, S. Matsuo, and H. Misawa, "Effect of refractive indexmismatch on laser microfabrication in silica glass," Appl. Phys. A 76, 257-260 (2003).

60. S. Hendy, "Light scattering in transparent glass ceramics," Appl. Phys. Lett. 81, 1171-1173 (2002).

61. L. V. Keldysh, "Diagram technique for nonequilibrium processes," Sov. Phys. JETP 20, 1018-1026 (1965).

62. I. Shchatsinin, "Free clusters and free molecules in strong, shaped laser fields," Doctoral dissertation (Freie Universität Berlin, 2009).

63. C. B. Schaffer, A. Brodeur, and E. Mazur, "Laser-induced breakdown and damage in bulk transparent materials induced by tightly focused femtosecond laser pulses," Meas. Sci. Technol. 12, 1784 (2001).

64. B. J. Buerke, "Accurate measurement of tunneling ionization rates of atoms in a high-intensity laser field," Doctoral dissertation (University of Rochester, 2000).

65. B. C. Stuart, M. D. Feit, A. M. Rubenchik, B. W. Shore, and M. D. Perry, "Laser-induced damage in dielectrics with nanosecond to subpicosecond pulses," Phys. Rev. Lett 74, 2248-2251 (1995).

66. I. Miyamoto, A. Horn, and J. Gottmann, "Local melting of glass material and its application to direct fusion welding by ps-laser pulses," J. Laser Micro Nanoen 2, 7-14 (2007).

67. I. Miyamoto, A. Horn, J. Gottmann, D. Wortmann, and F. Yoshino, "Fusion welding of glass using femtosecond laser pulses with high-repetition rates," J. Laser Micro Nanoen 2, 57-63 (2007).

68. G. H. Wannier, "The structure of electronic excitation levels in insulating crystals," Phys. Rev. 52, 191 (1937). 
69. M. Ueta, H. Kanzaki, K. Kobayashi, Y. Toyozawa, and E. Hanamura, Excitonic processes in solids (Springer series in solid state sciences, 1986).

70. J. D. Musgraves, K. Richardson, and H. Jain, "Laser-induced structural modification, its mechanisms, and applications in glassy optical materials," Opt Mater Express 1, 921-935 (2011).

71. M. Lancry, B. Poumellec, A. Chahid-Erraji, M. Beresna, and P. G. Kazansky, "Dependence of the femtosecond laser refractive index change thresholds on the chemical composition of doped-silica glasses," Opt. Mater. Express 1, 711-723 (2011).

72. R. Wang, K. Saito, and A. Ikushima, "Distributions of self-trapped hole continuums in silica glass," J. Appl. Phys 100, 013706 (2006).

73. C. Gee and M. Kastner, "Intrinsic-defect photoluminescence in amorphous $\mathrm{SiO}_{2}$," Phys. Rev. Lett 42 , 1765 (1979).

74. M. Watanabe, S. Juodkazis, H.-B. Sun, S. Matsuo, and H. Misawa, "Luminescence and defect formation by visible and near-infrared irradiation of vitreous silica," Phys. Rev. B 60, 9959-9964 (1999).

75. C. M. Gee and M. Kastner, "Intrinsic-defect photoluminescence in amorphous and crystalline $\mathrm{SiO}_{2}$," J. Non-Cryst. Solids 35, 927-932 (1980).

76. M. Lancry, B. Poumellec, R. Desmarchelier, and B. Bourguignon, "Oriented creation of anisotropic defects by IR femtosecond laser scanning in silica," Opt Mater Express 2, 1809-1821 (2012).

77. D. Grojo, M. Gertsvolf, S. Lei, T. Barillot, D. M. Rayner, and P. B. Corkum, "Exciton-seeded multiphoton ionization in bulk $\mathrm{SiO}_{2}, "$ Phys. Rev. B 81, 212301 (2010).

78. L. Sudrie, M. Franco, B. Prade, and A. Mysyrowicz, "Study of damage in fused silica induced by ultrashort IR laser pulses," Opt. Commun 191, 333-339 (2001).

79. A. Couairon, L. Sudrie, M. Franco, B. Prade, and A. Mysyrowicz, "Filamentation and damage in fused silica induced by tightly focused femtosecond laser pulses," Phys. Rev. B 71, 125435 (2005).

80. C. Hnatovsky, R. Taylor, P. Rajeev, E. Simova, V. Bhardwaj, D. Rayner, and P. Corkum, "Pulse duration dependence of femtosecond-laser-fabricated nanogratings in fused silica," Appl. Phys. Lett. 87, 014104 (2005).

81. R. Osellame, G. Cerullo, and R. Ramponi, Femtosecond laser micromachining: Photonic and microfluidic devices in transparent materials (Springer Science \& Business Media, 2012).

82. B. Poumellec, M. Lancry, A. Chahid-Erraji, and P. G. Kazansky, "Modification thresholds in femtosecond laser processing of pure silica: review of dependencies on laser parameters [Invited]," Opt Mater Express 1, 766-782 (2011).

83. M. Lancry, E. Régnier, and B. Poumellec, "Fictive temperature in silica-based glasses and its application to optical fiber manufacturing," Prog. Mater Sci. 57, 63-94 (2012).

84. V. Bhardwaj, P. Corkum, D. Rayner, C. Hnatovsky, E. Simova, and R. Taylor, "Stress in femtosecondlaser-written waveguides in fused silica," Opt. Lett 29, 1312-1314 (2004).

85. J. D. Mills, P. G. Kazansky, E. Bricchi, and J. J. Baumberg, "Embedded anisotropic microreflectors by femtosecond-laser nanomachining," Appl. Phys. Lett. 81, 196-198 (2002). 
86. M. Lancry, B. Poumellec, K. Cook, and J. Canning, "Nanogratings and molecular oxygen formation during femtosecond laser irradiation in silica," in Proceedings of the International Quantum Electronics Conference and Conference on Lasers and Electro-Optics Pacific Rim 2011, (Optical Society of America, 2011), C229.

87. E. Bricchi and P. G. Kazansky, "Extraordinary stability of anisotropic femtosecond direct-written structures embedded in silica glass," Appl. Phys. Lett. 88, 111119 (2006).

88. R. Desmarchelier, B. Poumellec, F. Brisset, S. Mazerat, and M. Lancry, "In the heart of femtosecond laser induced nanogratings: from porous nanoplanes to form birefringence," World J. Nano Sci. Eng. 5, 115 (2015).

89. T. Asai, Y. Shimotsuma, T. Kurita, A. Murata, S. Kubota, M. Sakakura, K. Miura, F. Brisset, B. Poumellec, and M. Lancry, "Systematic control of structural changes in $\mathrm{GeO}_{2}$ glass induced by femtosecond laser direct writing," J. Am. Ceram. Soc 98, 1471-1477 (2015).

90. F. Zimmermann, A. Plech, S. Richter, A. Tünnermann, and S. Nolte, "Ultrashort laser pulse induced nanogratings in borosilicate glass," Appl. Phys. Lett. 104, 211107 (2014).

91. W. Yang, E. Bricchi, P. G. Kazansky, J. Bovatsek, and A. Y. Arai, "Self-assembled periodic subwavelength structures by femtosecond laser direct writing," Opt. Express 14, 10117-10124 (2006).

92. F. Zimmermann, A. Plech, S. Richter, A. Tünnermann, and S. Nolte, "The onset of ultrashort pulseinduced nanogratings," Laser Photon. Rev. 10, 327-334 (2016).

93. E. N. Glezer, M. Milosavljevic, L. Huang, R. J. Finlay, T. H. Her, J. P. Callan, and E. Mazur, "Threedimensional optical storage inside transparentmaterials," Opt. Lett 21, 2023-2025 (1996).

94. H.-B. Sun, Y. Xu, S. Juodkazis, K. Sun, M. Watanabe, S. Matsuo, H. Misawa, and J. Nishii, "Arbitrarylattice photonic crystals created by multiphoton microfabrication," Opt. Lett 26, 325-327 (2001).

95. F. Zimmermann, M. Lancry, A. Plech, S. Richter, B. Hari Babu, B. Poumellec, A. Tünnermann, and S. Nolte, "Femtosecond laser written nanostructures in Ge-doped glasses," Opt. Lett 41, 1161-1164 (2016).

96. R. Graf, A. Fernandez, M. Dubov, H. Brueckner, B. Chichkov, and A. Apolonski, "Pearl-chain waveguides written at megahertz repetition rate," Appl. Phys. B 87, 21-27 (2007).

97. Y. Bellouard and M.-O. Hongler, "Femtosecond-laser generation of self-organized bubble patterns in fused silica," Opt. Express 19, 6807-6821 (2011).

98. S. Maruo, O. Nakamura, and S. Kawata, "Three-dimensional microfabrication with two-photonabsorbed photopolymerization," Opt. Lett 22, 132-134 (1997).

99. S. Kawata, H.-B. Sun, T. Tanaka, and K. Takada, "Finer features for functional microdevices," Nature 412, 697-698 (2001).

100. D. Umstadter, "Relativistic laser-plasma interactions," J. Phys. D: Appl. Phys. 36, R151 (2003).

101. A. Scott, Encyclopedia of nonlinear science (Routledge, 2006).

102. H. Daido, M. Nishiuchi, and A. S. Pirozhkov, "Review of laser-driven ion sources and their applications," Rep. Prog. Phys. 75, 056401 (2012).

103. F. Träger, Springer handbook of lasers and optics (Springer Science \& Business Media, 2007). 
104. A. L. Fussell, A. Isomaki, and C. J. Strachan, "Non-linear optical imaging-Introduction and pharmaceutical applications," Am Pharmaceut Rev 16, 54-63 (2013).

105. P. Pantazis, J. Maloney, D. Wu, and S. E. Fraser, "Second harmonic generating (SHG) nanoprobes for in vivo imaging," Proc. Natl. Acad. Sci. 107, 14535-14540 (2010).

106. "Quasi-phase Matching," https://www.rp-photonics.com/quasi phase matching.html, accessed 30 November 2016.

107. J. Butet, I. Russier-Antoine, C. Jonin, N. Lascoux, E. Benichou, and P.-F. Brevet, "Nonlinear Mie theory for the second harmonic generation in metallic nanoshells," J. Opt. Soc. Am. B 29, 2213-2221 (2012).

108. P. Franken, A. Hill, C. e. Peters, and G. Weinreich, "Generation of optical harmonics," Phys. Rev. Lett 7, 118 (1961).

109. P. S. Halasyamani and K. R. Poeppelmeier, "Noncentrosymmetric oxides," Chem. Mater. 10, 2753-2769 (1998).

110. J. H. Marburger, "Self-focusing: Theory," Prog Quant Electron 4, 35-110 (1975).

111. A. Couairon and A. Mysyrowicz, "Femtosecond filamentation in transparent media," Phys. Rep 441, 47-189 (2007).

112. A. Brodeur and S. L. Chin, "Ultrafast white-light continuum generation and self-focusing in transparent condensed media," J. Opt. Soc. Am. B 16, 637-650 (1999).

113. M. Soileau, W. E. Williams, N. Mansour, and E. W. Van Stryland, "Laser-induced damage and the role of self-focusing," Opt. Eng 28, 281133-281133 (1989).

114. S. Tzortzakis, L. Sudrie, M. Franco, B. Prade, A. Mysyrowicz, A. Couairon, and L. Bergé, "Self-guided propagation of ultrashort IR laser pulses in fused silica," Phys. Rev. Lett. 87, 213902 (2001).

115. P. G. Debenedetti and F. H. Stillinger, "Supercooled liquids and the glass transition," Nature 410, 259267 (2001).

116. H. Scholze, Glass: nature, structure, and properties (Springer Science \& Business Media, 2012).

117. "The Silica Group," http://www.quartzpage.de/gen mod.html, accessed 22 September 2016.

118. C. A. Angell, "Formation of glasses from liquids and biopolymers," Science 267, 1924 (1995).

119. M. I. Ojovan, "Viscosity and glass transition in amorphous oxides," Adv. Condens. Matter Phys. 2008(2009).

120. P. Hrma, "Glass viscosity as a function of temperature and composition: A model based on AdamGibbs equation," J. Non-Cryst. Solids 354, 3389-3399 (2008).

121. T. Rouxel and J.-C. Sanglebœuf, "The brittle to ductile transition in a soda-lime-silica glass," J. NonCryst. Solids 271, 224-235 (2000).

122. R. Brückner, "Properties and structure of vitreous silica. I," J. Non-Cryst. Solids 5, 123-175 (1970).

123. A. Mermillod-Blondin, I. M. Burakov, Y. P. Meshcheryakov, N. M. Bulgakova, E. Audouard, A. Rosenfeld, A. Husakou, I. V. Hertel, and R. Stoian, "Flipping the sign of refractive index changes in ultrafast and temporally shaped laser-irradiated borosilicate crown optical glass at high repetition rates," Phys. Rev. B 77, 104205 (2008). 
124. H. Zeng, B. Poumellec, C. Fan, G. Chen, A. Erraji-Chahid, and M. Lancry, Preparation of glass-ceramics with oriented nonlinear crystals: A review, Advances in Materials Science Research (Nova Science Publishers, 2012), Vol. 12, pp. 89-134.

125. K. Wasai, G. Kaptay, K. Mukai, and N. Shinozaki, "Modified classical homogeneous nucleation theory and a new minimum in free energy change: 1. A new minimum and Kelvin equation," FFE 254, 67-74 (2007).

126. S. B. Lee and N. J. Kim, "Kinetics of crystallization in continuously cooled BMG," Mater. Sci. Eng., A 404, 153-158 (2005).

127. V. M. Fokin, E. D. Zanotto, N. S. Yuritsyn, and J. W. Schmelzer, "Homogeneous crystal nucleation in silicate glasses: a 40 years perspective," J. Non-Cryst. Solids 352, 2681-2714 (2006).

128. M. Shimizu, M. Sakakura, S. Kanehira, M. Nishi, Y. Shimotsuma, K. Hirao, and K. Miura, "Formation mechanism of element distribution in glass under femtosecond laser irradiation," Opt. Lett 36, 21612163 (2011).

129. D. Uhlmann and H. Yinnon, "The formation of glasses," in Glass: Science and Technology, 1983$), 1$.

130. P. Soares, E. Zanotto, V. Fokin, and H. Jain, "TEM and XRD study of early crystallization of lithium disilicate glasses," J. Non-Cryst. Solids 331, 217-227 (2003).

131. S. Claus, H. Kleykamp, and W. Smykatz-Kloss, "Phase equilibria in the $\mathrm{Li}_{4} \mathrm{SiO}_{4} \cdot \mathrm{Li}_{2} \mathrm{SiO}_{3}$ region of the pseudobinary $\mathrm{Li}_{2} \mathrm{O} \cdot \mathrm{SiO}_{2}$ system," J. Nucl. Mater. 230, 8-11 (1996).

132. F. C. Kracek, "The binary system $\mathrm{Li}_{2} \mathrm{O}-\mathrm{SiO}_{2}$," J. Phys. Chem. 34, 2641-2650 (1929).

133. A. R. West and F. P. Glasser, "Crystallization of lithium trisilicate, $\mathrm{Li}_{2} \mathrm{Si}_{3} \mathrm{O}_{7}$, from $\mathrm{Li}_{2} \mathrm{O} \mid \mathrm{SiO}_{2}$ melts," Mater. Res. Bull. 5, 837-842 (1970).

134. R. I. Smith, R. A. Howie, A. R. West, A. Aragón-Piña, and M. E. Villafuerte-Castrejón, "The structure of metastable lithium disilicate, $\mathrm{Li}_{2} \mathrm{Si}_{2} \mathrm{O}_{5}$," Acta Cryst. C 46, 363-365 (1990).

135. M. Ibrahim and N. F. Bright, "The binary system $\mathrm{Nb}_{2} \mathrm{O}_{5}-\mathrm{SiO}_{2}$," J. Am. Ceram. Soc 45, 221-222 (1962).

136. N. Umesaki, M. Takahashi, M. Tatsumisago, and T. Minami, "Structure of rapidly quenched glasses in the system $\mathrm{Li}_{2} \mathrm{O}-\mathrm{SiO}_{2}$," J. Mater. Sci 28, 3473-3481 (1993).

137. J. Stanworth, "Tellurite glasses," Nature 169, 581-582 (1952).

138. T. Komatsu, "Design and control of crystallization in oxide glasses," J. Non-Cryst. Solids 428, 156-175 (2015).

139. M. P. F. Graça, M. G. Ferreira da Silva, and M. A. Valente, "Structural and electrical characteristics of $\mathrm{LiNbO}_{3}$ embedded in a 34\% $\mathrm{SiO}_{2}$ glass matrix," J. Eur. Ceram. Soc. 28, 1197-1203 (2008).

140. O. S. Maksimova, L. V. Korzunova, and Z. P. Milberg, "Properties and structure of glasses of the system $\mathrm{Li}_{2} \mathrm{O}-\mathrm{Nb}_{2} \mathrm{O}_{5}-\mathrm{SiO}_{2}$," Izv.Akad.Nauk Latv.SSR,Ser.Khim 5, 530-534 (1975).

141. A. Stone, M. Sakakura, Y. Shimotsuma, G. Stone, P. Gupta, K. Miura, K. Hirao, V. Dierolf, and H. Jain, "Formation of ferroelectric single-crystal architectures in $\mathrm{LaBGeO}_{5}$ glass by femtosecond vs. continuous-wave lasers," J. Non-Cryst. Solids 356, 3059-3065 (2010). 
142. T. Komatsu, R. Ihara, T. Honma, Y. Benino, R. Sato, H. Kim, and T. Fujiwara, "Patterning of non-linear optical crystals in glass by laser-induced crystallization," J. Am. Ceram. Soc 90, 699-705 (2007).

143. T. Honma, Y. Benino, T. Fujiwara, and T. Komatsu, "Transition metal atom heat processing for writing of crystal lines in glass," Appl. Phys. Lett. 88, 231105-231105 (2006).

144. T. Komatsu, K. Koshiba, and T. Honma, "Preferential growth orientation of laser-patterned $\mathrm{LiNbO}_{3}$ crystals in lithium niobium silicate glass," J. Solid State Chem. 184, 411-418 (2011).

145. R. Ihara, T. Honma, Y. Benino, T. Fujiwara, R. Sato, and T. Komatsu, "Writing of two-dimensional crystal curved lines at the surface of $\mathrm{Sm}_{2} \mathrm{O}_{3}-\mathrm{Bi}_{2} \mathrm{O}_{3}-\mathrm{B}_{2} \mathrm{O}_{3}$ glass by samarium atom heat processing," Solid State Commun. 136, 273-277 (2005).

146. M. Saito, T. Honma, Y. Benino, T. Fujiwara, and T. Komatsu, "Formation of nonlinear optical $\mathrm{KSm}_{(}\left(\mathrm{PO}_{3}\right)_{4}$ crystals in phosphate glasses by YAG laser irradiation," Solid State Sci. 6, 1013-1018 (2004).

147. S. Kawasaki, T. Honma, Y. Benino, T. Fujiwara, R. Sato, and T. Komatsu, "Writing of crystal-dots and lines by YAG laser irradiation and their morphologies in samarium tellurite glasses," J. Non-Cryst. Solids 325, 61-69 (2003).

148. N. Chayapiwut, T. Honma, Y. Benino, T. Fujiwara, and T. Komatsu, "Synthesis of Sm ${ }^{3+}$-doped strontium barium niobate crystals in glass by samarium atom heat processing," J. Solid State Chem. 178, 35073513 (2005).

149. F. Suzuki, T. Honma, and T. Komatsu, "Origin of periodic domain structure in $\mathrm{Er}^{3+}$-doped $\beta^{\prime}-$ $(\mathrm{Sm}, \mathrm{Gd})_{2}(\mathrm{MoO} 4)_{3}$ crystal lines patterned by laser irradiations in glasses," J. Solid State Chem 183, 909 914 (2010).

150. M. Abe, Y. Benino, T. Fujiwara, T. Komatsu, and R. Sato, "Writing of nonlinear optical $\mathrm{Sm}_{2}\left(\mathrm{MoO}_{4}\right)_{3}$ crystal lines at the surface of glass by samarium atom heat processing," J. Appl. Phys 97, 123516 (2005).

151. T. Honma, Y. Benino, T. Fujiwara, T. Komatsu, and R. Sato, "Technique for writing of nonlinear optical single-crystal lines in glass," Appl. Phys. Lett. 83, 2796-2798 (2003).

152. A. F. Maciente, V. R. Mastelaro, A. L. Martinez, A. C. Hernandes, and C. A. C. Carneiro, "Surface crystallization of $\beta-\mathrm{BaB}_{2} \mathrm{O}_{4}$ phase using a $\mathrm{CO}_{2}$ laser source," J. Non-Cryst. Solids 306, 309-312 (2002).

153. R. Vaish, V. Rodriguez, M. Maglione, J. Etourneau, and K. B. Varma, "Laser-induced periodic surface crystalline patterns on $\mathrm{SrO}-0.5 \mathrm{Li}_{2} \mathrm{O}-4.5 \mathrm{~B}_{2} \mathrm{O}_{3}$ and $\mathrm{BaO}-0.5 \mathrm{Na}_{2} \mathrm{O}-4.5 \mathrm{~B}_{2} \mathrm{O}_{3}$ glasses and optical second harmonic generation," Int J Appl Glass Sci 1, 350-357 (2010).

154. P. Gupta, H. Jain, D. B. Williams, T. Honma, Y. Benino, and T. Komatsu, "Creation of ferroelectric, single-crystal architecture in $\mathrm{Sm}_{0.5} \mathrm{La}_{0 .} \mathrm{BGeO}_{5}$ glass," J. Am. Ceram. Soc. 91, 110-114 (2008).

155. P. Gupta, H. Jain, D. B. Williams, J. Toulouse, and I. Veltchev, "Creation of tailored features by laser heating of $\mathrm{Nd}_{0.2} \mathrm{La}_{0.8} \mathrm{BGeO}_{5}$ glass," Opt. Mater 29, 355-359 (2006).

156. B. Zhu, Y. Dai, H. Ma, S. Zhang, G. Lin, and J. Qiu, "Femtosecond laser induced space-selective precipitation of nonlinear optical crystals in rare-earth-doped glasses," Opt. Express 15, 6069-6074 (2007). 
157. Y. Dai, B. Zhu, J. Qiu, H. Ma, B. Lu, and B. Yu, "Space-selective precipitation of functional crystals in glass by using a high repetition rate femtosecond laser," Chem. Phys. Lett. 443, 253-257 (2007).

158. H. Sugita, T. Honma, Y. Benino, and T. Komatsu, "Formation of $\mathrm{LiNbO}_{3}$ crystals at the surface of $\mathrm{TeO}_{2}$ based glass by YAG laser-induced crystallization," Solid State Commun. 143, 280-284 (2007).

159. V. Rodriguez, B. H. Venkataraman, N. S. Prasad, K. Varma, M. Maglione, R. Vondermuhll, and J. Etourneau, "Optical diffraction of second-harmonic signals in the $\mathrm{LiBO}_{2}-\mathrm{Nb}_{2} \mathrm{O}_{5}$ glasses induced by selforganized $\mathrm{LiNbO}_{3}$ crystallites," Appl. Phys. Lett. 87, 3 (2005).

160. T. Honma, K. Koshiba, Y. Benino, and T. Komatsu, "Writing of crystal lines and its optical properties of rare-earth ion $\left(\mathrm{Er}^{3+}\right.$ and $\mathrm{Sm}^{3+}$ ) doped lithium niobate crystal on glass surface formed by laser irradiation," Opt. Mater 31, 315-319 (2008).

161. Y. Ochi, T. Meguro, and K. Kakegawa, "Orientated crystallization of fresnoite glass-ceramics by using a thermal gradient," J. Eur. Ceram. Soc. 26, 627-630 (2006).

162. N. M. Ferreira, F. M. Costa, R. N. Nogueira, and M. P. F. Graça, "Lithium niobate bulk crystallization promoted by $\mathrm{CO}_{2}$ laser radiation," Appl. Surf. Sci. 258, 9457-9460 (2012).

163. K. Gerth, C. Rüssel, R. Keding, P. Schleevoigt, and H. Dunken, "Oriented crystallisation of lithium niobate containing glass ceramic in an electric field and determination of the crystallographic orientation by infrared spectroscopy," Phys. Chem. Glasses 40, 135-139 (1999).

164. F. Luo, G. Lin, H. Pan, Q. Chen, J. Qiu, Q. Zhao, and Z. Xu, "Elemental redistribution in glass induced by a 250-kHz femtosecond laser," J. Non-Cryst. Solids 357, 2384-2386 (2011).

165. J. del Hoyo, R. M. Vazquez, B. Sotillo, T. T. Fernandez, J. Siegel, P. Fernández, R. Osellame, and J. Solis, "Control of waveguide properties by tuning femtosecond laser induced compositional changes," Appl. Phys. Lett. 105, 131101 (2014).

166. T. T. Fernandez, M. Hernandez, B. Sotillo, S. Eaton, G. Jose, R. Osellame, A. Jha, P. Fernandez, and J. Solis, "Role of ion migrations in ultrafast laser written tellurite glass waveguides," Opt. Express 22, 15298-15304 (2014).

167. M. Sakakura, T. Kurita, M. Shimizu, K. Yoshimura, Y. Shimotsuma, N. Fukuda, K. Hirao, and K. Miura, "Shape control of elemental distributions inside a glass by simultaneous femtosecond laser irradiation at multiple spots," Opt. Lett 38, 4939-4942 (2013).

168. B. Matthias and J. Remeika, "Ferroelectricity in the ilmenite structure," Phys. Rev. 76, 1886 (1949).

169. P. Bordui, R. Norwood, D. Jundt, and M. Fejer, "Preparation and characterization of off-congruent lithium niobate crystals," J. Appl. Phys 71, 875-879 (1992).

170. L. O. Svaasand, M. Eriksrud, G. Nakken, and A. P. Grande, "Solid-solution range of LiNbO 3 ," J. Cryst. Growth 22, 230-232 (1974).

171. H. M. O'BRYAN, P. K. Gallagher, and C. Brandle, "Congruent composition and Li-rich phase boundary of $\mathrm{LiNbO}_{3}, "$ J. Am. Ceram. Soc 68, 493-496 (1985).

172. M. Wöhlecke, G. Corradi, and K. Betzler, "Optical methods to characterise the composition and homogeneity of lithium niobate single crystals," Appl. Phys. B 63, 323-330 (1996). 
173. B. Ausrine, P. Valentina, A. Adulfas, S. Sandra, M. Samuel, B. Pascal, T. Kobata, U. Yoshiaki, and G. Jerome, "Identification of $\mathrm{LiNbO}_{3}, \mathrm{LiNb}_{3} \mathrm{O}_{8}$ and $\mathrm{Li}_{3} \mathrm{NbO}_{4}$ phases in thin films synthesized with different deposition techniques by means of XRD and Raman spectroscopy," J. Phys. Condens. Matter 25, 205901 (2013).

174. M. Lundberg, "The crystal structure of $\mathrm{LiNb}_{3} \mathrm{O}_{8}$," Acta Chem. Scand. 25, 3337-3346 (1971).

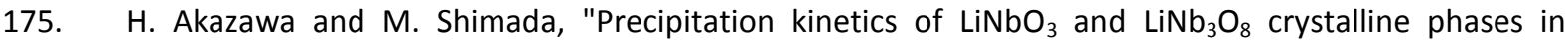
thermally annealed amorphous $\mathrm{LiNbO}_{3}$ thin films," Phys. Status Solidi A 203, 2823-2827 (2006).

176. R. Subasri and O. M. Sreedharan, "Thermodynamic stability of $\mathrm{Li}_{3} \mathrm{NbO}_{4}$ by emf measurements using a novel composite electrolyte," Solid State Ion. 93, 341-346 (1997).

177. N. Yabuuchi, M. Takeuchi, M. Nakayama, H. Shiiba, M. Ogawa, K. Nakayama, T. Ohta, D. Endo, T. Ozaki, T. Inamasu, K. Sato, and S. Komaba, "High-capacity electrode materials for rechargeable lithium batteries: $\mathrm{Li}_{3} \mathrm{NbO}_{4}$-based system with cation-disordered rocksalt structure," Proc Natl Acad Sci U S A 112, 7650-7655 (2015).

178. K. Ukei, H. Suzuki, T. Shishido, and T. Fukuda, "Structure of $\mathrm{Li}_{3} \mathrm{NbO}_{4}, "$ Acta Crystallogr., Sect. C: Cryst. Struct. Commun. 50, 655-656 (1994).

179. A. Glass, K. Nassau, and T. Negran, "Ionic conductivity of quenched alkali niobate and tantalate glasses," J. Appl. Phys 49, 4808-4811 (1978).

180. M. Tatsumisago, A. Hamada, T. Minami, and M. Tanaka, "Preparation and properties of quenched $\mathrm{Li}_{2} \mathrm{O}-\mathrm{BaO}-\mathrm{Nb}_{2} \mathrm{O}_{5}$ Glasses," J. Am. Ceram. Soc 65, 575-577 (1982).

181. M. Tatsumisago, A. Hamada, T. Minami, and M. Tanaka, "Structure and properties of rapidly quenched $\mathrm{Li}_{2} \mathrm{O}-\mathrm{Al}_{2} \mathrm{O}_{3}-\mathrm{Nb}_{2} \mathrm{O}_{5}$ glasses," J. Am. Ceram. Soc 66, 890-892 (1983).

182. "LiNbO 3 crystal basic properties," http://www.bbotech.com/product.php?cid=135, accessed 28 October 2016.

183. D. E. Zelmon, D. L. Small, and D. Jundt, "Infrared corrected Sellmeier coefficients for congruently grown lithium niobate and 5 mol.\% magnesium oxide-doped lithium niobate," J. Opt. Soc. Am. B 14, 3319-3322 (1997).

184. U. Schlarb and K. Betzler, "Refractive indices of lithium niobate as a function of temperature, wavelength, and composition: A generalized fit," Phys. Rev. B 48, 15613 (1993).

185. M. Weber, CRC handbook of laser science and technology. Optical materials, Part 1-Nonlinear optical properties/radiation damage (CRC press, 1986), Vol. 3.

186. M. Graça, M. Valente, and M. F. da Silva, "Electrical properties of lithium niobium silicate glasses," J. Non-Cryst. Solids 325, 267-274 (2003).

187. V. Sigaev, N. Golubev, S. Y. Stefanovich, T. Komatsu, Y. Benino, P. Pernice, A. Aronne, E. Fanelli, B. Champagnon, and V. Califano, "Second-order optical non-linearity initiated in $\mathrm{Li}_{2} \mathrm{O}-\mathrm{Nb}_{2} \mathrm{O}_{5}-\mathrm{SiO}_{2}$ and $\mathrm{Li}_{2} \mathrm{O}-\mathrm{ZnO}-\mathrm{Nb}_{2} \mathrm{O}_{5}-\mathrm{SiO}_{2}$ glasses by formation of polar and centrosymmetric nanostructures," J. Non-Cryst. Solids 354, 873-881 (2008).

188. C. Fan, "Contribution to nano or micro crystallization induction in silica-based glass by femtosecond laser irradiation," Doctoral dissertation (Université Paris Sud-Paris XI, 2013). 
189. C. Fan, B. Poumellec, H. Zeng, M. Lancry, W. Yang, B. Bourguignon, and G. Chen, "Directional writing dependence of birefringence in multicomponent silica-based glasses with ultrashort laser irradiation," J. Laser Micro Nanoen 6, 158-163 (2011).

190. E. Prasad, M. Sayer, and H. Vyas, "Li ${ }^{+}$conductivity in lithium niobate: silica glasses," J. Non-Cryst. Solids 40, 119-134 (1980).

191

"Vertical scanning

http://blog.nus.edu.sg/me4105precisionengineering2012/state-of-the-artresearch/interferometry/vertical-scanning-interferometry/, accessed 24 September 2016.

192. C. Blanc, Y. Roques, and G. Mankowski, "Application of phase shifting interferometric microscopy to studies of the behaviour of coarse intermetallic particles in 6056 aluminium alloy," Corros. Sci. 40, 1019-1035 (1998).

193. M. Sakakura, M. Shimizu, Y. Shimotsuma, K. Miura, and K. Hirao, "Temperature distribution and modification mechanism inside glass with heat accumulation during $250 \mathrm{kHz}$ irradiation of femtosecond laser pulses," Appl. Phys. Lett. 93, 231112 (2008).

194. M. Beresna, T. Gertus, R. Tomašiūnas, H. Misawa, and S. Juodkazis, "Three-dimensional modeling of the heat-affected zone in laser machining applications," Laser Chem. 2008(2008).

195. F. Hashimoto, S. Richter, S. Nolte, Y. Ozeki, and K. Itoh, "Time-resolved micro-Raman measurement of temperature dynamics during high-repetition-rate ultrafast laser microprocessing," J. Laser Micro Nanoen 10, 29 (2015).

196. "Lithium oxide," http://www-ferp.ucsd.edu/LIB/PROPS/PANOS/li2o.html, accessed 29 November 2016.

197. W. D. Kingery, "Thermal Conductivity: XII, Temperature dependence of conductivity for single-phase ceramics," J. Am. Ceram. Soc 38, 251-255 (1955).

198. K.-K. Wong, Properties of lithium niobate (IET, 2002).

199. R. A. Morgan, K. I. Kang, C. C. Hsu, C. L. Koliopoulos, and N. Peyghambarian, "Measurement of the thermal diffusivity of nonlinear anisotropic crystals using optical interferometry," Appl. Opt. 26, 52665271 (1987).

200. M. Shimizu, M. Sakakura, M. Ohnishi, Y. Shimotsuma, T. Nakaya, K. Miura, and K. Hirao, "Mechanism of heat-modification inside a glass after irradiation with high-repetition rate femtosecond laser pulses," J. Appl. Phys 108, 073533 (2010).

201. M. Lancry, B. Poumellec, and S. Guizard, "Comparison between plasma properties and damage thresholds in doped silica exposed to IR femtosecond laser," J. Laser Micro Nanoen 7, 217-225 (2012).

202. F. Zimmermann, M. Lancry, A. Plech, S. Richter, T. Ullsperger, B. Poumellec, A. Tünnermann, and S. Nolte, "Ultrashort pulse laser processing of silica at high repetition rates - from network change to residual strain," Int J Appl Glass Sci Accepted(2016).

203. M. Dussauze, E. Fargin, A. Malakho, V. Rodriguez, T. Buffeteau, and F. Adamietz, "Correlation of large SHG responses with structural characterization in borophosphate niobium glasses," Opt. Mater 28, 1417-1422 (2006). 
204.

"Microscope

configuration,"

http://www.olympusmicro.com/primer/techniques/polarized/configuration.html, accessed 10 September 2016.

205. "Birefringence

crystals

between

crossed

polarizers

http://nikon.magnet.fsu.edu/print/articles/polarized/birefringence-print.html, accessed 10 September 2016.

"The first order (full wave) retardation plate," http://www.olympusmicro.com/primer/techniques/polarized/firstorderplate.html, $\quad$ accessed 10 September 2016.

207. M. Shribak and R. Oldenbourg, "Techniques for fast and sensitive measurements of two-dimensional birefringence distributions," Appl. Opt. 42, 3009-3017 (2003).

208. R. Salh, Defect related luminescence in silicon dioxide network: a review (INTECH Open Access Publisher, 2011).

209. J. Goldstein, D. E. Newbury, D. C. Joy, C. E. Lyman, P. Echlin, E. Lifshin, L. Sawyer, and J. R. Michael, Scanning electron microscopy and X-ray microanalysis (Springer US, 2013).

210. "How EBSD measurements work," http://www.gm.univ-montp2.fr/spip.php?article104\&lang=fr, accessed 07 September 2016.

211. O. Engler and V. Randle, Introduction to texture analysis: macrotexture, microtexture, and orientation mapping (CRC press, 2009).

212. L. Brewer and J. Michael, "Risks of 'cleaning'electron backscatter diffraction data," Microsc. Today 18, 10-15 (2010).

213. S. I. Wright and M. M. Nowell, "EBSD image quality mapping," Microsc. Microanal. 12, $72-84$ (2006).

214. R. Petrov, L. Kestens, A. Wasilkowska, and Y. Houbaert, "Microstructure and texture of a lightly deformed TRIP-assisted steel characterized by means of the EBSD technique," Mater. Sci. Eng., A 447, 285-297 (2007).

215. "Electron microscopy," http://www.microscopy.ethz.ch/TEM.htm, accessed 08 September 2016.

216. "The transmission microscope," http://www.nobelprize.org/educational/physics/microscopes/tem/, accessed 10 September 2016.

217. "Transmission electron microscopy," http://labs.mete.metu.edu.tr/tem/TEMtext/TEMtext.html, accessed 08 September 2016.

218. C.-S. Kim, S.-H. Ahn, and D.-Y. Jang, "Review: Developments in micro/nanoscale fabrication by focused ion beams," Vacuum 86, 1014-1035 (2012).

219. R. Wirth, "Focused ion beam (FIB) combined with SEM and TEM: Advanced analytical tools for studies of chemical composition, microstructure and crystal structure in geomaterials on a nanometre scale," Chem. Geol. 261, 217-229 (2009).

220. A.-M. Flank, G. Cauchon, P. Lagarde, S. Bac, M. Janousch, R. Wetter, J.-M. Dubuisson, M. Idir, F. Langlois, and T. Moreno, "LUCIA, a microfocus soft XAS beamline," Nucl. Instr. Meth. Phys. Res. B 246, 269-274 (2006). 
221. D. R. Neuville, L. Cormier, and D. Caurant, Du verre au cristal: Nucléation, croissance et démixtion, de la recherche aux applications (EDP Sciences, 2013).

222. B. Fultz and J. M. Howe, Transmission electron microscopy and diffractometry of materials (Springer Science \& Business Media, 2012).

223. P. W. Hawkes and J. C. Spence, Science of microscopy (Wiley Online Library, 2007), Vol. 1.

224. Y. Llabador and P. Moretto, Applications of nuclear microprobe in the life sciences: An efficient analytical technique for the research in biology and medicine (World Scientific, 1998).

225. A. W. Miziolek, V. Palleschi, and I. Schechter, Laser induced breakdown spectroscopy (Cambridge University Press, 2006).

226. "What is LIBS?," http://www1.uwindsor.ca/people/rehse/15/what-is-libs, accessed 24 September 2016.

227. D. W. Hahn and N. Omenetto, "Laser-induced breakdown spectroscopy (LIBS), part II: review of instrumental and methodological approaches to material analysis and applications to different fields," Appl. Spectrosc. 66, 347-419 (2012).

228. B. Poumellec, M. Lancry, R. Desmarchelier, E. Hervé, F. Brisset, and J. Poulin, "Asymmetric orientational writing in glass with femtosecond laser irradiation," Opt. Mater. Express 3, 1586-1599 (2013).

229. I. Nakai, J. Akimoto, M. Imafuku, R. Miyawaki, Y. Sugitani, and K. Koto, "Characterization of the amorphous state in metamict silicates and niobates by EXAFS and XANES analyses," Phys. Chem. Miner. 15, 113-124 (1987).

230. C. Sugiura, M. Kitamura, and S. Muramatsu, "Niobium $L_{|| l}$ and $L_{||} X$-ray absorption-edge spectra of $\mathrm{Nb}_{2} \mathrm{O}_{5}$ and $\mathrm{NH}_{4} \mathrm{NbF}_{6}, "$ J. Phys. Chem. Solids 49, 1095-1099 (1988).

231. T. Vitova, "X-ray absorption spectroscopy investigation of structurally modified lithium niobate crystals," Doctoral dissertation (Universität Bonn, 2008).

232. D.-L. Zhang, J. Gao, P.-R. Hua, D.-Y. Yu, and E. Y.-B. Pun, "Appropriate solvent $\mathrm{NaVO}_{3}$ for composition analysis of $\mathrm{LiNbO}_{3}$ crystal using chemical method," Anal. Chem. 85, 1940-1944 (2013).

233. H. C. Zeng, K. Tanaka, K. Hirao, and N. Soga, "Crystallization and glass formation in $50 \mathrm{Li}_{2} \mathrm{O} \cdot 50 \mathrm{Nb}_{2} \mathrm{O}_{5} \mathrm{and}$ $25 \mathrm{Li}_{2} \mathrm{O} \cdot 25 \mathrm{Nb}_{2} \mathrm{O}_{5} \cdot 50 \mathrm{SiO}_{2}, "$ J. Non-Cryst. Solids 209, 112-121 (1997).

234. H. Vigouroux, "Etude de vitrocéramiques optiques pour le doublement de fréquence," Doctoral dissertation (Université Sciences et Technologies-Bordeaux I, 2012).

235. M. Beresna, M. Gecevičius, P. G. Kazansky, T. Taylor, and A. V. Kavokin, "Exciton mediated selforganization in glass driven by ultrashort light pulses," Appl. Phys. Lett. 101, 053120 (2012).

236. M. Beresna, M. Gecevičius, and P. G. Kazansky, "Polarization sensitive elements fabricated by femtosecond laser nanostructuring of glass [Invited]," Opt Mater Express 1, 783-795 (2011).

237. J. Wen, G.-D. Peng, W. Luo, Z. Xiao, Z. Chen, and T. Wang, "Gamma irradiation effect on Rayleigh scattering in low water peak single-mode optical fibers," Opt. Express 19, 23271-23278 (2011). 
238. S. Loranger, M. Gagné, V. Lambin-lezzi, and R. Kashyap, "Rayleigh scatter based order of magnitude increase in distributed temperature and strain sensing by simple UV exposure of optical fibre," Sci Rep 5 (2015).

239. M. Lines, "Scattering losses in optic fiber materials. I. A new parametrization," J. Appl. Phys 55, 40524057 (1984).

240. Y. Liao, W. Pan, Y. Cui, L. Qiao, Y. Bellouard, K. Sugioka, and Y. Cheng, "Formation of in-volume nanogratings with sub-100-nm periods in glass by femtosecond laser irradiation," Opt. Lett. 40, 36233626 (2015).

241. A. Rudenko, J.-P. Colombier, and T. E. Itina, "From random inhomogeneities to periodic nanostructures induced in bulk silica by ultrashort laser," Phys. Rev. B 93, 075427 (2016).

242. "Refractive index database," http://refractiveindex.info/?shelf=glass\&book=SCHOTT-BK\&page=N-BK7, accessed 07 June 2016.

243. A. Revcolevschi and G. Dhalenne, "Engineering oxide-oxide and metal-oxide microstructures in directionally solidified eutectics," Adv. Mater. 5, 657-662 (1993).

244. B. Poumellec, G. Dhalenne, M. Lancry, R. Saint Martin, and S. Ani-Joseph, Elaboration of a Specific Class of Metamaterial: Glass in Single Crystal (INTECH Open Access Publisher, 2012).

245. X. Ma and D. Li, "The multi-scale discrete crystal growth in the solidification of 20SiMnMo5 Steel," Cryst. Growth Des. 16, 3163-3169 (2016).

246. W. Yang, P. G. Kazansky, and Y. P. Svirko, "Non-reciprocal ultrafast laser writing," Nat Photon 2, 99-104 (2008).

247. M. Born and E. Wolf, Principles of optics: electromagnetic theory of propagation, interference and diffraction of light (CUP Archive, 2000).

248. I. H. Malitson, "Interspecimen comparison of the refractive index of fused silica*,†," J. Opt. Soc. Am. 55, 1205-1209 (1965).

249. E. Simova, C. Hnatovsky, R. S. Taylor, J. Liu, R. Pattathil, R. Bhardwaj, D. M. Rayner, and P. B. Corkum, "Rewritable nanogratings in fused silica using a focused femtosecond laser beam," in Conference on Lasers and Electro-Optics, (Optical Society of America, 2006), CMX4.

250. M. Rose, "A history of the laser: A trip through the light fantastic-Presenting a timeline of notable laser-related scientific accomplishments," Photonics Spectra 44, 58 (2010).

251. J. Hecht, "Short history of laser development," Opt. Eng 49, $091002-091002$ (2010).

252. R. Paschotta, " 'chirp' in the encyclopedia of laser physics and technology," Wiley-VCH, 15 September 2016 (2008).

253. K. L. Vora, "Three-dimensional nanofabrication of silver structures in polymer with direct laser writing," Doctoral dissertation (Harvard University, 2014).

254. L. Skuja, "Optically active oxygen-deficiency-related centers in amorphous silicon dioxide," J. NonCryst. Solids 239, 16-48 (1998). 
255. A. Zoubir, M. Richardson, L. Canioni, A. Brocas, and L. Sarger, "Optical properties of infrared femtosecond laser-modified fused silica and application to waveguide fabrication," J. Opt. Soc. Am. B 22, 2138-2143 (2005).

256. A. Zoubir, C. Rivero, R. Grodsky, K. Richardson, M. Richardson, T. Cardinal, and M. Couzi, "Laserinduced defects in fused silica by femtosecond IR irradiation," Phys. Rev. B 73, 224117 (2006).

257. A. Podlipensky, V. Grebenev, G. Seifert, and H. Graener, "Ionization and photomodification of Ag nanoparticles in soda-lime glass by 150 fs laser irradiation: a luminescence study," J. Lumin. 109, 135142 (2004).

258. R. Buividas, I. Aharonovich, G. Seniutinas, X. W. Wang, L. Rapp, A. V. Rode, T. Taniguchi, and S. Juodkazis, "Photoluminescence from voids created by femtosecond-laser pulses inside cubic-BN," Opt. Lett 40, 5711-5713 (2015).

259. C. Zhang, Z. Dong, G. You, R. Zhu, S. Qian, H. Deng, H. Cheng, and J. Wang, "Femtosecond pulse excited two-photon photoluminescence and second harmonic generation in ZnO nanowires," Appl. Phys. Lett. 89, 42117-42500 (2006).

260. Y. Duan, H. Pfeiffer, B. Li, I. C. Romero-Ibarra, D. C. Sorescu, D. R. Luebke, and J. W. Halley, "CO capture properties of lithium silicates with different ratios of $\mathrm{Li}_{2} \mathrm{O} / \mathrm{SiO}_{2}$ : an ab initio thermodynamic and experimental approach," Phys. Chem. Chem. Phys. 15, 13538-13558 (2013).

261. M. Tatsumisago, T. Minami, and M. Tanaka, "Rapid quenching technique using thermal-image furnace for glass preparation," J. Am. Ceram. Soc 64, C97-C98 (1981).

262. P. Sarjeant and R. Roy, "A new approach to the prediction of glass formation," Mater. Res. Bull. 3, 265279 (1968).

263. T. Suzuki and A.-M. Anthony, "Rapid quenching on the binary systems of high temperature oxides," Mater. Res. Bull. 9, 745-753 (1974).

264. "Seven crystal classes," http://encyclopedia2.thefreedictionary.com/crystallography, accessed 22 September 2016.

265.
"Michel-Levy
birefringence
chart," http://www.olympusmicro.com/primer/techniques/polarized/michel.html, accessed 12 September 2016. 


\section{Academic activity}

1) The European Conference on Lasers and Electro-Optics and the European Quantum Electronics Conference (CLEO ${ }^{\circledR} /$ Europe-EQEC), June 25-29, 2017, Munich, Germany (scheduled)

2) $\quad 2^{\text {nd }}$ Teli Forum, December 26-29, 2016, Beijing, China (full grant, oral presentation)

3) Les Journées "Verre" auront lieu à Bordeaux, November 17-18, 2016, Bordeaux, France (poster)

4) Invited talk in Wuhan University, November 10, 2016, Wuhan, China

5) $4^{\text {th }}$ International Symposium on Laser interaction with Matter, November 06-09, 2016, Chengdu, China (excellent presentation award)

6) High Level Zijin Forum, November 03-04, 2016, Nanjing, China (full grant, oral presentation)

7) International School on Light Sciences and Technology, IS-LiST, June 20-24, 2016, Santander, Spain (full grant)

8) Stanford PULSE Ultrafast X-ray Summer Seminar, June 12-16, 2016, SLAC national accelerator laboratory, Menlo Park, California, USA (travel grant from DOE)

9) Workshop on Materials for Optics and Optoelectronics, June 08-09, 2016, Gif sur Yvette, France (oral presentation)

10) Première journée de L'Ecole Doctorale 2 MIB de I'Université Paris-Saclay, November 24, 2015, Ecole Polytechnique, France (oral presentation)

11) $14^{\text {th }}$ International Conference on the Physics of Non-Crystalline Solids, September 20-25, 2015, Niagara Falls, NY, USA (oral presentation)

12) Advanced School on Glasses and Glass-Ceramics, 100 positions available for top-quality Masters and PhD students (50 Brazilians and 50 foreigner from all over the world), São Carlos, São Paulo, Brazil, August 1-9, 2015 (full grant)

13) $6^{\text {th }}$ Workshop for New Research in Glass Science and Technology, Montpellier, France, July 07-11, 2014 
Titre : Création et orientation de nano-cristaux par irradiation laser femtoseconde pour le contrôle de l'orientation des propriétés optiques non-linéaires dans des verres à base de silice

Mots clés : verre céramique; niobate de lithium; technique en trois dimensions écriture directe; processus ultrarapides; linéarités optiques de la matière condensée

Résumé : En raison d'un désordre aléatoire à longue distance, un verre présente une symétrie d'inversion telle que la génération de seconde harmonique (GSH) est interdite. Cependant, par irradiation avec un laser femtoseconde (fs) très focalisé, il est possible de précipiter des cristaux optiquement non linéaires, et de rompre la symétrie d'inversion et donc d'induire une GSH. De plus, ceci peut être réalisé localement en trois dimensions.

Pour la démonstration, on a appliqué, dans le système vitreux $\mathrm{Li}_{2} \mathrm{O}-\mathrm{Nb}_{2} \mathrm{O}_{5}-\mathrm{SiO}_{2}$ le mode opératoire décrit cidessous qui permet la formation de cristaux de $\mathrm{LiNbO}_{3}$, hautement optiquement non linéaire. La procédure est la suivante: 1) ajustement de la composition chimique du verre pour obtenir un verre suffisamment sensible au laser fs; 2 ) contrôle des paramètres du laser (durée d'impulsion, fréquence de répétition des impulsions, vitesse de balayage du faisceau, énergie d'impulsion...) pour obtenir des nanocristaux avec répartition spatiale et taille correcte. En outre, la taille de la zone affectée doit être limitée; 3) contrôle de l'orientation des nanocristaux. On montre qu'il est possible de satisfaire à cette condition, en contrôlant l'orientation de

la polarisation du laser. Ceci a été montré par la méthode de rétrodiffusion d'électrons de diffraction (EBSD). En d'autres termes, ce processus peut être contrôlé directement avec la lumière.

En outre, la spectroscopie par rayons $X$ à dispersion d'énergie couplée à la microscopie à transmission électronique à balayage (STEM /EDS) et la microscopie électronique à transmission a révélé une microstructure orientable similaire à celle appelée nanoréseaux formée dans silice. L'originalité est que les nanocristaux optiques non linéaires texturées noyées dans un réseau de "murs" vitreux, sont alignés perpendiculairement à la direction de polarisation du laser. Il en résulte que la biréfringence et la propriété optique non linéaire peuvent être maîtrisées ensemble. Ceci est une percée dans ce travail de thèse. Ces résultats mettent en évidence des modifications spectaculaires de verre par rayonnement laser fs. Avec de nouvelles améliorations dans les techniques de fabrication, l'application de ce travail est de parvenir à réaliser un guide d'ondes biréfringent doubleur ou changeur de fréquences.

Title : Creation and orientation of nano-crystals by femtosecond laser light for controlling optical non-linear response in silica-based glasses

Keywords : glass ceramic; Lithium niobate; three-dimensional direct writing technique; Ultra-fast processes; optical nonlinearities of condensed matter

Abstract : Due to random disorder, a glass exhibits inversion symmetry such that second harmonic generation (SHG) is forbidden. However, by irradiation with a tightly focused femtosecond (fs) laser, it is possible to induce nonlinear optical crystal precipitation, in order to break the inversion symmetry and thus to induce SHG. Moreover, this can be achieved locally in three dimensions.

For demonstration, we applied the procedure described below in the glass system $\mathrm{Li}_{2} \mathrm{O}-\mathrm{Nb}_{2} \mathrm{O}_{5}-\mathrm{SiO}_{2}$ that allows the formation of $\mathrm{LiNbO}_{3}$ crystal, a highly nonlinear optical one. The procedure is thus the following: 1) adjustment of the glass chemical composition for obtaining a glass sensitive enough to fs laser; 2) control of the laser parameters (pulse duration, pulse repetition rate, speed of beam scanning, pulse energy...) for obtaining nano-crystals with correct space distribution and size. In addition, the size of the affected zone has to be limited; 3) control of the orientation of the nano-crystals. We show that it is possible to fulfill this condition by

controlling the laser polarization orientation. This has been achieved by electron backscatter diffraction method (EBSD). In other words, this process can be controlled with light directly.

In addition, energy dispersive X-ray spectroscopy coupled to scanning transmission electron microscopy (STEM/EDS) and transmission electron microscopy revealed an orientable microstructure similar to the one called nanogratings form in silica. The originality here is a textured nonlinear optical nano-crystals embedded in a network of "walls" made of vitreous phase, aligned perpendicular to the laser polarization direction. It results that birefringence and nonlinear optical property can be mastered in the same time. This is a highly valuable aspect of the work.

These findings highlight spectacular modifications of glass by fs laser radiation. With further improvements in the fabrication techniques, the application of this work is to achieve SHG waveguide and birefringencebased devices. 